NIST NCSTAR 1-6D

Federal Building and Fire Safety Investigation of the World Trade Center Disaster

\title{
Global Structural Analysis of the Response of the World Trade Center Towers to Impact Damage and Fire (Chapter 4 - Appendix C)
}

Mehdi S. Zarghamee

Yasuo Kitane

Ömer O. Erbay

Therese P. McAllister

John L. Gross

NSTational Institute of Standards and Technology - Technology Administration • U.S. Department of Cammerce 



\section{Chapter 4 \\ GLOBAL ANALYSIS}

\subsection{CONVERSION FROM SAP2000 TO ANSYS}

Simpson, Gumpertz \& Heger, Inc. (SGH) performed global analysis of the WTC 1 and WTC 2 towers using global models of the two towers developed in ANSYS as described in Chapter 2. The models were subjected to the aircraft impact damage and the temperature time histories resulting from the ensuing fire environments. The global models described in this report, and the reference models in NIST NCSTAR 1 2A, used the same coordinate system. As illustrated in Fig. 4-1, the z-axis in this coordinate system is parallel to the vertical axis of the building, the $\mathrm{x}$ axis is parallel to the long direction of the building's central core, and the $y$-axis is parallel to the short direction of the central core. In the WTC 1 models, the origin of the coordinate system is located at the southeast corner of the building with the y-axis extending to the north. In the WTC 2 models, the origin is located at the northeast corner of the building with the yaxis extending west.
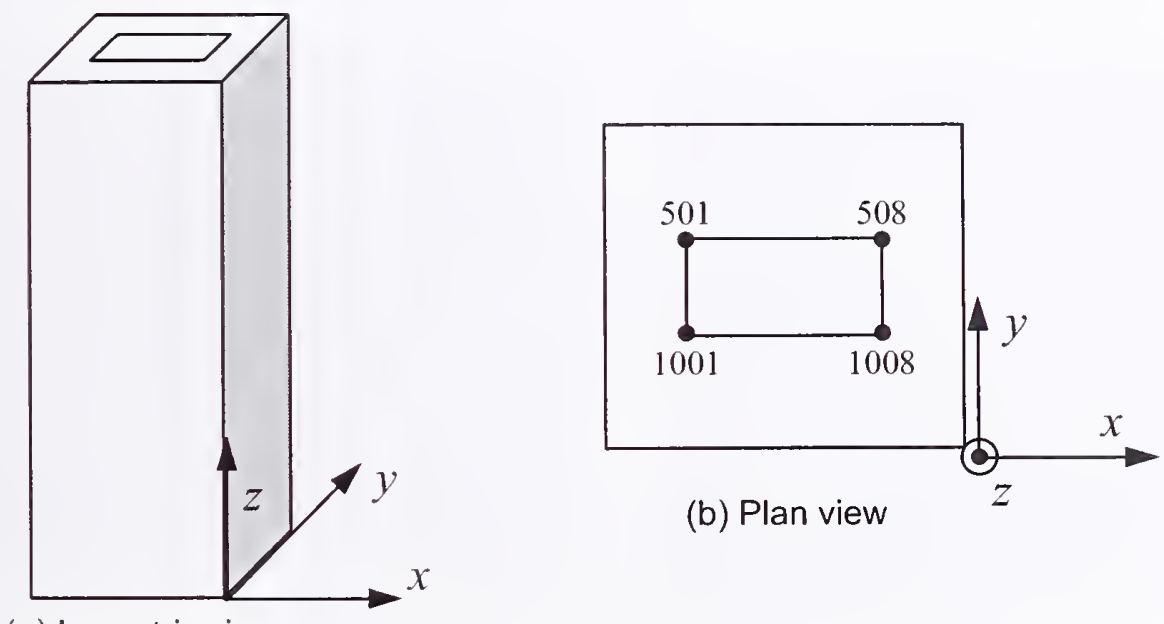

(a) Isometric view

(b) Plan view

Figure 4-1. Coordinate system used in the analysis models.

The SAP2000 global models in NIST NCSTAR 1-2A were linearly elastic and included interior and exterior columns, spandrels, hat truss members, and floor slabs at mechanical floors. Only the mechanical floors were individually modeled. The other floor slabs were modeled using a set of coupling equations in the software to slave the displacement of certain nodes to the displacement of other nodes. In this case, these coupling equations provided full rigidity within the plane of the floor slabs (the $x-y$ plane) and full flexibility out of this plane.

As most of the structural action that led to the collapse of the buildings took place within and above the aircraft-impacted floors, the reference models were truncated for each building below the lowest damaged floors. The model of WTC 1 was truncated at Floor 89, five floors below the zone of impact, and a series of equivalent vertical linear springs were introduced at the base of this truncated model to represent the 
stiffness of the interior eolumns and exterior walls beneath the level of truncation. Similarly, the model of WTC 2 was truneated at Floor 73.

The truncated SAP2000 models were converted to ANSYS and modified to capture the failure modes of floors and eolumns and to enhanee numerical effieieney. The global models included geometric and temperature-dependent material nonlinearities, ineluding creep.

\subsubsection{ANSYS Models}

The ANSYS models were the direet translation of the truncated SAP2000 models. During the translation of the models, the coordinates of the nodes, cross sectional properties of members, including orientation and offset of the cross sections, nodal loads, material properties, and member end releases were automatically converted from eaeh SAP2000 database into an ANSYS database. Table 4-1 summarizes the conversion used in translating the elcment types between SAP2000 and ANSYS. Comparison of the results obtained for the gravity analysis of WTC 1 and WTC 2 from the SAP2000 models and translated ANSYS models are discussed in the next section.

Table 4-1 summarizes the correspondence between element types containd in the SAP2000 model and those in the converted ANSYS model. Table 2-1 previously presented a description of the various ANSYS elements used in the global models. Gravity load analyses of the two buildings were then performed using both the truneated SAP2000 and eonverted ANSYS models to vcrify the fidelity of the conversion.

Table 4-1. Conversion from SAP2000 element types to ANSYS element types.

\begin{tabular}{|l|l|}
\hline \multicolumn{1}{|c|}{ SAP2000 Element Types } & \multicolumn{1}{c|}{ ANSYS Element Types } \\
\hline $\begin{array}{l}\text { Frame/Cable elements } \\
\text { Shell elements } \\
\text { Springs }\end{array}$ & BEAM188/189 later changed to BEAM24 \\
SHELL63 later changed to SHELL181 \\
\hline
\end{tabular}

Following these analyses, the linear, elastic material properties of the converted ANSYS models were replaced with temperature-dependent inelastic material properties as defined for all material types in Chapter 3 of NIST NCSTAR 1-6C. These material types were assigned to the elements according to their material and cross sectional properties and their locations in the buildings. In the SAP2000 global model, each eross seetion was assigned a yield value represcnting the material capacity for that eross section. During the translation to ANSYS, a different name was given to each cross section and material combination, which resulted in a unique material index. Using this material index, the material propcrties of all elements were replaced with temperature-dependent inelastic material properties. In the aetual buildings, the rectangular tube columns in the exterior wall were typically fabricated from four plates, Plate 1 (see Chapter 6 of NIST NCSTAR 1-6C for more information) and eould have a different specified yield strength than the rest (Plates 2 and 3). Examination of drawings in the region of interest showed that all these plates had the same yield strength. Therefore, in the translation process, all plates in the same column cross seetion were considered to be of the same material.

The ANSYS models were also modified to include representation of the floor slabs, which except at mechanical floors, were not ineluded in the SAP2000 models. Floor elements added into the ANSYS model included the core slabs, those eore beams that were framed with moment connections at their ends, 
and the office area slabs. Figure 4-2 shows the analytical representation of the core and office area floors and the core beams included in the models. Beams in the core that were framed without moment connections were not included in the model because they cannot transfer shear between columns without significant relative displacement. However, their axial stiffness was combined with in-plane stiffness of the floor slab, and then shell elements with this composite in-plane stiffness were used to model the floor.

An equivalent concrete thickness and modulus was calculated for the office area floors to match the inplane stiffness of the composite floor system which included the concrete slab, the floor trusses, and the floor seats. The thickness of the core slab was taken from the PANYNJ drawings, but the elastic modulus was adjusted to match the in-plane stiffness of the composite floor that included the steel beams and the concrete slab. Both core and office area floor slabs were modeled with linear-elastic material properties for lightweight concrete. Later analyses with these models indicated that these slabs, composite with their framing members, could redistribute load locally amongst neighboring columns and transfer lateral loads to the exterior walls as collapse initiated and WTC 2 began to tilt.

Floors in the global models also provided diaphragm stiffness at each level. However, the floors were not modeled with sufficient detail to capture such floor behaviors under elevated temperatures as sagging and failure of floor-wall connections. Instead, these effects were incorporated into the global analyses as fireinduced damage, as described in Section 2.5 .

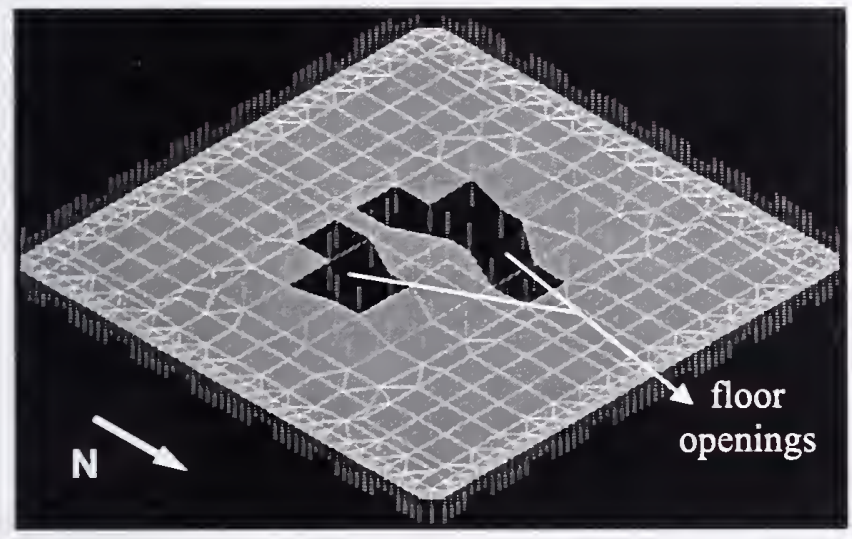

(a) Office and core area floor

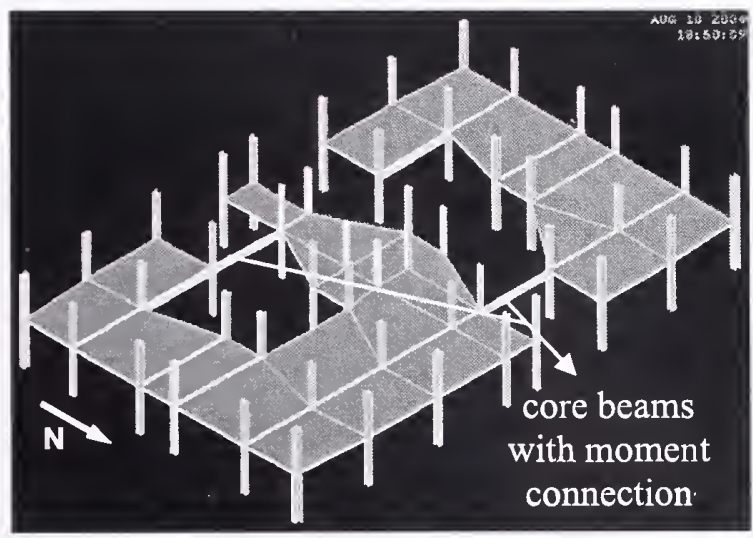

(b) Core area floor and core beams

Figure 4-2. Office and core area floors and core beams. 


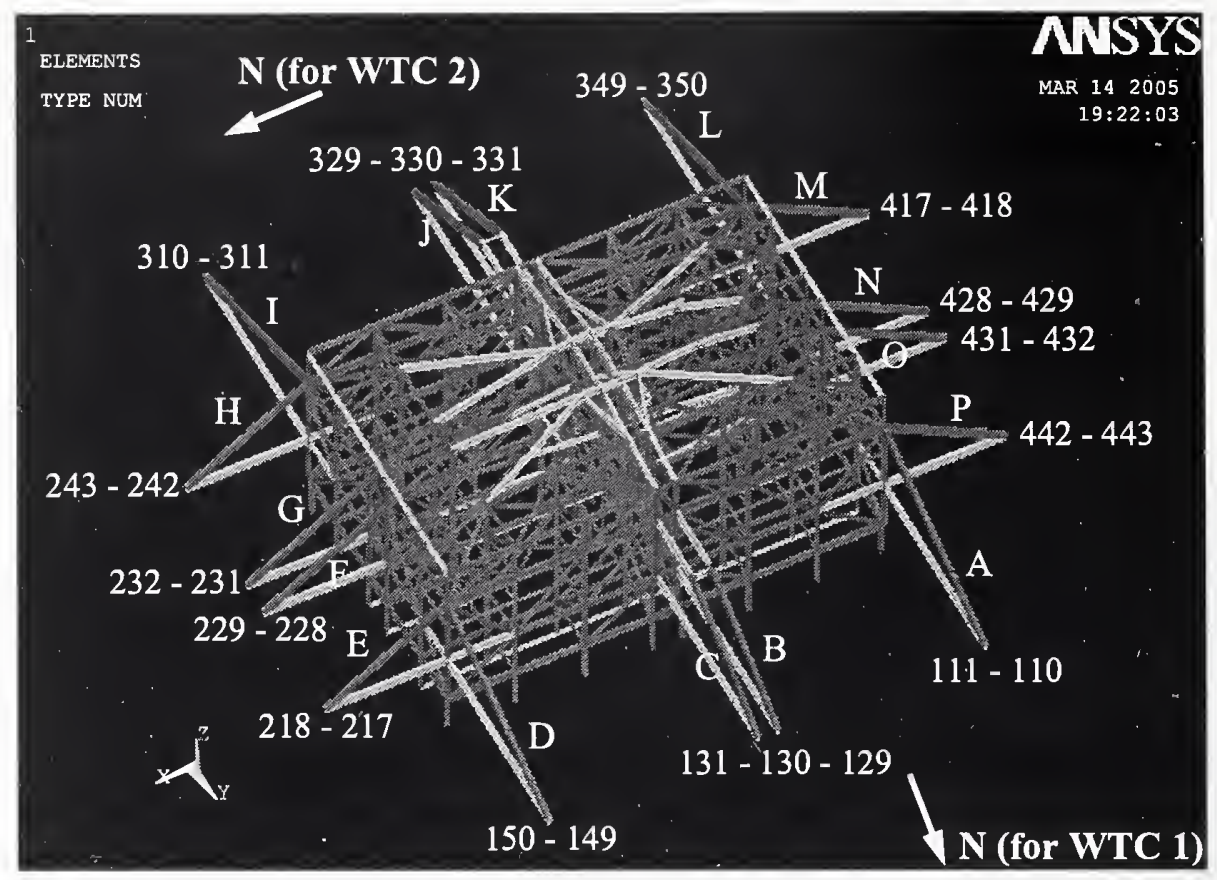

Figure 4-3. Location and IDs of outriggers and supporting columns.

Due to the limitations of the BEAM188 element used to model spandrels, columns, and truss members, a set of preliminary global analyses were conducted without including creep and inelastic buckling in the core and exterior wall columns, as discussed in Appendix C of this report. Plasticity and large deflection were the only nonlinearities included in those analyses. Later, the BEAM188 element was replaced with the BEAM24 element to eliminate the limitations on creep and inelastic buckling. As shown in the global analyses that included consideration of creep and buckling, described in Section 4.2, creep strains and inelastic column buckling played a very significant role in the collapse of the towers.

Figure 4-3 shows the hat trusses in the global models. The hat trusses connected the exterior walls and the core columns. As seen in the figure, four outrigger members on each face of the core extended outward from the core to the exterior walls. Hat truss members were provided with plasticity in the preliminary global models without creep and in the final WTC 1 global model with creep. The hat truss of the WTC 2 global model with creep was part of the "superstructure" where elastic properties were used. Further discussion of this is presented in Section 4.2.2. The hat trusses were not modeled with sufficient detail to capture the onset of buckling. However, the yield strength of the outrigger elements in the final WTC 1 model was set so that compressive yielding would occur when axial load reached the compressive capacity of the members.

\subsubsection{Validation of Translated ANSYS Global Models}

In order to verify the accuracy of the conversion of the building models from SAP2000 to ANSYS, prior to adding material nonlinearities and elements representing the floor slabs to the ANSYS models, the translated ANSYS models were subjected to gravity dead and live loads, and the ANSYS results were compared to the results of SAP2000 global models. The comparison included the calculated overall displaced shapes, the maximum displacements, vertical base reactions at each construction stage, and element forces for a set of randomly selected members from different parts of the buildings. 
The gravity analysis was performed in three stages to simulate construction sequencing in the actual erection of the buildings. In Stage 1 the portion of the towers up to and including Floor 106 was analyzed under self-weight. In Stage 2 the members above Floor 106 were added, and the analysis was performed for the effects of dead loads of the newly added members. In Stage 3, the supcrimposed dead load and 25 percent of the design live load was added at each floor level to obtain an estimate of the deformations and stresses in each building under normal occupancy conditions.

Figure 4-4 shows the deformed shape calculated for WTC 1 by the ANSYS model and the truncated SAP 2000 model, following Stage 3 analysis. Figure 4-5 provides a similar comparison for WTC 2.

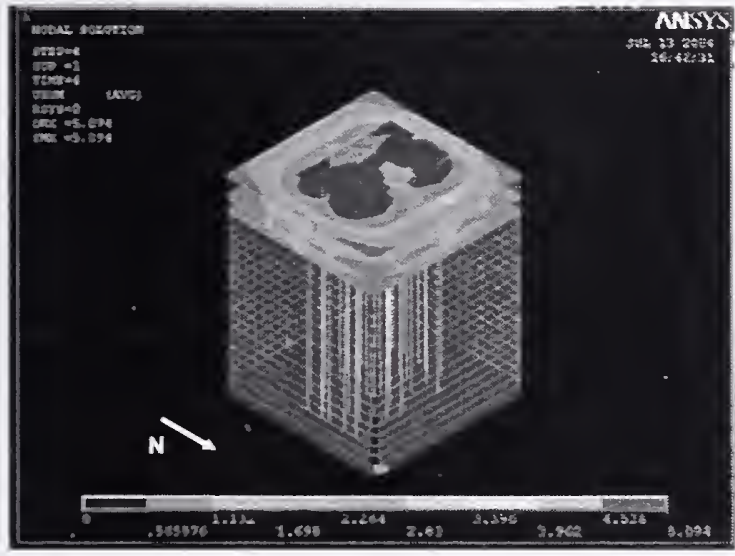

(a) WTC 1 ANSYS Model at Stage 3

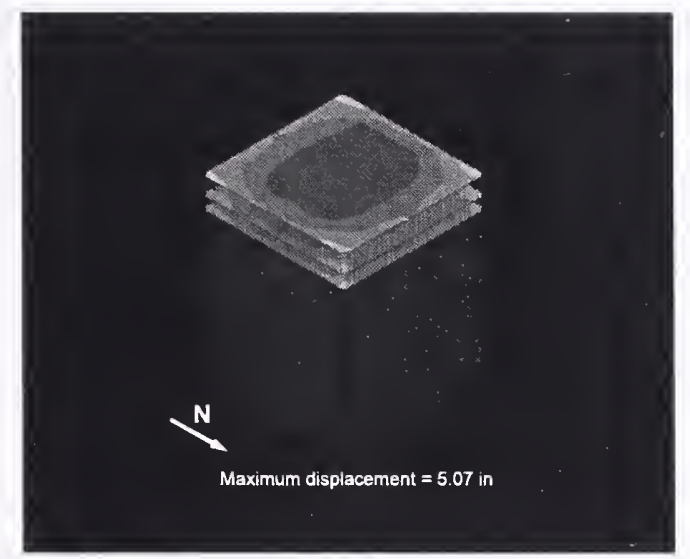

(b) WTC 1 SAP2000 Model at Stage 3 (Displacement is only shown on shell elements.)

Figure 4-4. Displaced shape of WTC 1 at the end of gravity analysis.

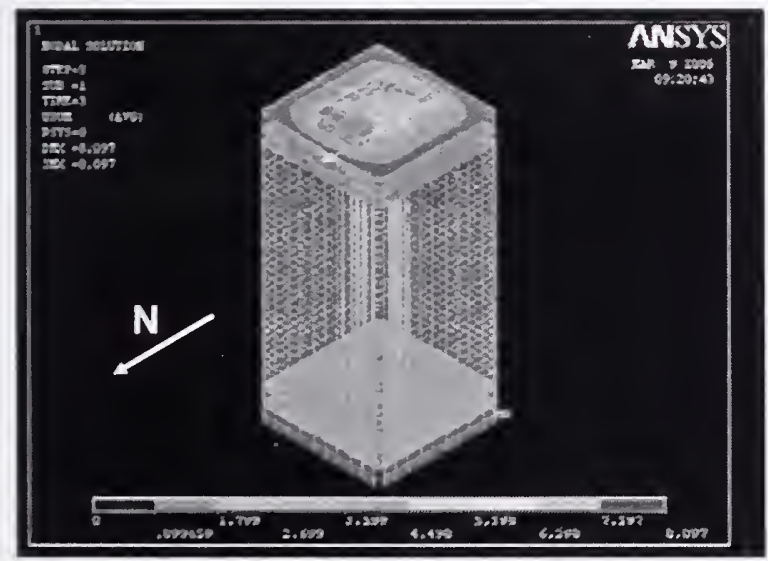

(a) WTC 2 ANSYS Model at Stage 3

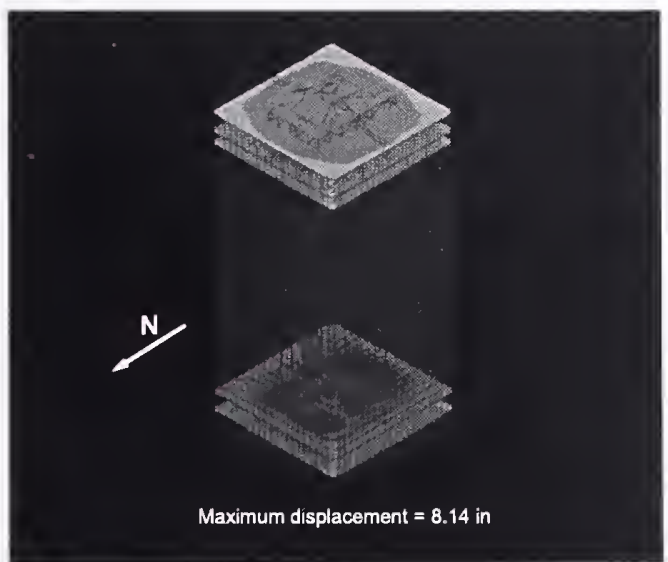

(b) WTC 2 SAP2000 Model at Stage 3 (Displacement is only shown on shell elements.)

\section{Figure 4-5. Displaced shape of WTC 2 at the end of gravity analysis.}

Table 4-2 summarizes the maximum displacement and vertical reactions at the base for all stages of the gravity analysis of WTC 1, and Table 4-3 presents similar data for WTC 2. As can be seen, the deformed shapes as well as the maximum displacements and vertical reactions obtained from the analyses performed with the ANSYS models agree well with the results of the similar analyses performed with 
SAP2000. The maximum difference in displacements between the two models was less than 1.4 percent for WTC 1 and 0.7 percent for WTC 2. The maximum difference between base reactions predicted by the two analyses was 1.2 percent for WTC 1 and 0.3 percent for WTC 2 .

Table 4-4 presents a comparison of the axial forces computed from the ANSYS and SAP2000 analyses for a randomly selected set of elements from different parts of WTC 1. Table 4-5 presents similar data for WTC 2. The agreement between the results obtained from the ANSYS and SAP2000 analyses is quite good.

Table 4-2. Comparison of maximum displacements and base reactions of WTC 1 from translated ANSYS and SAP2000 models.

\begin{tabular}{|c|c|c|c|c|c|c|}
\hline \multirow{2}{*}{ Stage } & \multicolumn{2}{|c|}{ Maximum Vertical Displacement (in.) } & \multicolumn{3}{c|}{ Base Reaction Sum (kip) } \\
\cline { 2 - 7 } & ANSYS & SAP2000 & $\begin{array}{c}\text { Percent } \\
\text { Difference }\end{array}$ & ANSYS & SAP2000 & $\begin{array}{c}\text { Percent } \\
\text { Difference }\end{array}$ \\
\hline 1 & -2.87 & -2.83 & 1.4 & 55,600 & 54,940 & 1.2 \\
\hline 2 & -4.76 & -4.74 & 0.4 & 98,470 & 97.850 & 0.6 \\
\hline 3 & -5.09 & -5.07 & 0.4 & 107.040 & 106,450 & 0.6 \\
\hline
\end{tabular}

Table 4-3. Comparison of maximum displacements and base reactions of WTC 2 obtained from translated ANSYS and SAP2000 models.

\begin{tabular}{|c|c|c|c|c|c|c|}
\hline \multirow{2}{*}{ Stage } & \multicolumn{2}{|c|}{ Maximum Vertical Displacement (in.) } & \multicolumn{3}{c|}{ Base Reaction Sum (kip) } \\
\cline { 2 - 7 } & ANSYS & SAP2000 & $\begin{array}{c}\text { Percent } \\
\text { Difference }\end{array}$ & ANSYS & SAP2000 & $\begin{array}{c}\text { Percent } \\
\text { Difference }\end{array}$ \\
\hline 1 & -5.87 & -5.91 & -0.7 & 125,050 & 124,680 & 0.3 \\
\hline 2 & -7.67 & -7.71 & -0.5 & 166,950 & 166,980 & -0.02 \\
\hline 3 & -8.10 & -8.14 & -0.5 & 180,250 & 180,360 & -0.06 \\
\hline
\end{tabular}

Table 4-4. Comparison of axial forces in randomly selected elements from WTC 1 model at the end of gravity analysis.

\begin{tabular}{|l|c|c|}
\hline \multirow{2}{*}{ Location and Type of Selected Element } & \multicolumn{2}{c|}{ Axial Force (kip) } \\
\cline { 2 - 3 } & ANSYS & SAP2000 \\
\hline Exterior Column 302 at Floor 104 & -77 & -69 \\
Spandrel between Columns 124 and 125 at Floor 102 & 0 & 0 \\
Outrigger member between at Floor 110 & -39 & -48 \\
Vertical hat truss member at 1005 core column line at Floor 109 & -74 & -91 \\
Horizontal hat truss member at Floor 107 & 21 & 19 \\
Horizontal hat truss member at Floor 108 & 170 & 150 \\
Core Column 602 at Floor 97 & -738 & -745 \\
Core Column 501 at Floor 93 & $-2,180$ & $-2,190$ \\
Core Column 1001 at Floor 89 & $-2,570$ & $-2,590$ \\
Spandrel between Columns 339 and 340 at Floor 100 & 0 & 0 \\
\hline
\end{tabular}


Table 4-5. Comparison of axial forces in randomly selected elements from WTC 2 model at the end of gravity analysis.

\begin{tabular}{|l|c|c|}
\hline \multirow{2}{*}{\multicolumn{1}{|c|}{ Location and Type of Selected Element }} & \multicolumn{2}{c|}{ Axial Force (kip) } \\
\cline { 2 - 3 } & ANSYS & SAP2000 \\
\hline Core beam at Floor 107 & 11 & 6 \\
Inclined truss member at hat truss at Floor 107 & -34 & -4 \\
Inclined truss member at hat truss at Floor 108 & 36 & -670 \\
Inclined truss member at hat truss at Floor 108 & -580 & 1,940 \\
Core Column 502 at Floor 87 & $-1,930$ & $-3,290$ \\
Core Column 1001 at Floor 82 & $-3,270$ & $-1,920$ \\
Core Column 1002 at Floor 87 & $-1,910$ & 3,520 \\
Core Column 1008 at Floor 82 & $-3,400$ & -608 \\
Core Column 1003 at Floor 107 & -590 & -313 \\
Exterior Column 122 at Floor 82 & -313 & -230 \\
Exterior Column 329 at Floor 82 & -228 & -202 \\
Exterior Column 130 at Floor 107 & -222 & 0 \\
Spandrel between Columns 138 and 139 at Floor 83 & 0 & 0 \\
Spandrel between Columns 447 and 448 at Floor 87 & 0 & \\
\hline
\end{tabular}

\subsection{GLOBAL ANALYSIS WITH CREEP}

\subsubsection{Introduction}

This section presents the results of analyses conducted with global models that included consideration of plasticity, geometric nonlinearity relating to large displacements, inelastic buckling, and creep, and are referred to hereafter as global models or global analysis with creep. As noted earlier, a preliminary set of global analyses were conducted on models that did not include creep and could not simulate inelastic buckling of columns with fidelity. The results of these preliminary analyses are presented in Appendix $\mathrm{C}$ of this report, and are referred to as global analysis without creep. Due to the limited non-linear features in the preliminary global models without creep, global instability could not be captured during temperature time history analyses.

The results of the global analyses with creep are presented in Section 4.2.4 for WTC 1 and in Section 4.2.5 for WTC 2.

\subsubsection{Modifications to the Global Model with Creep and Inelastic Buckling of Columns}

Preliminary global analyses with creep resulted in an unacceptably slow rate of convergence. This was due to the size of the models and the nonlinear effects of temperature-dependent material properties, especially creep. To reduce the size of the global models and to enhance the computational efficiency without adversely affecting the fidelity of the results, a set of modifications were made. These modifications and their effects on the analysis results are discussed in this section. Table 4-6 summarizes the number of degrees of freedom, number of elements, and number of constraint equations in WTC 1 and WTC 2 global models before and after the modifications. 
Table 4-6. Global model properties before and after modifications for computational efficiency.

\begin{tabular}{|l|c|c|c|c|}
\hline \multirow{2}{*}{\multicolumn{1}{|c|}{ Item }} & \multicolumn{2}{|c|}{ WTC 1 } & \multicolumn{2}{c|}{ WTC 2 } \\
\cline { 2 - 5 } & $\begin{array}{c}\text { Before } \\
\text { Modifications }\end{array}$ & $\begin{array}{c}\text { After } \\
\text { Modifications }\end{array}$ & $\begin{array}{c}\text { Before } \\
\text { Modifications }\end{array}$ & $\begin{array}{c}\text { After } \\
\text { Modifications }\end{array}$ \\
\hline Number of Degrees of Freedom & 291,670 & 265,760 & 487,260 & 196,900 \\
Number of Constraint Eqs. & 31,680 & 28,330 & 53,890 & 12,560 \\
Number of Elements & 63,880 & 57,680 & 106,460 & 38,130 \\
Number of Beam Elements & 48,200 & 43,600 & 81,280 & 32,540 \\
Number of Shell Elements & 15,680 & 14,080 & 25,180 & 5,490 \\
\hline
\end{tabular}

\section{Removal of Floors below Impact Zone}

The parts of structures below the impact zones (Floor 89 to Floor 91 in WTC 1 and Floor 73 to Floor 77 in WTC 2) contributed little to the overall behavior of the buildings. Previous analyses of subsystem models and preliminary global models showed that the elements below the impact zone did not experience plastic deformations or buckling. Therefore, they were eliminated to further reduce the size of the models. With this modification, the global model for WTC 2 was truncated at Floor 77 just above the mechanical floors and the global model for WTC 1 was truncated at Floor 91.

\section{Release of Axial Degree of Freedom of Spandrels}

The spandrel elements in the exterior wall were modeled using BEAM188 elements. These elements caused convergence problems when thermal expansion caused buckling of spandrels. Based on visual evidence, buckling of spandrels did not compromise their ability to transfer shear and bending moment and did not play a very important role in the collapse sequence. Therefore, the axial degree of freedom was released on these spandrel elements, avoiding buckling due to devclopment of large axial force in the spandrels. However, these BEAM188 elements could still transfer shear and bending moments. The coefficient of thermal expansion of spandrels was also removed so that the spandrels would not expand with temperatures.

\section{Removal of Coefficient of Thermal Expansion from the Slab in the Office Area}

Since trusses were not modeled individually, the office area slab buckled easily when thermal expansion was restrained by the exterior wall. Artificial buckling of the slab caused convergence problems in the global analysis. Since buckling of the slab in the actual buildings was resisted by the joists, and was not observed in any of the detailed full floor models, this slab buckling mode was considered unlikely to represent real building behavior. Consequently, the coefficient of thermal expansion for the slab in the office area was set to zero.

Neglecting the thermal expansion of the office area slabs did introduce small errors in the out-of-plane bending of columns extending between a hot floor and a cool floor, but such errors were small for columns extending between two hot floors. The error introduced by this modification was not expected to change failure modes or collapse sequence in the global analysis, because thermal expansion of floors was limited to less than a few inches (see Appendix A). The full floor models thermally expanded and pushed 
outward on the columns until the thermal expansion was overcome by the floor sagging and the floors pulled inward on the exterior columns..

\section{Use of Superelement in WTC 2}

The term "superelement" is used to indicate substructuring, in which a portion of a large model expected to remain elastic is condensed out from the model as a whole. In this technique, the stiffness, damping and mass matrices for the substructure elements are calculated once and used throughout the analysis without any further change. One can calculate stresses and strains in individual elements in the superelement allowing verification of the assumption that the substructure elements remain elastic, or nearly so. The WTC 2 model was suitable for such modification as earlier analyses indicated that the section of the building above Floor 86 would remain nearly elastic. Therefore, this portion of the structure was converted into a superelement.

The use of this superelement in the WTC 2 analyses reduced the solution time required to complete a single iteration by a factor of three. However, it was recognized that if the hat truss members became inelastic and highly nonlinear, such nonlinearities could not be captured. Moreover, when the superelement is used, the effects of construction sequence on the load distribution between core and wall elements cannot be represented, since the birth and death option cannot be used in a superelement. As shown below, the effect of not including construction sequence was evaluated and found to introduce an error of less than 12 percent for vertical displacement.

To evaluate whether any member in the hat truss exceeded its elastic limits, the stresses in all elements within the superelement were calculated at the end of each time interval. For this purpose, a separate model that included the elements at and above Floor 86 was created. In this model, the material properties of all elements were replaced with elastic material properties. At each time interval, the displacements that were obtained from the global model at Floor 86 were imposed on the new model at the same level. Dead and live loads were also applied on the model. Member forces were calculated and compared with their capacities. The results are discussed in Section 4.2.5.

\section{Change from BEAM188 to BEAM24 Elements}

In the global models without creep, columns were modeled by BEAM188 (3D linear finite strain beam) elements. The analysis could not be conducted with elements of this type capturing time dependent creep behavior of steel. Frequent convergence problems occurred when thermally induced creep and buckling of columns was in process. Different element types were tested to determine whether thermally induced buckling and creep could be captured. Finally, BEAM24 element was selected, and the element type for the columns was changed from BEAM188 to BEAM24 (3D thin-walled beam).

\section{Construction Sequence}

Construction sequence was not included in the global models with creep. The effect of neglecting construction sequence was examined for both buildings. When construction sequence was not included in the analysis, the total axial loads in columns along the exterior walls increased by 7 percent to 15 percent. Similarly, the total column loads supported by the core columns decreased by about 10 percent. 
Tables 4-7 and 4-8 indicate the differences in column loads when construction sequence either was or was not considered.

Table 4-7. Comparison of total column loads between WTC 1 models with and without construction sequence

\begin{tabular}{|c|c|c|c|c|c|c|c|c|c|c|}
\hline & $\begin{array}{c}\text { North } \\
\text { w/ Const } \\
\end{array}$ & w/o Const & $\begin{array}{c}\text { South } \\
\text { w/ Const } \\
\end{array}$ & w/o Const & $\begin{array}{c}\text { East } \\
\text { w/ Const } \\
\end{array}$ & w/o Const & $\begin{array}{c}\text { West } \\
\text { w/ Const } \\
\end{array}$ & w/o Const & $\begin{array}{c}\text { Core } \\
\text { w/ Const } \\
\end{array}$ & w/o Const \\
\hline Floor $93-94$ & 12,307 & 13,145 & 12,383 & 13,191 & 8,910 & 10,036 & 8,933 & 10,049 & 47,525 & 43,638 \\
\hline Floor $94-95$ & 11,922 & 12,760 & 11,999 & 12,806 & 8,580 & 9,708 & 8,605 & 9,723 & 45,573 & 41,682 \\
\hline Floor $95-96$ & 11,450 & 12.281 & 11,530 & 12,329 & 8,339 & 9,475 & 8,365 & 9,492 & 43,598 & 39,703 \\
\hline Floor $96-97$ & 11.065 & 11,895 & 11,145 & 11,944 & 8,012 & 9,151 & 8,040 & 9,170 & 41,633 & 37,735 \\
\hline Floor $97-98$ & 10,602 & 11,426 & 10,686 & 11,478 & 7,763 & 8,911 & 7,793 & 8,932 & 39,669 & 35,767 \\
\hline Floor $98-99$ & 10.217 & 11,040 & 10.302 & 11.092 & 7,439 & $8,59]$ & 7,471 & 8.613 & 37,714 & 33,808 \\
\hline
\end{tabular}

Note: Compression is positive. Units are in kip.

Table 4-8. Comparison of total column loads between WTC 2 models with and without construction sequence

\begin{tabular}{|c|c|c|c|c|c|c|c|c|c|c|}
\hline & $\begin{array}{c}\text { North } \\
\text { w/ Const } \\
\end{array}$ & w/o Const & $\begin{array}{c}\text { South } \\
\text { w/ Const } \\
\end{array}$ & w/o Const & $\begin{array}{c}\text { East } \\
\text { w/ Const } \\
\end{array}$ & w/o Const & $\begin{array}{c}\text { West } \\
\text { w/ Const } \\
\end{array}$ & w/o Const & $\begin{array}{c}\text { Core } \\
\text { w/ Const } \\
\end{array}$ & w/o Const \\
\hline Floor $78-79$ & 13,536 & 15,304 & 13,442 & 15,000 & 18,861 & 20,169 & 18,805 & 20,119 & 78,104 & 72,156 \\
\hline Floor $79-80$ & 12,965 & 14.723 & 12,877 & 14,424 & 18,650 & 19,970 & 18,596 & 19,923 & 76,197 & 70,246 \\
\hline Floor $80-81$ & 12,892 & 14,675 & 12,810 & 14,379 & 17,959 & 19,257 & 17,903 & 19,207 & 74,160 & 68,206 \\
\hline Floor $81-82$ & 12,367 & 14.141 & 12,292 & 13,851 & 17,728 & 19.036 & 17,673 & 18.989 & 71,824 & 65,866 \\
\hline Floor $82-83$ & 12,279 & 14,078 & 12.208 & 13,789 & 17,064 & 18.353 & 17,008 & 18,301 & 69,777 & 63,815 \\
\hline Floor $83-84$ & 11.775 & 13.567 & 11,712 & 13.284 & 16,816 & 18,114 & 16,761 & 18.114 & 67.793 & 61,828 \\
\hline
\end{tabular}

Note: Compression is positive. Units are in kip.

The calculations showed that the outriggers in the WTC 1 simulations were more highly stressed when the construction sequence was not considered. Since it was believed that the hat truss played an important role in transferring loads in WTC 1, the yield strengths of the materials for these outriggers in WTC 1 were artificially increased to account for the incorrect increase in compressive stresses when construction sequence was not considered.

The difference in maximum displacement calculated with and without consideration of construction sequence was within 12 percent for both WTC 1 and WTC 2. Figures 4-6 and 4-7 present the differences in calculated displacements for analyses in which construction sequence either was or was not considered, respectively, for WTC 1 and WTC 2. In these figures, the results presented for global analyses with creep for the state of the structure before aircraft impact are for the analyses in which construction sequence was neglected; they differ from the corresponding results presented in Appendix C for global analyses without creep as those analyses included the effects of the construction sequence. 


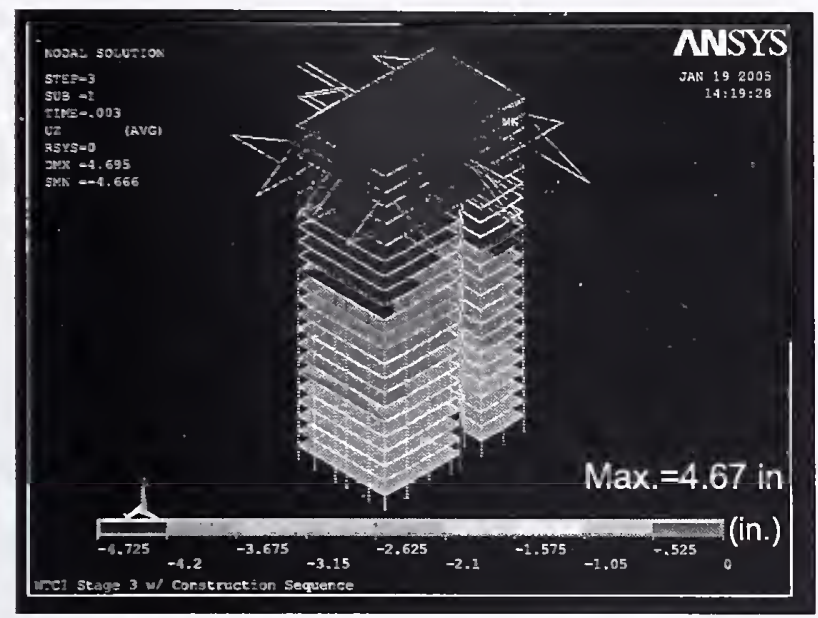

(a) Core w/ construction sequence

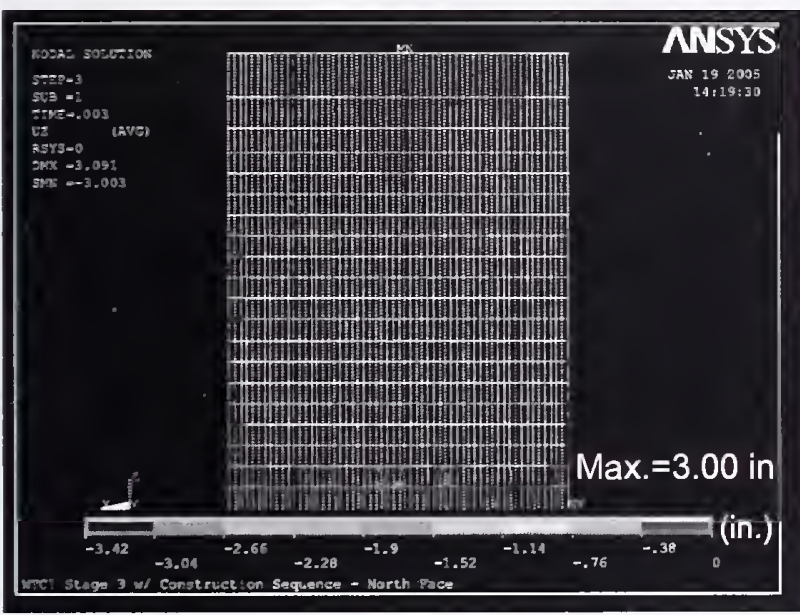

(c) North face w/ construction sequence

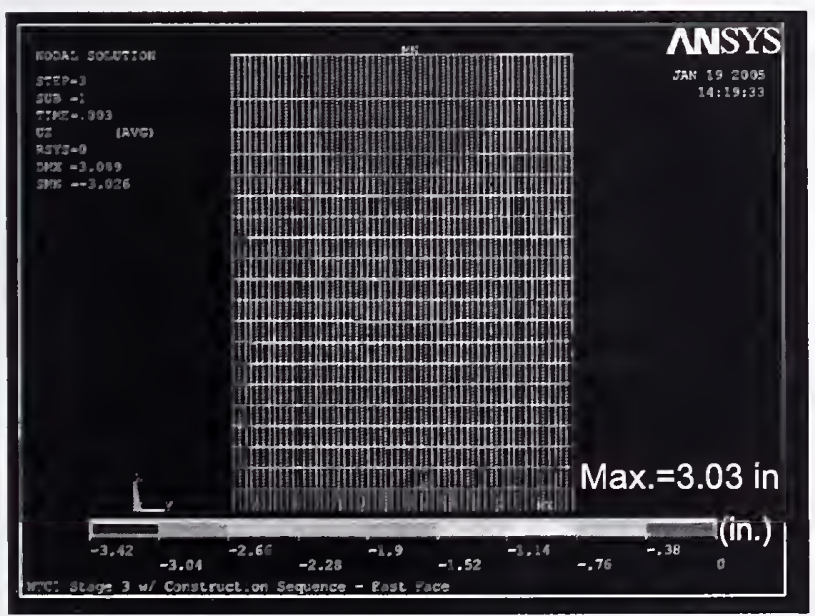

(e) East face w/ construction sequence

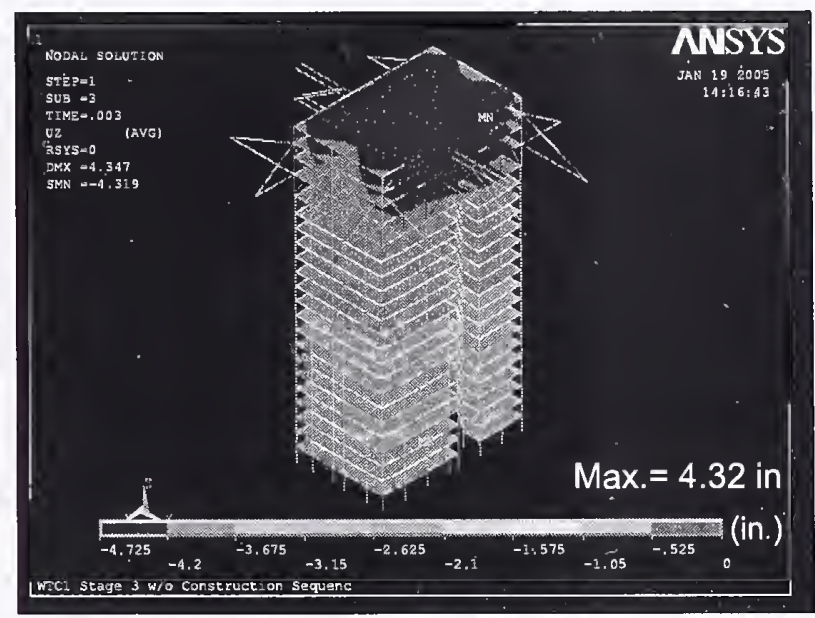

(b) Core w/o construction sequence

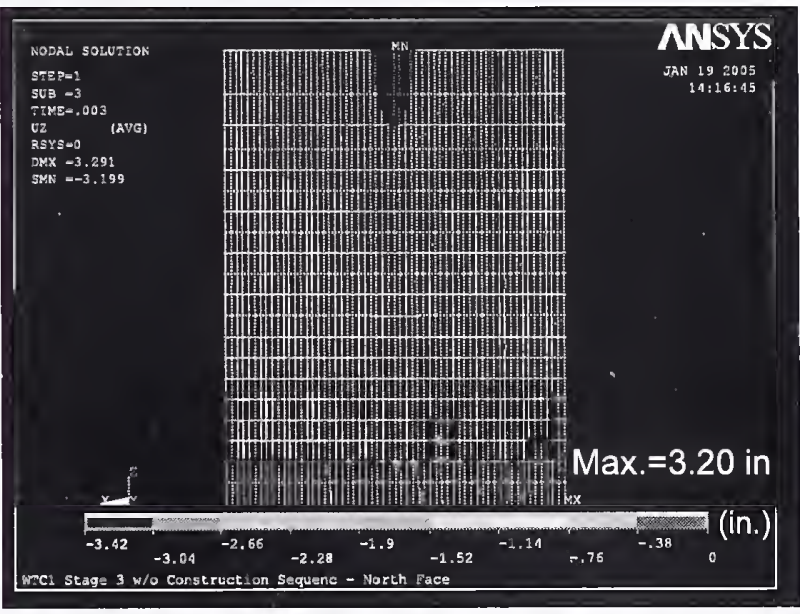

(d) North face w/o construction sequence

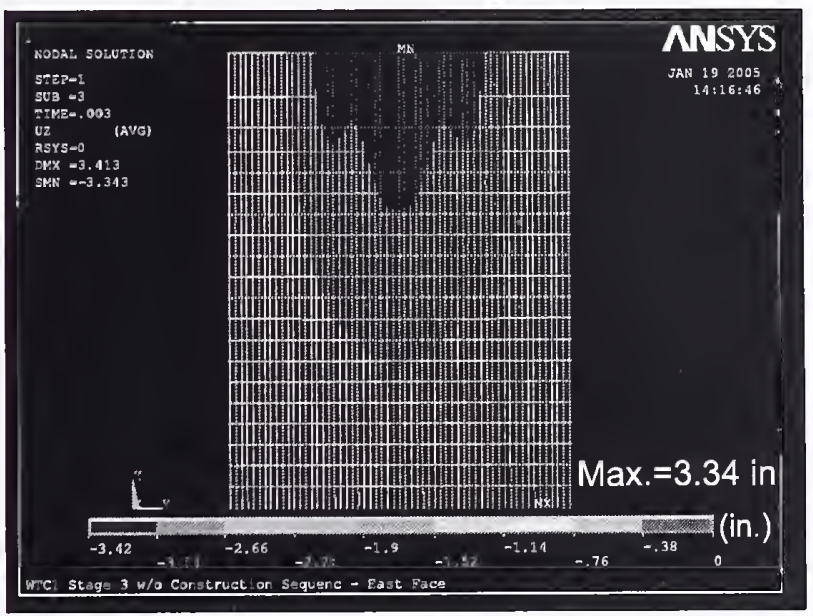

(f) East face w/o construction sequence

Figure 4-6. Comparison of vertical displacement between WTC 1 models with and without construction sequence. 


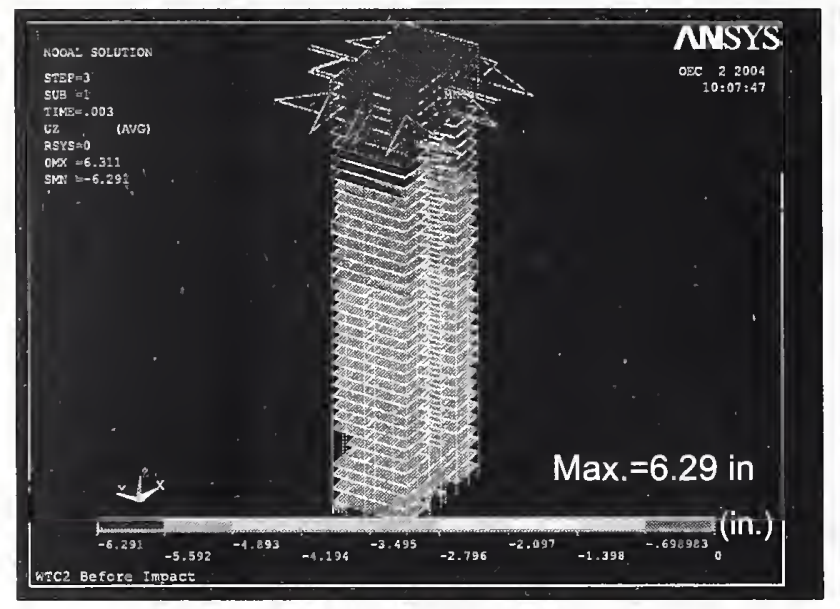

(a) Core w/ construction sequence

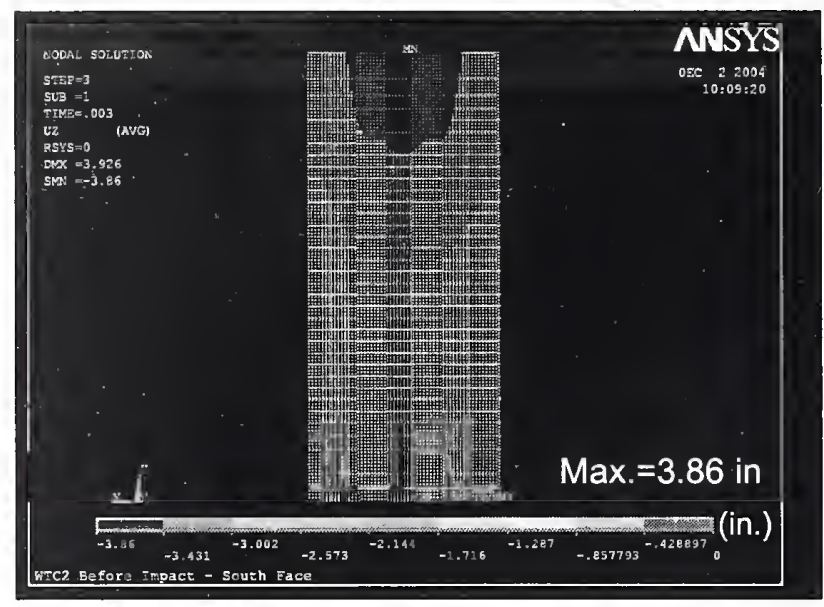

(c) South face w/ construction sequence

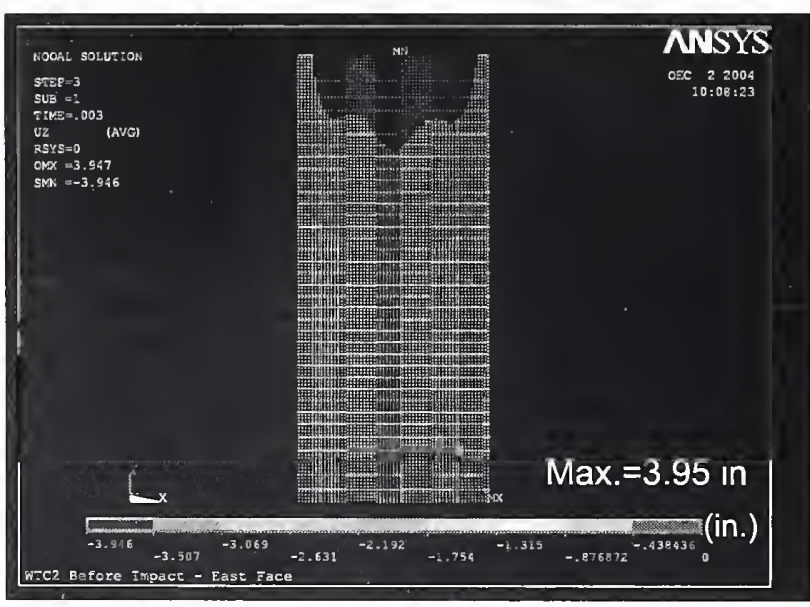

(e) East face w/ construction sequence

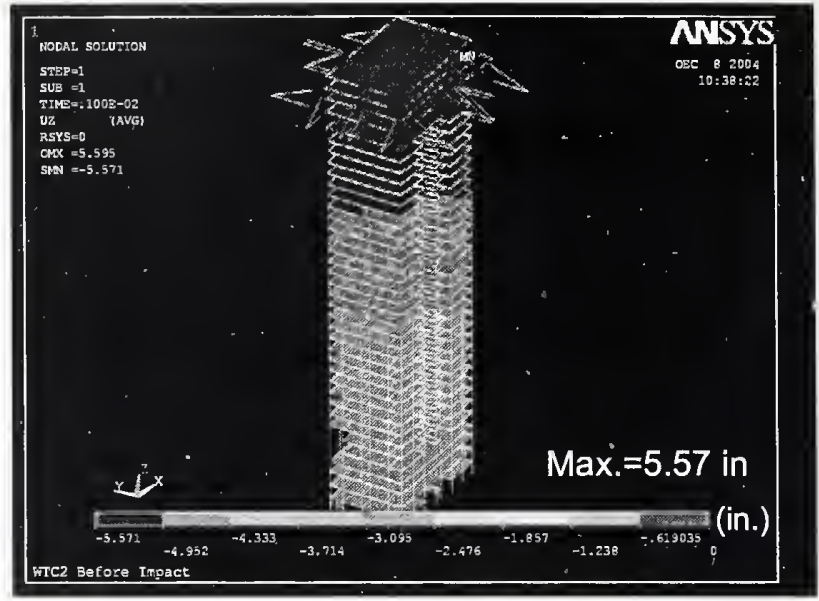

(b) Core w/o construction sequence

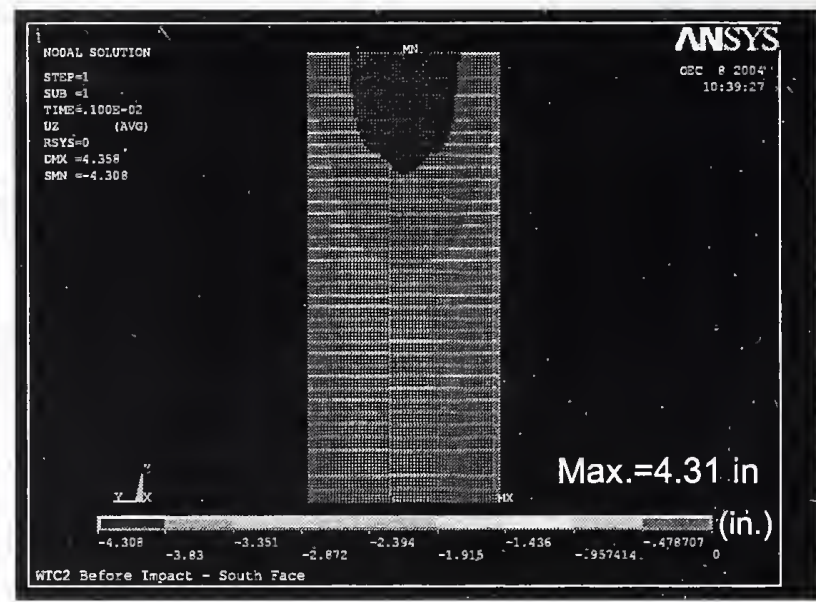

(d) South face w/o construction sequence

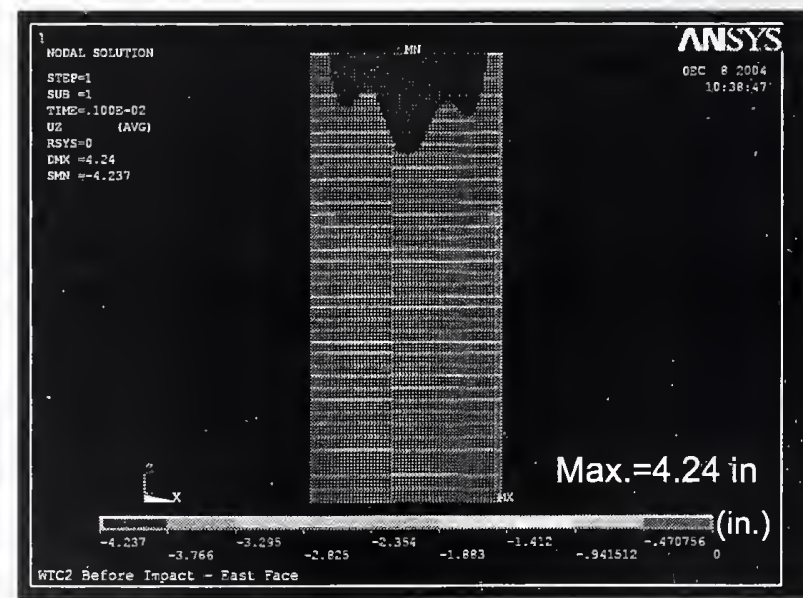

(f) East face w/o construction sequence

Figure 4-7. Comparison of vertical displacement between WTC 2 models with and without construction sequence. 


\subsubsection{Boundary Conditions and Loading Steps}

The global models with creep were vertically supported on elastic springs at Floor 91 for WTC 1 and Floor 77 for WTC 2. These elastic springs represented the axial flexibility of the portion of the building below these floors. The models were fixed against horizontal translation at these floors.

The collapse analysis of WTC 1 considered creep and was performed only for Case B damage conditions and temperature time histories; and that for WTC 2 was only performed for Case D. Section 2.2.3 of this report provides a description of these damage conditions. Severed and heavily damaged core columns were removed in the global model with creep, while only severed columns were removed in the global models without creep.

To reduce the size of the global models as much as possible, members that were predicted to have been severed or heavily damaged by the aircraft impact were removed from the models before applying the gravity load. The floor dead and live loads were applied at each column node at the floor levels. These forces were taken directly from the ANSYS global models converted from the reference SAP2000 global models. WTC 1 and WTC 2 global models were then subjected to Case B and Case D temperature conditions, respectively. NIST provided temperature data at $10 \mathrm{~min}$ intervals ranging from $0 \mathrm{~min}$ to $100 \mathrm{~min}$ for WTC 1 and to $60 \mathrm{~min}$ for WTC 2. For the first loading step of temperature analysis, temperatures of the structural elements were linearly ramped up from room temperature to the temperatures at $10 \mathrm{~min}$. After the first step, the temperatures were linearly ramped from the temperatures at the end of the previous time step to those at the end of current time step.

\subsubsection{Simulation of WTC 1 Collapse}

The global model of WTC 1, described in the previous section, was used to simulate the response of the building to aircraft impact damage and the ensuing fire environment. Studies performed on the isolated exterior wall and core models and on the full floor subsystem models indicated that the calculated response of these models to the Case B impact damage and temperature time history set, as described in Section 2.2.3, more closely matched the structural behavior observed in the visual evidence than did analyses using the Case A data set. Therefore, only the Case B impact damage and temperature time history set was used in the final global analyses.

The gravity loading, consisting of the structure's self weight, superimposed dead load, and 25 percent of the design live loads, was applied as a concentrated joint load at each column-floor node, without consideration of construction sequence effects. Then the temperature time history was applied in a series of load steps. Table 4-9 summarizes the sequence of analyses that were performed. The results of each analysis step were used as the initial conditions for the next analysis step. The steps consisted of alternate applications of fire damage, in the form of floor/wall disconnections or pull-in forces, followed by application of temperature change. Section 2.5 describes the methods by which floor/wall disconnections and pull-in forces were determined and applied.

After application of the Case B aircraft impact damage set, which included severe damage on the north face and to the north side of the core, the results of analysis showed slight tilting of the portion of the structure above the damaged area, to the north. In early stages of the application of the temperature time history, temperatures increased significantly in the core columns where thermal insulation was projected to be damaged. The resulting thermal expansion of these core columns was larger than the thermal 
expansion of the exterior walls which occurrcd at the same time. Therefore, as the core columns in the modcl expanded, they were restrained by the stiff hat truss, resulting in increased compressive forces in the core columns. This effect first became significant at $20 \mathrm{~min}$. By $50 \mathrm{~min}$ some of the core columns under the influence of the high temperatures and high compressive loads began to exhibit buckling and large creep strains. As buckling and creep strains accumulated, the core began to displace downward. Again, the movement of the tops of the core columns was restrained by the hat truss which now began to redistribute loads from the core columns to the exterior walls. At $80 \mathrm{~min}$, pull-in forces were applied to the south wall, based on the observed evidence. At $87 \mathrm{~min}$, the south wall started to bow inward significantly, initiating the buckling of the south wall that triggered the collapse sequence.

Table 4-9. Analysis steps of WTC 1 ANSYS global model.

\begin{tabular}{|c|c|}
\hline $\begin{array}{l}\text { Analysis } \\
\text { Step }\end{array}$ & Description \\
\hline 1 & Dead and 25 percent of the design live loads were applied on the model of WTC 1 with impact damage. \\
\hline 2 & $\begin{array}{l}\text { Floor/wall disconnections and pull-in forces that were projected to occur between } 0 \mathrm{~min} \text { and } 10 \mathrm{~min} \\
\text { were applied. }\end{array}$ \\
\hline 3 & $\begin{array}{l}\text { Column and spandrel temperatures were linearly ramped up from room temperature of } 20^{\circ} \mathrm{C} \text { to } \\
\text { temperatures at } 10 \mathrm{~min} \text {. }\end{array}$ \\
\hline 4 & $\begin{array}{l}\text { Floor/wall disconnections and pull-in forces that were projected to occur between } 10 \mathrm{~min} \text { and } 20 \mathrm{~min} \\
\text { were applied. }\end{array}$ \\
\hline 5 & Column and spandrel temperatures were linearly changed from temperatures at $10 \mathrm{~min}$ to $20 \mathrm{~min}$. \\
\hline 6 & $\begin{array}{l}\text { Floor/wall disconnections and pull-in forces that were projected to occur between } 20 \mathrm{~min} \text { and } 30 \mathrm{~min} \\
\text { were applied. }\end{array}$ \\
\hline 7 & Column and spandrel temperatures were linearly changed from temperatures at $20 \mathrm{~min}$ to $30 \mathrm{~min}$. \\
\hline 8 & $\begin{array}{l}\text { Floor/wall disconnections and pull-in forces that were projected to occur between } 30 \mathrm{~min} \text { and } 40 \mathrm{~min} \\
\text { were applied. }\end{array}$ \\
\hline 9 & Column and spandrel temperatures were linearly changed from temperatures at $30 \mathrm{~min}$ to $40 \mathrm{~min}$. \\
\hline 10 & $\begin{array}{l}\text { Floor/wall disconnections and pull-in forces that were projected to occur between } 40 \mathrm{~min} \text { and } 50 \mathrm{~min} \\
\text { were applied. }\end{array}$ \\
\hline 11 & Column and spandrel temperatures were linearly changed from temperatures at $40 \mathrm{~min}$ to $50 \mathrm{~min}$. \\
\hline 12 & $\begin{array}{l}\text { Floor/wall disconnections and pull-in forces that were projected to occur between } 50 \mathrm{~min} \text { and } 60 \mathrm{~min} \\
\text { were applied. }\end{array}$ \\
\hline 13 & Column and spandrel temperatures were linearly changed from temperatures at $50 \mathrm{~min}$ to $60 \mathrm{~min}$. \\
\hline 14 & $\begin{array}{l}\text { Floor/wall disconnections and pull-in forces that were projected to occur between } 60 \mathrm{~min} \text { and } 70 \mathrm{~min} \\
\text { were applied. }\end{array}$ \\
\hline 15 & Column and spandrel temperatures were linearly changed from temperatures at $60 \mathrm{~min}$ to $70 \mathrm{~min}$. \\
\hline 16 & $\begin{array}{l}\text { Floor/wall disconnections and pull-in forces that were projected to occur between } 70 \mathrm{~min} \text { and } 80 \mathrm{~min} \\
\text { were applied. }\end{array}$ \\
\hline 17 & Column and spandrel temperatures were linearly changed from temperatures at $70 \mathrm{~min}$ to $80 \mathrm{~min}$. \\
\hline 18 & $\begin{array}{l}\text { Floor/wall disconnections and pull-in forces that were projected to occur between } 80 \mathrm{~min} \text { and } 90 \mathrm{~min} \\
\text { were applied. }\end{array}$ \\
\hline 19 & Column and spandrel temperatures were linearly changed from temperatures at $80 \mathrm{~min}$ to $90 \mathrm{~min}$. \\
\hline 20 & $\begin{array}{l}\text { Floor/wall disconnections and pull-in forces that were projected to occur between } 90 \mathrm{~min} \text { and } 100 \mathrm{~min} \\
\text { were applied. }\end{array}$ \\
\hline 21 & Column and spandrel temperatures were linearly changed from temperatures at $90 \mathrm{~min}$ to $100 \mathrm{~min}$. \\
\hline
\end{tabular}


Figures 4-8 to 4-23 show the calculated vertical displacements for the exterior walls of WTC 1, before the aircraft impact, immediately after impact, at $80 \mathrm{~min}$ after impact (at the end of Step 17), and $100 \mathrm{~min}$ after impact. Figures 4-24 to 4-32 show similar data for the core columns. Figure 4-33 shows the calculated vertical displacement of Floor 99 before the aircraft impact. Figures 4-34 to 4-37 show changes in vertical displacement at Floor 99 from the state before the aircraft impact. Before aircraft impact, the maximum vertical displacements of the exterior walls and the core were respectively calculated as 3.3 in. and 4.2 in. (see Figs. 4-8 and 4-24). As indicated in Fig. 4-33, the core had larger vertical displacements by about 1 in. than the exterior walls at Floor 99. Since the construction sequence was not considered in the analysis, this difference in the vertical displacements changed initial loading conditions of columns. As shown in Table 4-7, the total column loads on the north and south walls increased by 7 percent at the impacted floors, the total column loads on the east and west walls increased by about 14 percent, and the total column loads on the core decreased by about 9 percent, by ignoring the construction sequence. Owing to impact damage on the north face and the north side of the core, WTC 1 tilted slightly to the north after the aircraft impact as can be seen in Fig. 4-34. The maximum calculated displacement of the north wall increased from 3.1 in. to 5.9 in. as shown in Figs. 4-12 and 4-13, and the maximum displacement of the south wall decreased from 3.2 in. to $3.0 \mathrm{in}$. as shown in Figs. 4-20 and 4-21. The calculated vertical displacement for the east and west walls only increased slightly.

In the early stages of thermal loading, the temperatures in the core area were rising. At $50 \mathrm{~min}$, the calculated downward displacement of the core from plasticity, creep, and buckling of core columns was 1.6 in. on average at Floor 99, as shown in Fig. 4-35. At 100 min, the downward displacement of the core was at $2.0 \mathrm{in}$. on average at Floor 99. Average increases of the downward vertical displacement at Floor 99 of the north, east, south, and west faces were $1.7 \mathrm{in.,}-0.24 \mathrm{in} .,-0.51 \mathrm{in}$., and $-0.24 \mathrm{in}$. at $100 \mathrm{~min}$, respectively. As the bowing of the south wall increased, a section of the south wall above the bowed-in area moved downward as shown in Fig. 4-37.

Figures 4-38 to 4-41 show the calculated out-of-plane displacements for the south wall before and after the aircraft impact, at $80 \mathrm{~min}$ (at the end of Step 17), and $100 \mathrm{~min}$ after impact. In these figures, inward displacement is shown as positive. Figure $4-42$ shows a plot of the variation of maximum calculated outof-plane displacement in the south wall between $80 \mathrm{~min}$ and $100 \mathrm{~min}$. Until pull-in forces were applied to the columns on the south wall over Floor 95 to Floor 99 at $80 \mathrm{~min}$ (Step 18), no inward bowing had occurred. This matches the available video evidence. After the pull-in forces were applied, the bow initiated and grew to $15.5 \mathrm{in}$. At about $87 \mathrm{~min}$ into the analysis, the inward bow began to increase significantly. By $90 \mathrm{~min}$, the rate of increase in inward bowing of the south wall slowed, and gravity loads were redistributed to the east and west walls and the core. The predicted inward bowing gradually increased to approximately $43 \mathrm{in}$. at $100 \mathrm{~min}$. However, the south wall did not show instability (buckling) at $100 \mathrm{~min}$.

Analyses of isolated exterior wall substructure models and of the global model showed that the amount of inward bowing predicted for the wall was highly sensitive to the magnitude of the applied pull-in forces. For a comparison, when pull-in forces were revised from the 5 kip magnitude used in the analyses discussed above to $4 \mathrm{kip}$, the predicted inward bowing of the south wall decreased dramatically from nearly $43 \mathrm{in}$. at $100 \mathrm{~min}$ to approximately $15 \mathrm{in}$. Minor upward adjustment of the pull-in forces, from the $5 \mathrm{kip}$ used in the analyses, would have produced wall instability by $100 \mathrm{~min}$. It is likely that the pull-in forces in the actual building increased with time, and that likely, this inward bowing of the south wall did trigger instability, which initiated the global structural collapse. 
Figures 4-44 to 4-55 show calculated axial loads in exterior columns before and after the aircraft impact, at $80 \mathrm{~min}$ (at the end of Step 17), and $100 \mathrm{~min}$ after impact. Figures 4-56 to 4-59 show the variation of calculated axial loads in exterior columns along the different building faces at Floor 98 at different points in time. Figures 4-60 to 4-63 show axial loads for the core columns before and after the aircraft impact, at $80 \mathrm{~min}$ (at the end of Step 17), and $100 \mathrm{~min}$. Figures 4-64 to 4-67 show the magnitude of axial load in each column at Floor 98; the size of axial load is proportional to the size of the circles. Tables 4-10 and 4-11 show the total column loads on each of the exterior walls and the core at Floor 98 and Floor 105, respcctively. Tables 4-12, 4-14, 4-16,4-18, and 4-20 show total column loads on each of the exterior walls and the core at different floors from Floor 93 to Floor 105. Tables 4-13, 4-15, 4-17, 4-19, and 4-21 show the predicted changes in the total column loads of each of the exterior walls and the core at various times in the analysis. Tables 4-22 to 4-24 show the difference in total column loads at Floor 98 and Floor 105 between the statcs bcfore and after aircraft impact, just aftcr aircraft impact to 80 min (at the end of Step 17), and between $80 \mathrm{~min}$ (at the end of Step 17) and $100 \mathrm{~min}$.

After aircraft impact, gravity loads that were previously carried by severed columns were redistributed to other columns within the building. For example, Table 4-10 shows that the exterior columns of the north wall at Floor 98 carried 10,974 kip load before the aircraft impact, and 10,137 kip just after aircraft impact, a net reduction of 837 kip. Table 4-11 shows that columns along the north wall at Floor 105 lost $732 \mathrm{kip}$ of column loads as a result of the impact damage. Therefore, 732 kip out of 837 kip was transferred by the hat truss to other portions of the structure, and the rest was redistributed to the adjacent walls by the Vierendeel behavior of the walls themselves. Table 4-22 shows this load redistribution. Due to the impact damage and the northward tilting of the building after impact, the south wall also lost gravity loads. Approximately $600 \mathrm{kip}$ was transferred from the south wall by the hat truss. The east and west walls and the core gained respectively gained $466 \mathrm{kip}, 472 \mathrm{kip}$, and $400 \mathrm{kip}$, respectively.

As described above, during early stages of the thermal analysis, thermal expansion of the core area was greater than that of the extcrior walls. As this expansion occurred, the total column loads in the core increased until $20 \mathrm{~min}$ into the analysis, as shown in Tables 4-20 and 4-21. After $20 \mathrm{~min}$, the core began to shed gravity loads as it displaced downward, under the influence of column creep and buckling. This behavior continued until the south wall initiated inward bowing. At $80 \mathrm{~min}$, about $6,800 \mathrm{kip}$ of the gravity load in the core was transferred by the hat truss to the exterior walls, as shown in Table 4-11. The north, east, south, and west walls respectively gained 1,234 kip, 2,470 kip, 1,063 kip, and 2,021 kip from this behavior. As shown in Table 4-23, the primary load redistribution path during the thermal loading up to 80 min was through the hat truss.

Figure 4-58 shows that after $80 \mathrm{~min}$, Columns 318 to 346 on the south wall unloaded as they bowed inward. The vertical displacement of the south wall simultaneously increased as shown in Figs. 4-23 and $4-37$, and the south wall shed 1,485 kip of the gravity load between $80 \mathrm{~min}$ and $100 \mathrm{~min}$. As a result, the east and west walls and the core all gained gravity loads. Figure 4-58 also shows load redistribution within the south wall. As the columns near the center of the south wall unloaded after $80 \mathrm{~min}$, the axial loads on the columns on the east and west sides of the south wall increased.

Figures 4-68 to 4-71 show the calculated axial load demand-to-capacity ratio of each core column before and after the aircraft impact, at $80 \mathrm{~min}$ (at the end of Step 17), and $100 \mathrm{~min}$. Compressive capacities of the core columns were calculated using Eq. E2-1 in the American Institutc of Steel Construction's Manual of Steel Construction Load and Resistance Factor Design (AISC LRFD) for inelastic buckling with an effective length factor, $\mathrm{K}$, of unity and a resistance factor of unity. At $100 \mathrm{~min}$, a large number of core 
columns $(501,601,603,606,701,703,705,707,801,804,806,807,901,903,904,905,906,908$, and 1001) exhibited demand-to-capacity ratios larger than 0.7 . Eight columns $(501,606,705,707,804,806$, 807 , and 908) exhibited ratios larger than 1.0. Although there is some significant uncertainty associated with calculation of both the load on these columns and the buckling capacity, this indicates that at this time step, the core had either initiated or was close to initiating buckling-induced failure.

Figures 4-72 to 4-77 show the maximum strains in each column between Floor 93 and Floor 99 before and after aircraft impact, at $10 \mathrm{~min}$ (at the end of Step 3), $40 \mathrm{~min}$ (at the end of Step 9), $80 \mathrm{~min}$ (at the end of Step 18), and $100 \mathrm{~min}$. These figures include elastic and inelastic strain, but no creep effects.

Figures 4-78 to 4-81 show the same data, but include the additional effects of creep. Before the aircraft impact, all of the columns were loaded within their elastic range. After the aircraft impact, columns close to removed columns (which included both severed and highly damaged columns) developed plastic strain. Plastic strain of the core columns increased significantly for the first $40 \mathrm{~min}$, and then remained almost constant until $100 \mathrm{~min}$. At $100 \mathrm{~min}$, the maximum elastic-plus-plastic strain was 0.98 percent at Column 603. From $80 \mathrm{~min}$ to $100 \mathrm{~min}$, plastic strain increased in almost all the bowed columns on the south face. However, creep strain was found to be far greater than plastic strain as can be seen in Figs. 4-78 to 4-81, especially in the core. At $40 \mathrm{~min}, 22$ of 38 core columns that were not severed or highly damaged by aircraft impact had creep strains larger than 1.0 percent. After $40 \mathrm{~min}$, the creep strains of core columns on the south side of the core slowly increased. The maximum elastic-plus-plastic-plus-creep strain at 100 min was 7.3 percent in Column 1006. As temperature increased on the south wall in the later times, creep strain in columns on the south wall also increased. By $100 \mathrm{~min}$, creep strain increased in about 20 columns on the south face; the maximum elastic-plus-plastic-plus-creep strain in the columns on the south face reached 2.9 percent.

At $100 \mathrm{~min}$, the core was weakened on the south side and had shortened by 1.6 in., and the south wall had bowed inward to approximately $43 \mathrm{in}$. from pull-in forces at Floors 95 to 99 and was unloading to the core and the adjacent east and west walls.

Based on the observations and the calculated results of the analysis, reported above, the following sequence of events likely occurred as the south wall reached instability and buckled:

- As columns buckled, they shed load through Vierendeel action to adjacent columns in the south wall, in turn buckling these columns.

- The inward bowing of the south wall increased as additional columns buckled.

- As a result of this behavior, instability progressed horizontally across the wall.

- When instability engulfed the entire south wall, the wall continued to shed load to the east and west walls and to the core.

The onset of this load redistribution can be found in Table 4-24. The section of the building above the impact zone began tilting to the south as column instability progressed rapidly from the south wall along the adjacent east and west walls, resulting in increase of the gravity load on the core columns and in turn contributing to the buckling failure of these columns and initiating global collapse of the structure. 
Since the global model did not include elements capable of capturing failure of column or hat truss element splices, nor buckling of hat truss outriggers, the conditions of the connections and the members in the primary load path to and through the hat truss were evaluated at diffcrent time intervals. This evaluation included the core column splices for tension, outriggers and supporting columns for compression, and the hat truss connections that were in the primary load path for tension.

Before the aircraft impact, core column splices were under compression. After the impact and with increasing plastic and creep strains, the core displaced downward, and some core columns became suspended from the hat truss. In fact, as shown in Table 4-26 and Fig. 4-82, Columns 503, 504, 505 , $602,603,604,605,702$, and 802 were calculated to be in tension at Floor 105 at $100 \mathrm{~min}$. To evaluate the condition of the core column splices at Floor 106, the tension capacities of these splices were calculated using the AISC LRFD procedures and compared to the calculated tensile forces at each time interval. Table 4-25 shows the calculated tension capacities of core column splices at Floor 106, and Fig. 4-83 shows tensile demand-to-capacity ratios for the core column splices at Floor 106 at 100 min. It was found that tension forces in core columns were less than the capacities of the splices.

Sixteen outrigger members (four on each face) were present and participated in transfer of gravity loads between the core columns and the exterior walls, as shown in Fig. 4-3. In the global model, each of these outriggers was represented by one BEAM24 element; therefore, buckling of the member could not be captured, although material nonlinearity was included. Table 4-27 presents axial load demand-tocapacity ratios of the outrigger members. Capacities of outriggers were calculated using AISC LRFD Eq. E2-1 with an effective length factor $\mathrm{K}=0.75$ and a resistance factor of unity. To check against calculated capacities, axial loads obtained from the global model were adjusted for the additional axial load caused by ignoring construction sequence. Analyses predicted that Outrigger E reached its axial load capacity (see Fig. 4-3 for designations of outrigger members). Because the material properties of the outriggers in the global model were set so that they would yield when the axial load in the outrigger reached its compressive capacity, the axial load in Outrigger E did not change after reaching its capacity as unloading in the post-buckling regime was not modeled. This may have resulted in an underestimate of the force redistribution to the other outriggers.

Table 4-28 shows the computed demand-to-capacity ratios for axial loads of exterior columns supporting the outriggers. Each of these exterior columns was also modeled by only one element (BEAM188) in the global model; therefore, buckling of the columns were not captured in the analysis. Compressive capacities of these columns were also calculated using AISC LRFD Eq. E2-1 with an effective length factor $\mathrm{K}=0.75$ and a resistance factor of unity. This analysis showed that the axial loads in these columns were well below their inelastic buckling capacities.

For effective load transfer from the core columns to the outriggers to occur, the capacities of the hat truss connections in the intermediate load path could not be exceeded. To investigate this issue, the hat truss connections that were in this load path were identified, and their capacities were compared to the calculated forces transferred through them. The load path was identified by selecting the hat truss members that were predicted to have an absolute axial stress of $25 \mathrm{ksi}$ or more. The hat truss stresses were evaluated at $80 \mathrm{~min}$ (at the end of Step 17) as at this time they had reached their predicted maxima. Only the connections that were transferring tensile forces were evaluated. Figure 4-84 shows the members determined to be in the primary load path. Figure 4-85 shows the location of the critical hat truss connections that were evaluated. The capacities of the connections were calculated using the AISC LRFD procedures. Table 4-29 summarizes capacities, demands, and the conditions of the connections 
identified in Fig. 4-85. As can be seen in Table 4-29, none of the connections exceeded their capacities. The state of the outriggers and core column splices at Floor 105 were discussed in the earlier paragraphs.

Based on these evaluations, it can be stated that even though one outrigger reached its capacity, the hat truss was capable of redistributing loads between the core and the exterior walls, and therefore, the evaluations described above are valid. 


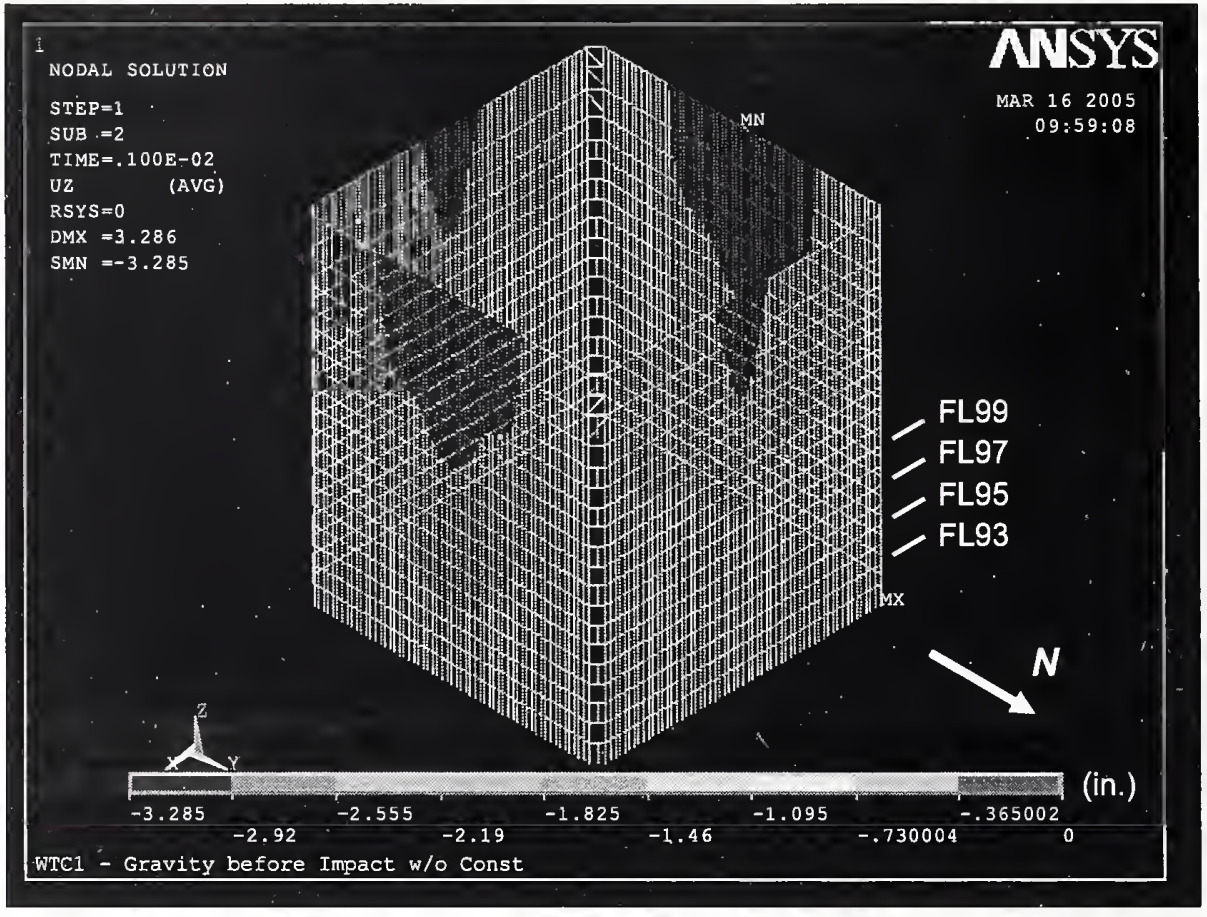

(a) North and east sides

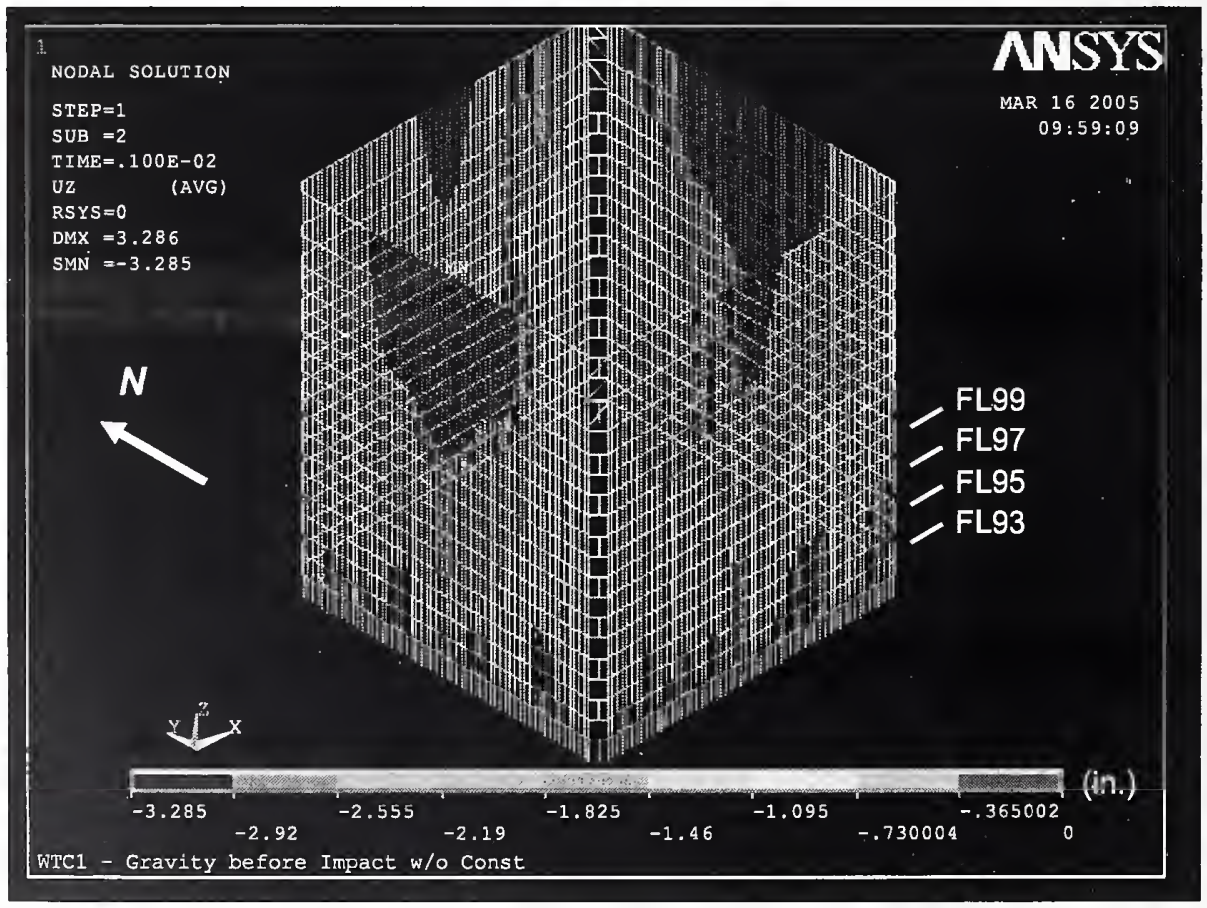

(b) South and west sides

Figure 4-8. Vertical displacement of exterior wall of WTC 1 before aircraft impact (downward displacement is negative). 


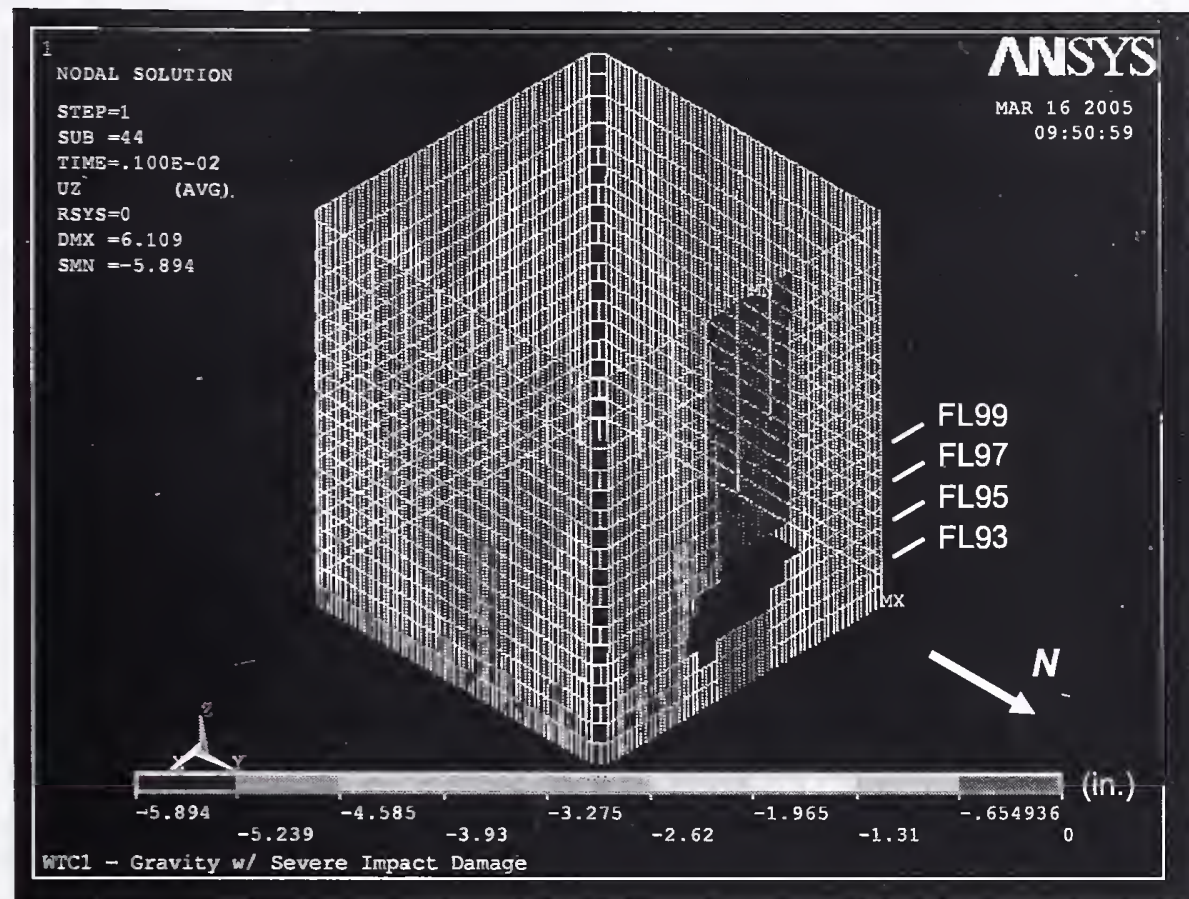

(a) North and east sides

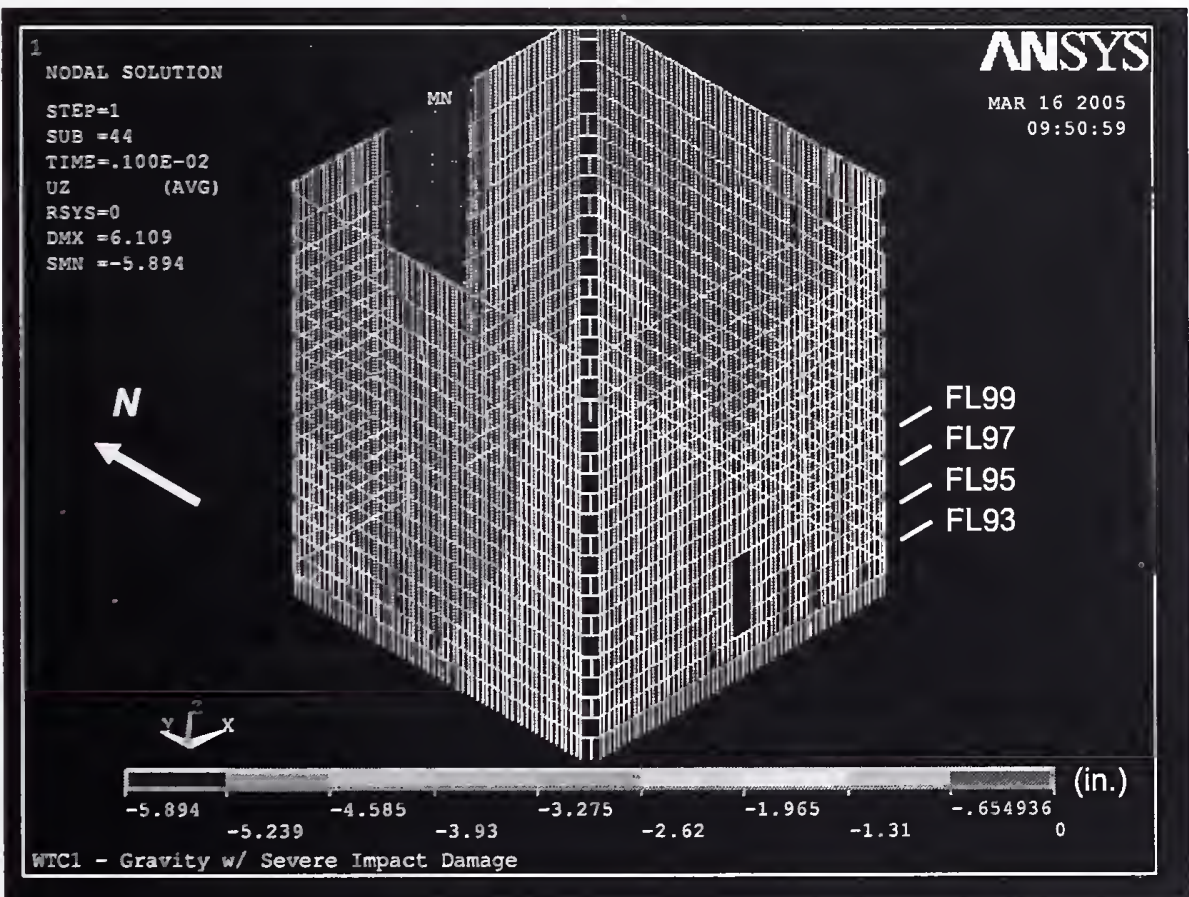

(b) South and west sides

Figure 4-9. Vertical displacement of exterior wall of WTC 1 after aircraft impact for Case B conditions (downward displacement is negative). 


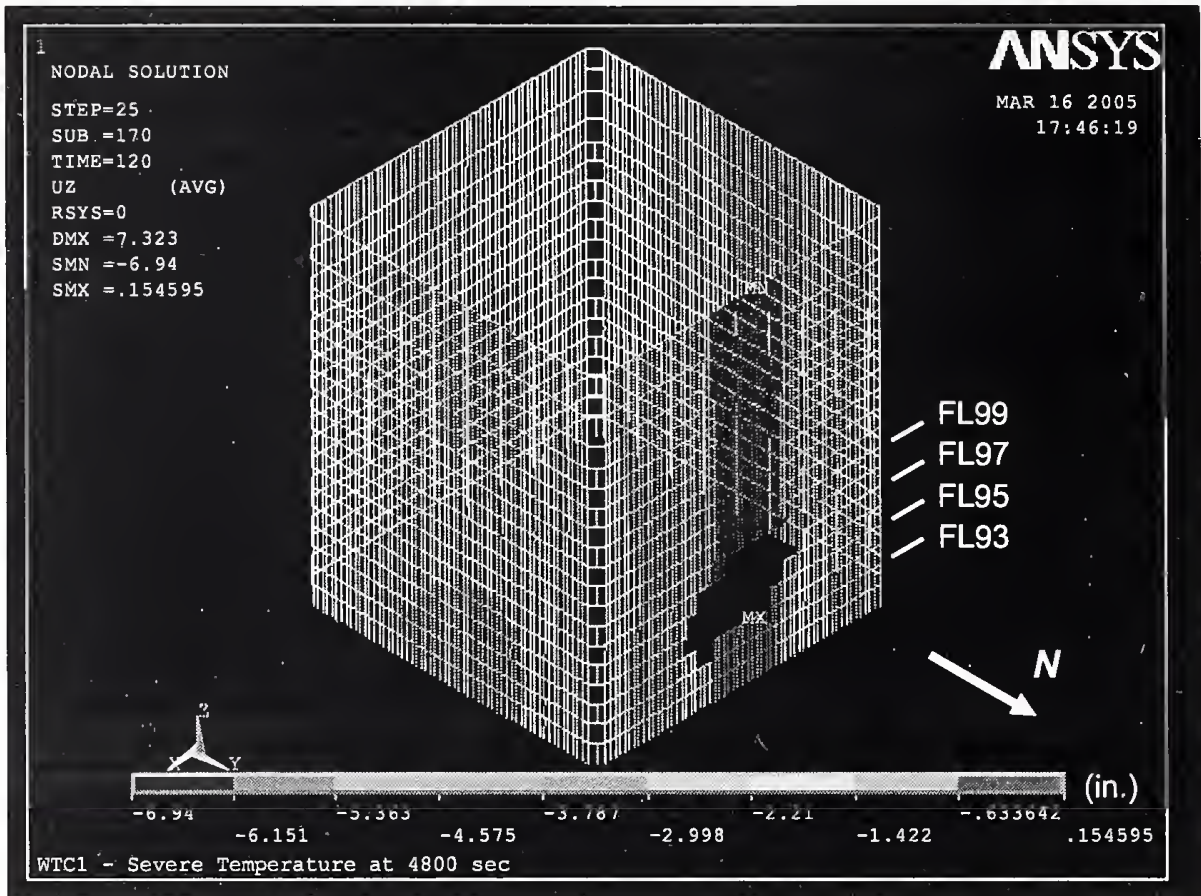

(a) North and east sides

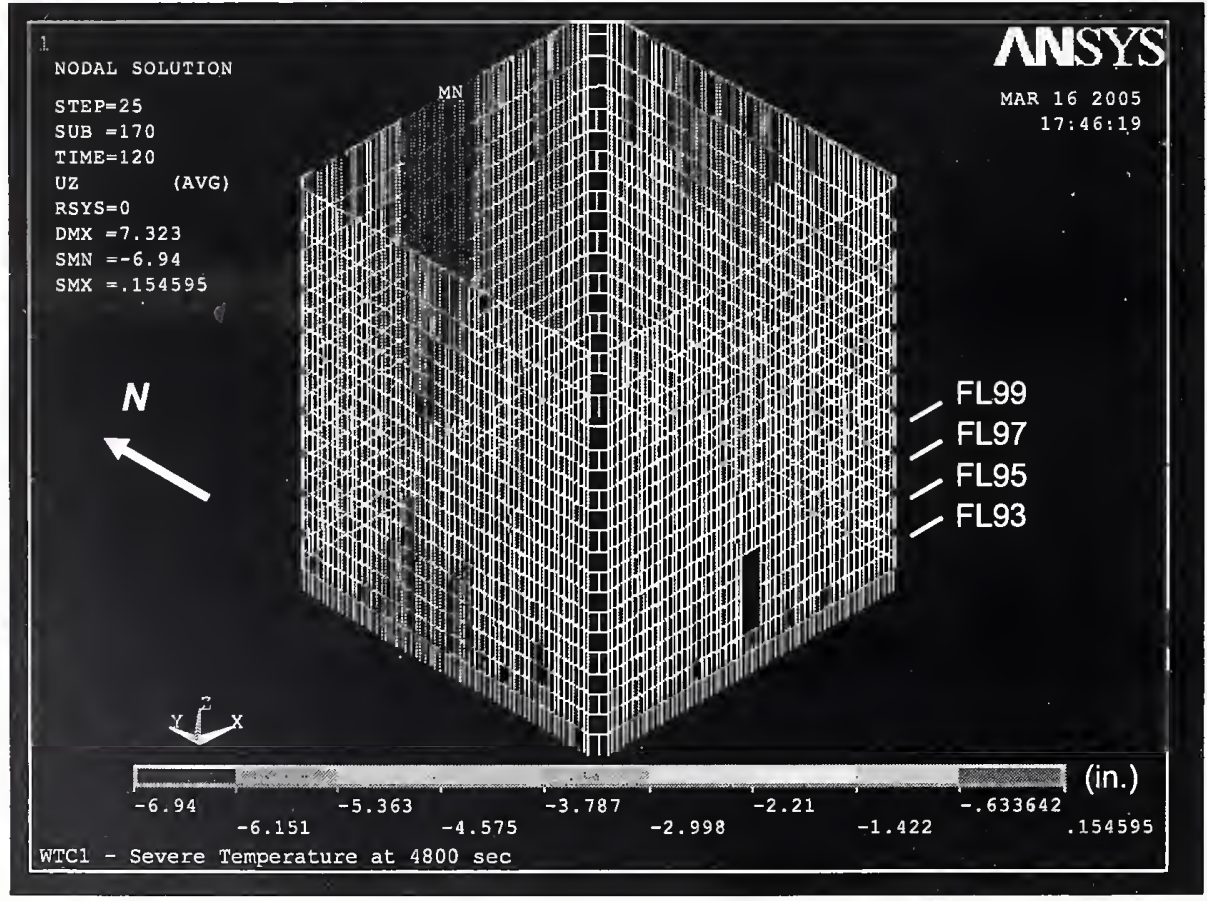

(b) South and west sides

Figure 4-10. Vertical displacement of exterior wall of WTC 1 at $80 \mathrm{~min}$ for Case B conditions (downward displacement is negative). 


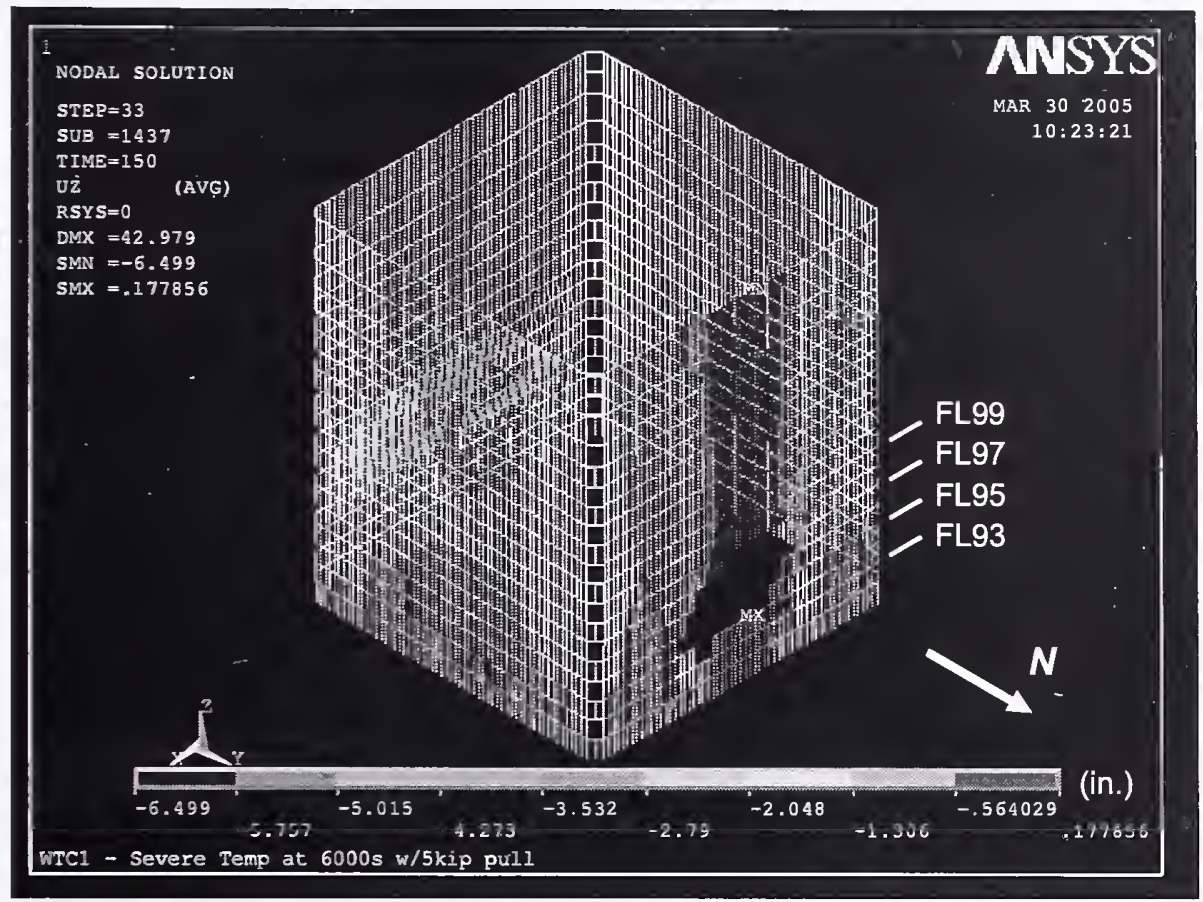

(a) North and east sides

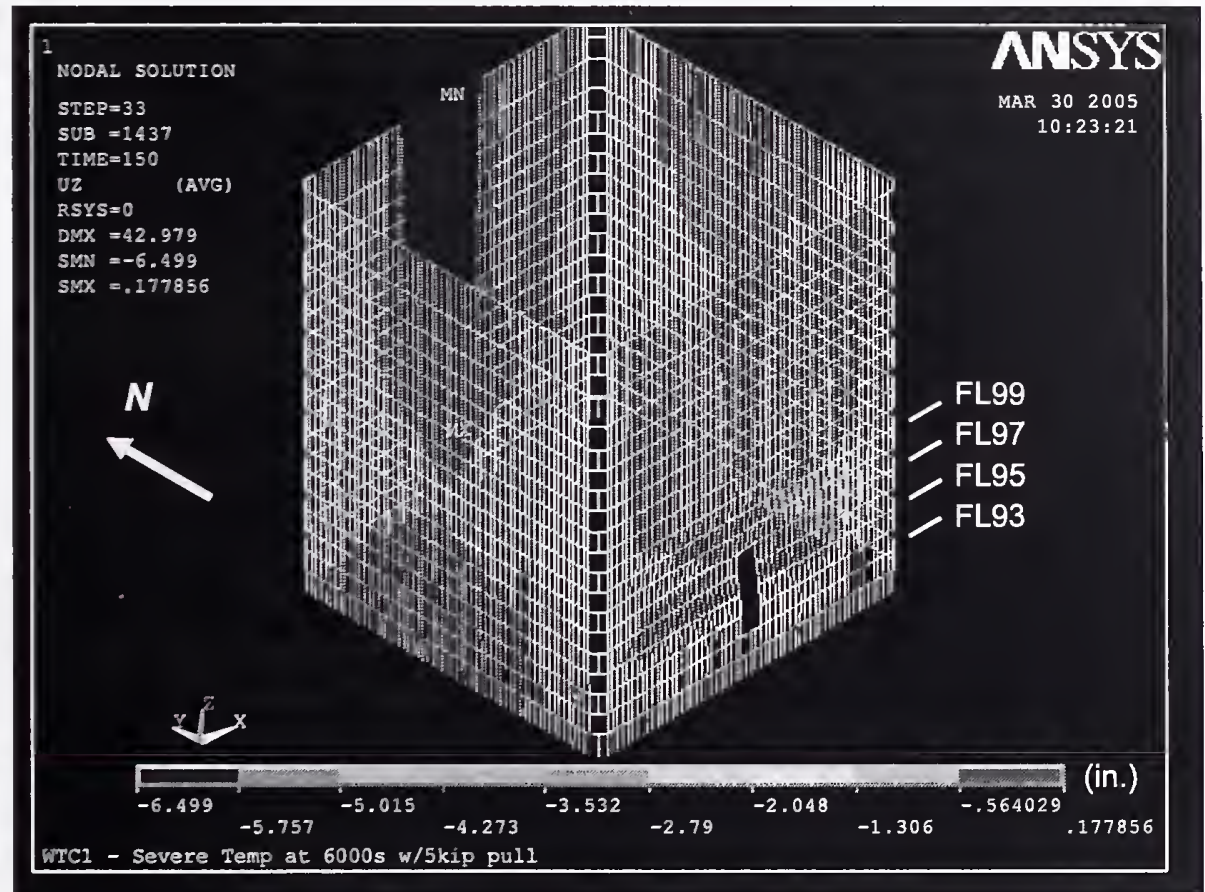

(b) South and west sides

Figure 4-11. Vertical displacement of exterior wall of WTC 1 at $100 \mathrm{~min}$ for Case B conditions (downward displacement is negative). 


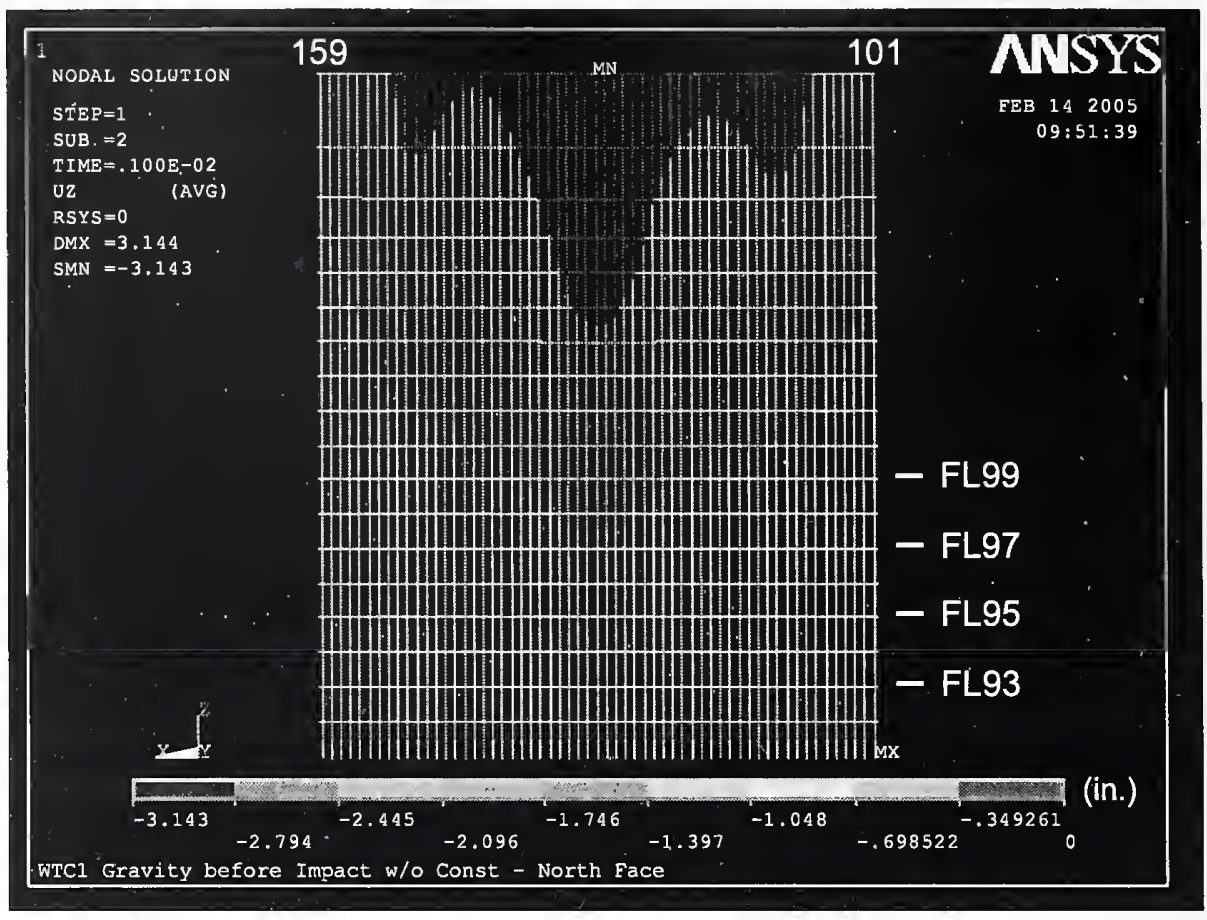

Figure 4-12. Vertical displacement of north wall of WTC 1 before aircraft impact.

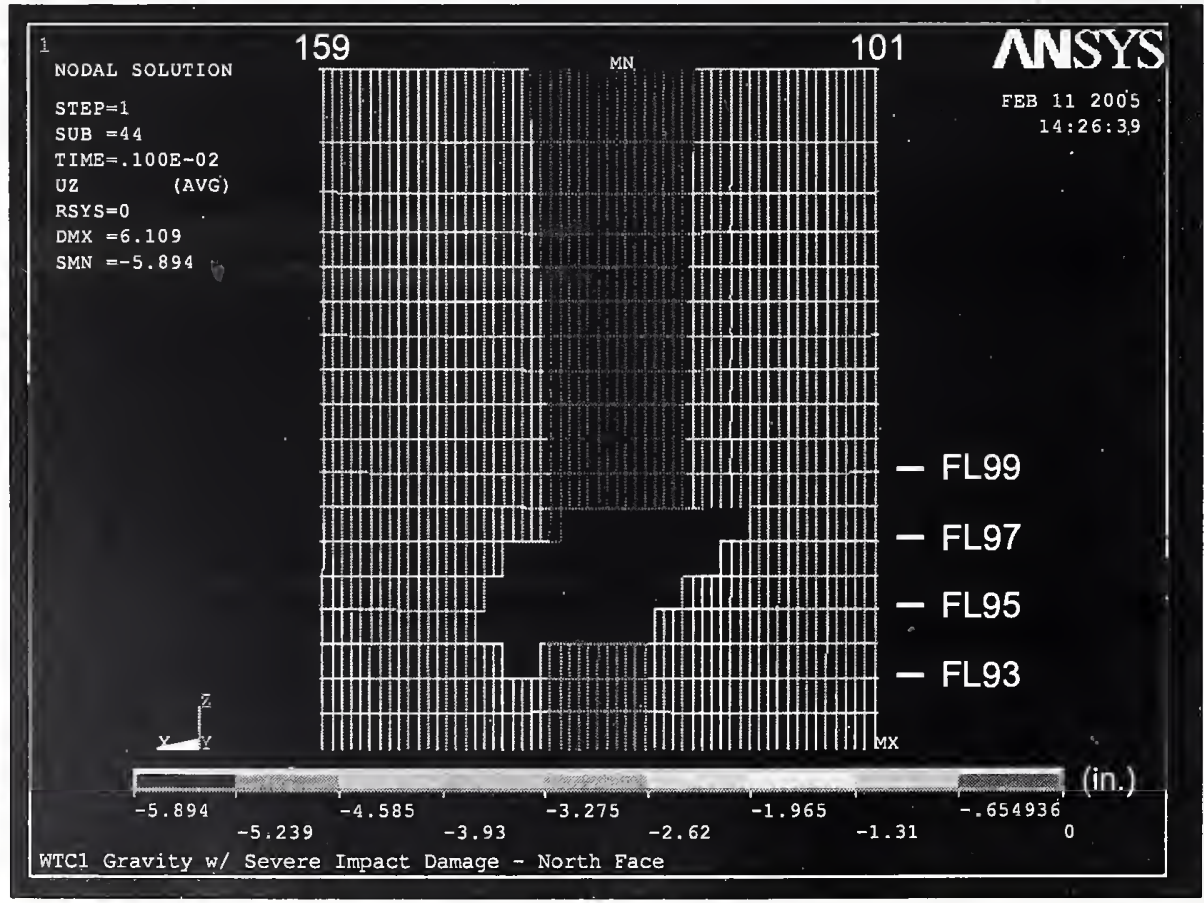

Figure 4-13. Vertical displacement of north wall of WTC 1 after aircraft impact for Case B conditions (downward displacement is negative). 


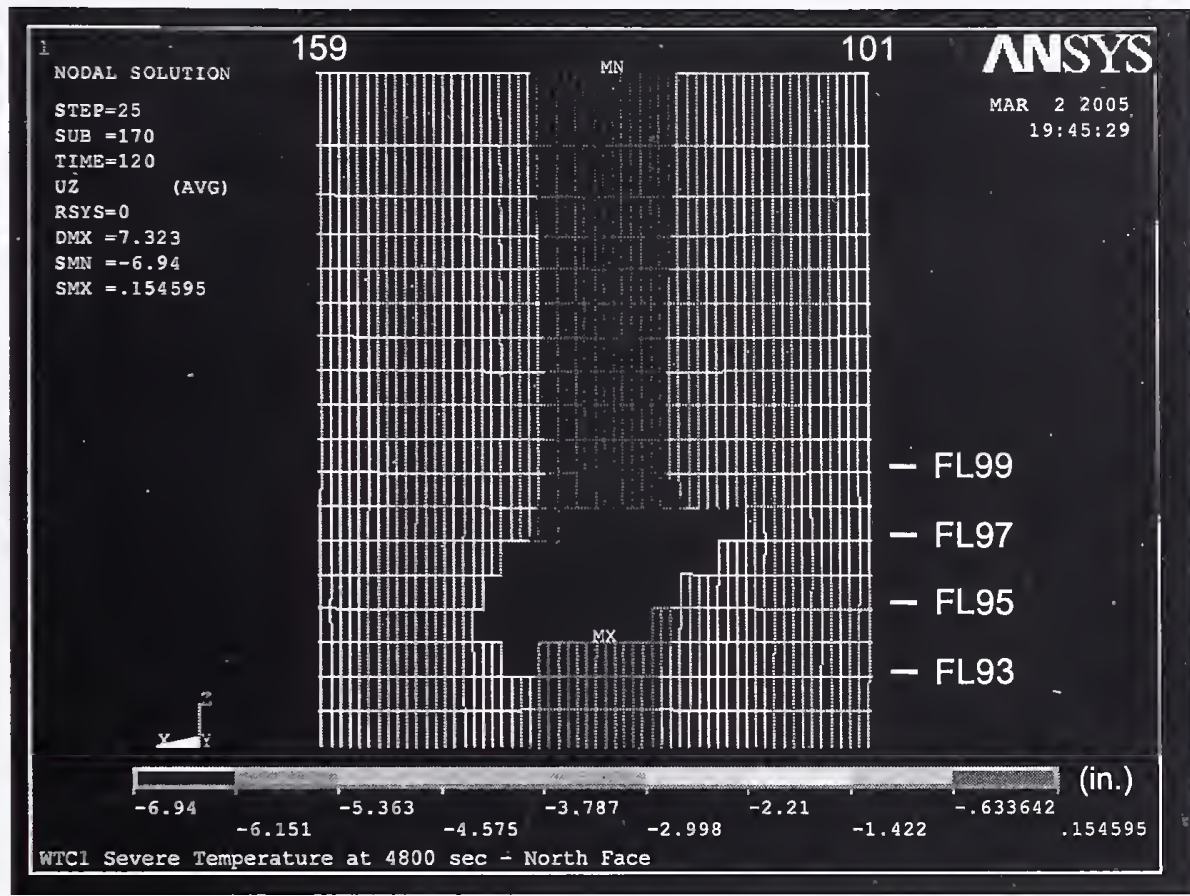

Figure 4-14. Vertical displacement of north wall of WTC 1 at $80 \mathrm{~min}$ for Case B conditions (downward displacement is negative).

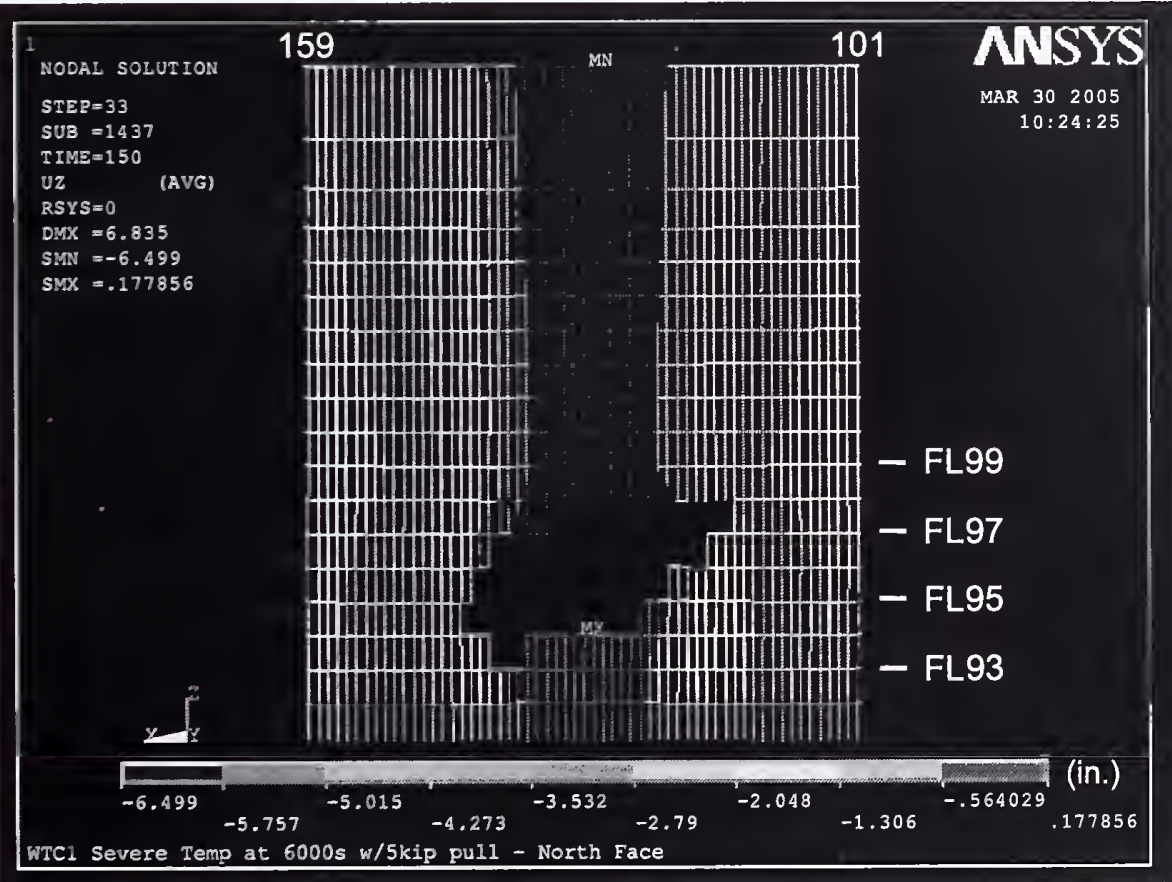

Figure 4-15. Vertical displacement of north wall of WTC 1 at 100 min for Case B conditions with 5 kip pull-in forces (downward displacement is negative). 


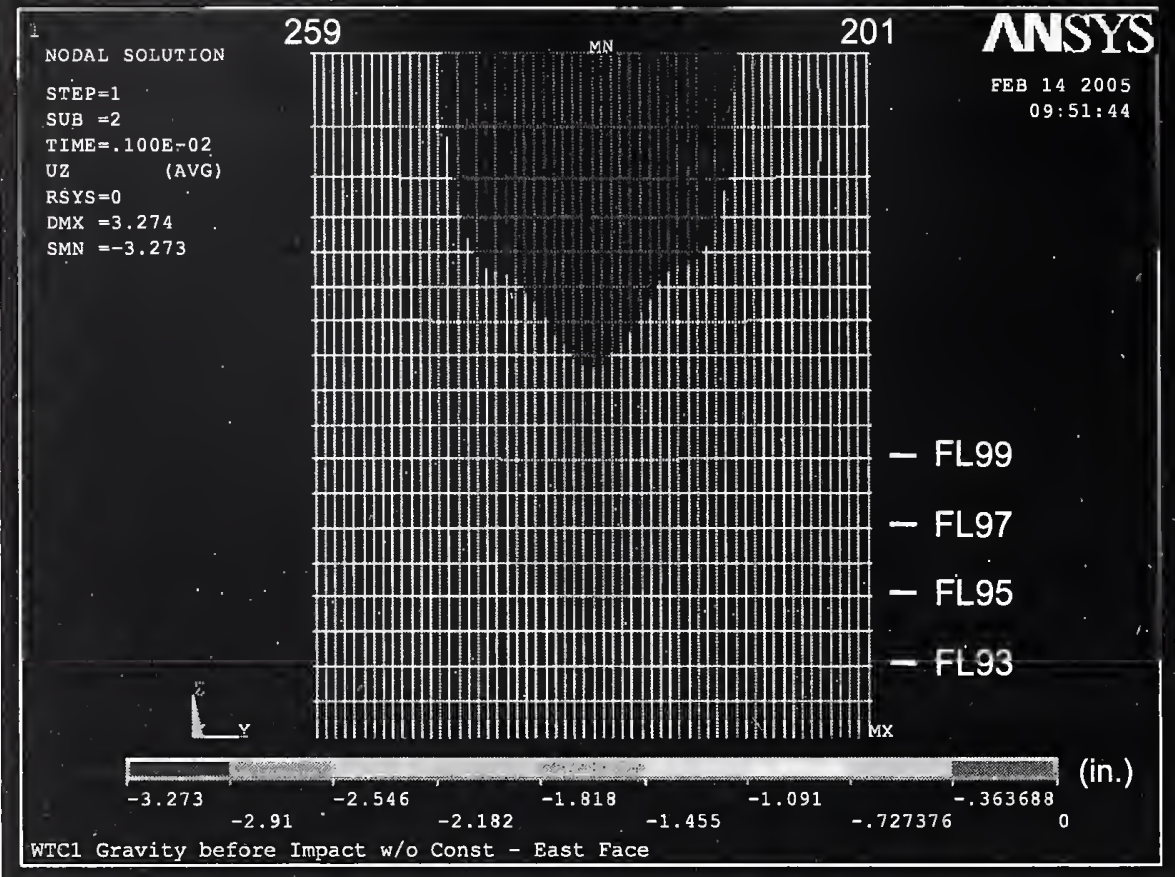

Figure 4-16. Vertical displacement of east wall of WTC 1 before aircraft impact (downward displacement is negative).

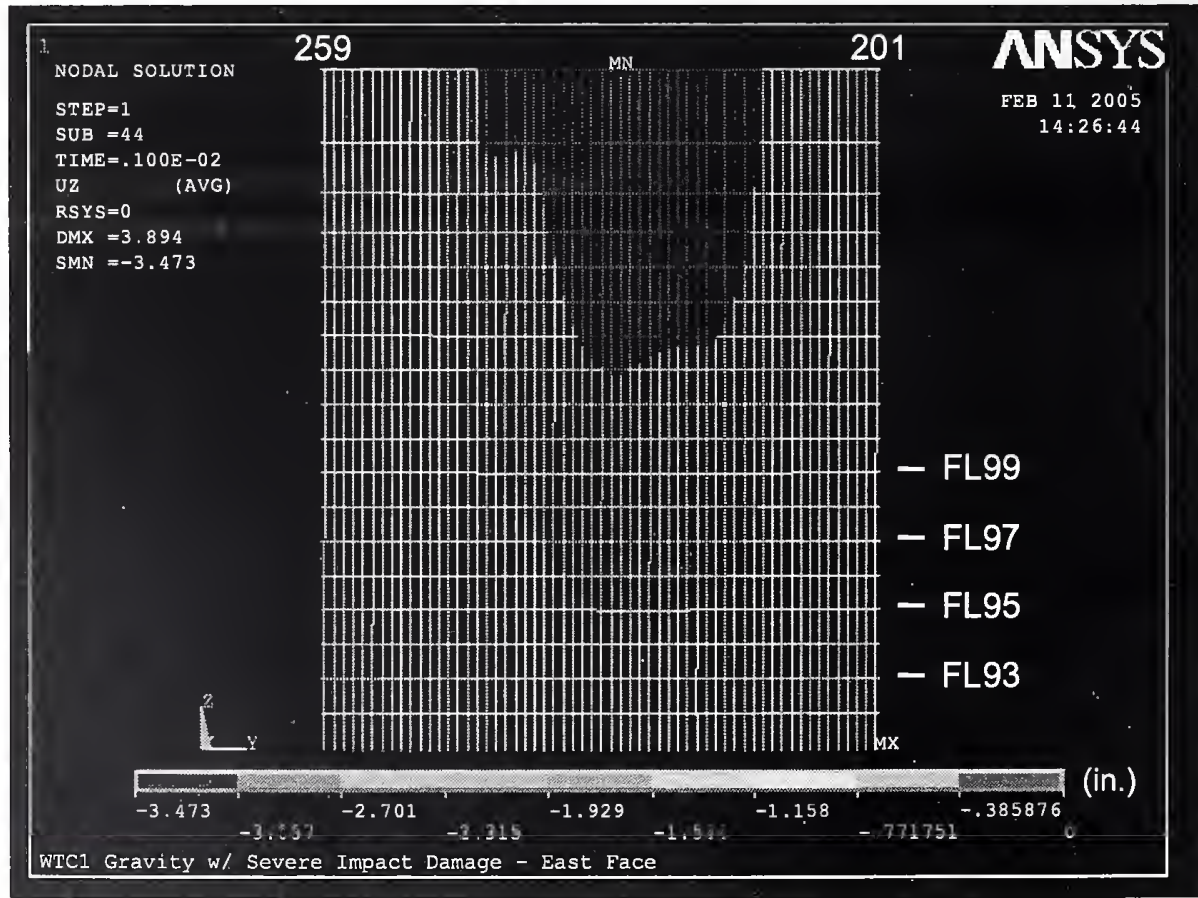

Figure 4-17. Vertical displacement of east wall of WTC 1 after aircraft impact for Case B conditions (downward displacement is negative). 


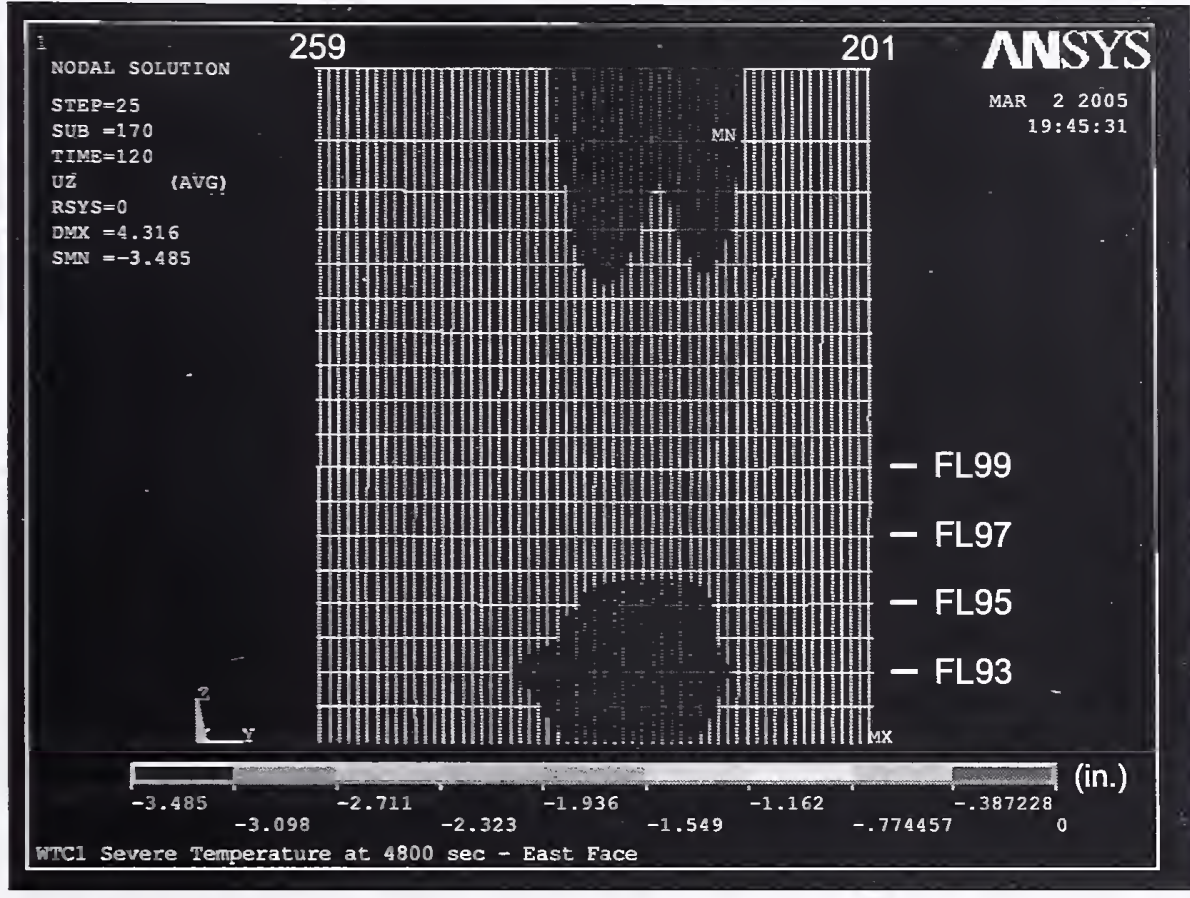

Figure 4-18. Vertical displacement of east wall of WTC 1 at $80 \mathrm{~min}$ for Case B conditions (downward displacement is negative).

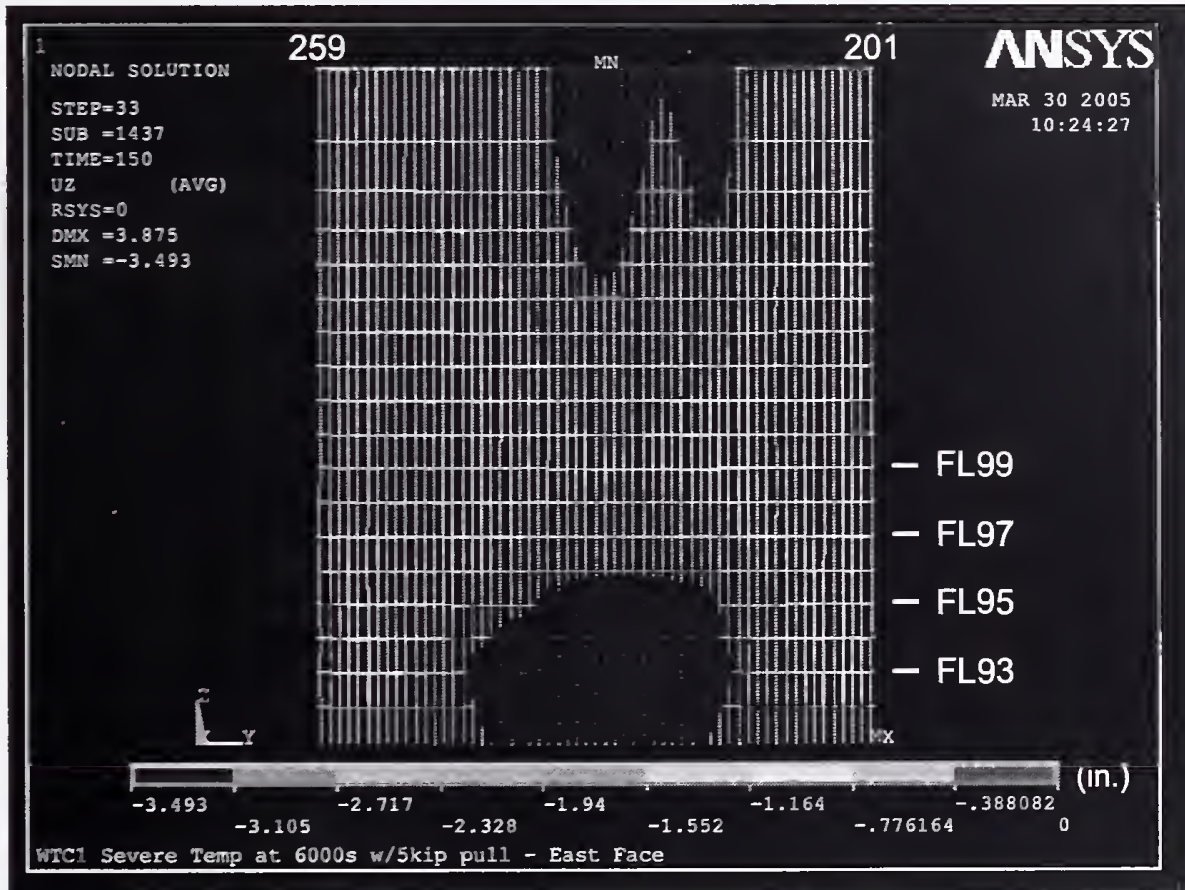

Figure 4-19. Vertical displacement of east wall of WTC 1 at $100 \mathrm{~min}$ for Case B conditions with 5 kip pull-in forces (downward displacement is negative). 


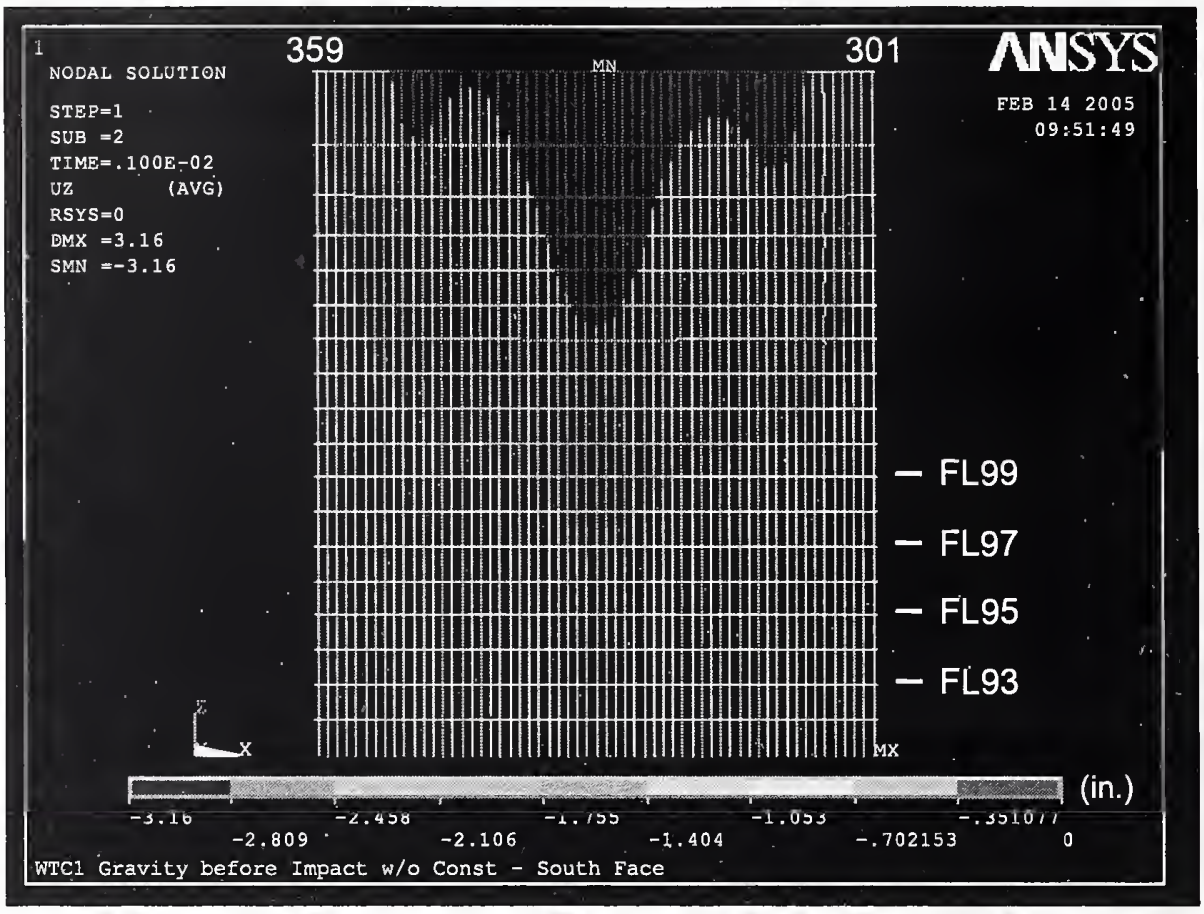

Figure 4-20. Vertical displacement of south wall of WTC 1 before aircraft impact (downward displacement is negative).

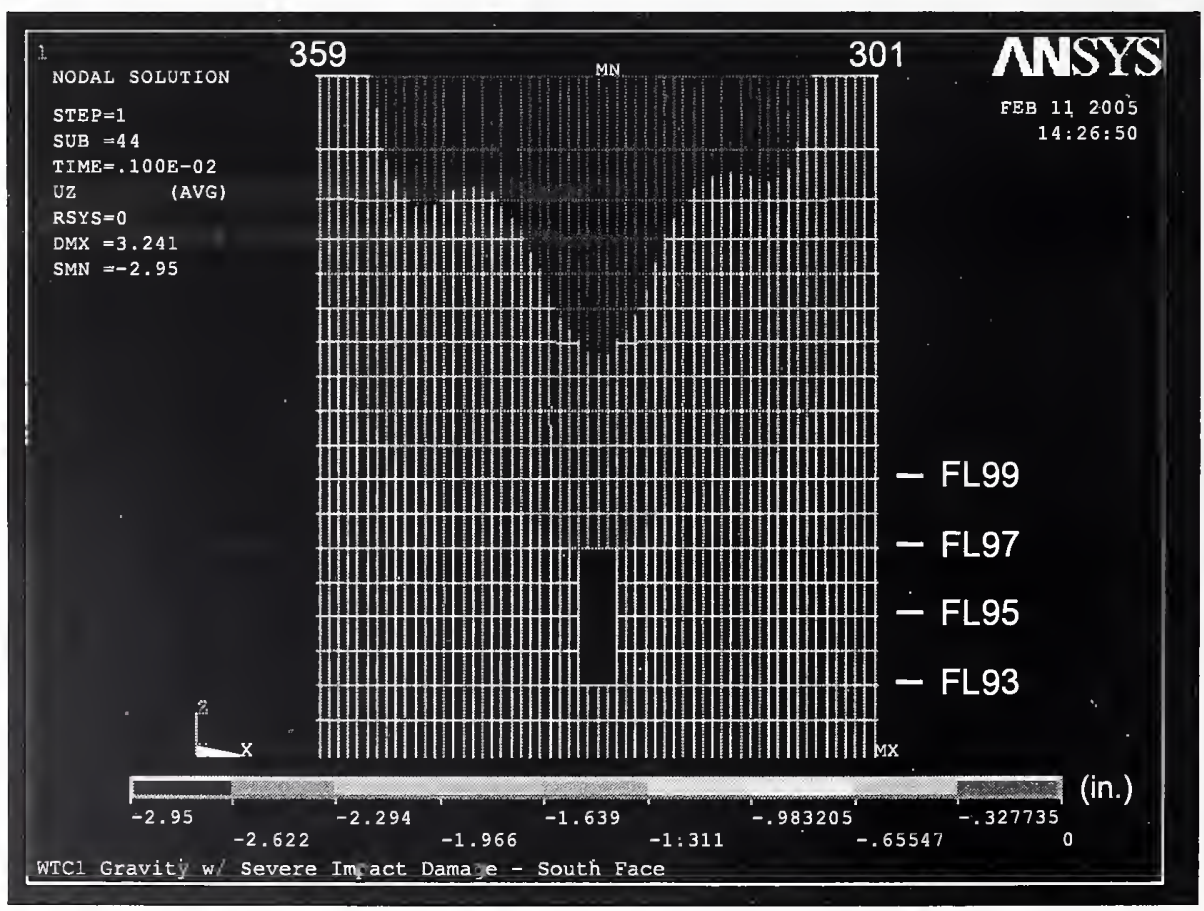

Figure 4-21. Vertical displacement of south wall of WTC 1 after aircraft impact for Case $B$ conditions (downward displacement is negative). 


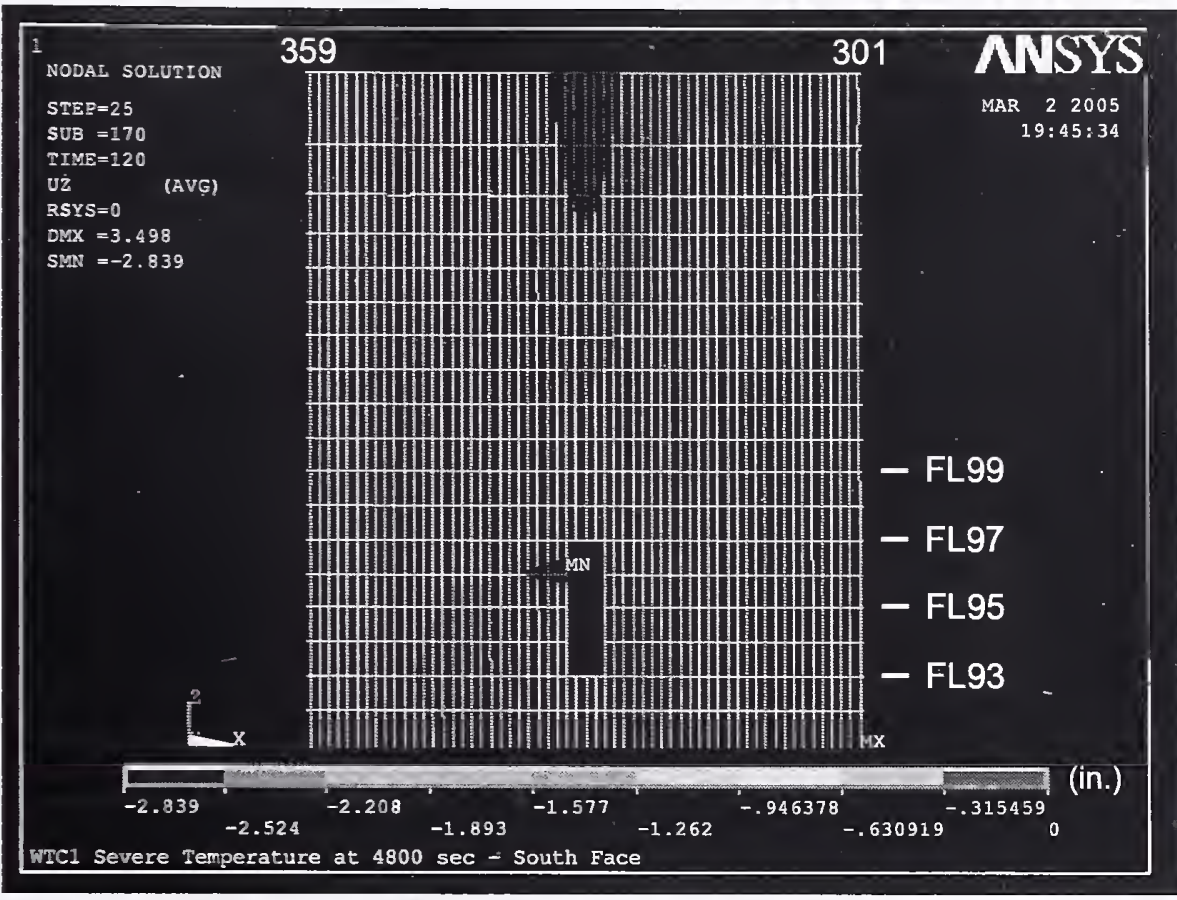

Figure 4-22. Vertical displacement of south wall of WTC 1 at $80 \mathrm{~min}$ for Case B conditions (downward displacement is negative).

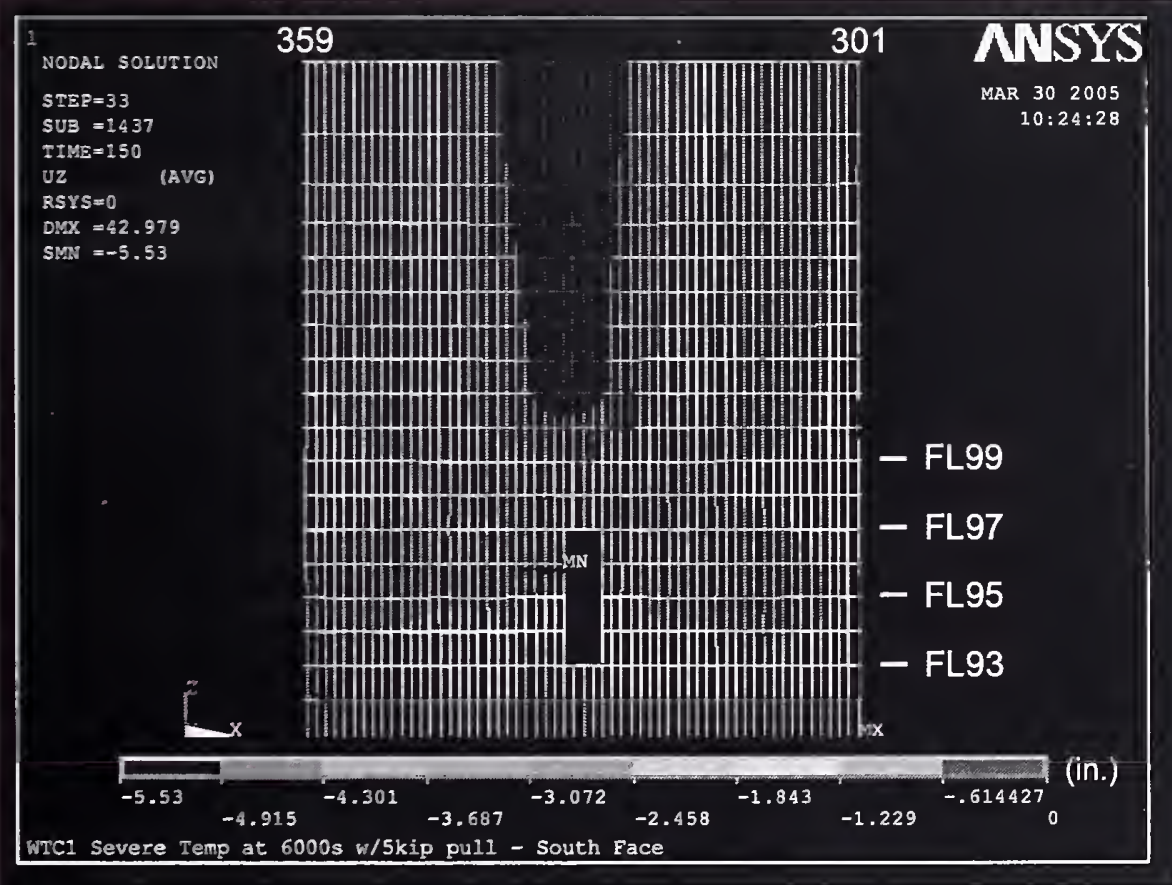

Figure 4-23. Vertical displacement of south wall of WTC 1 at $100 \mathrm{~min}$ for Case B conditions with 5 kip pull-in forces (downward displacement is negative). 


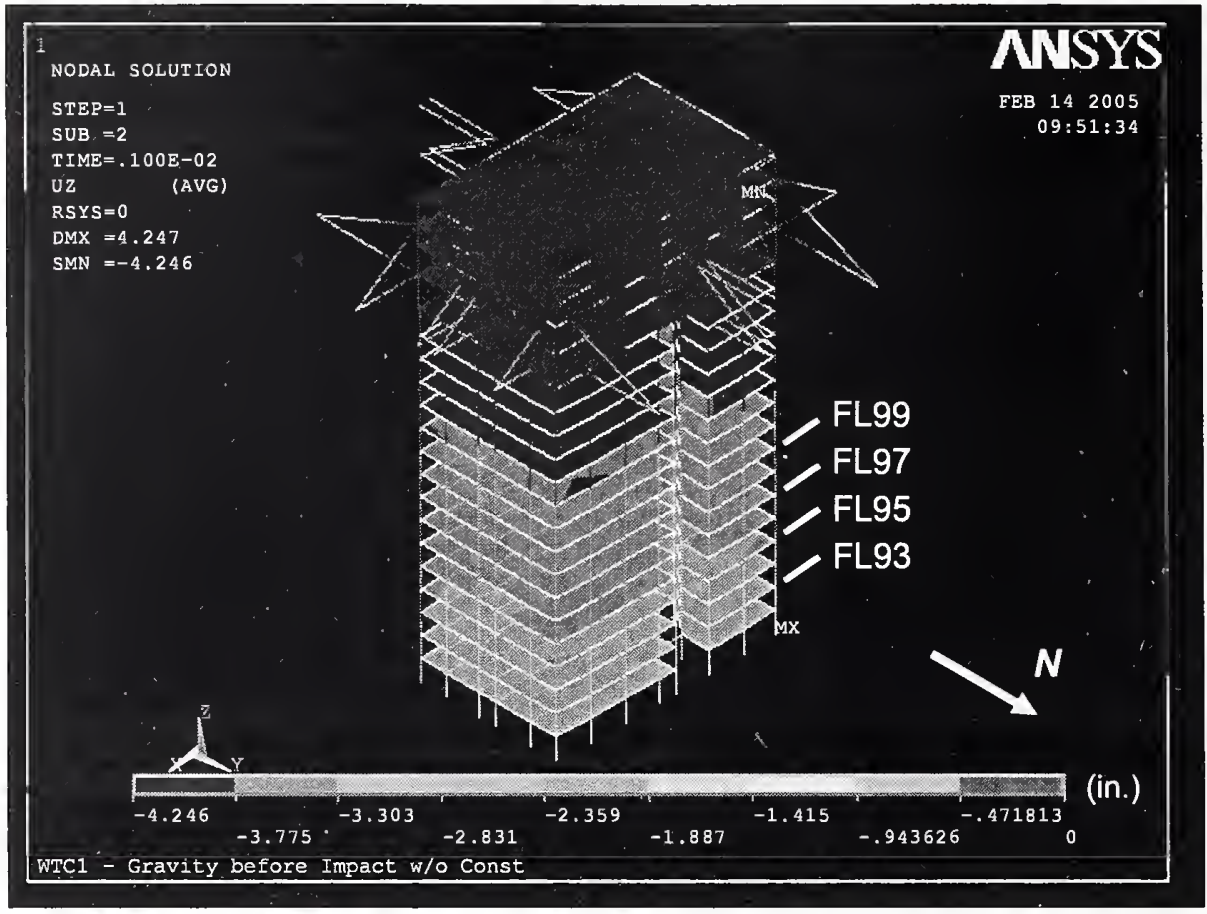

(a) North and east sides

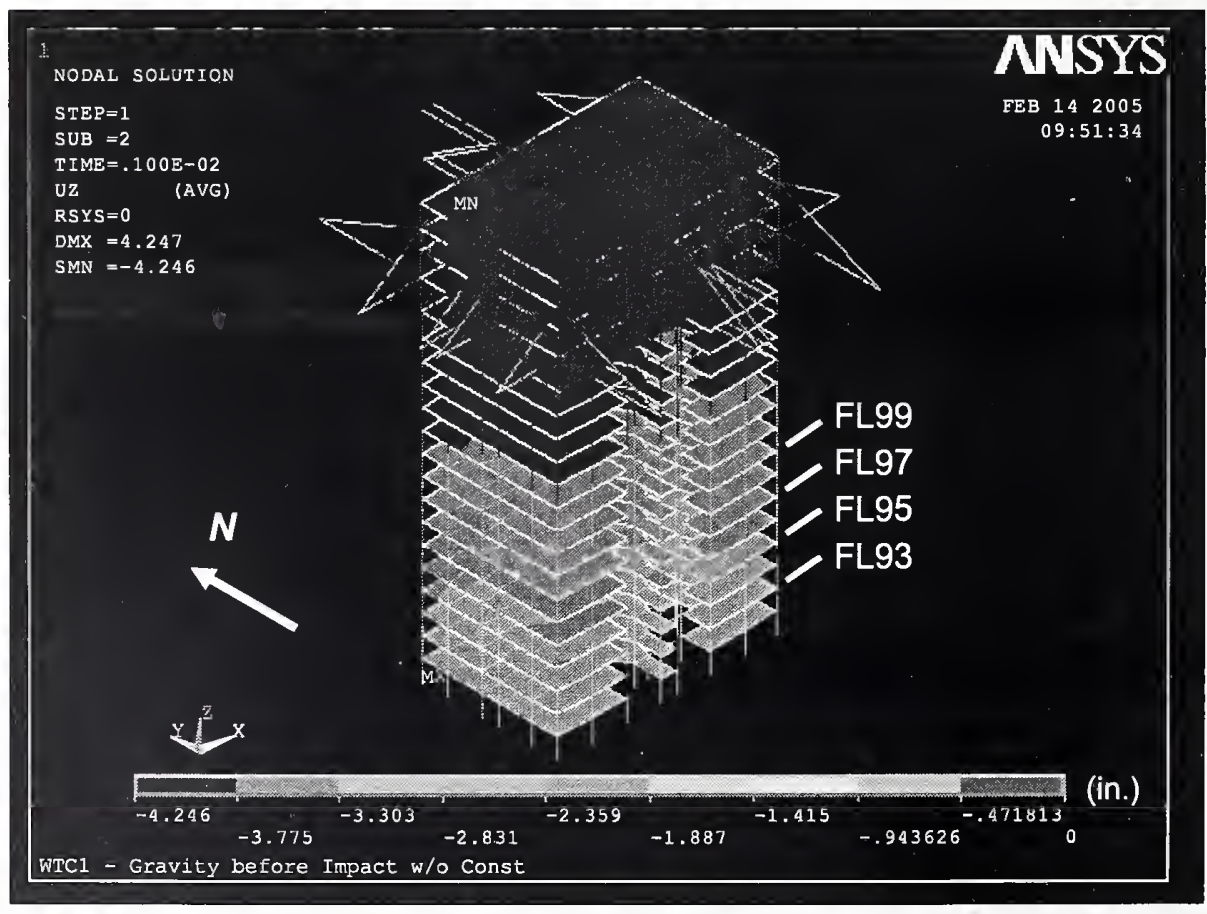

(b) South and west sides

Figure 4-24. Vertical displacement of core of WTC 1 before aircraft impact (downward displacement is negative). 


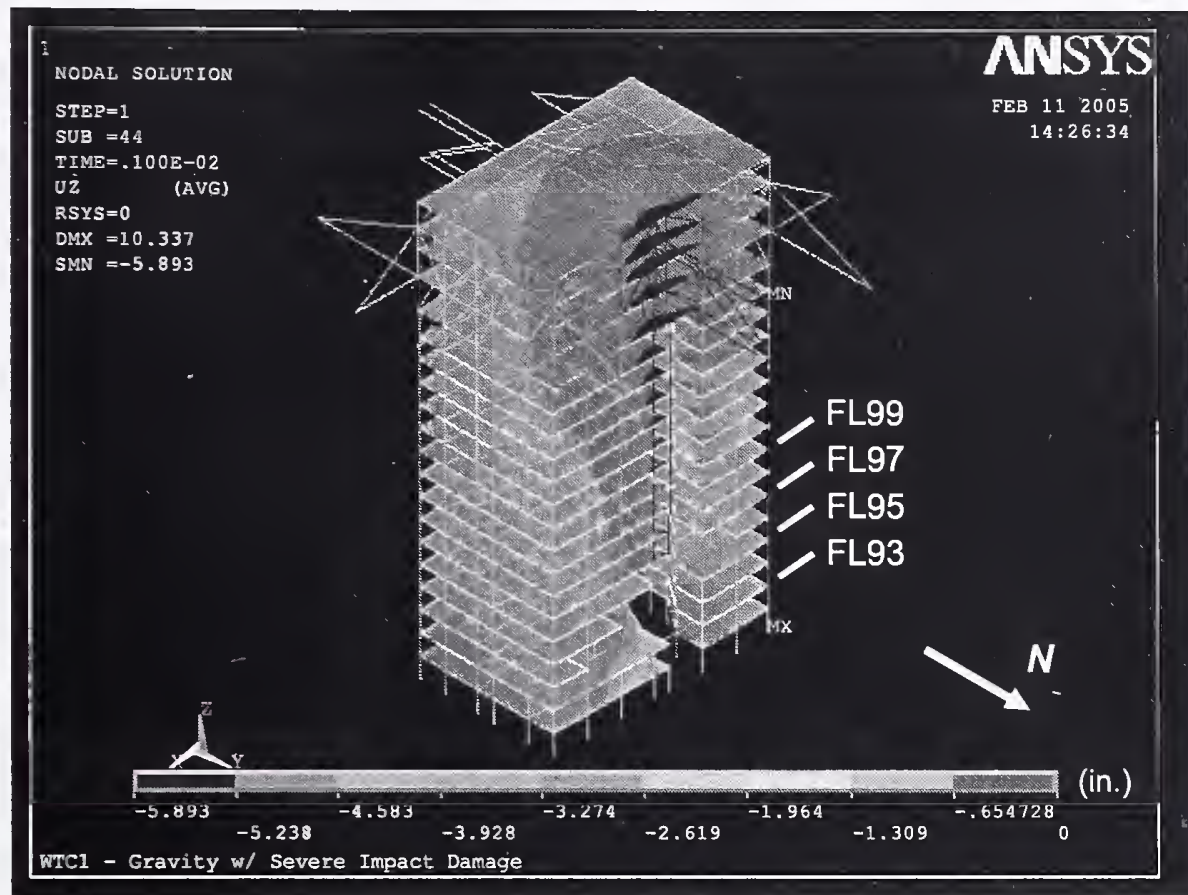

(a) North and east sides

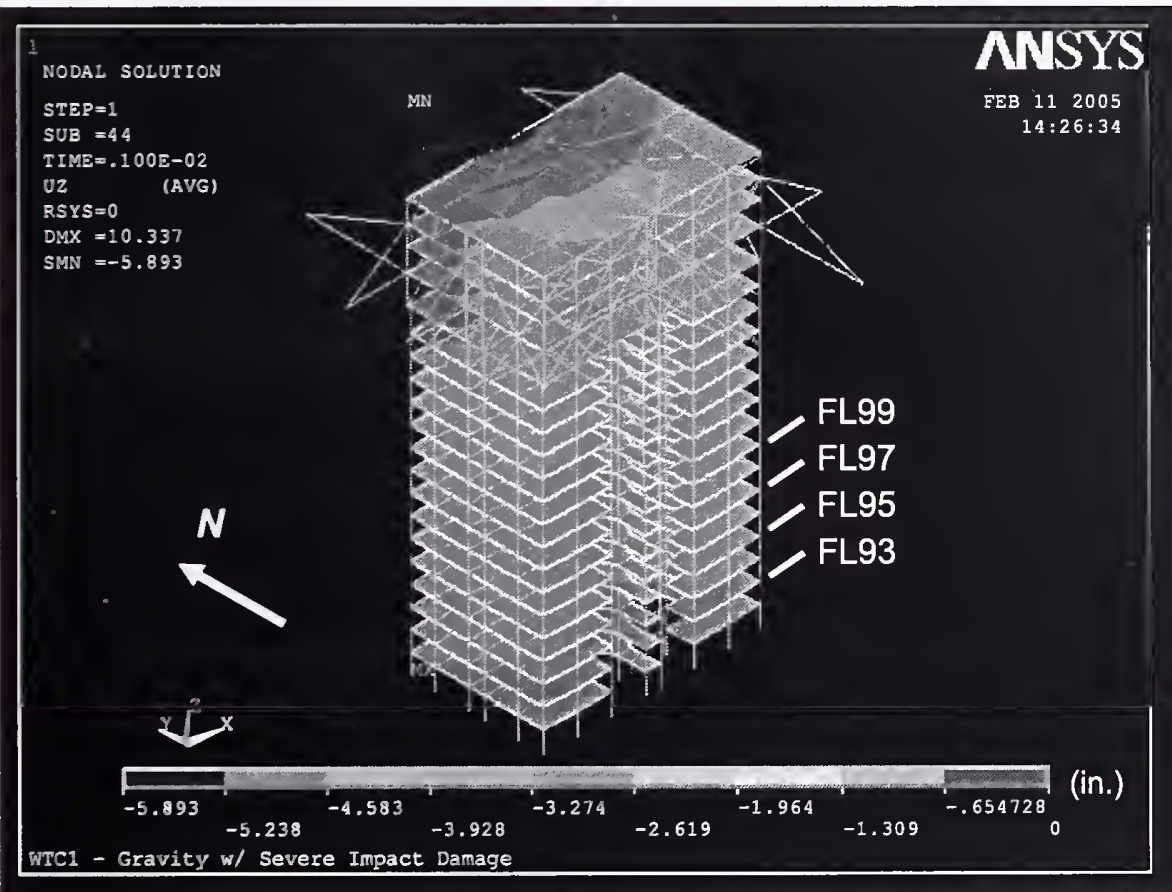

(b) South and west sides

Figure 4-25. Vertical displacement of core of WTC 1 after aircraft impact for Case B conditions (downward displacement is negative). 


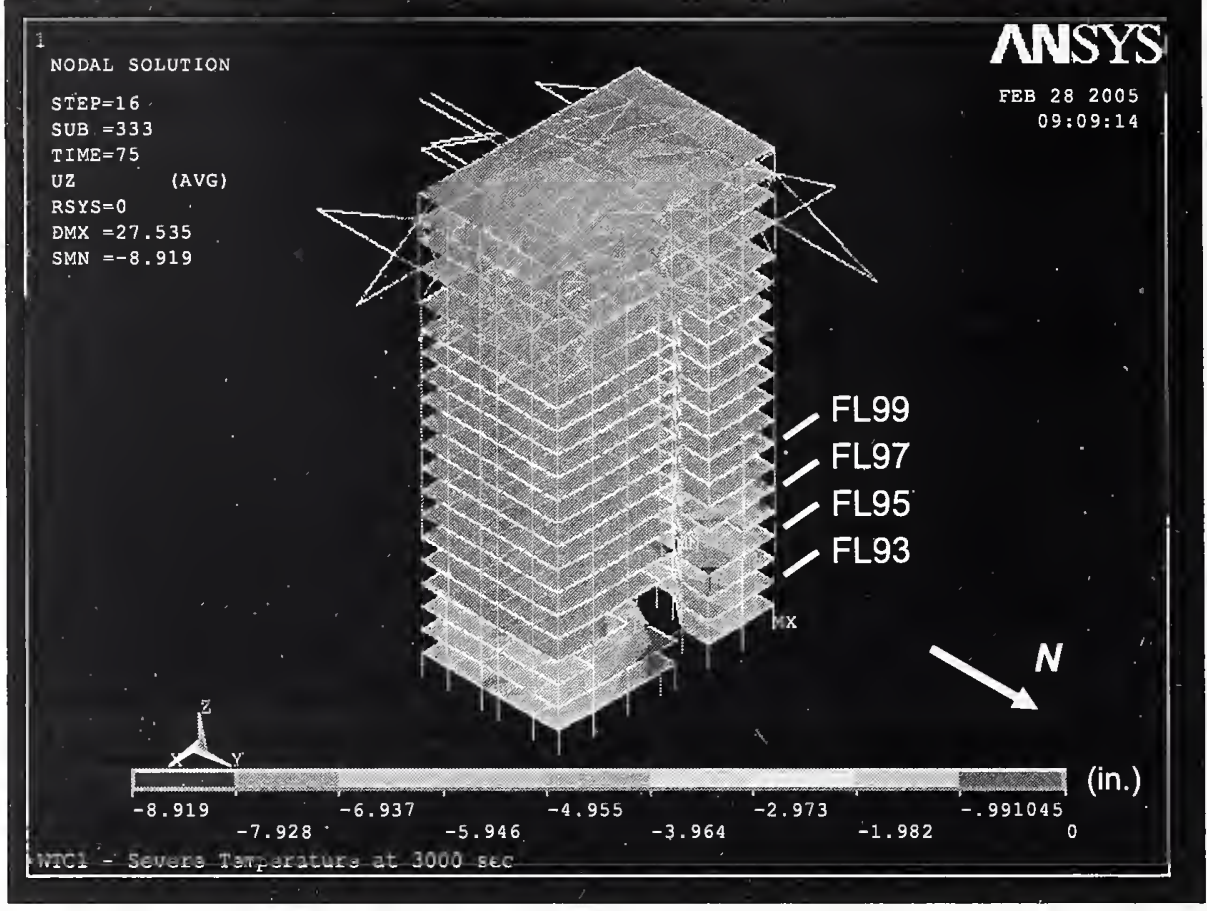

(a) North and east sides

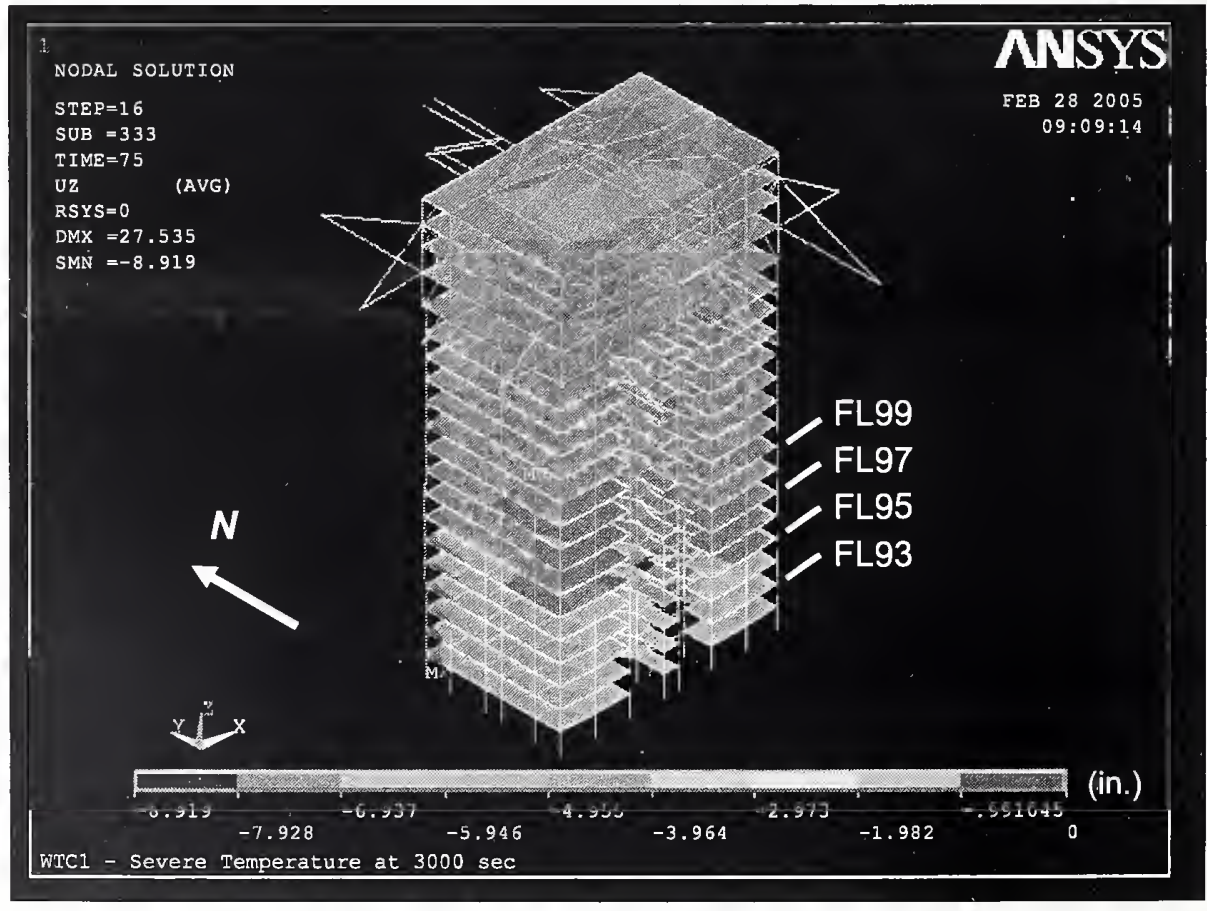

(b) South and west sides

Figure 4-26. Vertical displacement of core of WTC 1 at 50 min for Case B conditions (downward displacement is negative). 


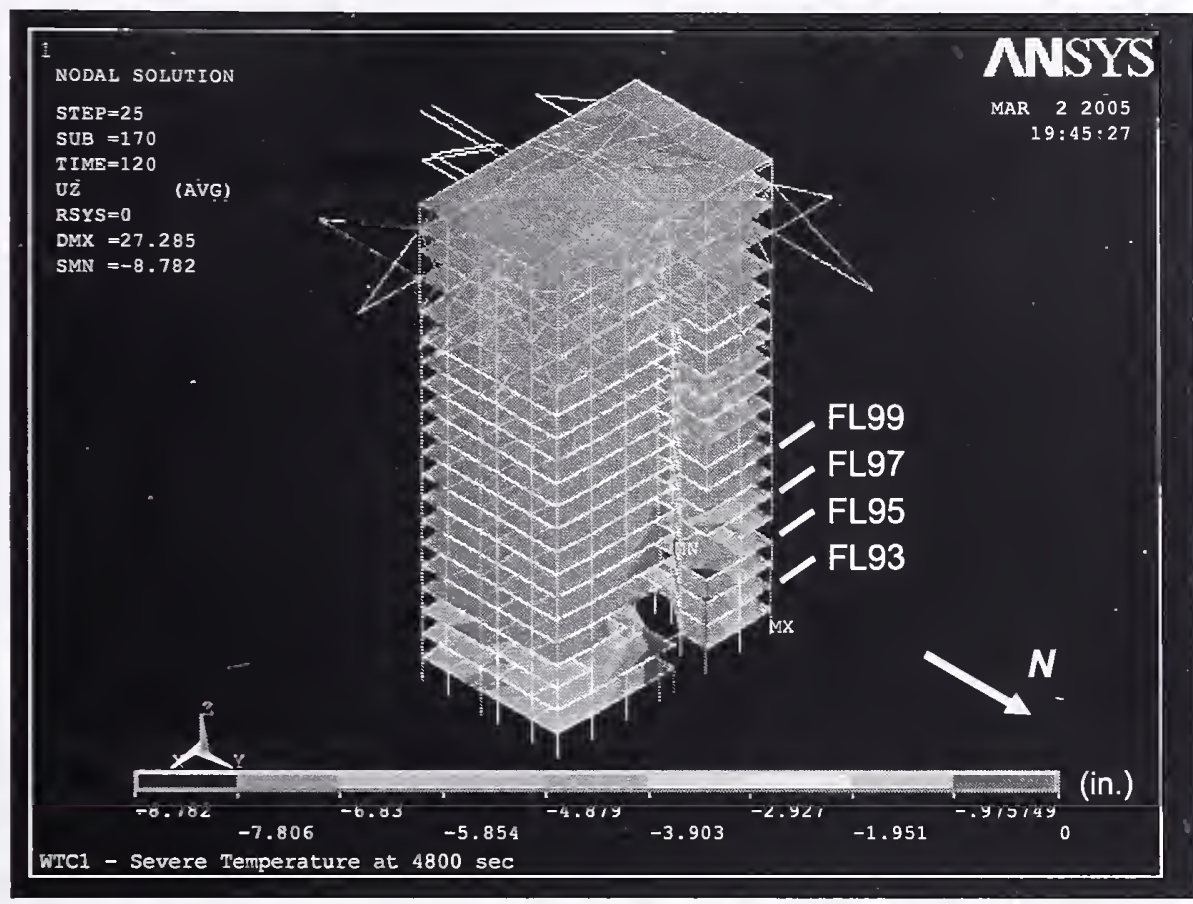

(a) North and east sides

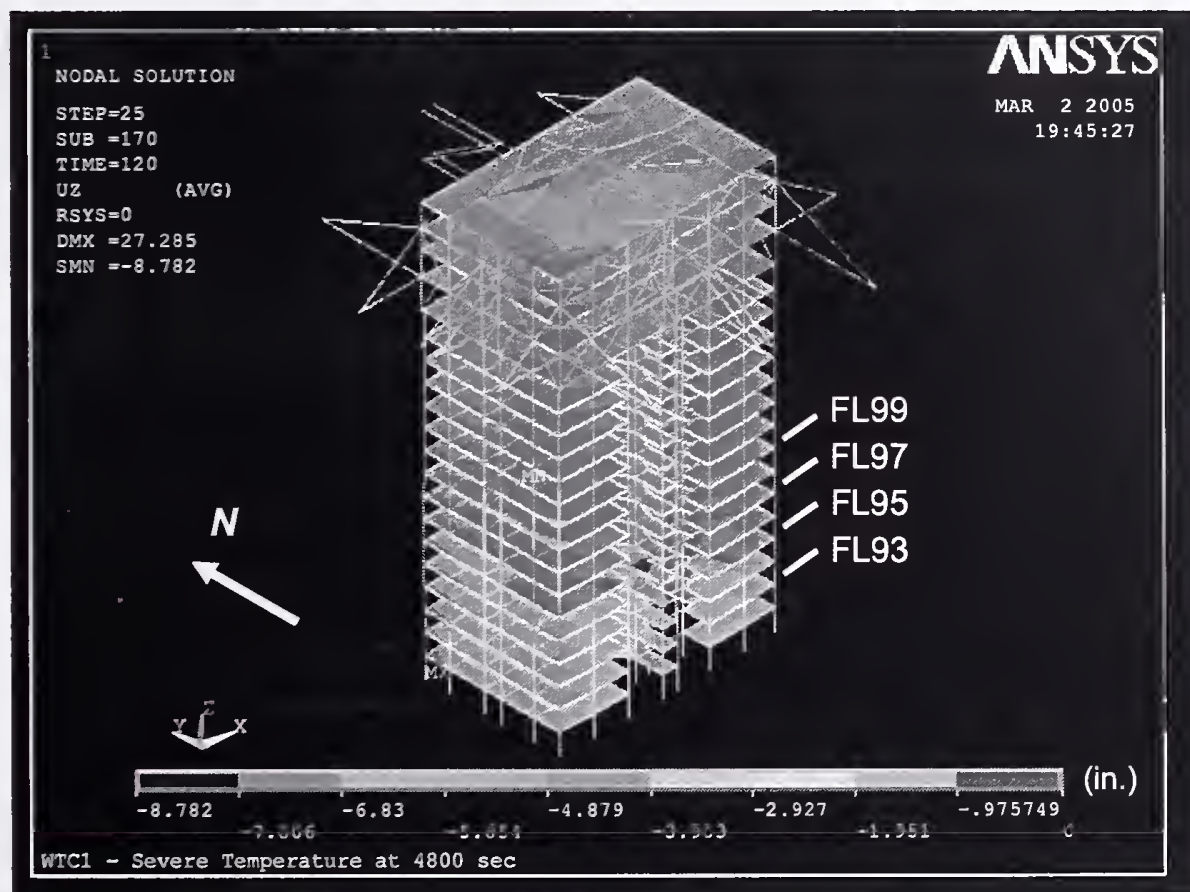

(b) South and west sides

Figure 4-27. Vertical displacement of core of WTC 1 at $80 \mathrm{~min}$ for Case B conditions (downward displacement is negative). 


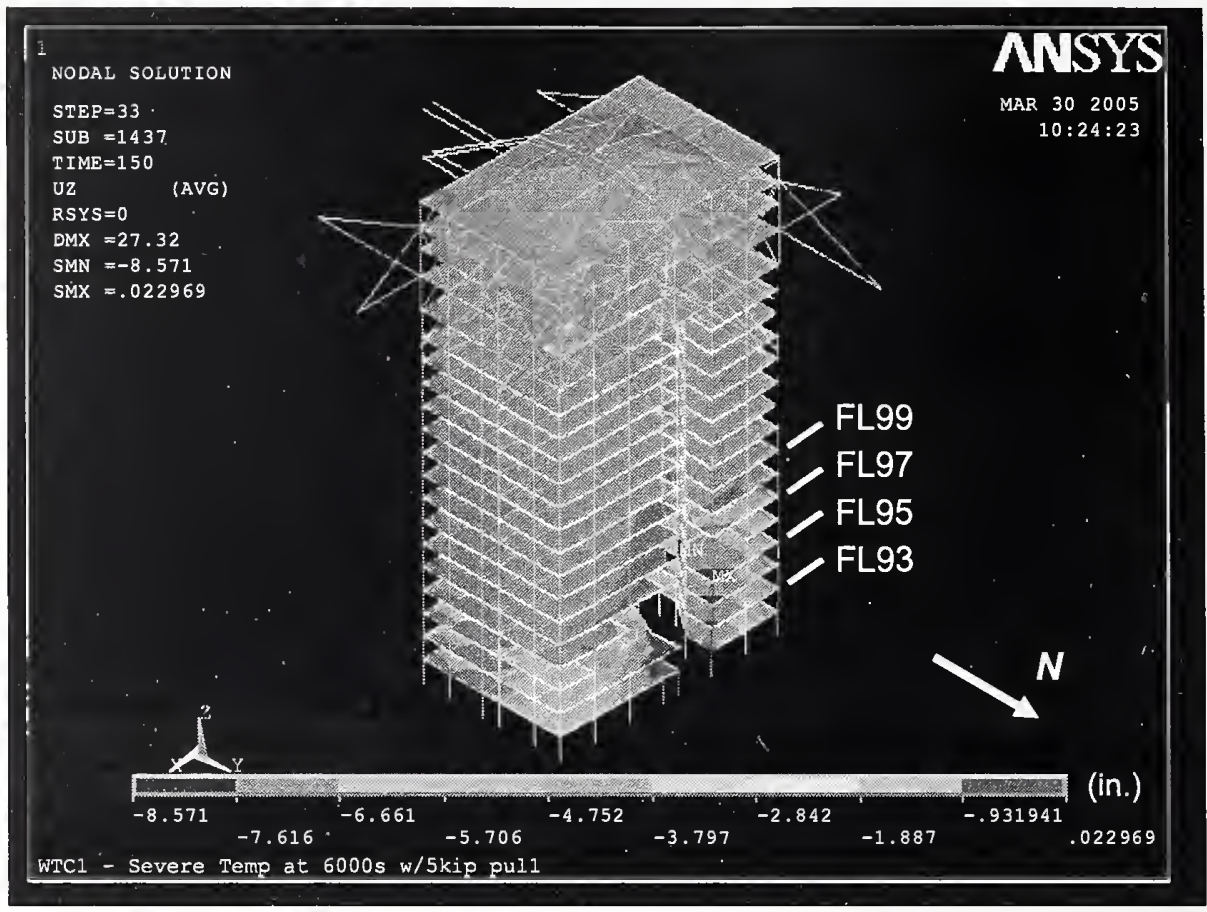

(a) North and east sides

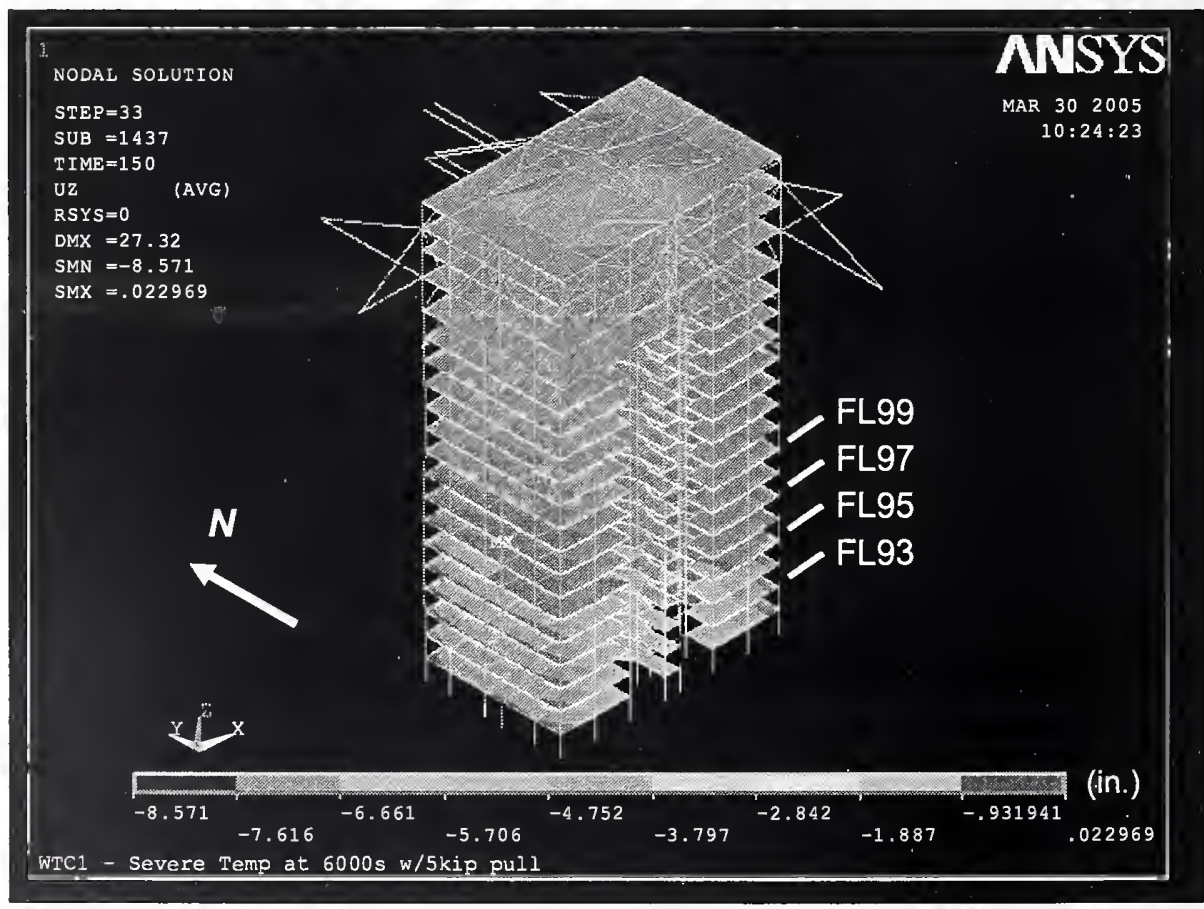

(b) South and west sides

Figure 4-28. Vertical displacement of core of WTC 1 at $100 \mathrm{~min}$ for Case B conditions with 5 kip pull-in forces (downward displacement is negative). 


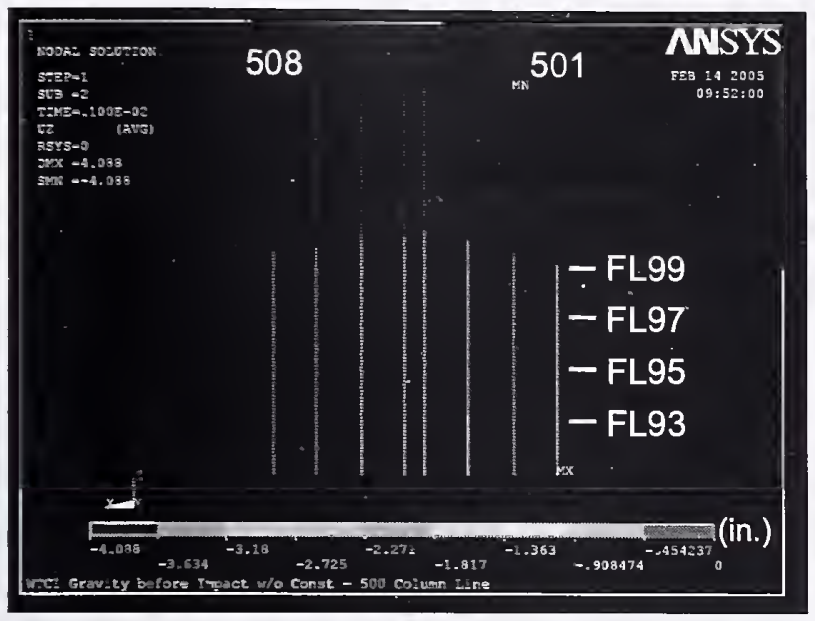

(a) 500 series columns

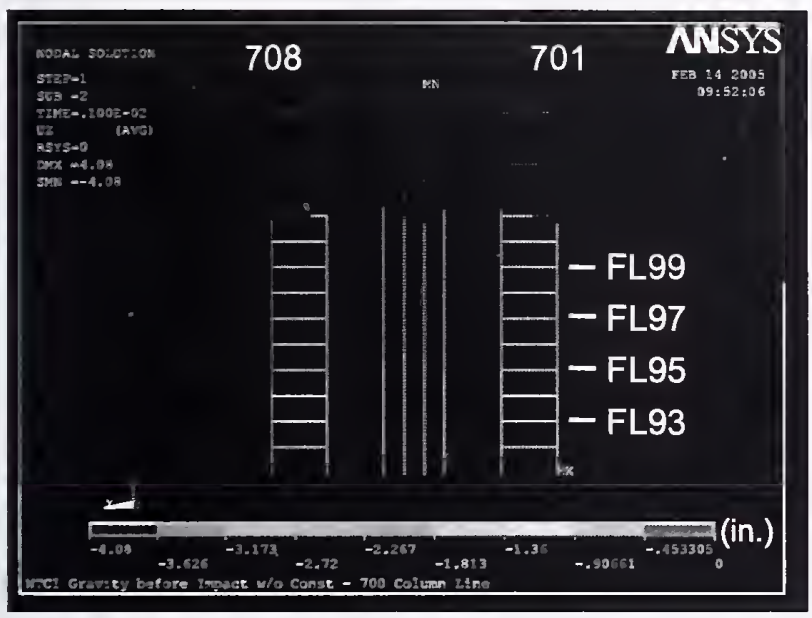

(c) 700 series columns

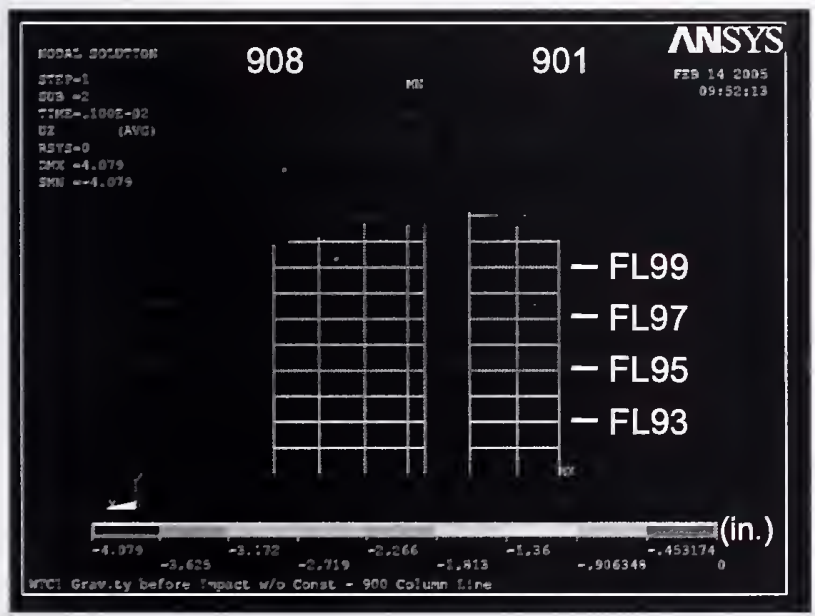

(e) 900 series columns

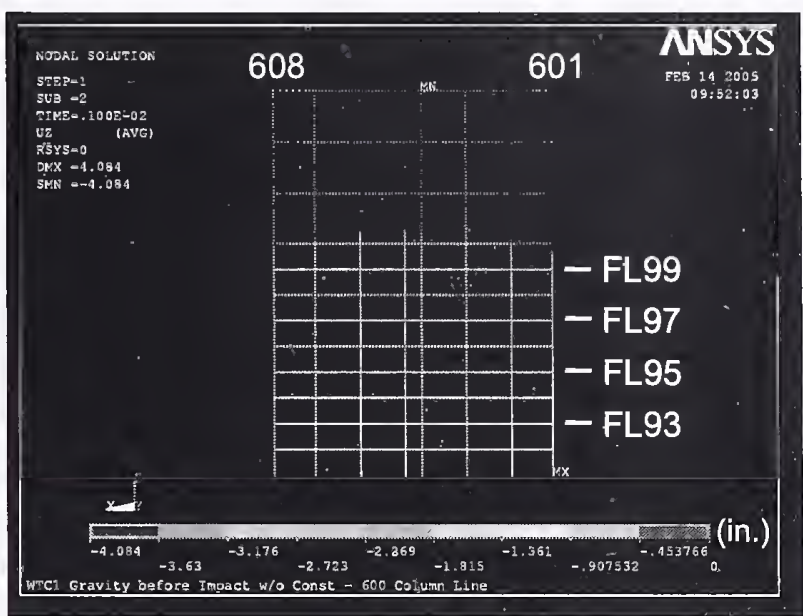

(b) 600 series columns

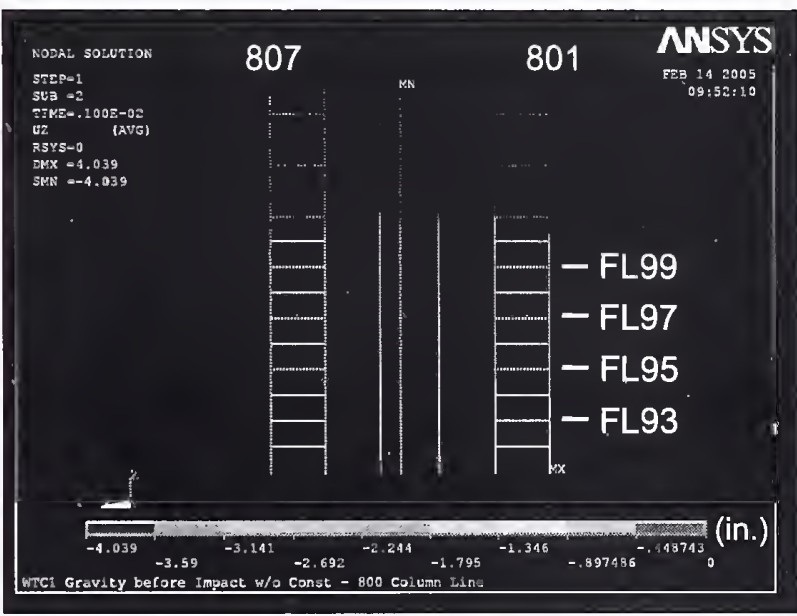

(d) 800 series columns

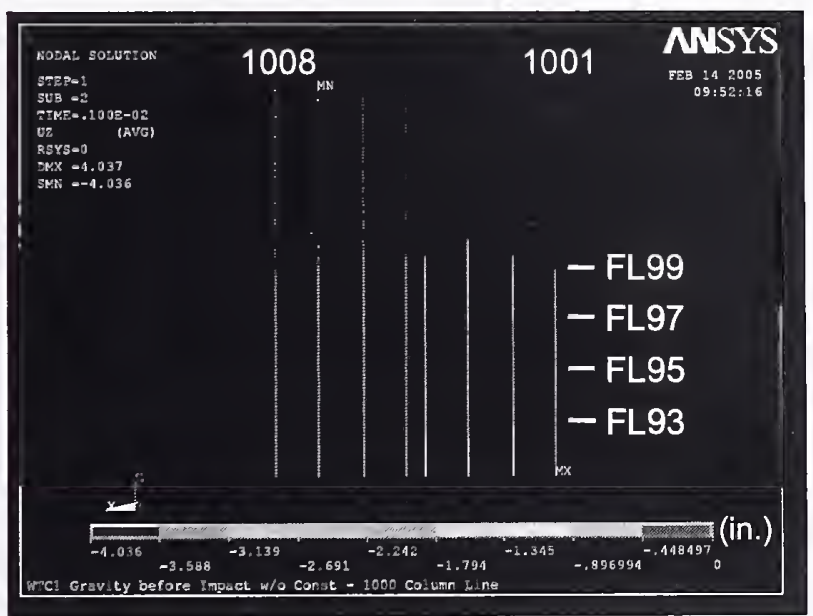

(f) 1000 series columns

Figure 4-29. Vertical displacement of core columns of WTC 1 before aircraft impact (downward displacement is negative). 


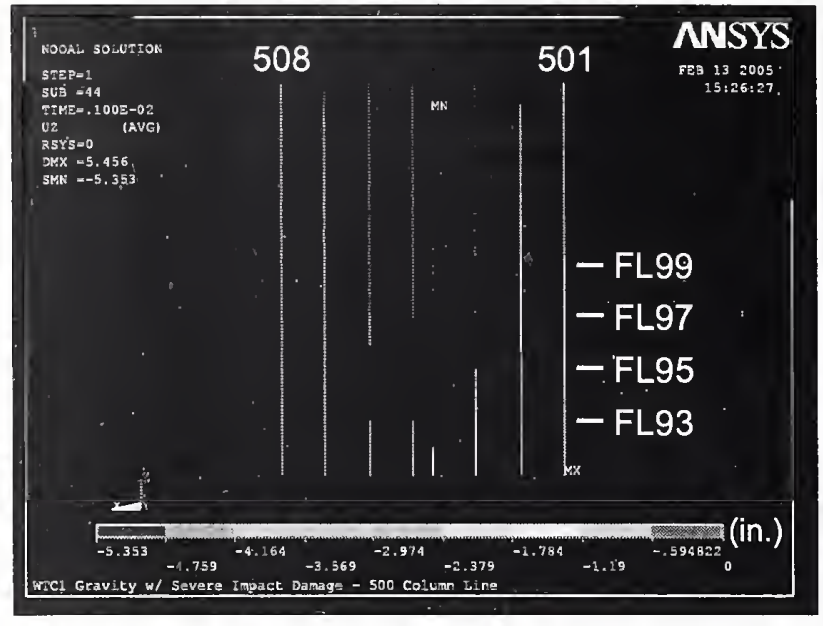

(a) 500 series columns

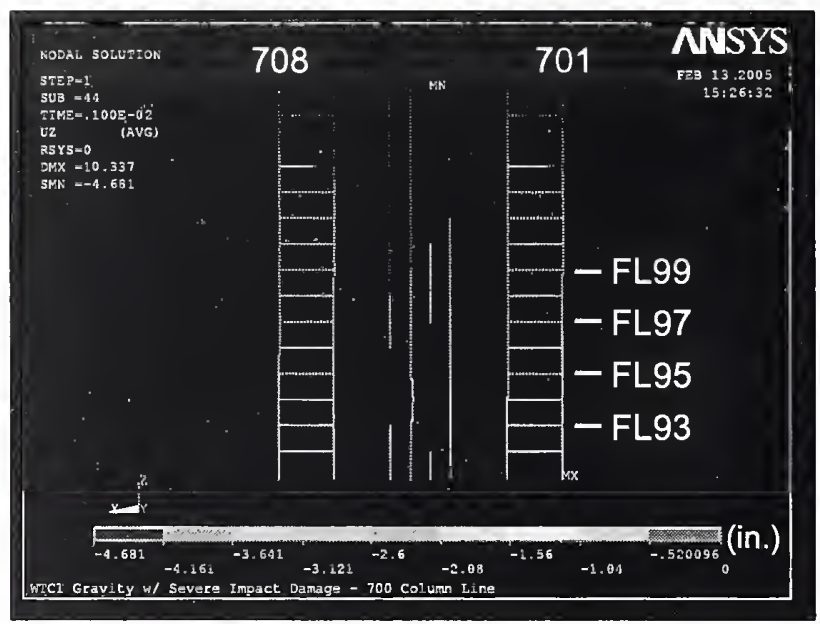

(c) 700 series columns

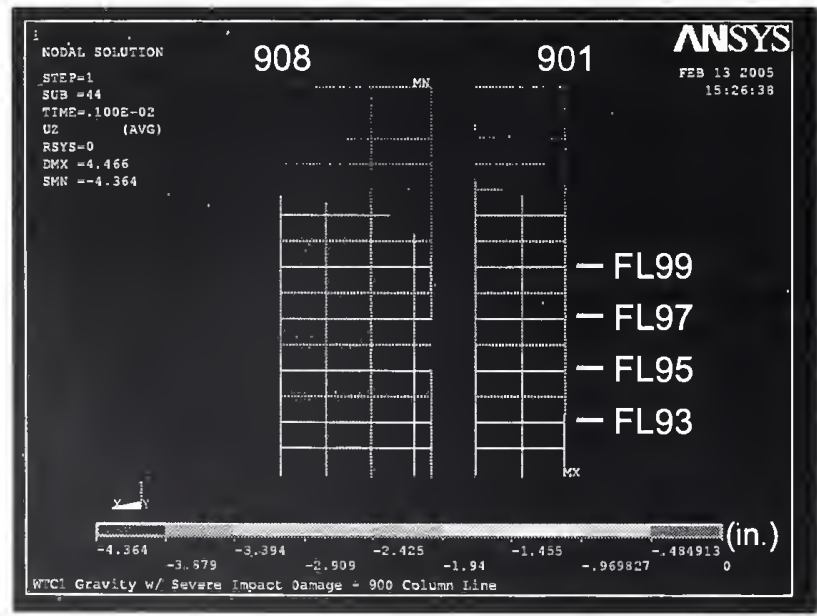

(e) 900 series columns

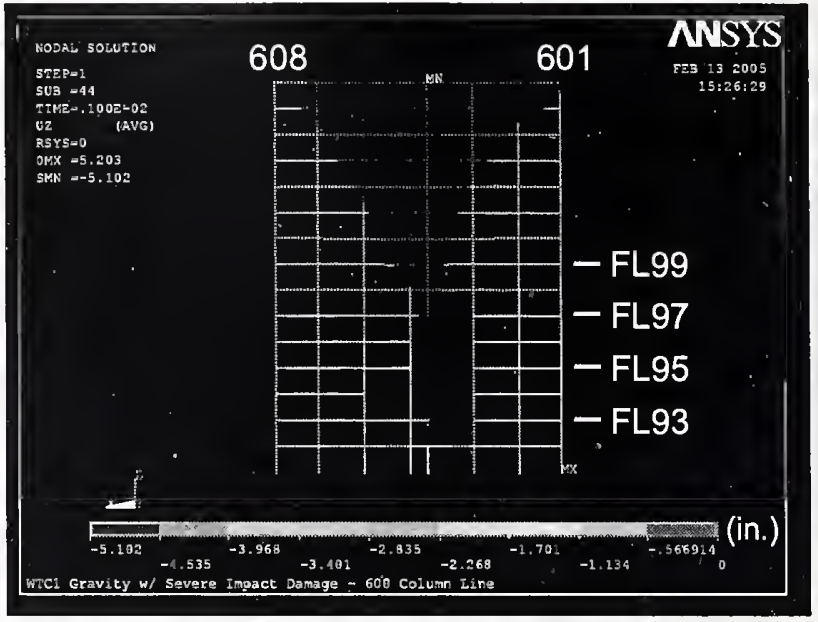

(b) 600 series columns

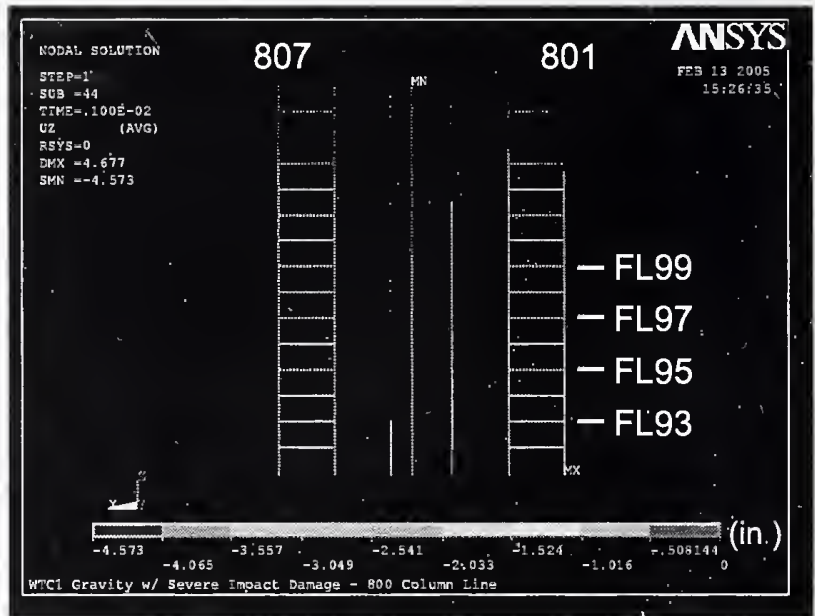

(d) 800 series columns

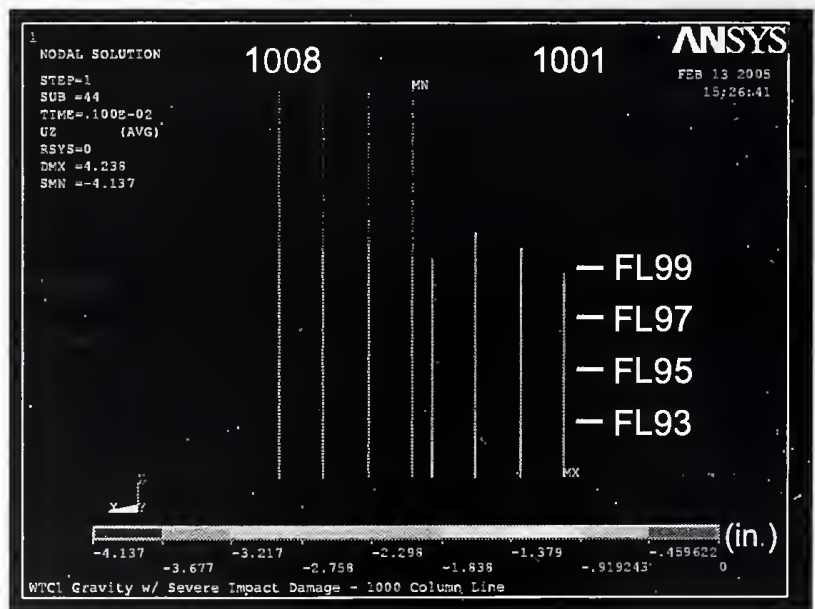

(f) 1000 series columns

Figure 4-30. Vertical displacement of core columns of WTC 1 after aircraft impact for Case B conditions (downward displacement is negative). 


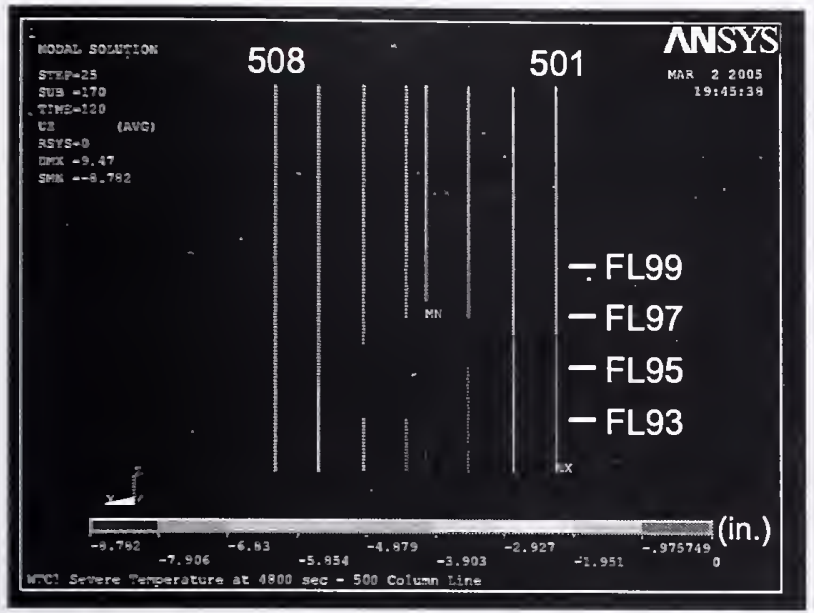

(a) 500 series columns

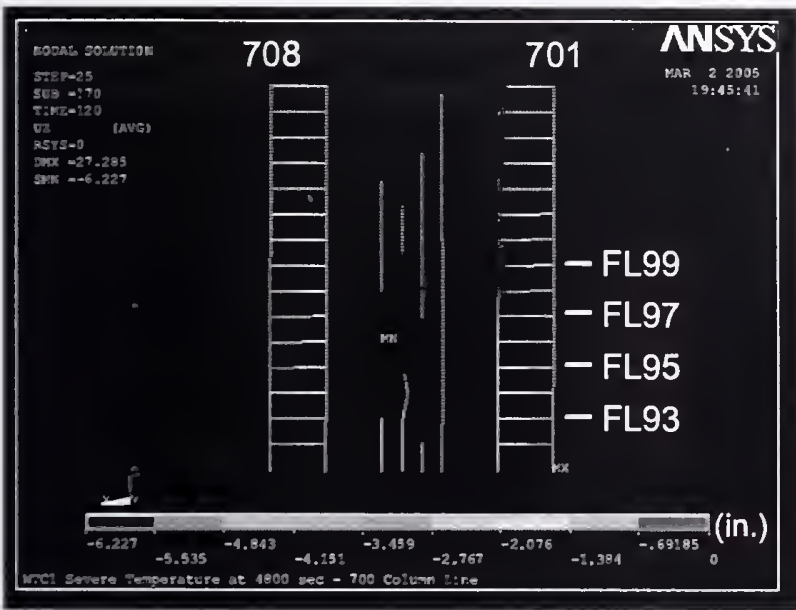

(c) 700 series columns

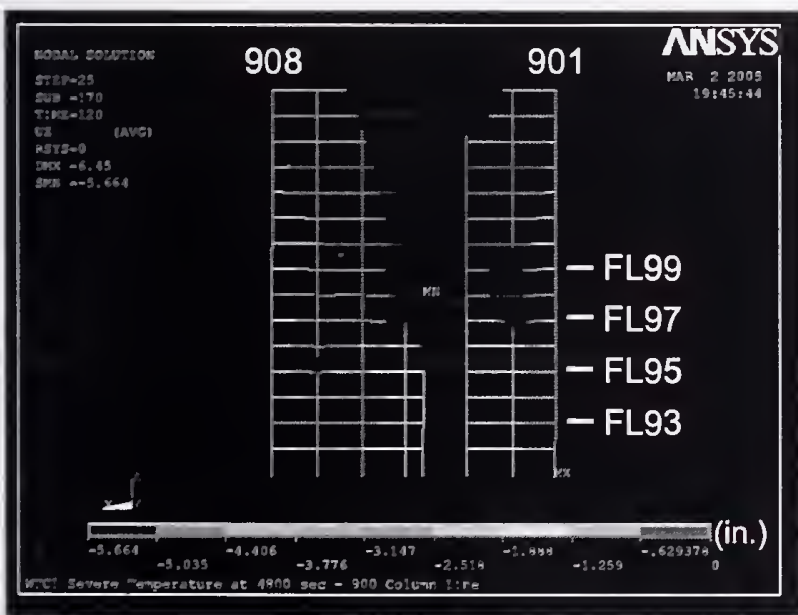

(e) 900 series columns

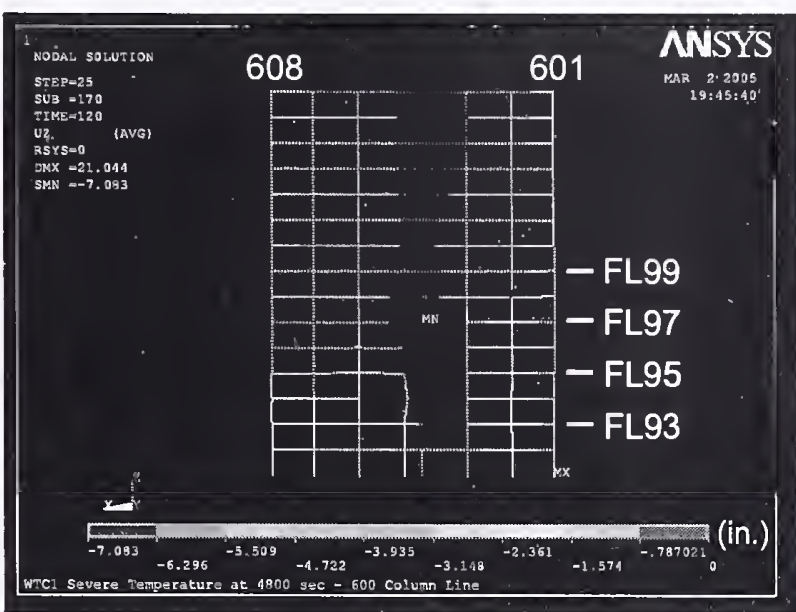

(b) 600 series columns

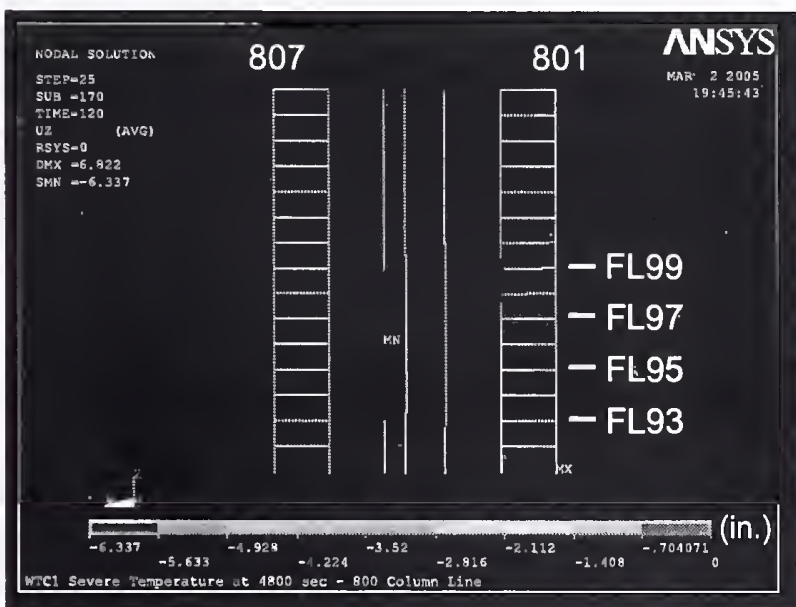

(d) 800 series columns

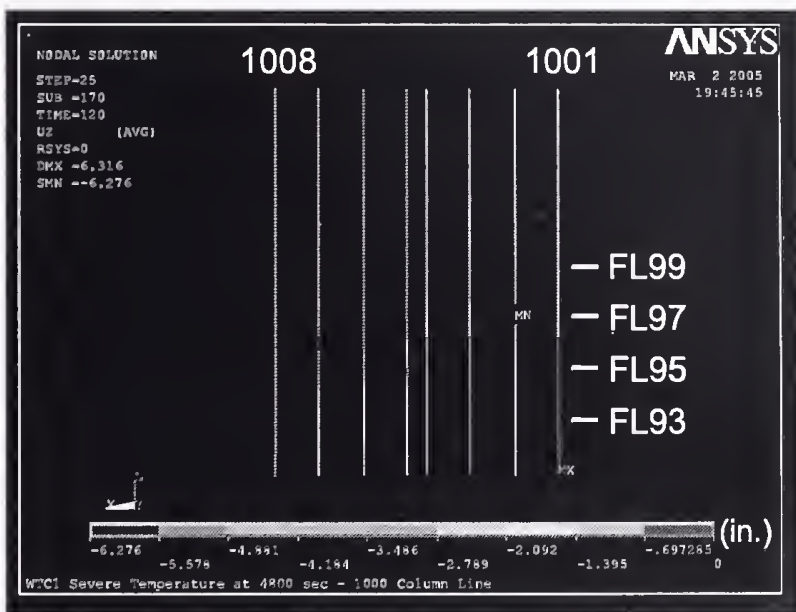

(f) 1000 series columns

Figure 4-31. Vertical displacement of core columns of WTC 1 at $80 \mathrm{~min}$ for Case B conditions (downward displacement is negative). 


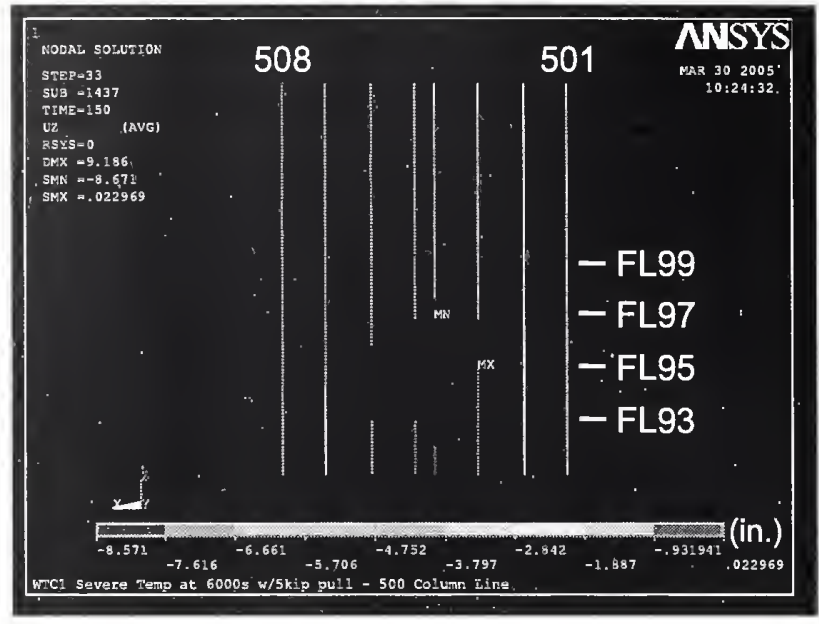

(a) 500 series columns

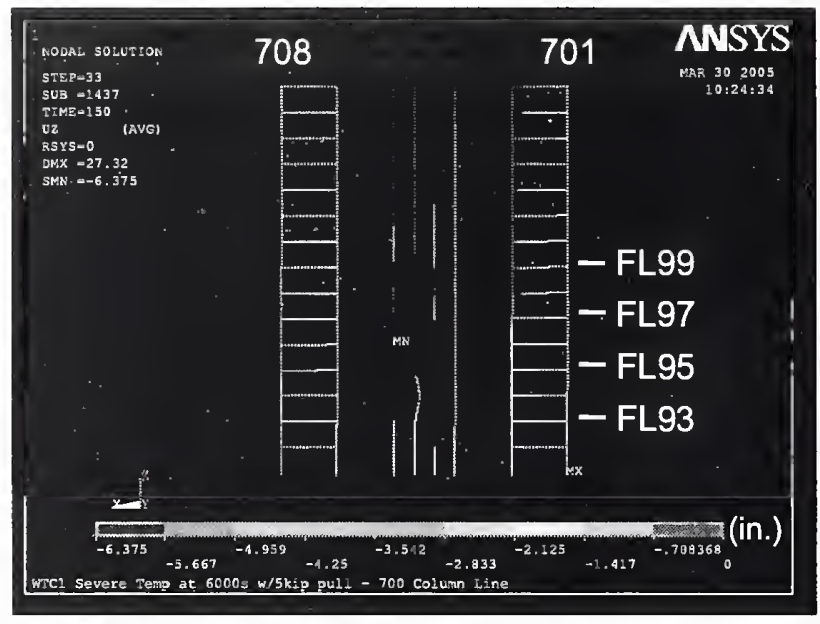

(c) 700 series columns

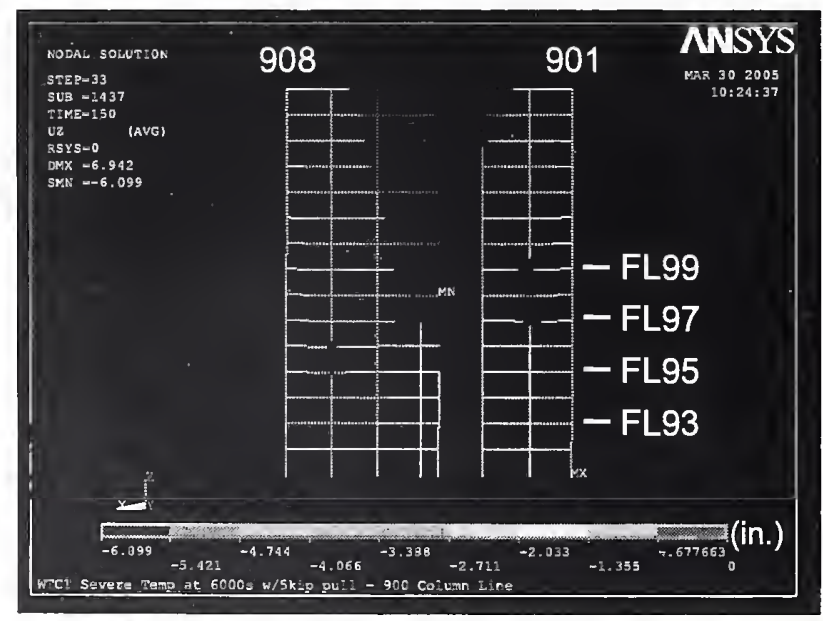

(e) 900 series columns

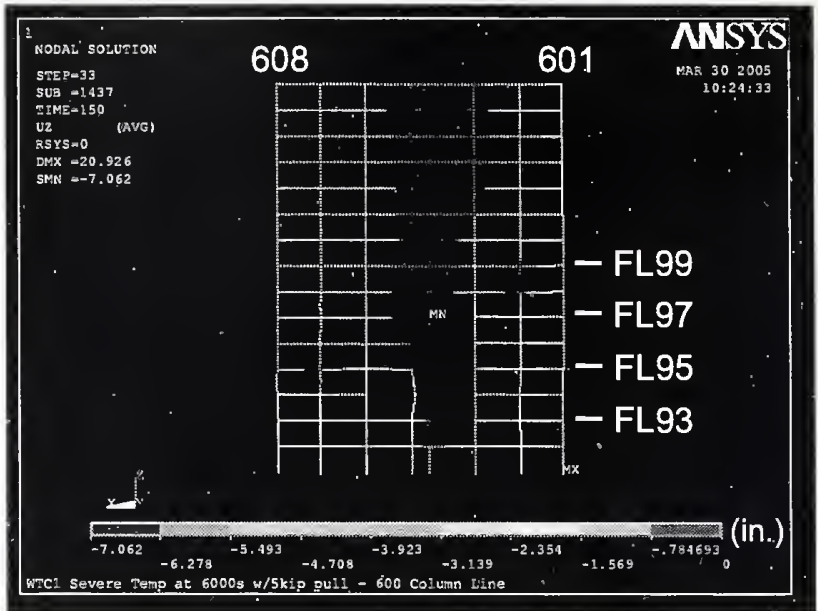

(b) 600 series columns

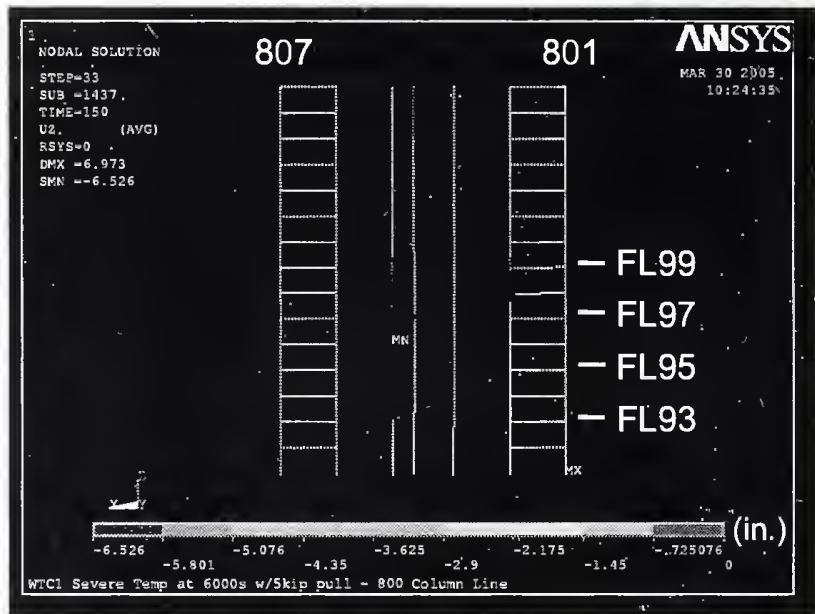

(d) 800 series columns

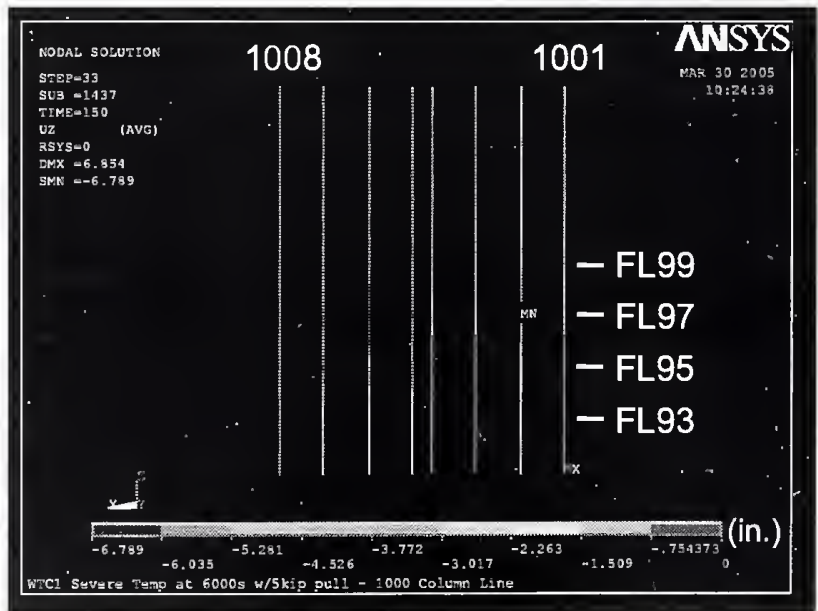

(f) 1000 series columns

Figure 4-32. Vertical displacement of core columns of WTC 1 at 100 min for Case B conditions with 5 kip pull-in forces (downward displacement is negative). 


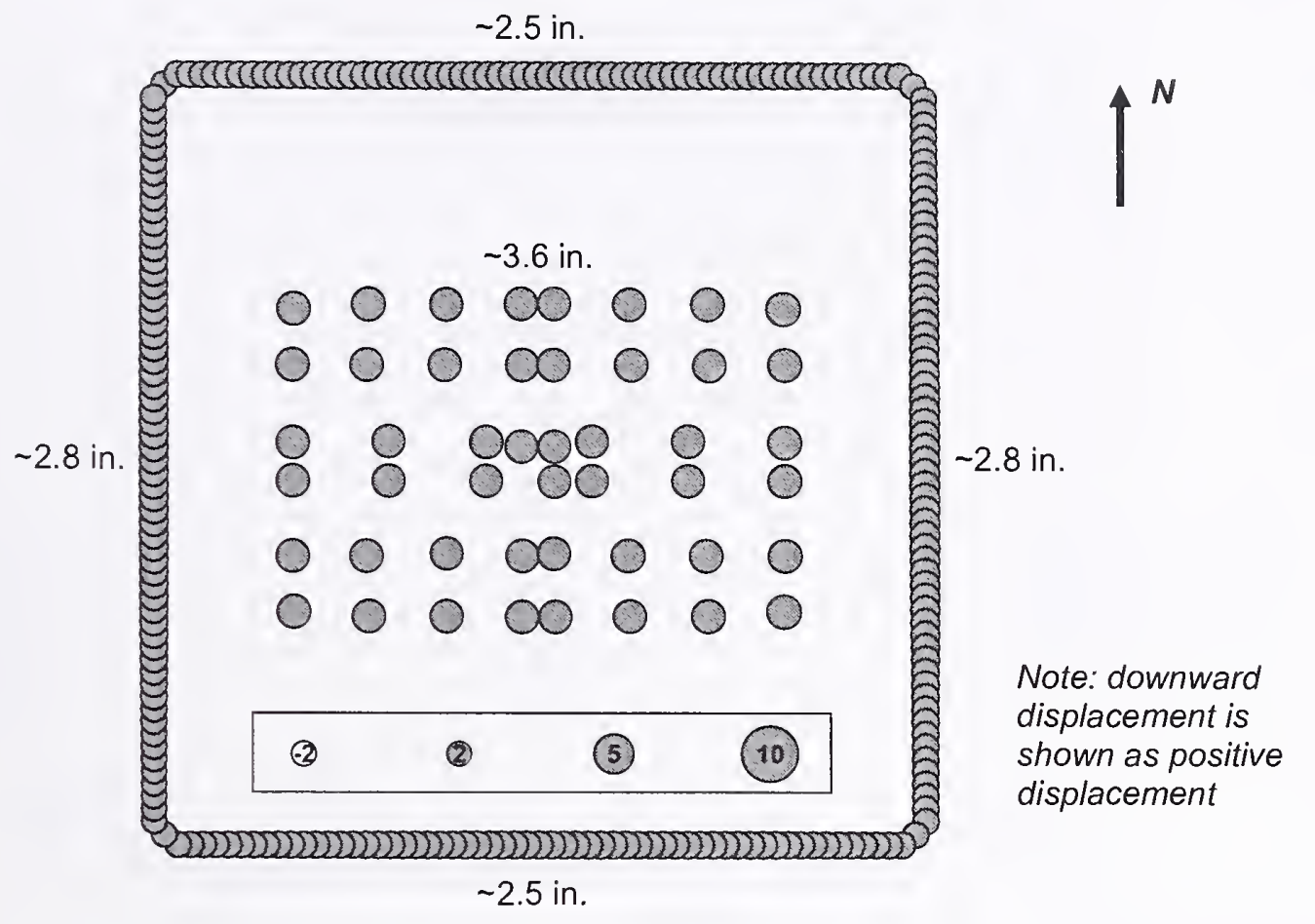

Figure 4-33. Vertical displacement at Floor 99 of WTC 1 before aircraft impact.

-+3.2 in.

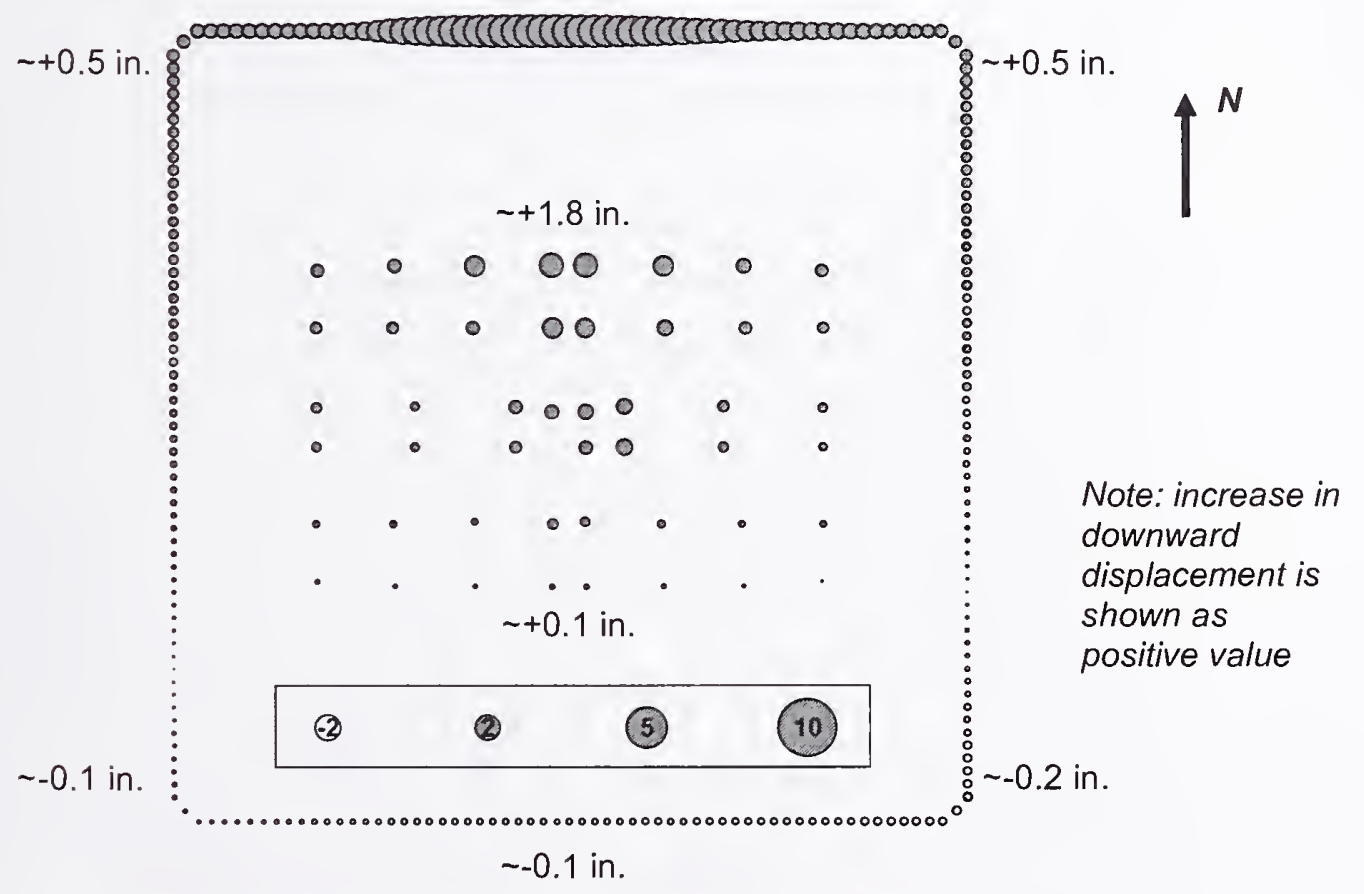

Figure 4-34. Change in vertical displacement at Floor 99 of WTC 1 from the time before impact to the time after impact for Case B conditions. 
$\sim+4.1$ in.

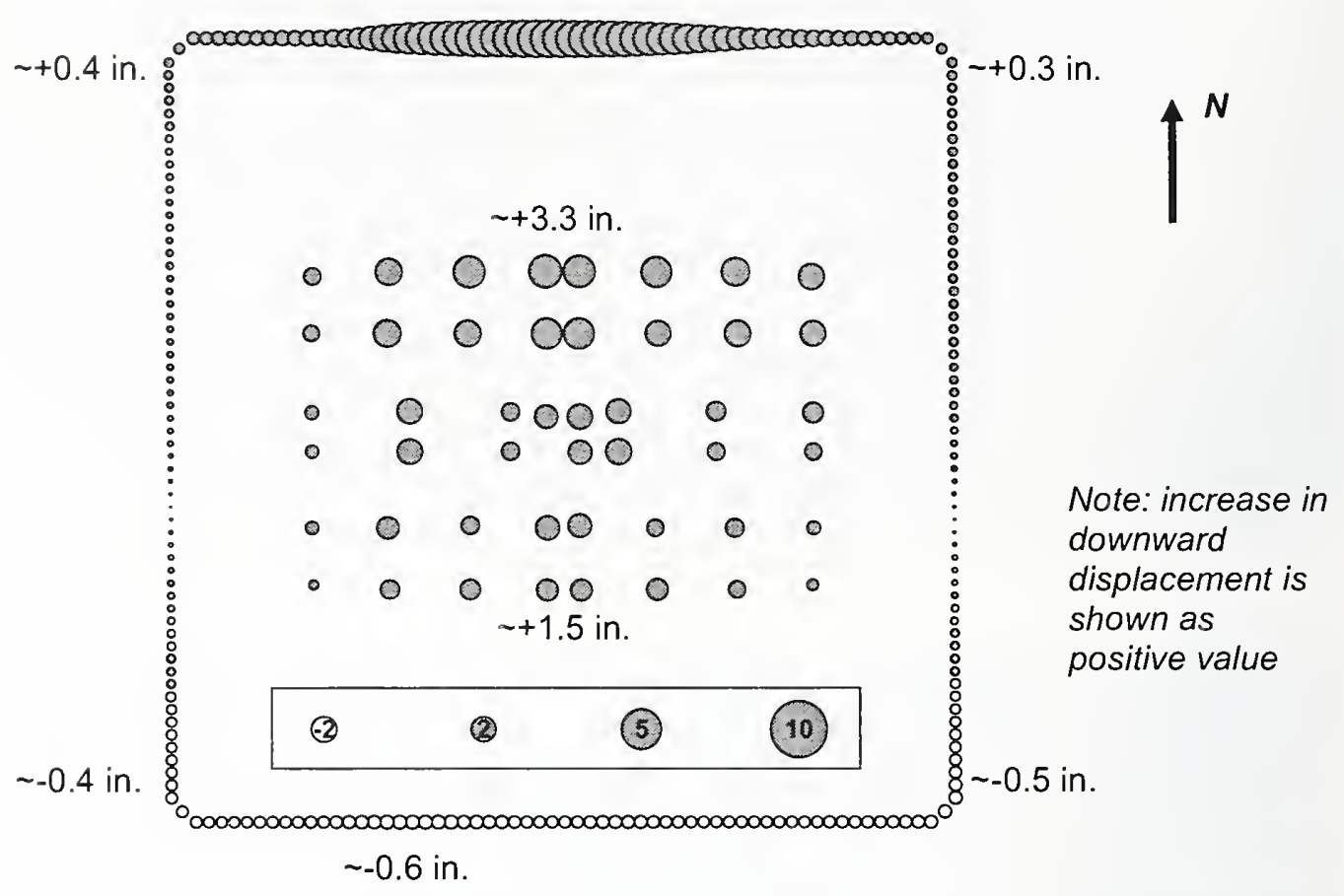

Figure 4-35. Change in vertical displacement at Floor 99 of WTC 1 from the time before impact to $50 \mathrm{~min}$ for Case B conditions.

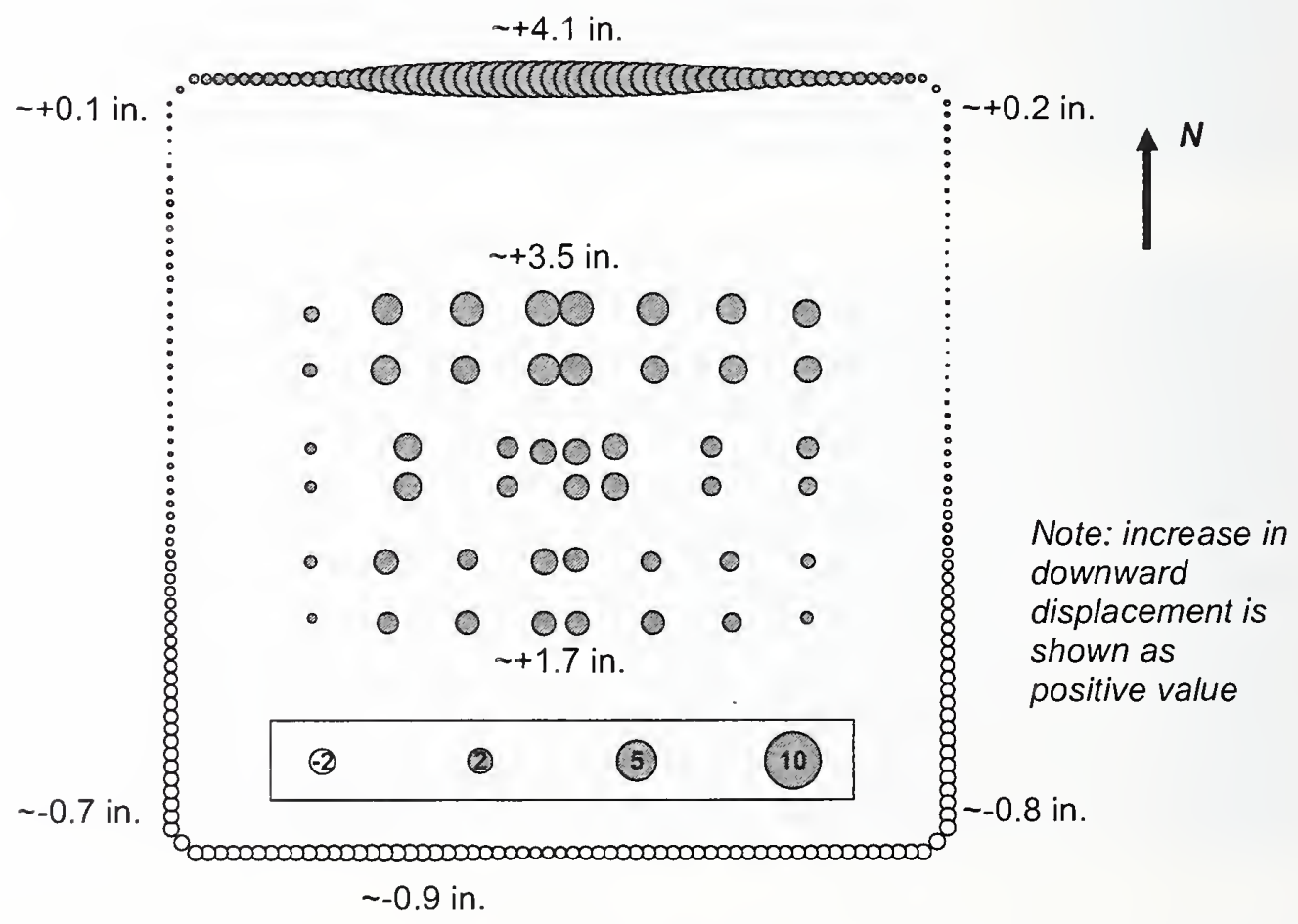

Figure 4-36. Change in vertical displacement at Floor 99 of WTC 1 from the time before impact to 80 min for Case B conditions. 
-+3.7 in.

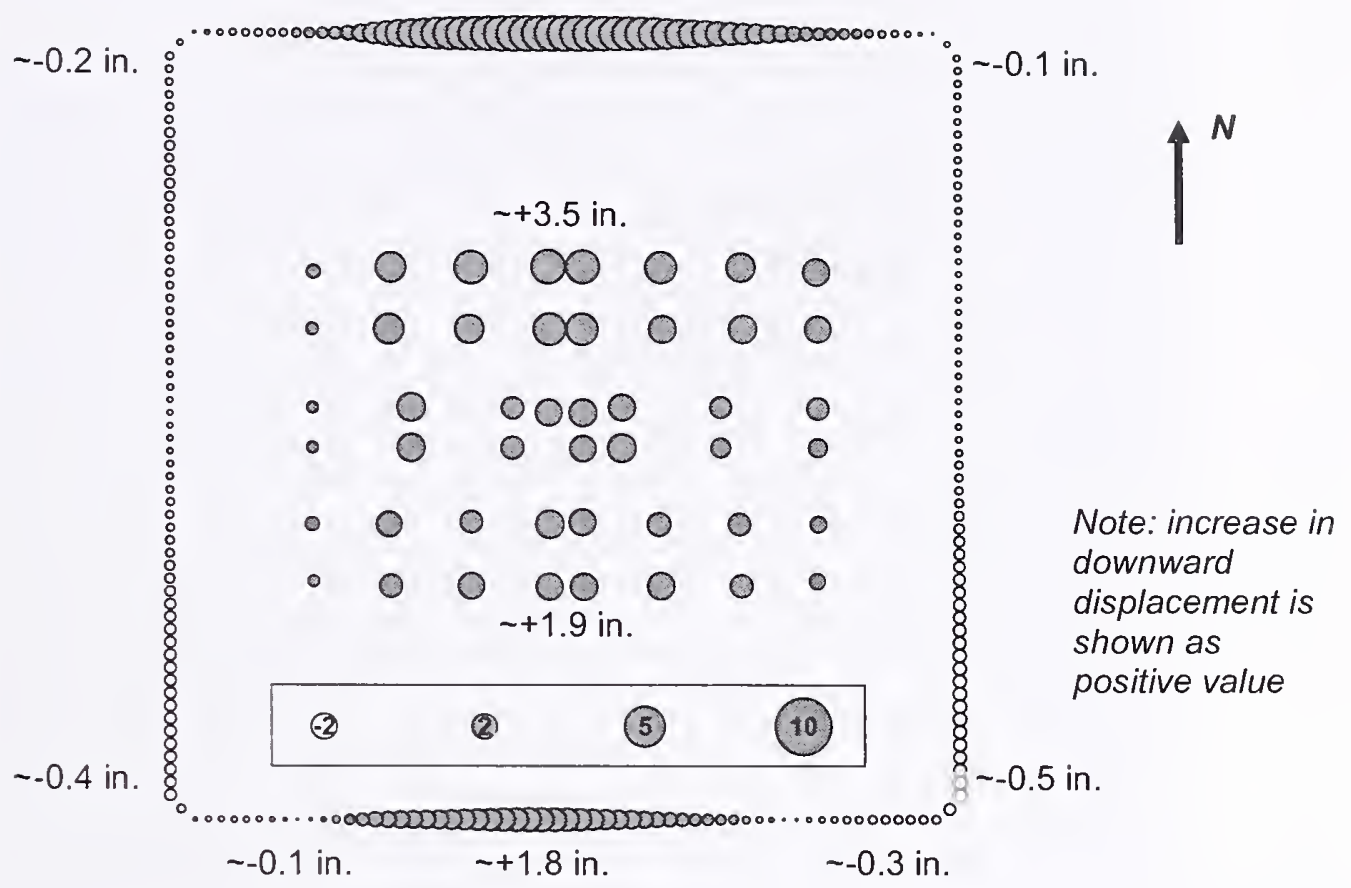

Figure 4-37. Change in vertical displacement at Floor 99 of WTC 1 from the time before impact to $100 \mathrm{~min}$ for Case $B$ conditions with $\mathbf{5}$ kip pull-in forces. 


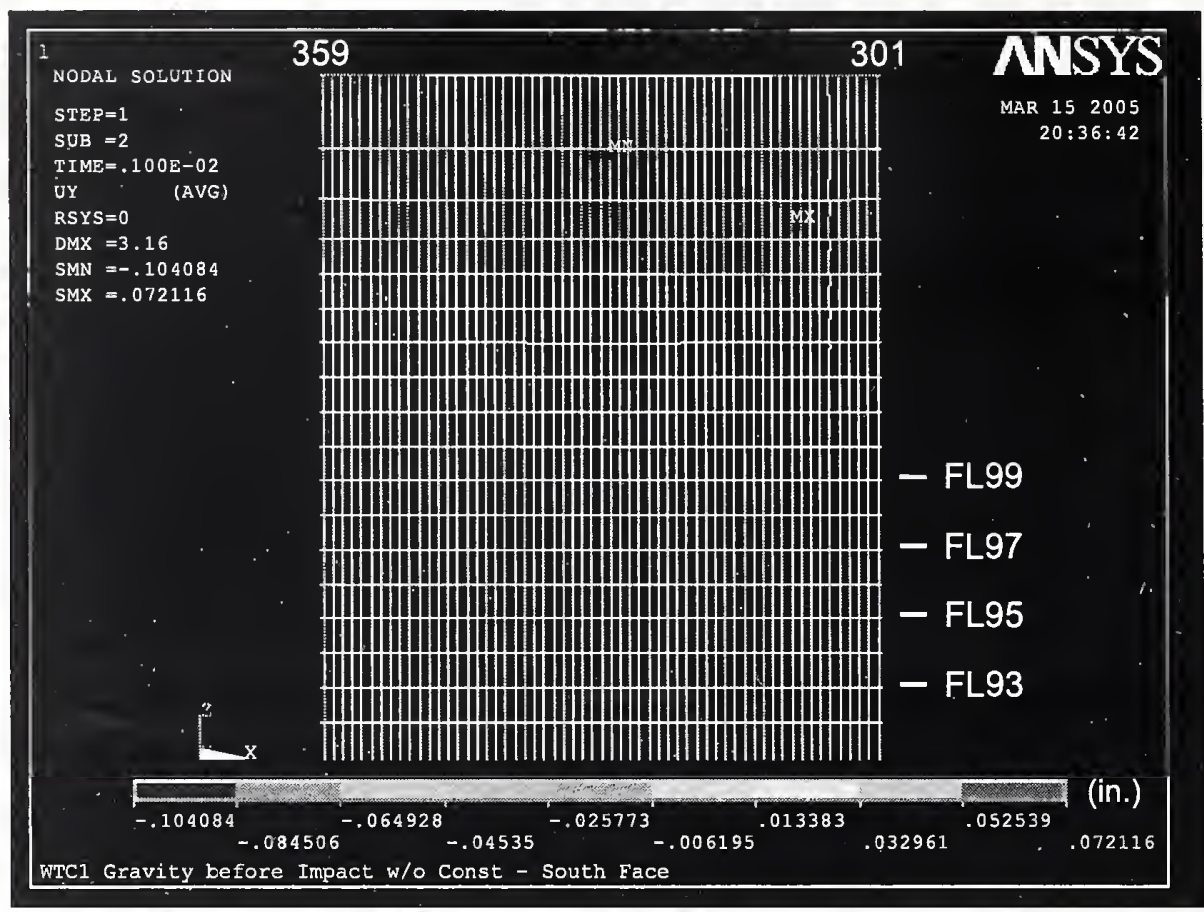

Figure 4-38. Out-of-plane displacement of south wall of WTC 1 before aircraft impact (inward displacement is positive).

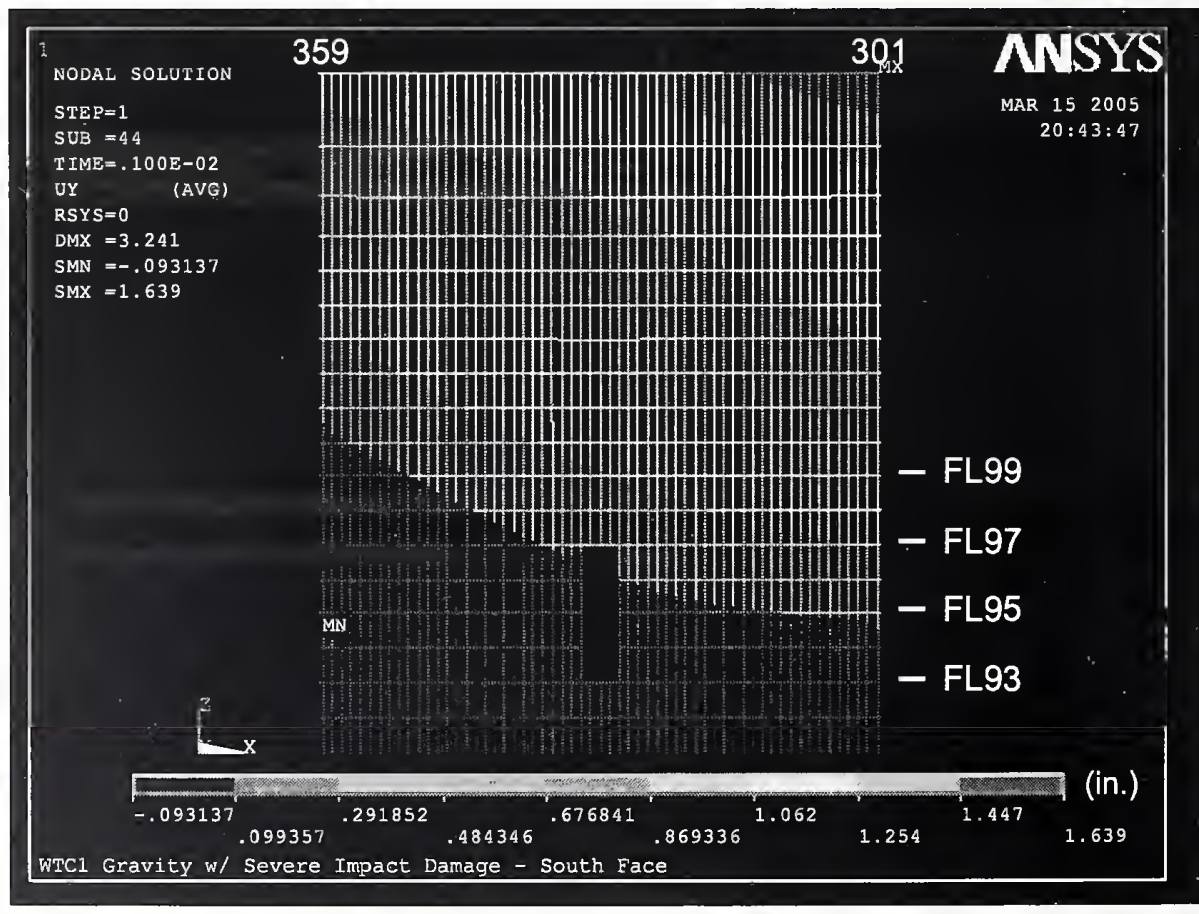

Figure 4-39. Out-of-plane displacement of south wall of WTC 1 after aircraft impact for Case B conditions (inward displacement is positive). 


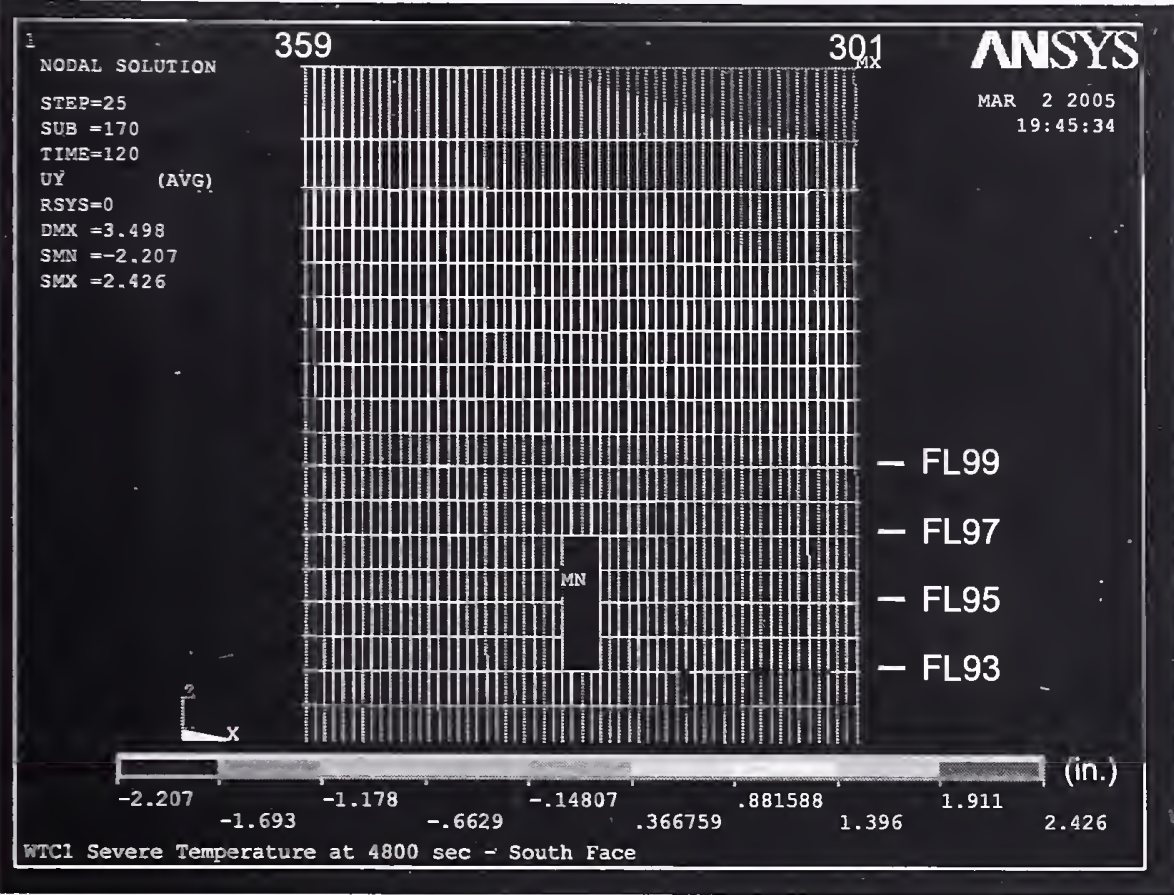

Figure 4-40. Out-of-plane displacement of south wall of WTC 1 at 80 min (at the end of Analysis Step 17) for Case B conditions (inward displacement is positive).

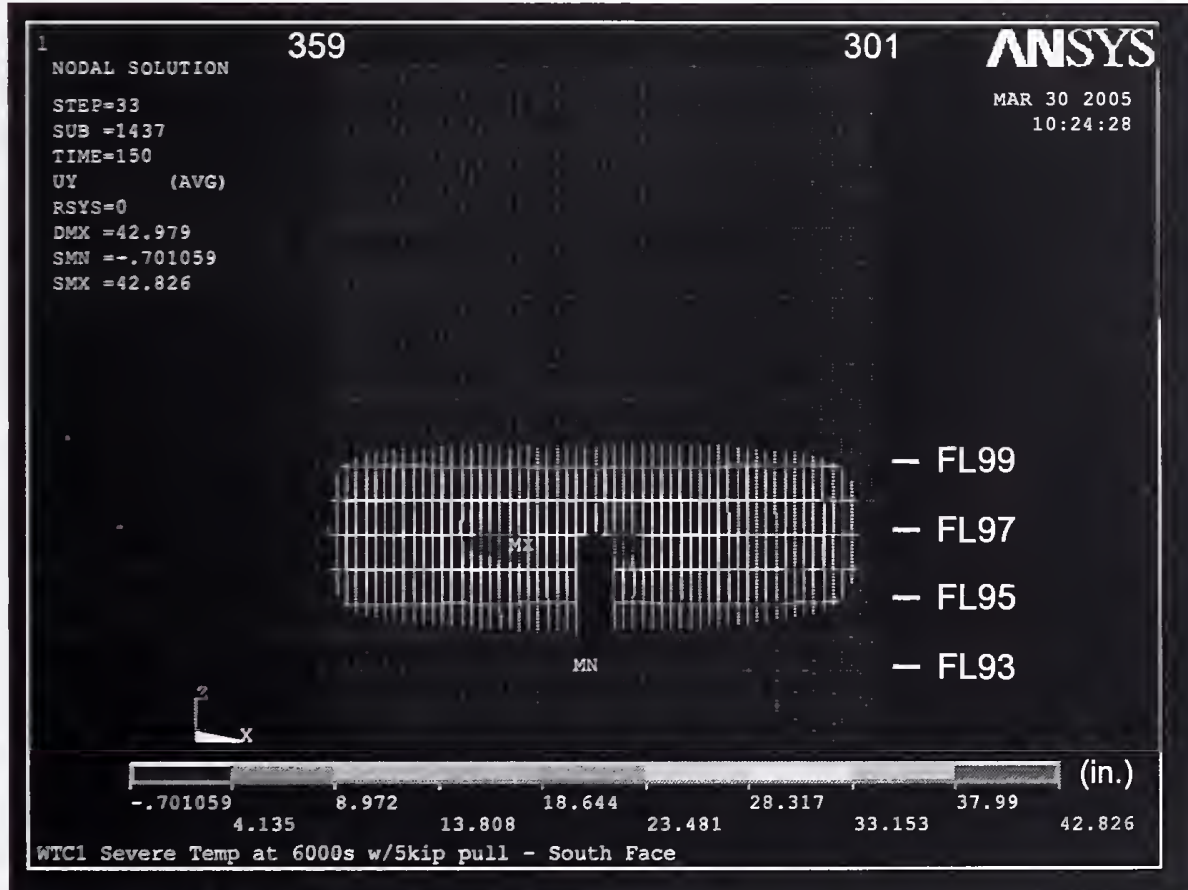

Figure 4-41. Out-of-plane displacement of south wall of WTC 1 at $100 \mathrm{~min}$ for Case B conditions with 5 kip pull-in forces (inward displacement is positive). 


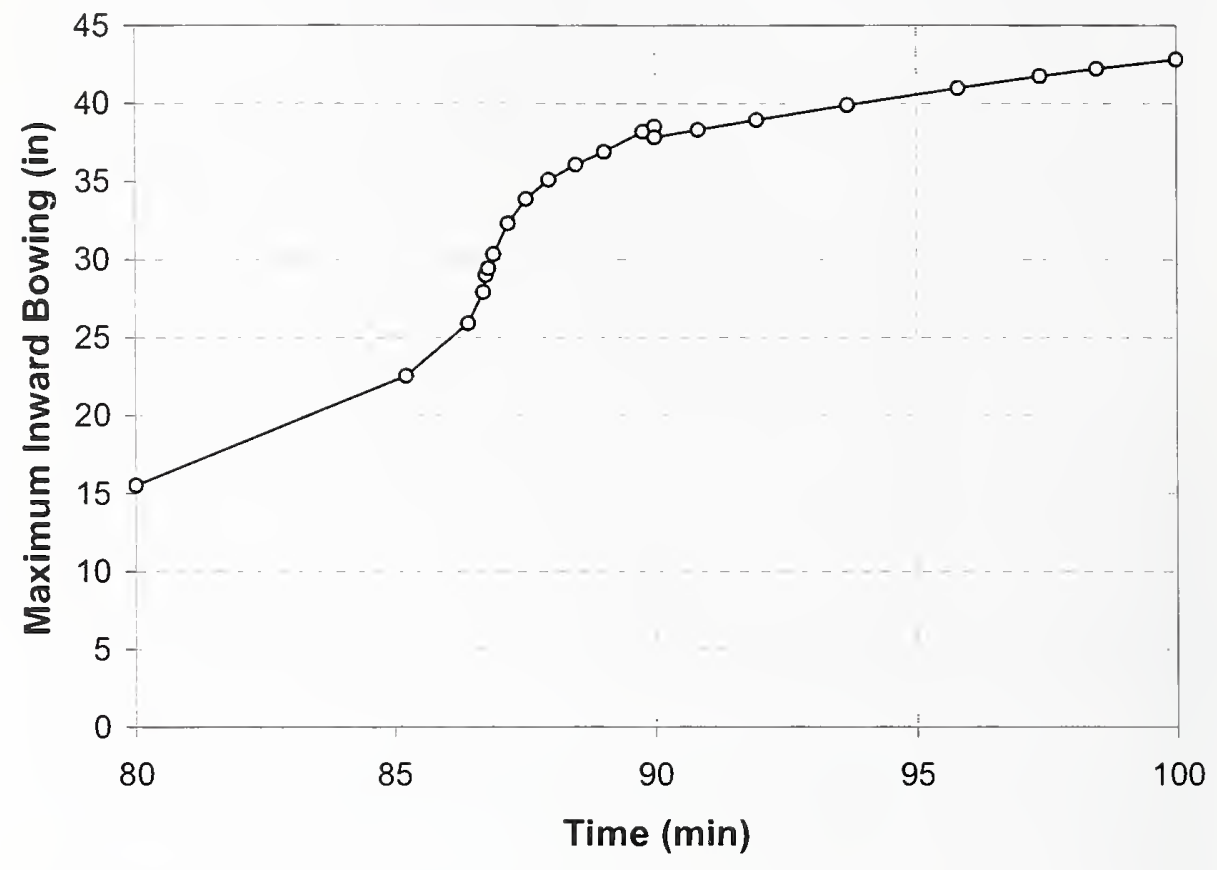

Figure 4-42. Time history of maximum out-of-plane displacement of south wall of WTC 1 for Case B conditions with 5 kip pull-in forces (from Analysis Step 18 to Step 21).

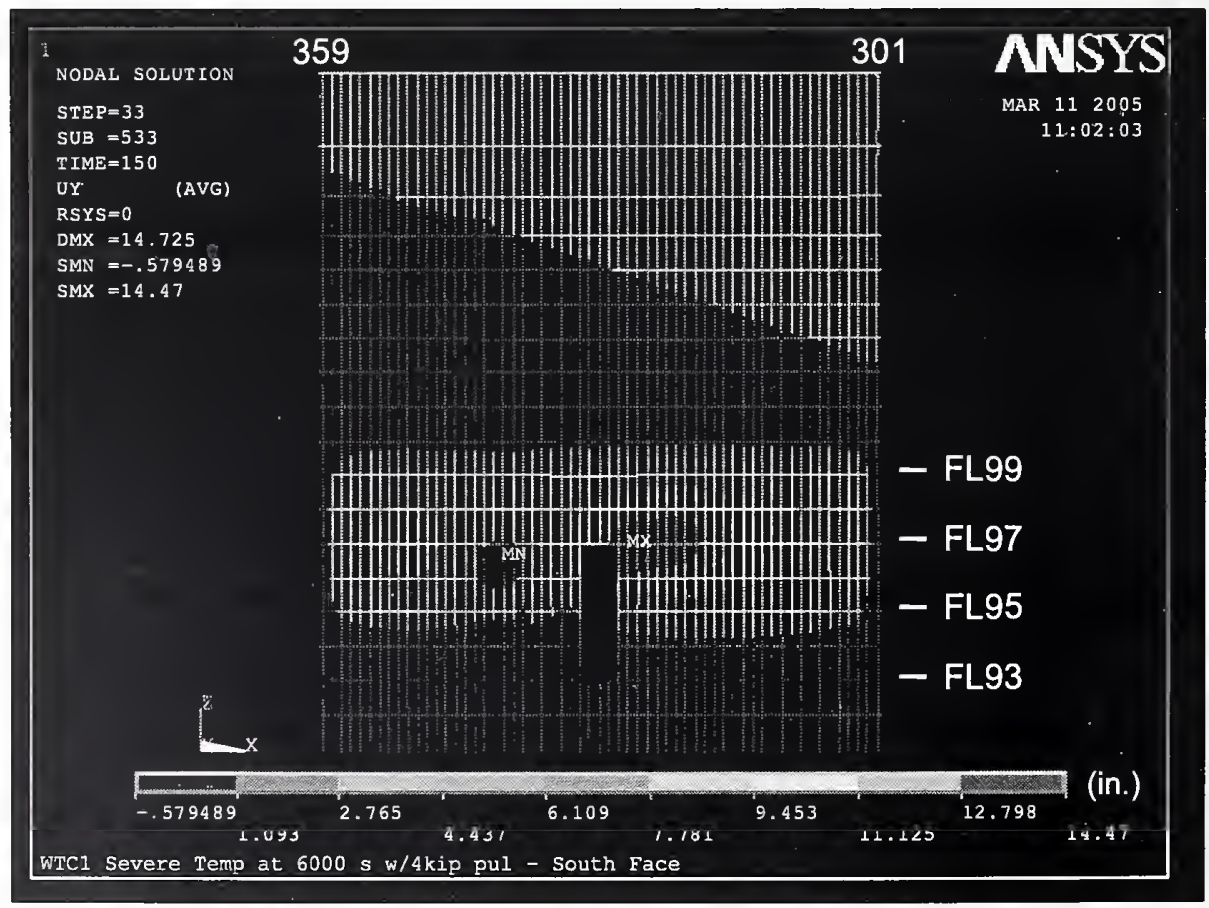

Figure 4-43. Out-of-plane displacement of south wall of WTC 1 at $100 \mathrm{~min}$ for Case B conditions with 4 kip pull-in forces (inward displacement is positive). 


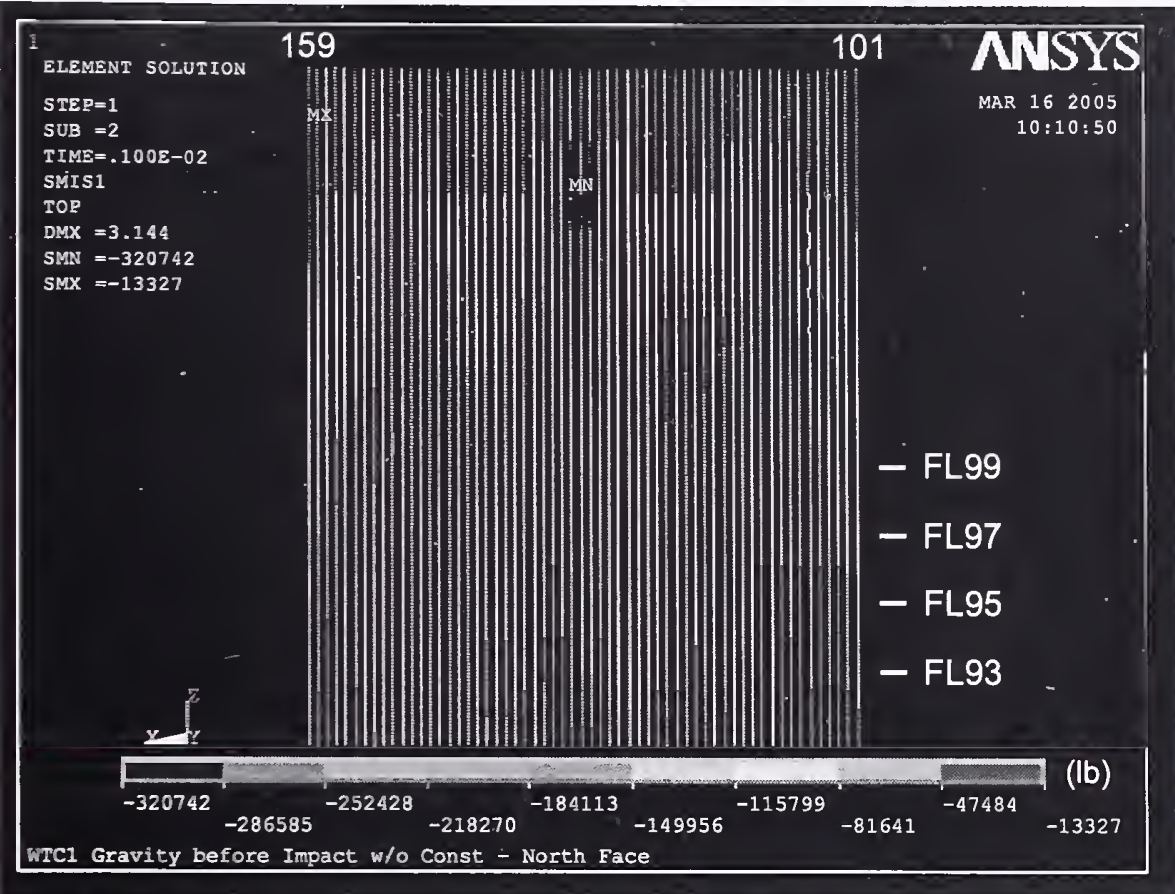

Figure 4-44. Axial load in exterior columns of north wall of WTC 1 before aircraft impact (compression is negative).

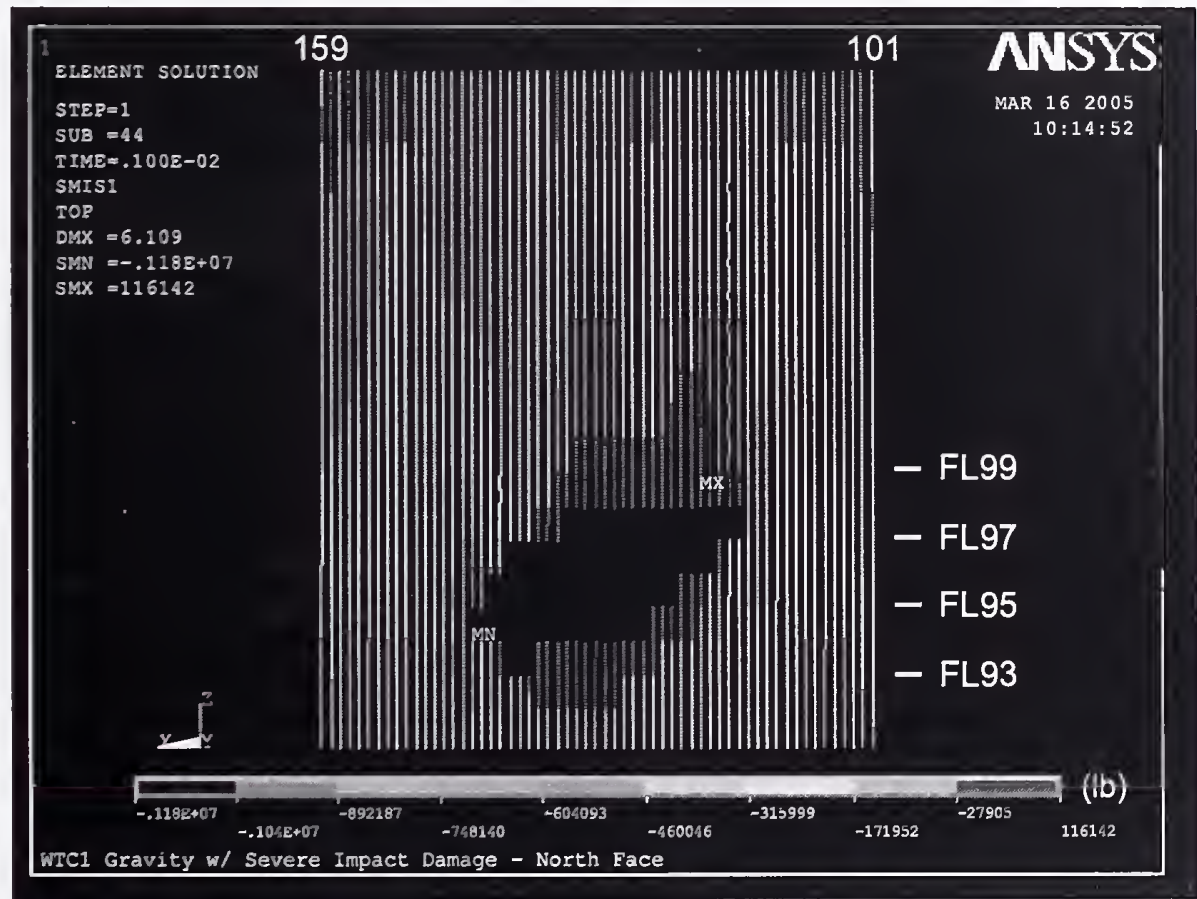

Figure 4-45. Axial load in exterior columns of north wall of WTC 1 after aircraft impact for Case B conditions (compression is negative). 


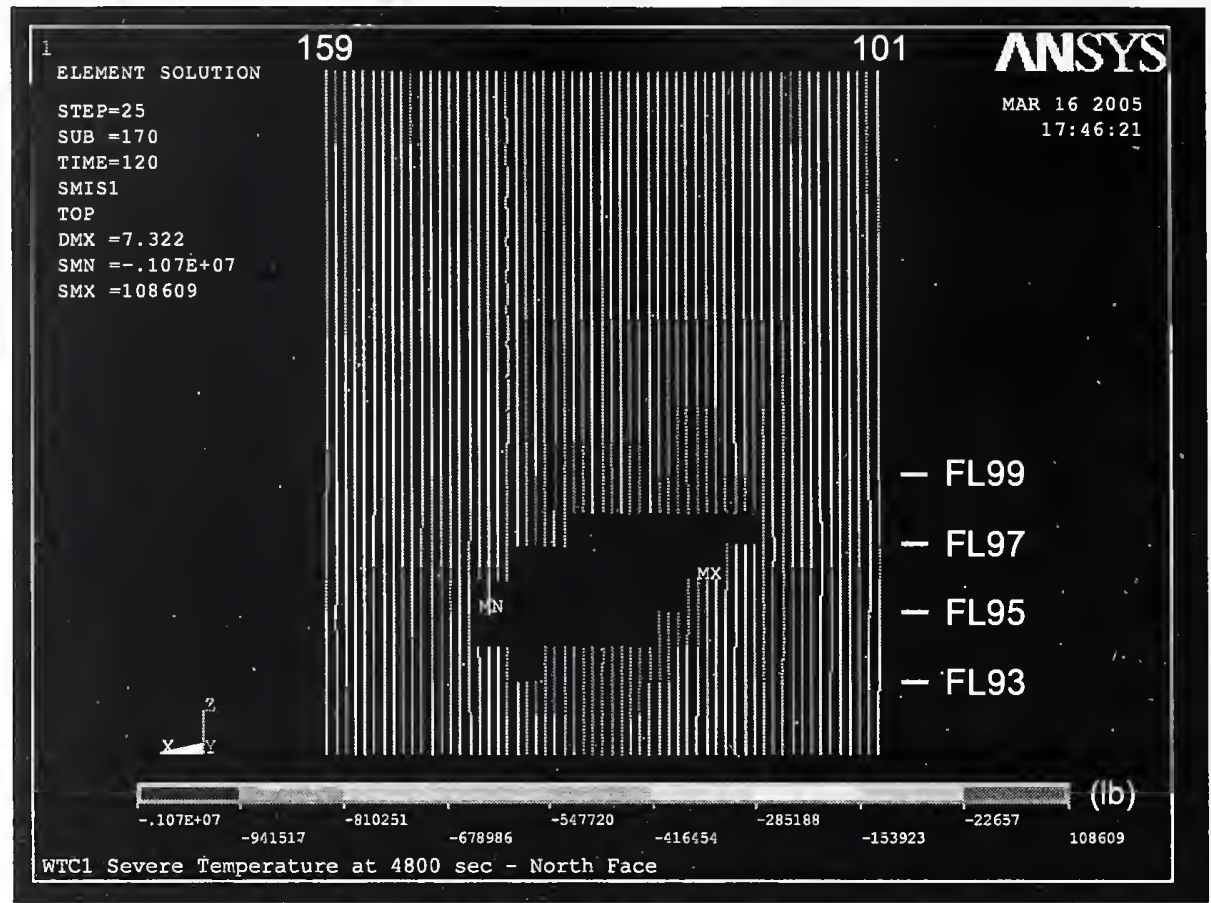

Figure 4-46. Axial load in exterior columns of north wall of WTC 1 at 80 min for the Case $B$ conditions (compression is negative).

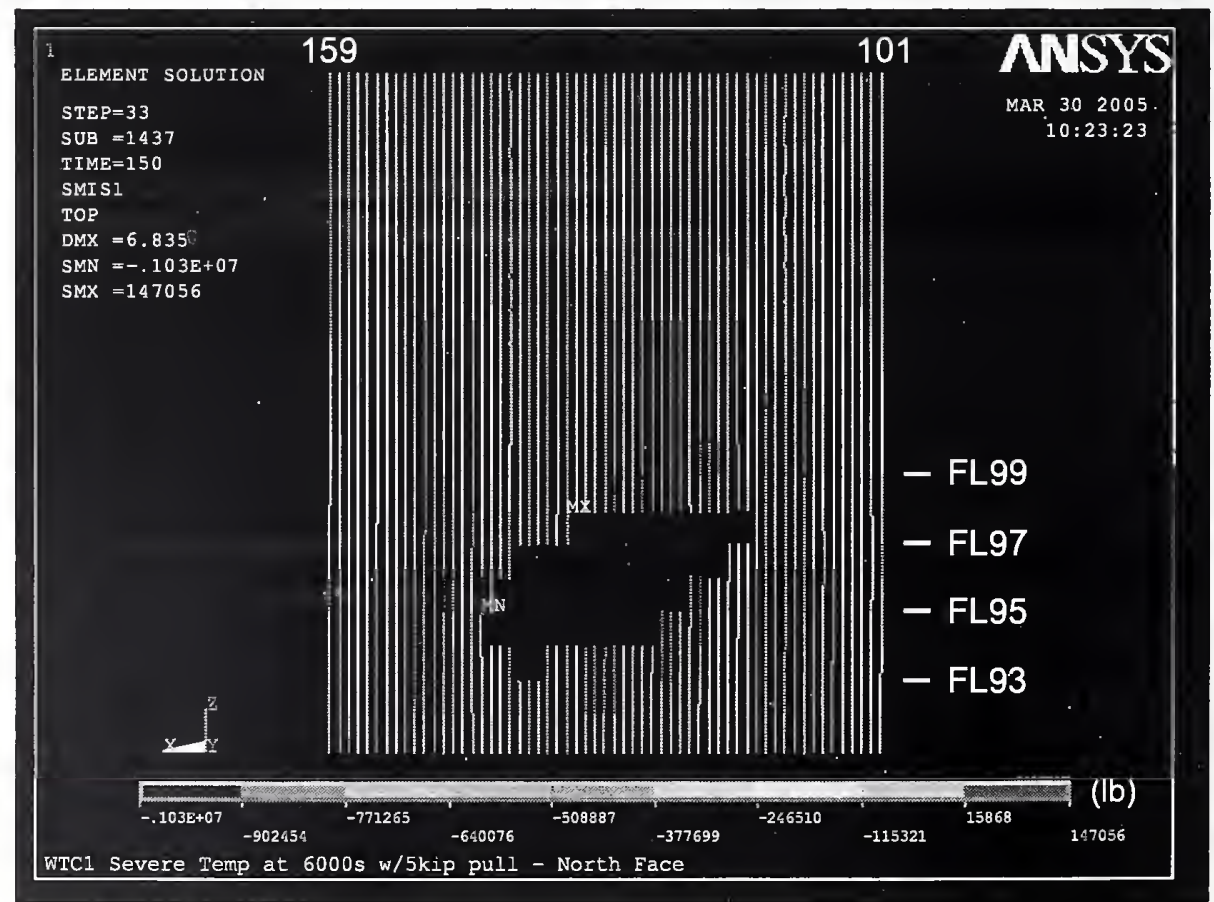

Figure 4-47. Axial load in exterior columns of north wall of WTC 1 at 100 min for Case B conditions with 5 kip pull-in forces (compression is negative). 


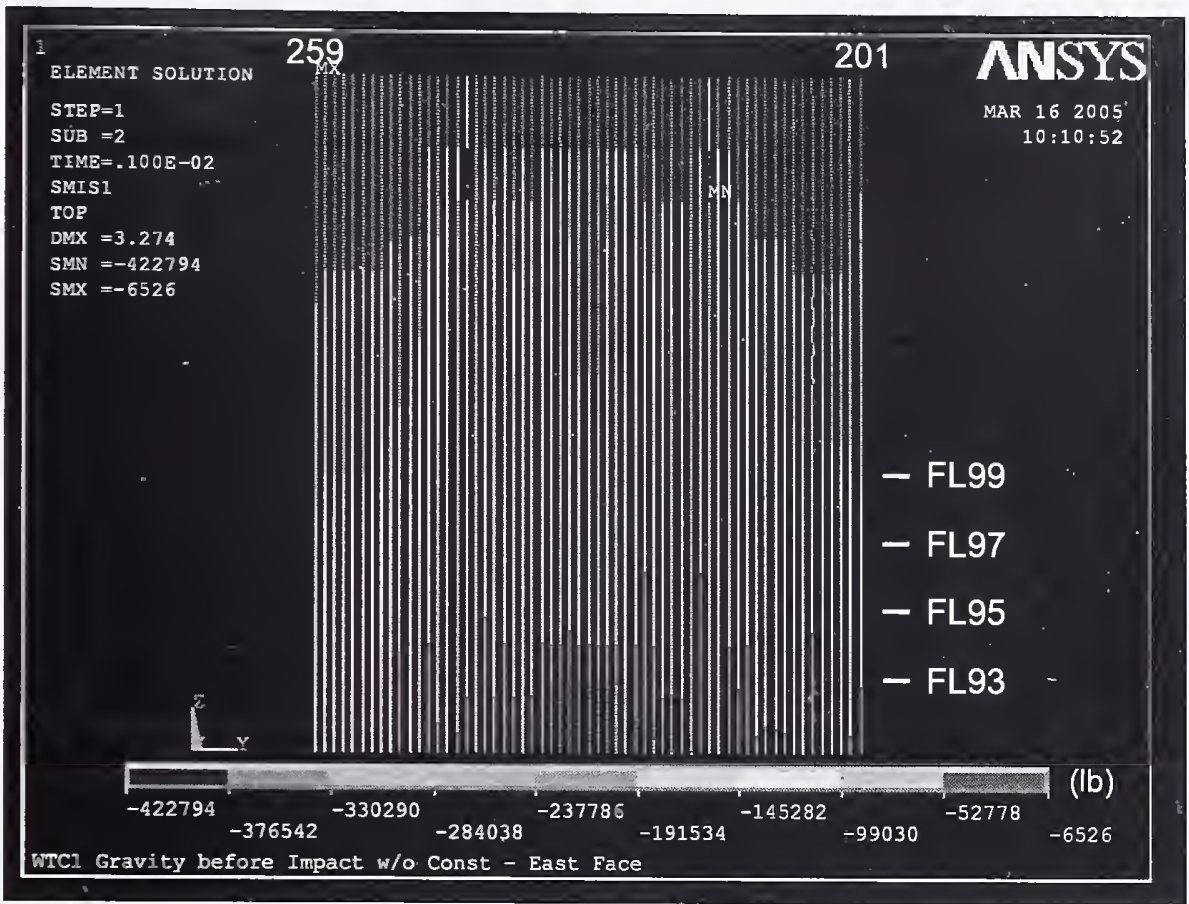

Figure 4-48. Axial load in exterior columns of east wall of WTC 1 before aircraft impact (compression is negative).

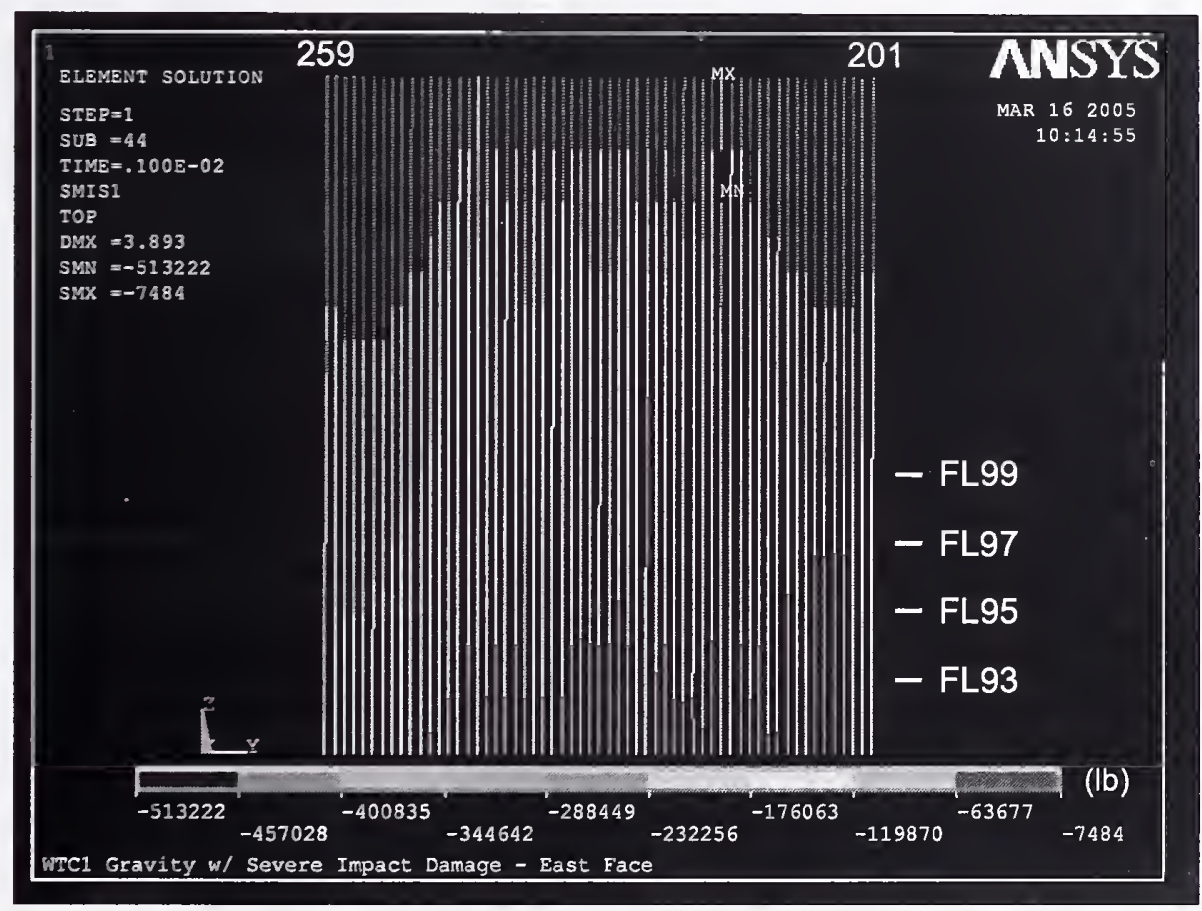

Figure 4-49. Axial load in exterior columns of east wall of WTC 1 after aircraft impact for Case $B$ conditions (compression is negative). 


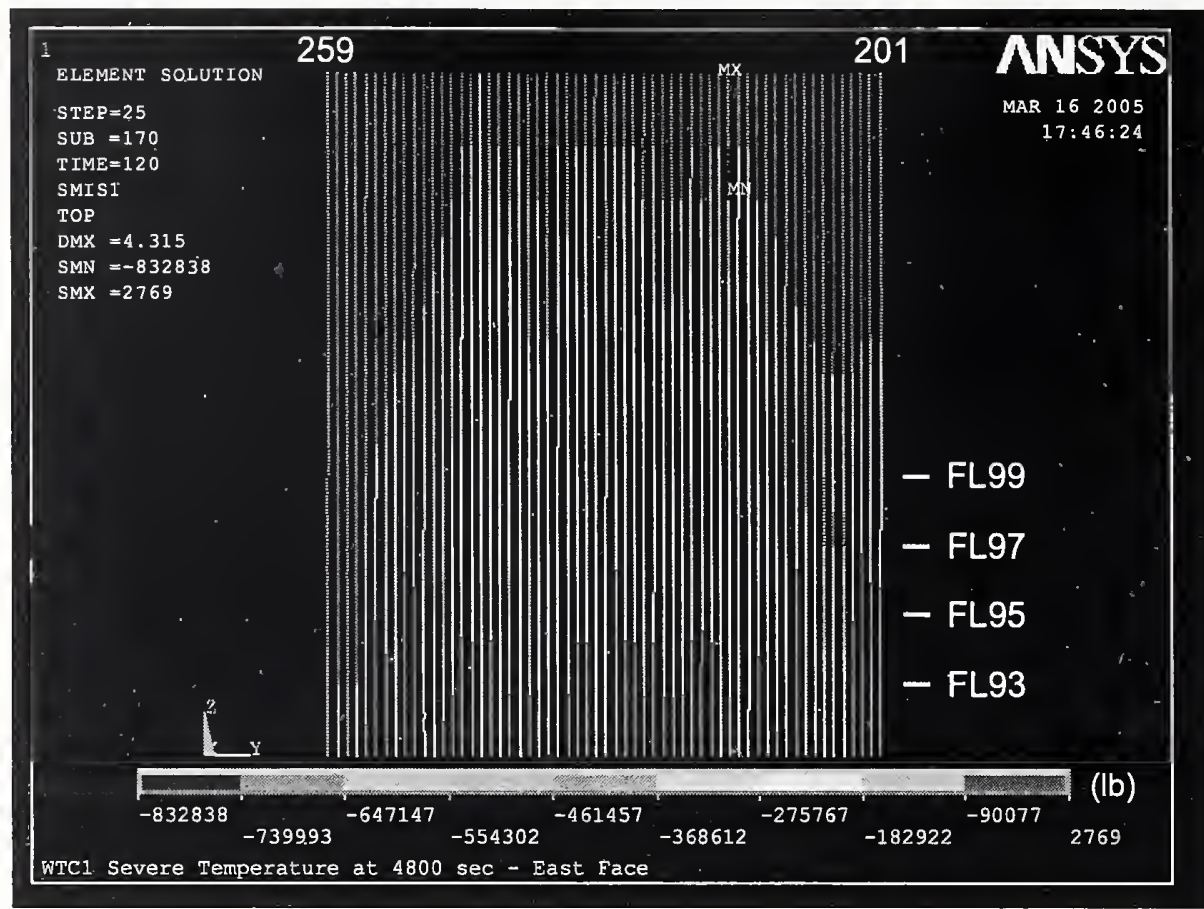

Figure 4-50. Axial load in exterior columns of east wall of WTC 1 at $80 \mathrm{~min}$ for the Case $B$ conditions (compression is negative).

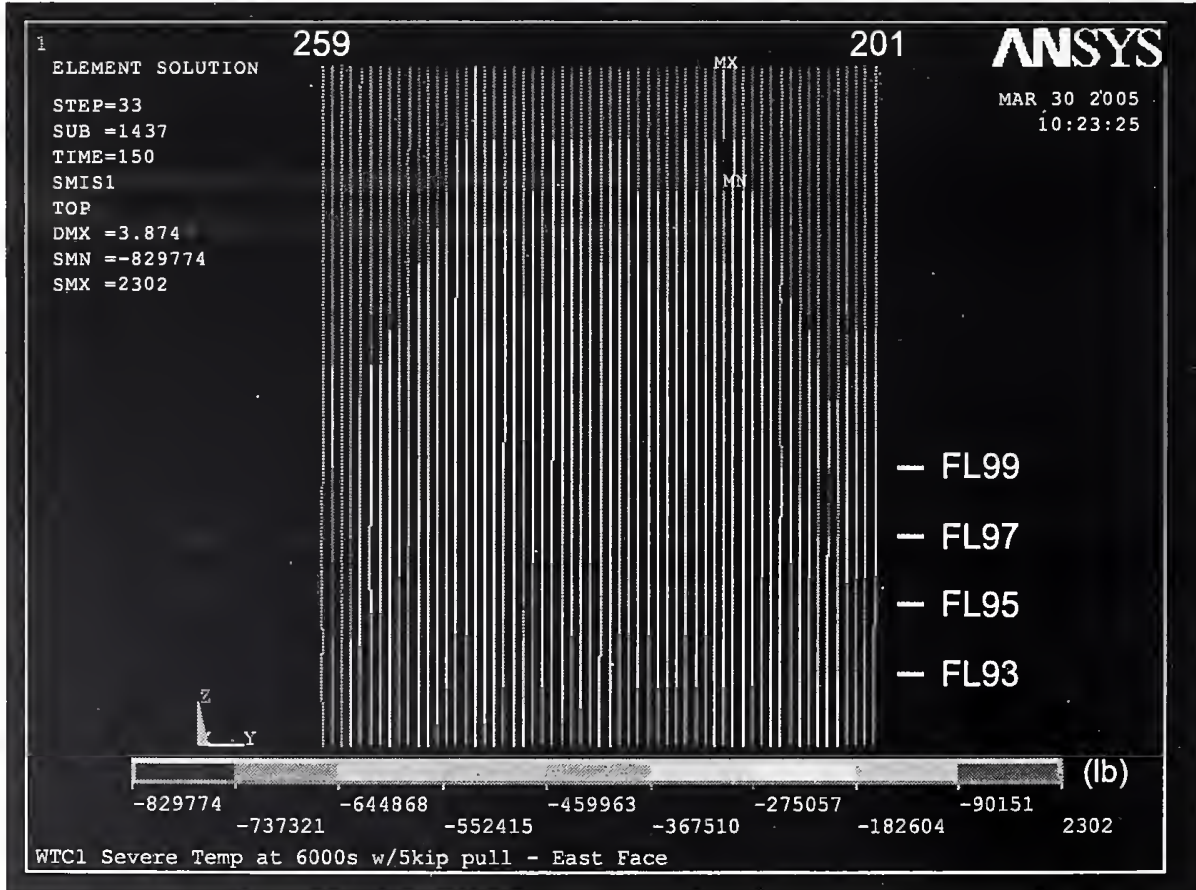

Figure 4-51. Axial load in exterior columns of east wall of WTC 1 at $100 \mathrm{~min}$ for the Case B conditions with 5 kip pull-in forces (compression is negative). 


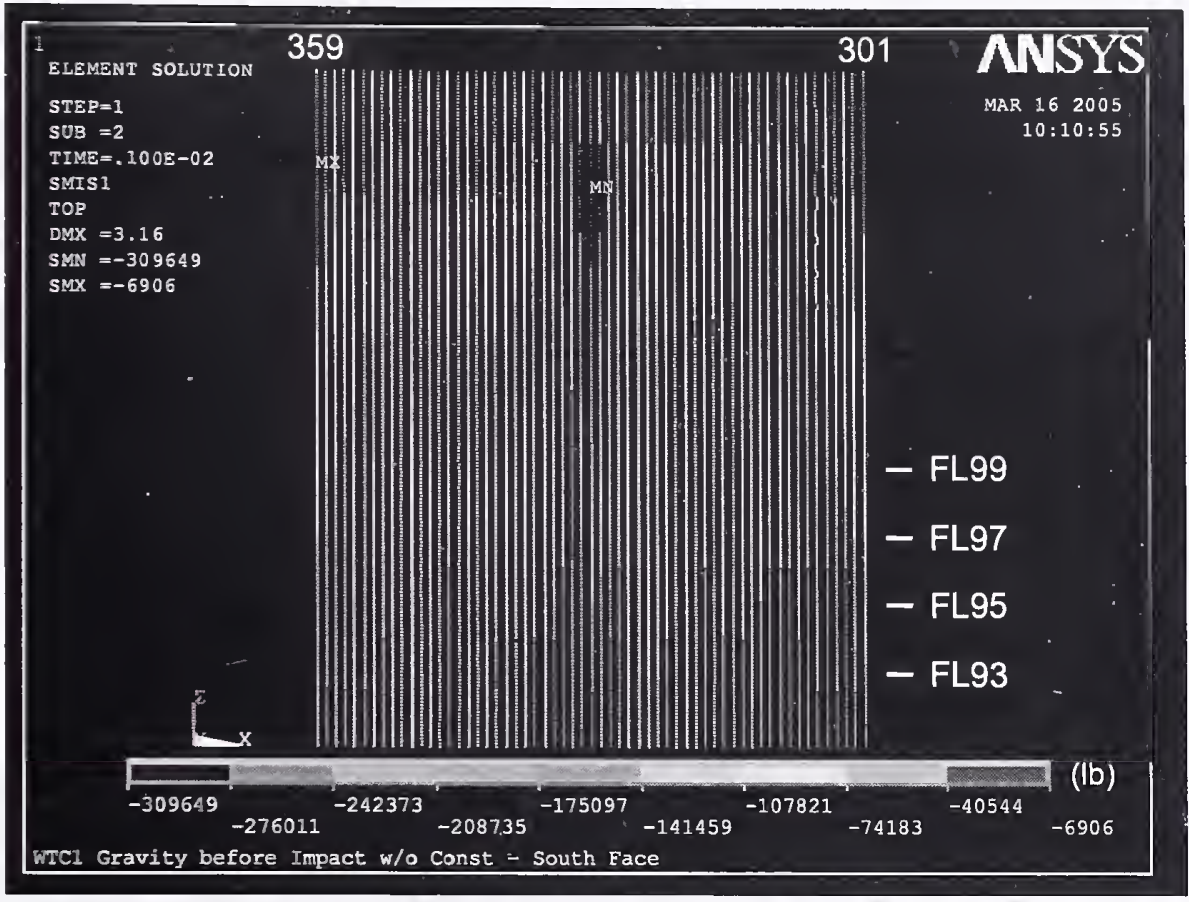

Figure 4-52. Axial load in exterior columns of south wall of WTC 1 before aircraft impact (compression is negative).

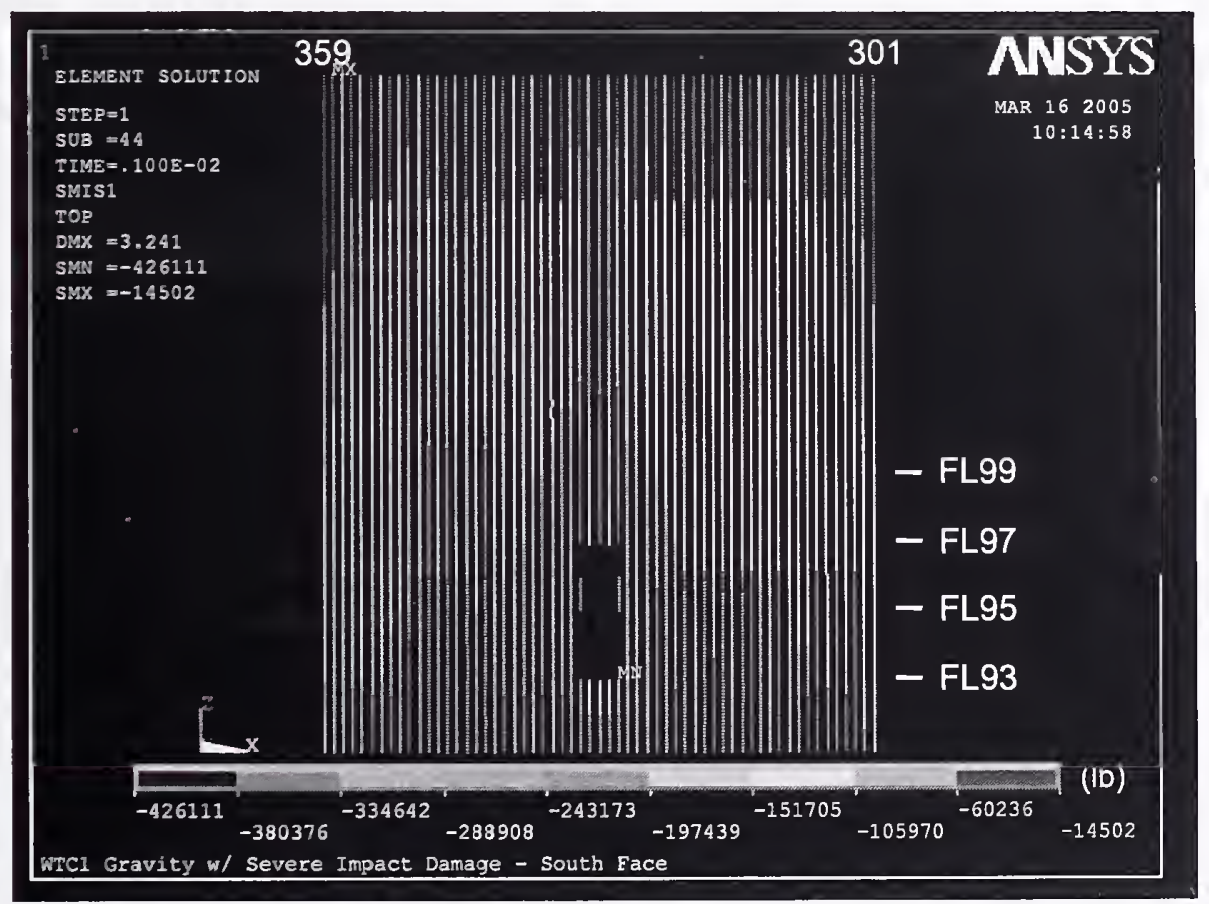

Figure 4-53. Axial load in exterior columns of south wall of WTC 1 after aircraft impact for Case B conditions (compression is negative). 


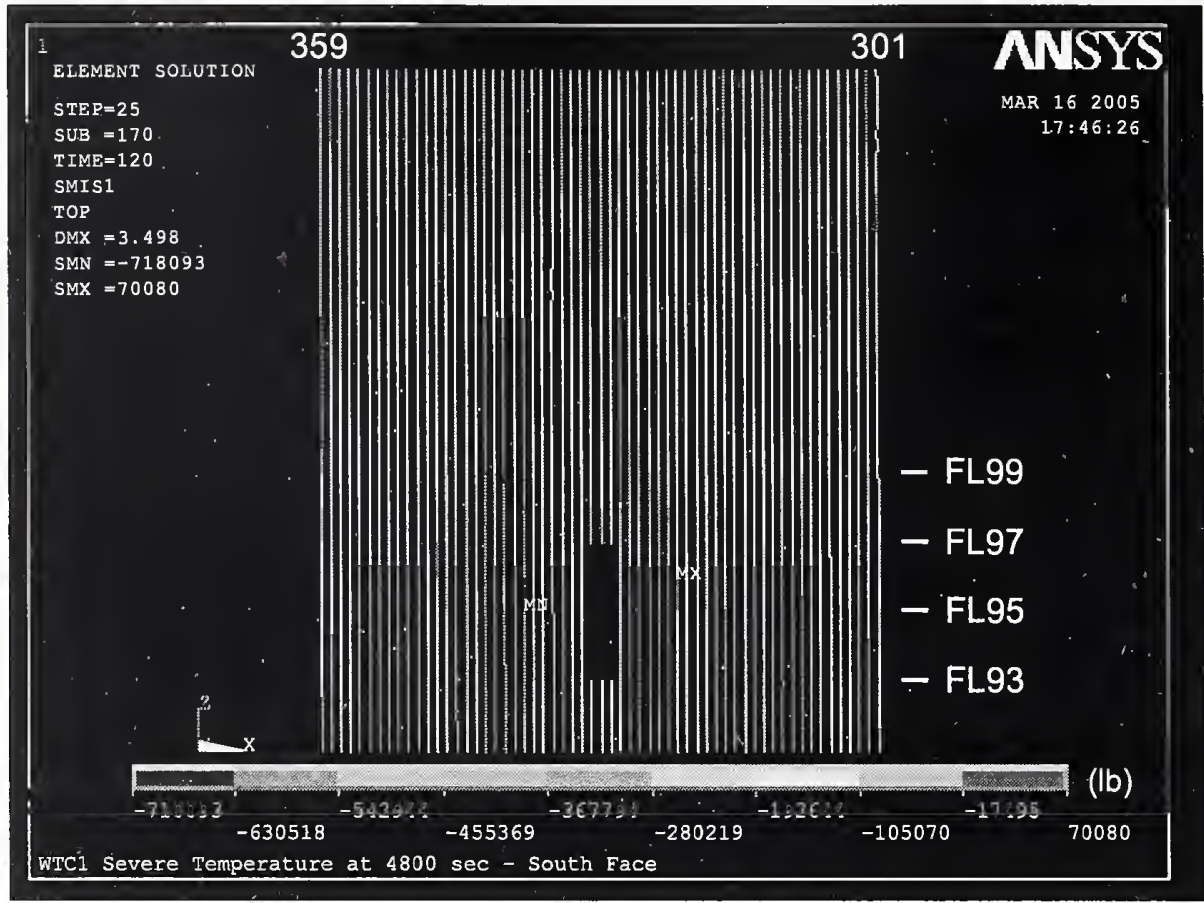

Figure 4-54. Axial load in exterior columns of south wall of WTC 1 at 80 min for Case B conditions (compression is negative).

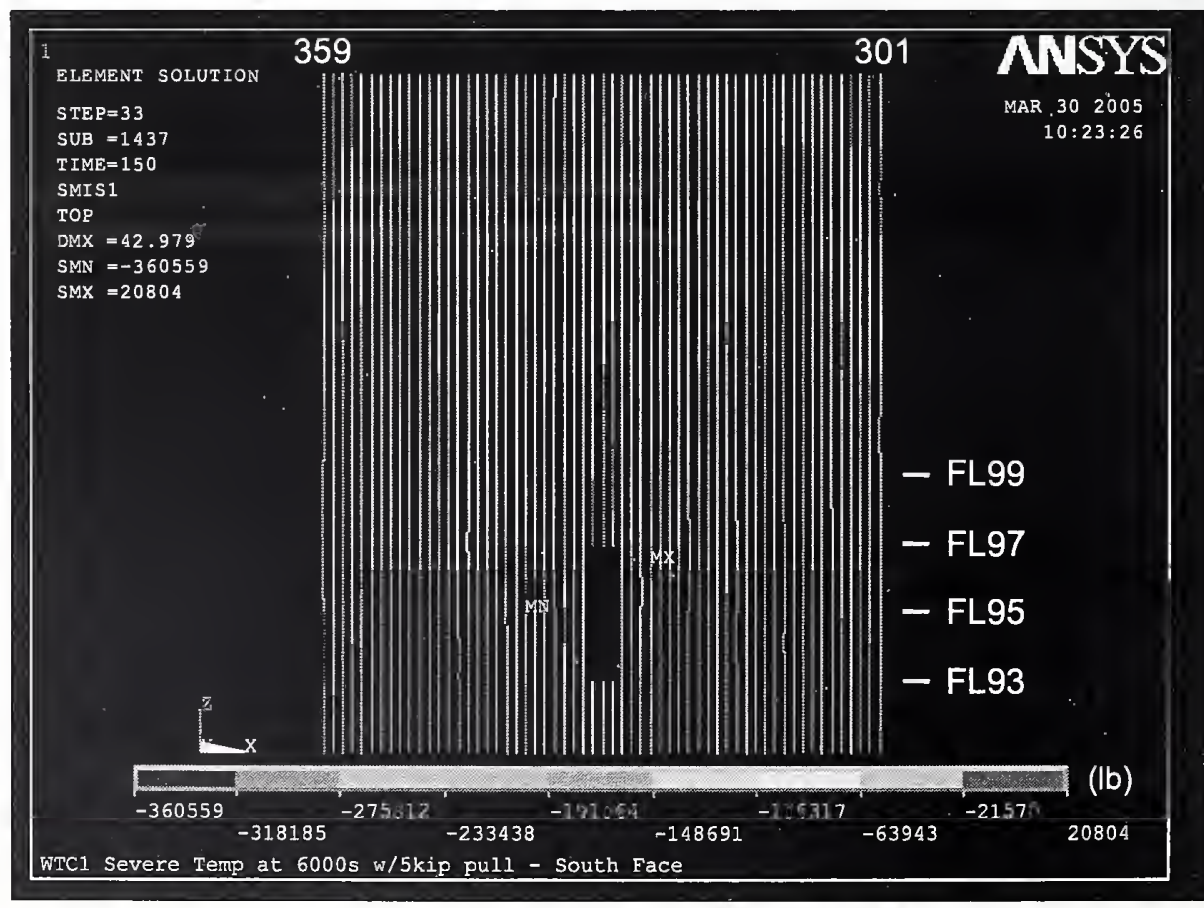

Figure 4-55. Axial load in exterior columns of south wall of WTC 1 at 100 min for Case B conditions with 5 kip pull-in forces (compression is negative). 


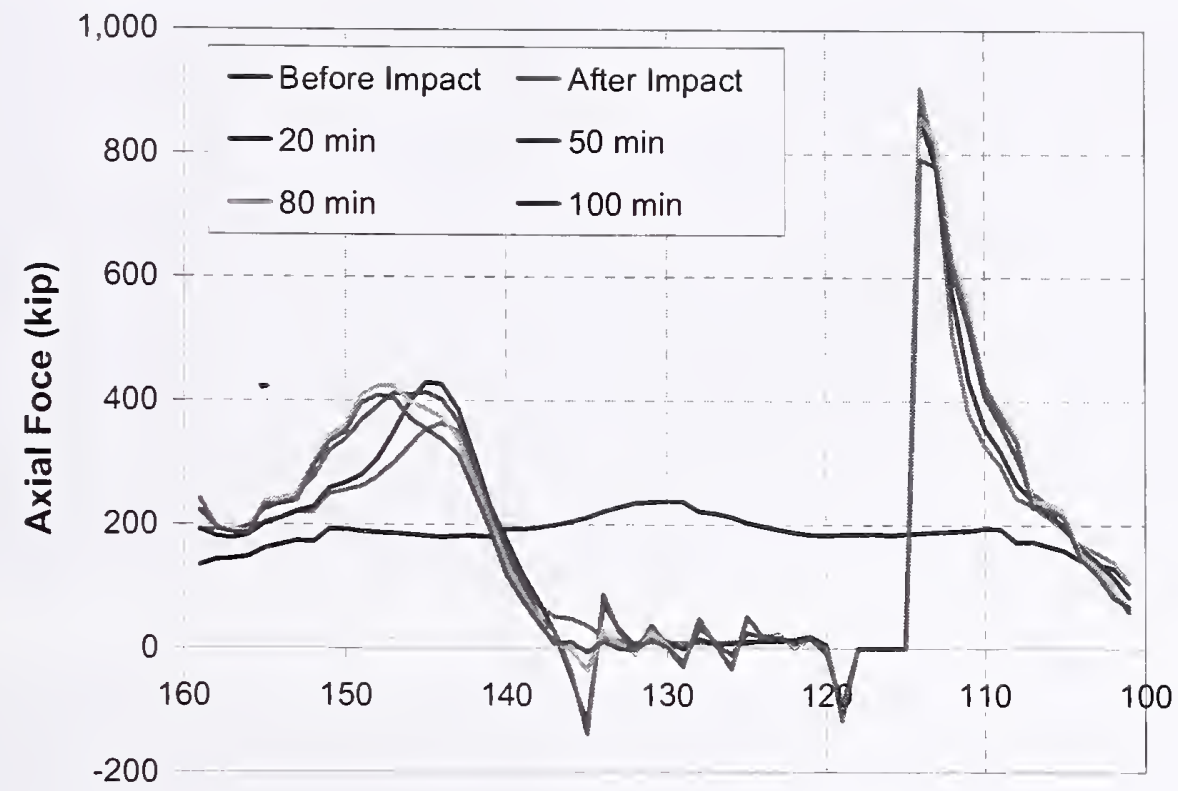

Column ID

Figure 4-56. Variation of axial load in exterior columns at Floor 98 of north wall of WTC 1 for Case B conditions with 5 kip pull-in forces (compression is positive).

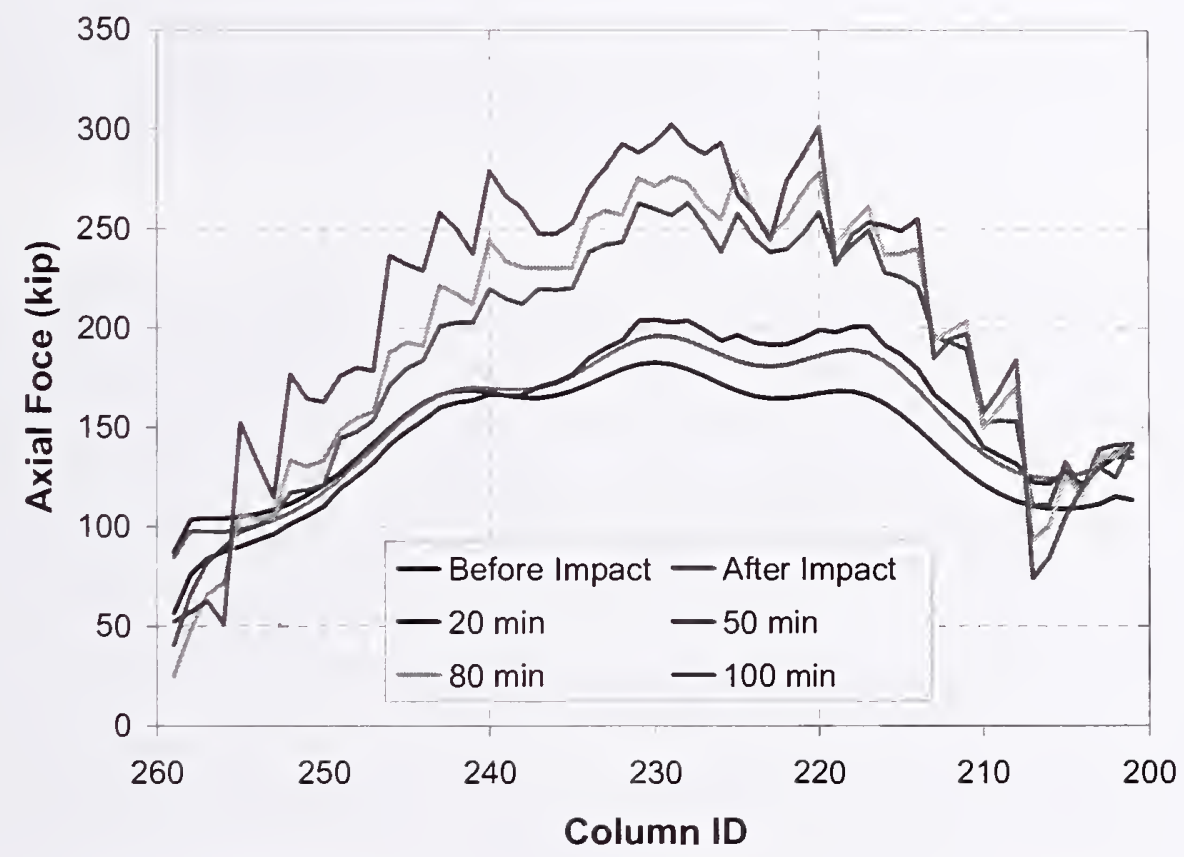

Figure 4-57. Variation of axial load in exterior columns at Floor 98 of east wall of WTC 1 for Case B conditions with 5 kip pull-in forces (compression is positive). 


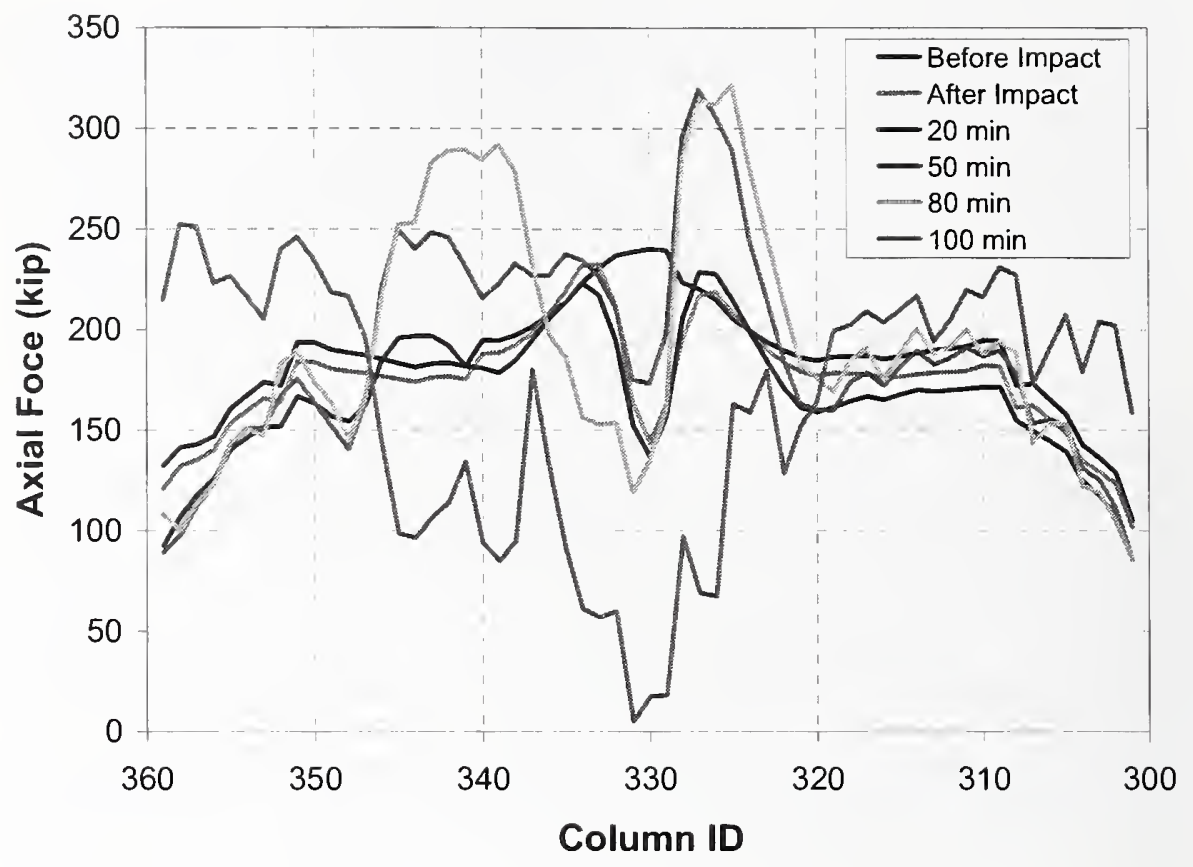

Figure 4-58. Variation of axial load in exterior columns at Floor 98 of south wall of WTC 1 for Case B conditions with 5 kip pull-in forces (compression is positive).

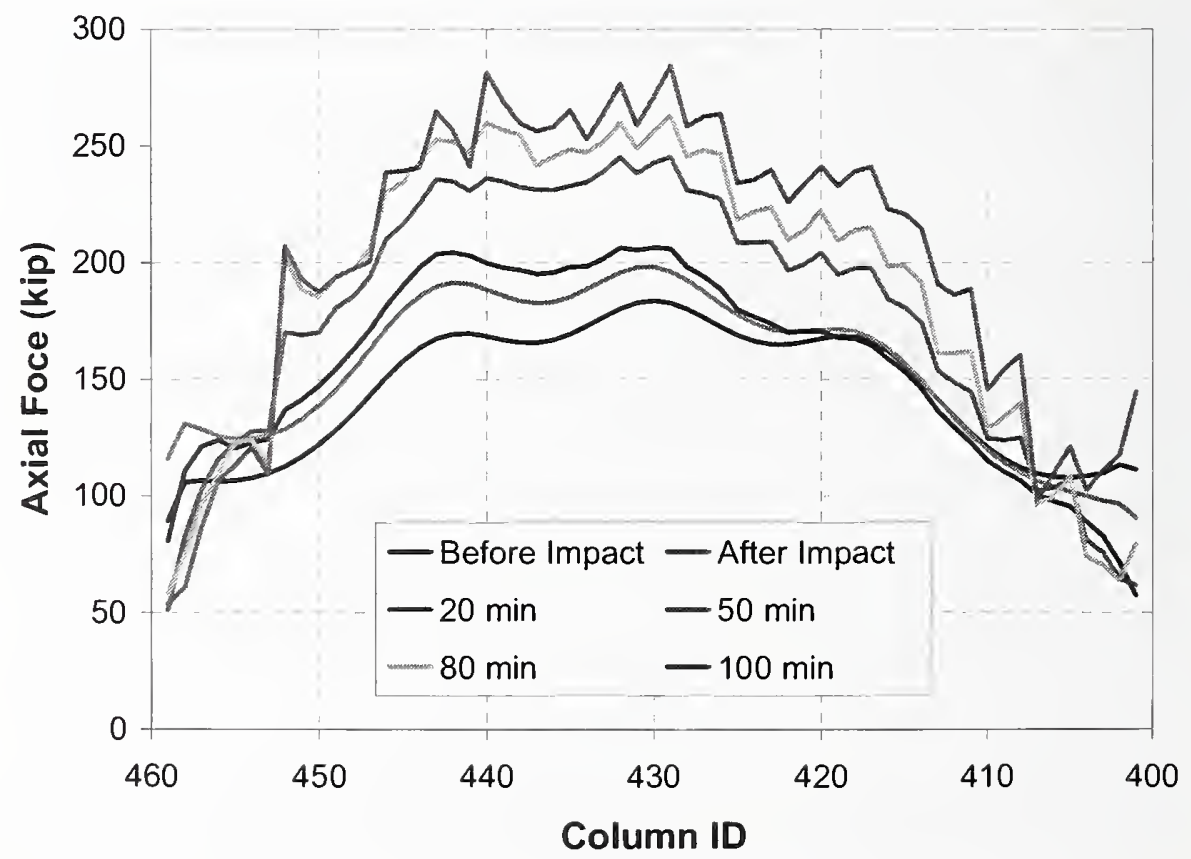

Figure 4-59. Variation of axial load in exterior columns at Floor 98 of west wall of WTC 1 for Case B conditions with $\mathbf{5}$ kip pull-in forces (compression is positive). 


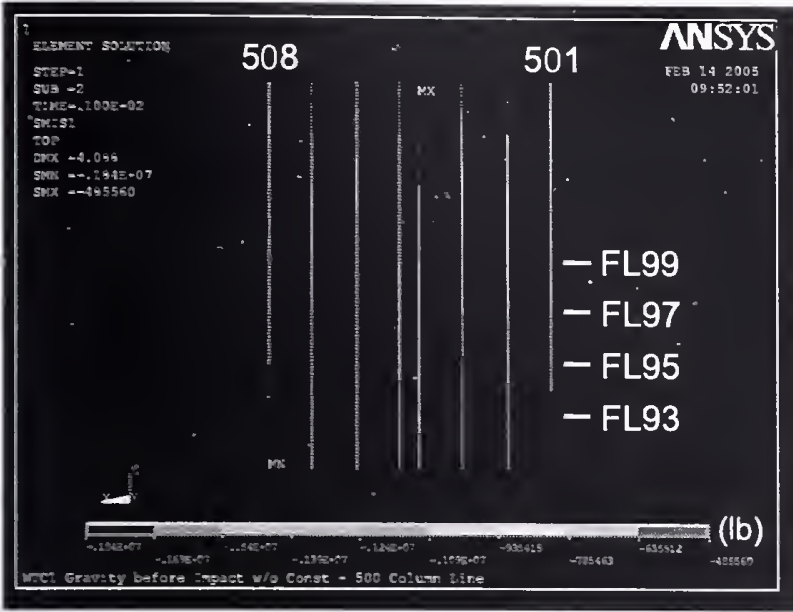

(a) 500 series columns

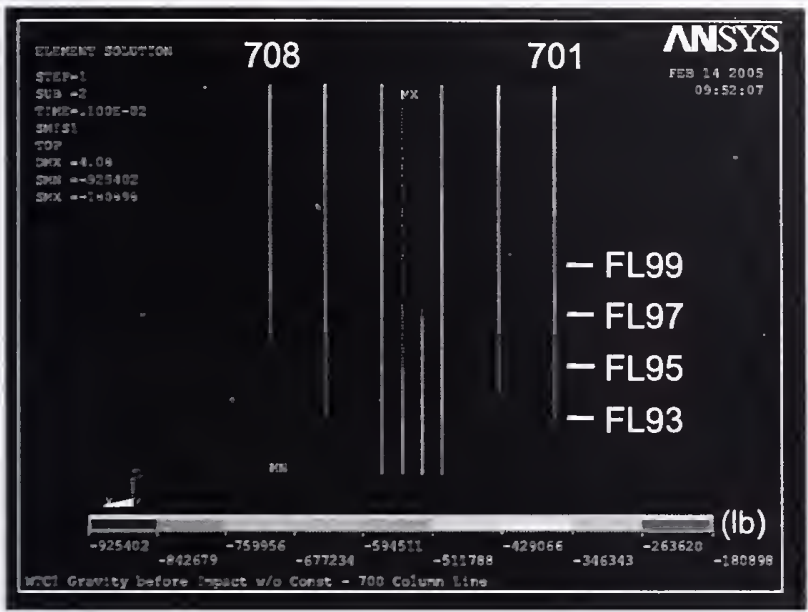

(c) 700 series columns

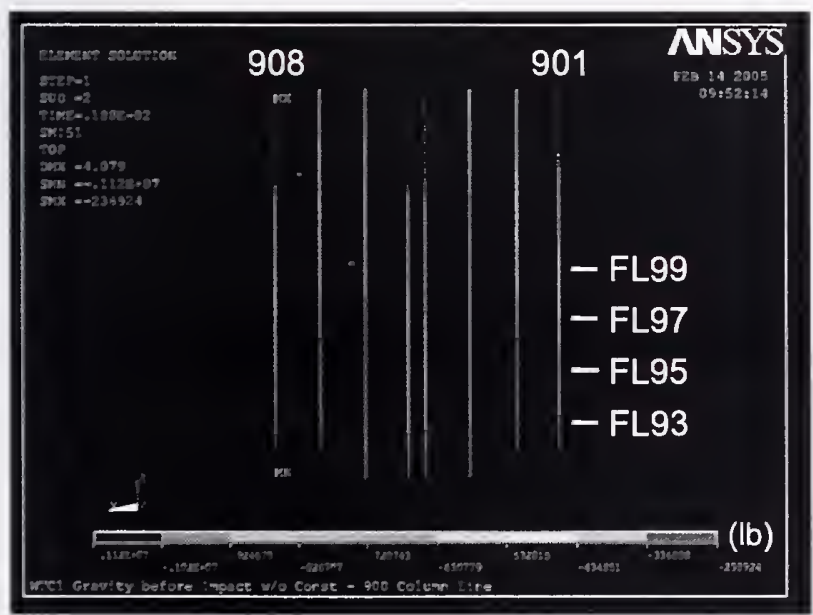

(e) 900 series columns

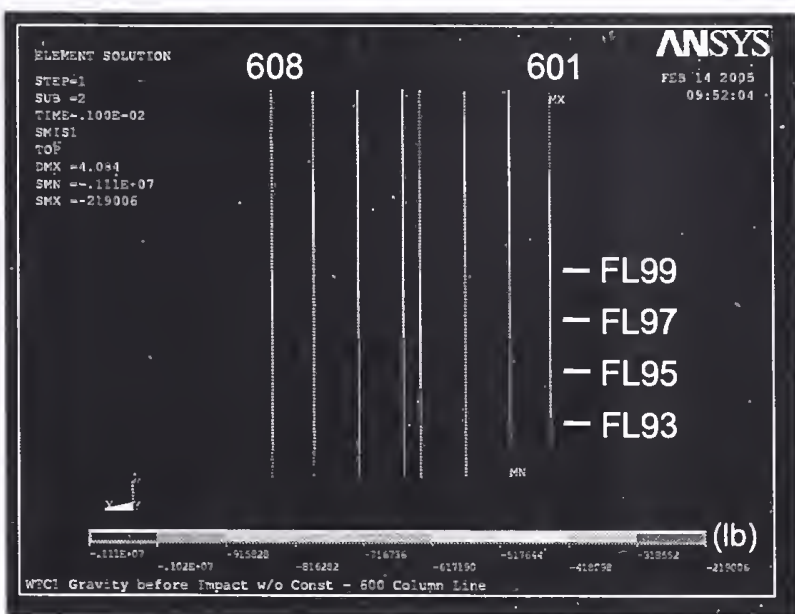

(b) 600 series columns

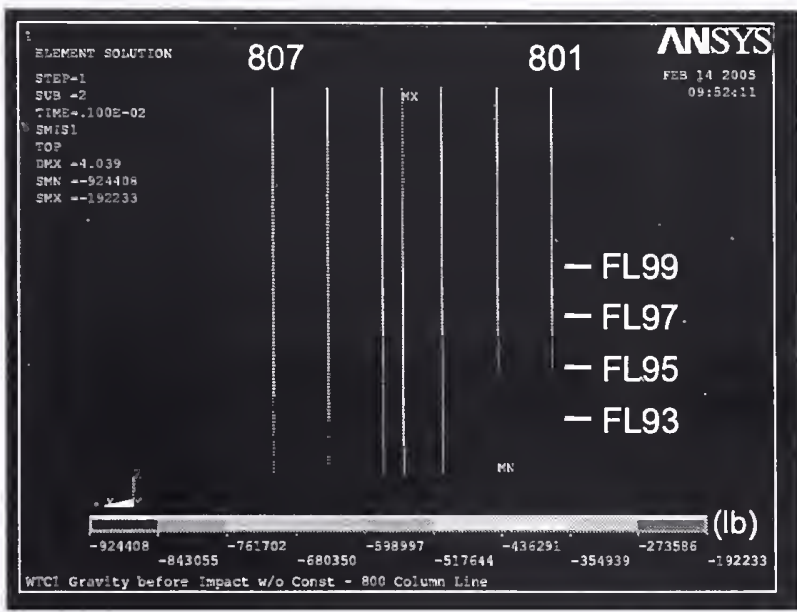

(d) 800 series columns

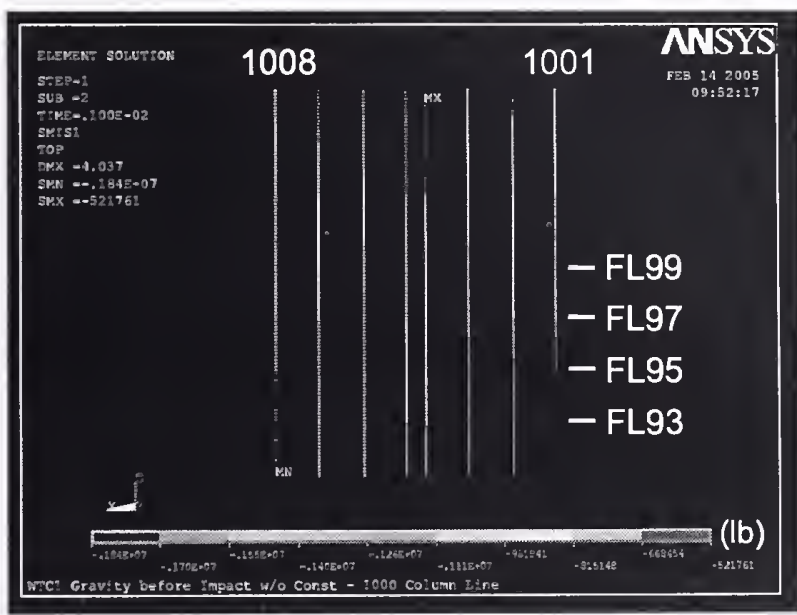

(f) 1000 series columns

Figure 4-60. Axial load in core columns of WTC 1 before aircraft impact (compression is negative). 


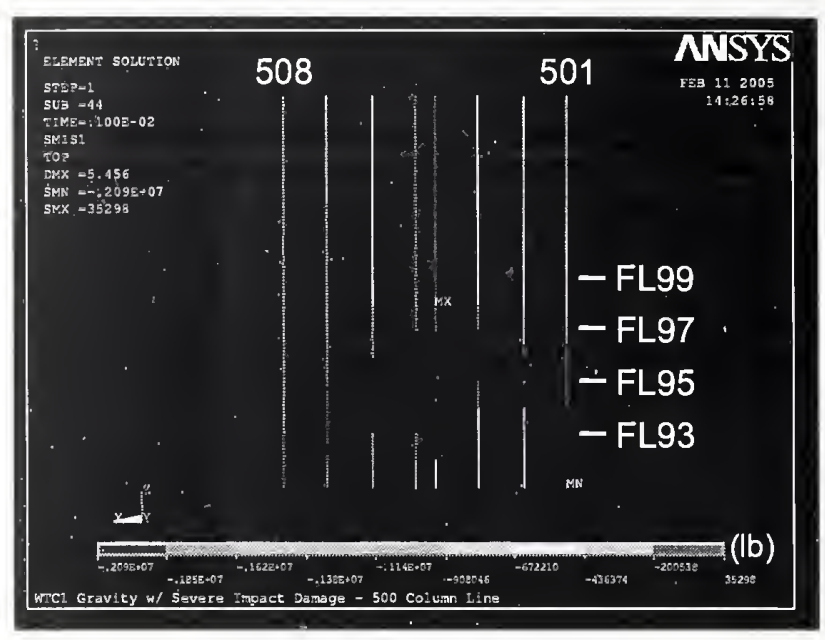

(a) 500 series columns

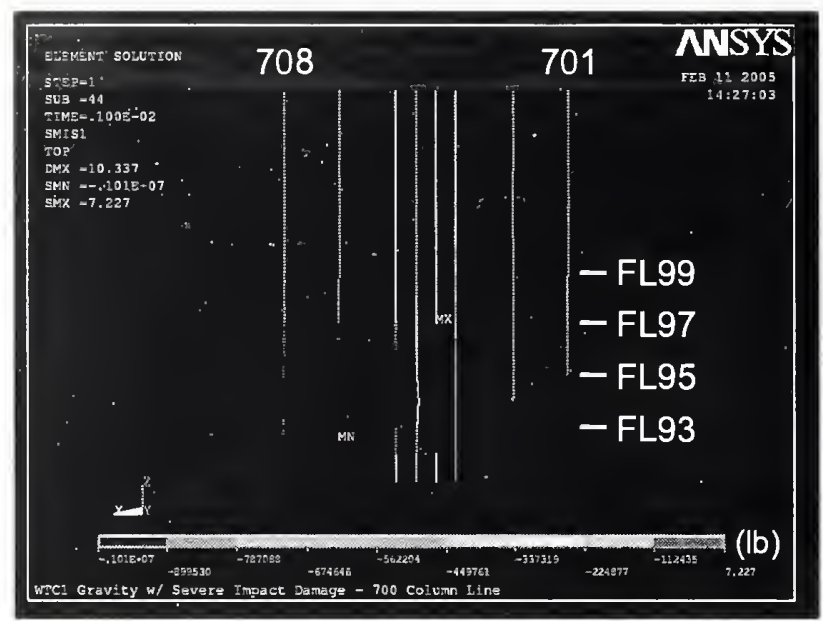

(c) 700 series columns

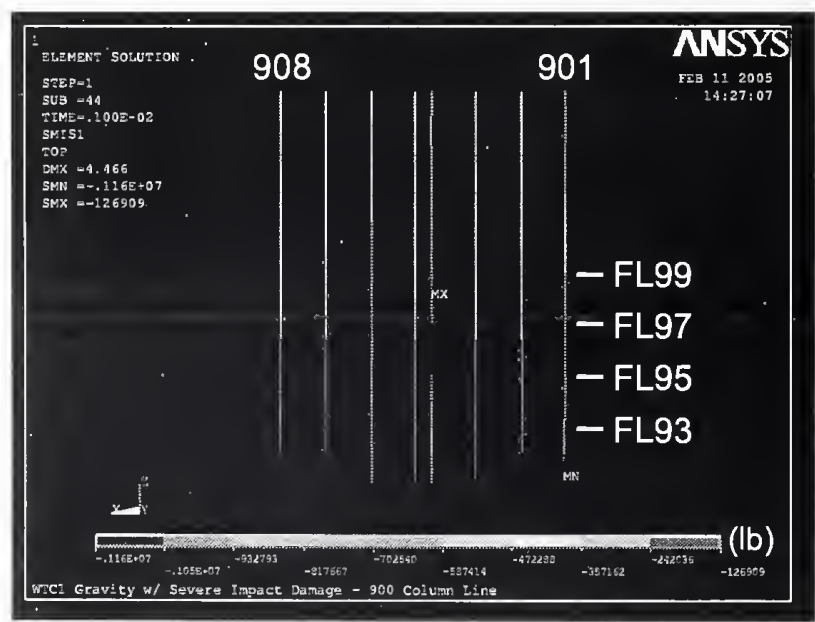

(e) 900 series columns

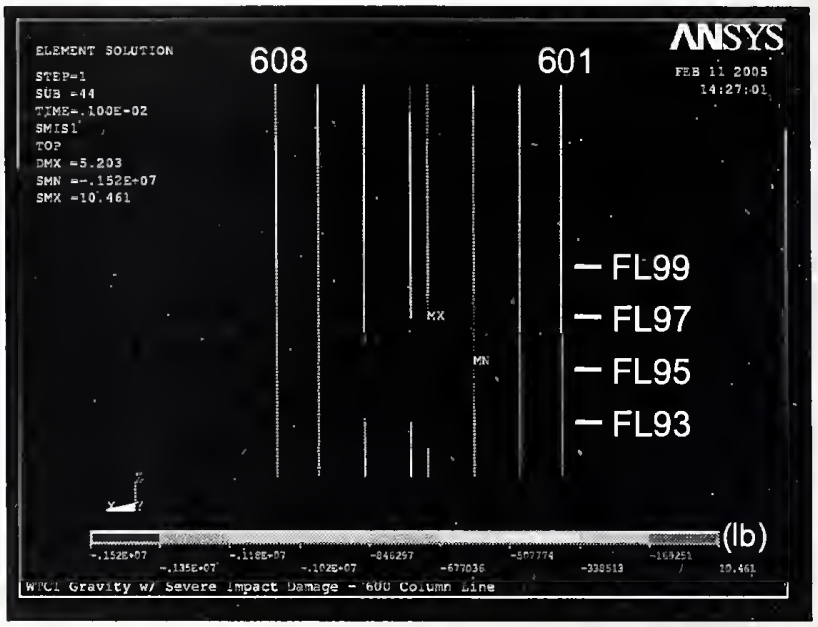

(b) 600 series columns

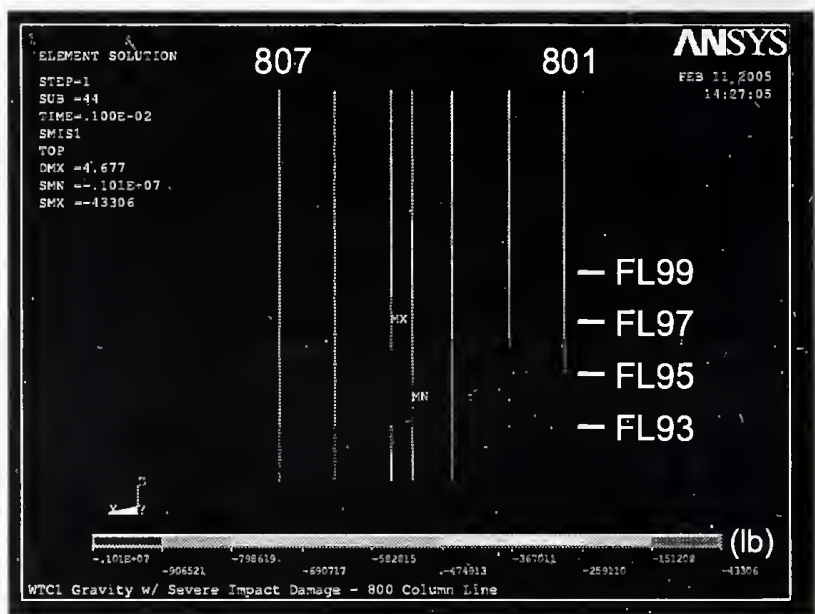

(d) 800 series columns

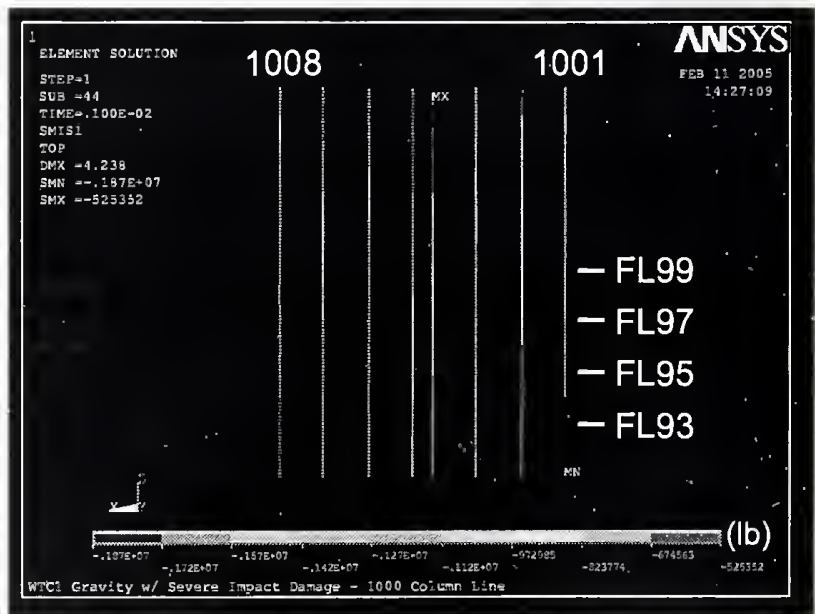

(f) 1000 series columns

Figure 4-61. Axial load in core columns of WTC 1 after aircraft impact for Case B conditions (compression is negative). 


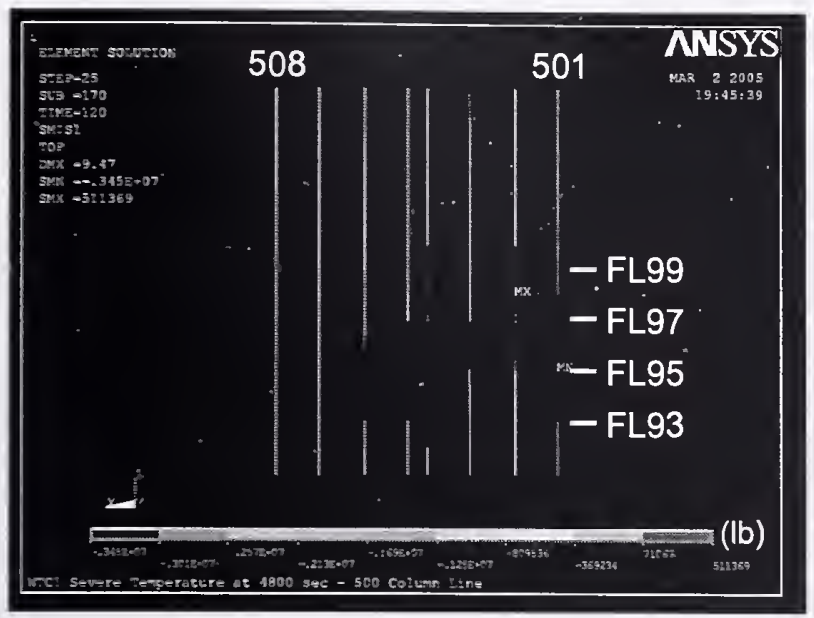

(a) 500 series columns

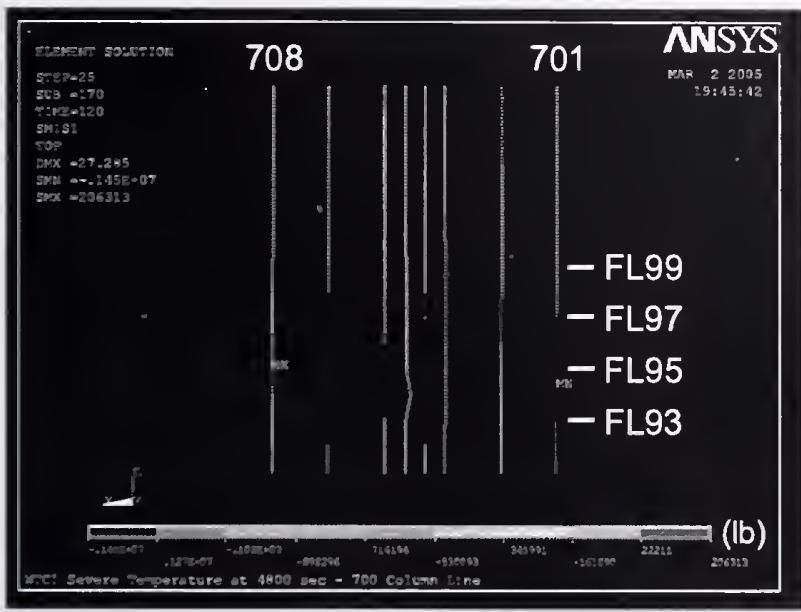

(c) 700 series columns

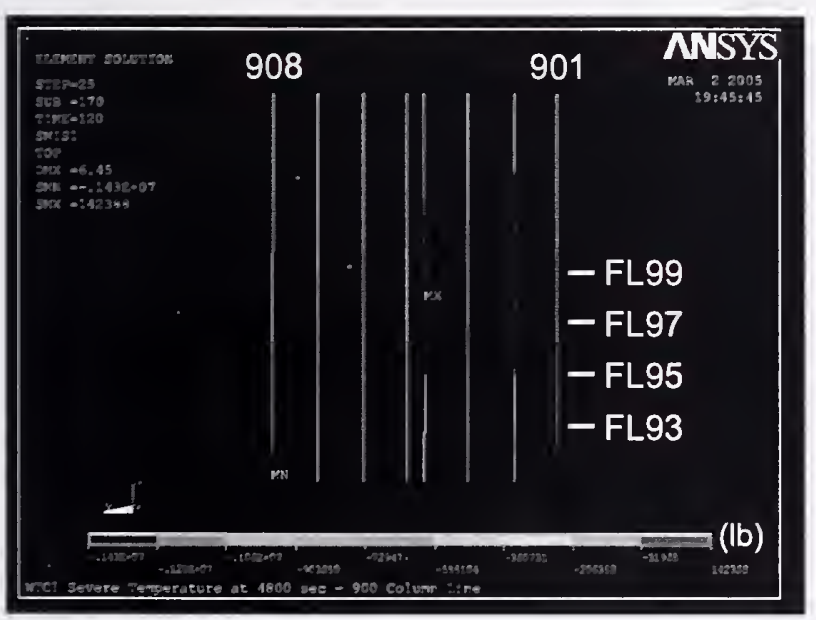

(e) 900 series columns

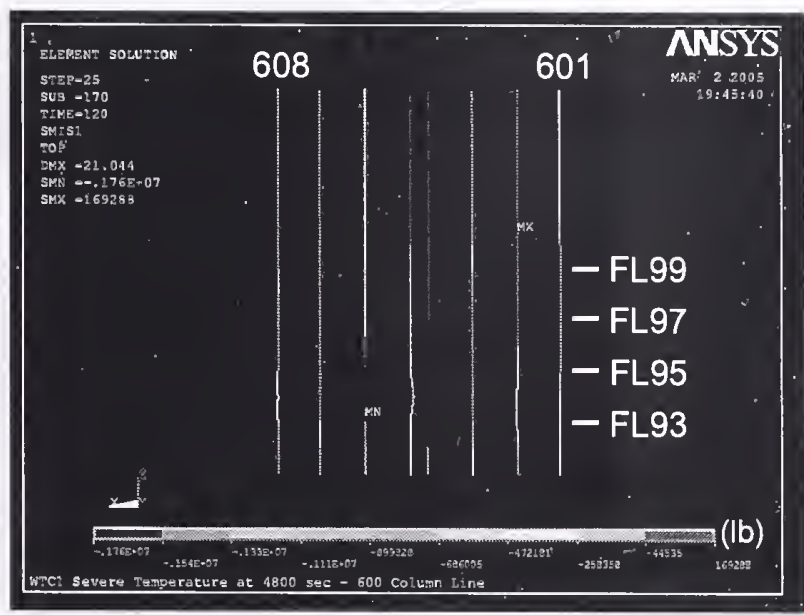

(b) 600 series columns

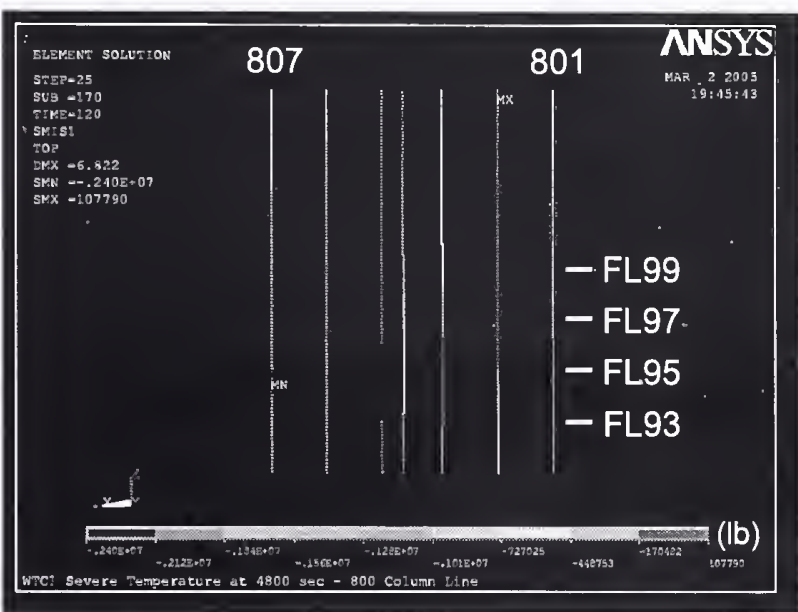

(d) 800 series columns

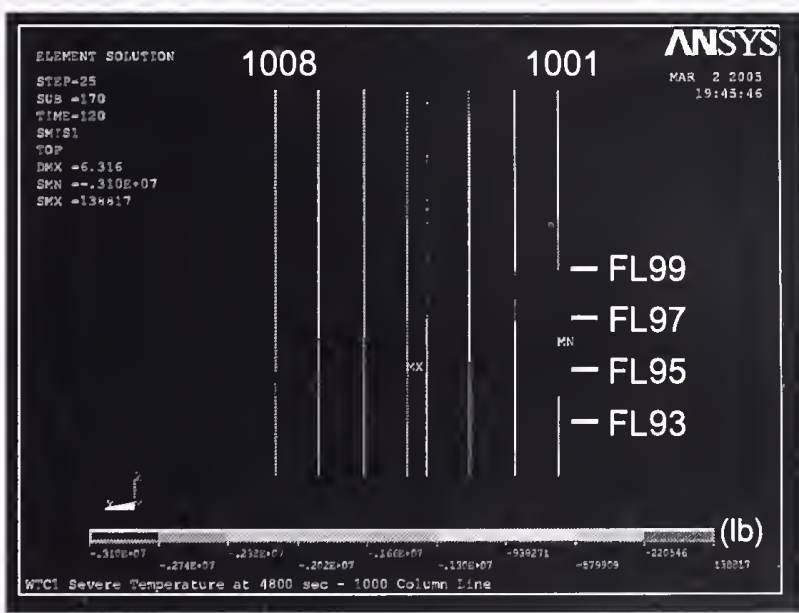

(f) 1000 series columns

Figure 4-62. Axial load in core columns of WTC 1 at $80 \mathrm{~min}$ for Case B conditions (compression is negative). 


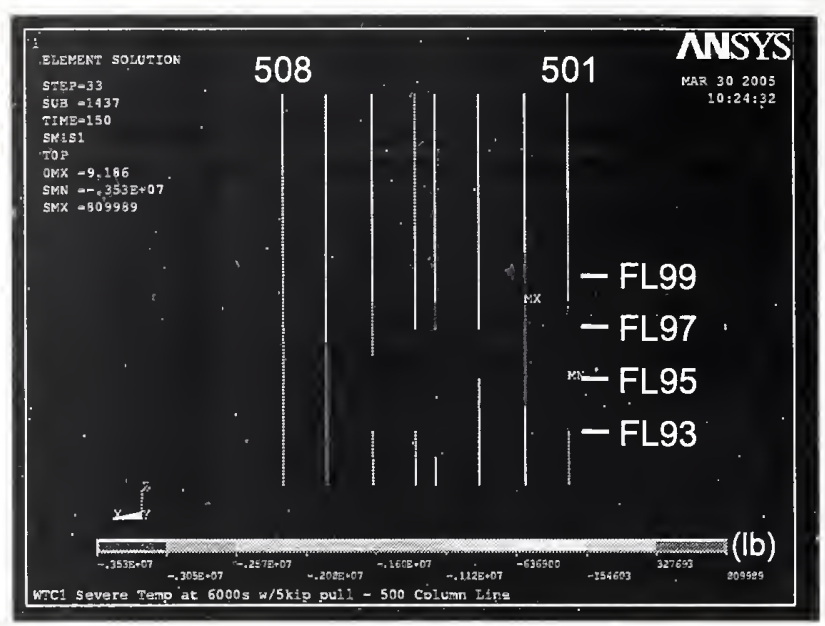

(a) 500 series columns

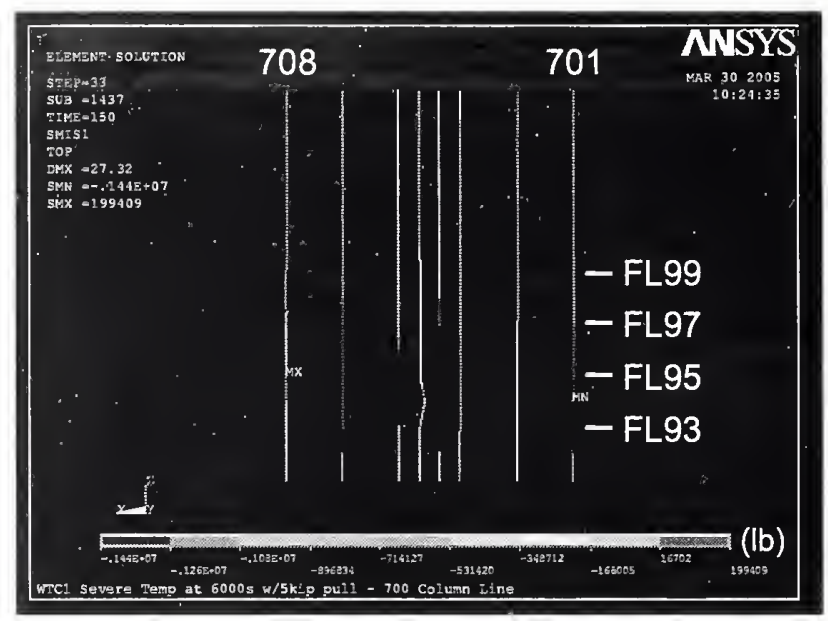

(c) 700 series columns

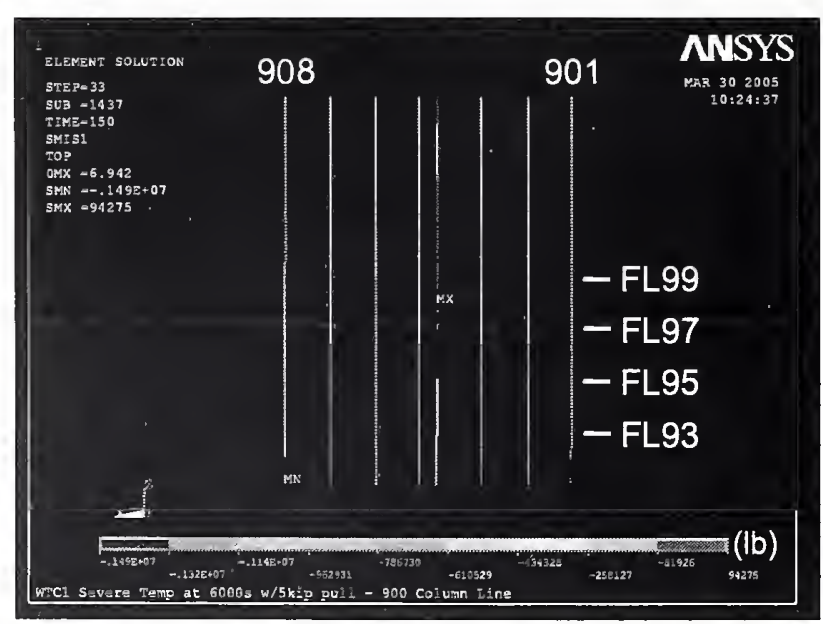

(e) 900 series columns

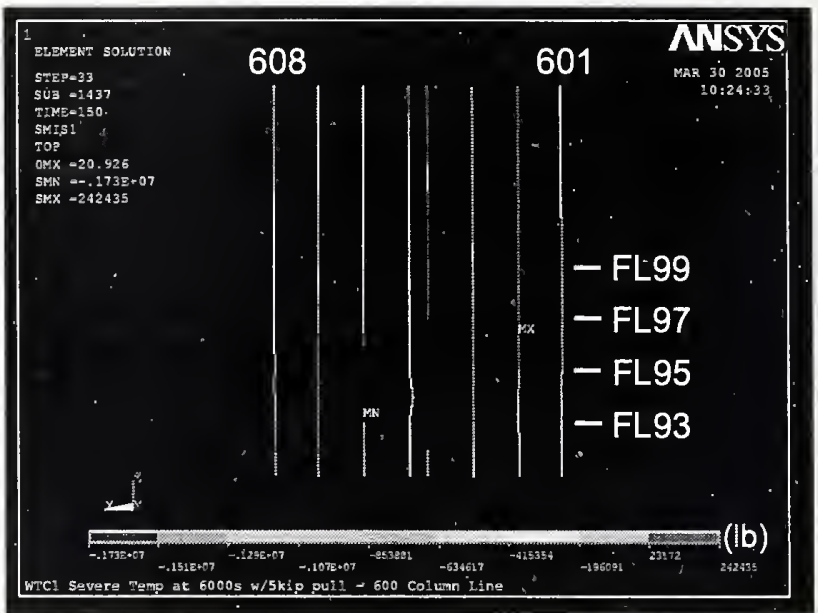

(b) 600 series columns

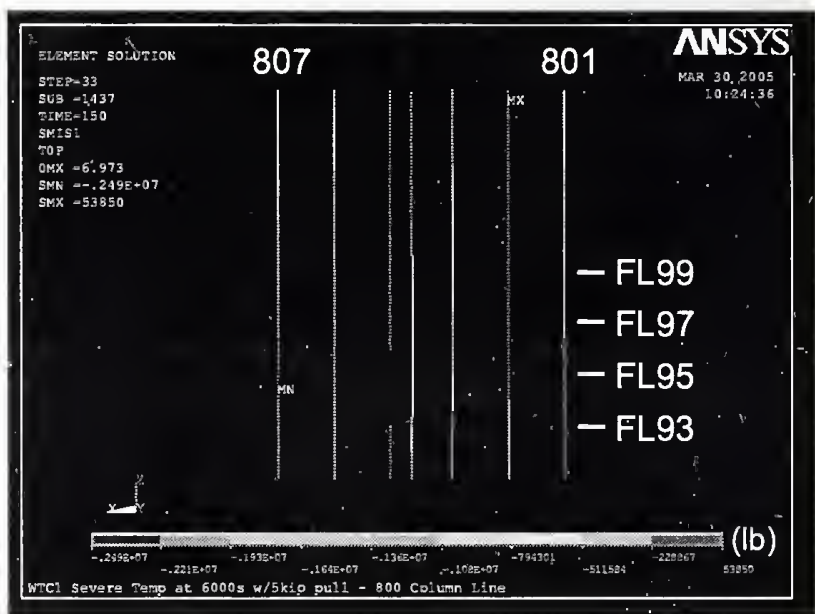

(d) 800 series columns

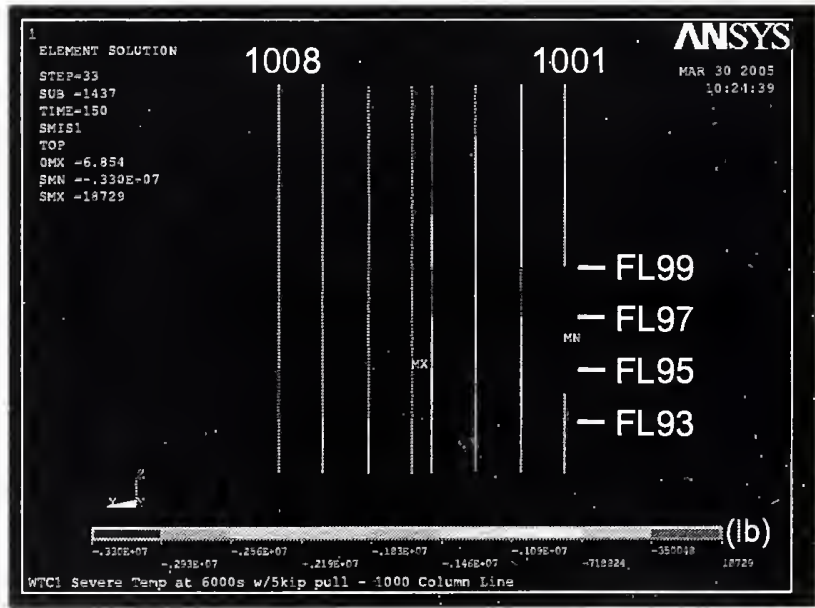

(f) 1000 series columns

Figure 4-63. Axial load in core columns of WTC 1 at $100 \mathrm{~min}$ for Case B conditions with 5 kip pull-in forces (compression is negative). 


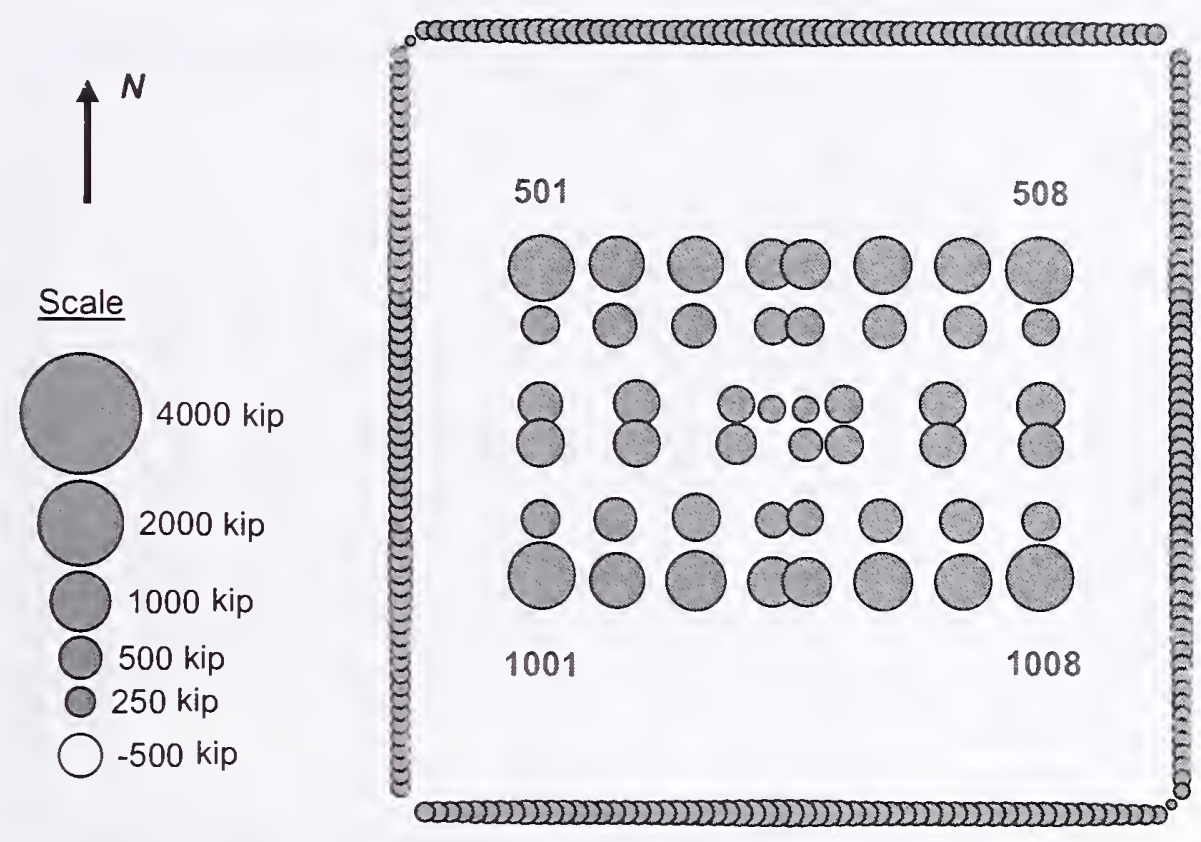

Figure 4-64. Axial load in columns at Floor 98 of WTC 1 before aircraft impact (compression is positive).
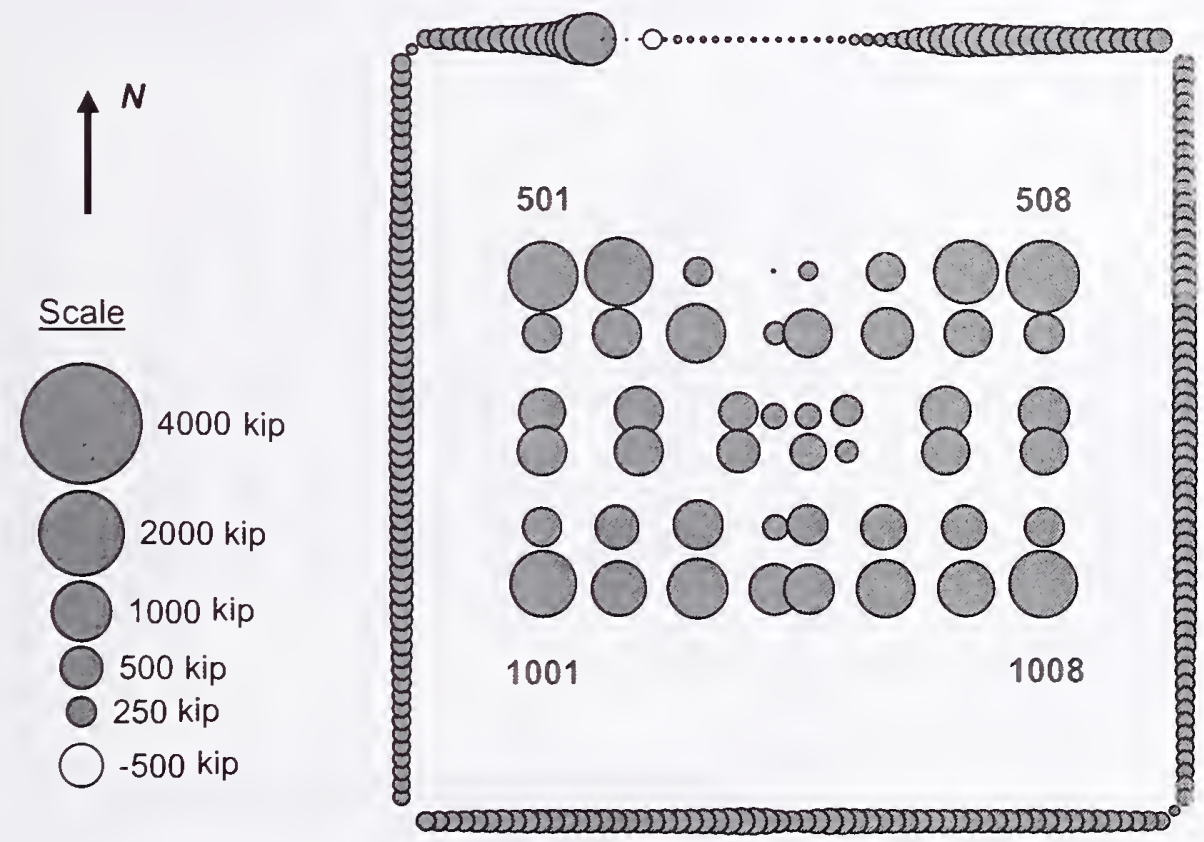

Figure 4-65. Axial load in columns at Floor 98 of WTC 1 after aircraft impact for Case B conditions (compression is positive). 

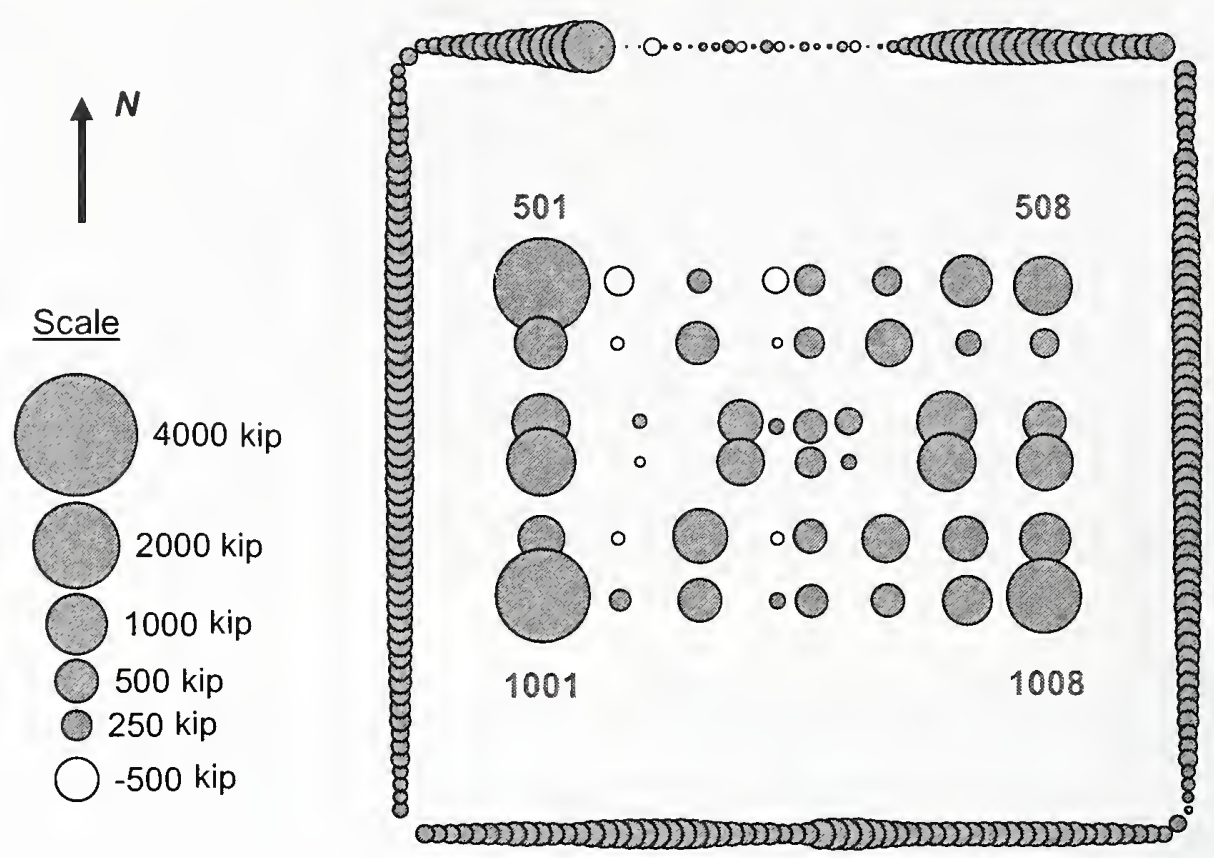

Figure 4-66. Axial load in columns at Floor 98 of WTC 1 at $80 \mathrm{~min}$ for Case B conditions (compression is positive).
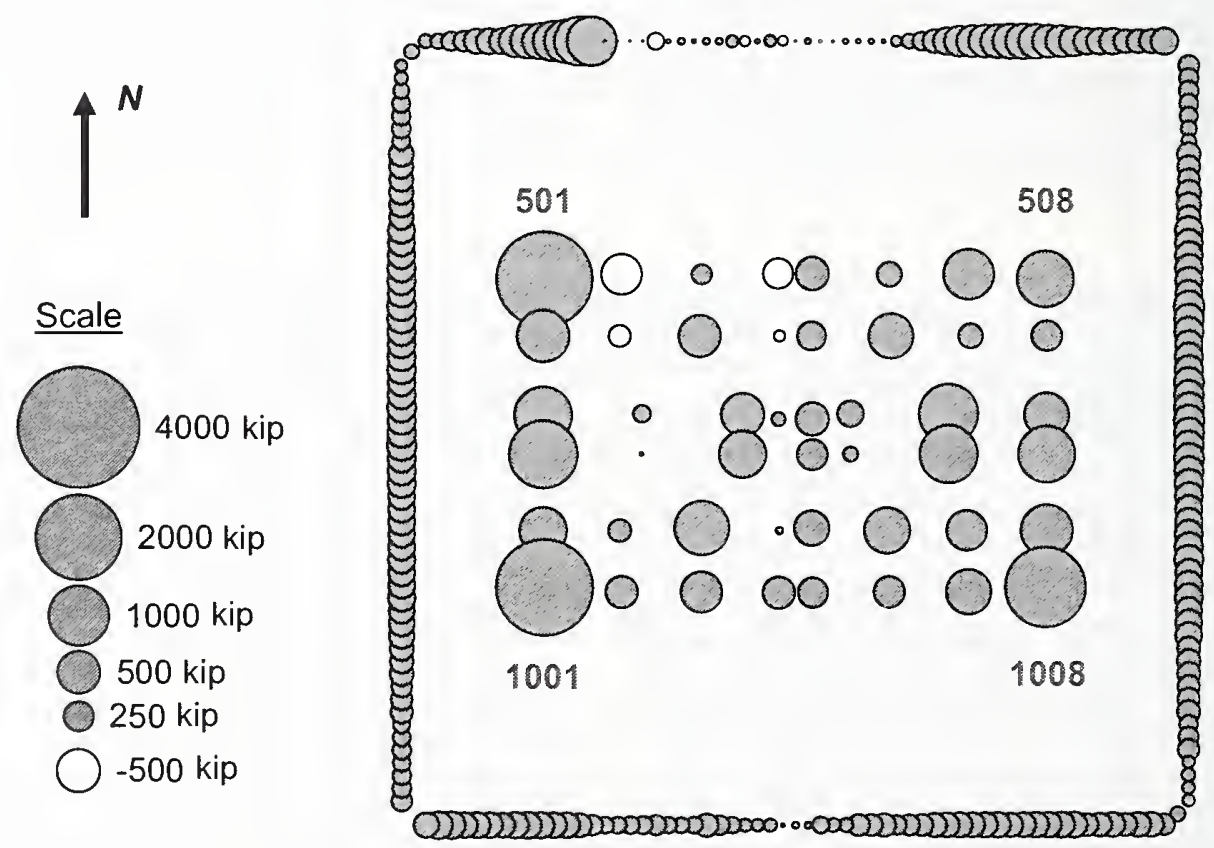

Figure 4-67. Axial load in columns at Floor 98 of WTC 1 at $100 \mathrm{~min}$ for Case B conditions with 5 kip pull-in forces (compression is positive). 
Table 4-10. Total column loads at Floor 98 of WTC 1 for Case B conditions.

\begin{tabular}{|c|c|cccccc|}
\hline Row & Analysis Step & North & East & South & West & Core & Total \\
\hline \hline$(1)$ & Before Impact & 10,974 & 8,545 & 11,025 & 8,572 & 34,029 & 73,144 \\
$(2)$ & After Impact & 10,137 & 9,071 & 10,356 & 9,146 & 34,429 & 73,139 \\
$(3)$ & $10 \mathrm{~min}$ & 9,796 & 8,490 & 9,848 & 8,536 & 36,473 & 73,143 \\
$(4)$ & $20 \mathrm{~min}$ & 10,437 & 9,108 & 9,900 & 9,202 & 34,495 & 73,143 \\
$(5)$ & $30 \mathrm{~min}$ & 10,913 & 10,034 & 10,420 & 9,715 & 32,060 & 73,142 \\
$(6)$ & $40 \mathrm{~min}$ & 11,068 & 10,599 & 11,004 & 10,178 & 30,294 & 73,142 \\
$(7)$ & $50 \mathrm{~min}$ & 11,149 & 10,908 & 11,192 & 10,458 & 29,435 & 73,141 \\
$(8)$ & $60 \mathrm{~min}$ & 11,205 & 11,168 & 11,285 & 10,716 & 28,766 & 73,141 \\
$(9)$ & $70 \mathrm{~min}$ & 11,286 & 11,366 & 11,343 & 10,939 & 28,205 & 73,138 \\
$(10)$ & $80 \mathrm{~min}$ & 11,376 & 11,555 & 11,409 & 11,119 & 27,681 & 73,140 \\
$(11)$ & $90 \mathrm{~min}$ & 10,916 & 11,991 & 9,949 & 11,657 & 28,587 & 73,099 \\
$(12)$ & $100 \mathrm{~min}$ & 10,828 & 12,249 & 9,638 & 11,905 & 28,478 & 73,098 \\
\hline$(13)$ & $(2)-(1)$ & -837 & 526 & -668 & 574 & 400 & -5 \\
$(14)$ & $(10)-(2)$ & 1,239 & 2,484 & 1,052 & 1,973 & $-6,748$ & 1 \\
$(15)$ & $(12)-(2)$ & 692 & 3,178 & -719 & 2,759 & $-5,951$ & -41 \\
$(16)$ & $(12)-(10)$ & -548 & 694 & $-1,771$ & 786 & 797 & -42 \\
\hline
\end{tabular}

Note: Compression is positive. Units are in kip.

Table 4-11. Total column loads at Floor 105 of WTC 1 for Case B conditions.

\begin{tabular}{|c|c|cccccc|}
\hline Row & Analysis Step & North & East & South & West & Core & Total \\
\hline \hline$(1)$ & Before Impact & 8,026 & 6,562 & 8,092 & 6,604 & 20,361 & 49,645 \\
$(2)$ & After Impact & 7,294 & 7,028 & 7,488 & 7,076 & 20,761 & 49,646 \\
$(3)$ & $10 \mathrm{~min}$ & 6,944 & 6,461 & 6,981 & 6,469 & 22,790 & 49,646 \\
$(4)$ & $20 \mathrm{~min}$ & 7,551 & 7,075 & 7,057 & 7,158 & 20,806 & 49,647 \\
$(5)$ & $30 \mathrm{~min}$ & 8,020 & 7,998 & 7,569 & 7,685 & 18,377 & 49,648 \\
$(6)$ & $40 \mathrm{~min}$ & 8,193 & 8,571 & 8,129 & 8,147 & 16,608 & 49,649 \\
$(7)$ & $50 \mathrm{~min}$ & 8,285 & 8,878 & 8,315 & 8,428 & 15,743 & 49,650 \\
$(8)$ & $60 \mathrm{~min}$ & 8,351 & 9,130 & 8,414 & 8,687 & 15,069 & 49,650 \\
$(9)$ & $70 \mathrm{~min}$ & 8,435 & 9,319 & 8,481 & 8,914 & 14,502 & 49,651 \\
$(10)$ & $80 \mathrm{~min}$ & 8,528 & 9,497 & 8,551 & 9,097 & 13,978 & 49,651 \\
$(11)$ & $90 \mathrm{~min}$ & 8,096 & 9,847 & 7,327 & 9,506 & 14,876 & 49,652 \\
$(12)$ & $100 \mathrm{~min}$ & 8,023 & 10,076 & 7,066 & 9,720 & 14,767 & 49,653 \\
\hline$(13)$ & $(2)-(1)$ & -732 & 466 & -604 & 472 & 400 & 1 \\
$(14)$ & $(10)-(2)$ & 1,234 & 2,470 & 1,063 & 2,021 & $-6,783$ & 5 \\
$(15)$ & $(12)-(2)$ & 730 & 3,048 & -422 & 2,644 & $-5,993$ & 7 \\
$(16)$ & $(12)-(10)$ & -504 & 579 & $-1,485$ & 623 & 790 & 2 \\
\hline
\end{tabular}

Note: Compression is positive. Units are in kip. 


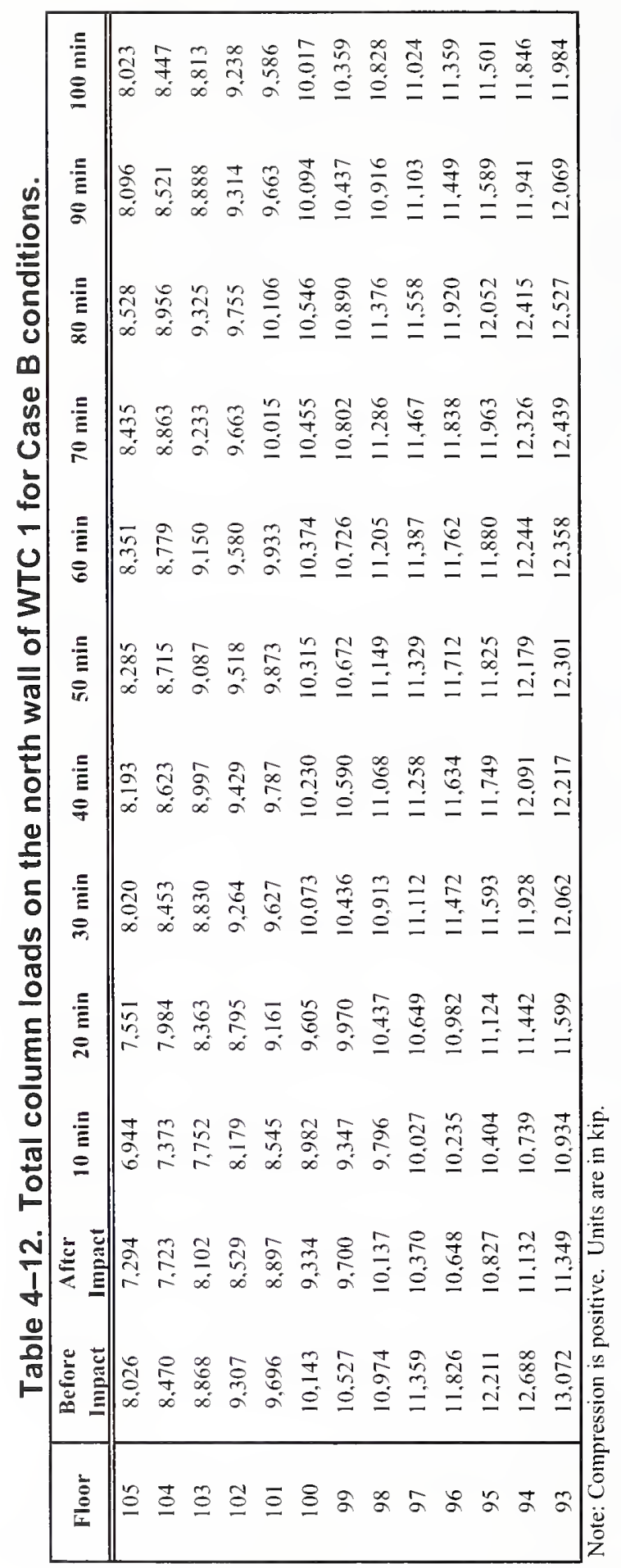

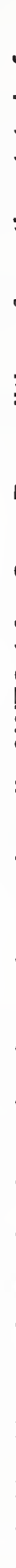

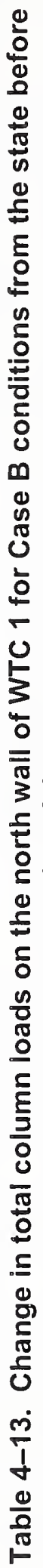

\begin{tabular}{|c|c|}
\hline & \\
\hline 玄 & 队구 \\
\hline 言春 & 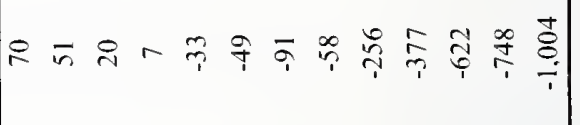 \\
\hline 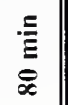 & 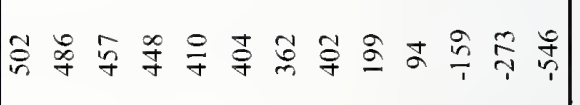 \\
\hline 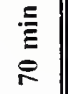 & 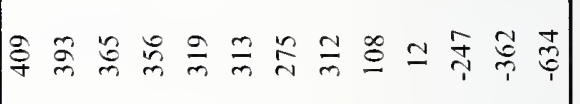 \\
\hline 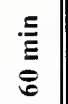 & 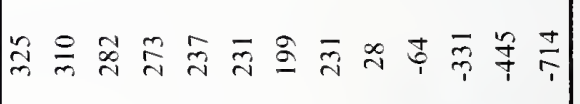 \\
\hline 毫 & 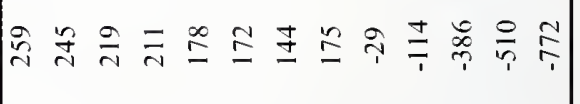 \\
\hline 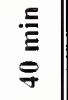 & 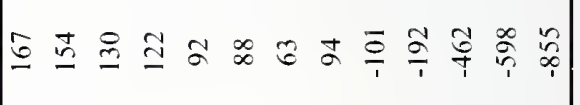 \\
\hline 訔 & 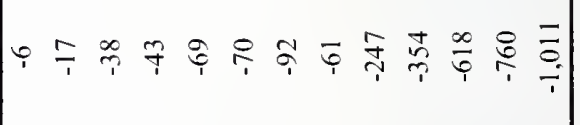 \\
\hline 言 & 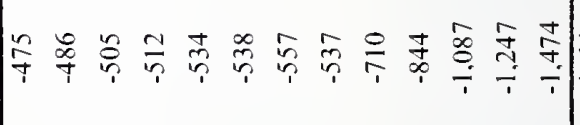 \\
\hline 裹 & 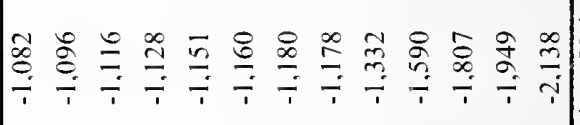 \\
\hline 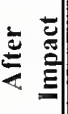 & 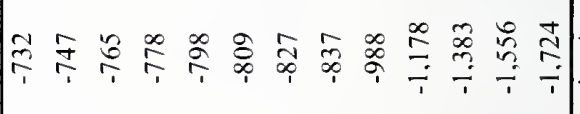 \\
\hline 产 & 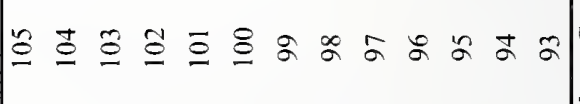 \\
\hline
\end{tabular}



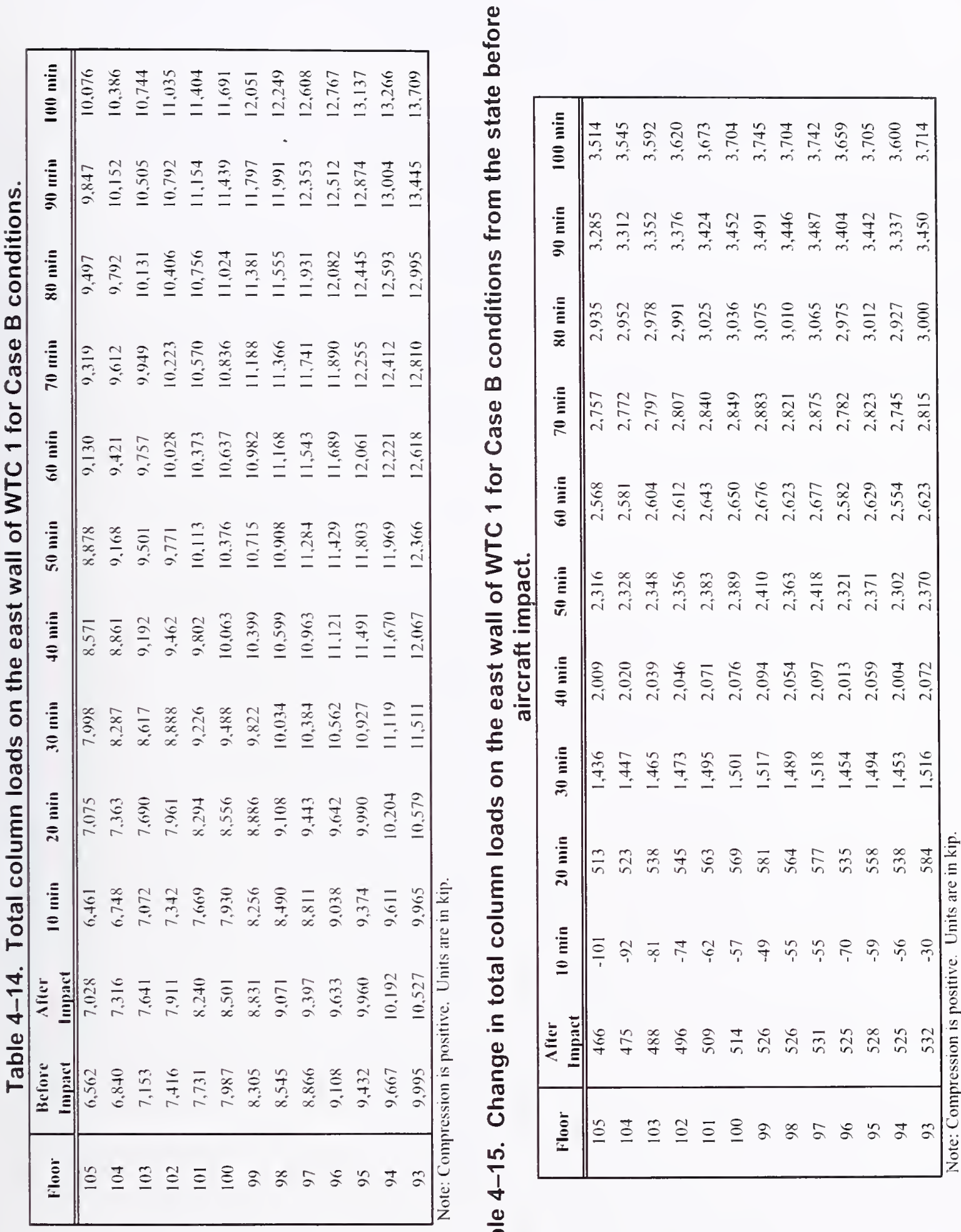


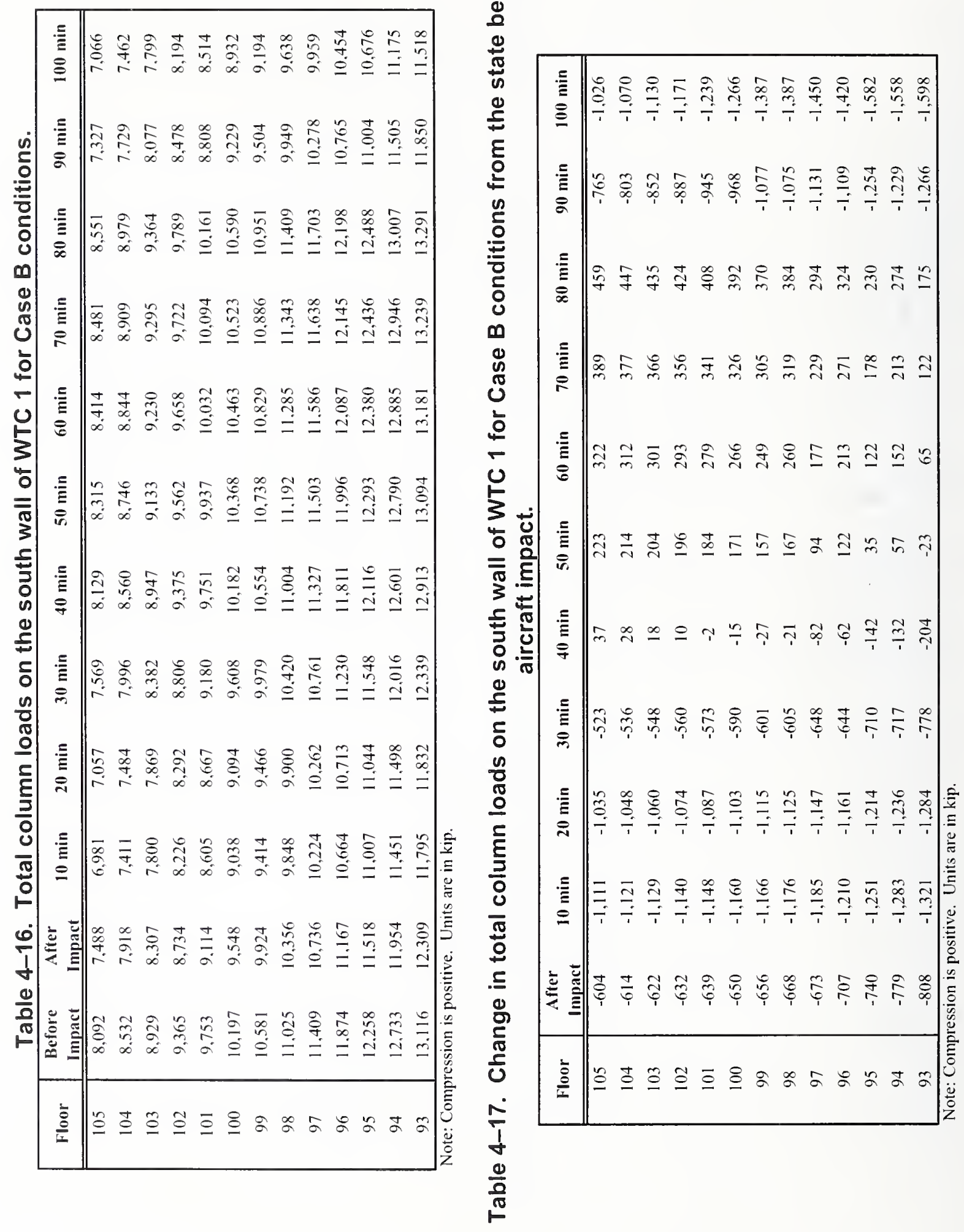




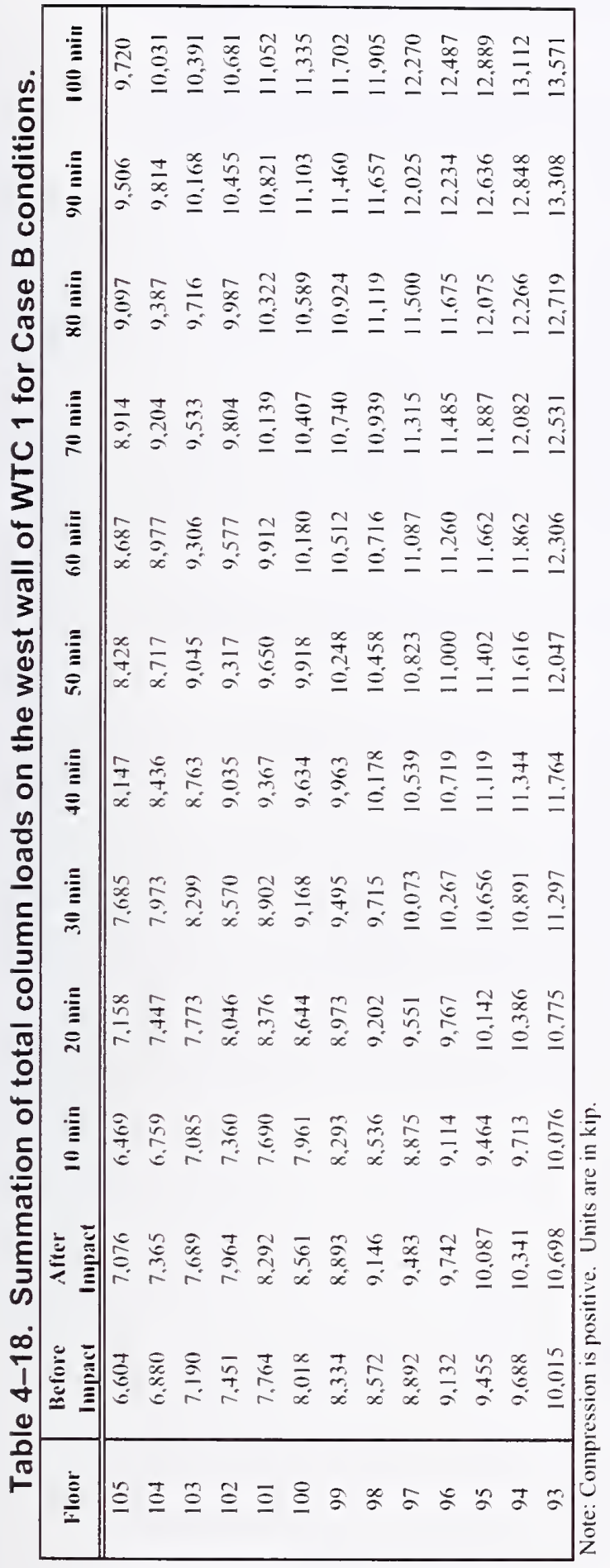

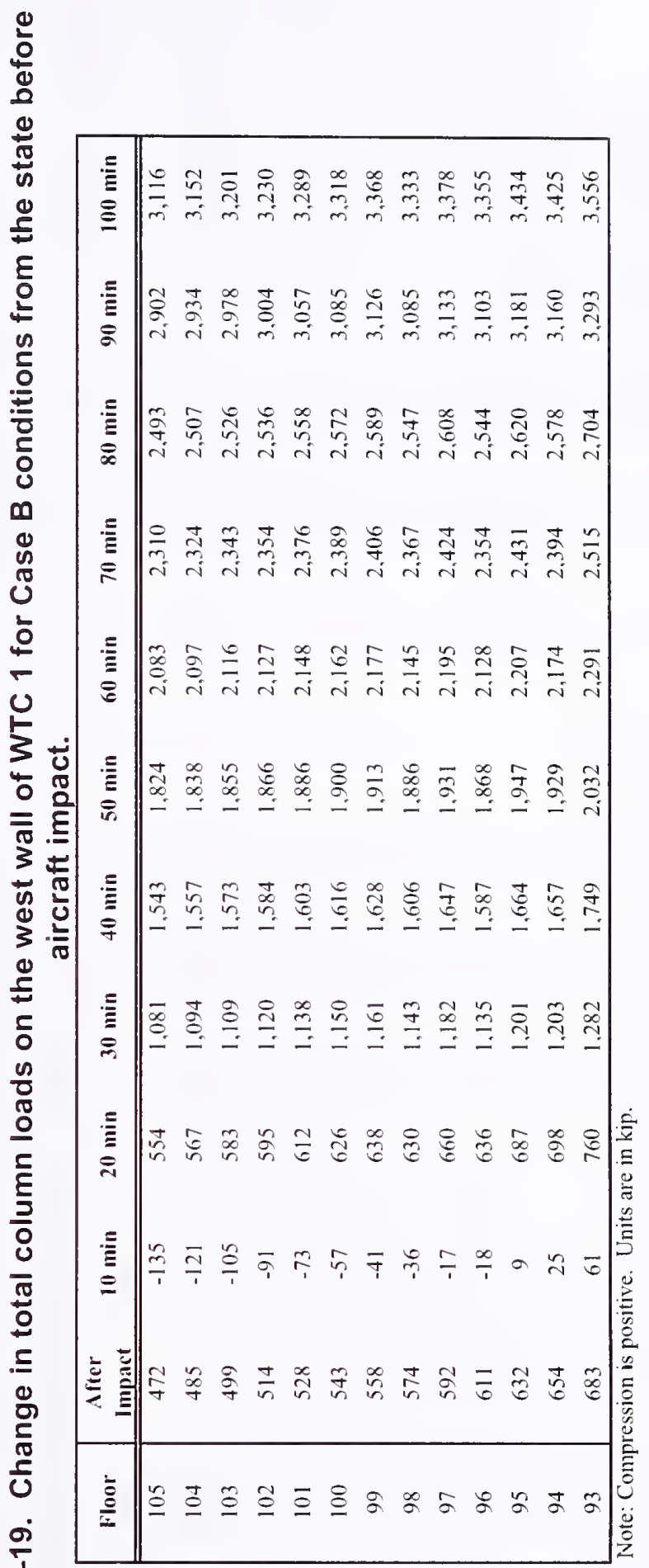



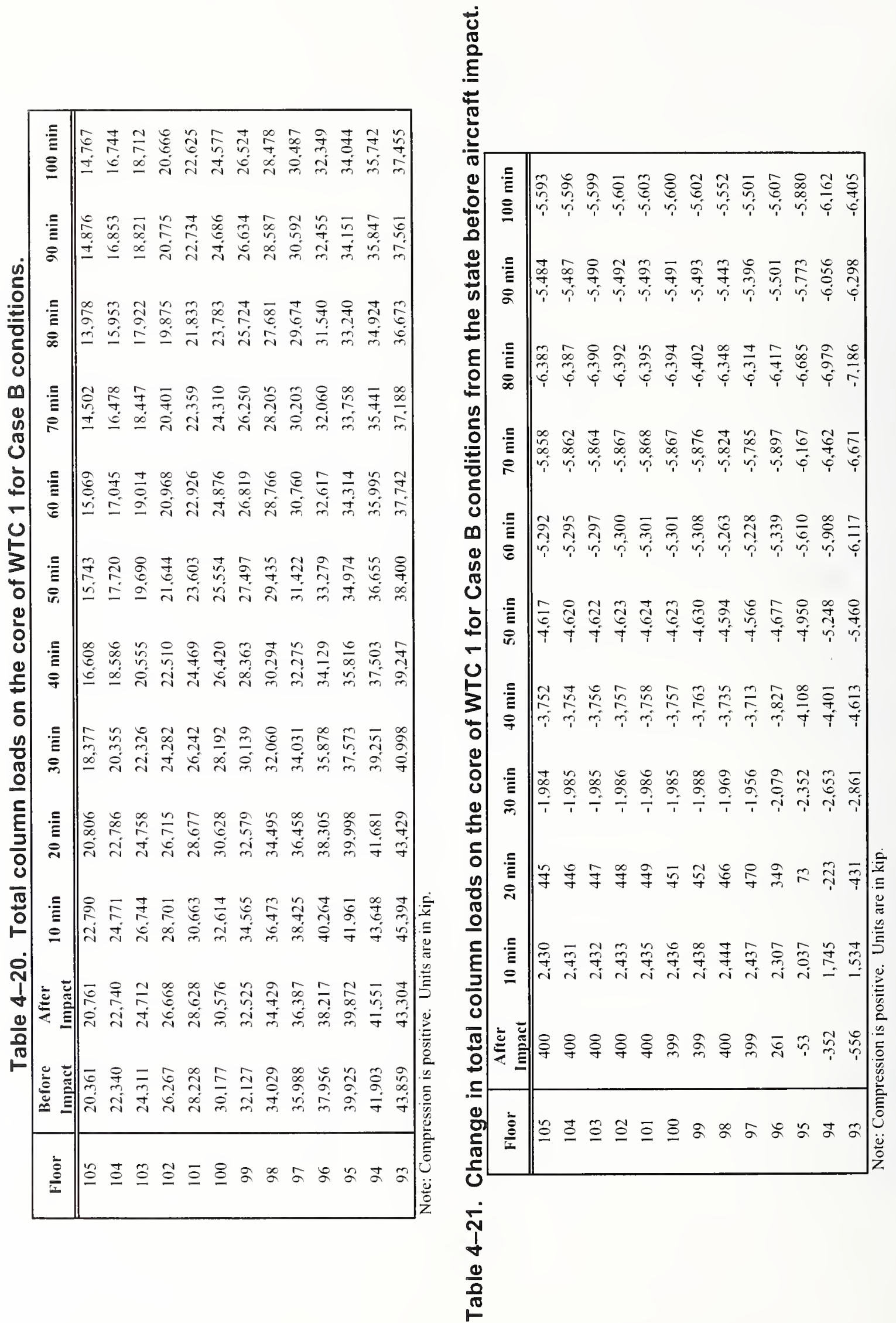
Table 4-22. Change in total column loads before and after aircraft impact. (Loads after impact) - (Loads before impact)

\begin{tabular}{|c|c|ccccc|}
\hline Row & Floor & North & East & South & West & Core \\
\hline \hline$(1)$ & Floor 98 & -837 & 526 & -668 & 574 & 400 \\
$(2)$ & Floor 105 & -732 & 466 & -604 & 472 & 400 \\
\hline (3) & $(2)-(1)$ & 105 & -60 & 64 & -103 & 0 \\
\hline
\end{tabular}

Note: Increase in compression is shown as positive. Units are in kip.

Table 4-23. Change in total column loads after aircraft impact and at $80 \mathrm{~min}$ for Case B conditions.

(Loads at $80 \mathrm{~min}$ ) - (Loads After Impact)

\begin{tabular}{|c|c|ccccc|}
\hline Row & Floor & North & East & South & West & Core \\
\hline \hline$(1)$ & Floor 98 & 1,239 & 2,484 & 1,052 & 1,973 & $-6,748$ \\
$(2)$ & Floor 105 & 1,234 & 2,470 & 1,063 & 2,021 & $-6,783$ \\
\hline$(3)$ & $(2)-(1)$ & -5 & -15 & 11 & 48 & -35 \\
\hline
\end{tabular}

Note: Increase in compression is shown as positive. Units are in kip.

Table 4-24. Change in total column loads at $80 \mathrm{~min}$ and at $100 \mathrm{~min}$ for Case B conditions.

(Loads at $100 \mathrm{~min}$ ) - (Loads at $80 \mathrm{~min}$ )

\begin{tabular}{|c|c|ccccc|}
\hline Row & Floor & North & East & South & West & Core \\
\hline \hline (1) & Floor 98 & -548 & 694 & $-1,771$ & 786 & 797 \\
(2) & Floor 105 & -504 & 579 & $-1,485$ & 623 & 790 \\
\hline (3) & $(2)-(1)$ & 44 & -115 & 285 & -163 & -7 \\
\hline
\end{tabular}

Note: Increase in compression is shown as positive. Units are in kip. 


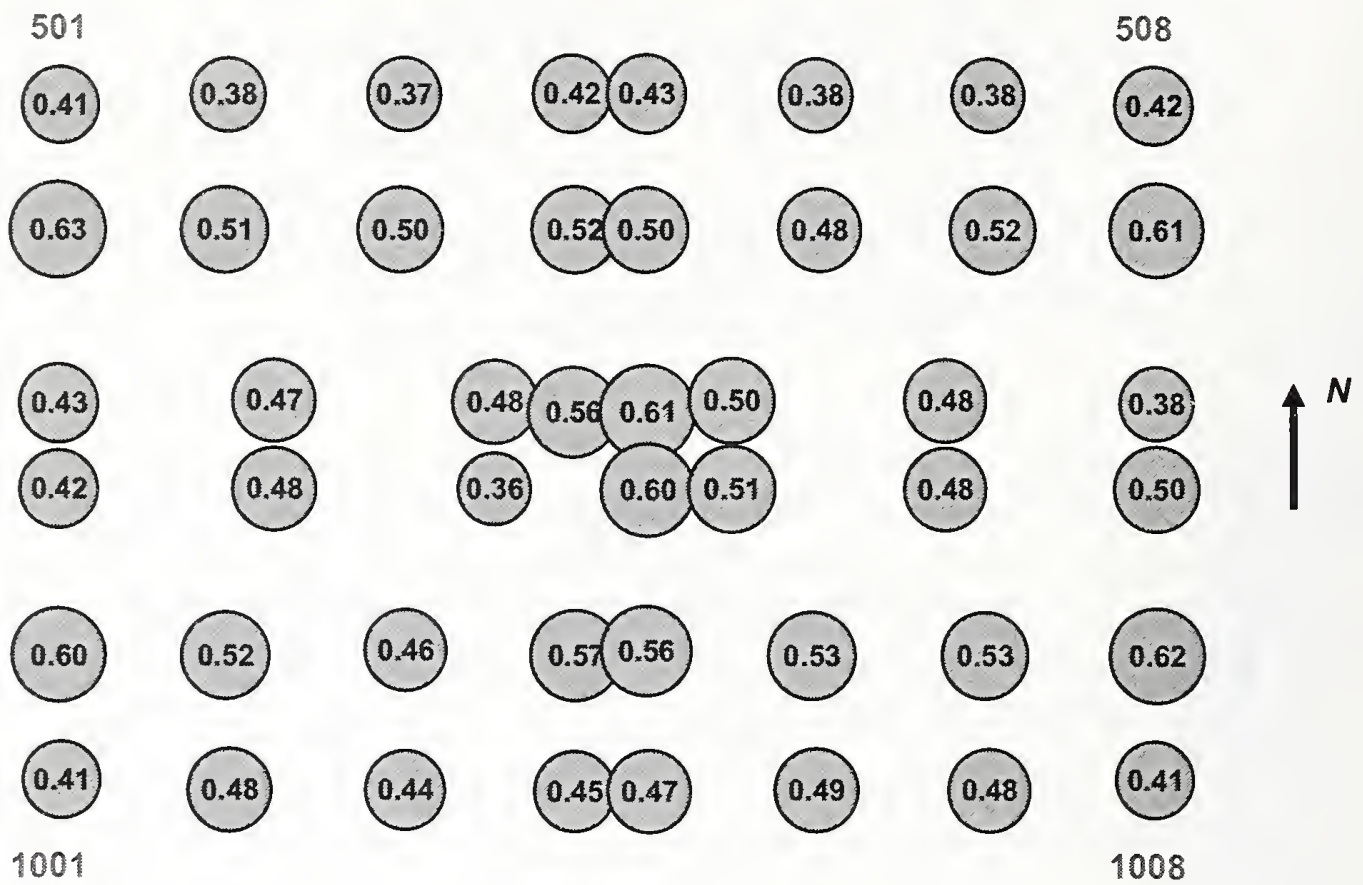

Figure 4-68. Maximum demand-to-capacity ratio for axial load in core columns between Floor 93 and Floor 99 of WTC 1 before aircraft impact.

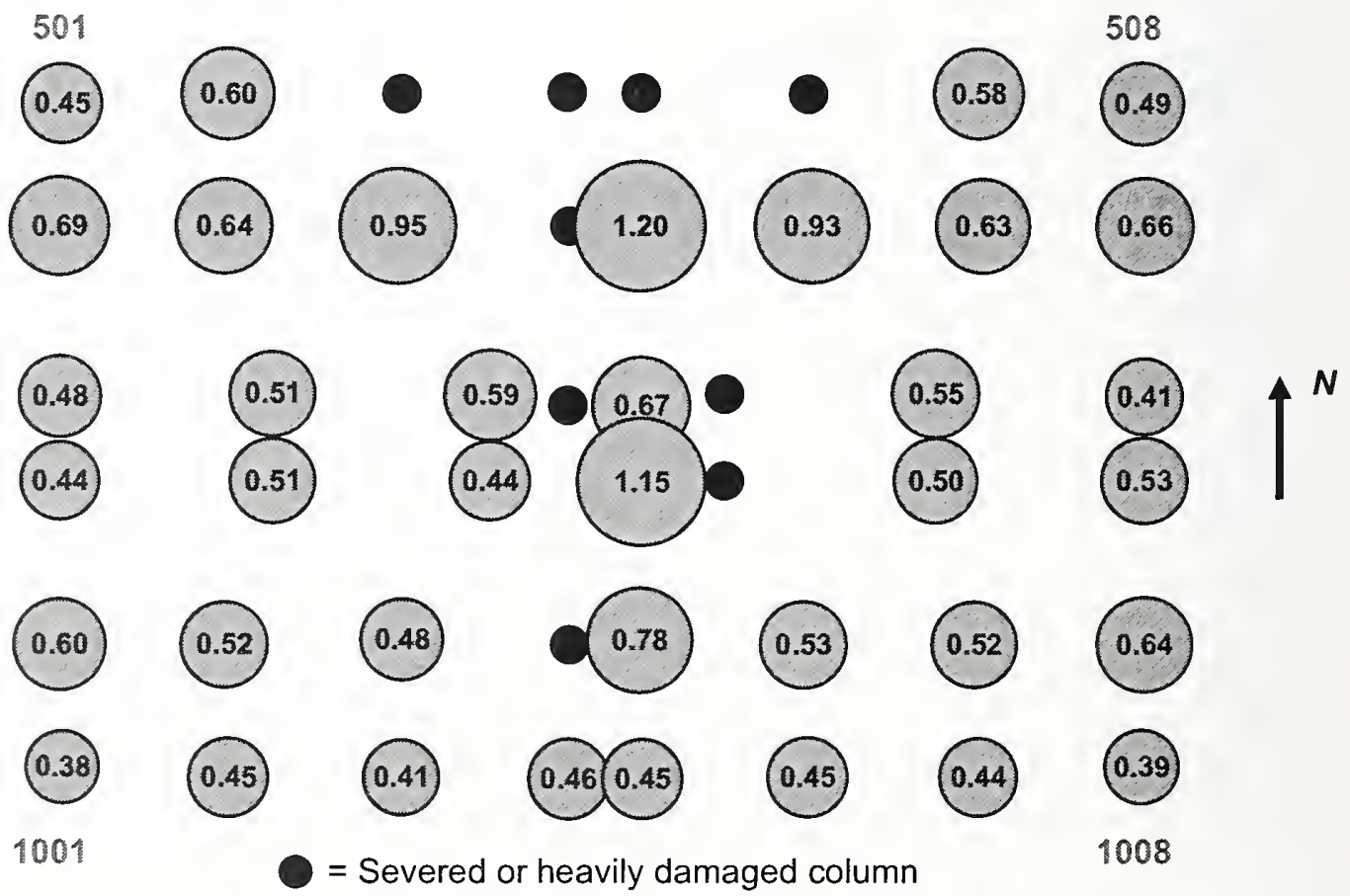

Figure 4-69. Maximum demand-to-capacity ratio for axial load in core columns between Floor 93 and Floor 99 of WTC 1 after aircraft impact for Case B conditions. 


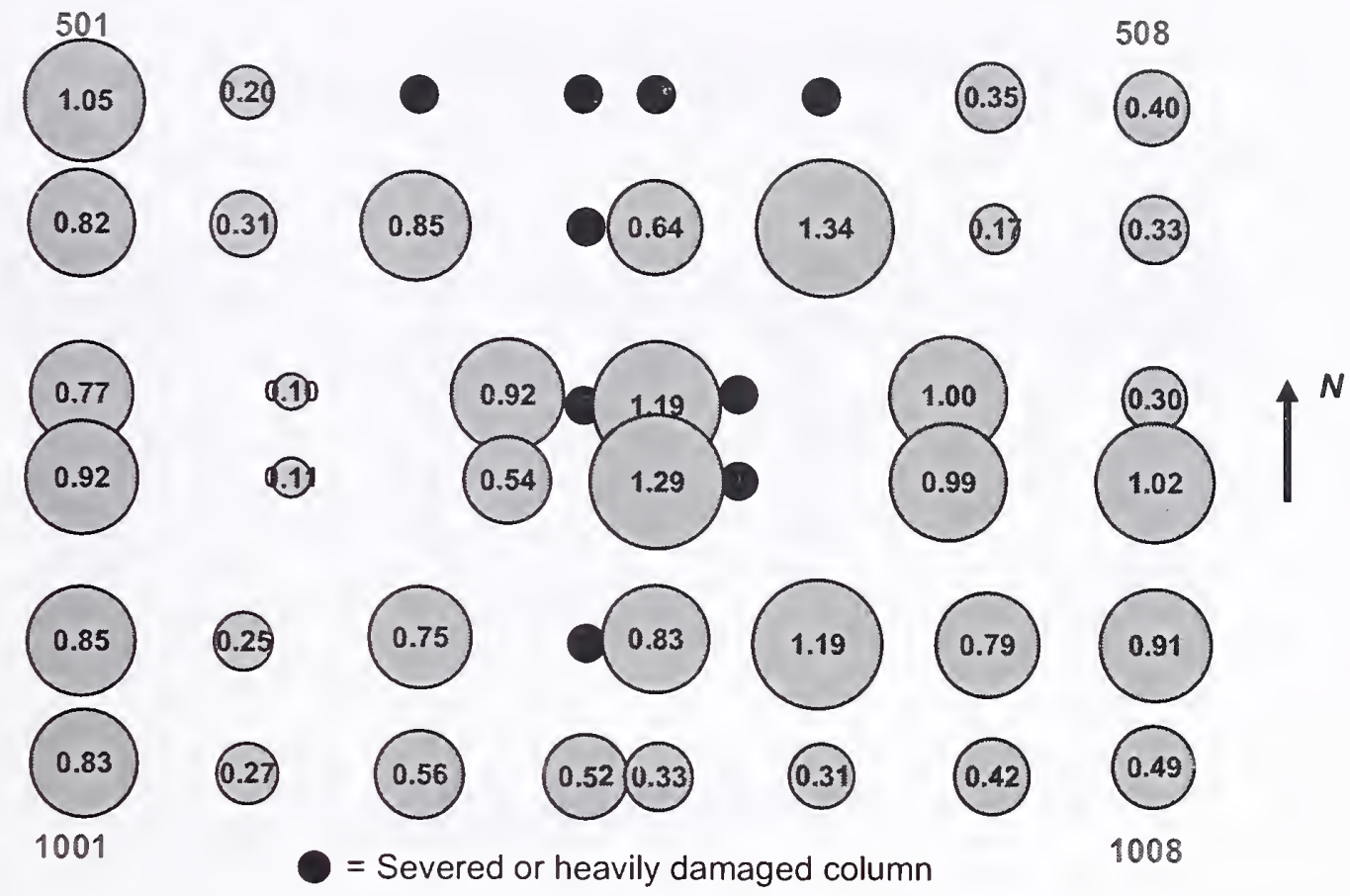

Figure 4-70. Maximum demand-to-capacity ratio for axial load in core columns between Floor 93 and Floor 99 of WTC 1 at $80 \mathrm{~min}$ for Case B conditions.

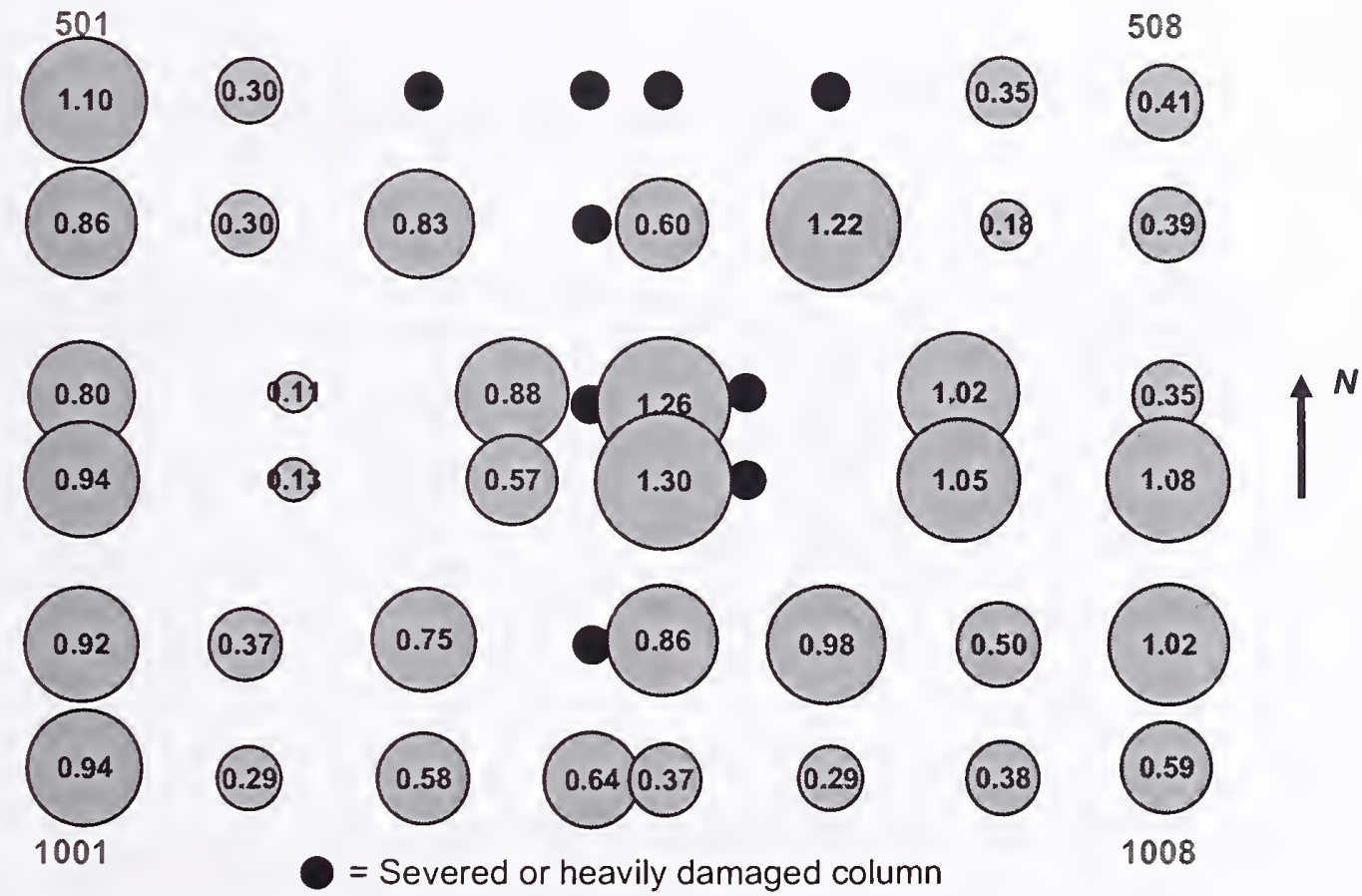

Figure 4-71. Maximum demand-to-capacity ratio for axial load in core columns between Floor 93 and Floor 99 of WTC 1 at 100 min for Case B conditions with 5 kip pull-in forces. 

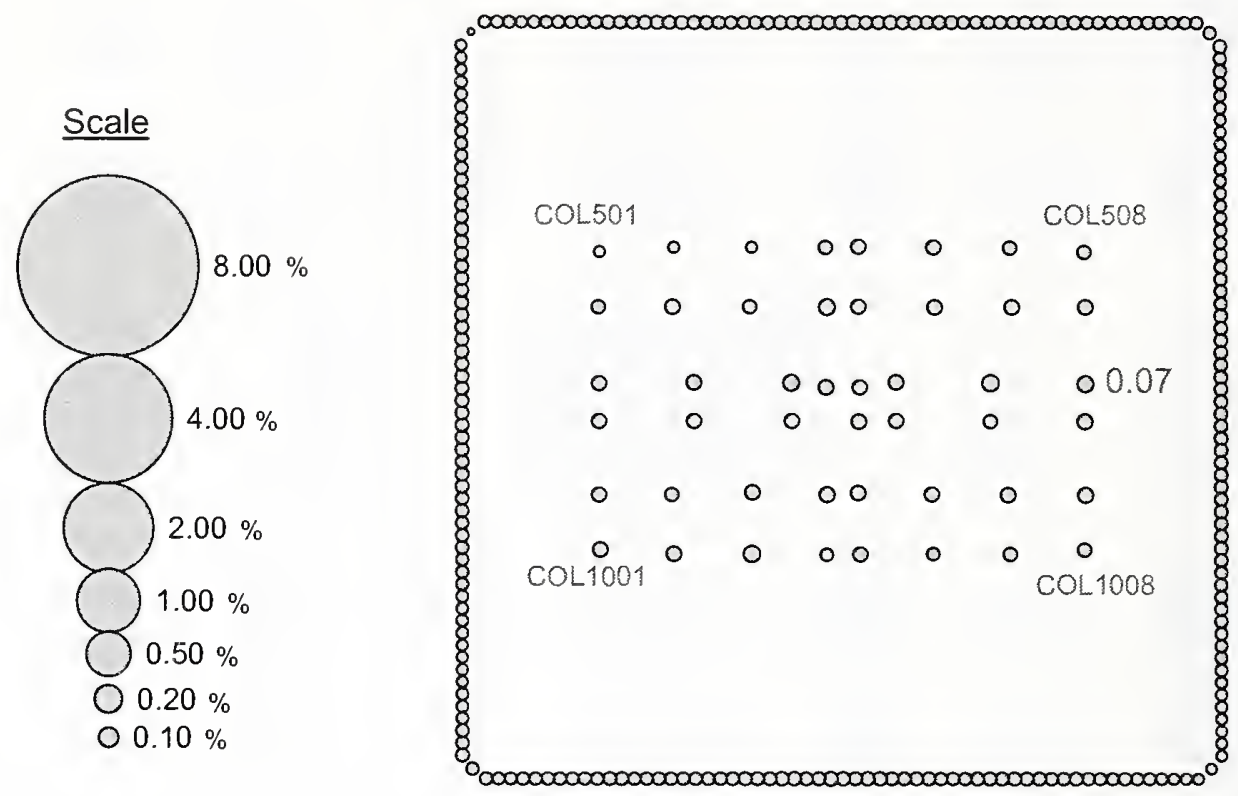

Figure 4-72. Maximum elastic-plus-plastic strain for columns between Floor 93 and Floor 99 of WTC 1 before aircraft impact (compressive strain is positive; strain values are in percent).
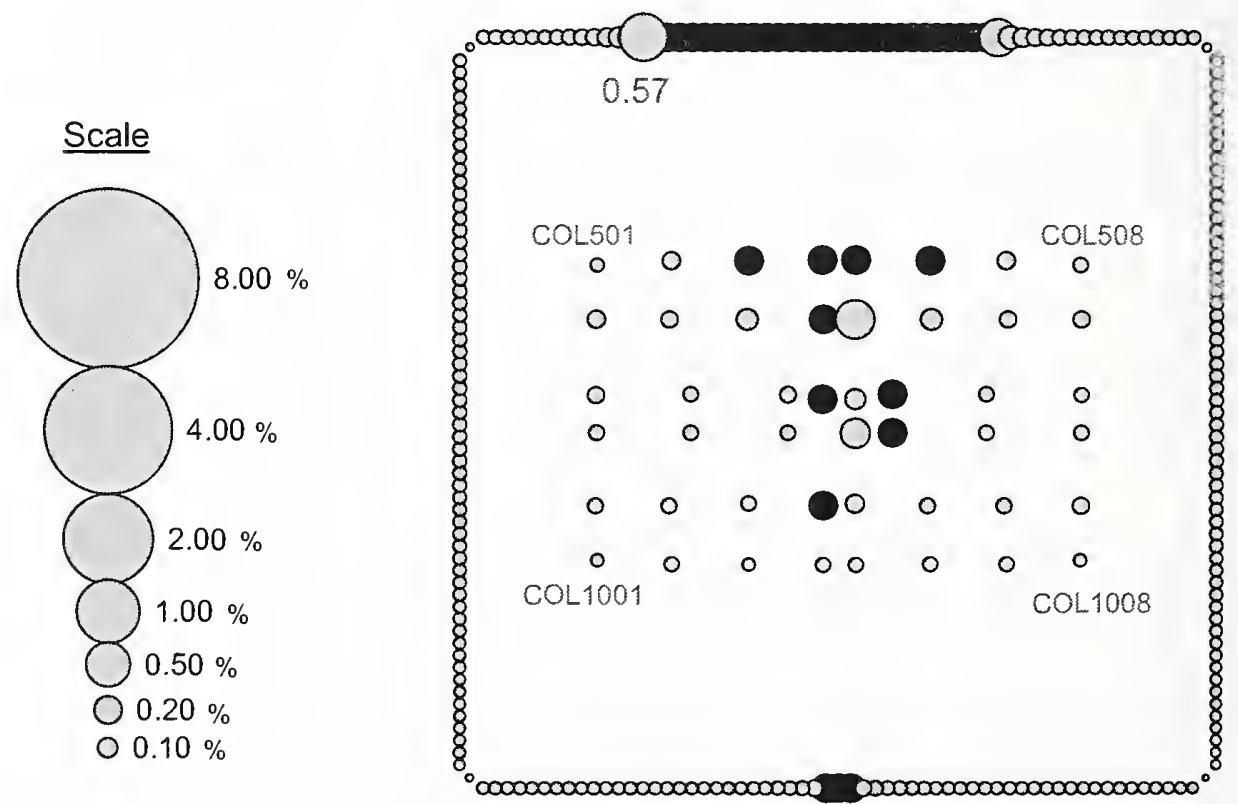

= Severed or heavily damaged column

Figure 4-73. Maximum elastic-plus-plastic strain for columns between Floor 93 and Floor 99 of WTC 1 after aircraft impact for Case B conditions (compressive strain is positive; strain values are in percent). 


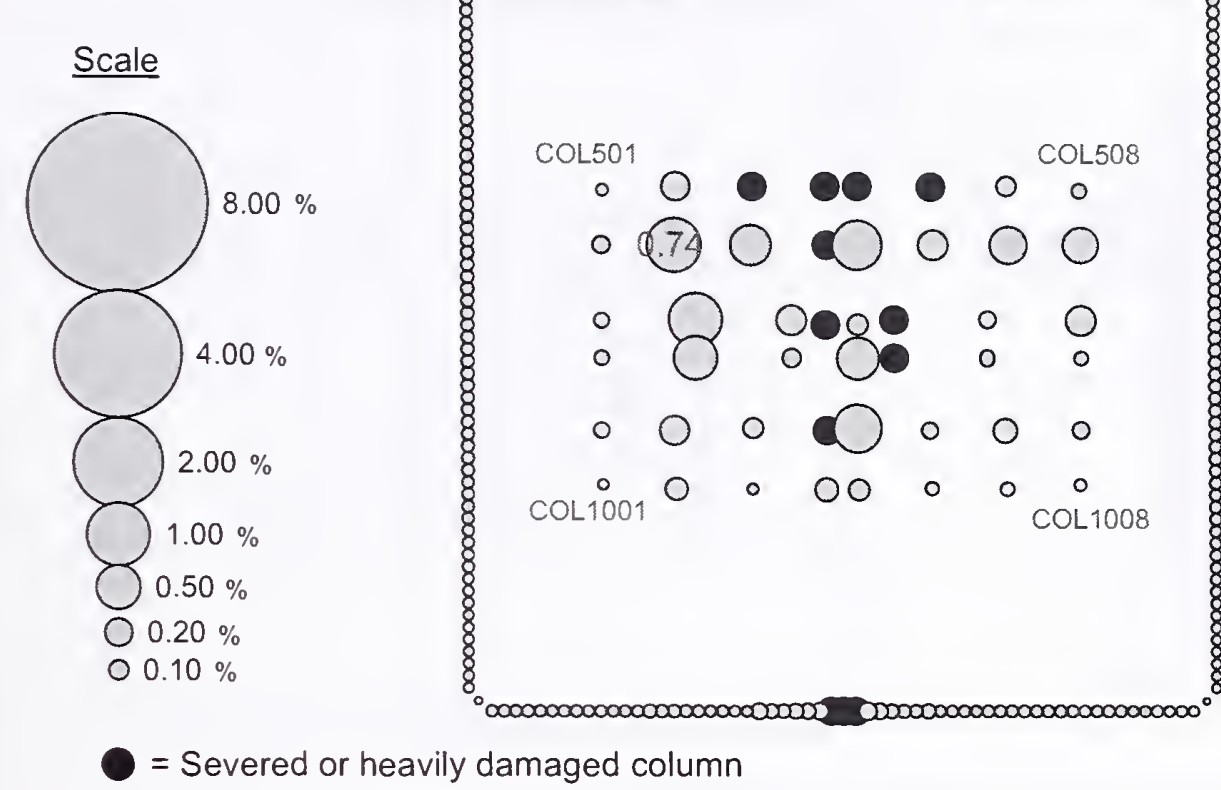

Figure 4-74. Maximum elastic-plus-plastic strain for columns between Floor 93 and Floor 99 of WTC 1 at $10 \mathrm{~min}$ for Case B conditions (compressive strain is positive; strain values are in percent).
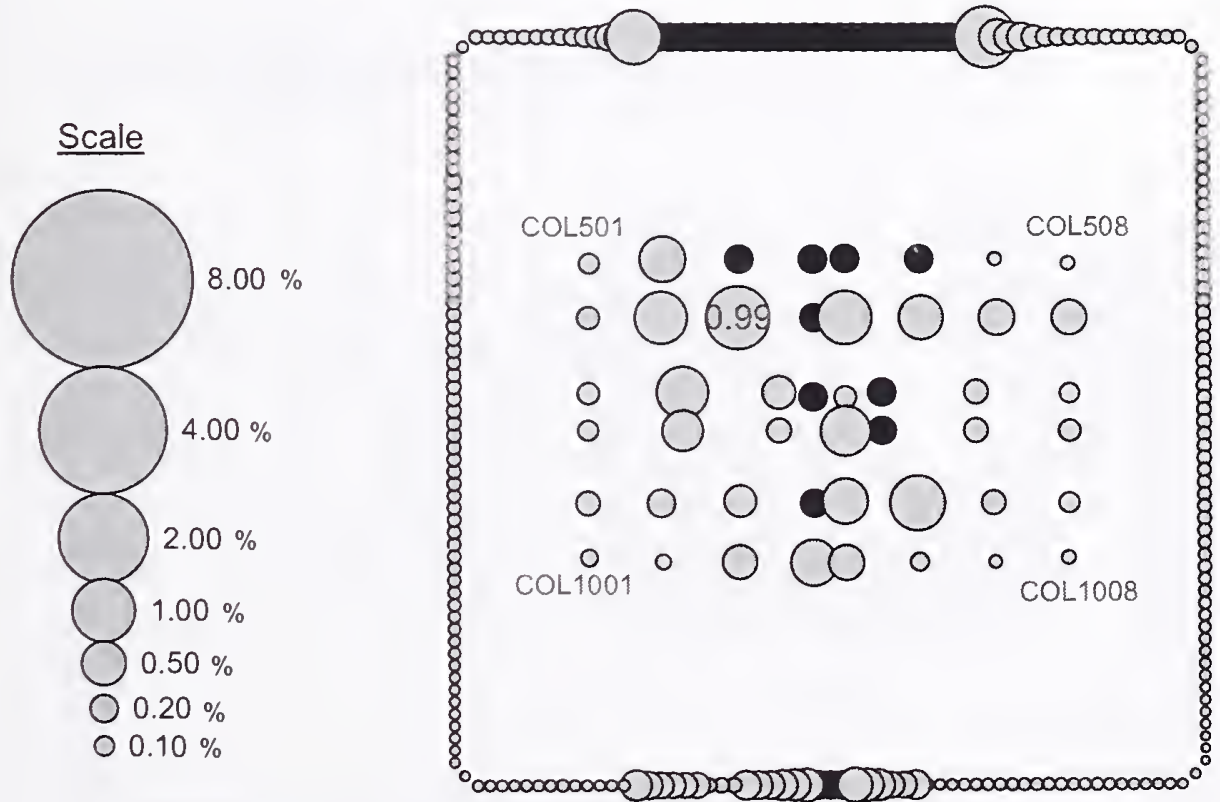

$=$ Severed or heavily damaged column

Figure 4-75. Maximum elastic-plus-plastic strain for columns between Floor 93 and Floor 99 of WTC 1 at $40 \mathrm{~min}$ for Case B conditions (compressive strain is positive; strain values are in percent). 

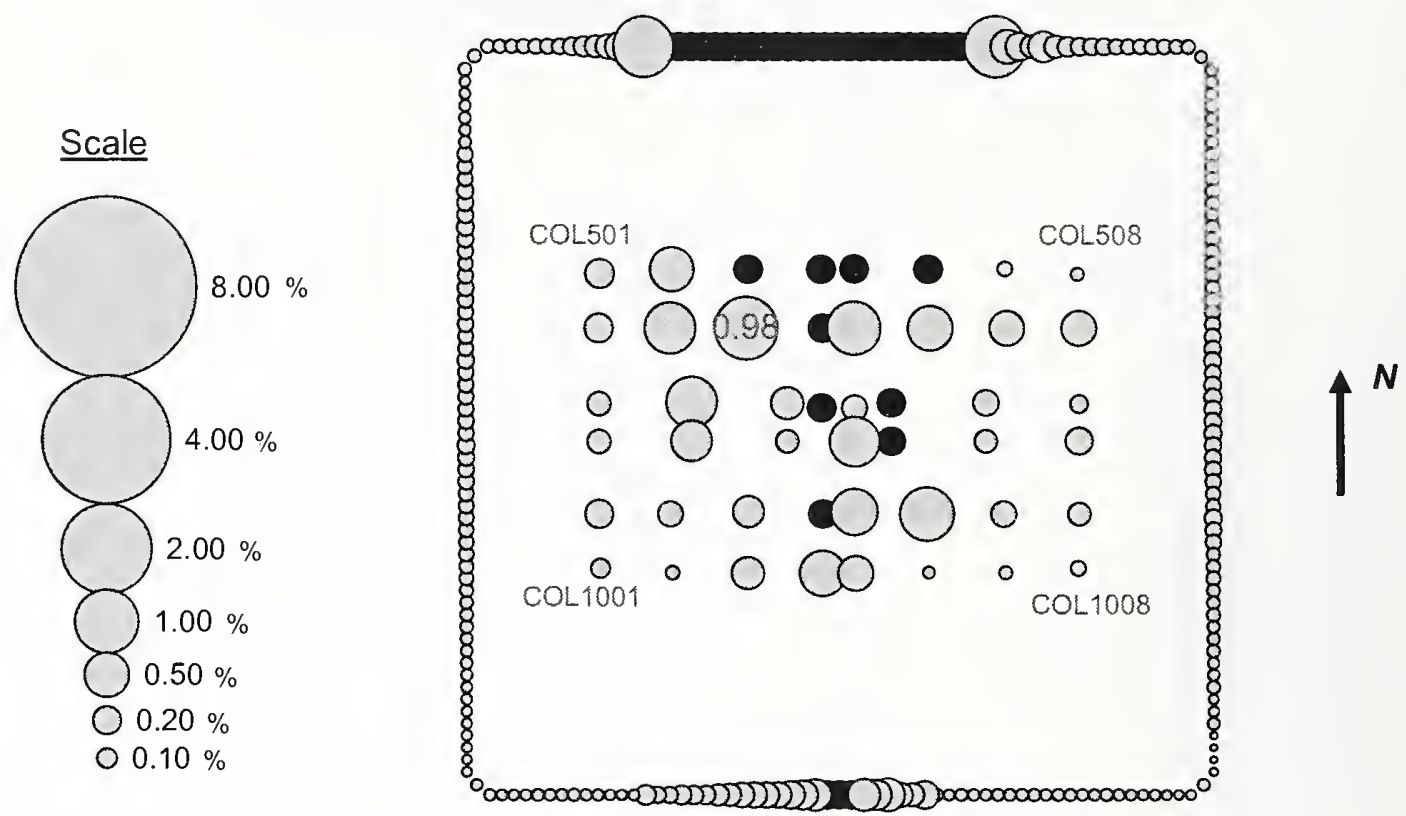

Figure 4-76. Maximum elastic-plus-plastic strain for columns between Floor 93 and Floor 99 of WTC 1 at 80 min for Case B conditions (compressive strain is positive; strain values are in percent).
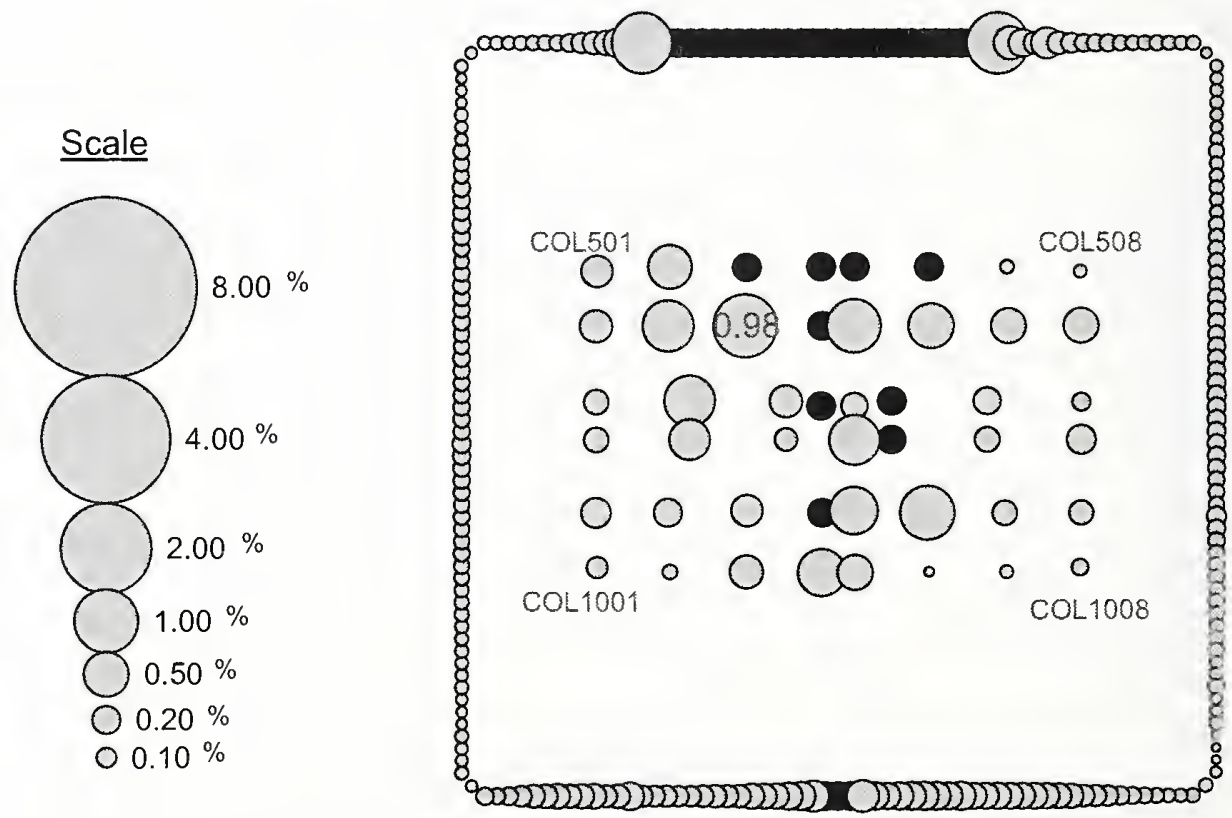

= Severed or heavily damaged column

Figure 4-77. Maximum elastic-plus-plastic strain for columns between Floor 93 and Floor 99 of WTC 1 at 100 min for Case B conditions with 5 kip pull-in forces (compressive strain is positive; strain values are in percent). 


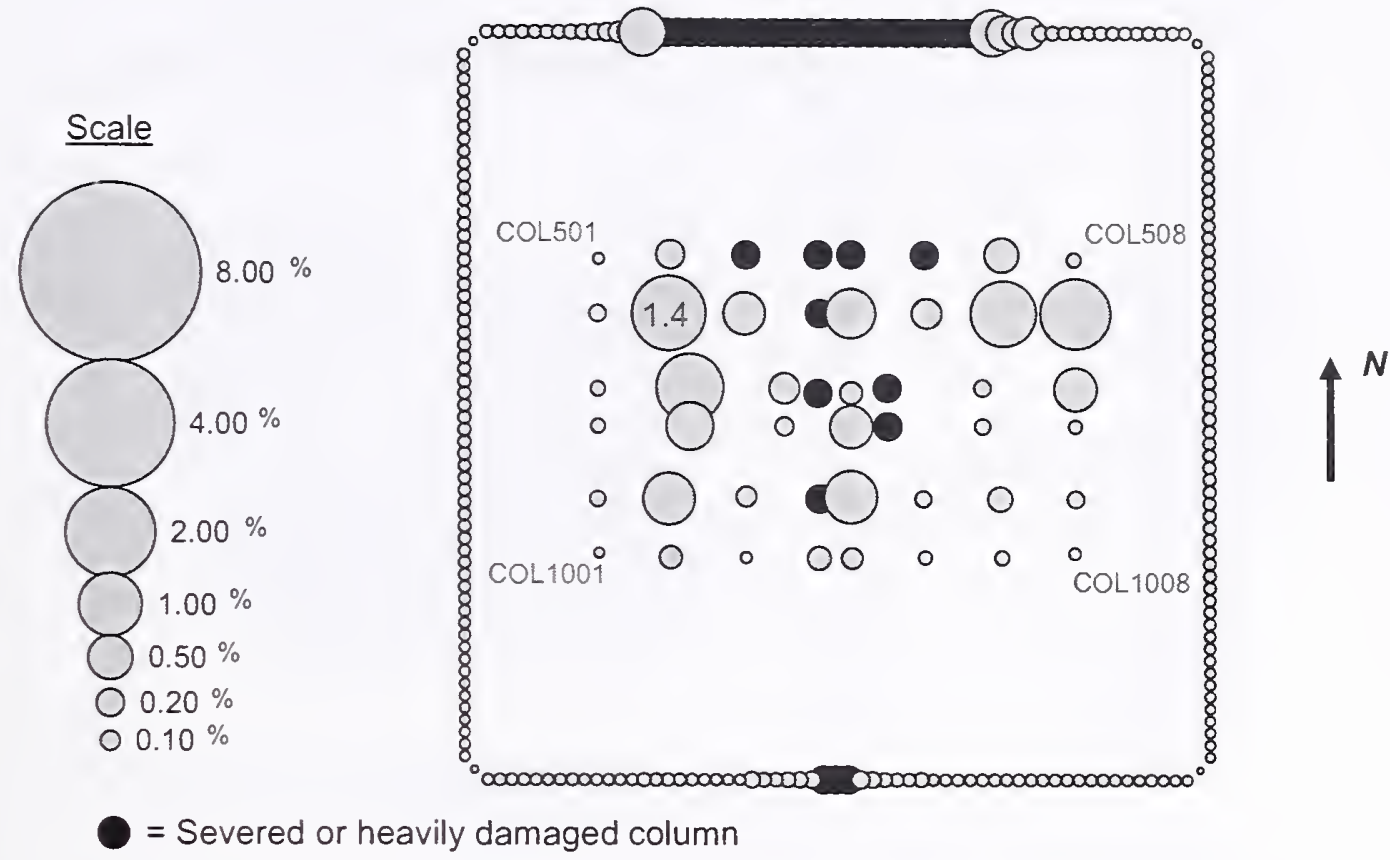

Figure 4-78. Maximum elastic-plus-plastic-plus-creep strain for columns between Floor 93 and Floor 99 of WTC 1 at 10 min for Case B conditions (compressive strain is positive; strain values are in percent).
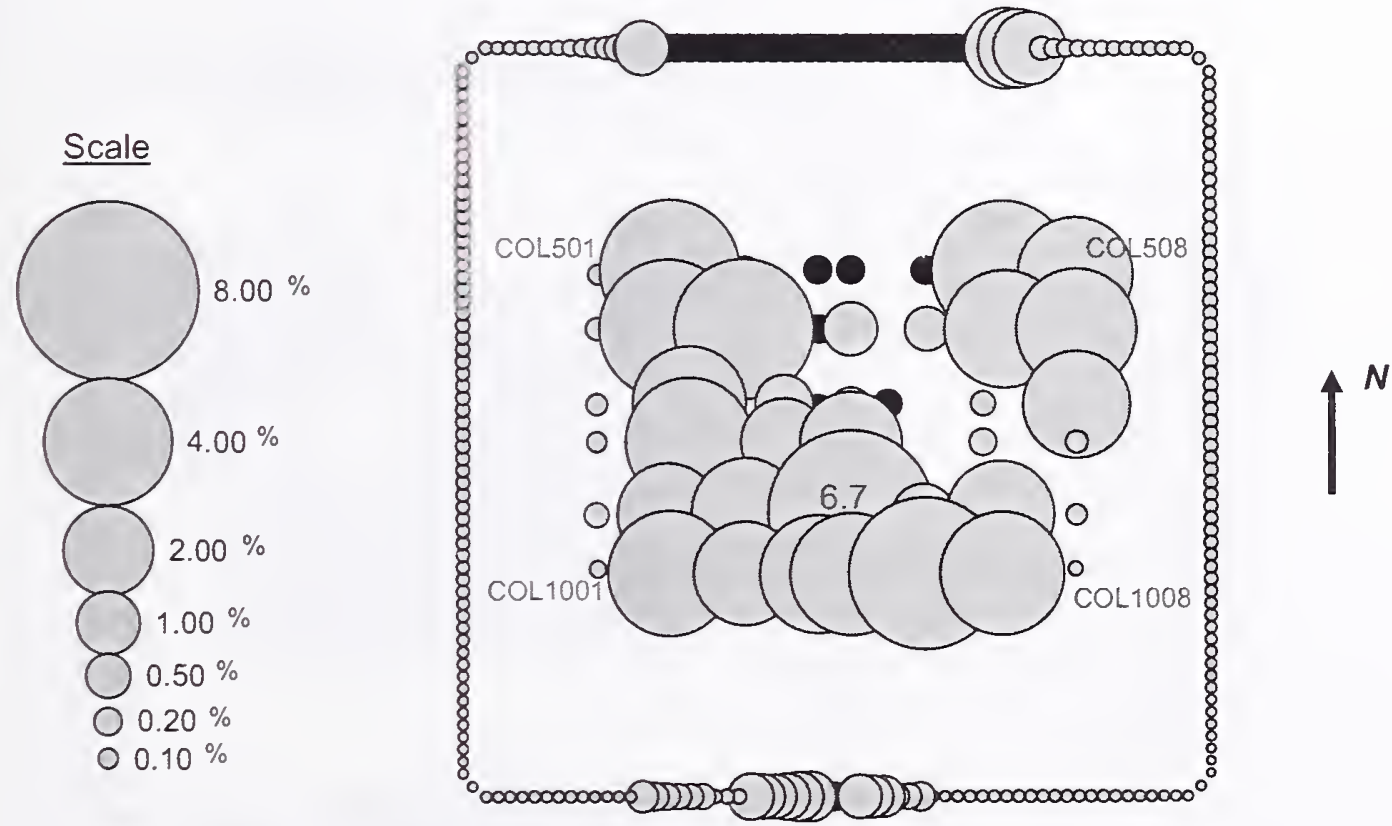

= Severed or heavily damaged column

Figure 4-79. Maximum elastic-plus-plastic-plus-creep strain for columns between Floor 93 and Floor 99 of WTC 1 at 40 min for Case B conditions (compressive strain is positive; strain values are in percent). 


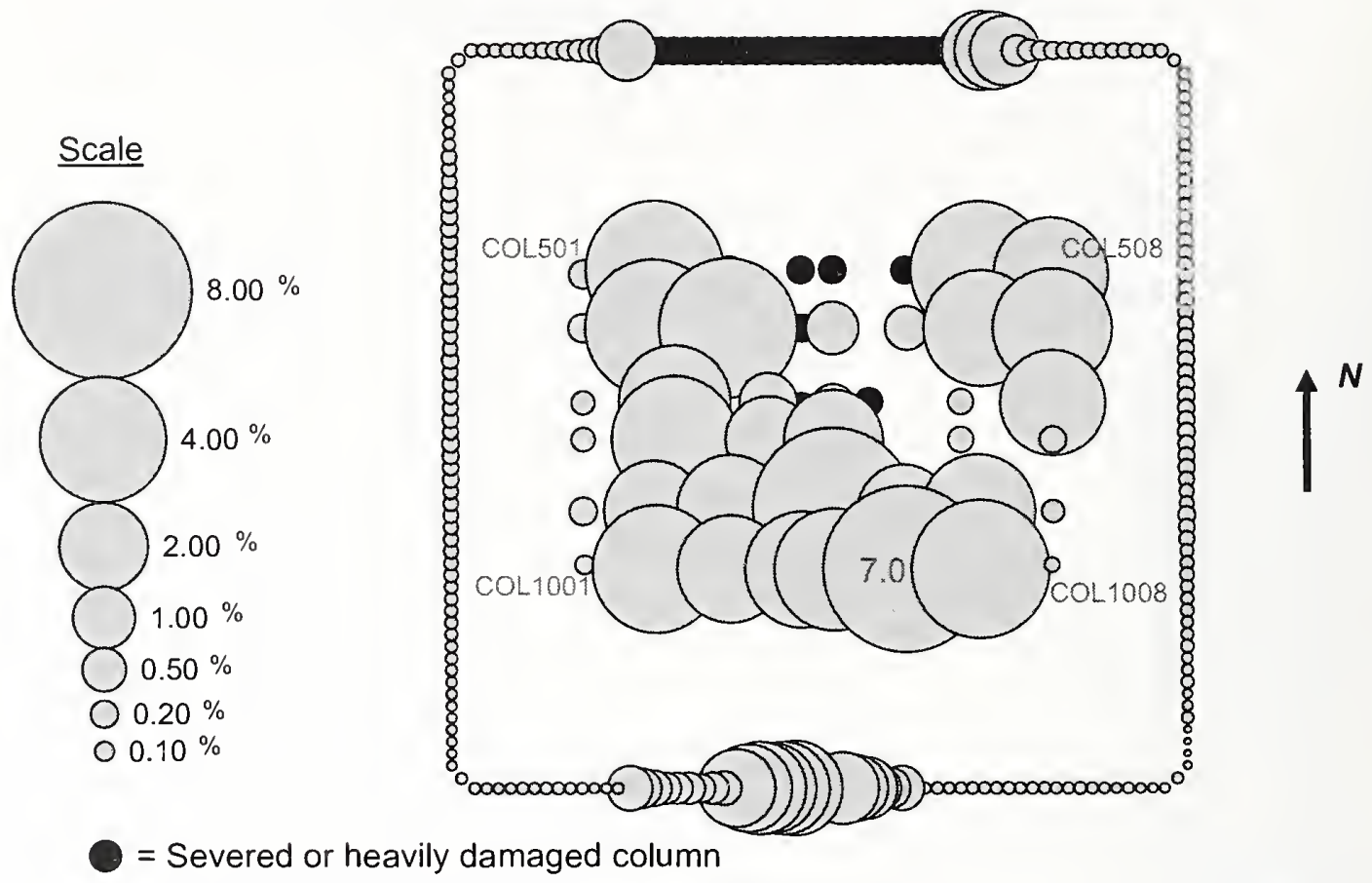

Figure 4-80. Maximum elastic-plus-plastic-plus-creep strain for columns between Floor 93 and Floor 99 of WTC 1 at 80 min for Case B conditions (compressive strain is positive; strain values are in percent).
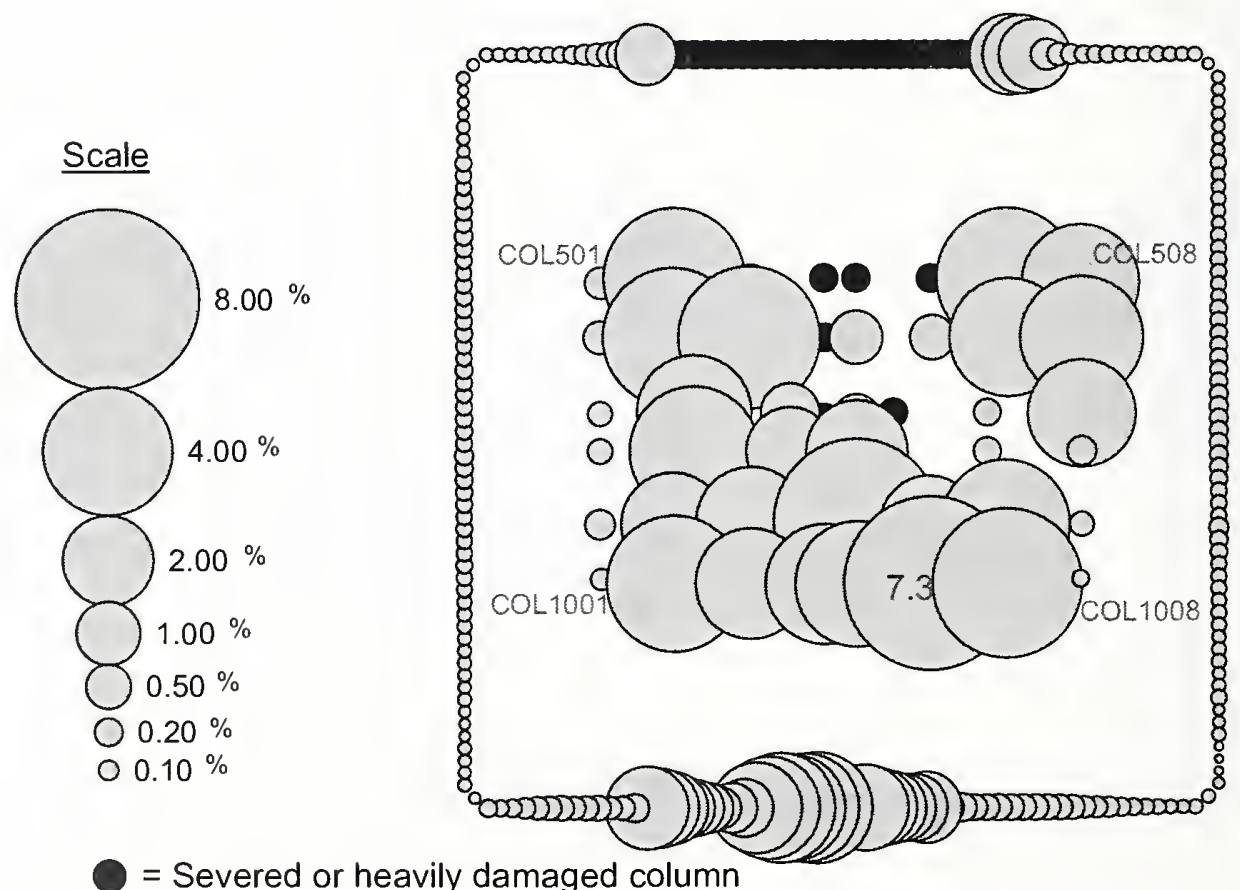

= Severed or heavily damaged column

Figure 4-81. Maximum elastic-plus-plastic-plus-creep strain for columns between Floor 93 and Floor 99 of WTC 1 at 100 min for Case B conditions with 5 kip pull-in forces (compressive strain is positive; strain value are in percent). 
Table 4-25. Tension capacity of core column splices at Floor 106

\begin{tabular}{|c|cccccc|}
\hline \multirow{2}{*}{ Col \# } & \multicolumn{7}{|c|}{ Column Line } \\
\cline { 2 - 7 } & $\mathbf{5 0 0}$ & $\mathbf{6 0 0}$ & $\mathbf{7 0 0}$ & $\mathbf{8 0 0}$ & $\mathbf{9 0 0}$ & $\mathbf{1 0 0 0}$ \\
\hline \hline $\mathbf{1}$ & 460 & 335 & 335 & 335 & 335 & 460 \\
$\mathbf{2}$ & 335 & 335 & 335 & 335 & 335 & 335 \\
$\mathbf{3}$ & 335 & 335 & 335 & 335 & 460 & 460 \\
$\mathbf{4}$ & 335 & 335 & 335 & 335 & 335 & 335 \\
$\mathbf{5}$ & 335 & 335 & 335 & 335 & 335 & 335 \\
$\mathbf{6}$ & 335 & 335 & 335 & 335 & 335 & 335 \\
$\mathbf{7}$ & 335 & 335 & 335 & 335 & 335 & 335 \\
$\mathbf{8}$ & 460 & 335 & 335 & & 335 & 460 \\
\hline
\end{tabular}

Note: Units are in kip. 
Table 4-26. Axial load in core columns at Floor 105 of WTC 1 for Case B conditions

\begin{tabular}{|c|c|c|c|c|c|c|c|c|c|c|c|c|}
\hline Column ID & $\begin{array}{l}\text { Before } \\
\text { Impact }\end{array}$ & $\begin{array}{c}\text { After } \\
\text { Impact }\end{array}$ & $10 \mathrm{~min}$ & $20 \mathrm{~min}$ & $30 \mathrm{~min}$ & $40 \mathrm{~min}$ & $50 \mathrm{~min}$ & $60 \mathrm{~min}$ & $70 \mathrm{~min}$ & $80 \mathrm{~min}$ & $90 \mathrm{~min}$ & $100 \mathrm{~min}$ \\
\hline 501 & 753 & 1,033 & 976 & 1,326 & 1,504 & 1,634 & 1,690 & 1,703 & 1,661 & 1,635 & 1,595 & 1,563 \\
\hline 502 & 567 & 751 & 996 & 569 & 416 & 353 & 303 & 266 & 210 & 156 & 90 & 44 \\
\hline 503 & 632 & 469 & 631 & 312 & 129 & 57 & 23 & -13 & -63 & -118 & -190 & -240 \\
\hline 504 & 486 & 1 & -49 & -17 & -34 & -39 & -32 & -34 & -36 & -44 & -70 & -82 \\
\hline 505 & 576 & 82 & 27 & 18 & 3 & -8 & -8 & -8 & -8 & -16 & -42 & -52 \\
\hline 506 & 629 & 512 & 544 & 311 & 311 & 324 & 320 & 313 & 314 & 315 & 281 & 266 \\
\hline 507 & 558 & 676 & 774 & 466 & 328 & 313 & 304 & 296 & 303 & 312 & 284 & 275 \\
\hline 508 & 743 & 1,057 & 1,185 & 1,108 & 587 & 493 & 469 & 455 & 448 & 430 & 399 & 389 \\
\hline 601 & 219 & 272 & 239 & 341 & 383 & 413 & 423 & 432 & 432 & 427 & 427 & 420 \\
\hline 602 & 395 & 524 & 612 & 310 & 206 & 153 & 103 & 69 & 30 & -5 & -21 & -45 \\
\hline 603 & 408 & 475 & 525 & 315 & 118 & 15 & -28 & -64 & -94 & -126 & -130 & -137 \\
\hline 604 & 275 & 131 & 85 & 45 & 7 & -16 & -28 & -38 & -45 & -51 & -50 & -52 \\
\hline 605 & 320 & 149 & 70 & 20 & -3 & -17 & -23 & -28 & -34 & -38 & -36 & -37 \\
\hline 606 & 408 & 428 & 415 & 334 & 327 & 325 & 313 & 303 & 295 & 291 & 298 & 299 \\
\hline 607 & 371 & 447 & 470 & 350 & 286 & 268 & 247 & 232 & 224 & 222 & 230 & 235 \\
\hline 608 & 224 & 296 & 335 & 255 & 165 & 149 & 134 & 123 & 116 & 113 & 123 & 128 \\
\hline 701 & 355 & 418 & 363 & 518 & 594 & 658 & 684 & 705 & 718 & 722 & 733 & 733 \\
\hline 702 & 395 & 529 & 652 & 232 & 145 & 85 & 17 & -34 & -73 & -98 & -88 & -89 \\
\hline 703 & 266 & 288 & 380 & 456 & 456 & 425 & 407 & 387 & 368 & 351 & 344 & 334 \\
\hline 704 & 224 & 221 & 237 & 214 & 189 & 165 & 147 & 132 & 121 & 113 & 119 & 117 \\
\hline 705 & 181 & 159 & 146 & 135 & 125 & 117 & 110 & 103 & 99 & 96 & 98 & 95 \\
\hline 706 & 276 & 227 & 195 & 178 & 167 & 161 & 153 & 146 & 141 & 137 & 138 & 133 \\
\hline 707 & 386 & 447 & 472 & 514 & 524 & 514 & 491 & 471 & 456 & 445 & 448 & 449 \\
\hline 708 & 391 & 491 & 540 & 420 & 448 & 470 & 462 & 451 & 446 & 445 & 470 & 482 \\
\hline 801 & 398 & 450 & 374 & 536 & 630 & 706 & 742 & 762 & 777 & 788 & 818 & 834 \\
\hline 802 & 398 & 473 & 622 & 167 & 110 & 69 & 1 & -55 & -91 & -108 & -73 & -54 \\
\hline 803 & 298 & 298 & 417 & 542 & 521 & 470 & 445 & 423 & 402 & 383 & 389 & 383 \\
\hline 804 & 192 & 173 & 179 & 166 & 146 & 136 & 125 & 119 & 119 & 116 & 127 & 127 \\
\hline 805 & 329 & 188 & 145 & 105 & 85 & 85 & 76 & 72 & 73 & 71 & 86 & 86 \\
\hline 806 & 395 & 454 & 502 & 567 & 631 & 652 & 642 & 635 & 630 & 620 & 626 & 624 \\
\hline 807 & 362 & 432 & 439 & 399 & 449 & 480 & 489 & 493 & 497 & 498 & 528 & 540 \\
\hline 901 & 253 & 273 & 217 & 280 & 343 & 398 & 422 & 436 & 450 & 460 & 496 & 517 \\
\hline 902 & 385 & 425 & 470 & 268 & 241 & 193 & 152 & 112 & 95 & 88 & 135 & 167 \\
\hline 903 & 523 & 606 & 833 & 847 & 653 & 491 & 435 & 393 & 358 & 336 & 358 & 349 \\
\hline 904 & 298 & 287 & 397 & 322 & 192 & 116 & 71 & 47 & 43 & 42 & 76 & 77 \\
\hline 905 & 278 & 271 & 332 & 259 & 150 & 96 & 66 & 55 & 60 & 59 & 89 & 89 \\
\hline 906 & 406 & 464 & 507 & 646 & 616 & 516 & 477 & 455 & 409 & 354 & 351 & 319 \\
\hline 907 & 413 & 457 & 485 & 534 & 513 & 468 & 456 & 458 & 455 & 441 & 438 & 425 \\
\hline 908 & 239 & 274 & 242 & 274 & 321 & 350 & 366 & 378 & 387 & 392 & 425 & 438 \\
\hline 1001 & 762 & 735 & 605 & 721 & 915 & 1,112 & 1,212 & 1,301 & 1,370 & 1,405 & 1,595 & 1,685 \\
\hline 1002 & 614 & 611 & 759 & 560 & 527 & 432 & 405 & 382 & 367 & 353 & 453 & 465 \\
\hline 1003 & 745 & 744 & 855 & 1,079 & 990 & 555 & 426 & 286 & 214 & 182 & 299 & 271 \\
\hline 1004 & 522 & 525 & 809 & 719 & 416 & 194 & 106 & 46 & 15 & 15 & 148 & 150 \\
\hline 1005 & 534 & 546 & 830 & 685 & 359 & 176 & 94 & 50 & 27 & 20 & 135 & 132 \\
\hline 1006 & 637 & 634 & 682 & 904 & 661 & 408 & 348 & 321 & 288 & 242 & 273 & 230 \\
\hline 1007 & 587 & 578 & 582 & 727 & 624 & 500 & 478 & 476 & 463 & 435 & 438 & 408 \\
\hline 1008 & 758 & 785 & 687 & 770 & 902 & 991 & 1,030 & 1.055 & 1,067 & 1,070 & 1,216 & 1,274 \\
\hline
\end{tabular}

Note: Compression is positive. Units are in kip.

Note: Shading indicates when core columns were in tension. 
Col 501

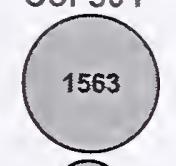

$\bigodot_{44} \bigcirc_{-240} \bigcirc_{-82} \quad-52$

(266)

(275)

Col 508

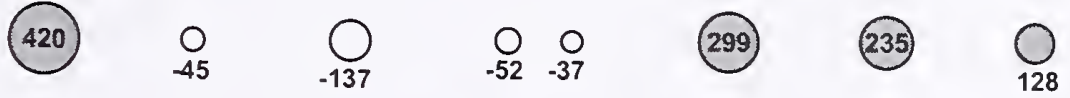

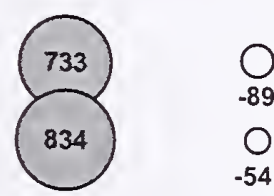

\begin{tabular}{lll}
$(334)$ & $\bigcirc$ & $\bigcirc$ \\
117 & $\bigcirc 5$ & 133 \\
& $\bigodot_{127}$ & $\bigcirc$ \\
\hline
\end{tabular}
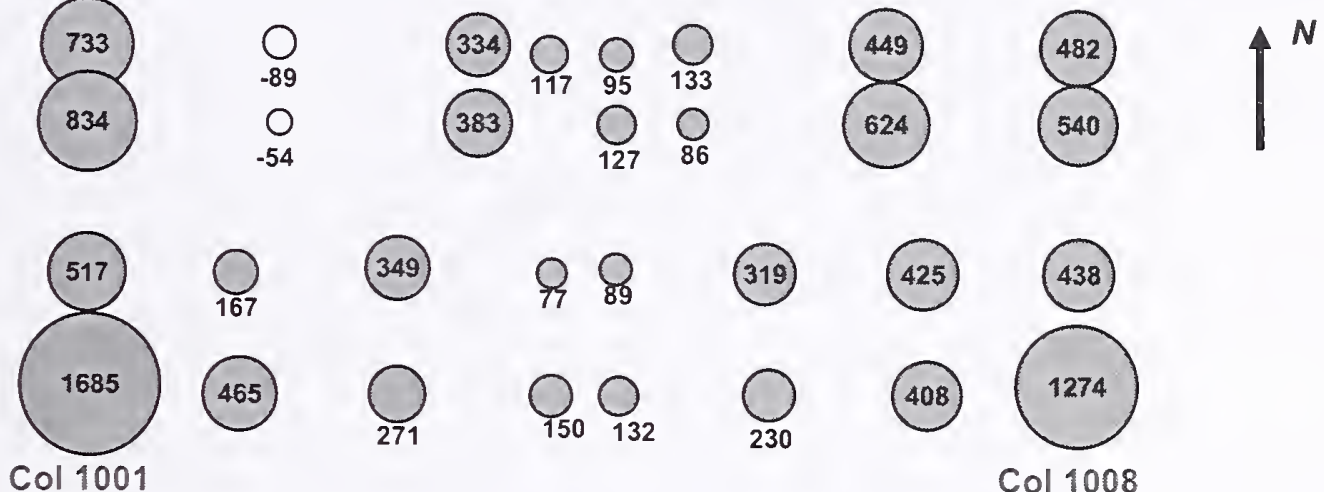

(349)

98

(319)

(425)

438

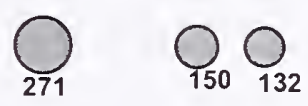

$\bigcirc_{230}$

(408)

1274

Col 1001

Figure 4-82. Axial load (kip) in core columns at Floor 105 of WTC 1 at $100 \mathrm{~min}$ for Case B conditions with $\mathbf{5}$ kip pull-in forces (compression is positive).

Col 501

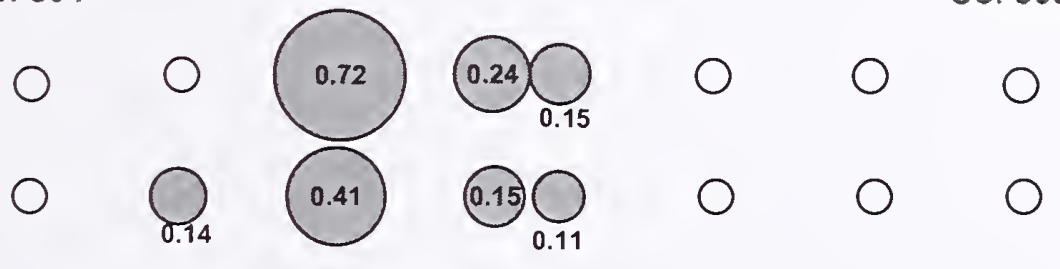

O

(0.27) 0000

$\bigcirc$

(0.16)

0

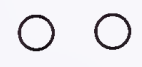

O

$\bigcirc$

Col 1008

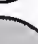

\section{.}


Table 4-27. Demand-to-capacity ratio for axial load in outriggers of WTC 1 for Case B conditions (outrigger IDs are indicated in Fig. 4-3).

\begin{tabular}{|c|c|c|c|c|c|c|c|c|c|c|c|c|}
\hline Outrigger ID & Bfr Imp & Aftr Imp & $10 \mathrm{~min}$ & $20 \min$ & $30 \mathrm{~min}$ & $40 \mathrm{~min}$ & $50 \min$ & $60 \mathrm{~min}$ & $70 \mathrm{~min}$ & $80 \mathrm{~min}$ & $90 \min$ & $100 \mathrm{~min}$ \\
\hline \multicolumn{13}{|l|}{ North } \\
\hline A & 0.26 & 0.35 & 0.26 & 0.40 & 0.49 & 0.53 & 0.56 & 0.59 & 0.62 & 0.65 & 0.54 & 0.52 \\
\hline B & 0.22 & -0.07 & -0.13 & -0.04 & 0.02 & 0.04 & 0.06 & 0.07 & 0.08 & 0.09 & 0.03 & 0.03 \\
\hline C & 0.21 & -0.05 & -0.11 & -0.02 & 0.04 & 0.06 & 0.08 & 0.08 & 0.09 & 0.11 & 0.05 & 0.04 \\
\hline D & 0.25 & 0.35 & 0.29 & 0.45 & 0.61 & 0.67 & 0.70 & 0.72 & 0.73 & 0.75 & 0.65 & 0.63 \\
\hline \multicolumn{13}{|l|}{ East } \\
\hline $\mathrm{E}$ & 0.32 & 0.48 & 0.38 & 0.56 & 0.75 & 0.83 & 0.88 & 0.92 & 0.94 & 0.97 & 0.95 & 0.96 \\
\hline $\mathrm{F}$ & 0.23 & 0.28 & 0.21 & 0.30 & 0.41 & 0.48 & 0.52 & 0.55 & 0.57 & 0.59 & 0.62 & 0.64 \\
\hline G & 0.23 & 0.26 & 0.20 & 0.26 & 0.35 & 0.42 & 0.46 & 0.49 & 0.51 & 0.54 & 0.58 & 0.61 \\
\hline $\mathrm{H}$ & 0.33 & 0.31 & 0.23 & 0.25 & 0.36 & 0.46 & 0.51 & 0.55 & 0.58 & 0.60 & 0.72 & 0.77 \\
\hline \multicolumn{13}{|l|}{ South } \\
\hline I & 0.25 & 0.12 & 0.04 & 0.03 & 0.12 & 0.20 & 0.23 & 0.26 & 0.27 & 0.28 & 0.28 & 0.28 \\
\hline J & 0.21 & 0.10 & 0.01 & 0.03 & 0.13 & 0.24 & 0.28 & 0.29 & 0.30 & 0.31 & 0.04 & -0.02 \\
\hline $\mathrm{K}$ & 0.21 & 0.10 & 0.01 & 0.03 & 0.13 & 0.24 & 0.27 & 0.28 & 0.30 & 0.31 & 0.02 & -0.04 \\
\hline $\mathrm{L}$ & 0.26 & 0.12 & 0.02 & 0.05 & 0.11 & 0.20 & 0.24 & 0.27 & 0.29 & 0.31 & 0.19 & 0.18 \\
\hline \multicolumn{13}{|l|}{ West } \\
\hline M & 0.33 & 0.33 & 0.22 & 0.29 & 0.36 & 0.44 & 0.49 & 0.53 & 0.56 & 0.58 & 0.69 & 0.73 \\
\hline $\mathrm{N}$ & 0.24 & 0.27 & 0.20 & 0.28 & 0.34 & 0.40 & 0.44 & 0.47 & 0.50 & 0.52 & 0.56 & 0.58 \\
\hline $\mathrm{o}$ & 0.24 & 0.29 & 0.22 & 0.31 & 0.38 & 0.43 & 0.47 & 0.50 & 0.53 & 0.55 & 0.59 & 0.61 \\
\hline $\mathrm{P}$ & 0.33 & 0.48 & 0.39 & 0.54 & 0.64 & 0.71 & 0.75 & 0.79 & 0.83 & 0.87 & 0.88 & 0.90 \\
\hline
\end{tabular}

Table 4-28. Demand to capacity ratio for axial load in exterior columns supporting outriggers at Floor 107 of WTC 1 for Case B conditions.

\begin{tabular}{|c|c|c|c|c|c|c|c|c|c|c|c|c|}
\hline Column ID & Bfr Imp & Aftr I mp & $10 \mathrm{~min}$ & $20 \mathrm{~min}$ & $30 \mathrm{~min}$ & 40 min & 50 min & $60 \mathrm{~min}$ & $70 \mathrm{~min}$ & $80 \mathrm{~min}$ & $90 \mathrm{~min}$ & $100 \mathrm{~min}$ \\
\hline \multicolumn{13}{|l|}{ North } \\
\hline 110 & 0.30 & 0.40 & 0.35 & 0.43 & 0.48 & 0.51 & 0.53 & 0.54 & 0.56 & 0.58 & 0.52 & 0.51 \\
\hline 111 & 0.24 & 0.31 & 0.28 & 0.33 & 0.36 & 0.37 & 0.38 & 0.39 & 0.40 & 0.41 & 0.37 & 0.37 \\
\hline 129 & 0.28 & 0.00 & -0.05 & 0.02 & 0.08 & 0.10 & 0.11 & 0.11 & 0.12 & 0.14 & 0.09 & 0.08 \\
\hline 130 & 0.30 & 0.04 & 0.00 & 0.06 & 0.11 & 0.13 & 0.14 & 0.15 & 0.16 & 0.17 & 0.12 & 0.11 \\
\hline 131 & 0.30 & 0.02 & -0.03 & 0.04 & 0.10 & 0.12 & 0.13 & 0.13 & 0.14 & 0.16 & 0.10 & 0.10 \\
\hline 149 & 0.25 & 0.30 & 0.28 & 0.33 & 0.39 & 0.41 & 0.42 & 0.43 & 0.43 & 0.44 & 0.41 & 0.40 \\
\hline 150 & 0.25 & 0.31 & 0.29 & 0.36 & 0.43 & 0.46 & 0.48 & 0.49 & 0.49 & 0.50 & 0.45 & 0.44 \\
\hline \multicolumn{13}{|l|}{ East } \\
\hline 217 & 0.20 & 0.26 & 0.23 & 0.29 & 0.38 & 0.41 & 0.43 & 0.45 & 0.46 & 0.47 & 0.46 & 0.47 \\
\hline 218 & 0.25 & 0.34 & 0.28 & 0.38 & 0.51 & 0.57 & 0.60 & 0.63 & 0.65 & 0.67 & 0.66 & 0.67 \\
\hline 228 & 0.30 & 0.35 & 0.28 & 0.36 & 0.47 & 0.53 & 0.57 & 0.59 & 0.62 & 0.64 & 0.67 & 0.69 \\
\hline 229 & 0.22 & 0.25 & 0.20 & 0.26 & 0.34 & 0.38 & 0.41 & 0.43 & 0.45 & 0.46 & 0.49 & 0.50 \\
\hline 231 & 0.22 & 0.25 & 0.20 & 0.25 & 0.32 & 0.37 & 0.40 & 0.42 & 0.43 & 0.45 & 0.48 & 0.49 \\
\hline 232 & 0.30 & 0.33 & 0.27 & 0.33 & 0.42 & 0.48 & 0.52 & 0.55 & 0.57 & 0.59 & 0.63 & 0.65 \\
\hline 242 & 0.25 & 0.25 & 0.20 & 0.21 & 0.28 & 0.33 & 0.36 & 0.39 & 0.40 & 0.42 & 0.50 & 0.53 \\
\hline 243 & 0.20 & 0.20 & 0.17 & 0.18 & 0.22 & 0.25 & 0.27 & 0.29 & 0.30 & 0.31 & 0.36 & 0.38 \\
\hline \multicolumn{13}{|l|}{ South } \\
\hline 310 & 0.30 & 0.23 & 0.19 & 0.19 & 0.23 & 0.28 & 0.29 & 0.31 & 0.31 & 0.32 & 0.33 & 0.33 \\
\hline 311 & 0.24 & 0.20 & 0.18 & 0.17 & 0.20 & 0.22 & 0.23 & 0.24 & 0.24 & 0.25 & 0.25 & 0.25 \\
\hline 329 & 0.27 & 0.17 & 0.10 & 0.12 & 0.20 & 0.29 & 0.32 & 0.33 & 0.33 & 0.34 & 0.10 & 0.05 \\
\hline 330 & 0.34 & 0.23 & 0.14 & 0.16 & 0.26 & 0.37 & 0.40 & 0.41 & 0.41 & 0.43 & 0.13 & 0.07 \\
\hline 331 & 0.27 & 0.18 & 0.10 & 0.12 & 0.21 & 0.30 & 0.32 & 0.32 & 0.33 & 0.34 & 0.10 & 0.05 \\
\hline 349 & 0.24 & 0.20 & 0.17 & 0.18 & 0.20 & 0.22 & 0.23 & 0.25 & 0.25 & 0.26 & 0.22 & 0.22 \\
\hline 350 & 0.31 & 0.23 & 0.18 & 0.20 & 0.23 & 0.27 & 0.30 & 0.31 & 0.33 & 0.33 & 0.28 & 0.28 \\
\hline \multicolumn{13}{|l|}{ West } \\
\hline 417 & 0.21 & 0.21 & 0.17 & 0.19 & 0.22 & 0.25 & 0.27 & 0.29 & 0.30 & 0.31 & 0.35 & 0.37 \\
\hline 418 & 0.25 & 0.25 & 0.19 & 0.23 & 0.27 & 0.32 & 0.34 & 0.37 & 0.38 & 0.40 & 0.46 & 0.49 \\
\hline 428 & 0.30 & 0.34 & 0.27 & 0.35 & 0.41 & 0.46 & 0.49 & 0.52 & 0.55 & 0.56 & 0.60 & 0.62 \\
\hline 429 & 0.23 & 0.26 & 0.20 & 0.26 & 0.31 & 0.35 & 0.37 & 0.40 & 0.41 & 0.43 & 0.46 & 0.47 \\
\hline 431 & 0.23 & 0.26 & 0.21 & 0.27 & 0.32 & 0.36 & 0.39 & 0.41 & 0.43 & 0.44 & 0.47 & 0.48 \\
\hline 432 & 0.30 & 0.35 & 0.28 & 0.37 & 0.43 & 0.48 & 0.51 & 0.54 & 0.57 & 0.59 & 0.62 & 0.64 \\
\hline 442 & 0.25 & 0.34 & 0.28 & 0.37 & 0.44 & 0.48 & 0.51 & 0.54 & 0.57 & 0.59 & 0.60 & 0.62 \\
\hline 443 & 0.21 & 0.26 & 0.23 & 0.29 & 0.33 & 0.36 & 0.37 & 0.39 & 0.41 & 0.43 & 0.43 & 0.44 \\
\hline
\end{tabular}




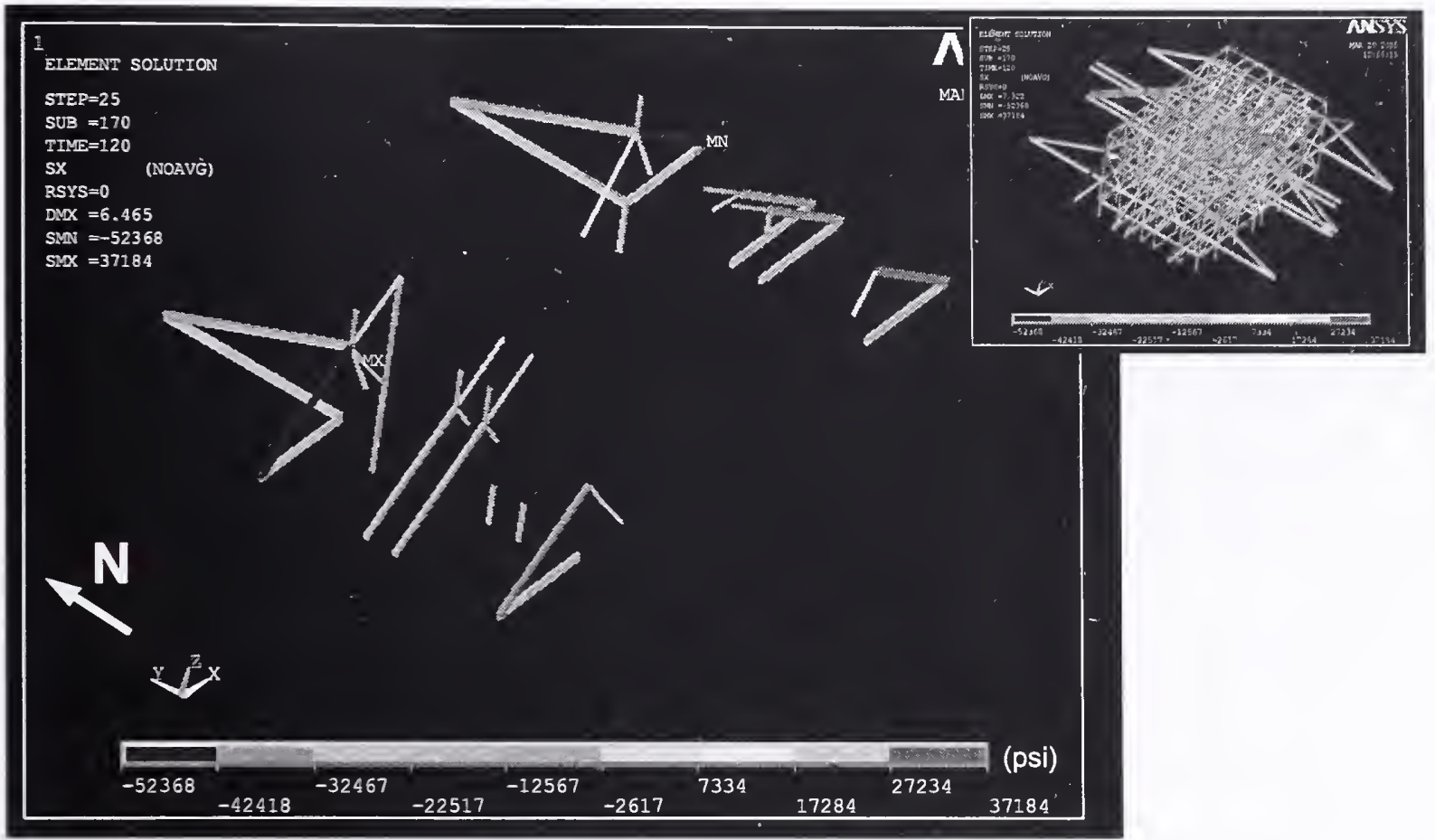

Figure 4-84. Primary load path within the hat truss of WTC 1.

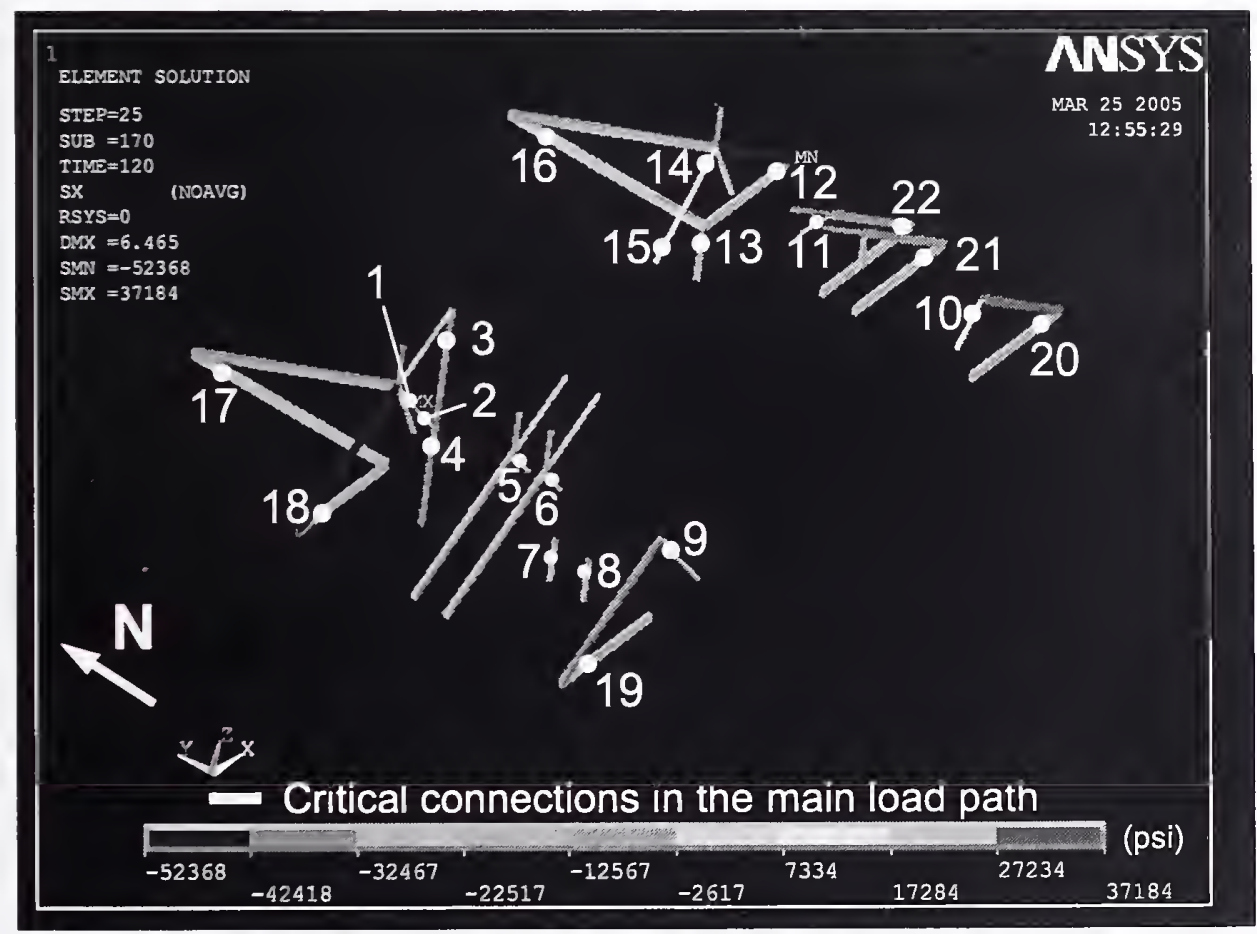

Figure 4-85. Location of the hat truss connections in the primary load path. 
Table 4-29. Demand and capacity of the hat truss connections (kip) in the primary load path at $80 \mathrm{~min}$ (connection IDs are shown in Fig. 4-85).

\begin{tabular}{|c|c|c|c|c|c|c|}
\hline \multirow{2}{*}{$\begin{array}{c}\text { Connection } \\
\text { ID }\end{array}$} & \multicolumn{2}{|c|}{ Location } & \multicolumn{2}{|c|}{ Tension Capacity } & \multirow{2}{*}{$\begin{array}{c}\text { Demand Force } \\
\text { (tension) }\end{array}$} & \multirow[t]{2}{*}{ State } \\
\hline & Floor & Column & Yield & Ultimate & & \\
\hline 1 & 110 & 501 & 1,250 & 1,250 & 680 & Safe \\
\hline 2 & 108 & 502 & 1,250 & 1,250 & 680 & Safe \\
\hline 3 & 111 & 502 & 1,930 & 1,930 & 80 & Safe \\
\hline 4 & 108 & 502 & 520 & 520 & 100 & Safe \\
\hline 5 & 110 & 701 & 1,340 & 1,340 & 390 & Safe \\
\hline 6 & 107 & 702 & 950 & 950 & 370 & Safe \\
\hline 7 & 110 & 801 & 1,340 & 1,340 & 140 & Safe \\
\hline 8 & 107 & 802 & 335 & 260 & 140 & Safe \\
\hline 9 & 110 & 1001 & 1,250 & 1,250 & 400 & Safe \\
\hline 10 & 110 & 1008 & 1,250 & 1,250 & 290 & Safe \\
\hline 11 & 110 & 708 & 1,340 & 1,340 & 360 & Safe \\
\hline 12 & 108 & $217-218$ & 4,520 & 5,880 & 1,810 & Safe \\
\hline 13 & 108 & 508 & 870 & 760 & 410 & Safe \\
\hline 14 & 110 & 508 & 1,250 & 1,250 & 530 & Safe \\
\hline 15 & 108 & 507 & 1,250 & 1,250 & 530 & Safe \\
\hline 16 & 108 & $149-150$ & 2,640 & 2,640 & 1,310 & Safe \\
\hline 17 & 108 & $110-111$ & 2,640 & 2,640 & 1,170 & Safe \\
\hline 18 & 108 & $442-443$ & 4,520 & 5,880 & 1,650 & Safe \\
\hline 19 & 108 & $417-418$ & 4,520 & 5,880 & 1,200 & Safe \\
\hline 20 & 108 & $242-243$ & 4,520 & 5,880 & 1,230 & Safe \\
\hline 21 & 108 & $231-232$ & 3,010 & 3,920 & 1,070 & Safe \\
\hline 22 & 108 & $228-229$ & 3,010 & 3,920 & 1,150 & Safe \\
\hline
\end{tabular}




\subsubsection{Simulation of WTC 2 Collapse}

The global model of WTC 2, as described in Sections 4.1.1 to 4.2.3, was used to simulate the response of the building to aircraft impact damage and the ensuing fire environment. Studies performed on the isolated exterior wall and core models and on the full floor subsystem models indicated that the calculated response of these models to the Case D damage and temperature time history set more closely matched the structural behavior observable in photographic and video evidence than did analyses using Case C. Therefore, in this global analysis only the Case D damage and temperature time history set were used. Section 2.2 of this report provides a description of the Case D structural impact damage set. The analysis started with dead and 25 percent of the design live loads taken directly from the reference SAP2000 models and applied as concentrated loads at each column-floor node. Table 4-30 summarizes the sequence of analyses that were performed. Section 2.5 gives the locations of floor/wall disconnections and pull-in forces at different analysis steps. The results of each analysis step were used as the initial conditions for the next analysis step.

Table 4-30. Analysis Steps of WTC 2 ANSYS global model with Case D conditions.

\begin{tabular}{|c|c|}
\hline Analysis Step & Description \\
\hline 1 & $\begin{array}{l}\text { Dead and } 25 \text { percent of the design live loads were applied on the model of WTC } 2 \text { with impact } \\
\text { damage. }\end{array}$ \\
\hline 2 & $\begin{array}{l}\text { Floor/wall disconnections and pull-in forces that were projected to occur between } 0 \text { min and } 10 \\
\text { min were applied. }\end{array}$ \\
\hline 3 & $\begin{array}{l}\text { Column and spandrel temperatures were linearly ramped up from room temperature of } 20^{\circ} \mathrm{C} \text { to } \\
\text { temperatures at } 10 \mathrm{~min} \text {. }\end{array}$ \\
\hline 4 & $\begin{array}{l}\text { Floor/wall disconnections and pull-in forces that were projected to occur between } 10 \mathrm{~min} \text { and } 20 \\
\text { min were applied. }\end{array}$ \\
\hline 5 & Column and spandrel temperatures were linearly changed from temperatures at $10 \mathrm{~min}$ to $20 \mathrm{~min}$. \\
\hline 6 & $\begin{array}{l}\text { Floor/wall disconnections and pull-in forces that were projected to occur between } 20 \text { min and } 30 \\
\text { min were applied. }\end{array}$ \\
\hline 7 & Column and spandrel temperatures were linearly changed from temperatures at $20 \mathrm{~min}$ to $30 \mathrm{~min}$. \\
\hline 8 & $\begin{array}{l}\text { Floor/wall disconnections and pull-in forces that were projected to occur between } 30 \mathrm{~min} \text { and } 40 \\
\text { min were applied. }\end{array}$ \\
\hline 9 & Column and spandrel temperatures were linearly changed from temperatures at $30 \mathrm{~min}$ to $40 \mathrm{~min}$. \\
\hline 10 & $\begin{array}{l}\text { Floor/wall disconnections and pull-in forces that were projected to occur between } 40 \text { min and } 50 \\
\text { min were applied. }\end{array}$ \\
\hline 11 & Column and spandrel temperatures were linearly changed from temperatures at $40 \mathrm{~min}$ to $50 \mathrm{~min}$. \\
\hline
\end{tabular}

Figures 4-86 through 4-133 summarize the results of the global analyses with creep for the analysis steps described in Table 4-30. The vertical displacements of the exterior wall and the core area are presented in Figs. 4-86 through 4-98. For the exterior wall, the vertical displacements were about 2.0 in. to 3.0 in. before the aircraft impact. As indicated in Fig. 4-88, the core had larger vertical displacements by about 1 in. than the exterior walls at Floor 83 before aircraft impact. Since construction sequence was not considered in the analysis, this difference in the vertical displacements changed initial loading conditions of the columns. As shown in Table 4-8, the total column loads on the east and west walls increased by 7 percent at the impacted floors, the total column loads on the north and south walls increased by about 13 percent, and the total column loads on the core decreased by about 8 percent, by ignoring the construction sequence. After aircraft impact, the vertical displacements increased to $7.4 \mathrm{in}$. on the south wall 
(Fig. 4-86). Due to thermal expansion of columns, the vertical downward displacements recovered slightly initially and eventually increased as shortening due to plastic and creep deformations became larger than the thermal expansion. However, the vertical displacements remained around 7.5 in. until 43 min at which point they increased rapidly to $11.3 \mathrm{in}$. on the east and south faces with the bowing of the east wall. When the east wall reached instability and buckled, the northwest corner of the exterior wall lifted up about $2.0 \mathrm{in}$., which indicated that the tower was tilting toward the southeast around an axis passing through the southwest and the northeast corners (Fig. 4-87). The tilting of the tower is also illustrated in Figs. 4-88 and 4-89, where the vertical displacements at Floor 83 are presented. As can be seen, with increasing time the displacements on the southeast comer increased, whereas the displacements on the northwest corner decreased. The likely axis about which the tower was tilted is indicated in Fig. 4-89. The axis location in Fig. 4-89 was based upon the distribution of plastic and creep strains, which is presented later in this Section. Figure 4-90 shows the total displacements above Floor 86 when the east wall buckled and collapse initiated. For reference, the undeformed tower is also shown. The tilt toward the southeast is clearly visible.

Analysis of the structure without aircraft impact damage indicates vertical displacements at the top of the building of approximately 4 in. (Figs. 4-91, 4-93, 4-95, and 4-97). Analysis with the estimated aircraft impact damage in place indicates that vertical displacements at the southeast corner of the core increased to approximately $10 \mathrm{in}$. (Figs. 4-91 and 4-97). As with displacements on the exterior wall, vertical downward displacement of the core was predicted to be somewhat recovered in the initial periods of the fire, due to thermal expansion, and then increased with time as inelastic and creep deformations of the heated and heavily loaded columns increased substantially. When the east wall reached instability and buckled, at $43 \mathrm{~min}$, the core displacements suddenly increased to $13 \mathrm{in}$. at the southeast corner (Figs. 492, 4-94, 4-96, and 4-98).

Figures 4-99 through 4-101 show the out-of-plane displacements of the east wall at various analysis steps. As shown in Fig. 4-99, there was no out-of-plane displacement on the east wall before the aircraft impact. After aircraft impact, the south side of the east wall at Floor 86 displaced outward about 2.0 in., whereas the north side at the same floor remained approximately at the same displacement as before aircraft impact. This shows a slight twist about the z-axis. With increasing time and temperatures, the east wall bowed and the inward displacements increased (Fig. 4-100). At 20 min the maximum inward displacement reached $9.5 \mathrm{in}$. toward the center of the east wall. This maximum inward displacement agrees well with the maximum inward displacement ( $\sim 10 \mathrm{in}$.) that was measured from the photographs and videos at the same time (at 9:21 am which is approximately 18 minutes after the aircraft impact). After $20 \mathrm{~min}$, the inward displacements steadily increased until at $43 \mathrm{~min}$ they rapidly increased to $62 \mathrm{in}$. (Figs. 4-100 and 4-101). At this point, the east wall reached instability and buckled over the entire width of the wall.

Figures 4-102 and 4-103 show the lateral displacements of the exterior wall above Floor 86 after aircraft impact and at the instant of the east wall buckling. After aircraft impact, Floor 110 moved toward the east about $5.0 \mathrm{in}$. and toward the south about $5.1 \mathrm{in}$. There was also a slight twist about the z-axis of the tower which was about 0.0007 radian at Floor 110 . The twist about the $z$-axis was calculated by taking the difference between the average in-plane displacement of the two opposing exterior walls (such as the east and the west walls) at Floor 110 and dividing the result by the distance between these walls ( $200 \mathrm{ft})$. With the buckling of the east wall, the lateral displacements increased rapidly. The lateral displacements 
of the north exterior wall increased to 15.2 in. toward the east, and of the south exterior wall increased to 6.7 in. toward south. The twist around the z-axis of the tower increased to 0.0010 radian at Floor 110.

Figures 4-104 through 4-11 show the variation of axial loads on the exterior wall columns at different analysis steps. Typically, before aircraft impact, the load distribution along the width of the walls was symmetric with respect to the centerline of each wall. After aircraft impact, the loads in the severed columns redistributed. As a result of this redistribution, the column loads on the south side of the east and west walls and on the east side of south wall increased. This was due to leaning of the tower toward the southeast as the aircraft impact severed columns on the east side of the south wall and on the southeast comer of the core (Figs. 4-104, 4-106, 4-108, 4-110, and 4-111). After redistribution, at Floor 83 the column loads on the south wall increased from about 250 kip before aircraft impact to 400 kip to 800 kip in general and to 1,500 kip at the edges of the severed area after aircraft impact. The column loads on the south side of the east wall at Floor 83 increased from about 350 kip before aircraft impact to about 450 kip after aircraft impact. The column loads on the east side of the north wall at Floor 83 did not change significantly; however, the column loads on the west side of the north wall at Floor 83 decreased from about $250 \mathrm{kip}$ before aircraft impact to about $200 \mathrm{kip}$ after aircraft impact. The column loads on the south side of the west wall at Floor 83 increased from about 300 kip before aircraft impact to about 380 kip after aircraft impact. The column loads on the north side of the west wall at Floor 83, however, decreased from about $300 \mathrm{kip}$ before aircraft impact to $250 \mathrm{kip}$ after aircraft impact. Over the duration of the fires the column load distributions remained constant except for some localized changes due to relative temperatures. When the east wall buckled, the load distribution significantly changed. The columns over nearly the whole width of the east wall unloaded about 400 kip on the average at Floor 83 . Similarly, the columns on the west face unloaded about $65 \mathrm{kip}$ on the average. This was due to the increased tilting of the tower toward the east, as the east wall buckled. A part of the loads from the east and the west walls were redistributed to the east side of the south and the north walls. The column loads on the east side of the south wall increased from about $500 \mathrm{kip}$ to $800 \mathrm{kip}$ after aircraft impact, and to $800 \mathrm{kip}$ to $1,100 \mathrm{kip}$ after east wall buckling. The column loads on the east side of the north wall increased from about $200 \mathrm{kip}$ to $250 \mathrm{kip}$ after aircraft impact, and to $300 \mathrm{kip}$ to $500 \mathrm{kip}$ after east wall buckling.

Figures 4-112 through 4-119 show the core column loads at different analysis steps. Before the aircraft impact the loads on the core columns were distributed symmetrically with respect to the center of the core. The slight difference between corner columns on the south side (Columns 501 and 1001) and north side (Columns 508 and 1008) of the core was due to slightly higher dead and live loads in the north side columns. After aircraft impact, some portion of the loads in the severed columns was redistributed to the adjacent intact columns within the core. The tilt toward the southeast also influenced the load redistribution within the core. Columns 506, 507, 508, and 1008 at the northwest and northeast corners unloaded; other intact core columns increased in load (Fig. 4-118). The maximum load in the 800 series core columns increased from about 1,400 kip before aircraft impact to about 1,700 kip after aircraft impact (Fig. 4-112). The maximum load in the 900 series core columns increased from about 1,250 kip before aircraft impact to about 1,900 kip after aircraft impact (Fig. 4-114). Although the maximum load in the 1000 series core columns reduced from about 4,300 kip before aircraft impact to about 3,000 kip after aircraft impact (Fig. 4-116), the average load on the remaining intact 1000 series columns increased about 360 kip at Floor 83 (Fig. 4-118).

Over the duration of the fires, some of the core columns, especially the ones on the east side of the core, unloaded due to yielding and creeping at high tcmperatures. Loads in softening columns were 
redistributed to the adjacent columns with lower temperatures. During buckling of the east wall, the loads in the core columns, especially the ones at the northeast corner of the core, increased significantly. For instance, at Floor 83 the load in core column 1008 increased from 2,820 kip after aircraft impact to 5,320 kip at $43 \mathrm{~min}$, the load in core column 907 increased from 1,290 kip to 2,330 kip, and the load in core column 805 increased from 950 kip to 1,480 kip (Figs. 4-118 and 4-119).

Figure 4-120 shows the axial loads in the columns at Floor 83 before aircraft impact and after the buckling of the east wall. This figure illustrates the load redistribution among the exterior wall and core columns. The tilting of the building about an axis likely located through the shaded area occurred after the buckling of the east wall and weakening of the core. Comparison of column loads before aircraft impact and after the east wall buckled clearly shows the unloading over the entire width of the east wall and increased loads at the east side of the south and north walls.

Figures 4-121, 4-122, and 4-123 show the maximum of the elastic-plus-plastic strains and elastic-plusplastic-plus-creep strains in the columns between Floor 78 and Floor 83 for different analysis steps. The elastic-plus-plastic strains, which were less than 0.05 percent before the aircraft impact, reached about 0.60 percent in the exterior columns and about 0.35 percent in the core columns after the aircraft impact. With increasing temperatures the plastic strains increased, especially on the east wall and the east side of the core. When the east wall buckled, the elastic-plus-plastic strains reached their maximum of 2.2 percent in the east wall and 0.9 percent in the east side core columns. The creep strains also increased with increasing temperatures to the same level as the elastic-plus-plastic strains in the east wall ( 1.0 percent to 2.0 percent), to about 2 to 6 times that in the core columns ( 2.0 percent to 6.0 percent), and to about 10 times that in the east side of the north wall ( 4.0 percent to 5.0 percent).

The state of the hat truss members and the connections were checked as the global model did not include break elements to capture column and hat truss splice failures or sufficient beam elements to capture buckling of hat truss outriggers. The condition of the connections and the members in the primary load path of the hat truss was evaluated at different time intervals. Evaluations were performed for the core column splices for tensile capacity, outriggers and supporting columns for compressive capacity, and hat truss connections in the primary load path for tensile capacity. The demand forces that were used in the evaluation were obtained from the "superelement" since the hat truss was part of it for the WTC 2 global model with creep. The results for elements within the superelement were obtained by back-substitution. For this purpose the elastic model that was used to generate the stiffness matrix for the superelement was used. This elastic model essentially represents the portion of the building from Floor 86 and above and is referred to as the "top model" hereinafter. The displacements obtained at the interface nodes between the "superelement" and the nonlinear portion of the building (Floor 86 and below) were applied to the base of the "top model" at the end of each analysis step together with the nodal loads representing the dead and the 25 percent of the design live loads.

Figures 4-124 and 4-125 show the loads on the core column splices at the hat truss level at different analysis steps. As can be noticed, each splice was under compressive load before the aircraft impact. After the aircraft impact, the splices at severed core column lines started to carry tensile loads. To evaluate the condition of the core column splices at Floor 106, the tensile capacities of these splices were determined using the AISC LRFD procedures and were compared to the tensile forces obtained at $40 \mathrm{~min}$, since at this time point the tensile forces at core column splices were at their maxima. The evaluation of core column splices required an iterative procedure as splice failures were not modeled in the "top model". The iteration procedure was required due to the slight nonlinearity of the problem where the 
failure of a few splices did not alter boundary conditions of the "top model" significantly. In the first iteration, the state of the "top model" was calculated using the interface nodal displacements at $40 \mathrm{~min}$. Once equilibrium was reached, the columns exceeding their splice capacity were identified (in the first iteration Columns 1001 and 1002 were identified (see Fig. 4-126) and removed). This removal represented the splice failure at that column location. Before removing the columns, the displacement boundary conditions applied at the bottom of these column lines (at Floor 86) were replaced with the reaction forces that were obtained at the end of the first iteration. This conversion from displacement to force boundary condition allowed the remaining portion of the column lines to displace in the vertical direction when the columns were removed at Floor 105 to simulate splice failure. After the removal of the columns, the "top model" was reanalyzed to redistribute the released tensile force due to splice failure. Once equilibrium was reached, the remaining core column splices were reevaluated and any additional splice failures were identified. This iterative procedure was repeated until none of the remaining splices exceeded their tension capacity. A stable state was reached at the end of the fourth iteration. Figure 4126 summarizes the iteration sequence and the splices that failed at each iteration. Figure 4-127 shows the state of the core column splices at the end of the fourth iteration.

In the global analyses, none of the splice failures were represented as those elements were part of the "superelement" which remained elastic throughout the analysis. However, the inclusion of splice failures is not expected to significantly affect the load redistribution in the global analysis. The loads on columns with failed splices would have redistributed through the core slab and core beams with moment connections to adjacent core columns, which in turn would transfer these extra loads to the hat truss. To quantify the amount of load that was redistributed to the hat truss through the adjacent core columns, the total column loads on each face and at the core area were extracted at Floor 105 at the end of each iteration. Table 4-31 summarizes these total column loads together with the magnitude of total tensile load released and total tensile load retransferred to the hat truss at each iteration. In Table 4-31, the Column "TTLRSF" represents the total tensile load that was released due to splice failure and is equal to the summation of the load carried by the circled columns in Fig. 4-126 up to and including the current iteration. In Table 4-31, the Column "Core" between Rows 6 and 9 represents the load that was transferred to the base of the "top model" through the core columns. The sum of Column "TTLRSF" and Column "Core" represents the total tensile load that was transferred to the hat truss after the splice failures (labeled as "TTLRHT" in Table 4-31). As can be seen, about 73 percent (=2,728 kip /3,717 kip) of the released tension load was transferred to the hat truss after the fourth iteration. The core slab and the core beams with moment connections were the primary components that transferred the released load to the adjacent core columns and to the hat truss. Capacity calculations have shown that the core slab and the core beams in the top 20 stories had enough cumulative capacity to redistribute the released load to the adjacent core columns. As a result of load redistribution, the loads on the southeast corner outriggers were reduced, but the loads on adjacent outriggers increased.

Figures 4-128 through 4-130 show the axial loads and stresses in the hat truss members at different analysis steps. With the aircraft impact, the maximum axial load on the outriggers, which was about 1,900 kip before the aircraft impact, increased to about 3,400 kip at the southeast corner. This increase remained almost constant over the duration of the fires. After the buckling of the east wall the maximum load slightly increased to $3,500 \mathrm{kip}$. The axial stresses in the outriggers also increased as a result of the aircraft impact. The maximum axial stress of $28.4 \mathrm{ksi}$ before aircraft impact increased to $55.0 \mathrm{ksi}$ after the buckling of the east wall. The specified grade of steel for the outriggers was $50 \mathrm{ksi}$. The tests conducted on this grade of steel showed an average yield strength of about $54 \mathrm{ksi}$. Considering this yield 
strength and the 10 percent increase in the hat truss forces due to neglecting construction sequence, it was concluded that the outriggers of the hat truss did not exceed their elastic limits. To check against buckling, the buckling capacities of these outriggers as well as the supporting columns were calculated. The buckling calculations were performed using the AISC LRFD Eqs. E2-1 and E2-3 with an effective length factor "K" of 0.75 and a resistance factor of 1.0. The calculated capacities were then compared with the axial compressive forces obtained from the analysis and corrected to account for the load increase due to construction sequence. The location of the outriggers and the supporting columns are shown in Fig. 4-3. Tables 4-32 and 4-33 summarize the resulting demand-to-capacity ratios for the outriggers and the supporting columns, respectively. Except for one outrigger, designated Outrigger L, none of the outriggers or supporting columns was predicted to buckle.

Outrigger L was located at the southeast corner of the core, and above the severed core columns. With the splice failures in Columns 1001, 1002, and adjacent core columns, the load on this outrigger reduced and the load redistributed to other outriggers. With the load redistribution after splice failures, the demand-to-capacity ratio on Outrigger L reduced from 1.3 to 1.1 ( $\mathrm{Col}$ " $40 *$ min" in Table 4-32). To investigate whether the buckling of the Outrigger $\mathrm{L}$ would cause additional outriggers to buckle, the element representing Outrigger L was removed from the "top model." Removal of Outrigger L from the "top model" represents an upper bound solution as the load in the outrigger would not drop to zero after buckling. After removal of Outrigger L, the adjacent outriggers increased in load however, as presented in Col "40** min" of Table 4-32, none of the remaining outriggers exceeded their buckling or yield capacities.

In the global analysis, it was assumed that the connections in the hat truss area were adequate to transfer the loads from the core columns to the outriggers. The structural adequacy of the "primary" connections, those hat truss connections between members that were predicted to have axial tensile or compressive stress of $25 \mathrm{ksi}$ or more, was evaluated. To perform this evaluation, the results at $40 \mathrm{~min}$ were used, since at $40 \mathrm{~min}$ the stresses in the hat truss members were at their maxima. Only connections that transferred tensile forces were evaluated. Figure 4-132 shows the members in the primary load path. Figure 4-133 shows the location of the critical hat truss connections that were evaluated in this study. The connections between the horizontal tie-backs and the outriggers were not evaluated as they were found to have significant capacity in the WTC 1 connection evaluations. Connection capacities were calculated using AISC LRFD procedures. Table 4-34 summarizes capacities, demands, and the predicted condition of the evaluated connections. As can be seen in Table 4-34, before redistribution of load due to column splice failure, none of the connections exceeded their capacities except the connections associated with the 1001 core column line. After load redistribution, the demand was less than the yield capacities for all connections. Based on this, and the other evaluations of column splices and hat truss members discussed above, it was concluded that the hat truss was capable of transferring loads from core columns to the outriggers without any connection failures.

Tables 4-35 and 4-36 summarize the total column loads at each exterior face and the core for Floor 83 and Floor 105. The first seven rows of Tables 4-35 and 4-36 provide the total loads at each analysis step. The rows from 8 through 13 give the change in total column loads at each analysis step with respect to the total column loads before aircraft impact. For instance, Row 8 gives the difference between the total column loads before and after aircraft impact. A positive value between Row 8 and Row 13 indicates that the total compressive load has increased in that analysis stage as compared to the total compressive load before the aircraft impact. Based on these tables, after the aircraft impact, the core and the north wall 
unloaded and their loads were redistributed to the south, west. and especially to the east wall at Floor 83. A similar load redistribution pattern was found for Floor 105. The comparison of the total column loads before and after aircraft impact at Floor 83 and Floor 105 is presented in Table 4-37. The second row in this table represents the amount of load redistributed through the hat truss, and the third row represents the amount of load redistributed through the spandrels and the floors. Based on this table, it can be stated that about 94 percent ( $3.740 \mathrm{kip} / 4,000 \mathrm{kip})$ of the load that was unloaded from the core was redistributed through the hat truss to the east, south. and west walls, and the remaining 6 percent was redistributed through the floors to the exterior walls. A similar calculation for the east wall indicates that about 62 percent (2.699 kip/4.368 kip) of the load increase on the east wall came through the hat truss and the remaining 38 percent came through the Vierendeel action of the wall. Rows 8, 9, 10, and 11 of Table 4 35. show that the loads in the various walls and the core itself did not significantly change until the core unloaded at about $30 \mathrm{~min}$. At $30 \mathrm{~min}$ the total column loads on the east wall increased by about 1,200 kip (from 5,567 kip to 4,368 kip) and the core unloaded about $850 \mathrm{kip}$ (from 4,861 kip to 4,007 kip) and the north wall unloaded about $420 \mathrm{kip}$ (from 1,797 kip to 1,374 kip) at Floor 83. Similarly, at Floor 105 and at $30 \mathrm{~min}$ the total column loads in the various walls and core remained almost constant until initiation of buckling of the east wall. After $40 \mathrm{~min}$, the east wall suddenly unloaded about 8,540 kip, the west wall unloaded about $2.860 \mathrm{kip}$, the core experienced a load increase of about 5,600 kip, the north wall increased by about $2,310 \mathrm{kip}$, and the south wall increased by about $2.820 \mathrm{kip}$ at Floor 83 (Table 4-38). Comparison of the load redistribution that took place at Floor 105 with the one at Floor 83 indicates that about 100 percent of the additional core load came from the east and the west walls through the hat truss. For the east wall, about 46 percent $(3,901 \mathrm{kip} / 8,539 \mathrm{kip})$ of the relieved load was redistributed through the hat truss to the core, and the remainder was redistributed through Vierendeel action to the south and north walls. After the redistribution, at $43 \mathrm{~min}$, the load in the core had recovered to the same level as the total load before the aircraft impact.

Tables 4-39 through 4-43 summarize the loads in the east, south, north, west walls, and the core at several floor levels. The tables also indicate the change in column load at each stage of the analysis, compared to the load present before aircraft impact. These load changes are provided in Columns 8 through 13. As described before, the changes in loads at Floor 105 represent the load redistribution through the hat truss. The changes in loads along the height of the tower indicate the load redistribution through the exterior wall and the floor system. In referring to the changes in loads after the aircraft impact ( $\mathrm{Col} 8$ in the tables). it can be concluded that the total column loads along the height of the core and the west wall remained almost constant. This indicates that almost all load redistribution from and to these portions of the tower went through the hat truss. The total column loads in the east wall increased at the hat truss level and continued to increase with distance from the hat truss. The north wall showed the opposite trend; at the hat truss level the wall unloaded and continued to unload further with distance from the hat truss. The unloading took place primarily through Vierendeel action, to the east wall. After the buckling of the east wall, the change in loads (Col 13 in the tables) indicates that the hat truss was the primary load path for the portion of the load picked up by the core. The east and west walls unloaded at the hat truss level and at lower floors. Both walls steadily picked up more load at lower floors through the action of spandrels.

During the load redistribution, the floor slabs played a significant role in restraining the core in the lateral direction. The aircraft impact damage to the core and the ensuing fire environment caused the core to lean toward the south and east. In fact, calculations show that the isolated core would not have been stable. The resistance to core leaning was provided by the exterior wall. The load was transferred to the 
exterior walls by shear either through slabs or hat truss. To identify the contribution of the floor slabs to the lateral restraint of the core, the moment of the core column loads around the center of the building in the $\mathrm{x}$ - and $\mathrm{y}$-directions at Floor 83 and Floor 105 were computed for WTC 2. Before impact, there was no eccentricity in the load, and the moments thus calculated were small. After impact and at the end of temperature histories, the moment in the x-direction was 5,905,640 kip-in at Floor 83 and 2,282,320 kip-in at Floor 105. Similarly, the moment in the y-direction was 5,051,130 kip-in at Floor 83 and 2,196,440 kip-in at Floor 105. The change in moment from Floors 83 to 105 was due to the lateral resistance of provided by the slabs and the exterior walls to the core. The results of calculations show that the overturning moment reduced by about 55 percent to 60 percent along the height of the tower. This reduction was due to the lateral resistance provided by the floor slabs. The remaining 40 percent to 45 percent was resisted by the hat truss. 


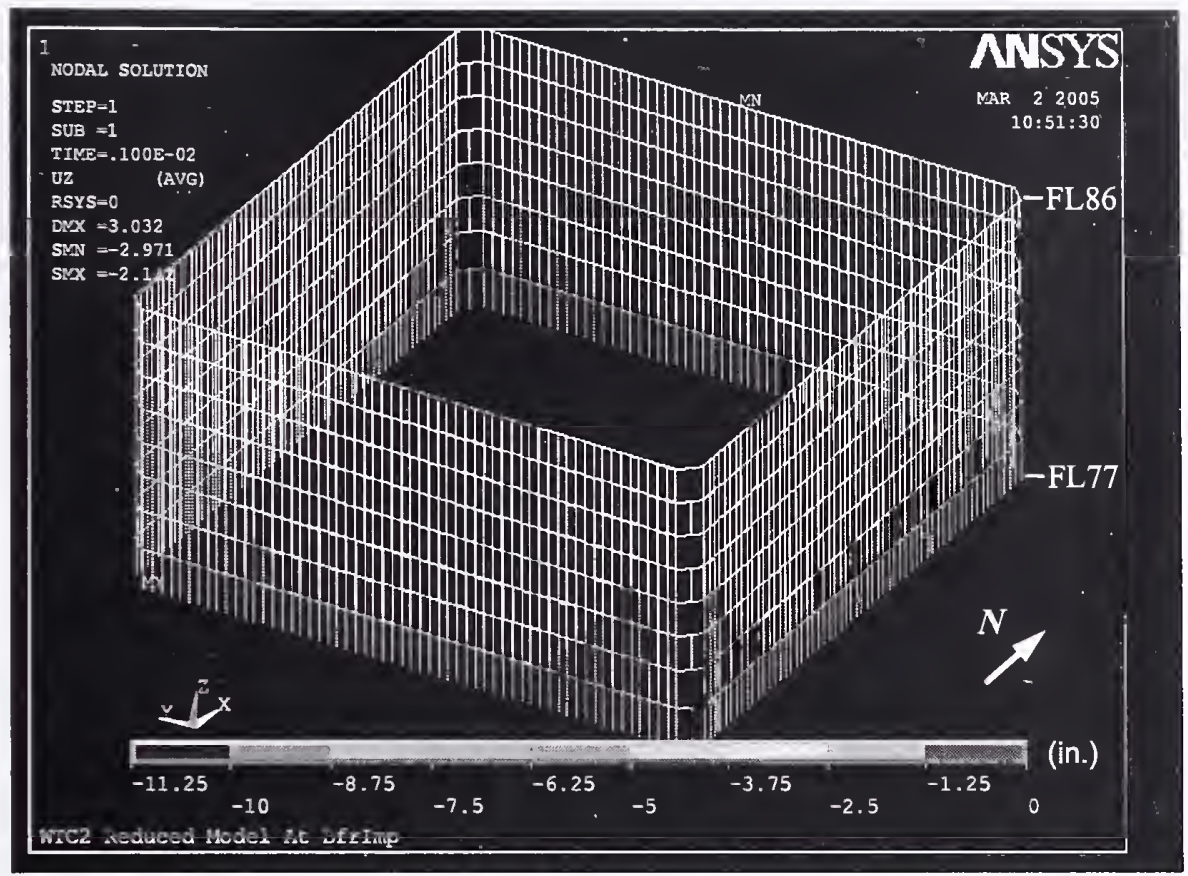

(a) Before impact

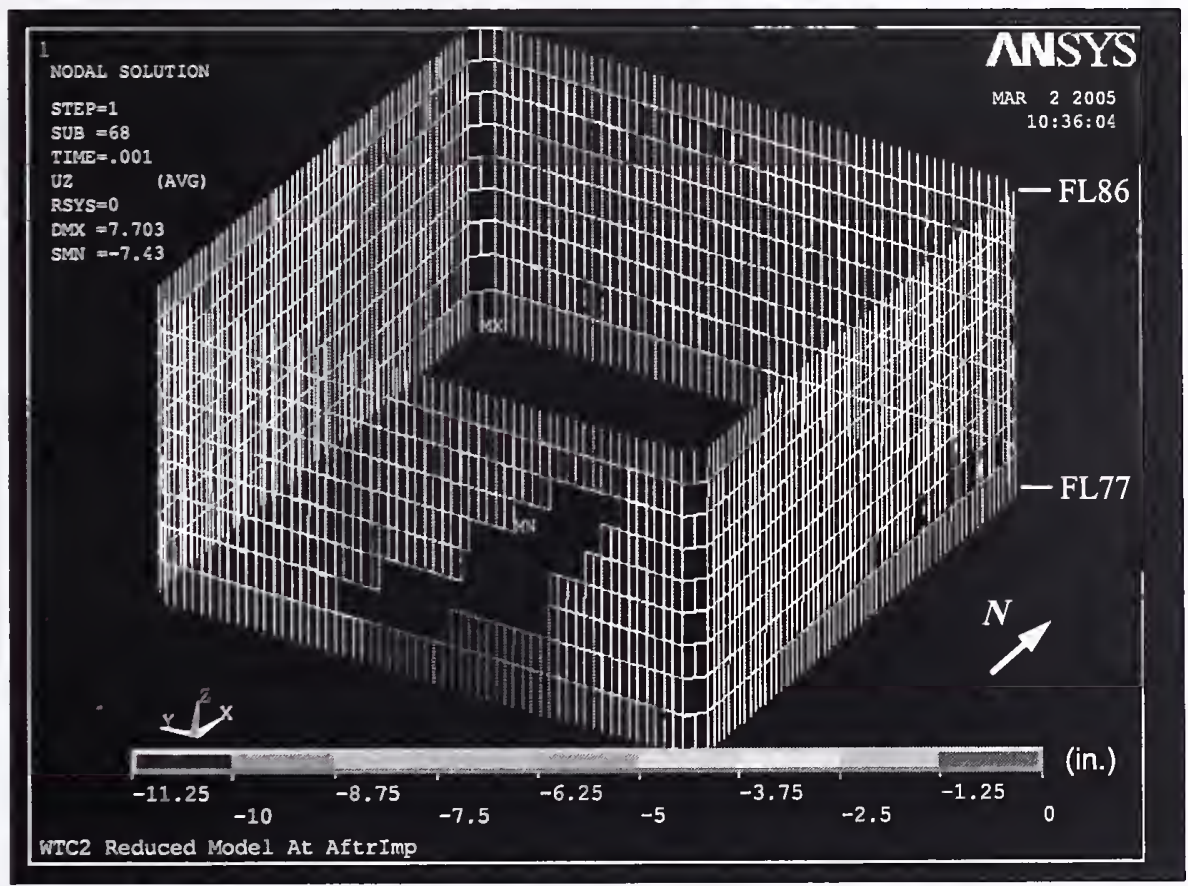

(b) After impact

Figure 4-86. Vertical displacement of exterior wall of WTC 2 for Case D conditions (downward displacement is negative). 


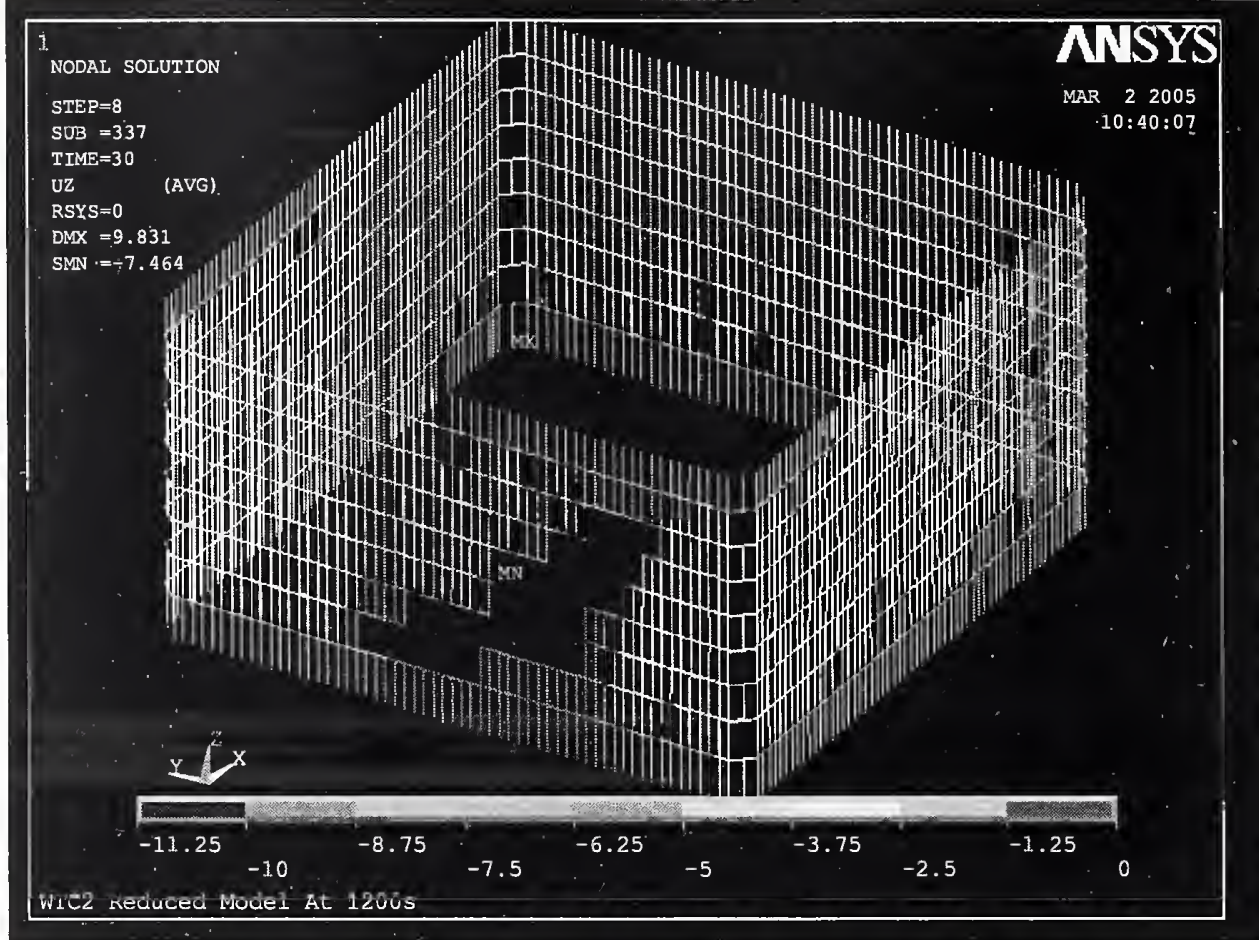

(a) $20 \mathrm{~min}$

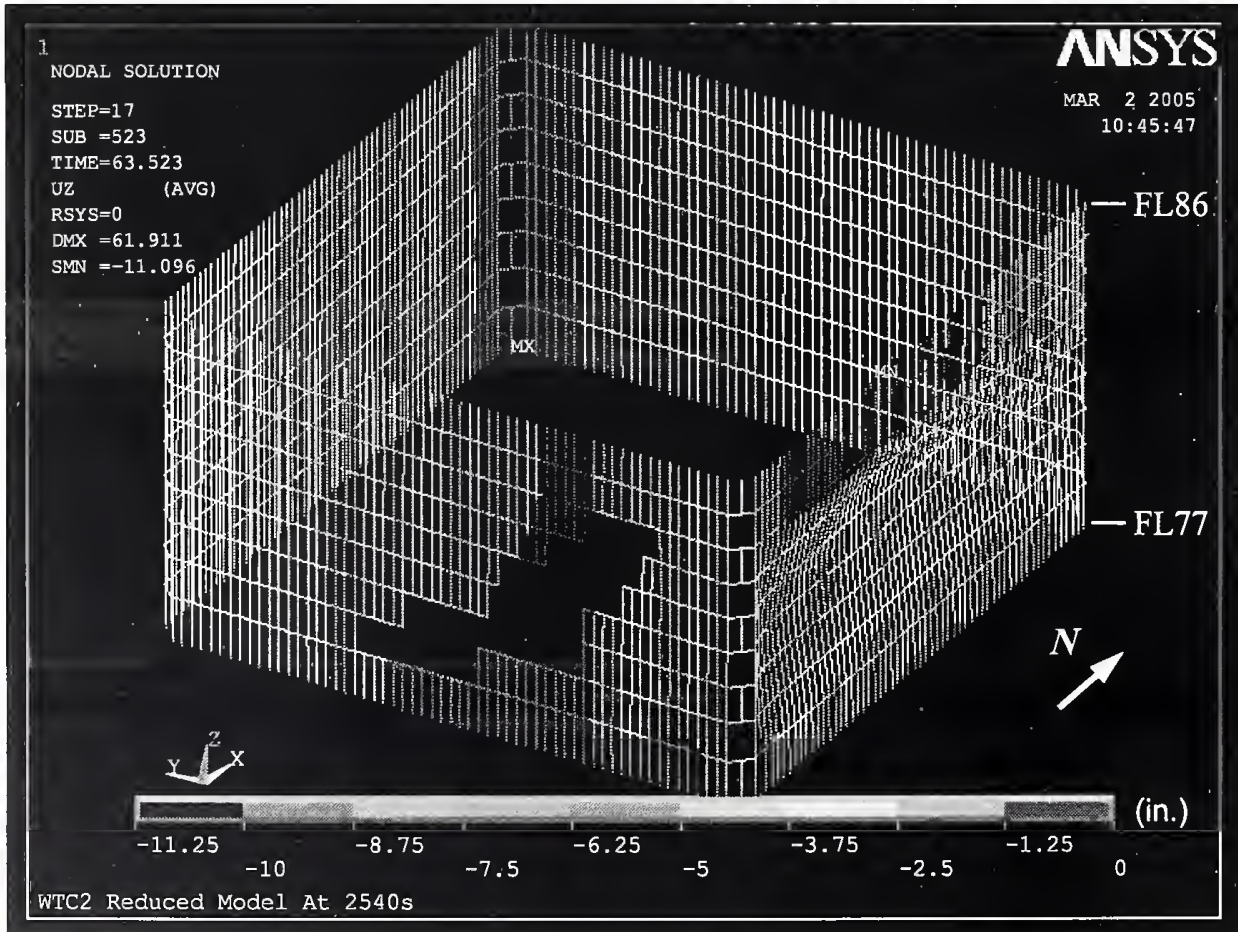

(b) $43 \mathrm{~min}$

Figure 4-87. Vertical displacement of exterior wall of WTC 2 for Case D conditions (downward displacement is negative). 


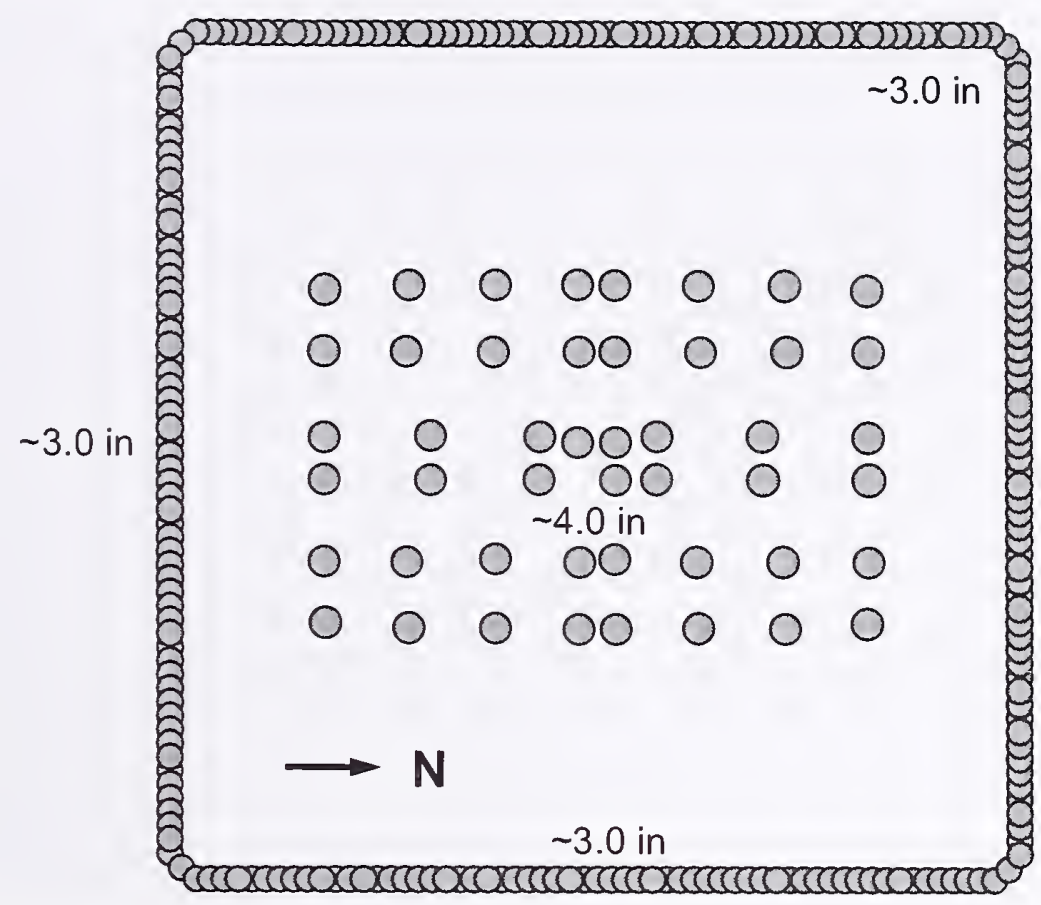

(a) Before impact

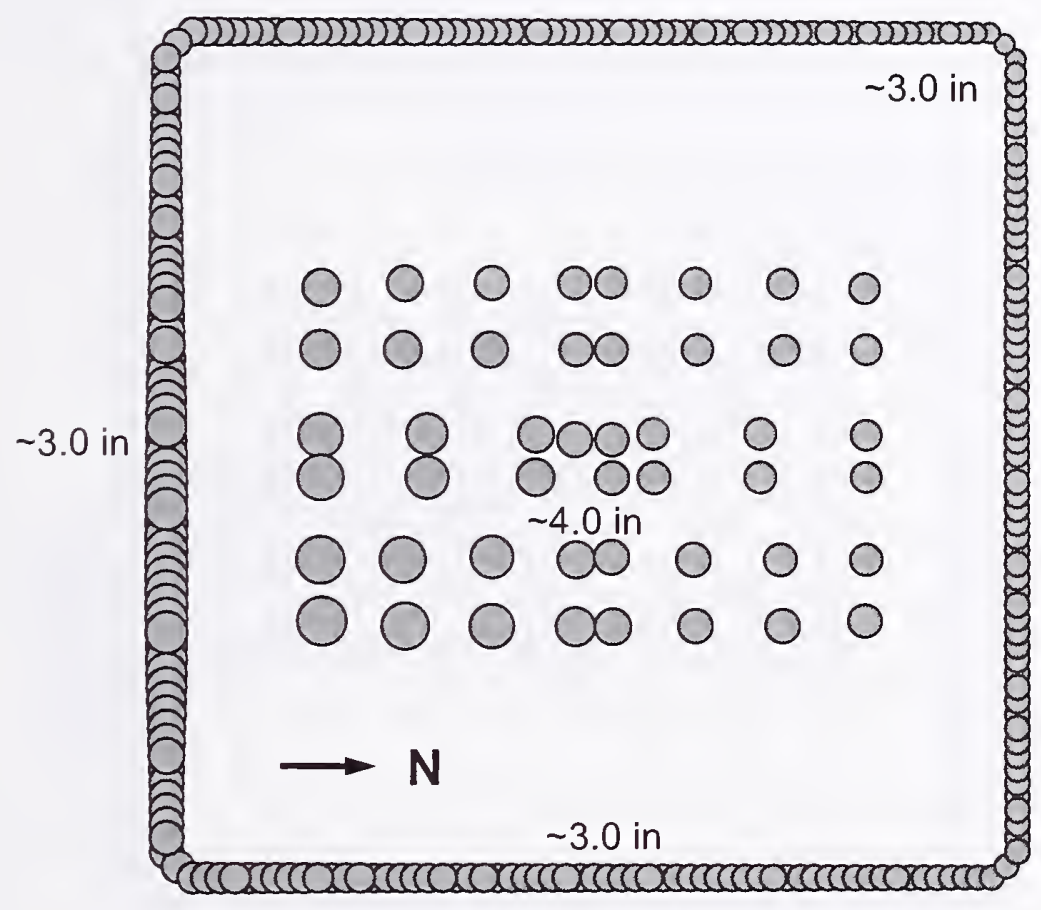

(b) After impact

Figure 4-88. Vertical displacement at Floor 83 of WTC 2 for Case D conditions (downward displacement is positive). 


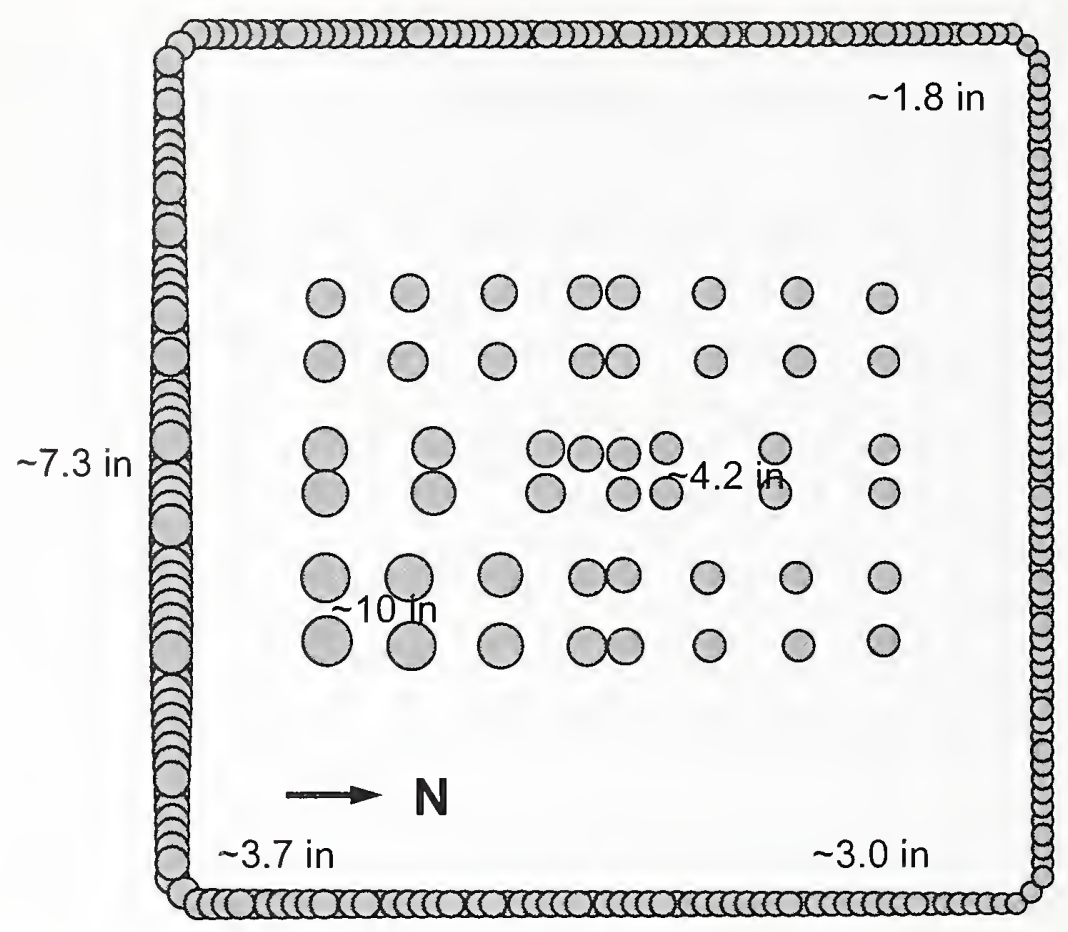

(a) $20 \mathrm{~min}$

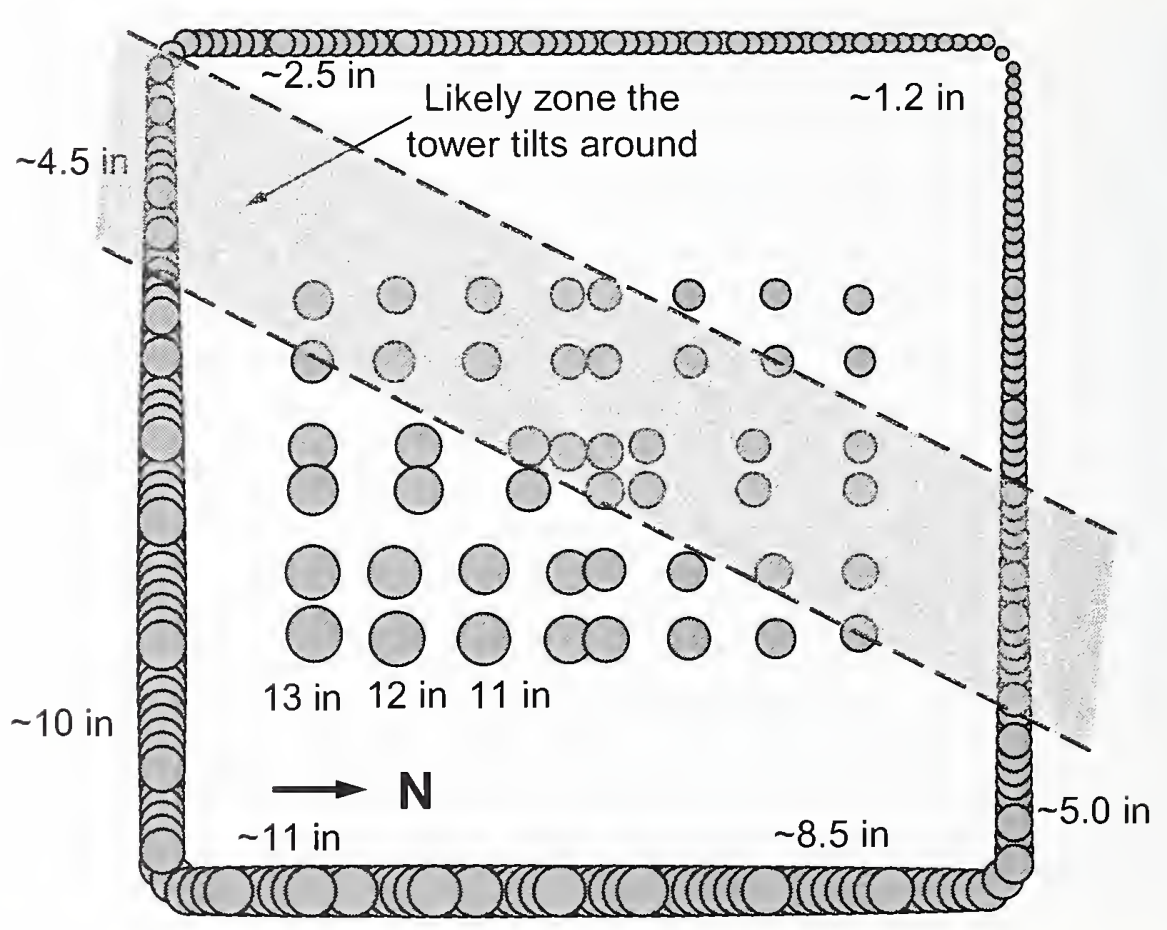

(b) $43 \mathrm{~min}$

Figure 4-89. Vertical displacement at Floor 83 of WTC 2 for Case D conditions (downward displacement is positive; note the tilt toward east and south). 


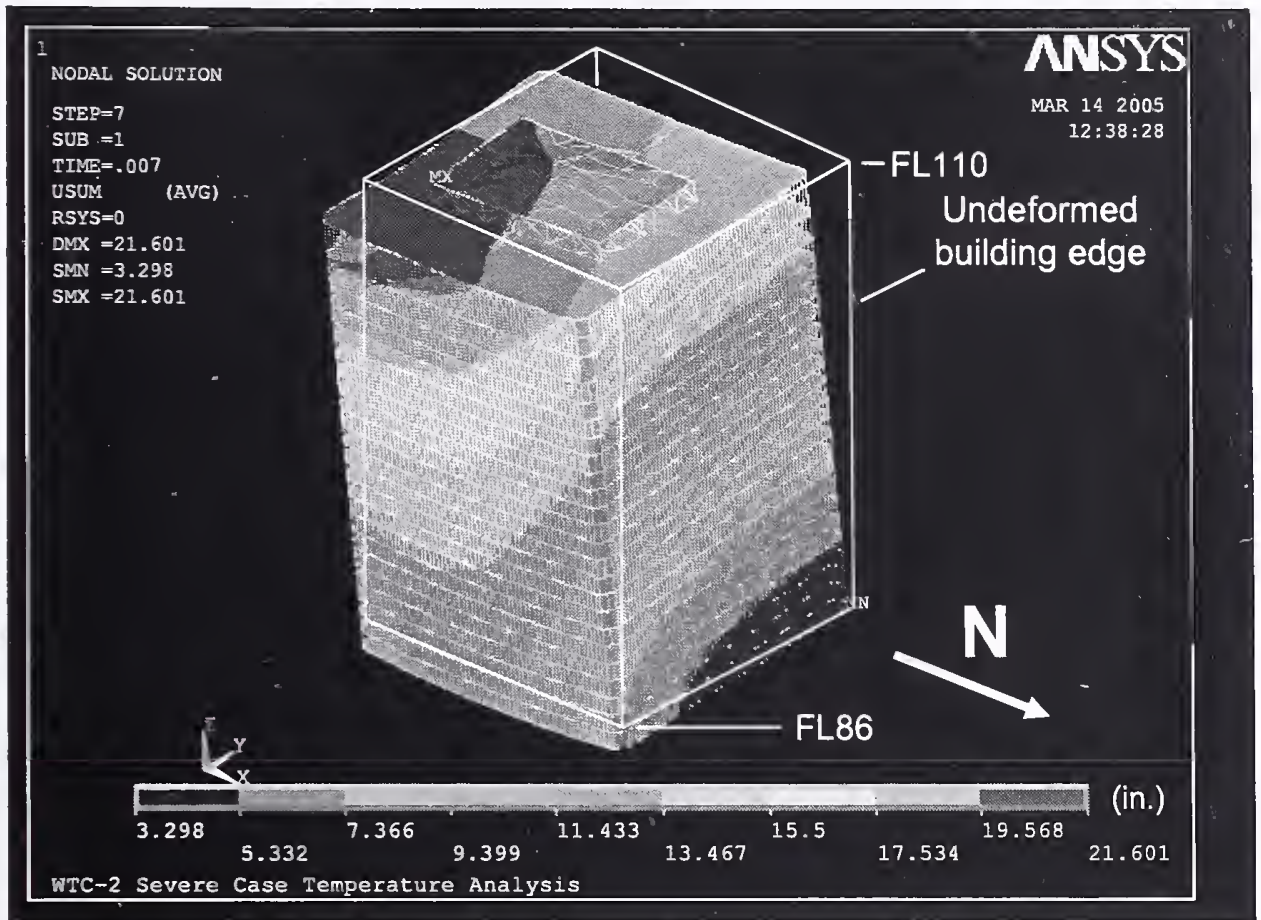

Figure 4-90. Total displacements of WTC 2 above Floor 86 at 43 min of Case D conditions (deformed shape magnified 20 times). Note the tilt toward east and south. 


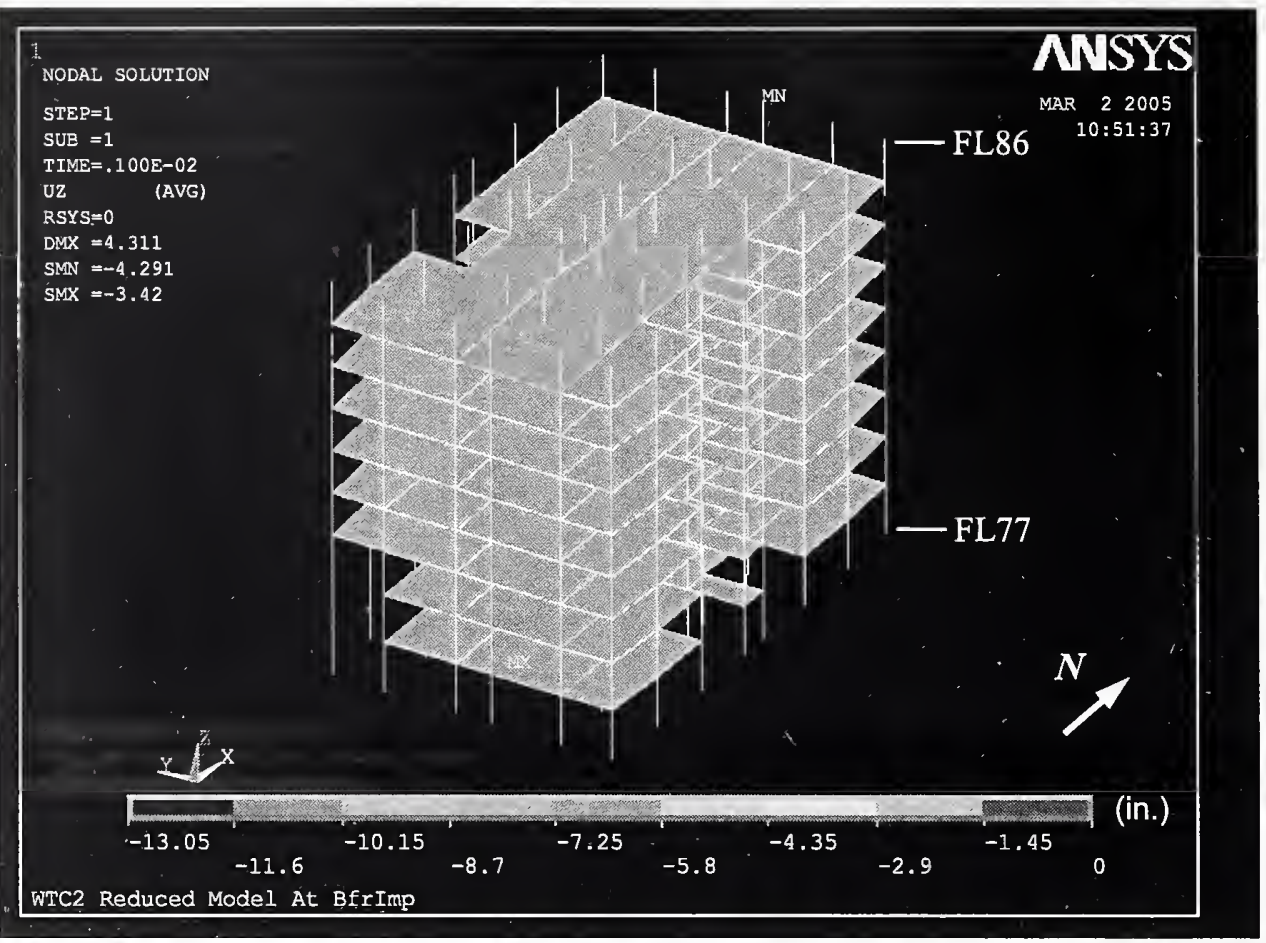

(a) Before impact

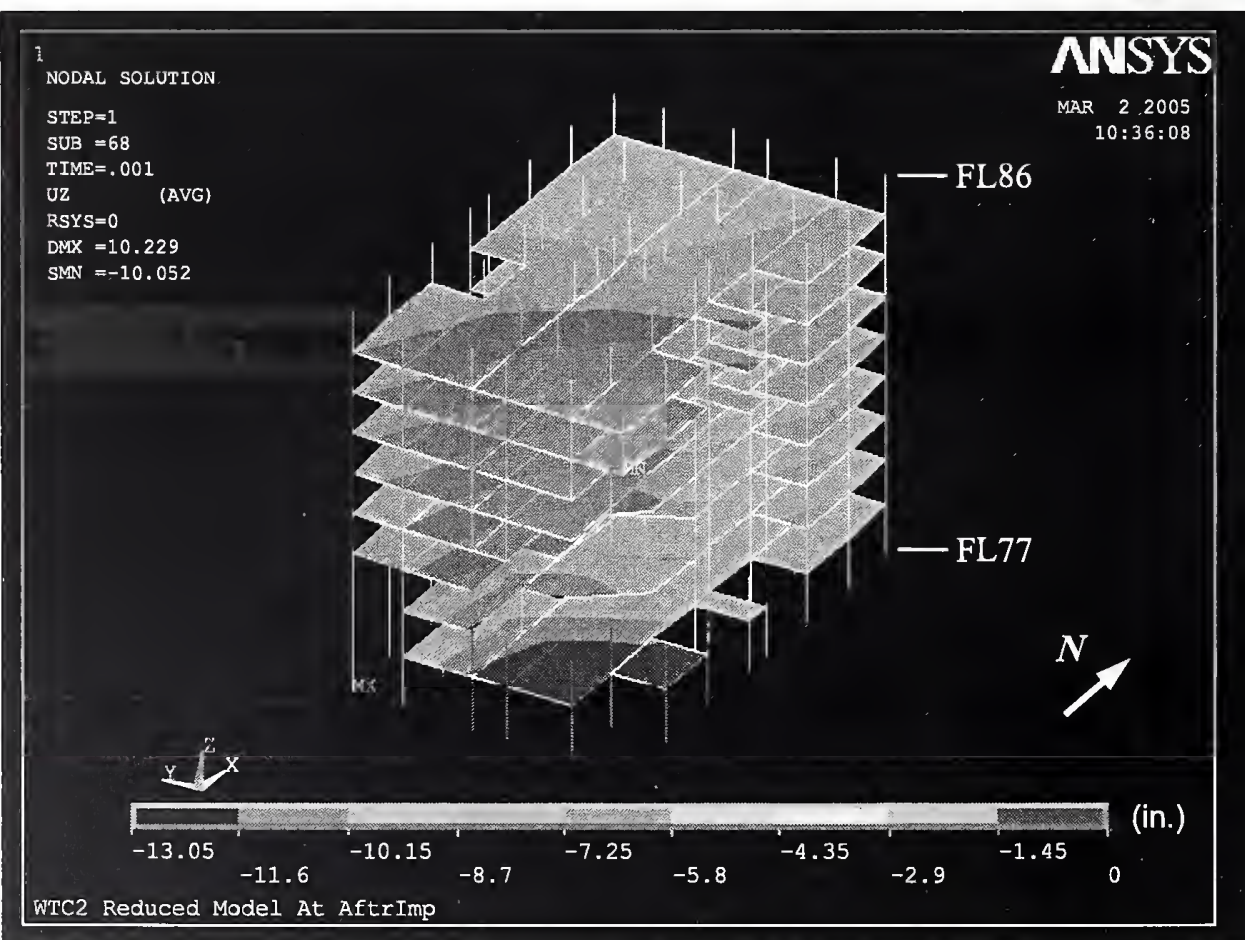

(b) After impact

Figure 4-91. Vertical displacement of core of WTC 2 for Case D conditions (downward displacement is negative). 


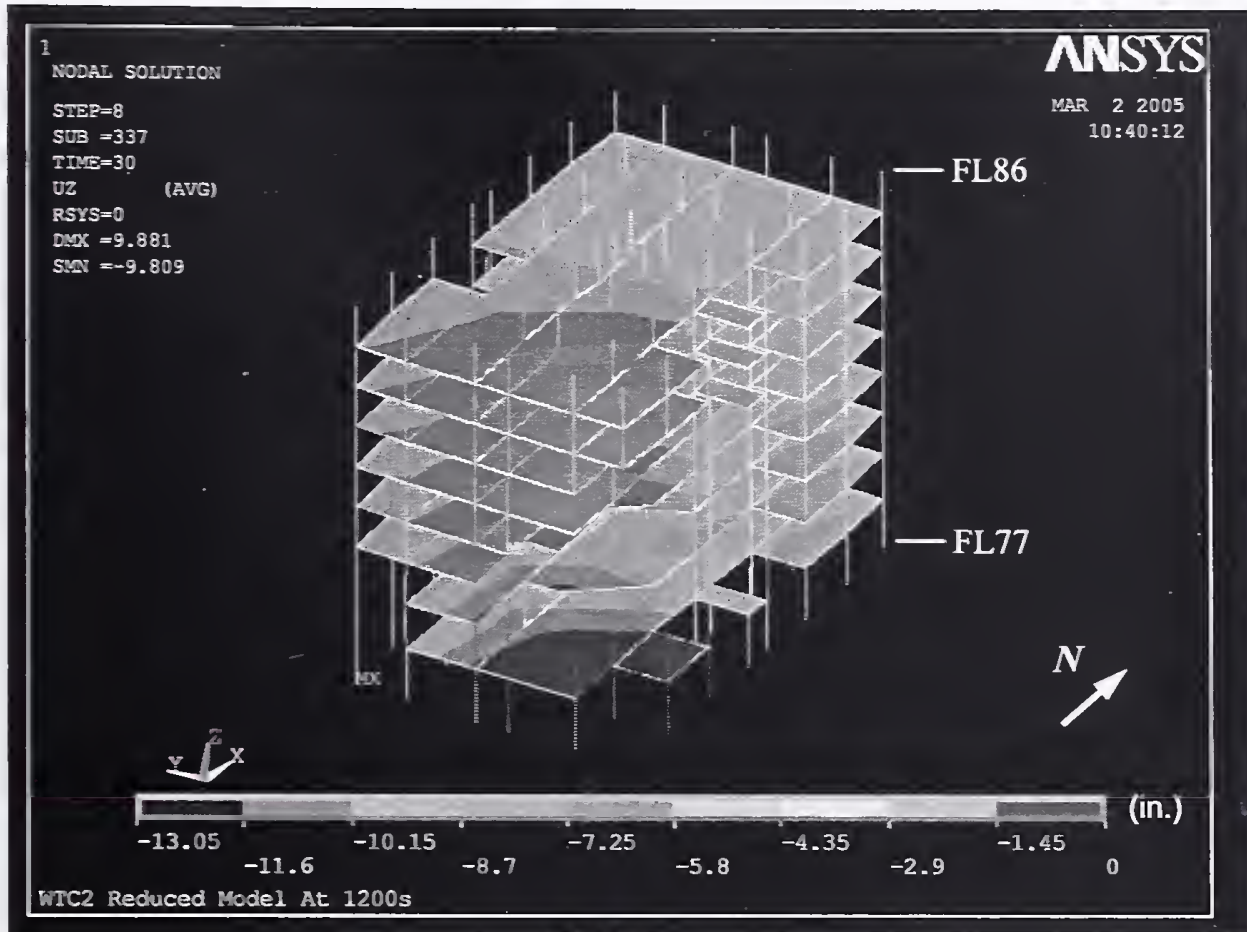

(a) $20 \mathrm{~min}$

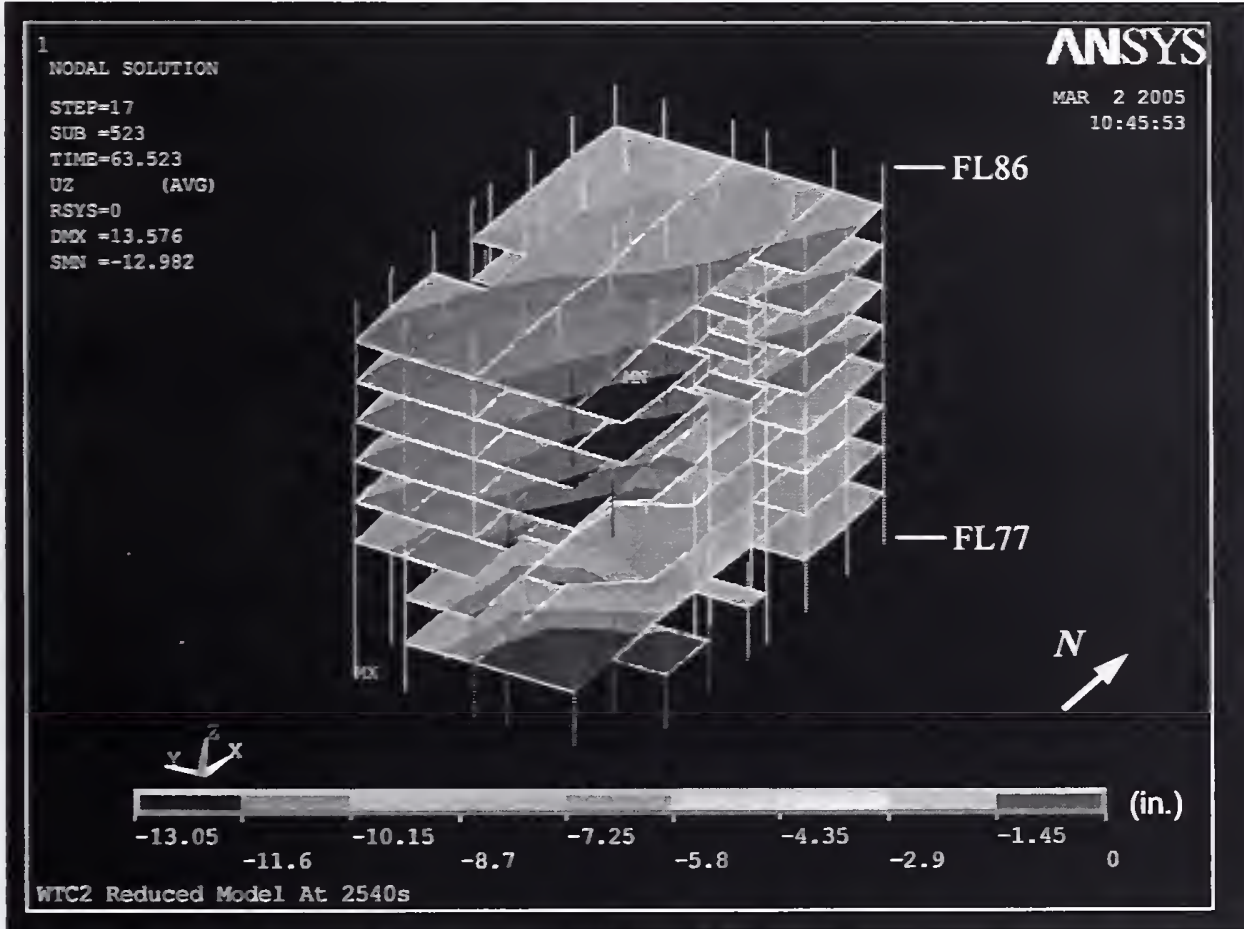

(b) $43 \mathrm{~min}$

Figure 4-92. Vertical displacement of core of WTC 2 for Case D conditions (downward displacement is negative). 


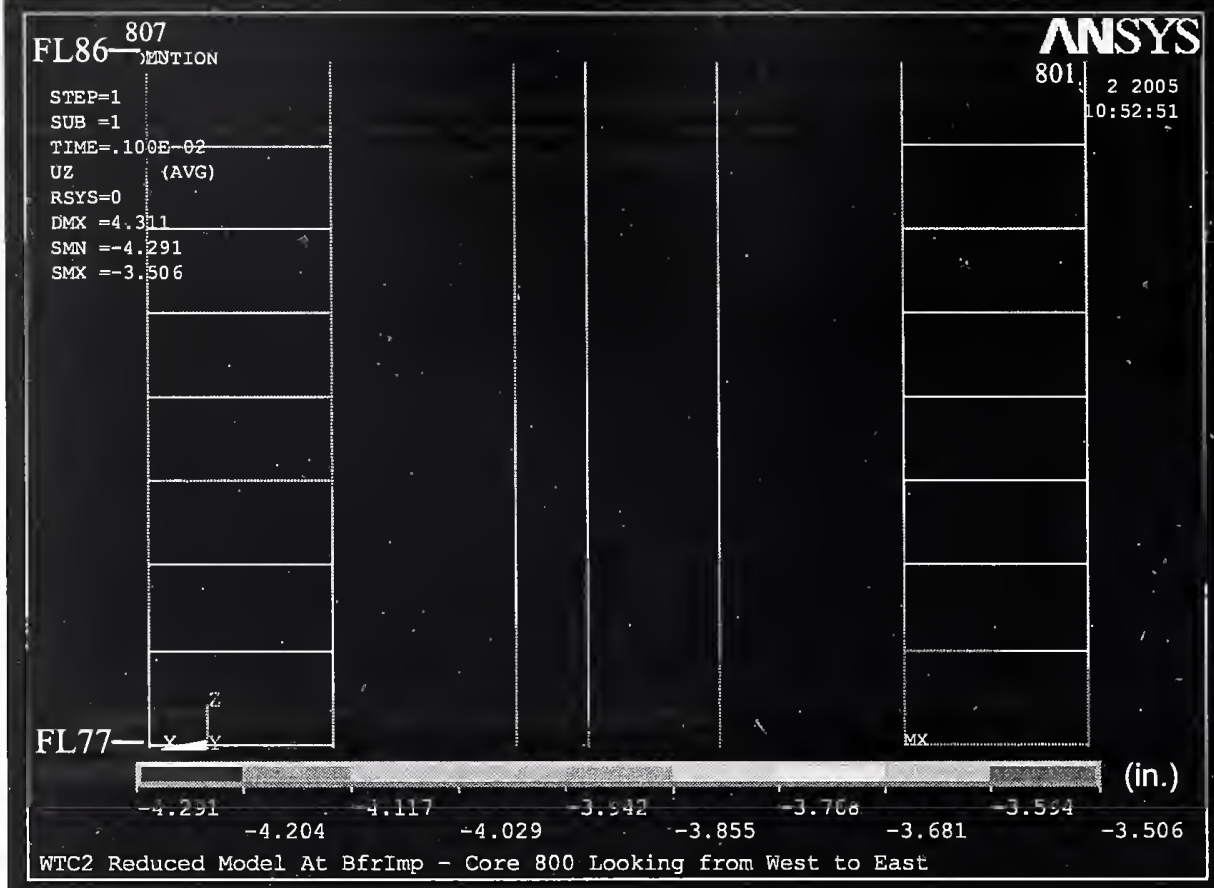

(a) Before impact

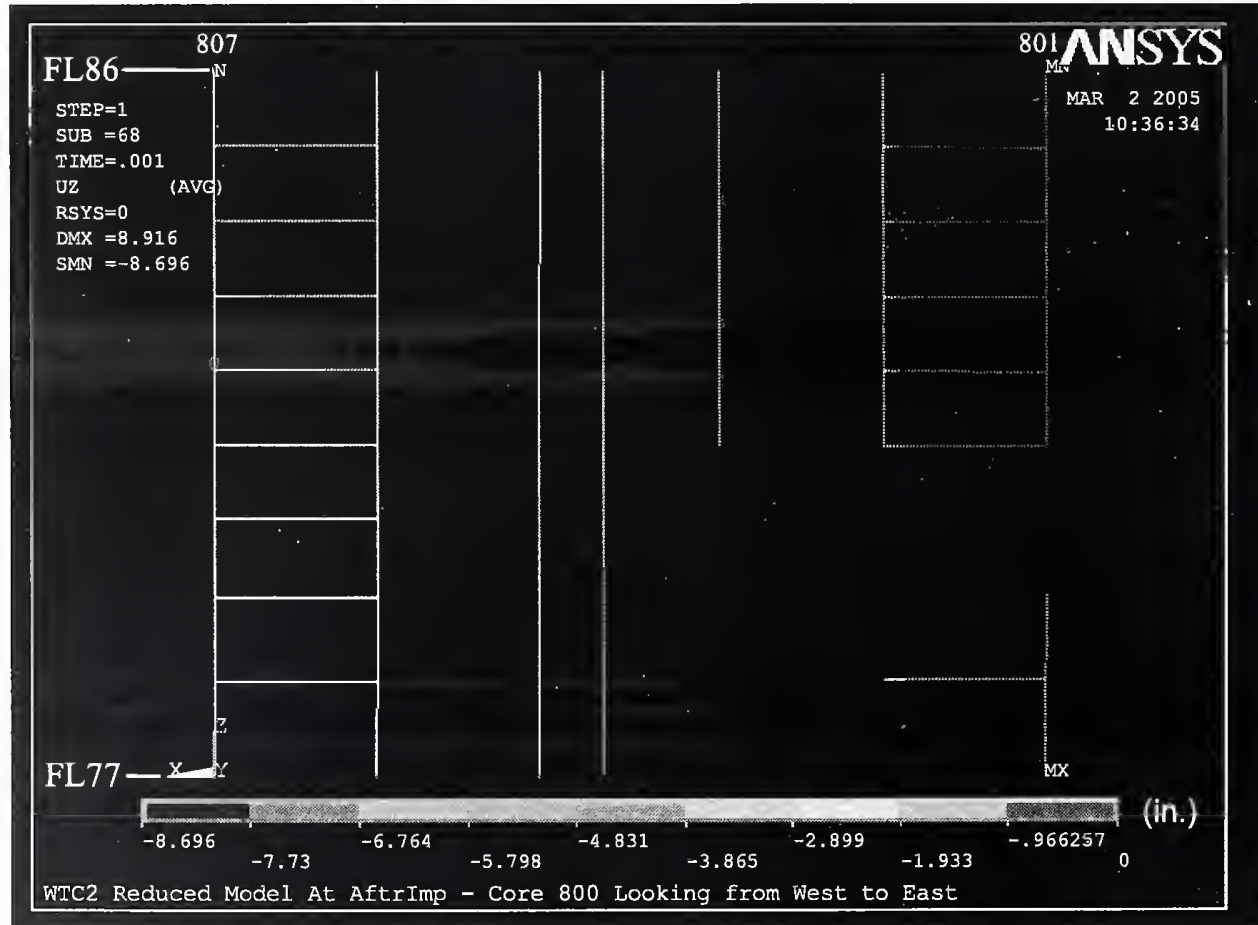

(b) After impact

Figure 4-93. Vertical displacement of 800 series core columns of WTC 2 for Case D conditions (downward displacement is negative). 


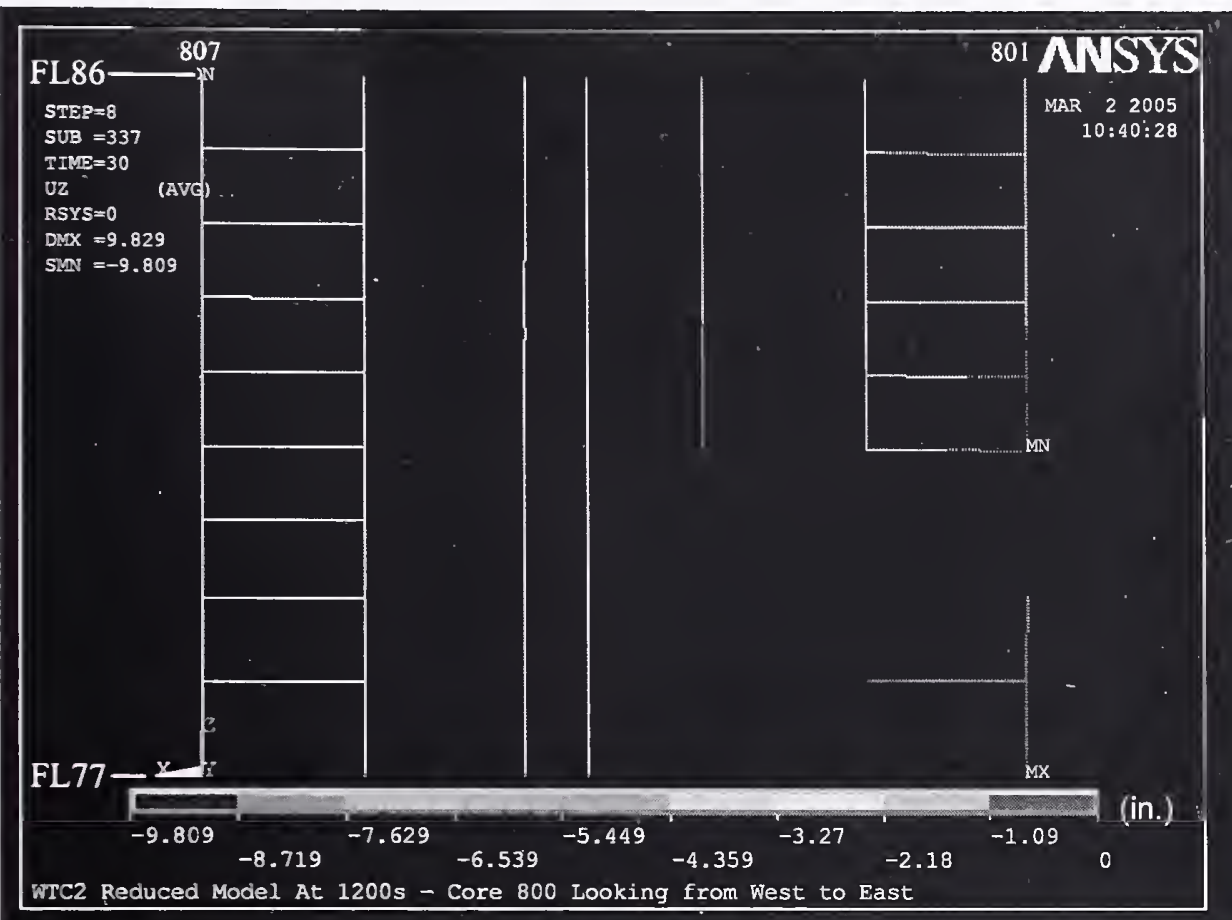

(a) $20 \mathrm{~min}$

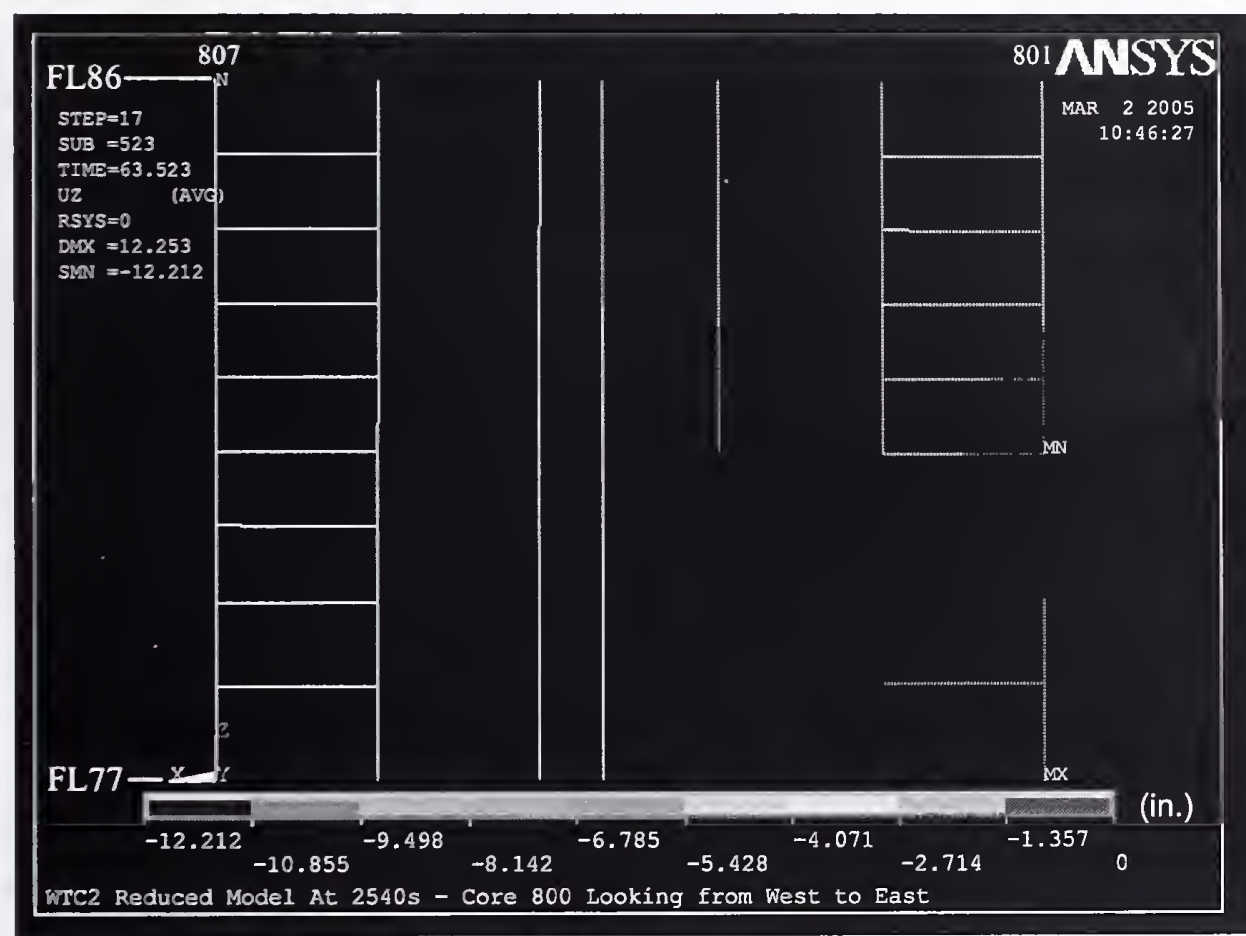

(b) $43 \mathrm{~min}$

Figure 4-94. Vertical displacement of 800 series core columns of WTC 2 for Case D conditions (downward displacement is negative). 


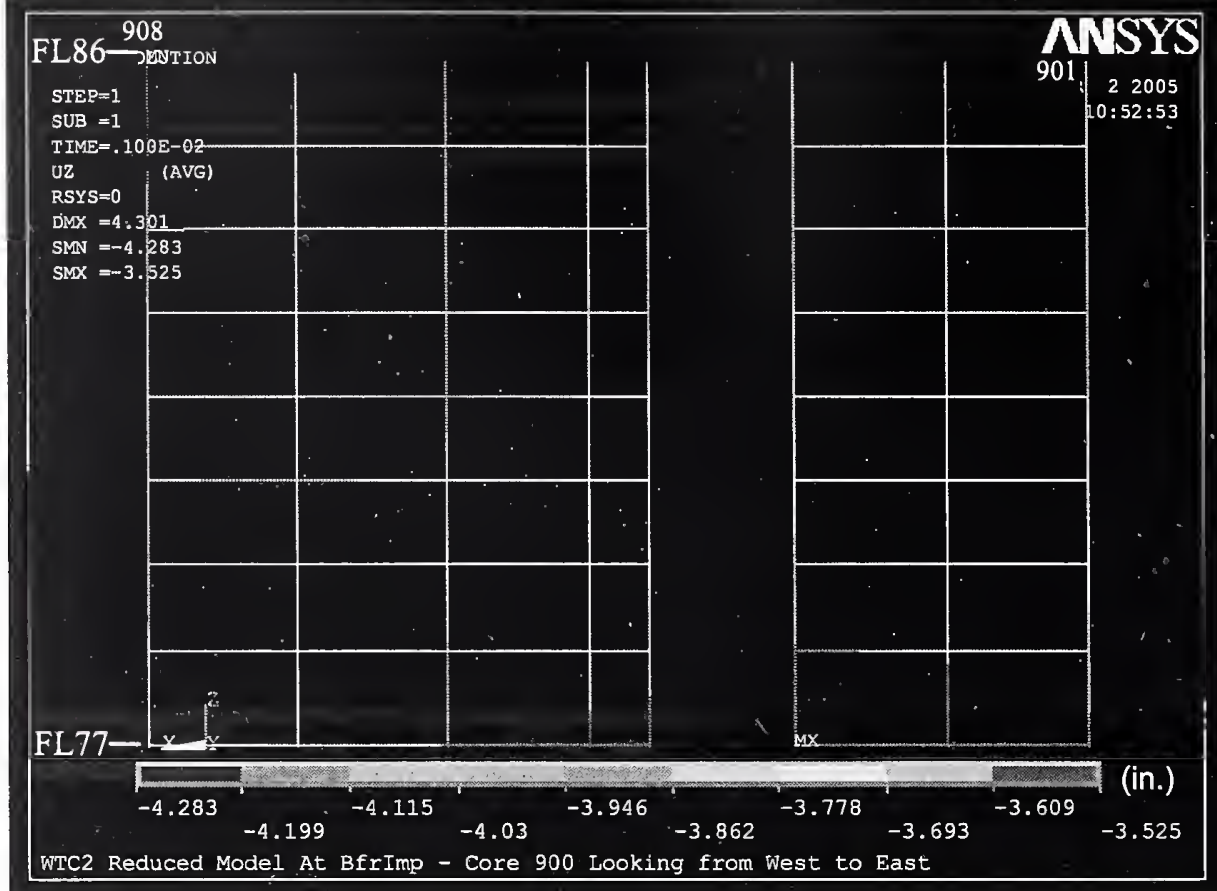

(a) Before impact

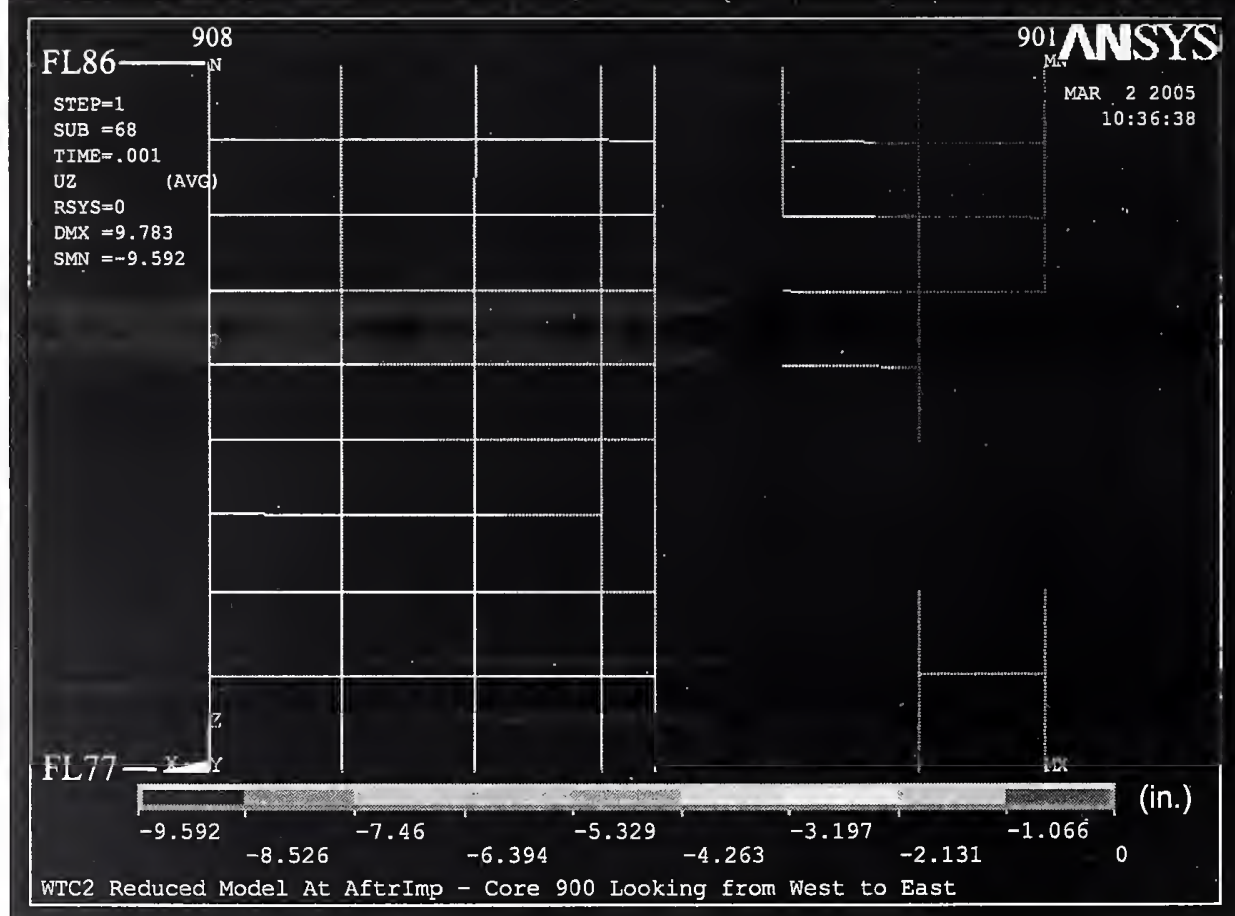

(b) After Impact

Figure 4-95. Vertical displacement of 900 series core columns of WTC 2 for Case D conditions (downward displacement is negative). 


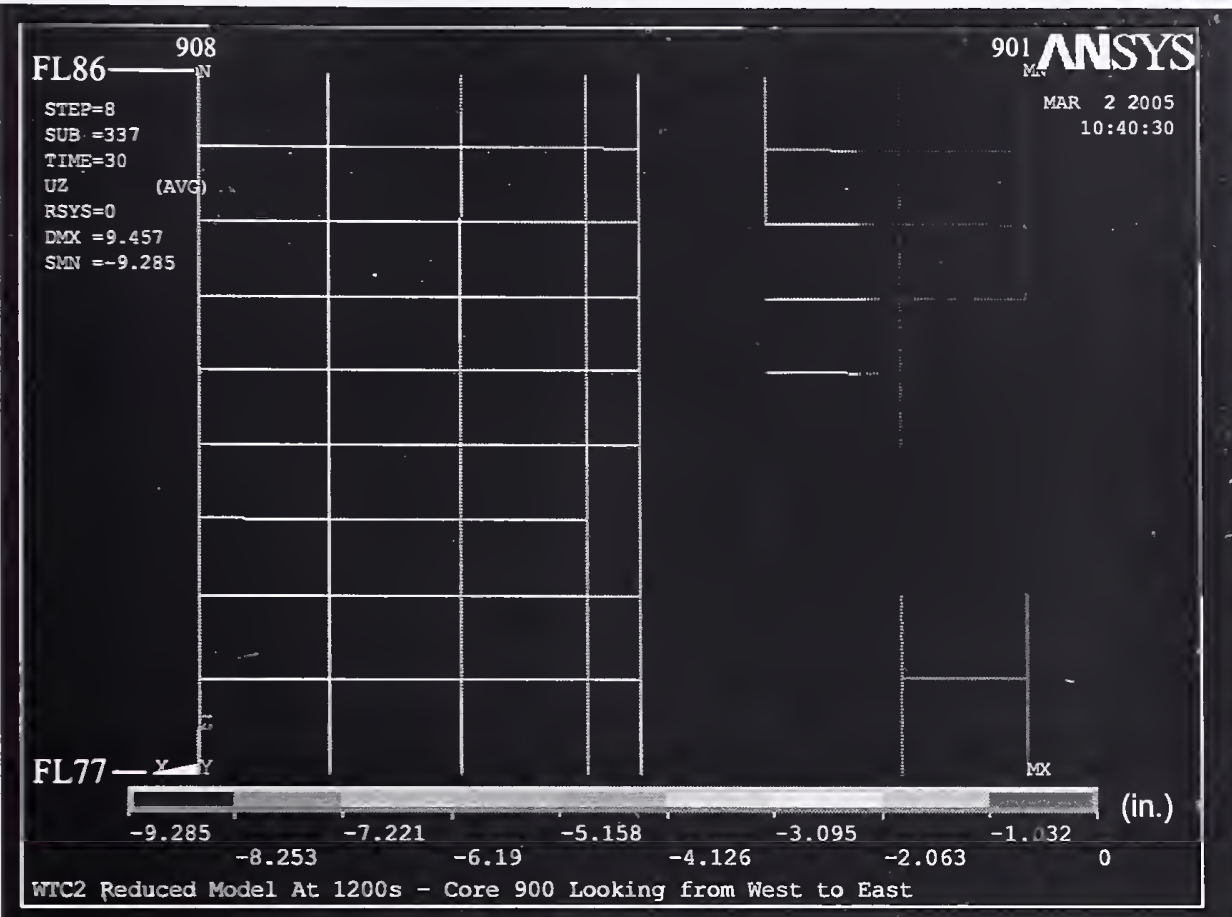

(a) $20 \mathrm{~min}$

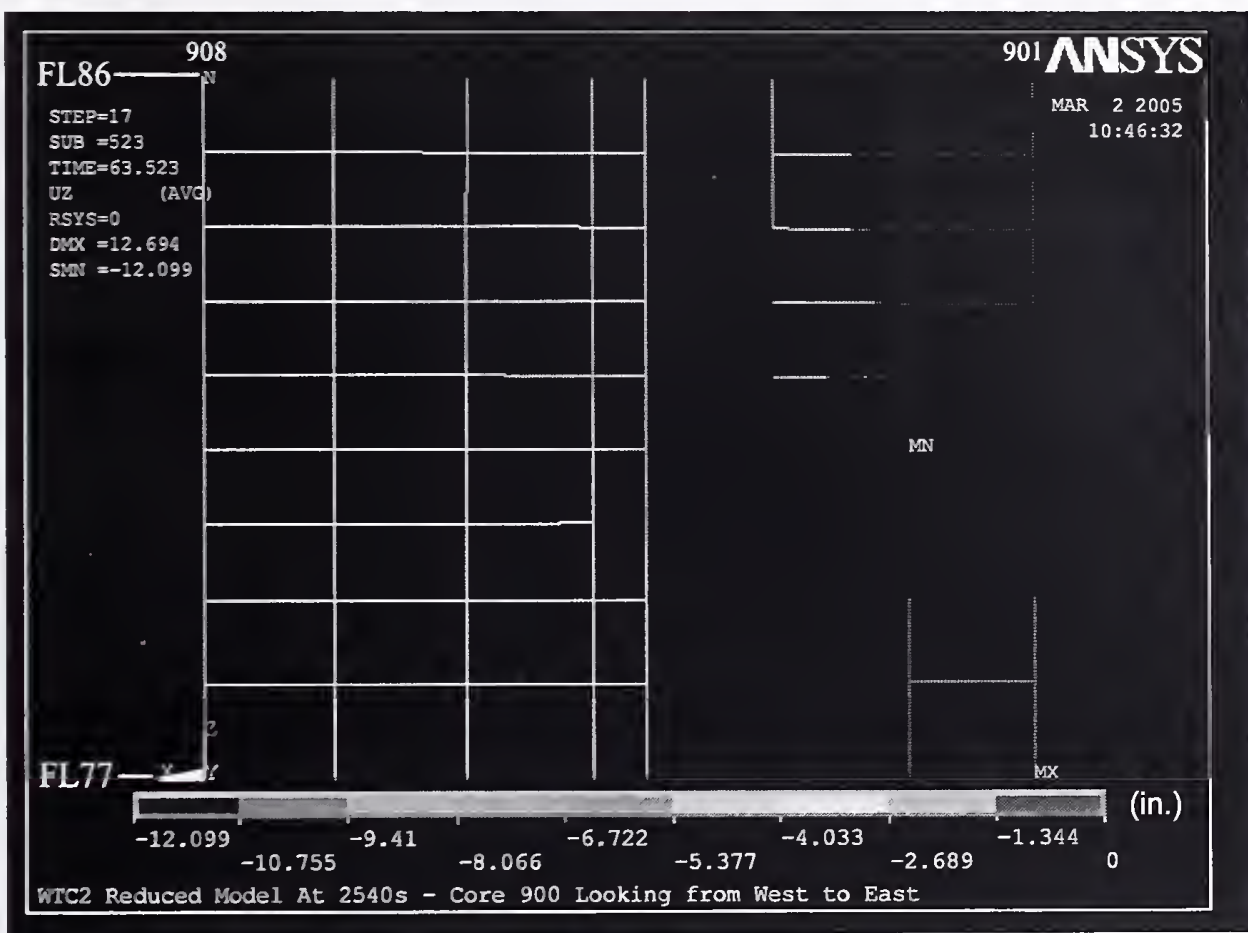

(b) $43 \mathrm{~min}$

Figure 4-96. Vertical displacement of 900 series core columns of WTC 2 for Case D conditions (downward displacement is negative). 


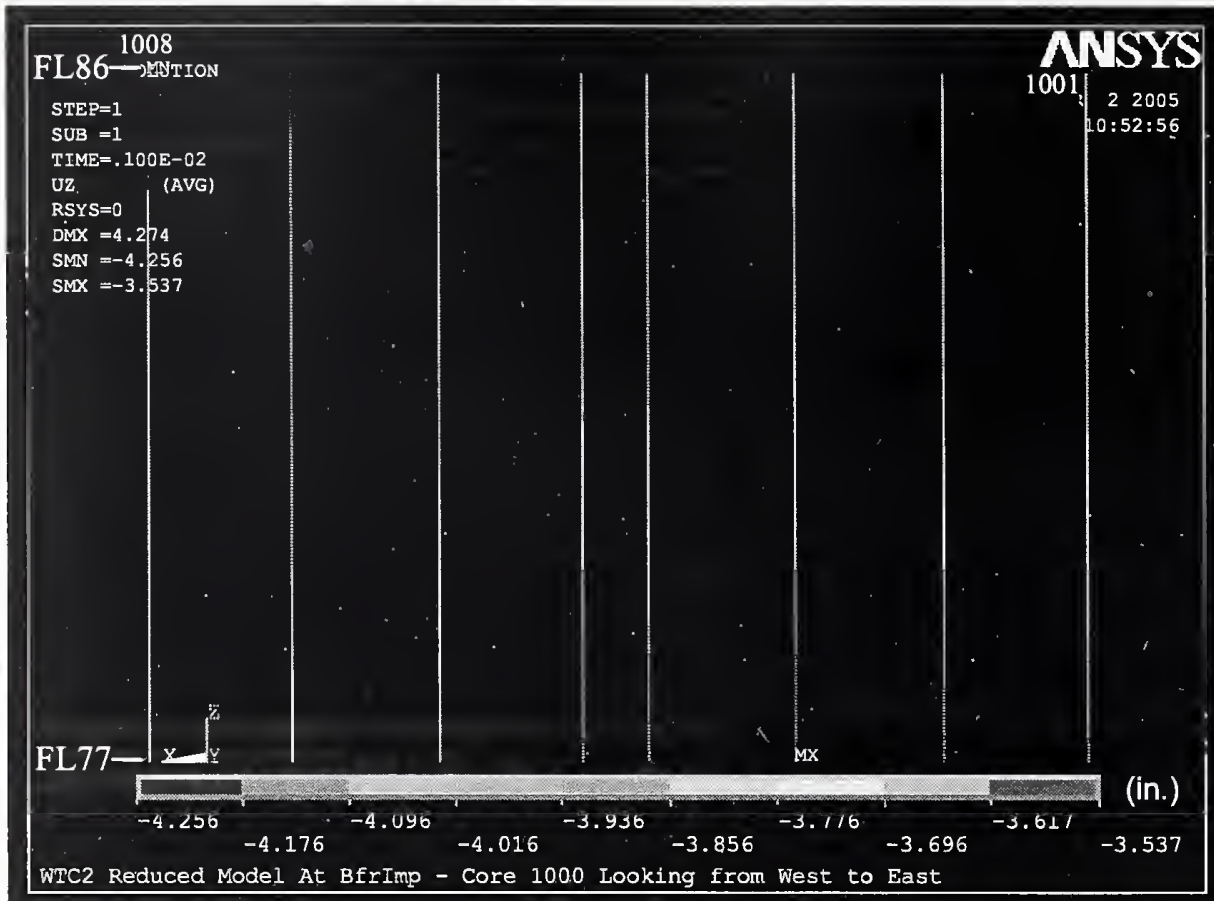

(a) Before impact

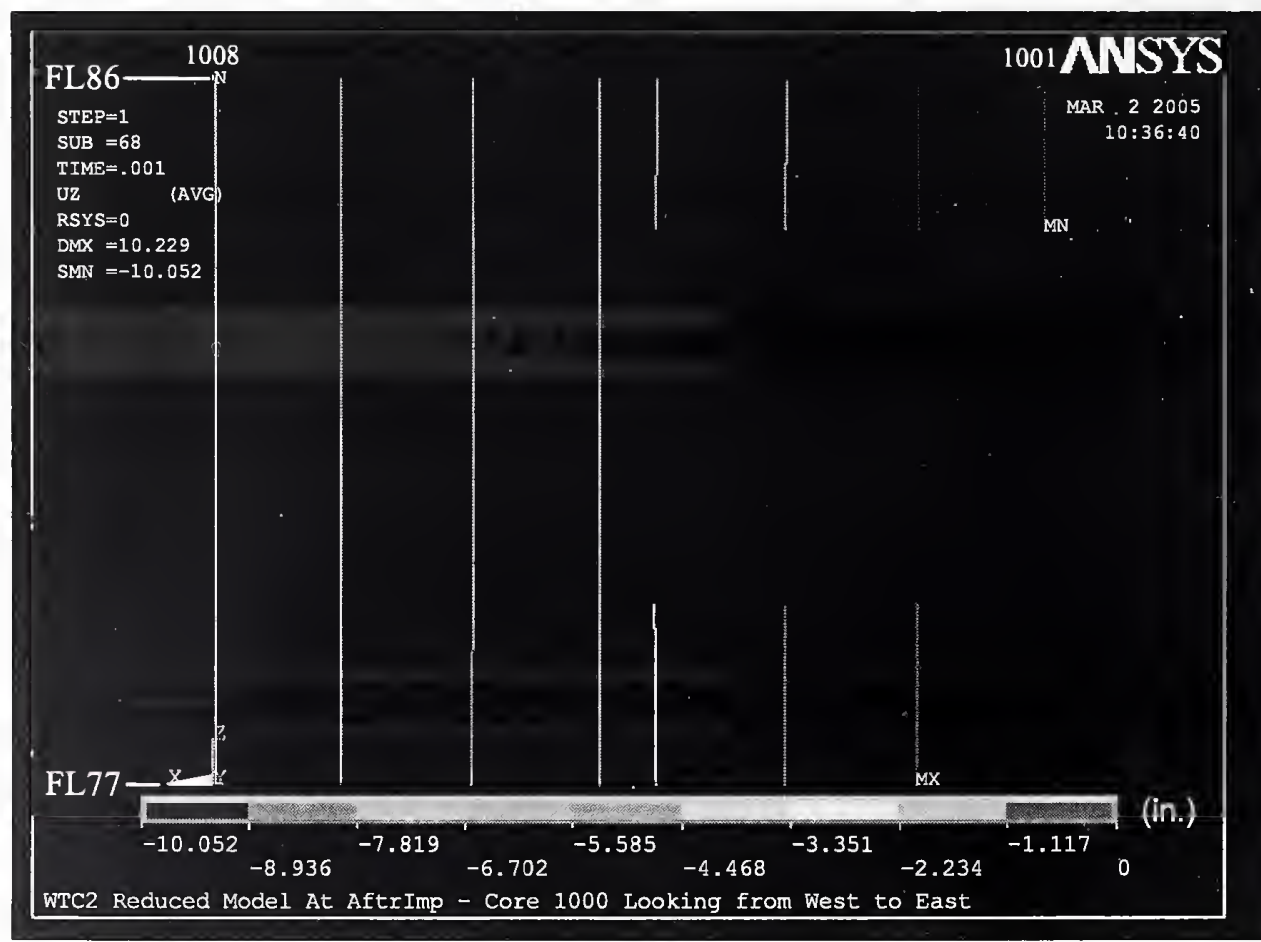

(b) After impact

Figure 4-97. Vertical displacement of 1000 series core columns of WTC 2 for Case D conditions (downward displacement is negative). 


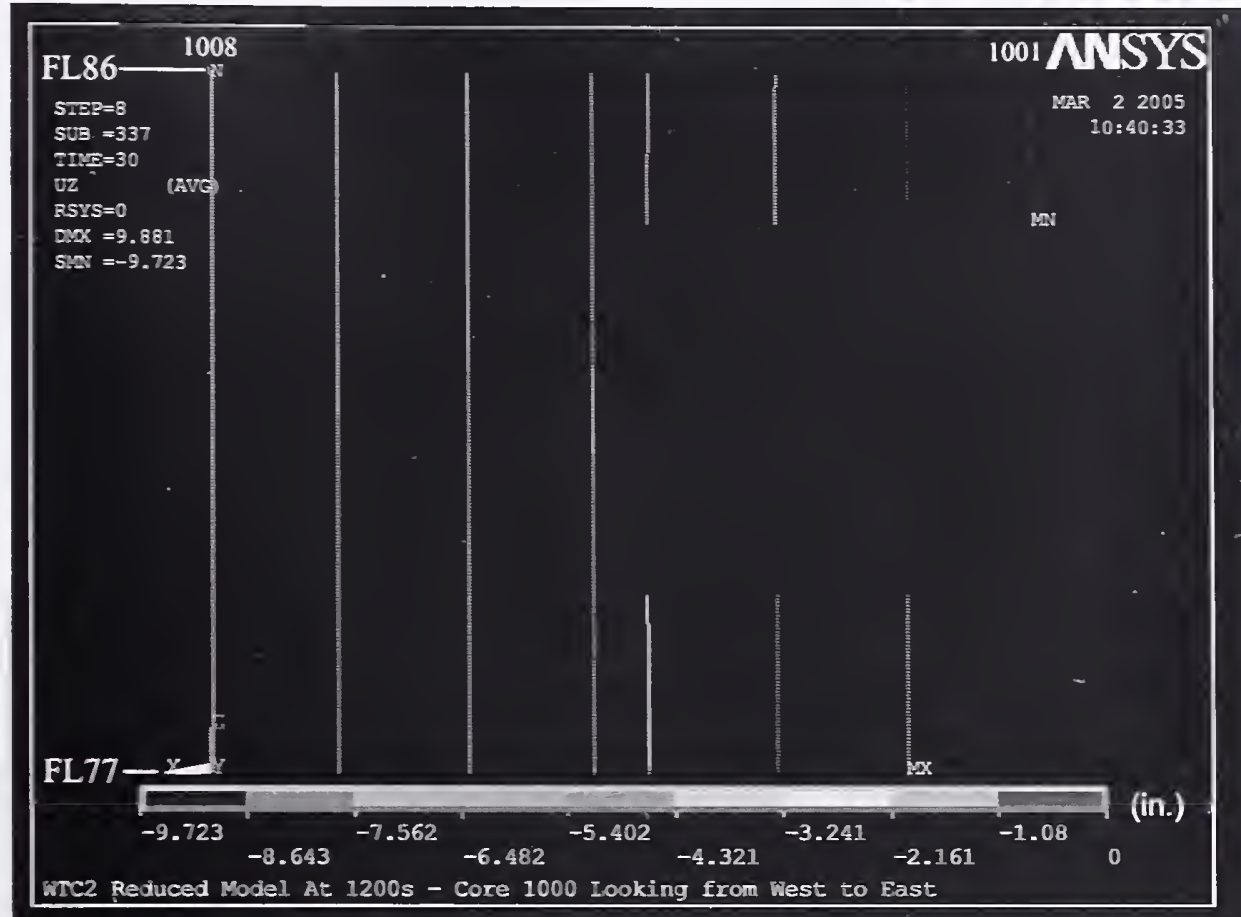

(a) $20 \mathrm{~min}$

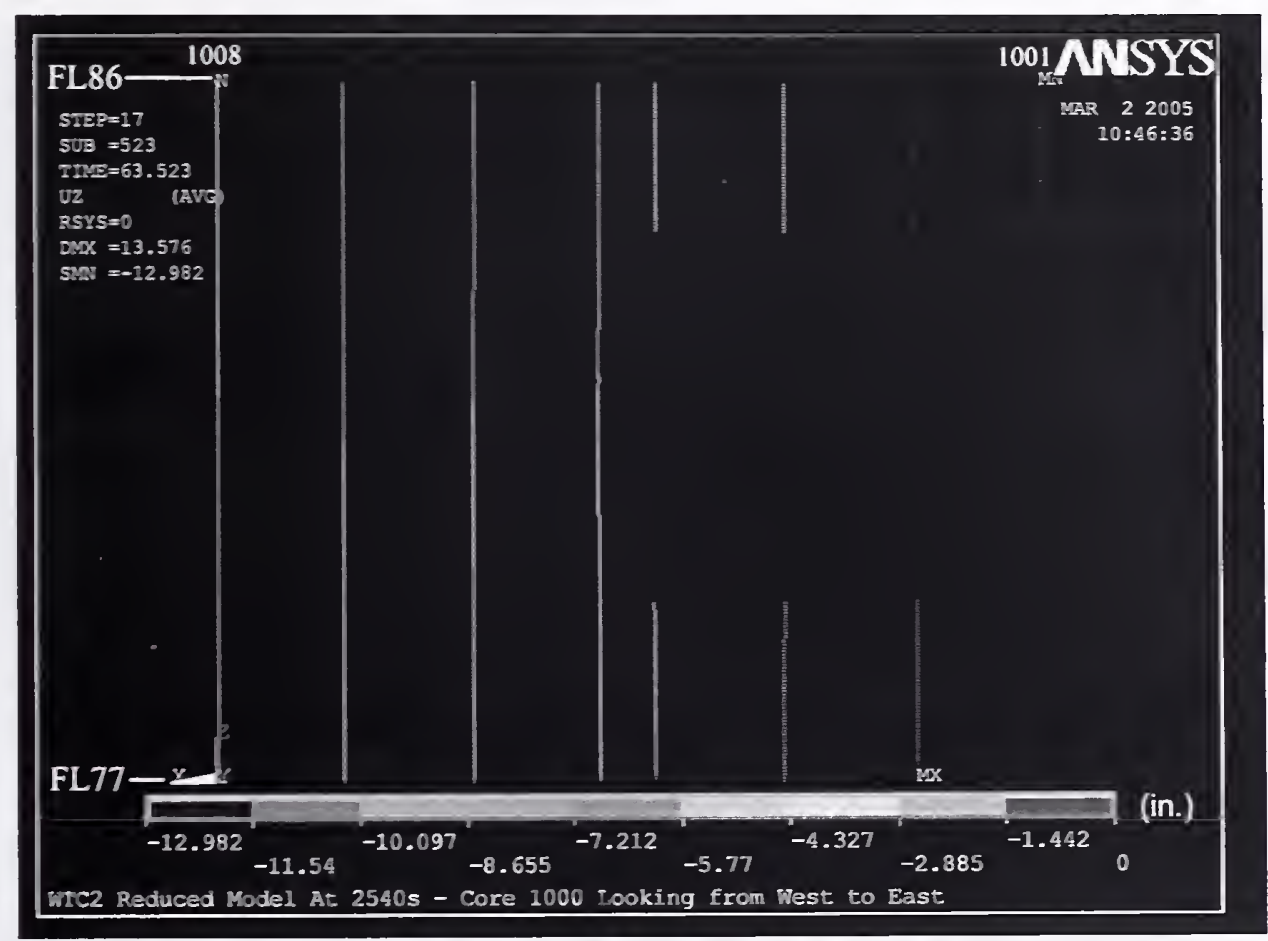

(b) $43 \mathrm{~min}$

Figure 4-98. Vertical displacement of 1000 series core columns of WTC 2 for Case D conditions (downward displacement is negative). 


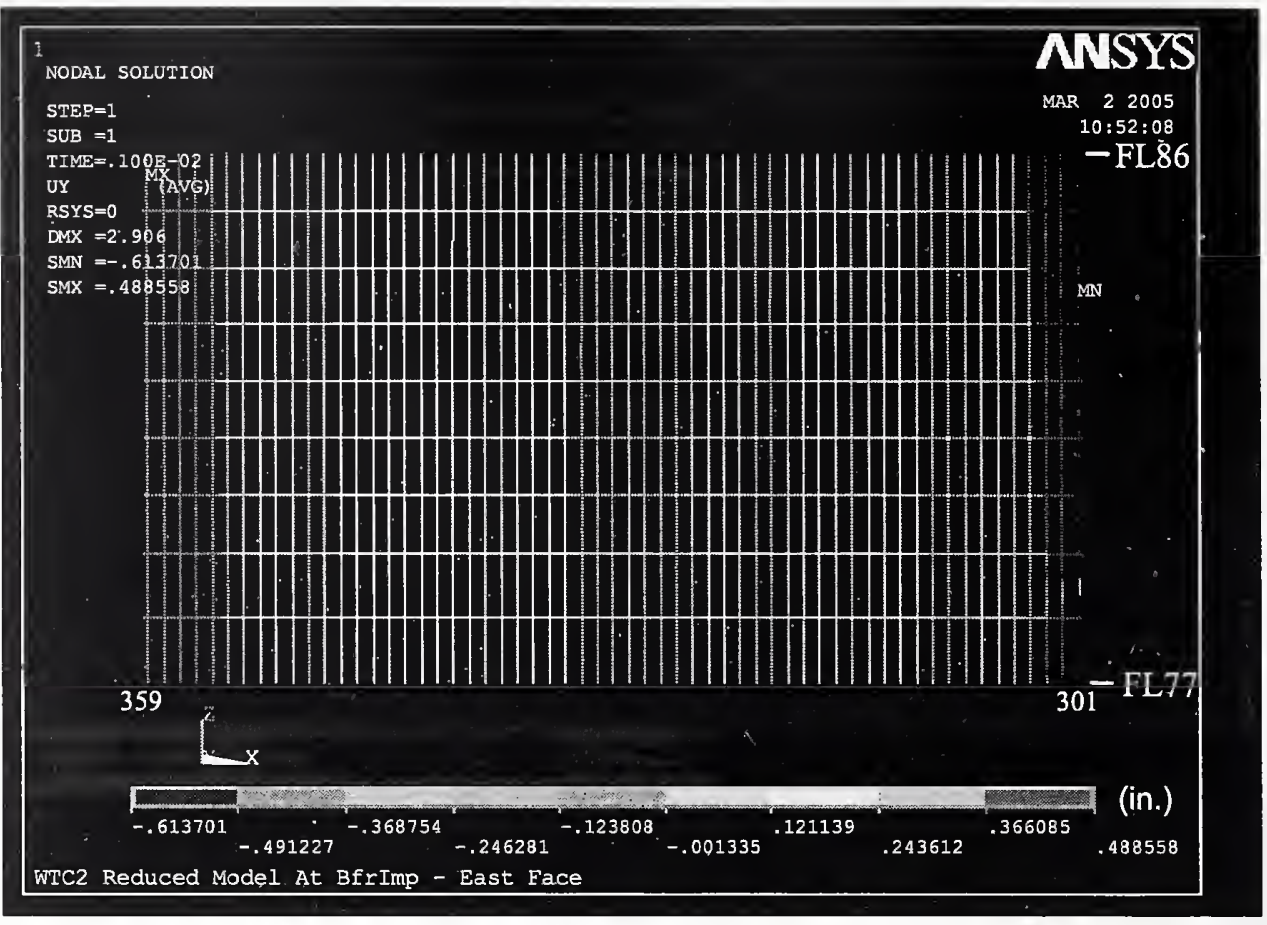

(a) Before impact

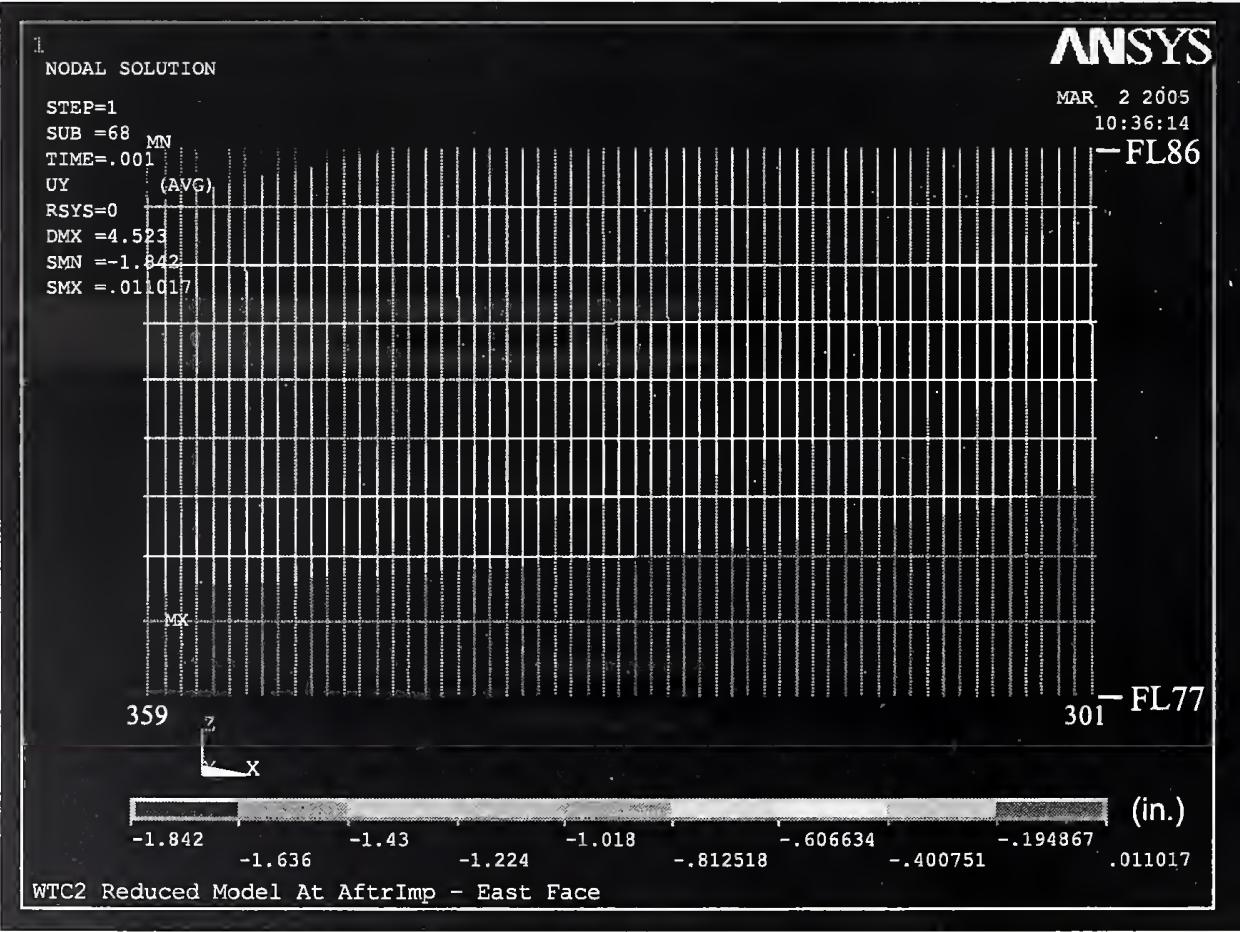

(b) After impact

Figure 4-99. Out-of-plane displacement of the east wall of WTC 2 for Case D conditions (inward displacement is positive). 


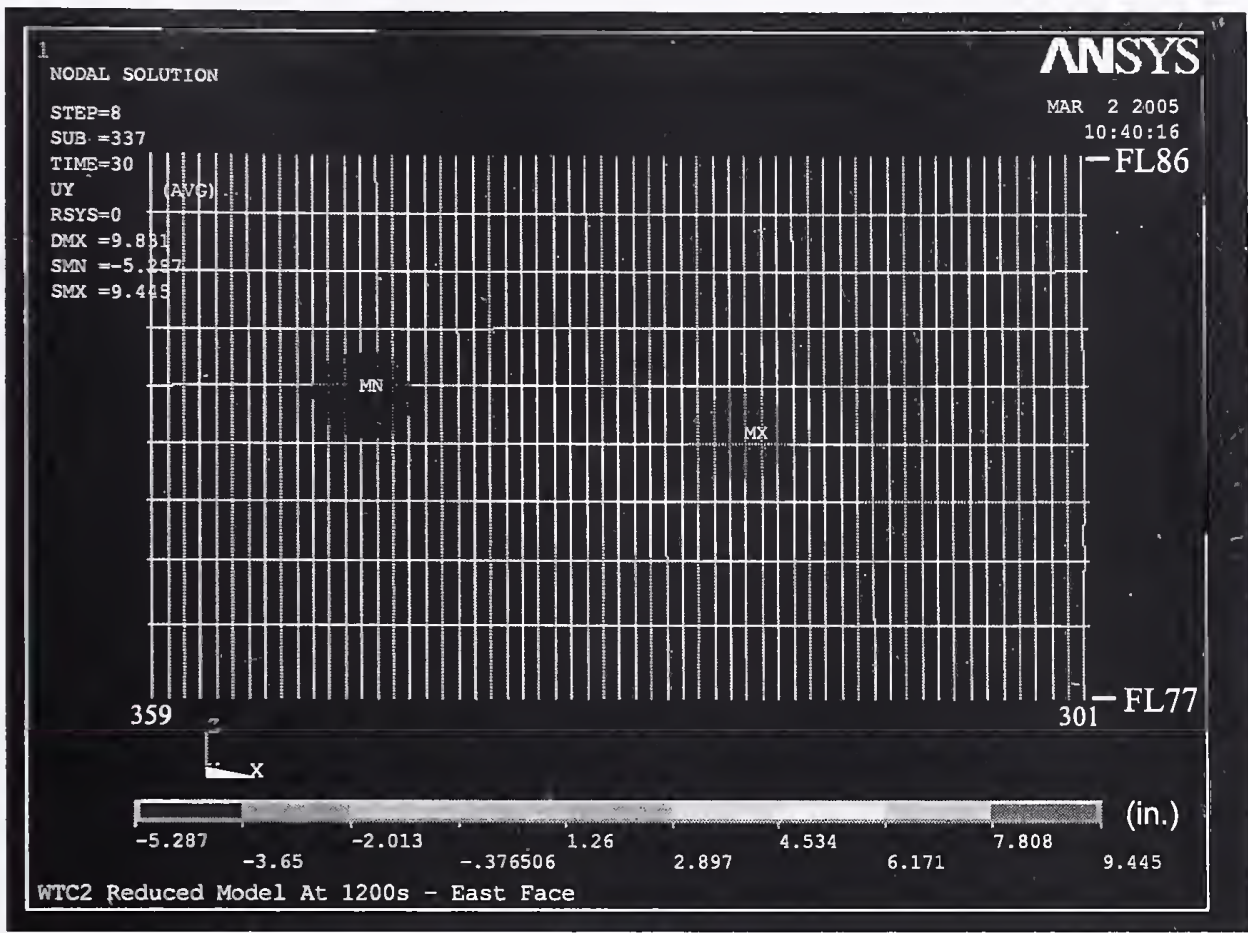

(a) $20 \mathrm{~min}$

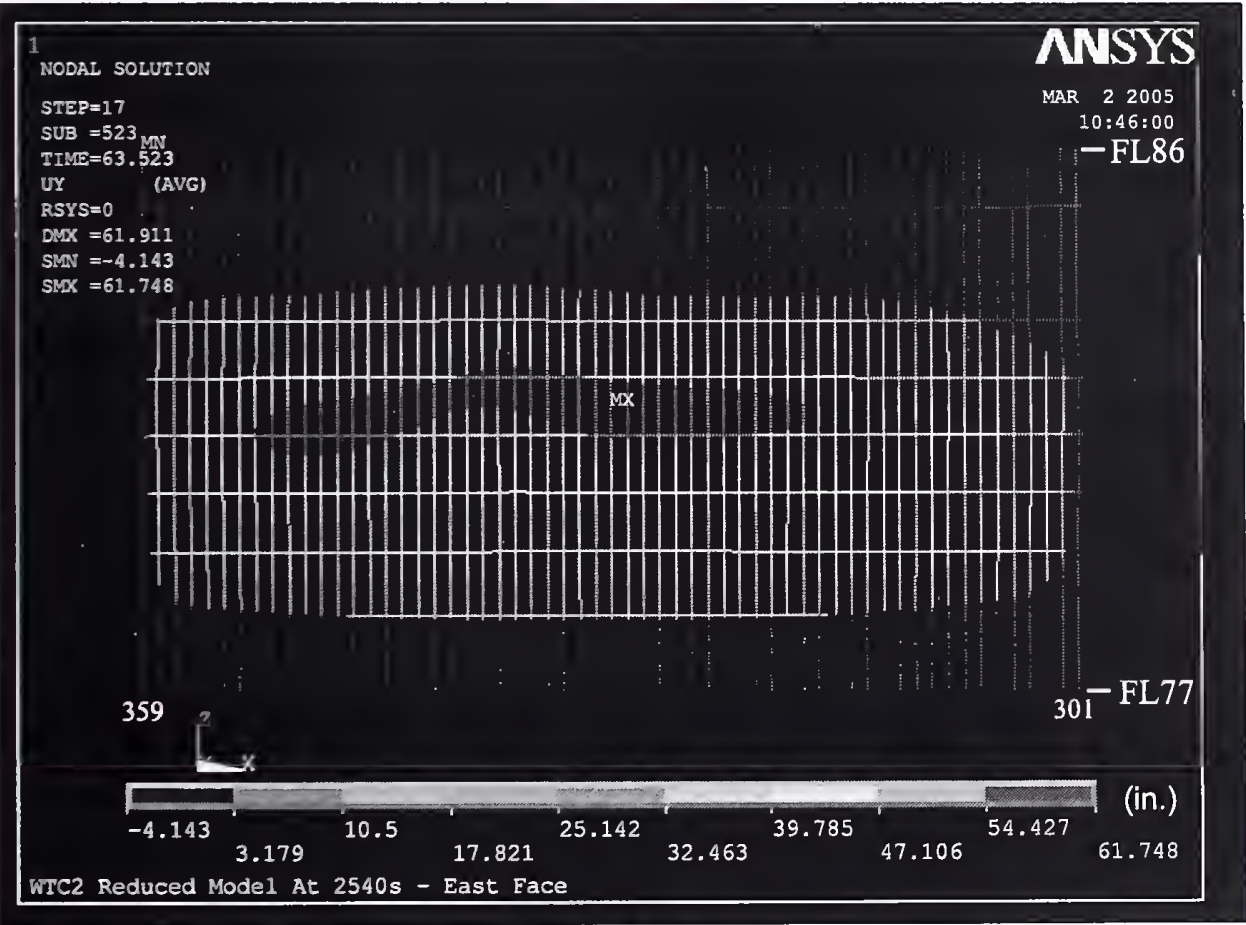

(b) $43 \mathrm{~min}$

Figure 4-100. Out-of-plane displacement of the east wall of WTC 2 for Case D conditions (inward displacement is positive). 


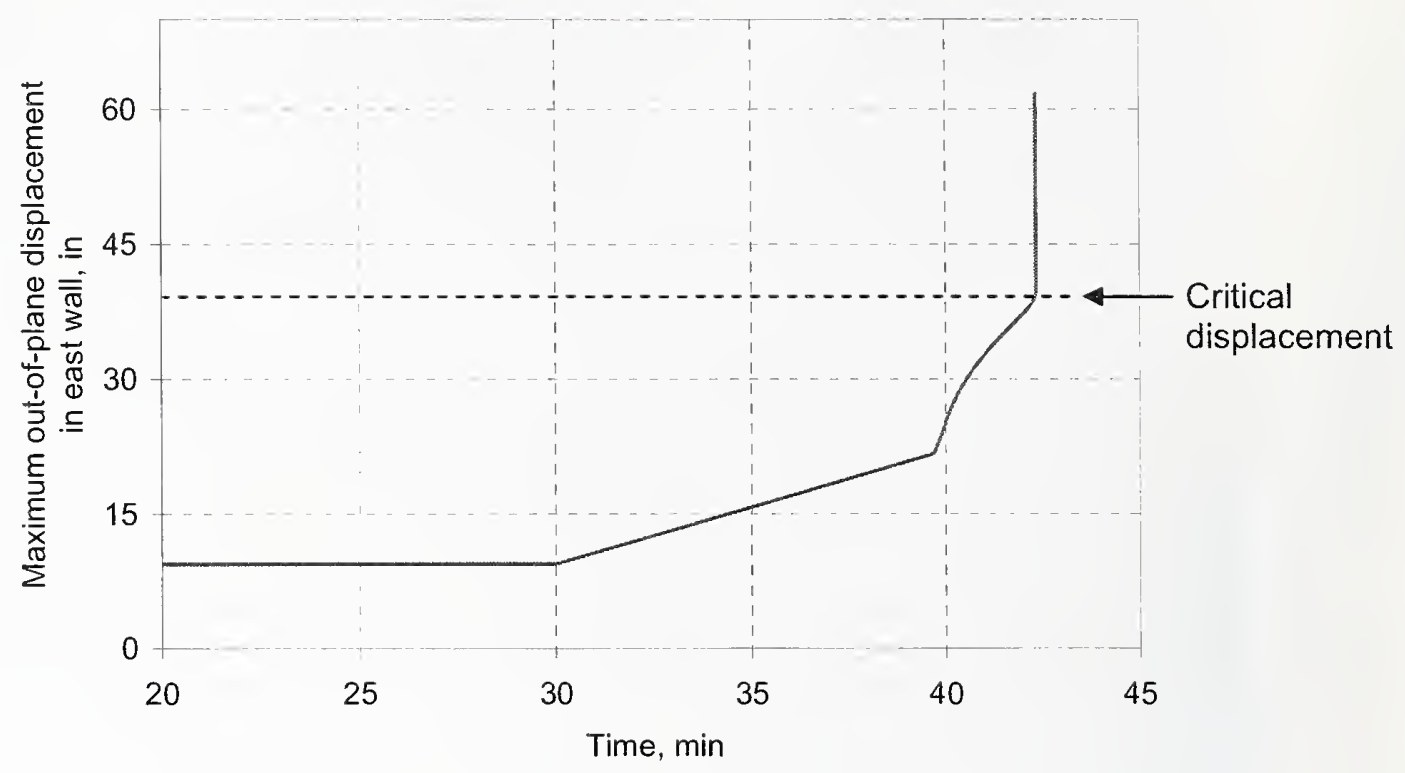

Figure 4-101. Variation of maximum inward bowing of the east wall of WTC 2 over time for Case D conditions. 


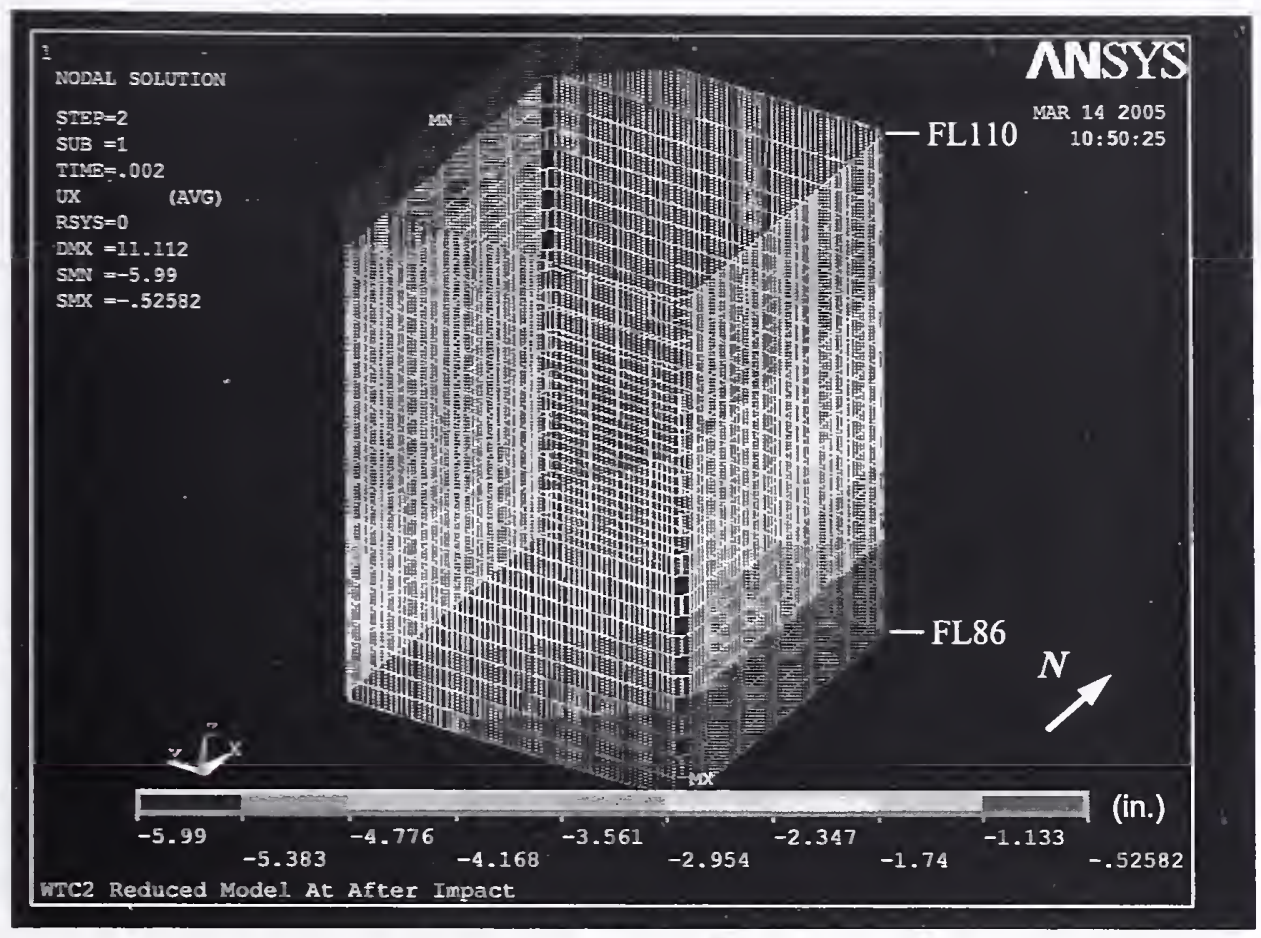

(a) After impact

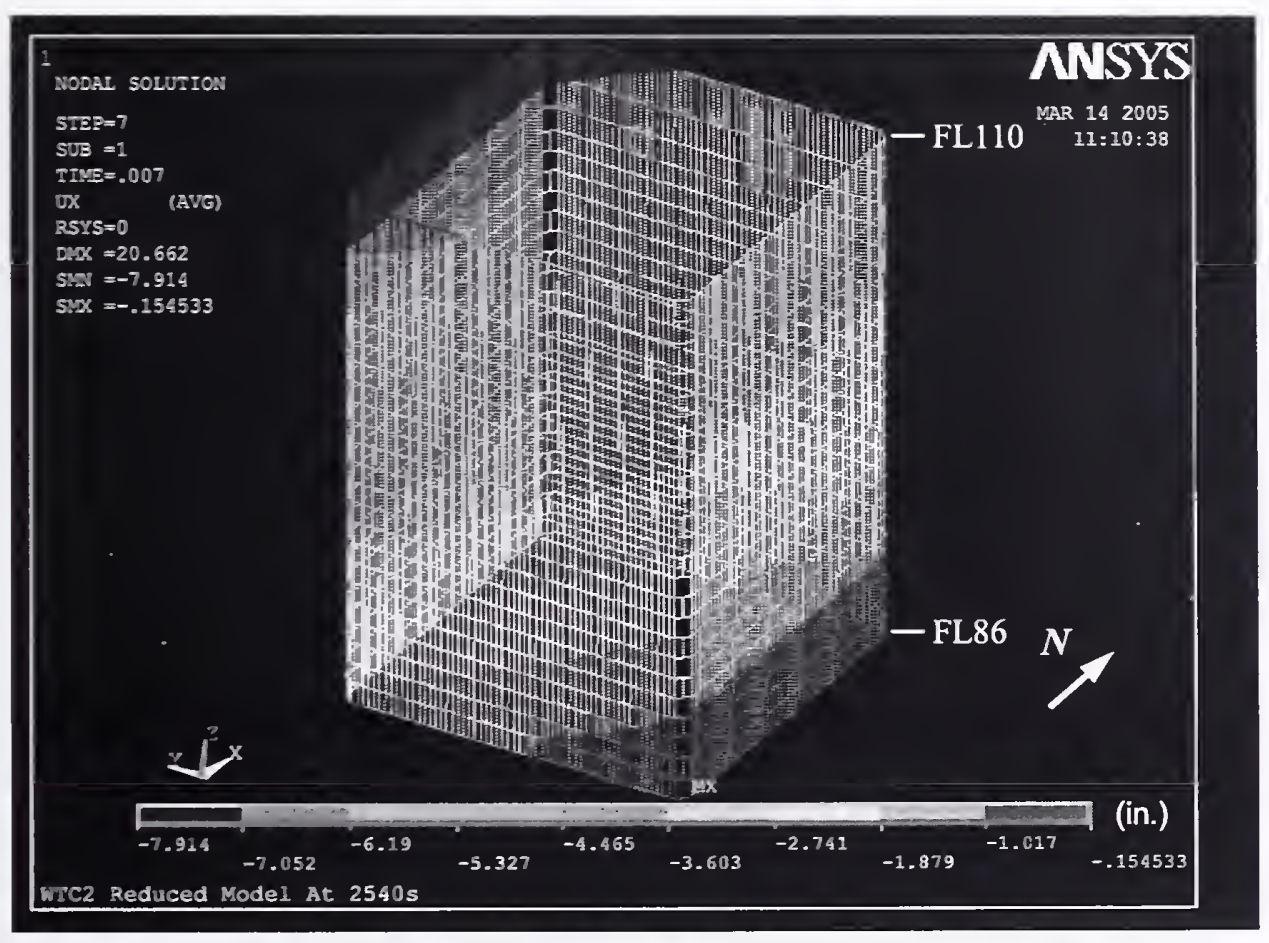

(b) $43 \mathrm{~min}$

Figure 4-102. Lateral displacements above Floor 86 of WTC 2 in the x-direction (northsouth) for Case D conditions. 


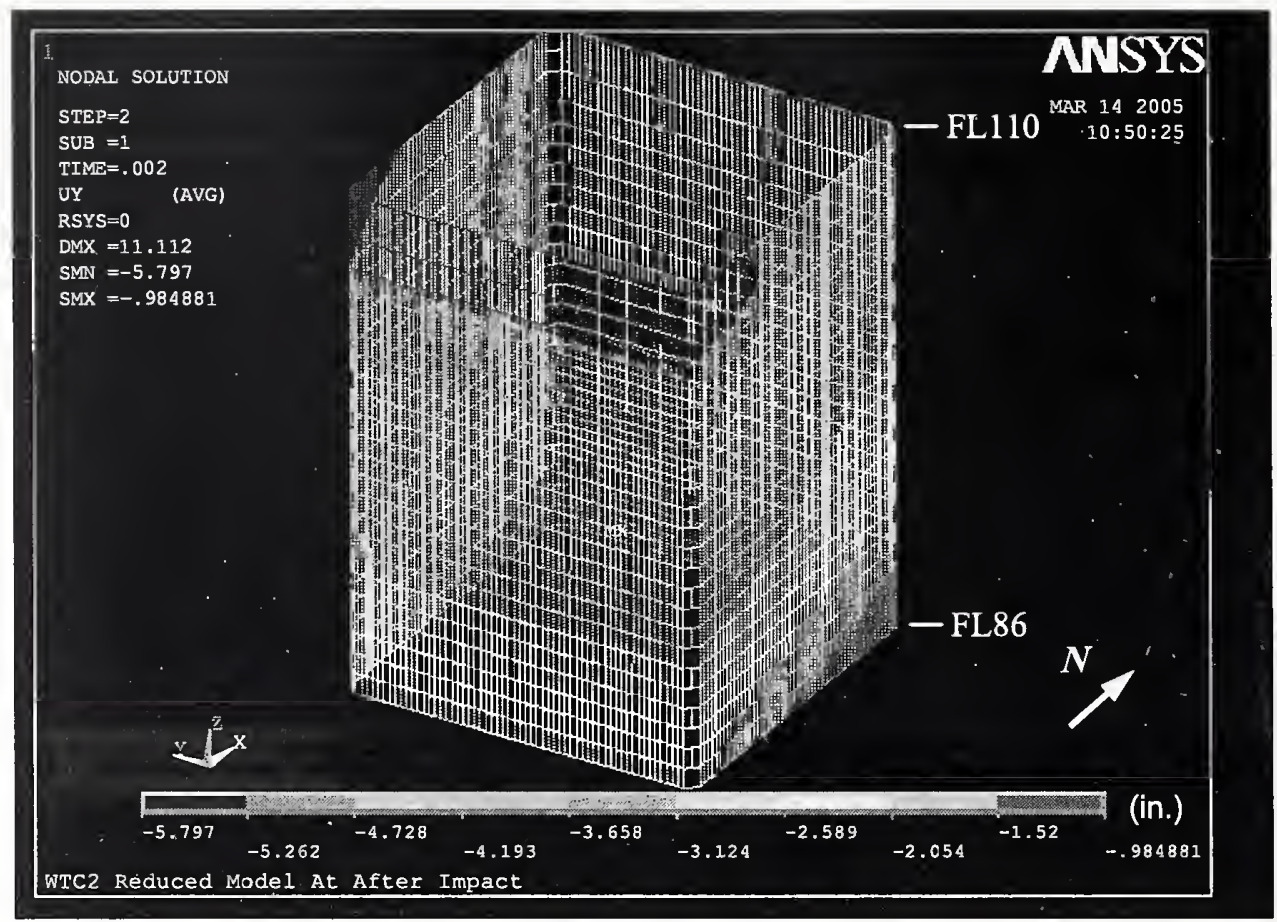

(a) After impact

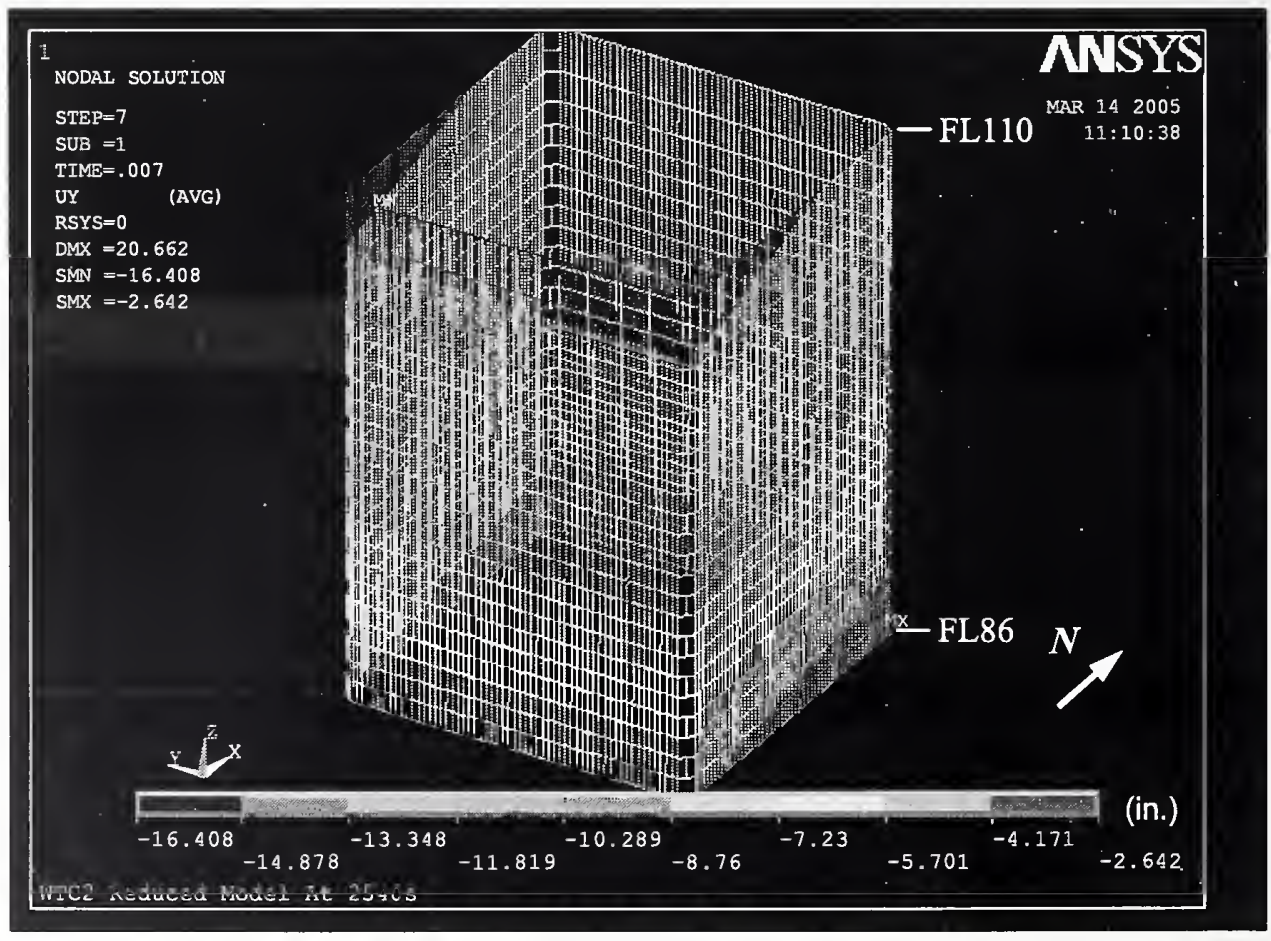

(b) $43 \mathrm{~min}$

Figure 4-103. Lateral displacements above Floor 86 of WTC 2 in the $y$-direction (eastwest) for Case D conditions. 


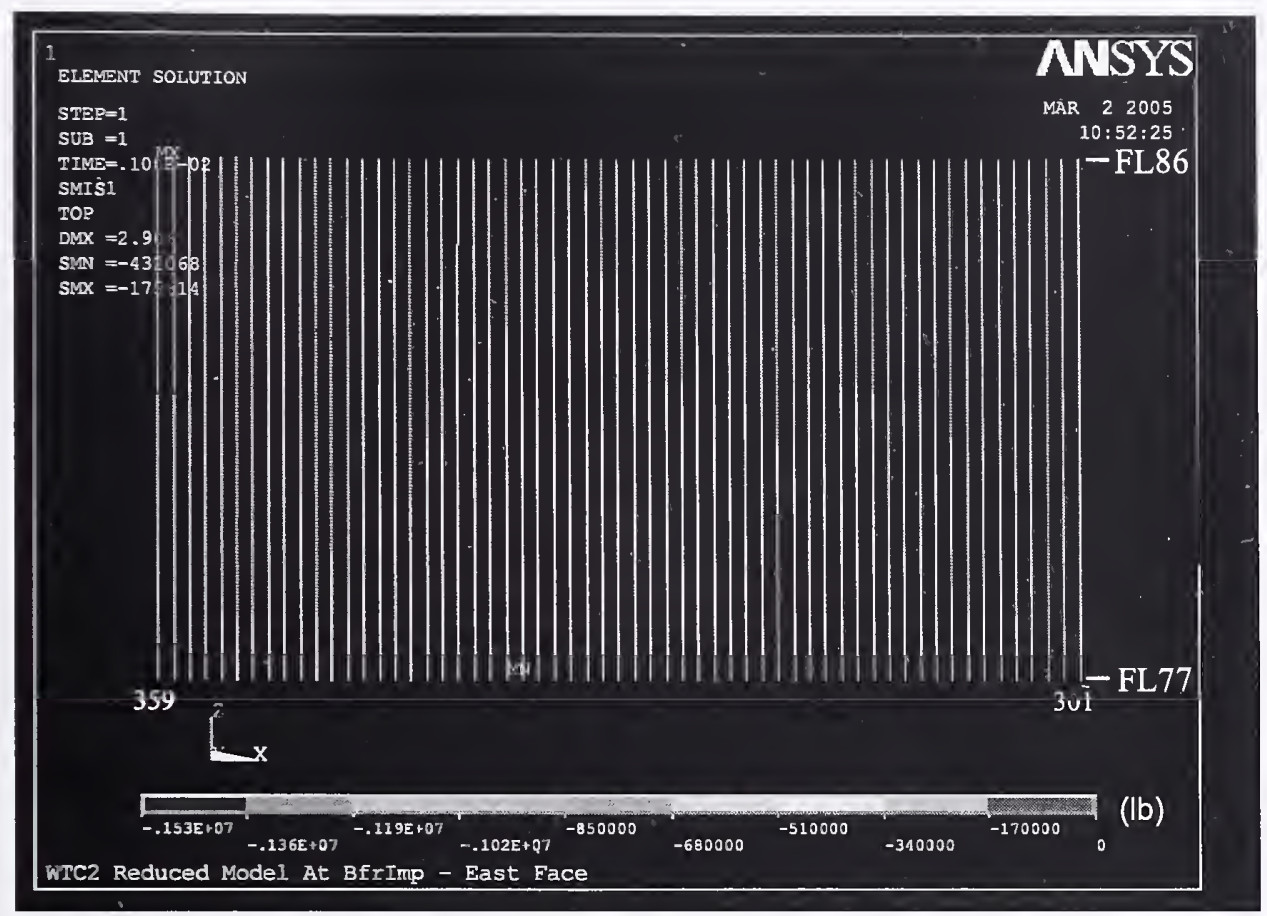

(a) Before impact

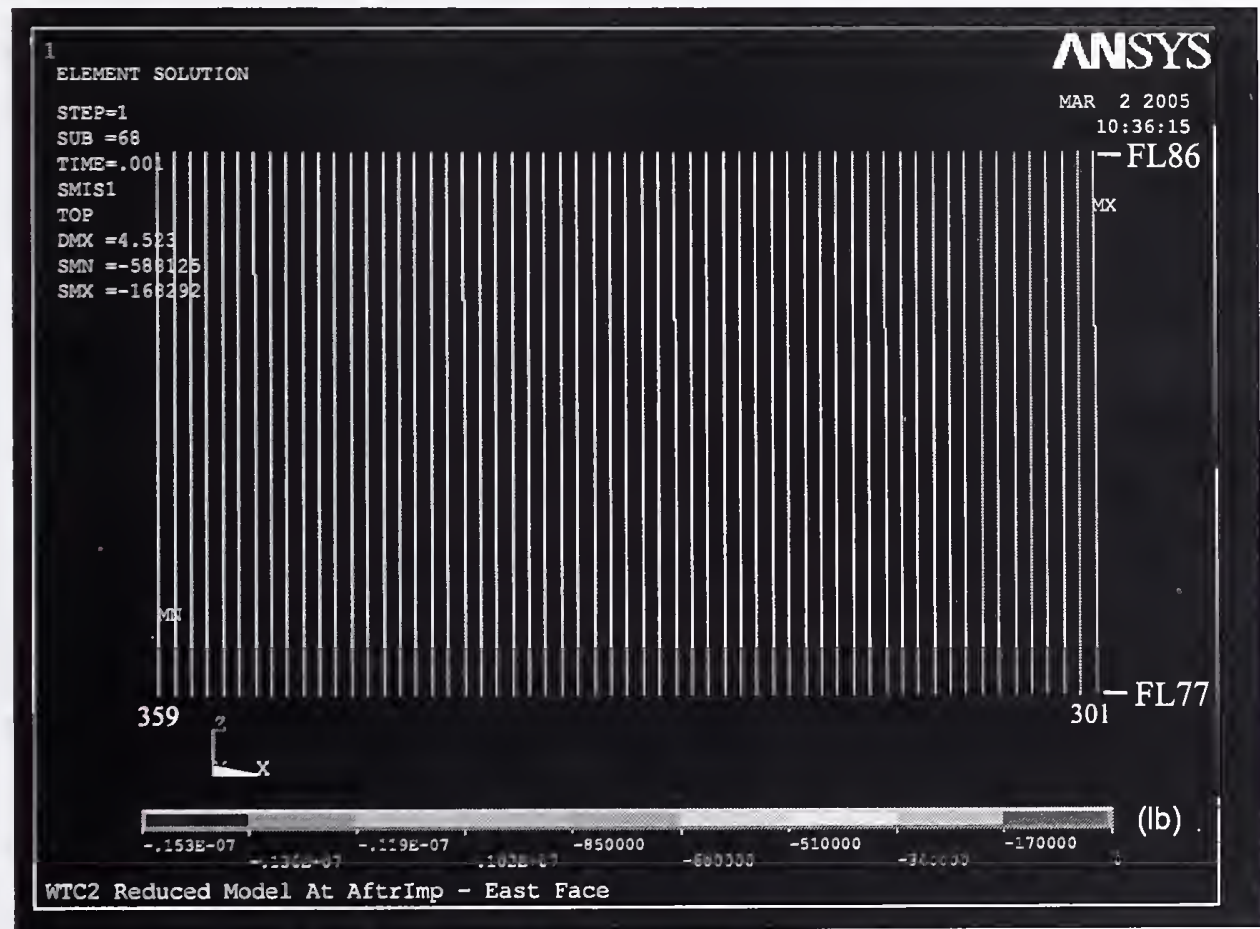

(b) After impact

Figure 4-104. Axial load in the east wall of WTC 2 for Case D conditions (compression is negative). 


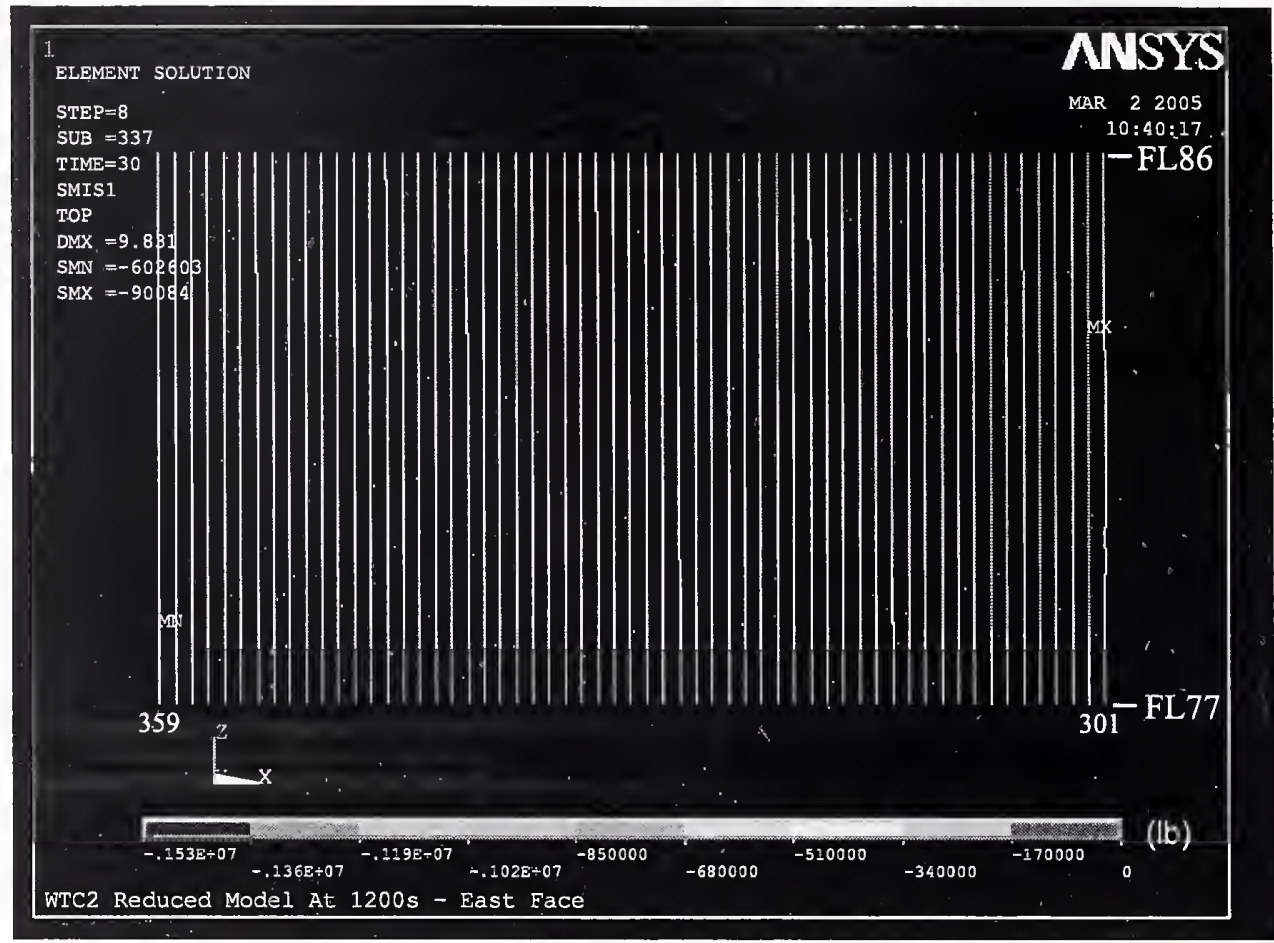

(a) $20 \mathrm{~min}$

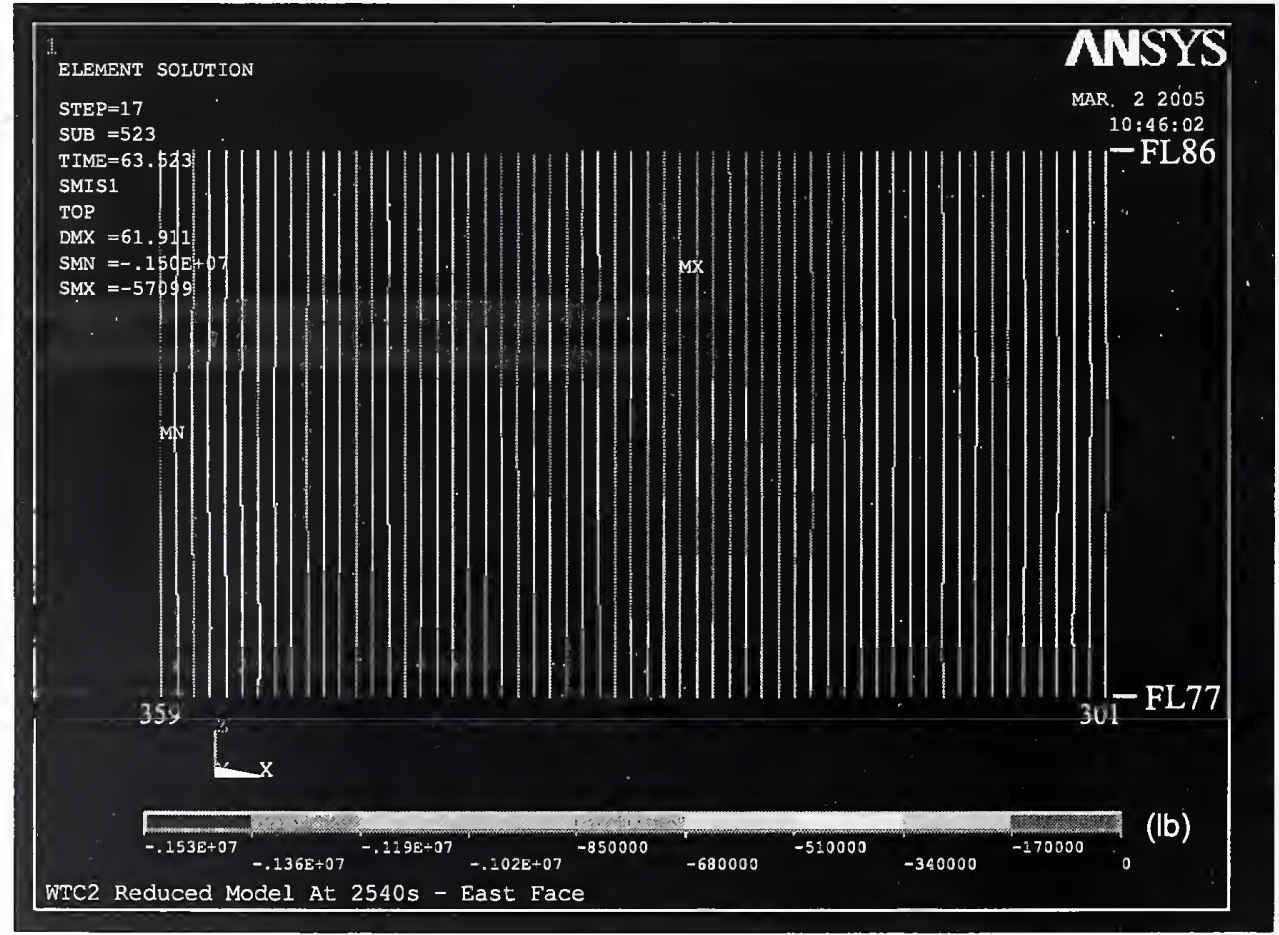

(b) $43 \mathrm{~min}$

Figure 4-105. Axial load in the east wall of WTC 2 for Case D conditions (compression is negative). 


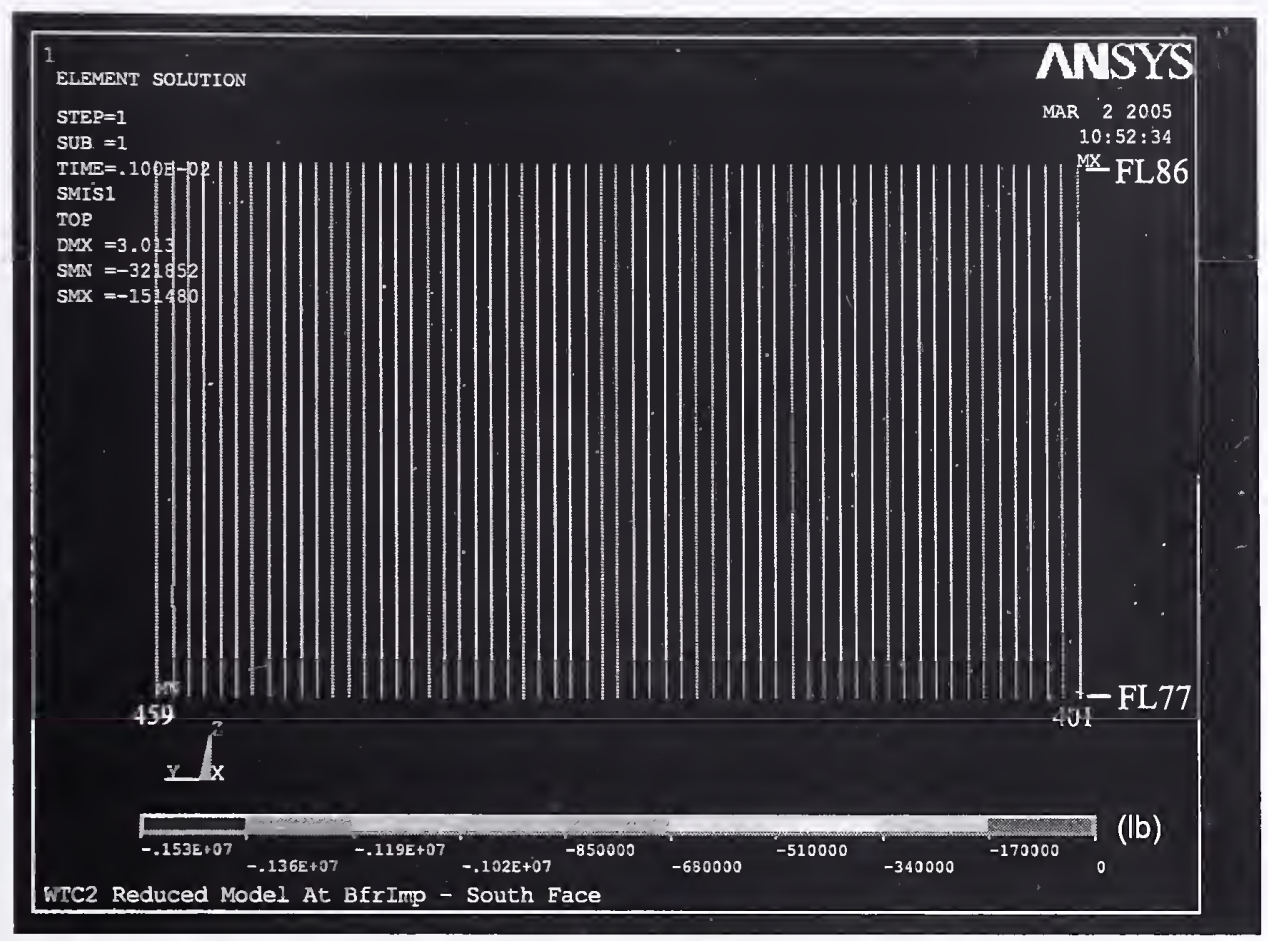

(a) Before impact

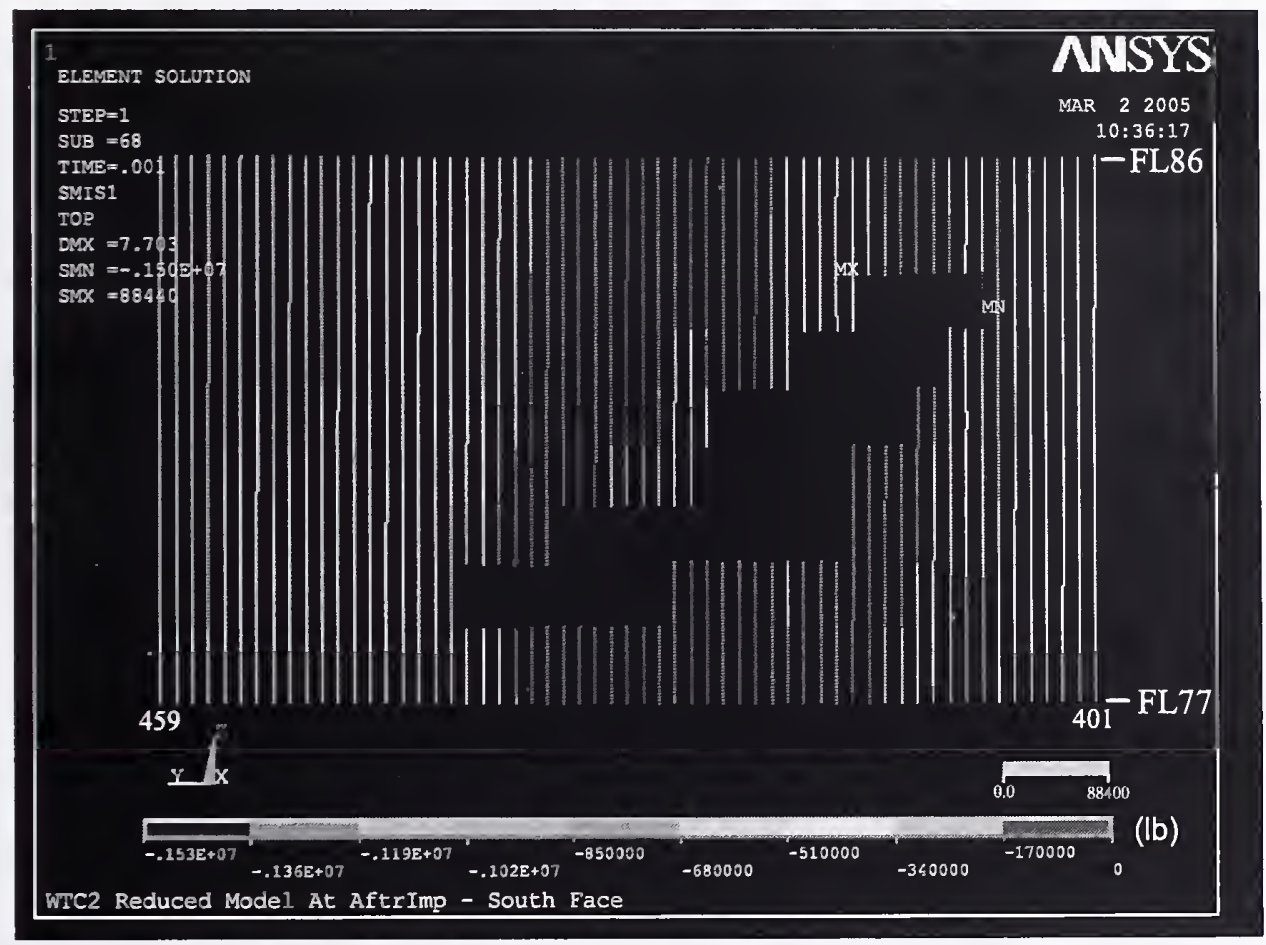

(b) After impact

Figure 4-106. Axial load in the south wall of WTC 2 for Case D conditions (compression is negative). 


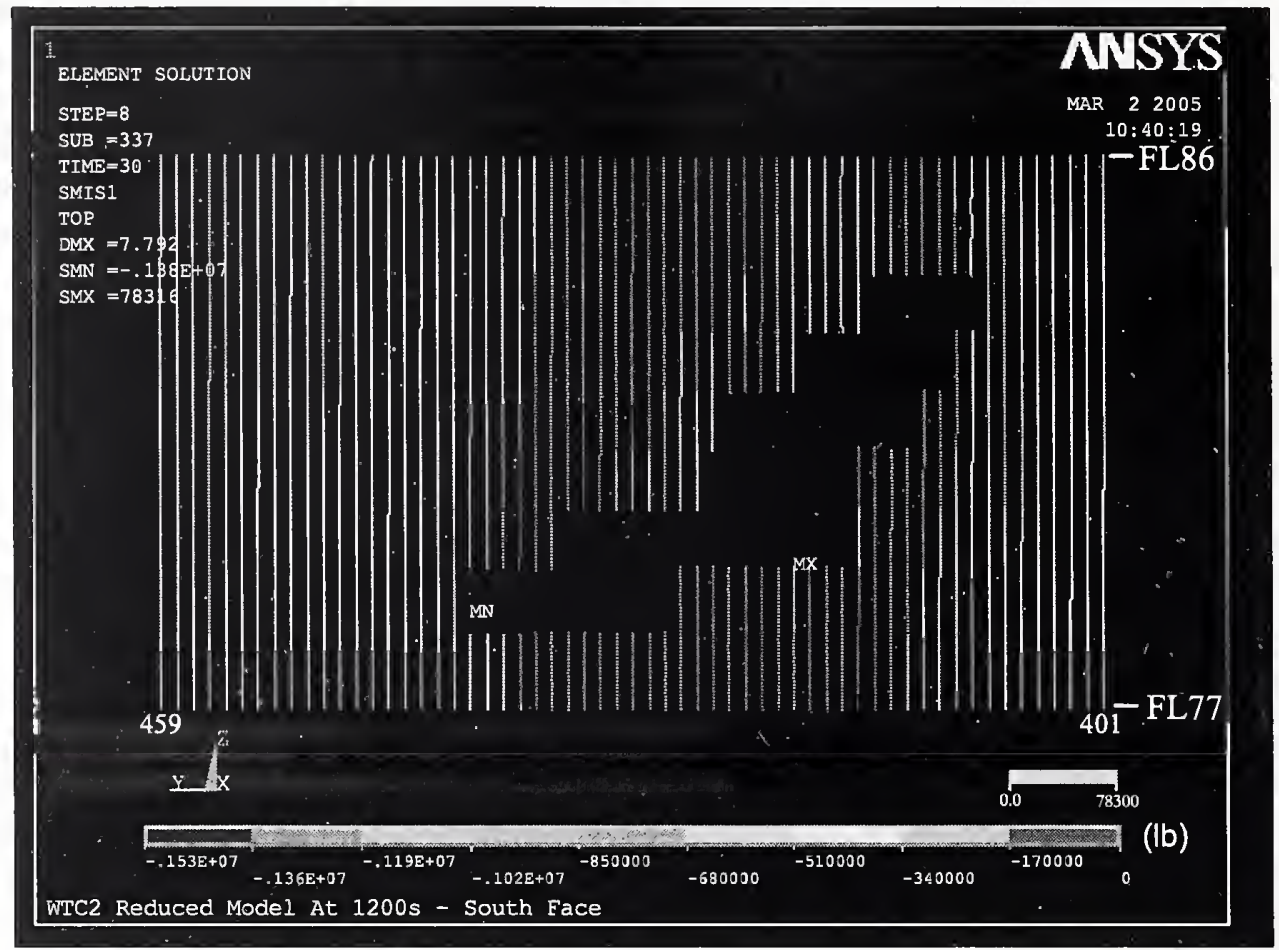

(a) $20 \mathrm{~min}$

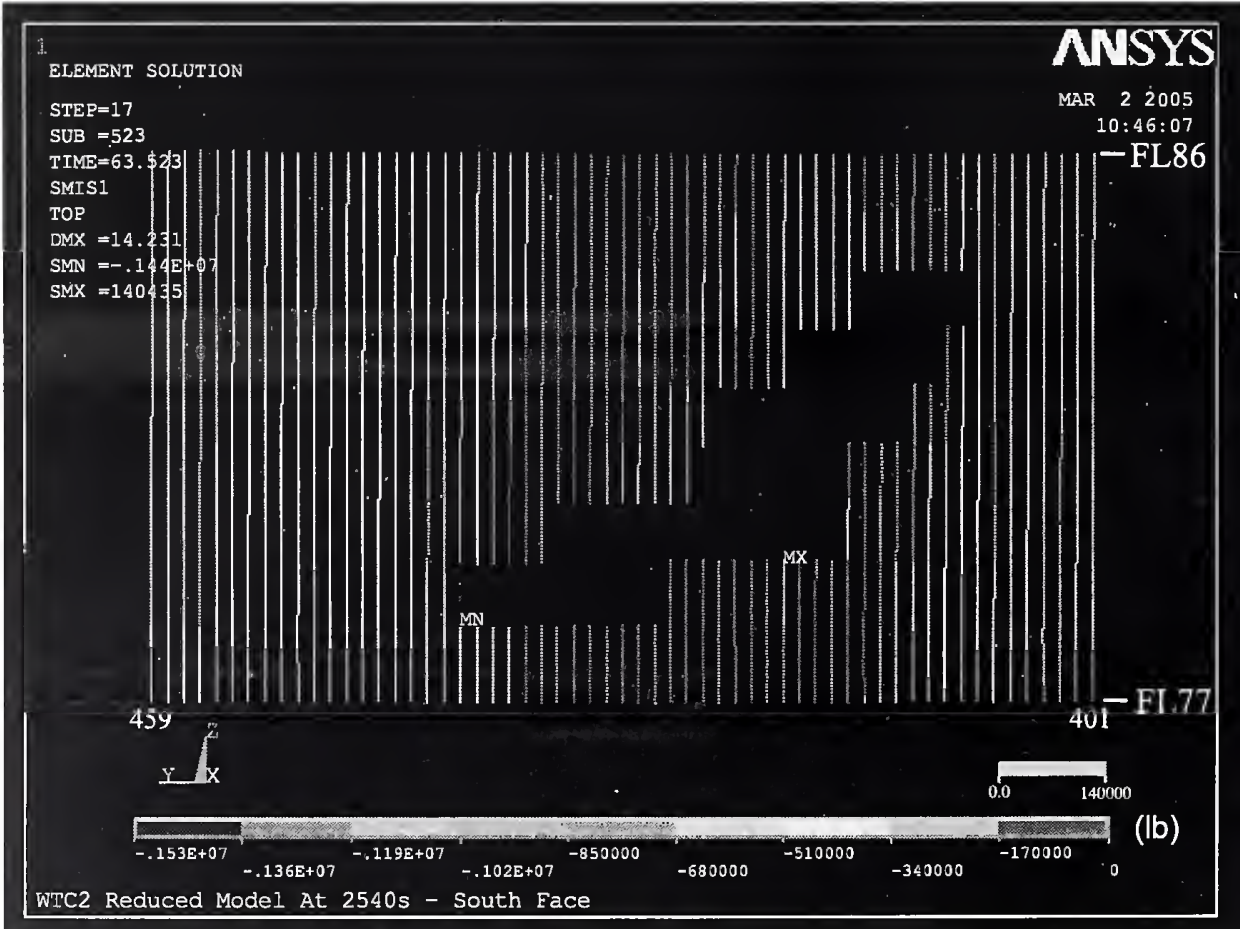

(b) $43 \mathrm{~min}$

Figure 4-107. Axial load in the south wall of WTC 2 for Case D conditions (compression is negative). 


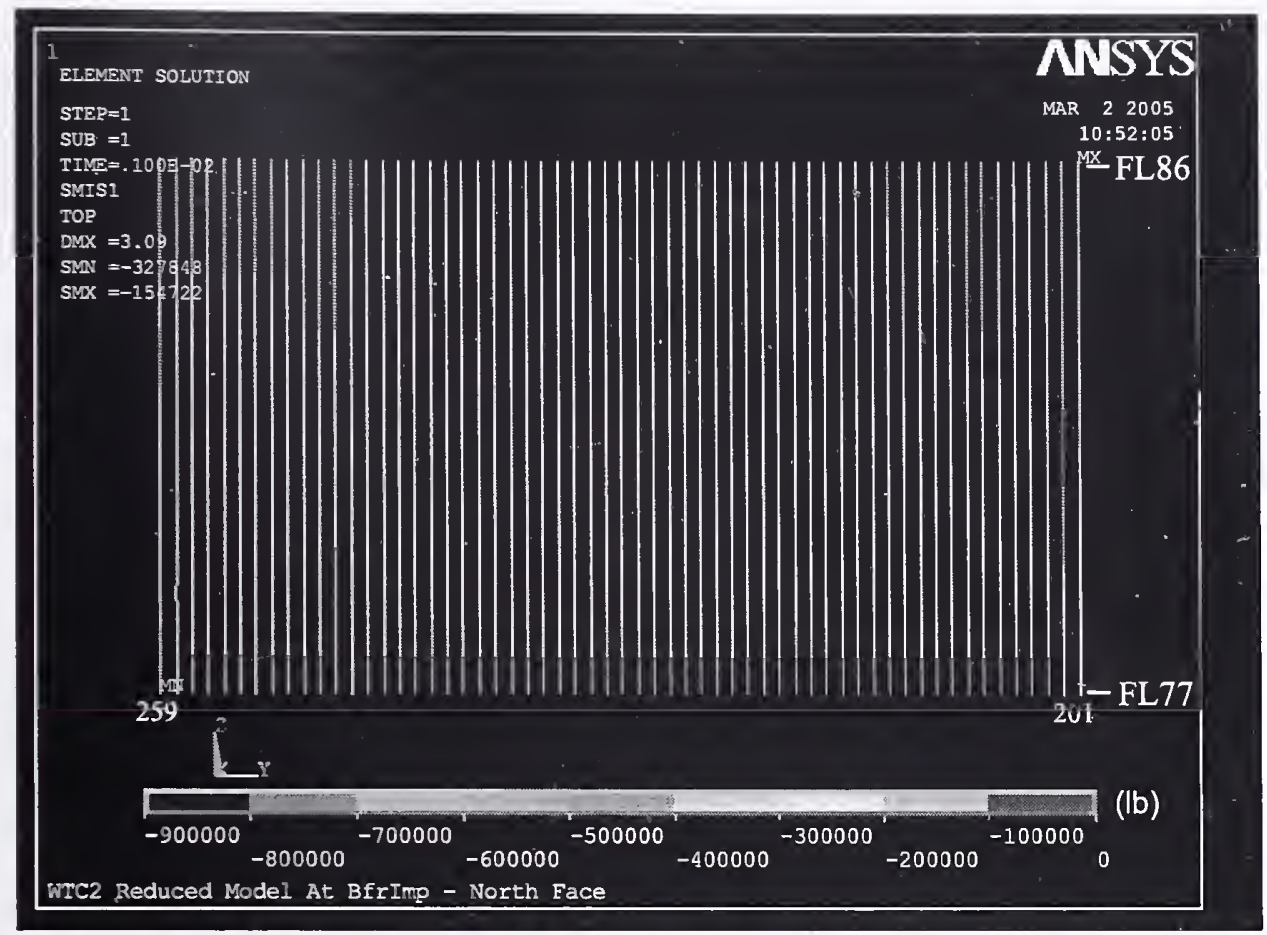

(a) Before impact

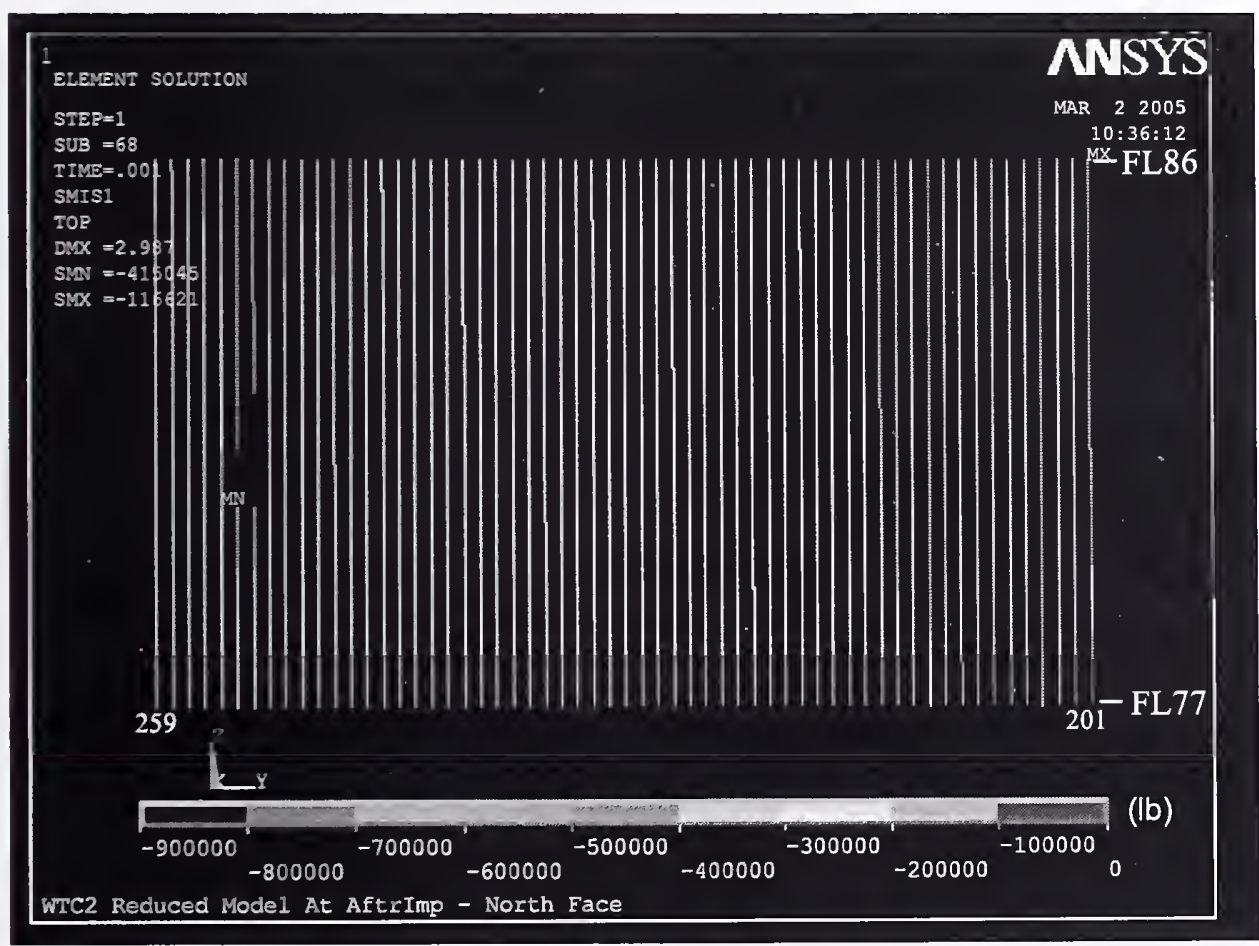

(b) After impact

Figure 4-108. Axial load in the north wall of WTC 2 for Case D conditions (compression is negative). 


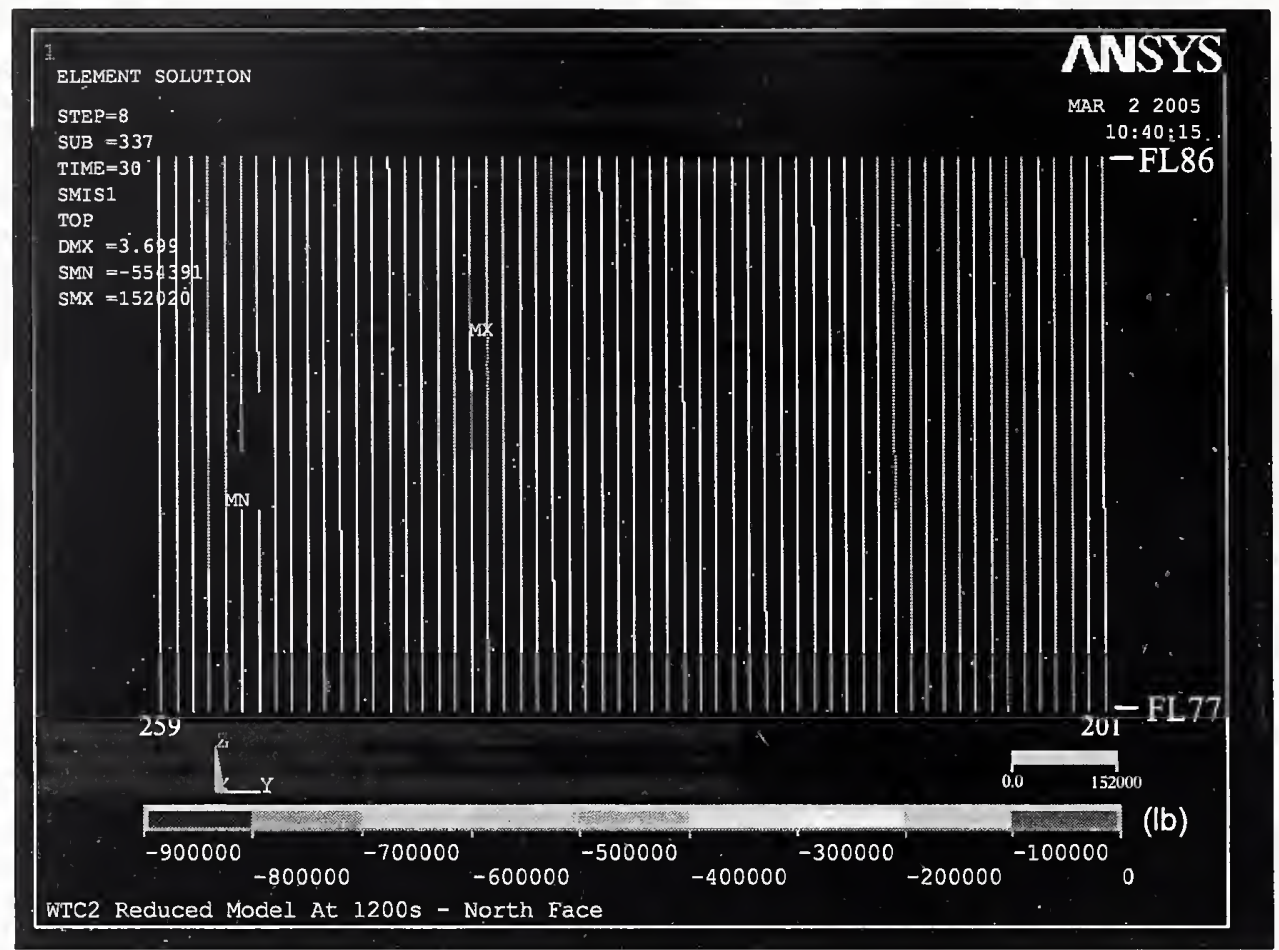

(a) $20 \mathrm{~min}$

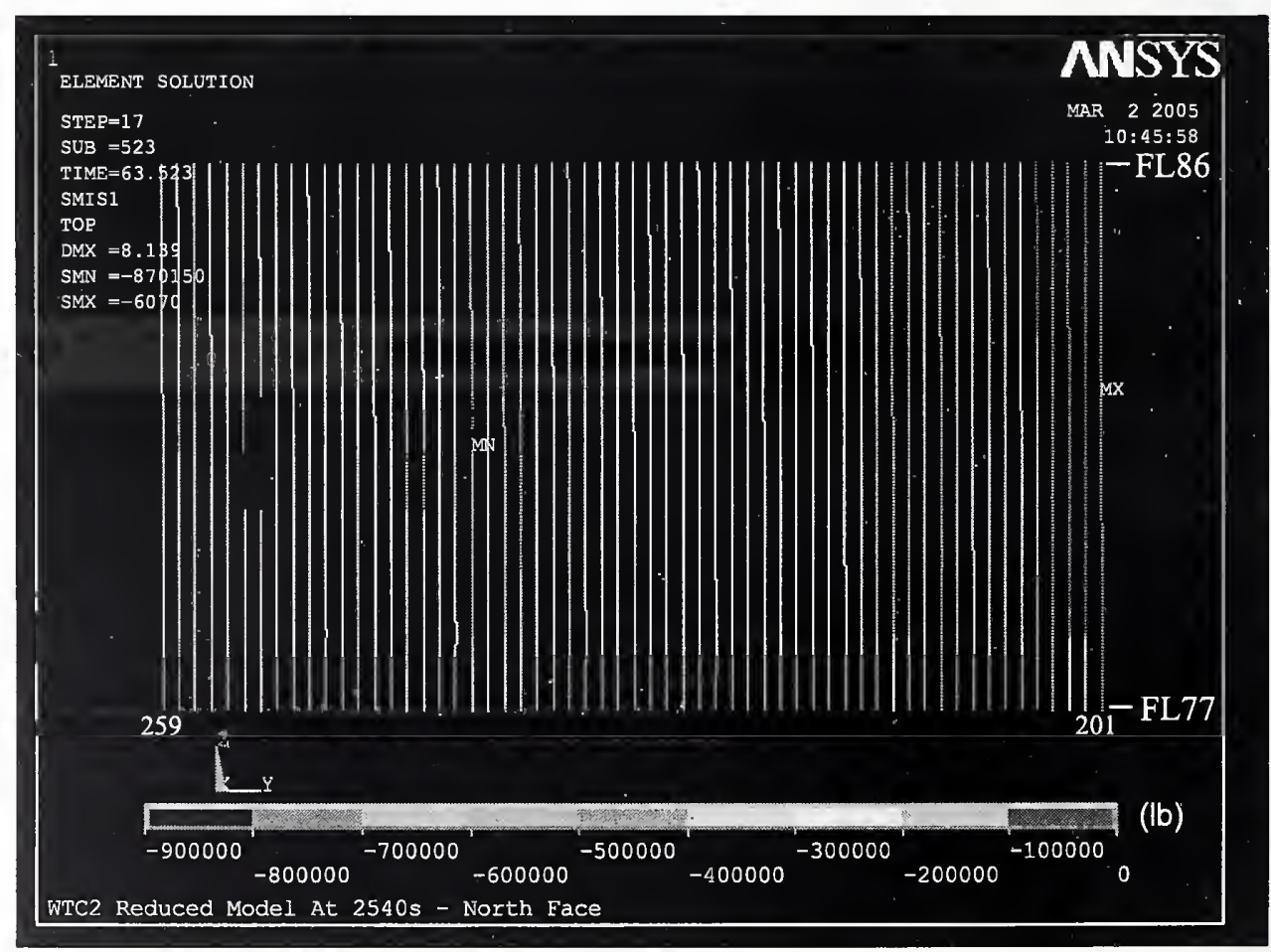

(b) $43 \mathrm{~min}$

Figure 4-109. Axial load in the north wall of WTC 2 for Case D conditions (compression is negative). 


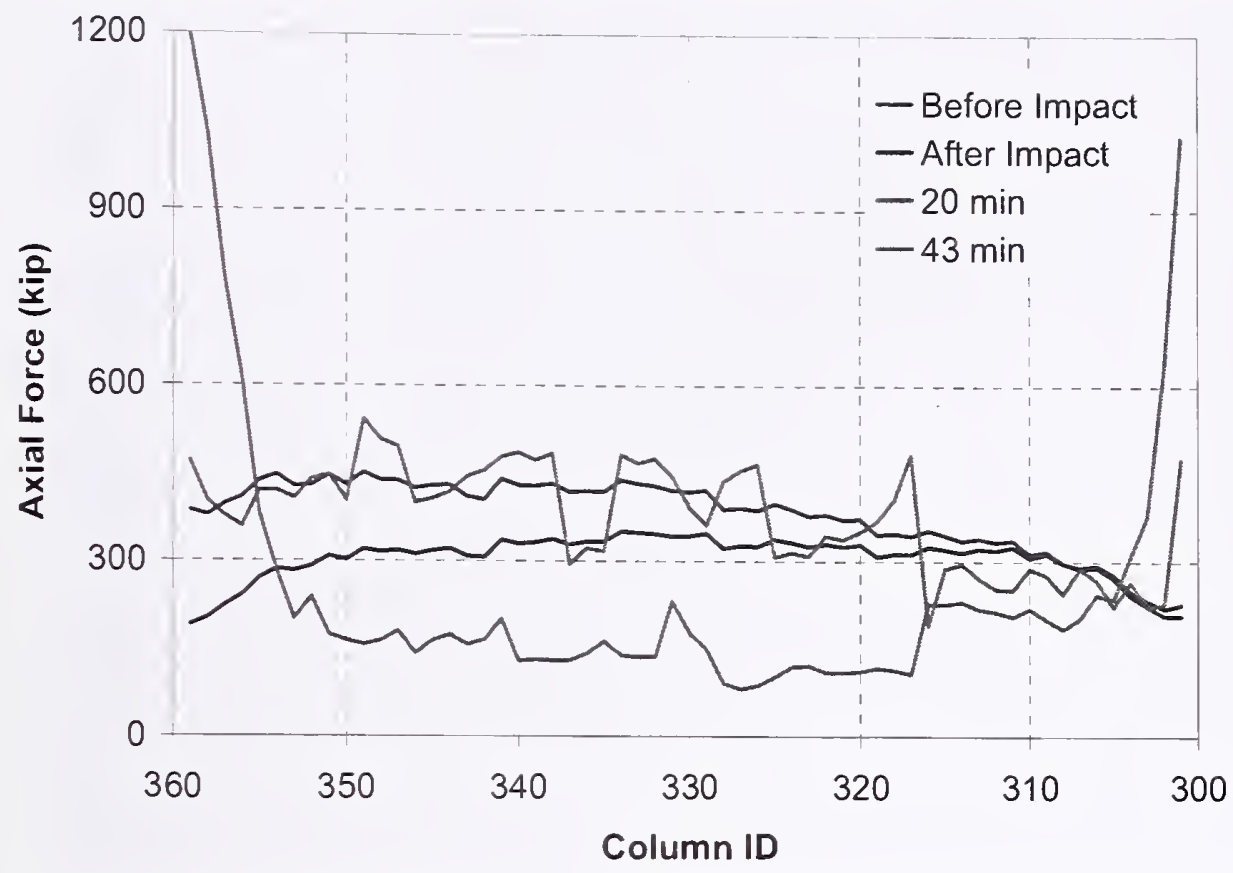

(a) East wall

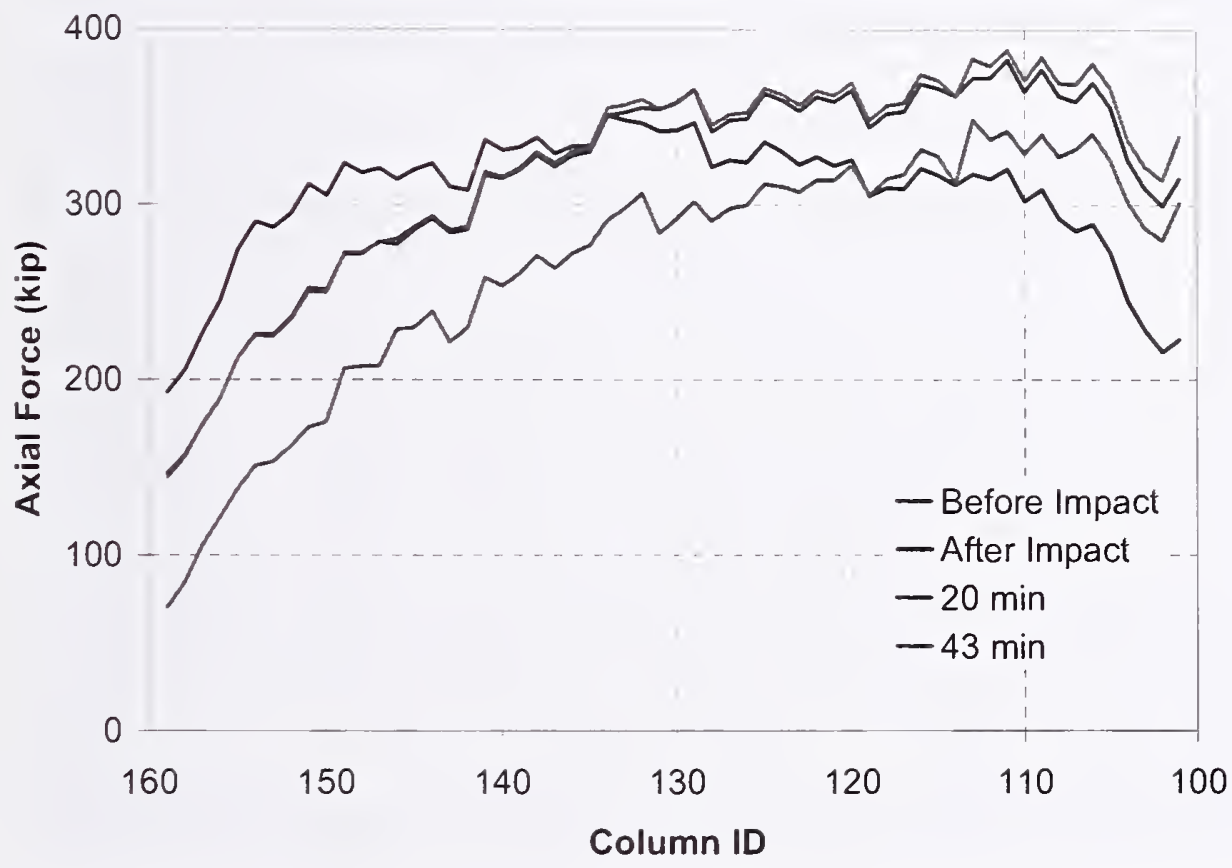

(b) West wall

Figure 4-110. Axial load in the east and the west wall columns at Floor 83 of WTC 2 for Case D conditions (compression is positive). 


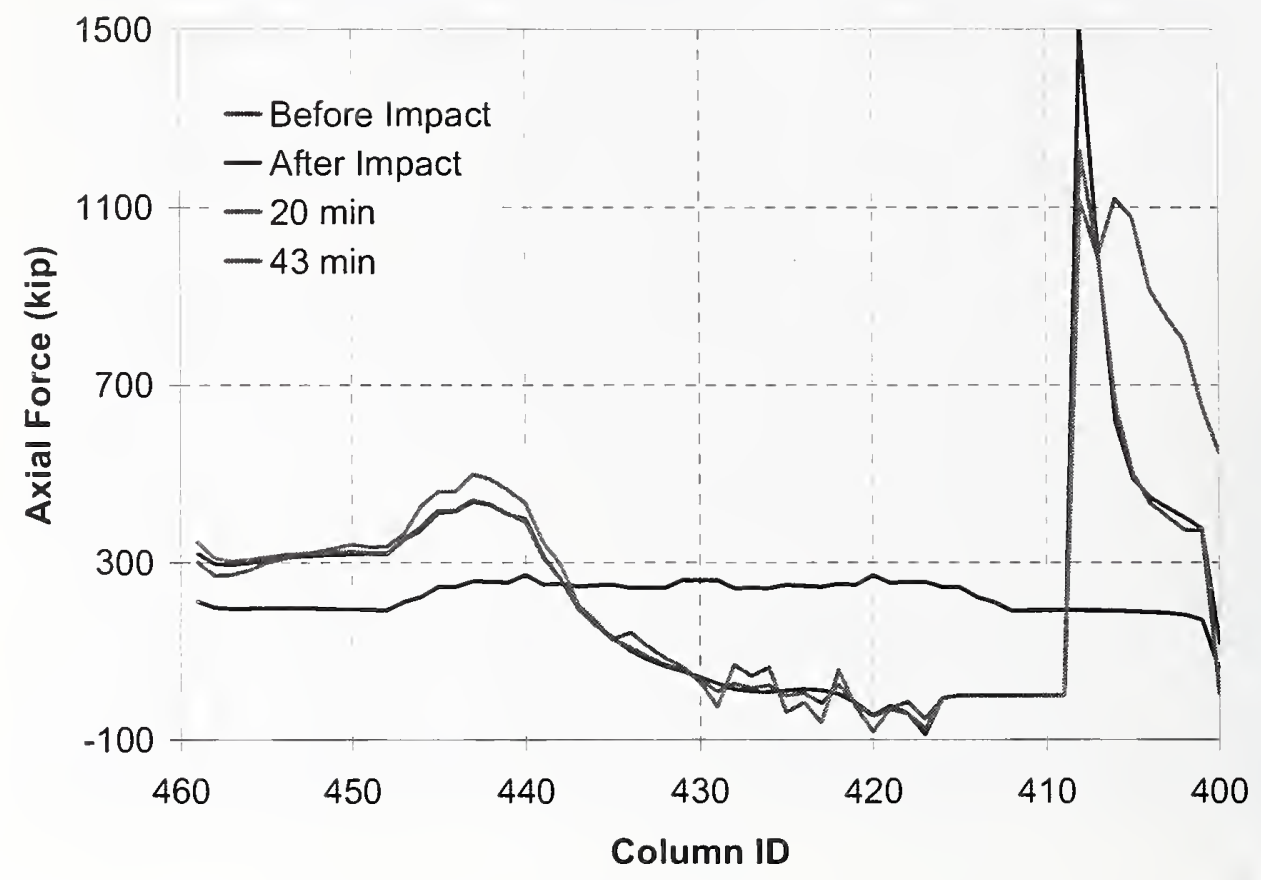

(a) South wall

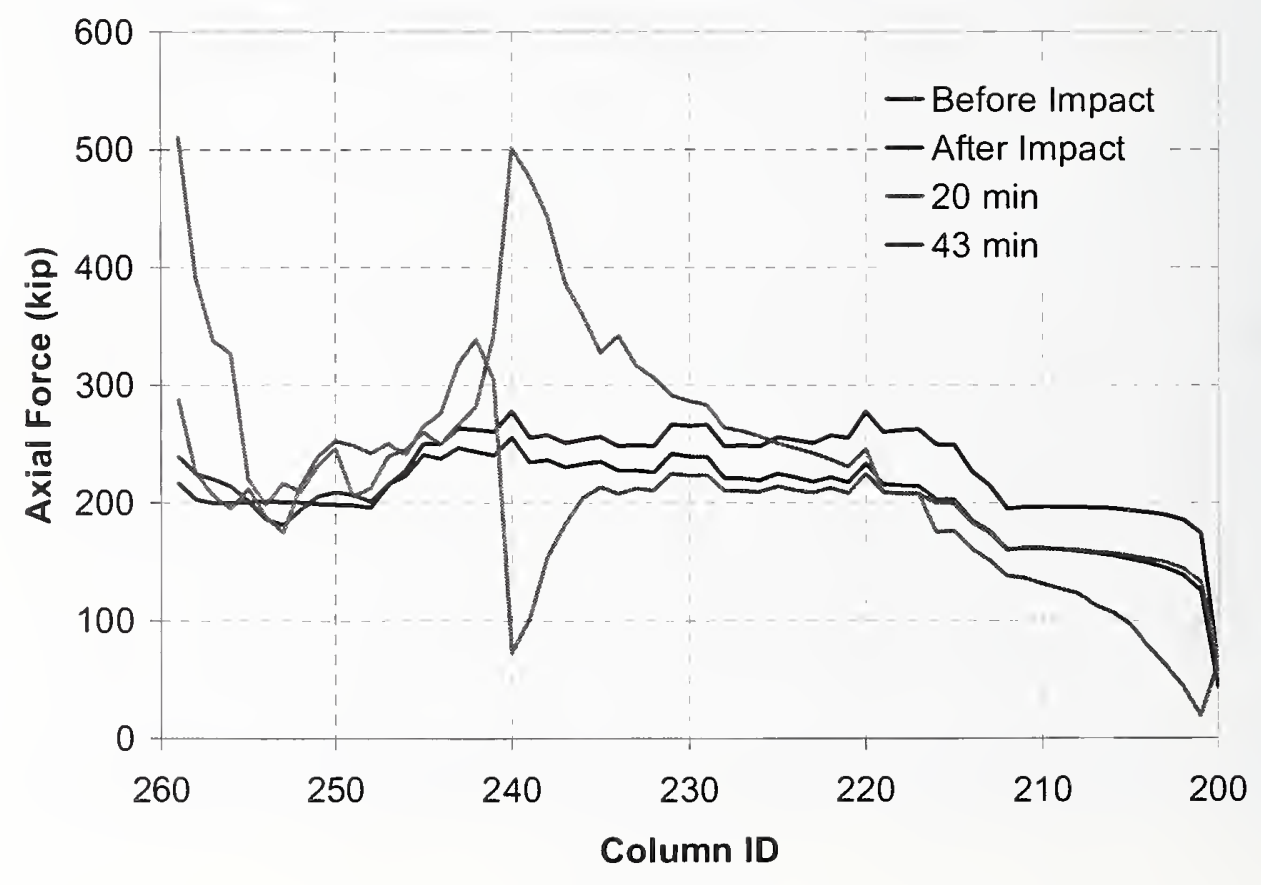

(b) North wall

Figure 4-111. Axial load in the south and the north wall columns at Floor 83 of WTC 2 for Case $D$ conditions (compression is positive). 


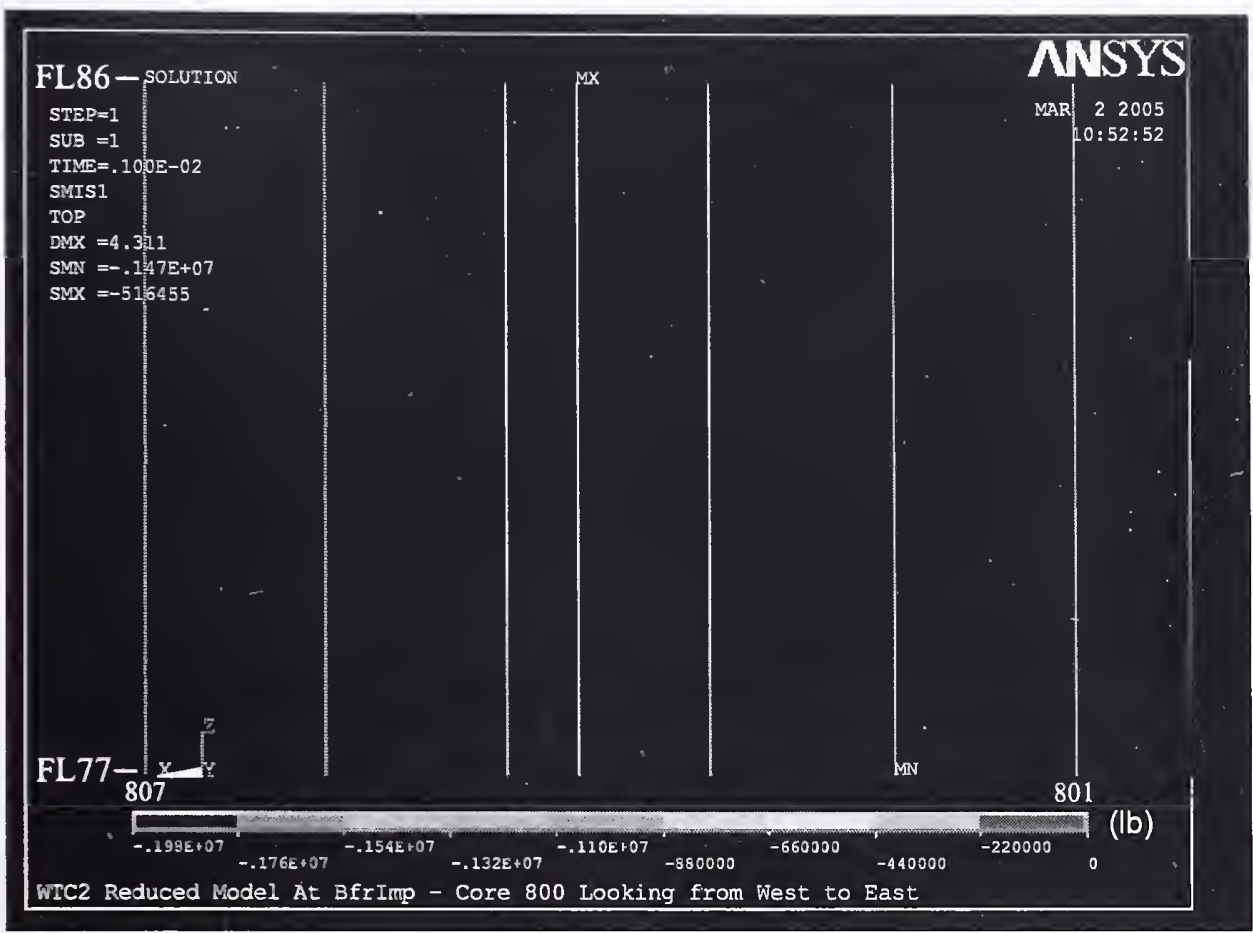

(a) Before impact

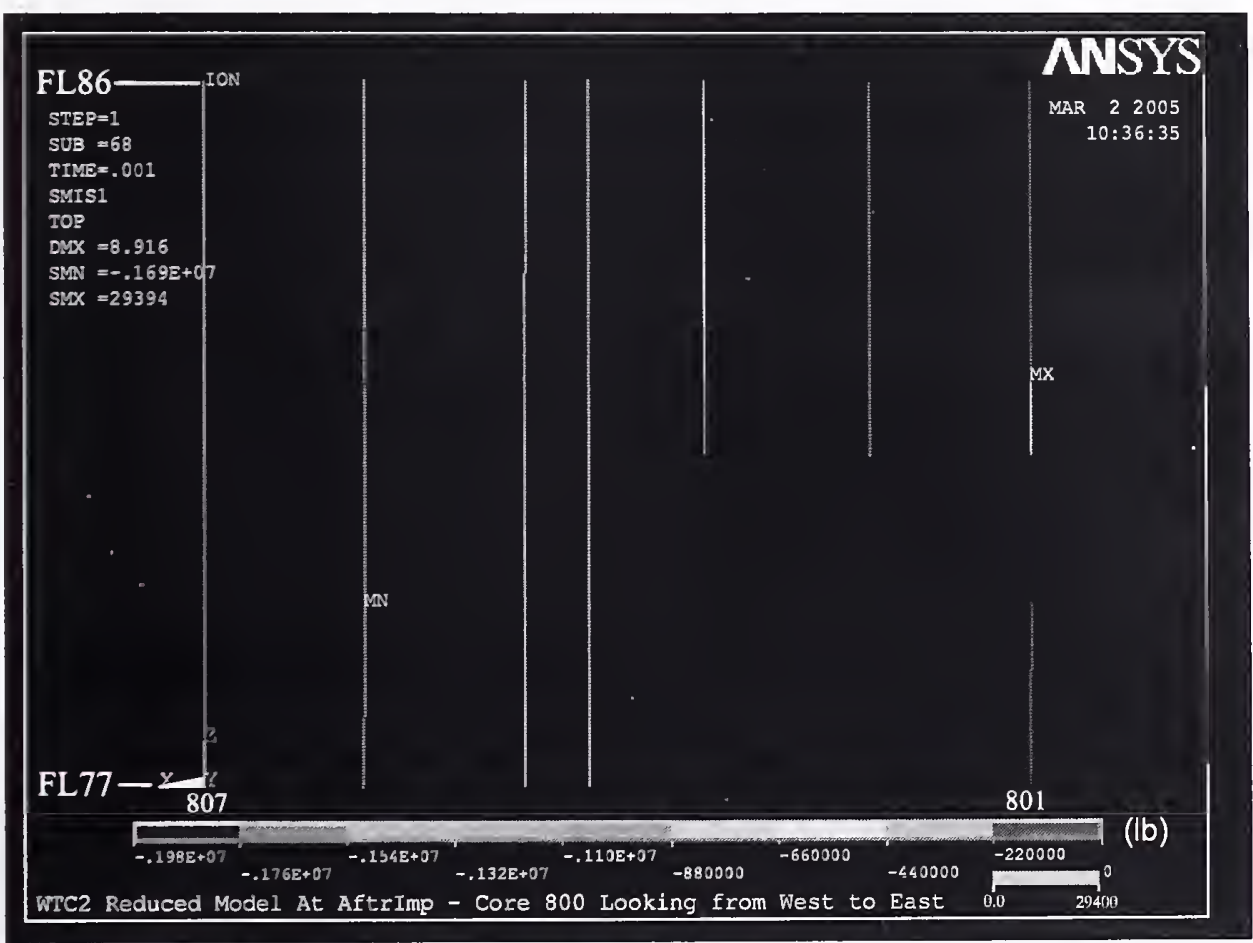

(b) After impact

Figure 4-112. Axial load in 800 series core columns of WTC 2 for Case D conditions (compression is negative). 


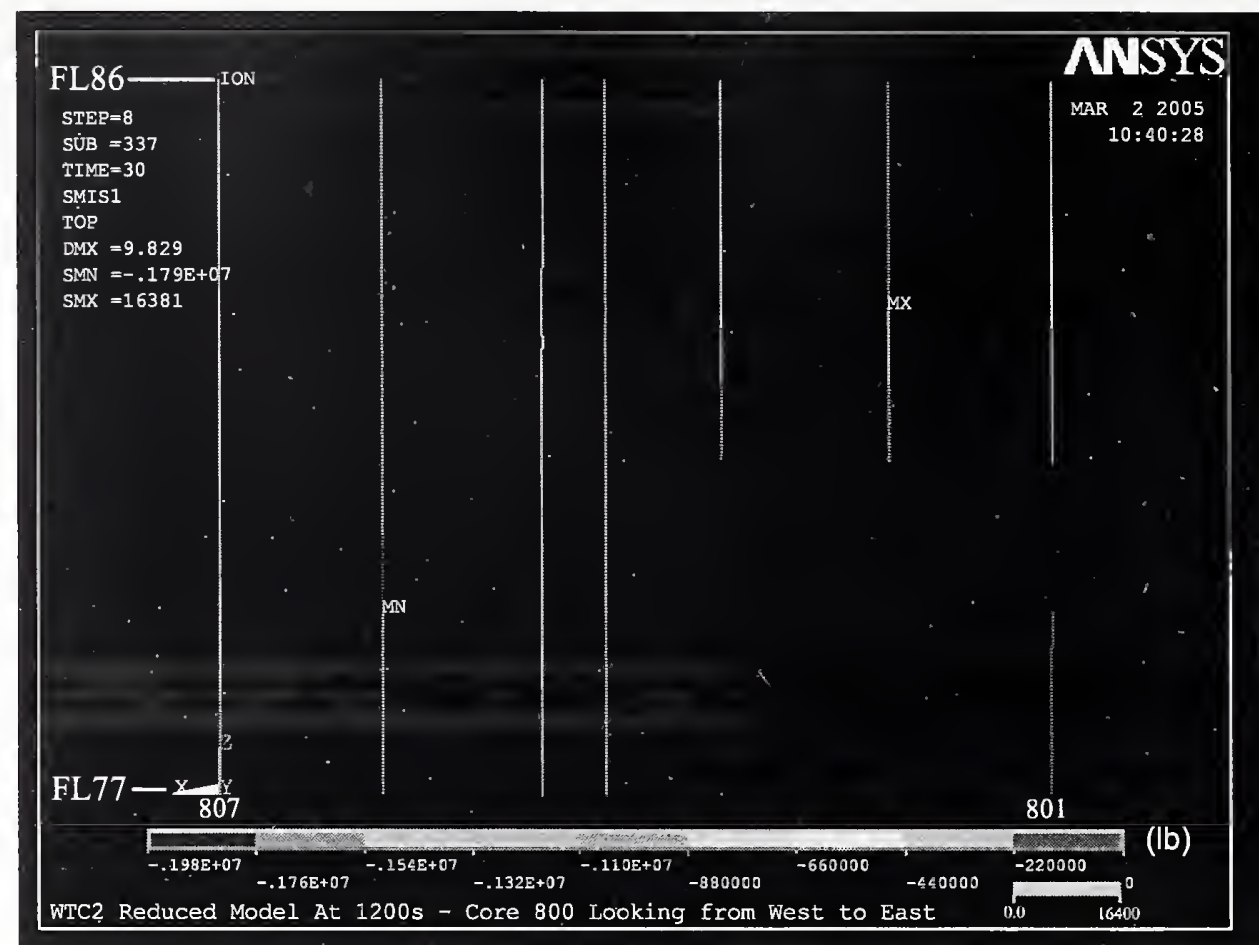

(a) $20 \mathrm{~min}$

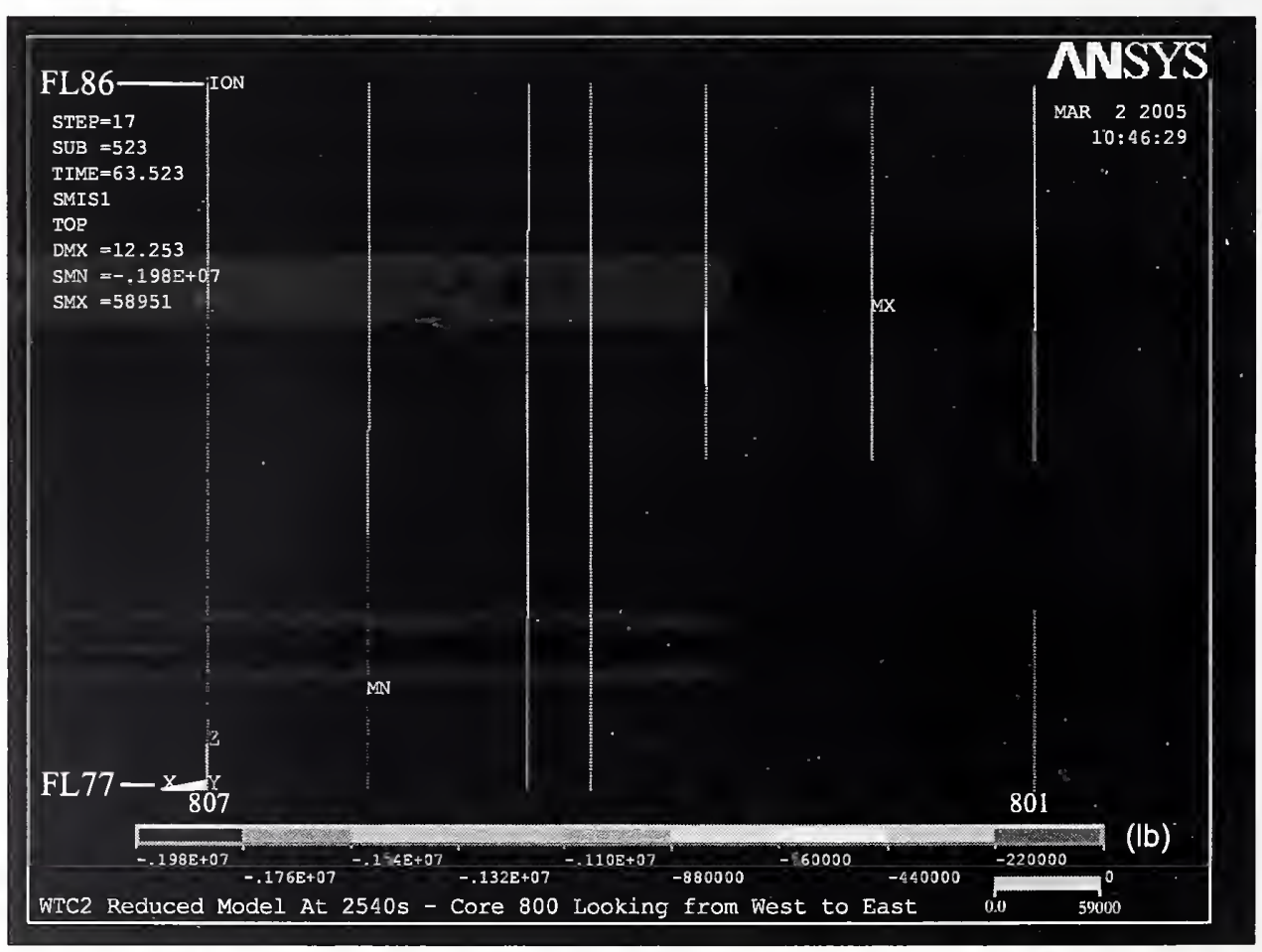

(b) $43 \mathrm{~min}$

Figure 4-113. Axial load in 800 series core columns of WTC 2 for Case D conditions (compression is negative). 


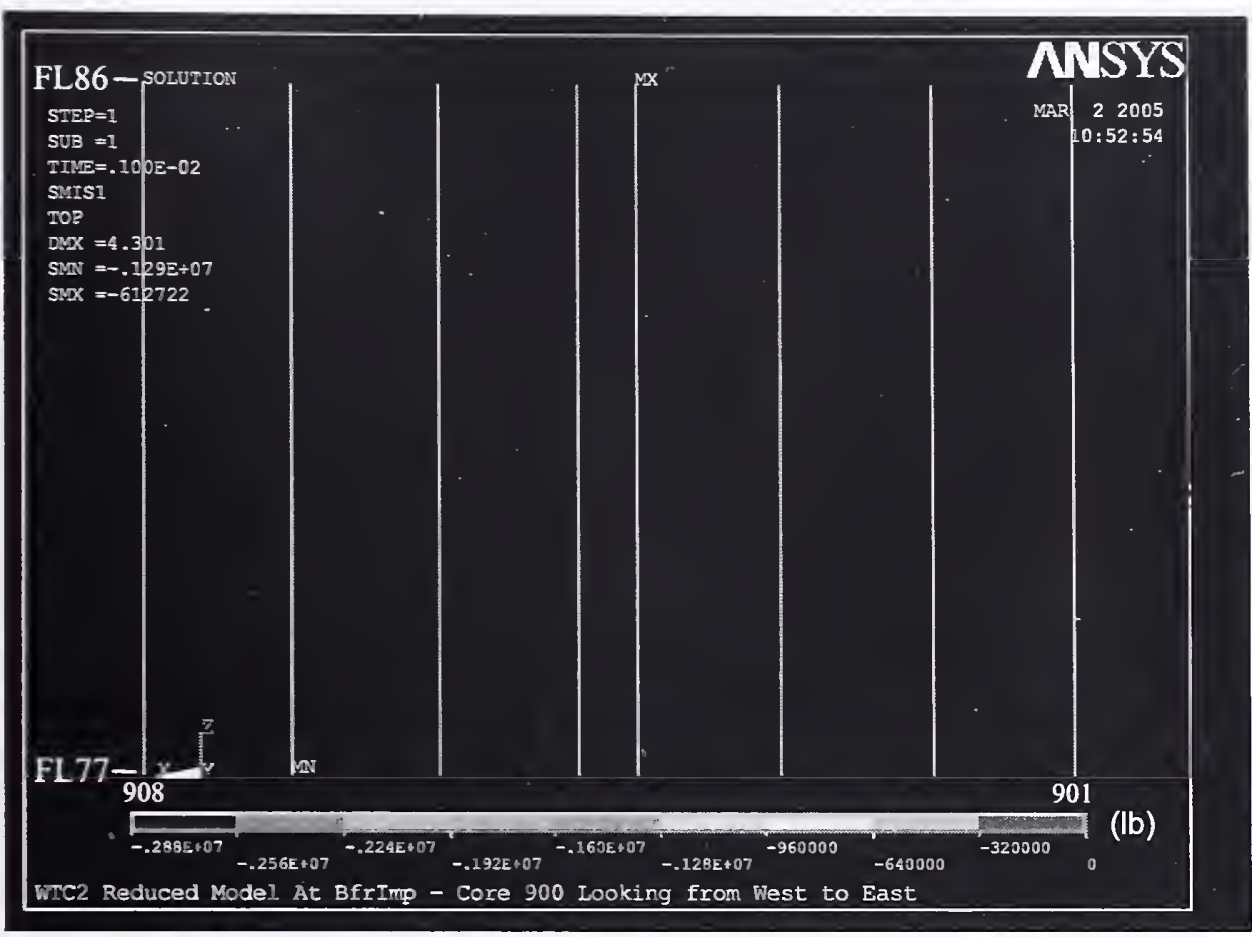

(a) Before impact

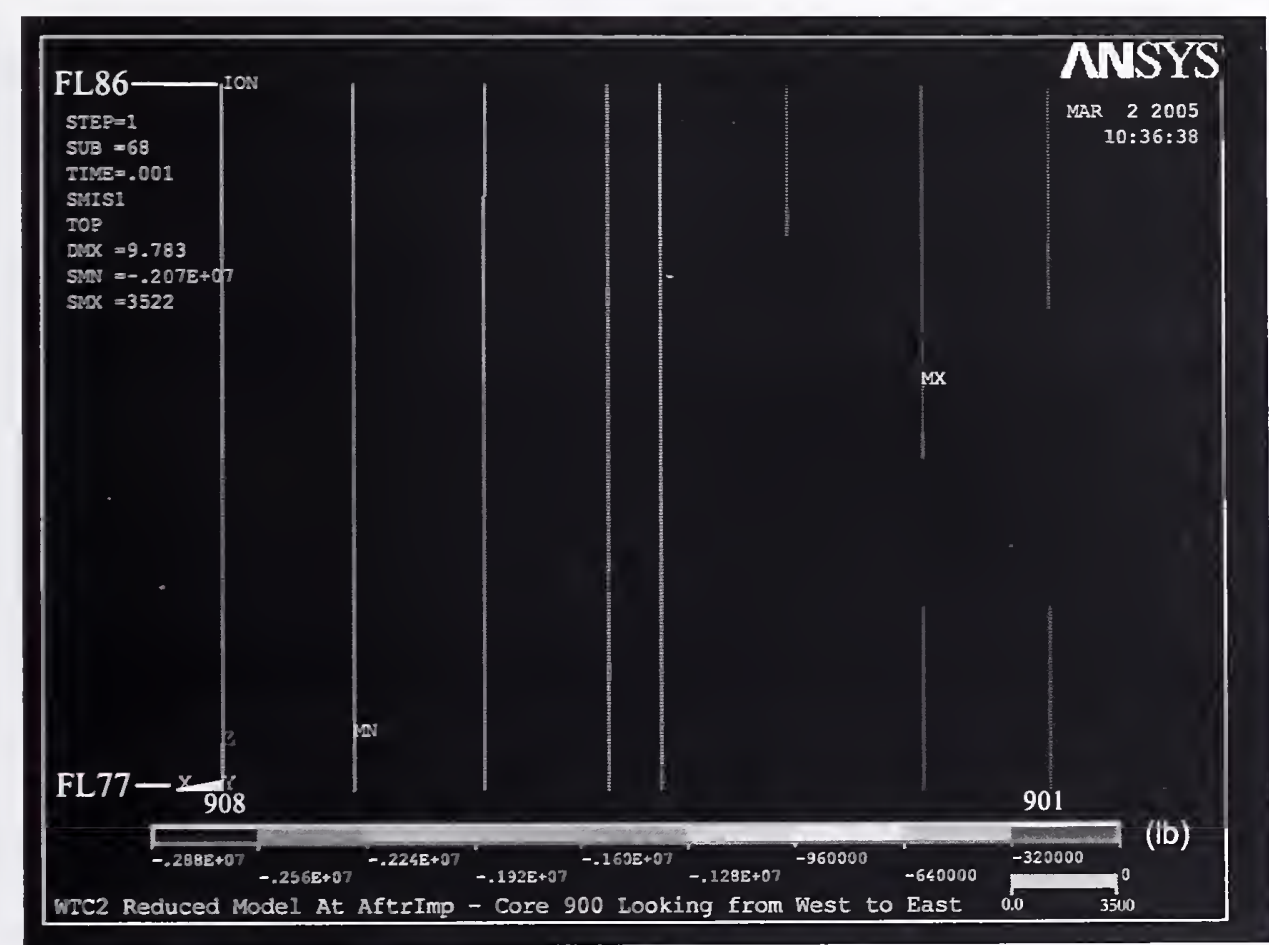

(b) After impact

Figure 4-114. Axial load in 900 series core columns of WTC 2 for Case D conditions (compression is negative). 


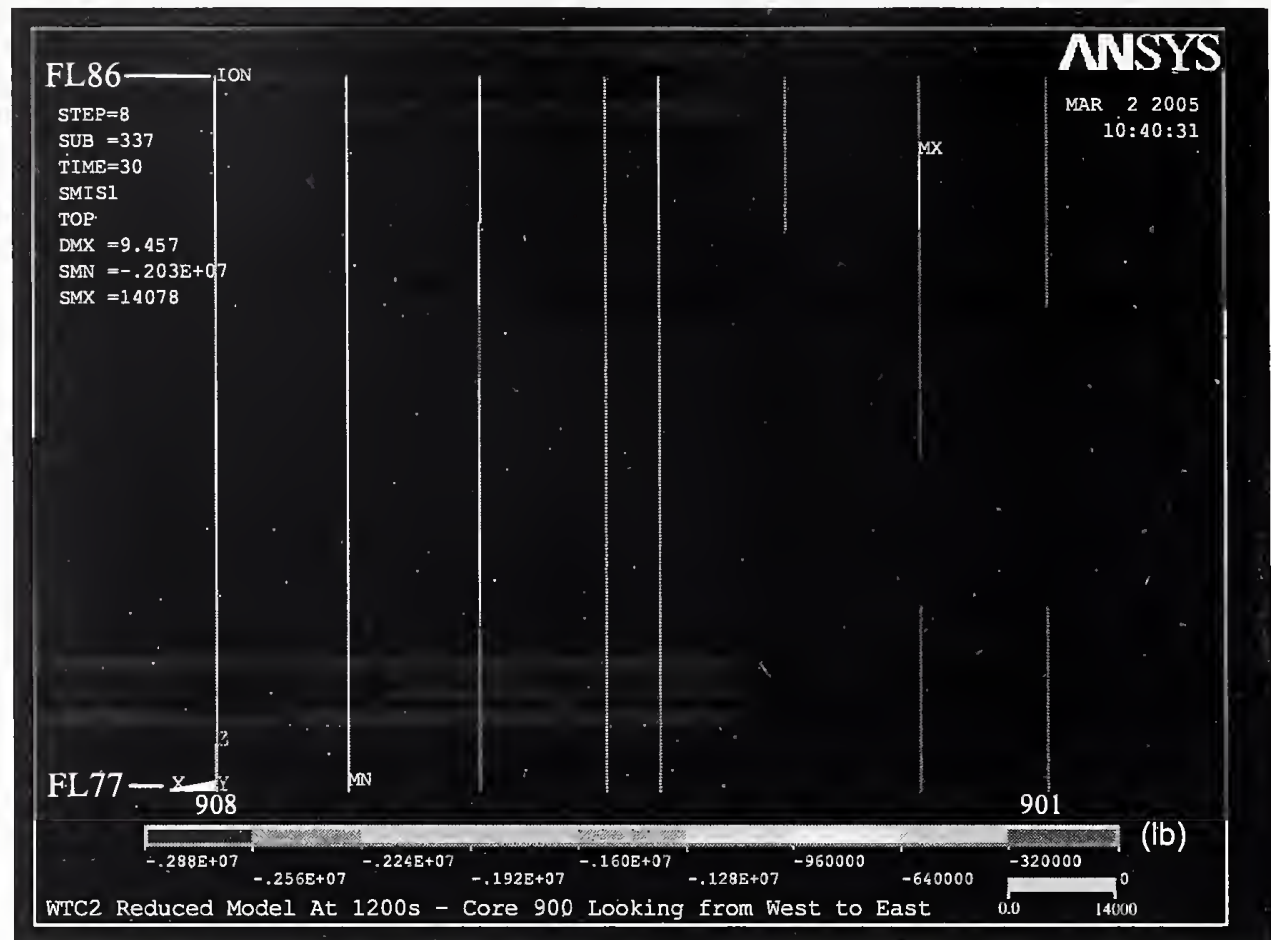

(a) $20 \mathrm{~min}$

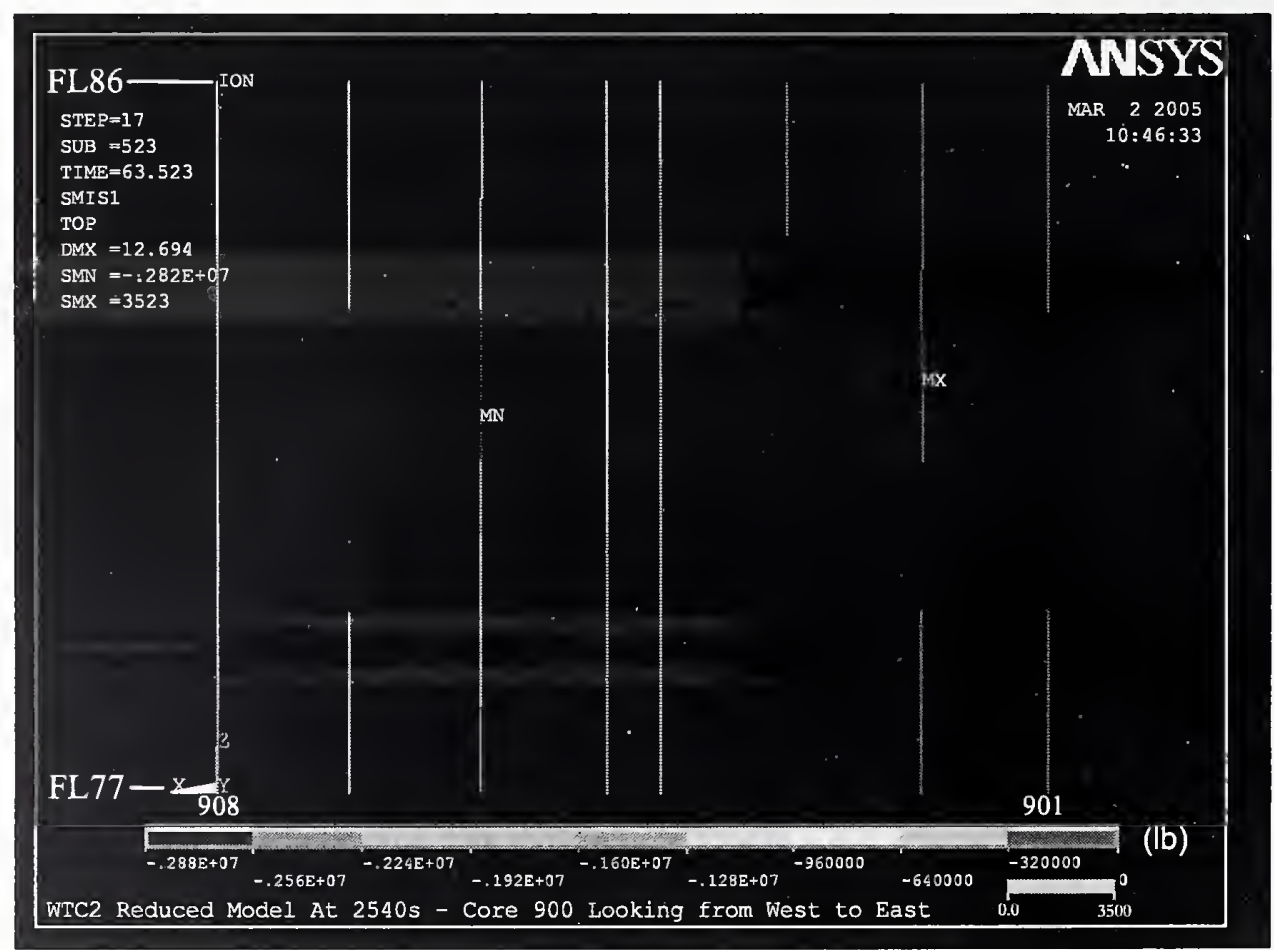

(b) $43 \mathrm{~min}$

Figure 4-115. Axial load in 900 series core columns of WTC 2 for Case D conditions (compression is negative). 


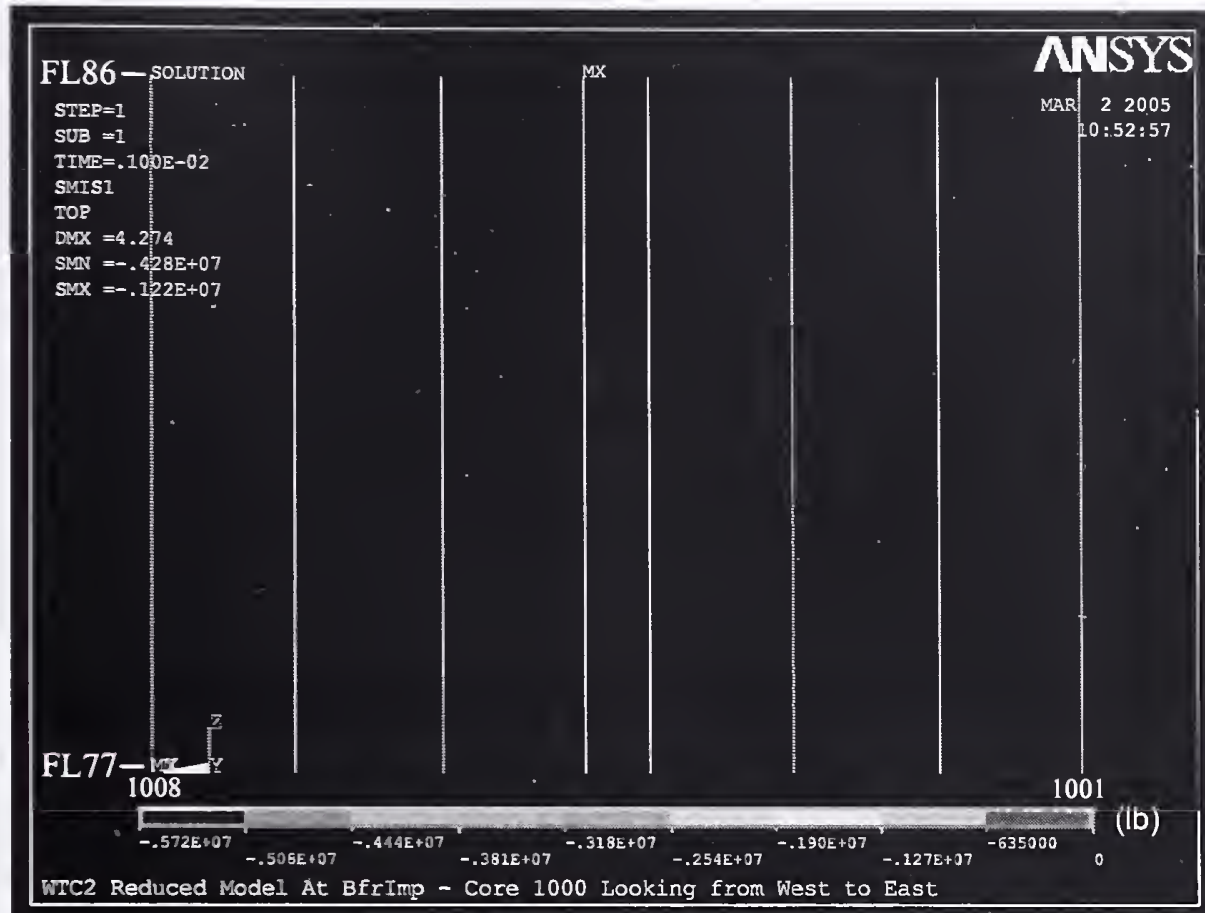

(a) Before impact

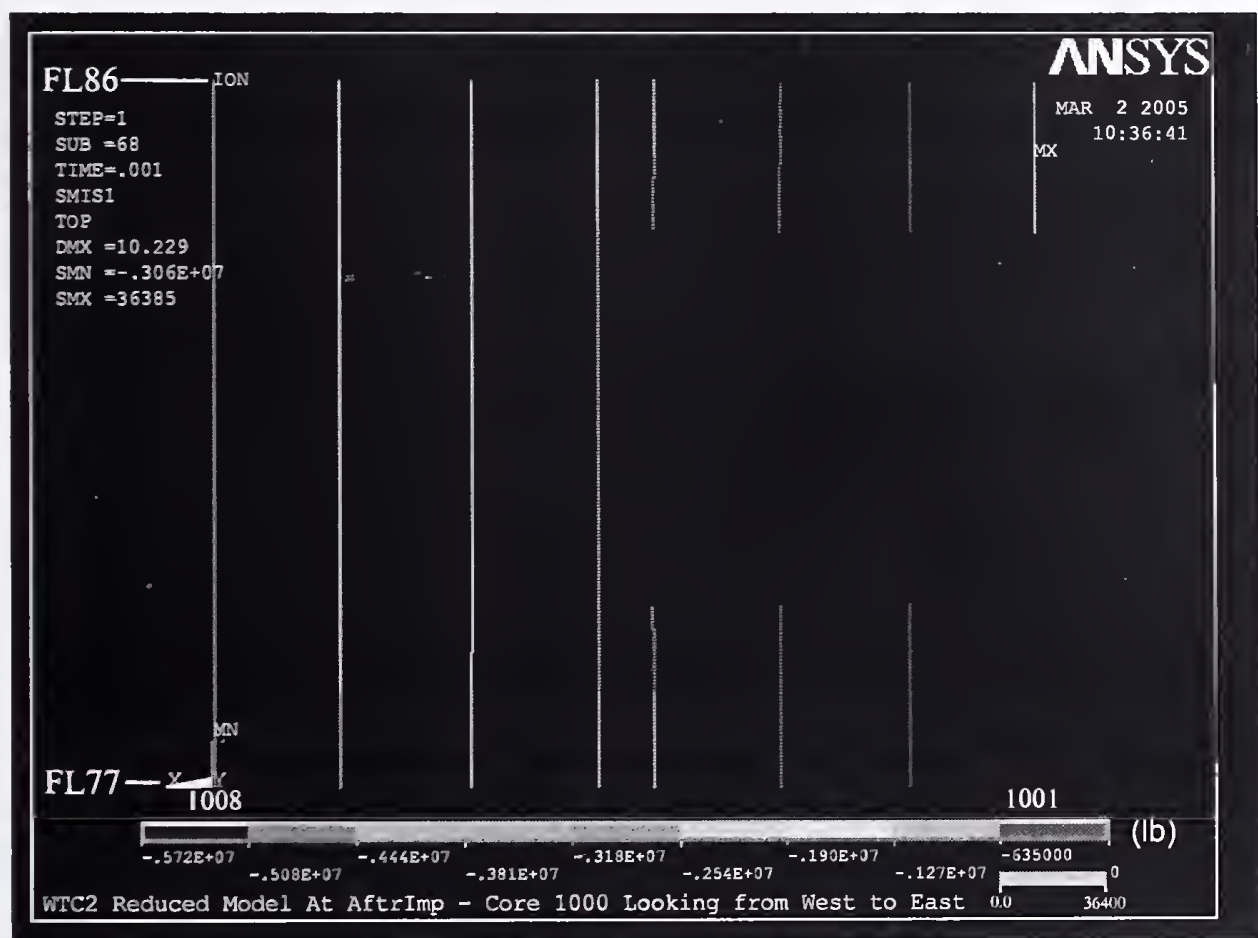

(b) After impact

Figure 4-116. Axial load in 1000 series core columns of WTC 2 for Case D conditions (compression is negative). 


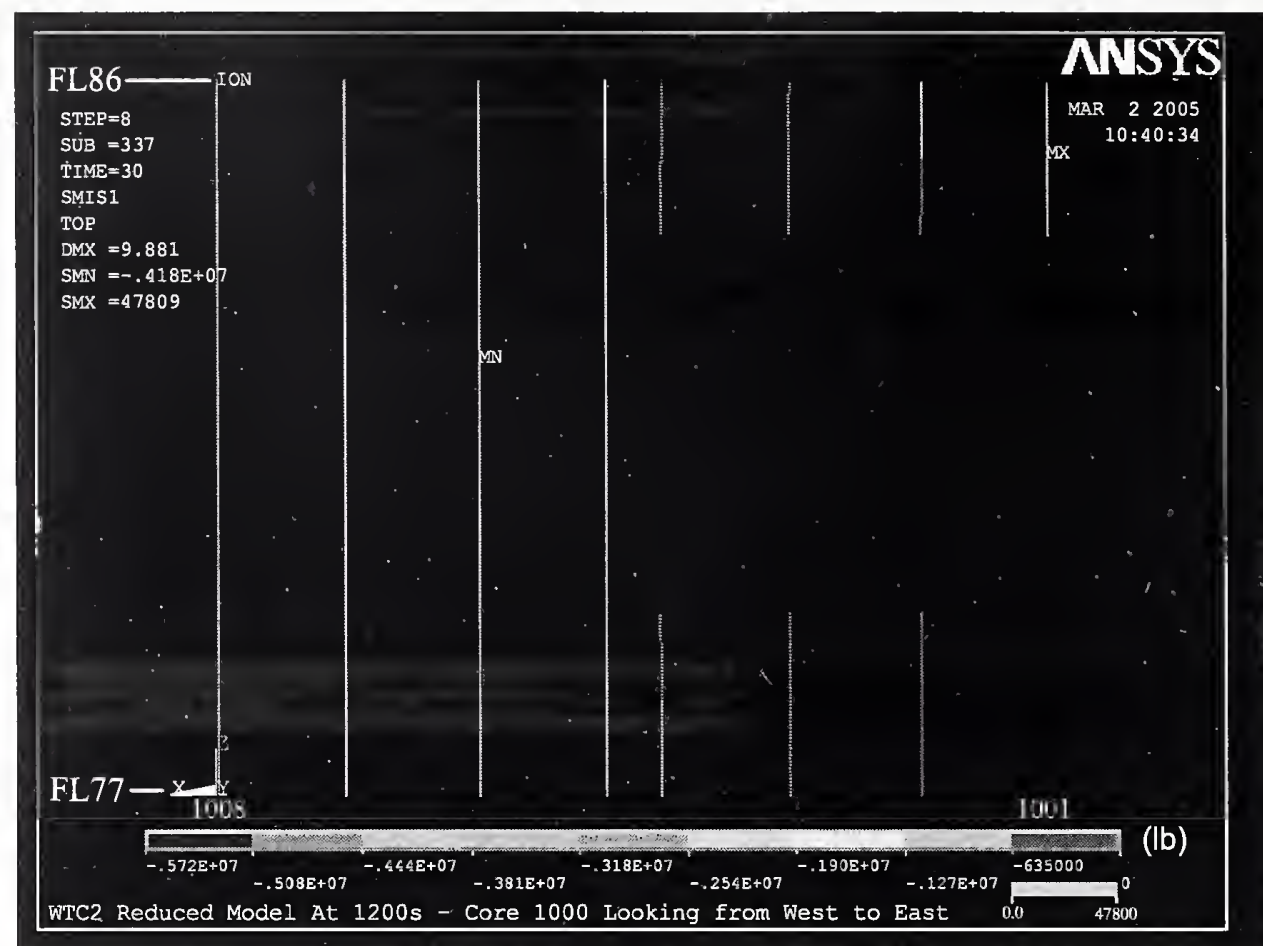

(a) $20 \mathrm{~min}$

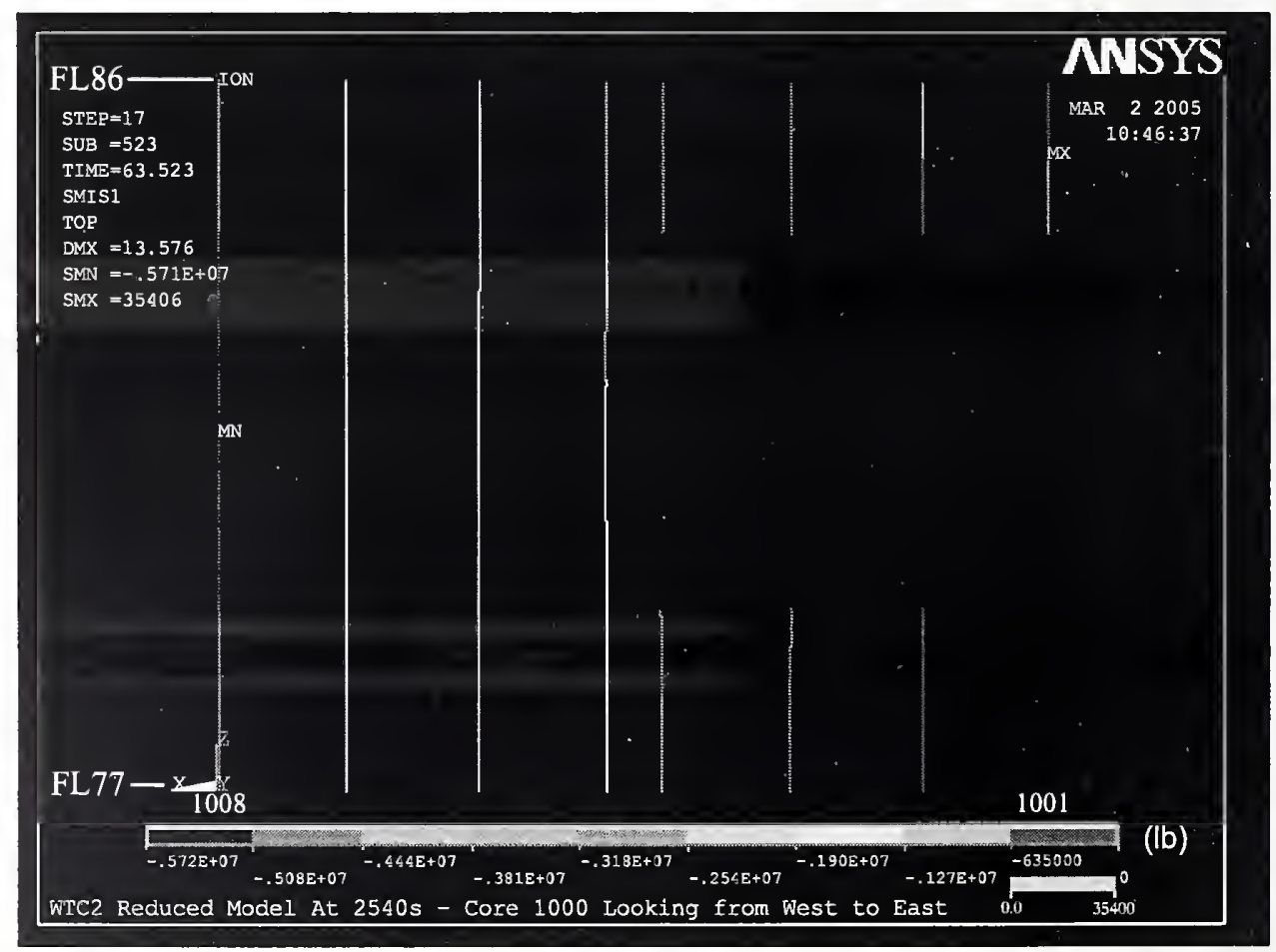

(b) $43 \mathrm{~min}$

Figure 4-117. Axial load in 1000 series core columns of WTC 2 for Case D conditions (compression is negative). 

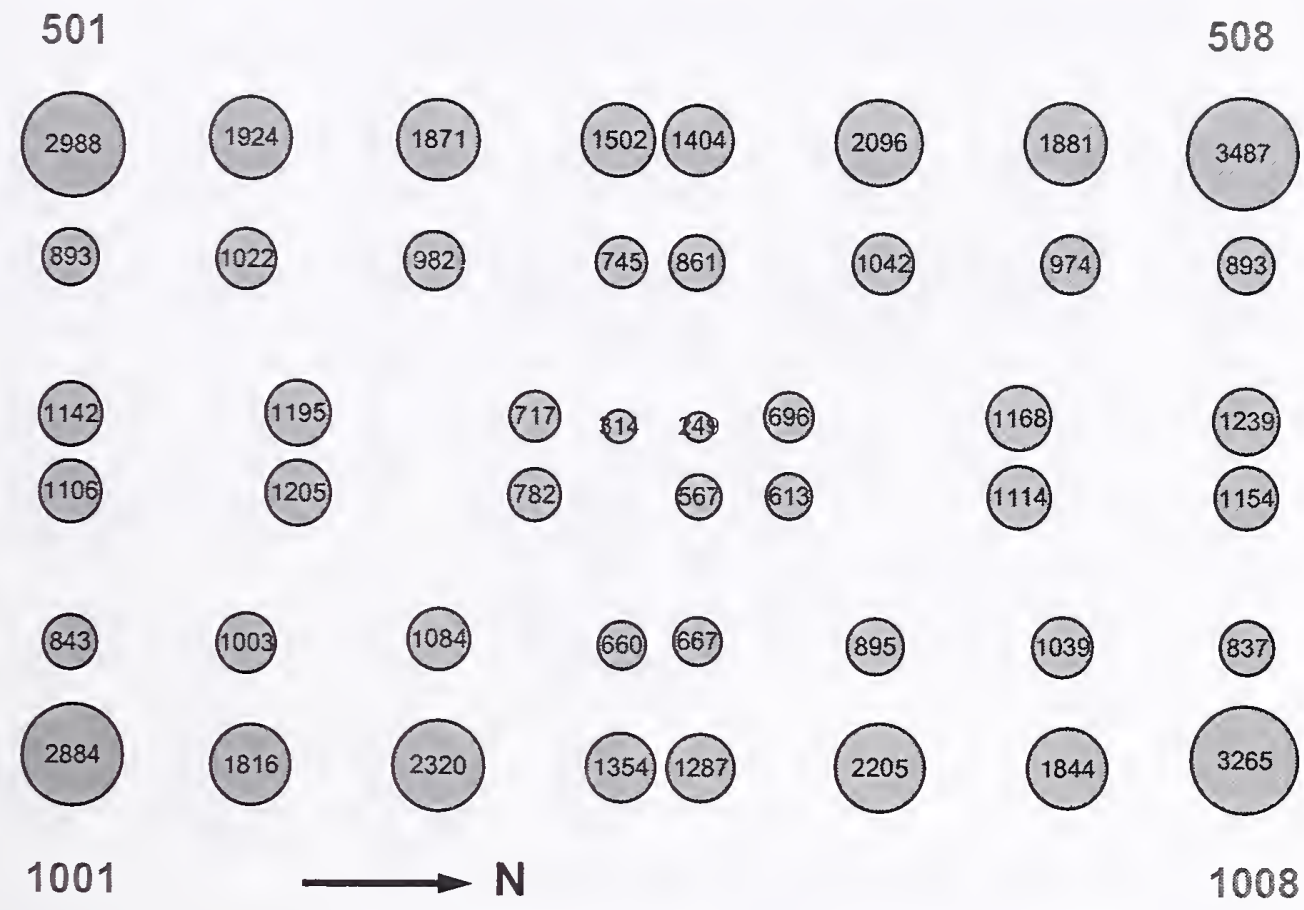

(a) Before impact

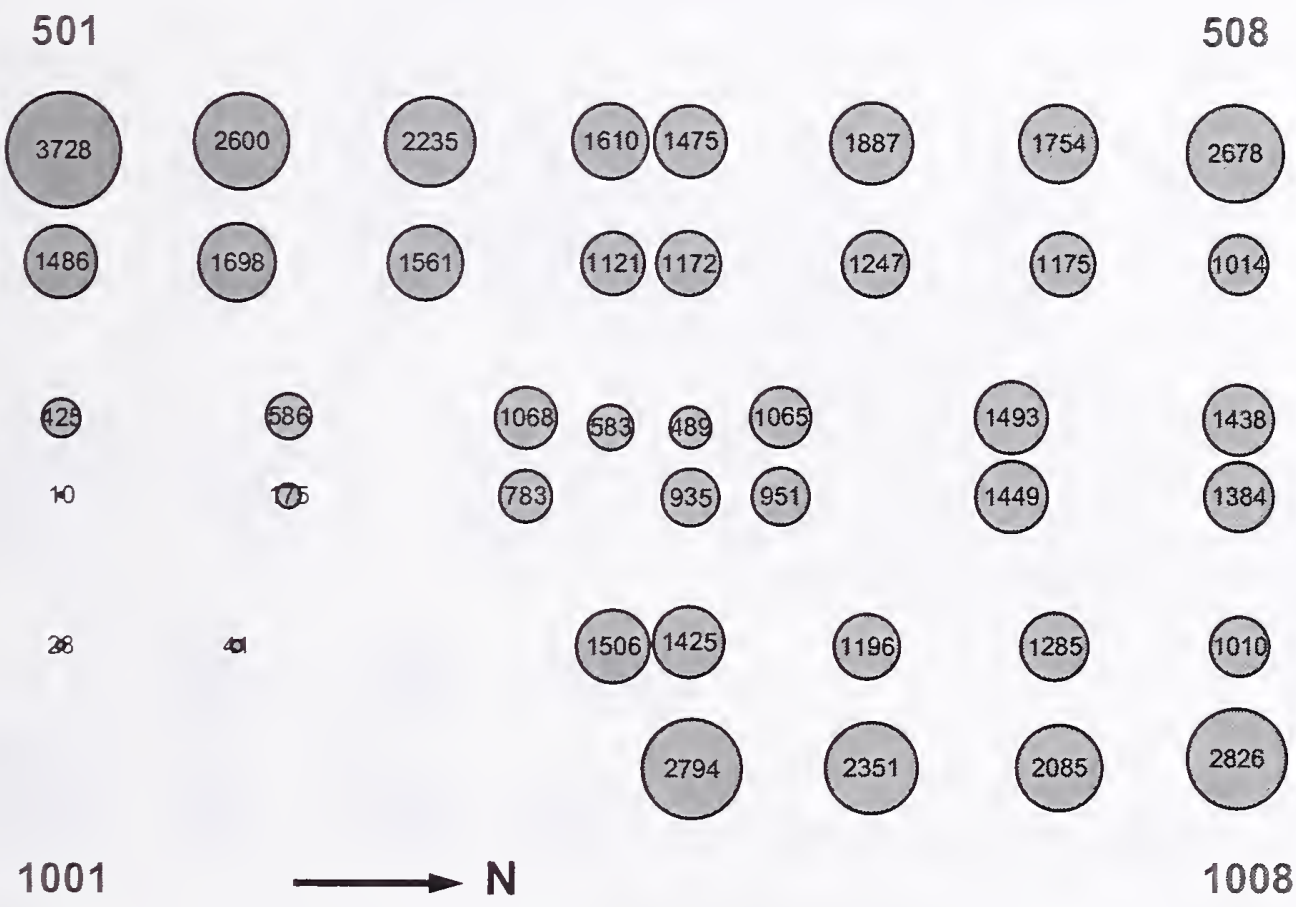

(b) After impact

Figure 4-118. Core column loads (kip) at Floor 83 of WTC 2 for Case D conditions (compression is positive). 
501

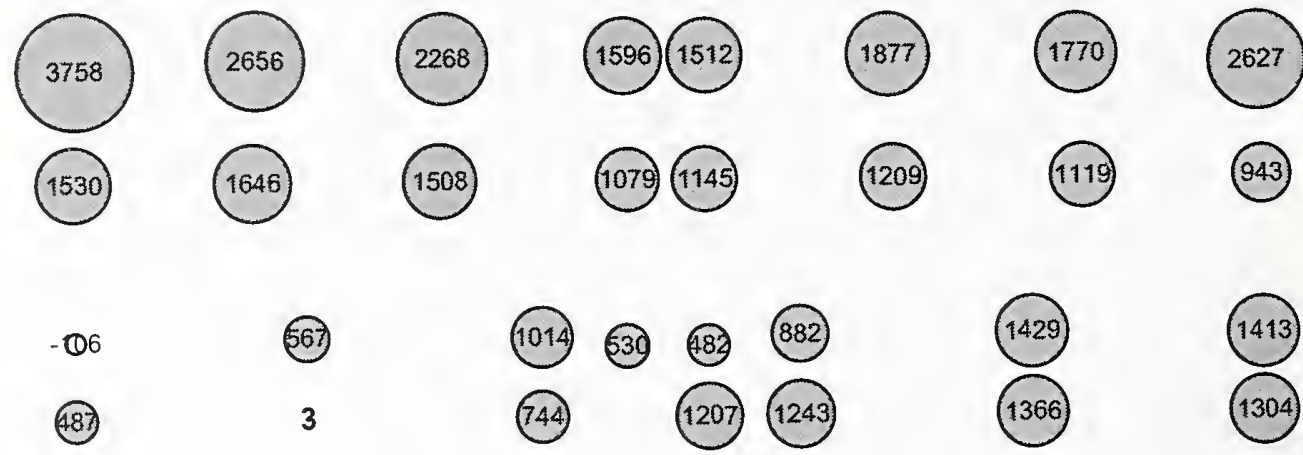

2

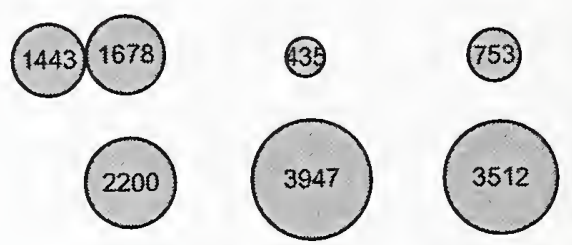

604

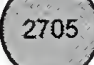

1001

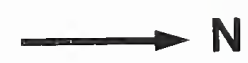

$N$

1008

(a) $20 \mathrm{~min}$

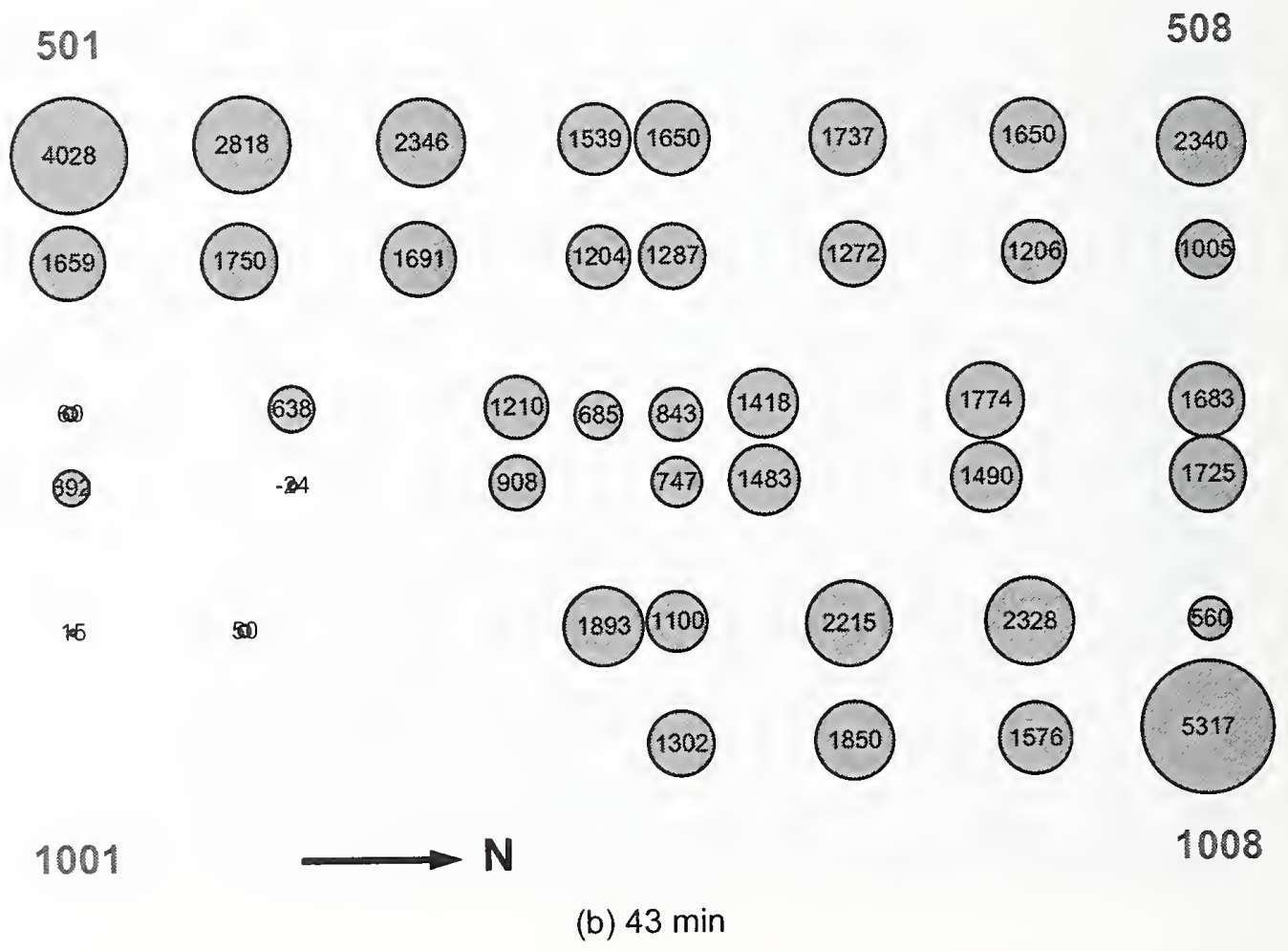

Figure 4-119. Core column loads (kip) at Floor 83 of WTC 2 for Case D conditions (compression is positive). 


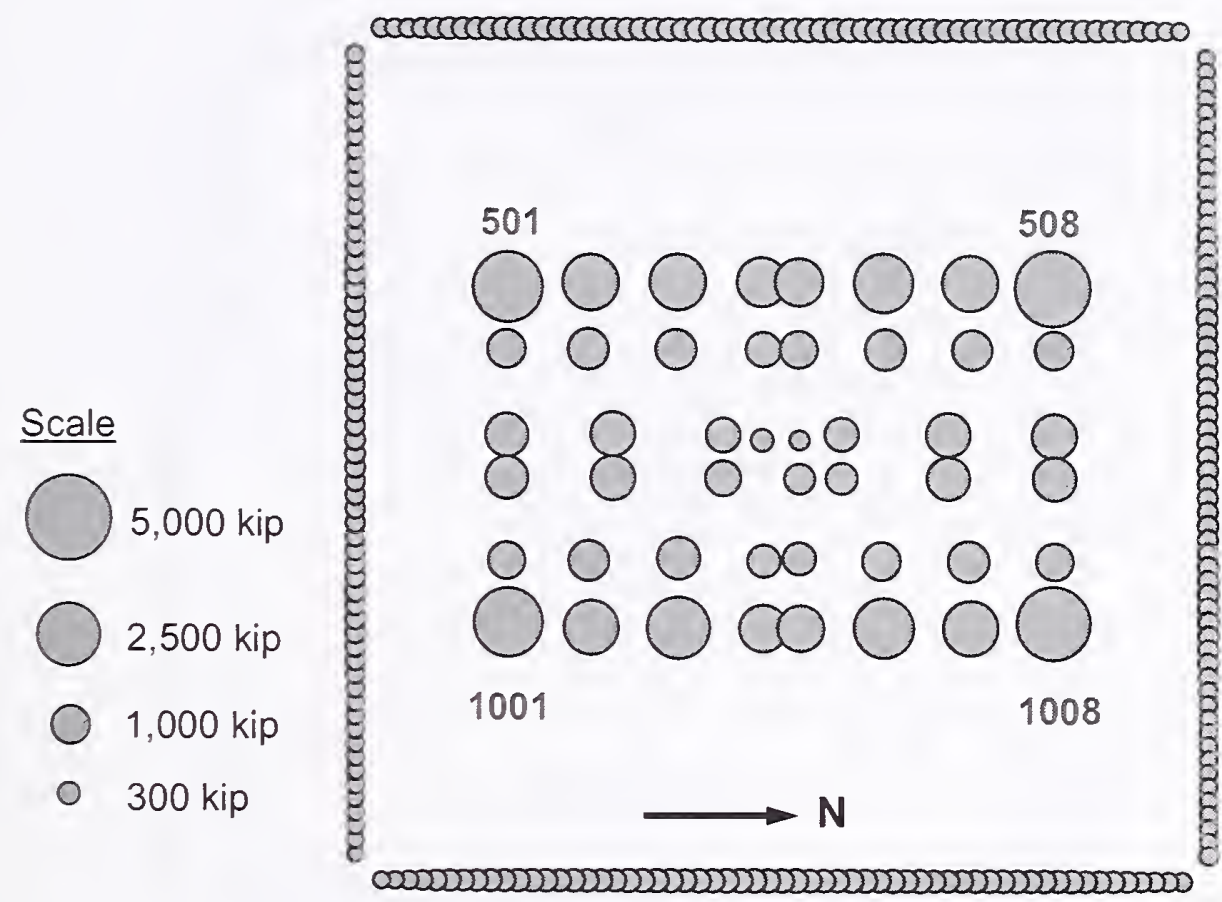

(a) Before impact

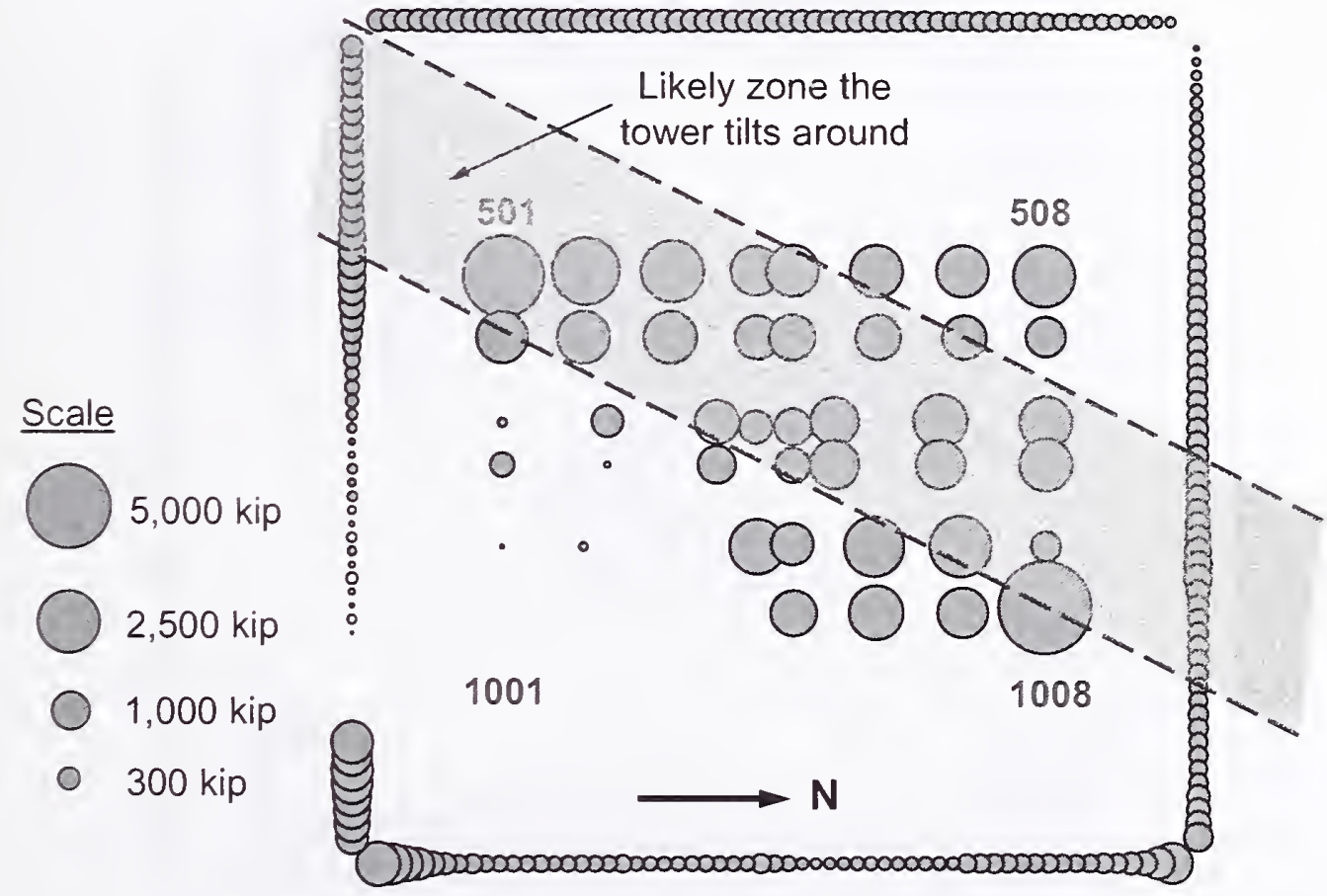

(b) $43 \mathrm{~min}$

Figure 4-120. Axial load in Floor 83 columns of WTC 2 for Case D conditions (compression is positive). 


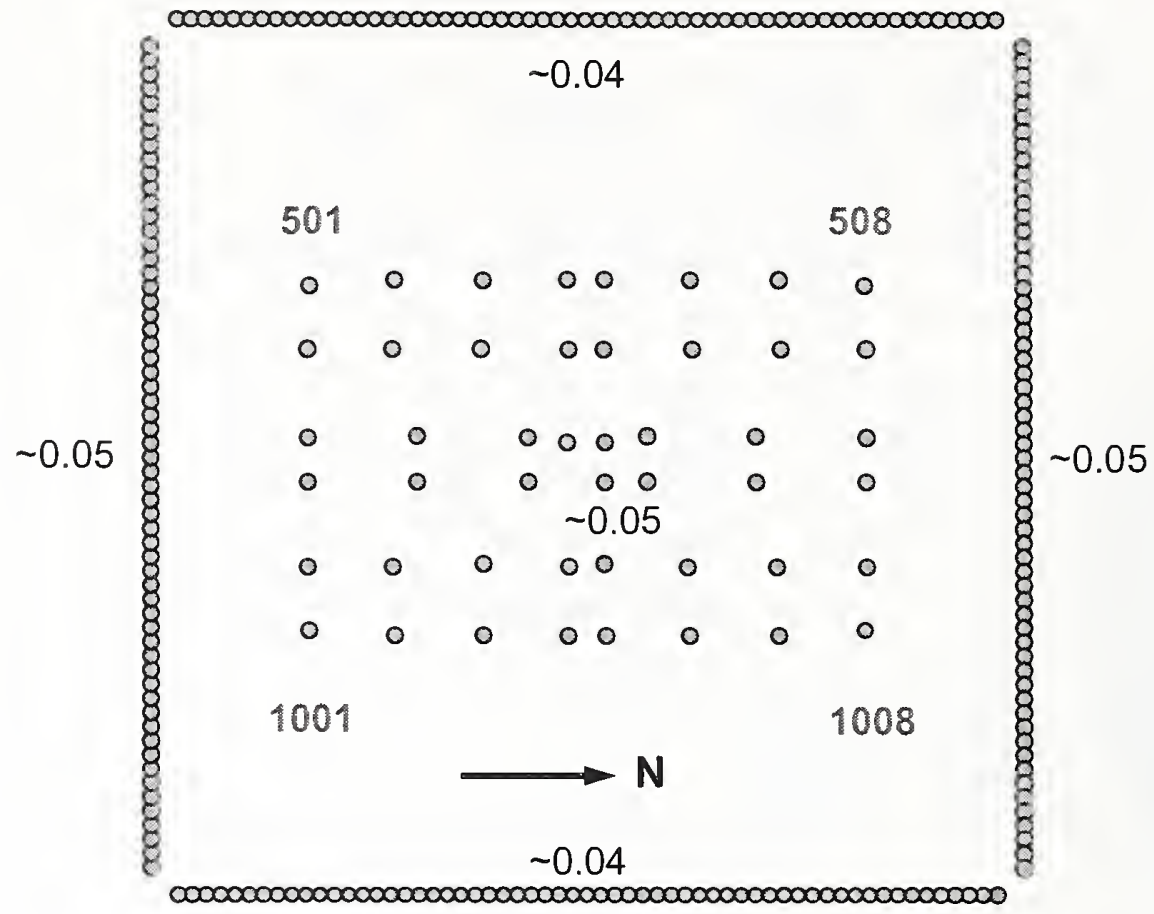

(a) Before impact

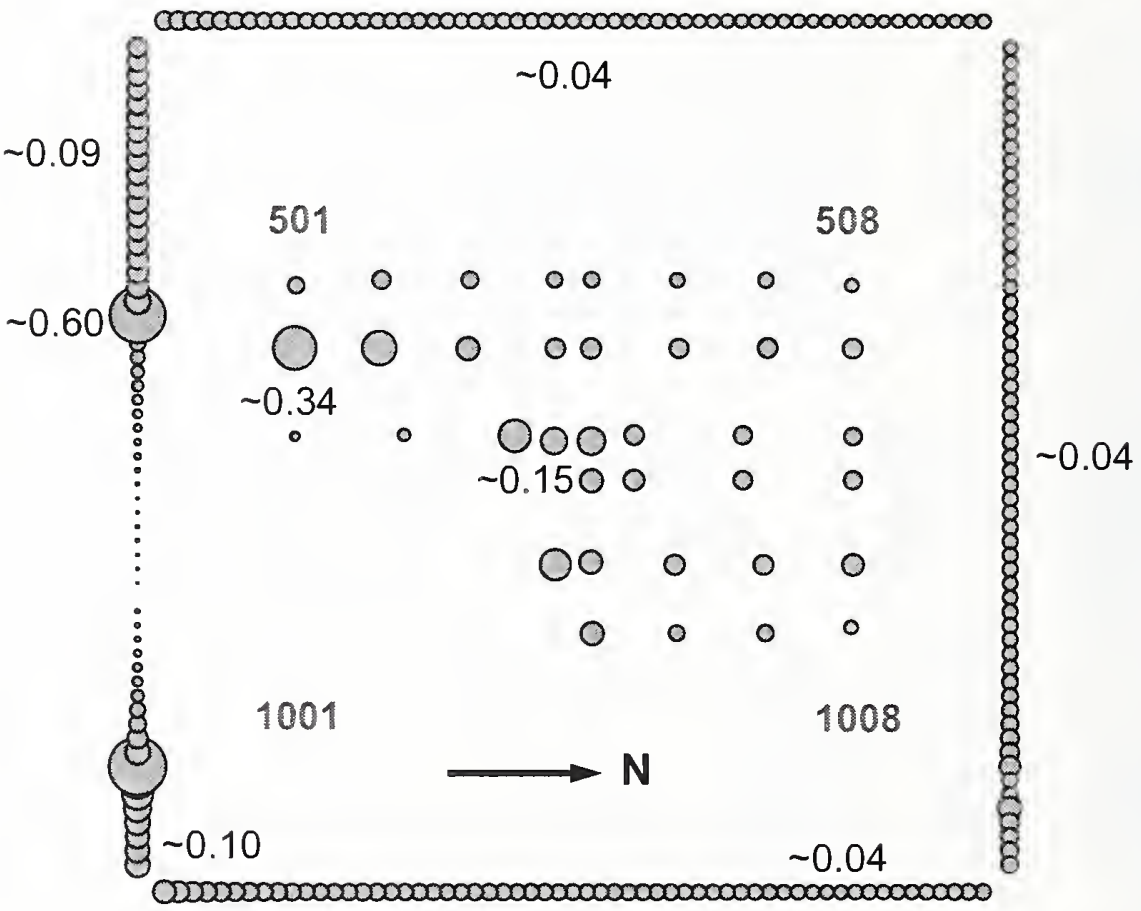

(b) After impact

Figure 4-121. Maximum elastic-plus-plastic strains for columns between Floor 78 and Floor 83 of WTC 2 for Case D conditions (compressive strain is positive; strain values are in percent). 


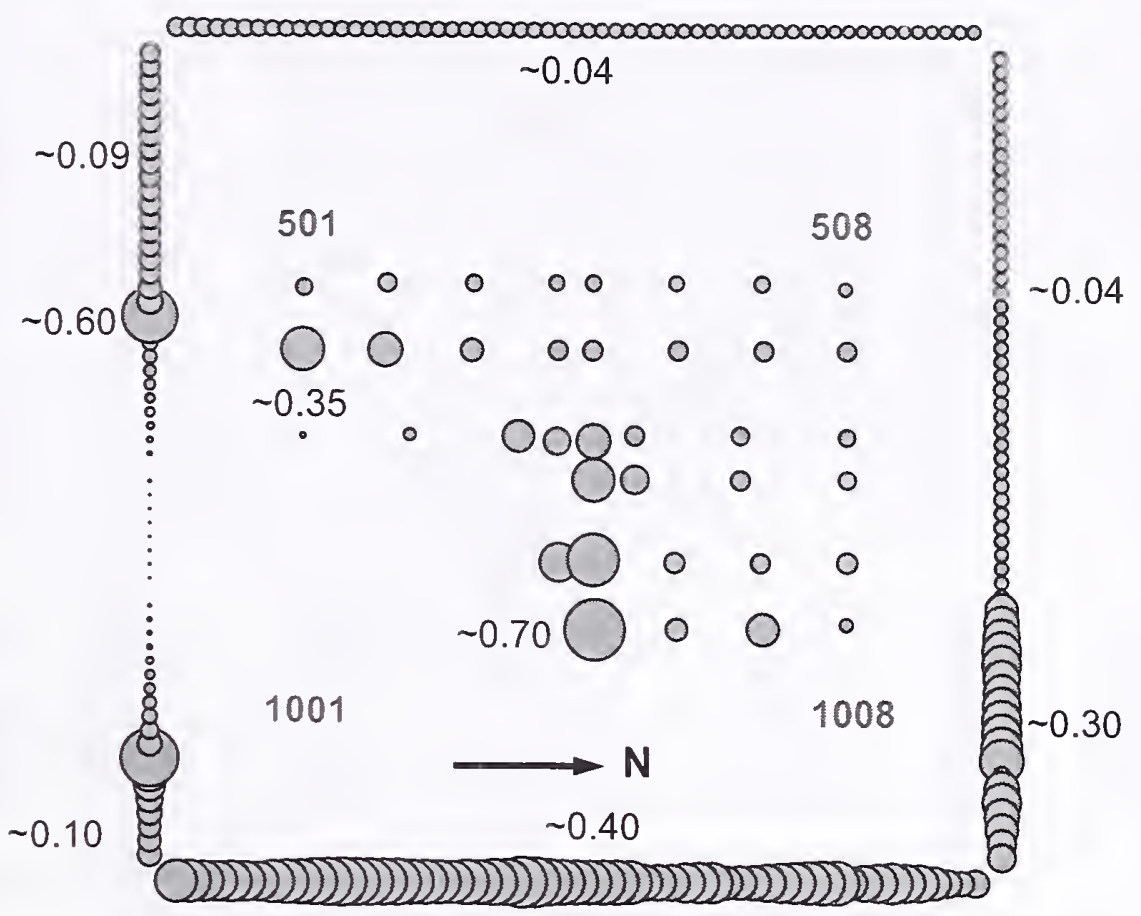

(a) $20 \mathrm{~min}$

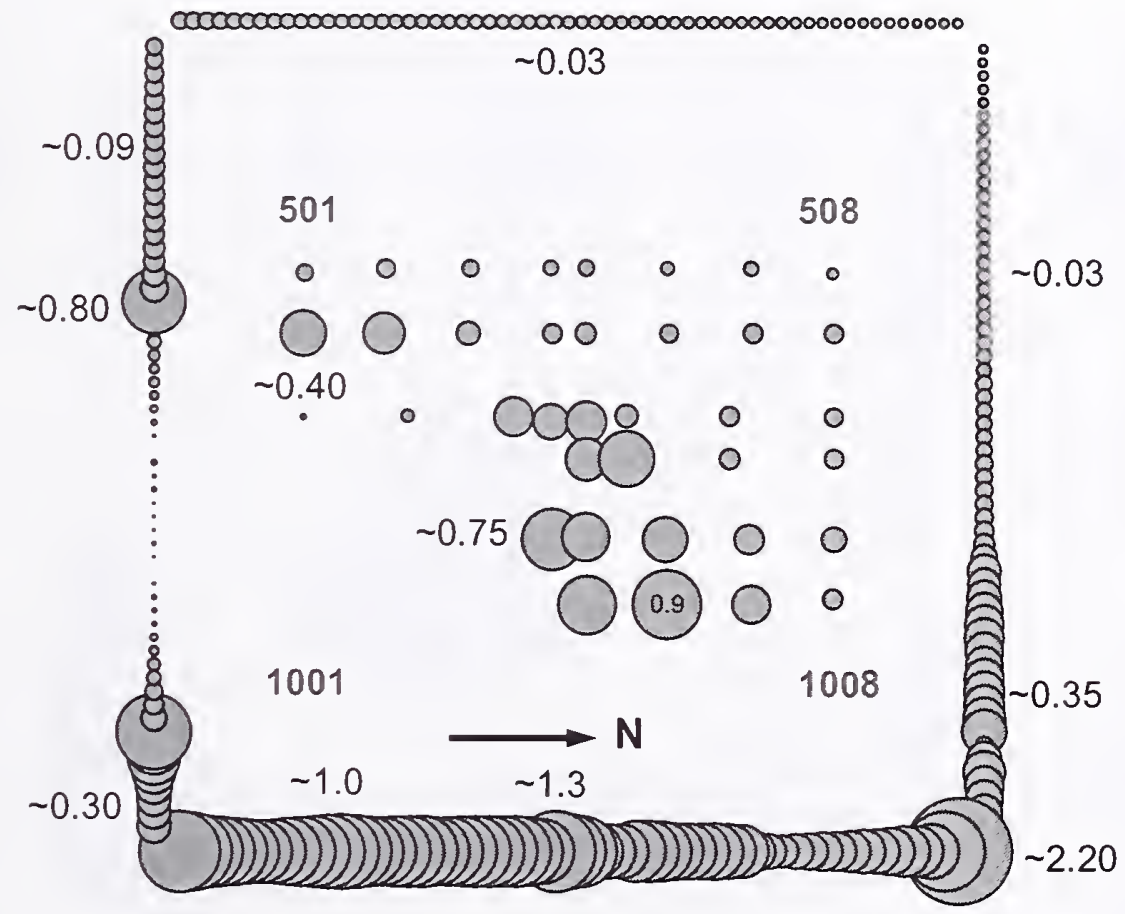

(b) $43 \mathrm{~min}$

Figure 4-122. Maximum elastic-plus-plastic strains for columns between Floor 78 and Floor 3 of WTC 2 for Case D conditions (compressive strain is positive; strain values are in percent). 


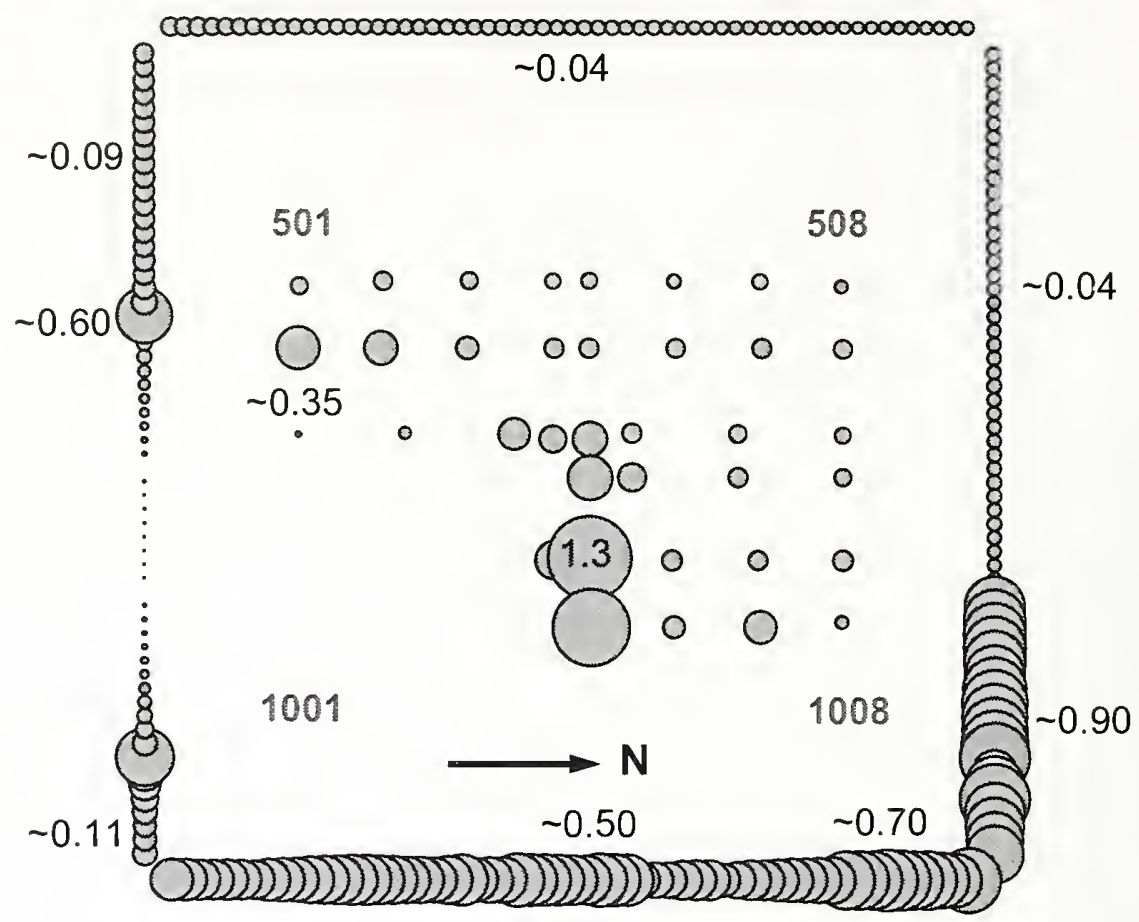

(a) $20 \mathrm{~min}$

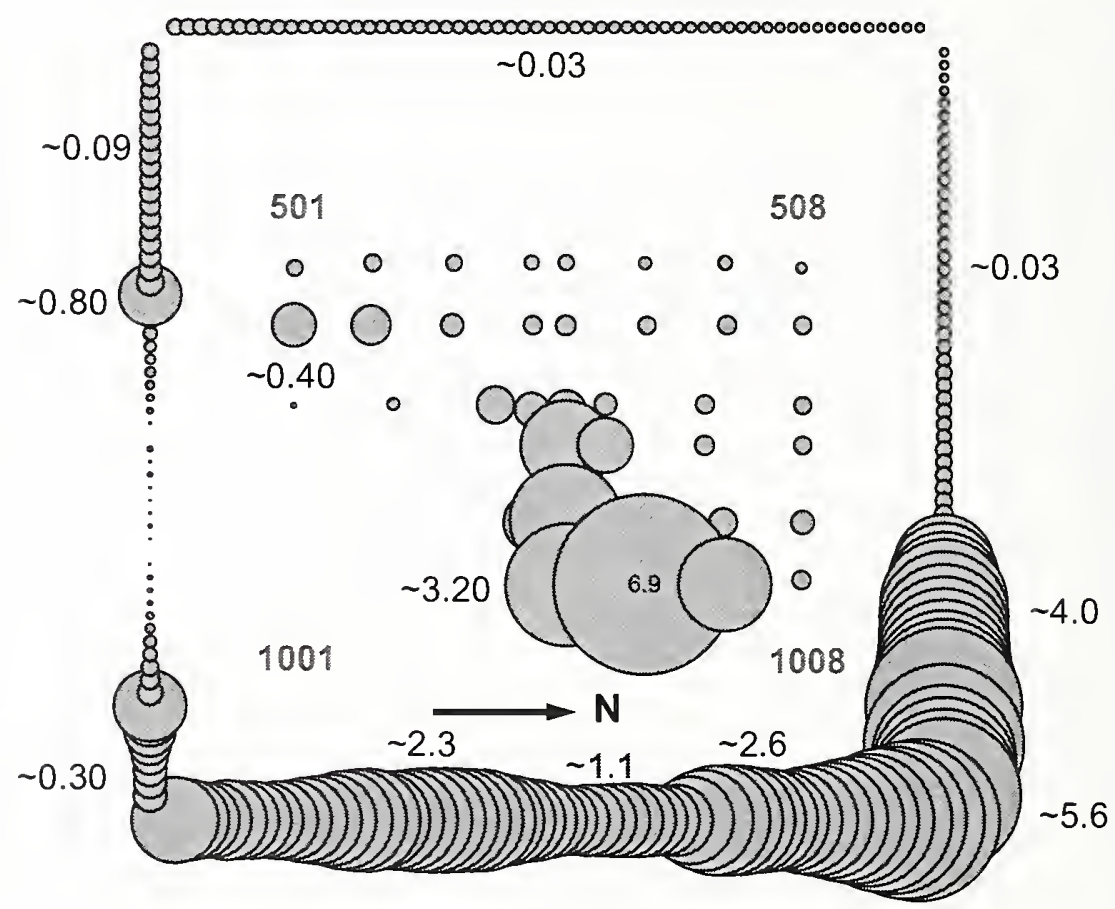

(b) $43 \mathrm{~min}$

Figure 4-123. Maximum elastic-plus-plastic-plus-creep strains for columns between Floor 78 and Floor 83 of WTC 2 for Case D conditions (compressive strain is positive; strain values are in percent). 

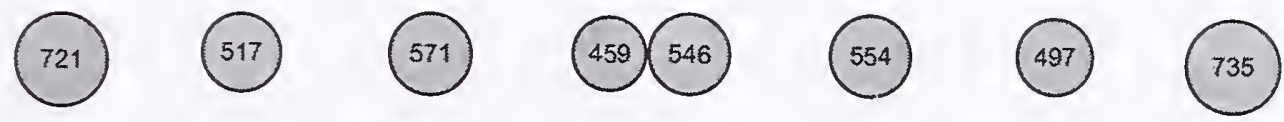

185

(338)

(357)

(237) (279)

(330)

(289)

157

252

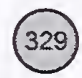

(218) (165) (214)

(285)

(17)

(292)

(320)

(241)

(13) (248)

(271)

166

(207)
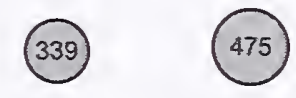

(246) 223

(330)

(317)

153
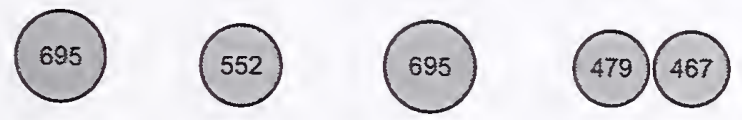

(560)

(507)

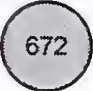

1001

N

1008

(a) Before impact

501

508
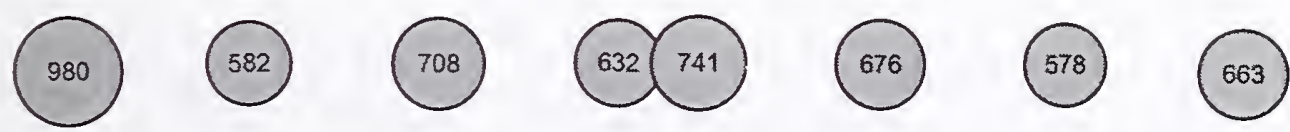

(170)

303

(366)

(350) 408

(439)

(351)

(168)

(7)

03

(226) (180) (185)

(395)

(229)

(18)

(2)

(300)

(17)

(367)

(383)

(210)

(233)

(10) 9

(245) (227)

(455)

(380)

(14)

$-939$

$-378$

(282) 418

708

(598)

589

1001

N

1008

(b) After impact

Figure 4-124. Axial load in core columns (kip) at Floor 105 (at hat truss level) of WTC 2 for Case $\mathrm{D}$ conditions (compression is positive). 


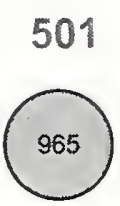

(163)

(8)

(189)

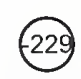

$-920$

1001

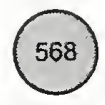

(292)

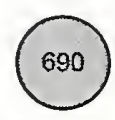

(351)

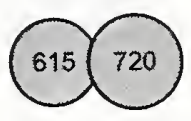

(344) 405

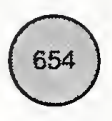

(433)

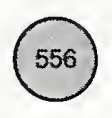

(341)
610

(154)

(212)

199

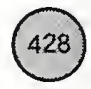

(15)

679

(297) 864

(717)

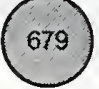

1008

(a) $20 \mathrm{~min}$

501

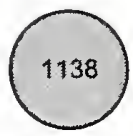

(216)

(5)

(14)

(20)

-779

1001<smiles>c1ccccc1</smiles>

(359)

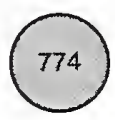

(443)

N
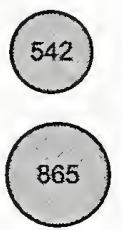

(387)

(396)

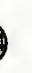




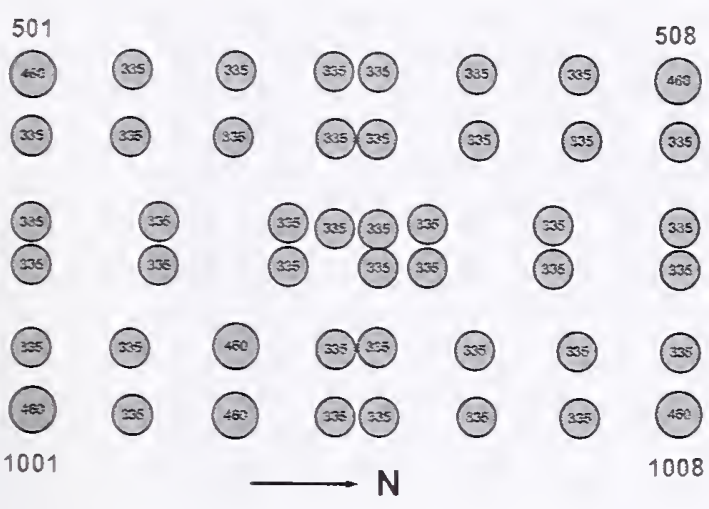

(a) Tension capacities of core column splices

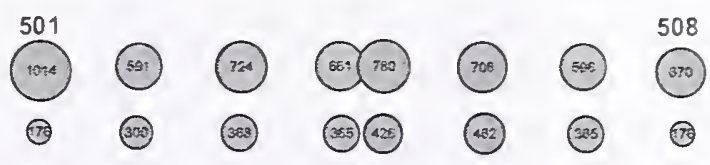

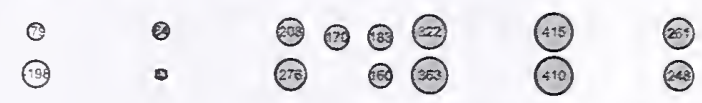

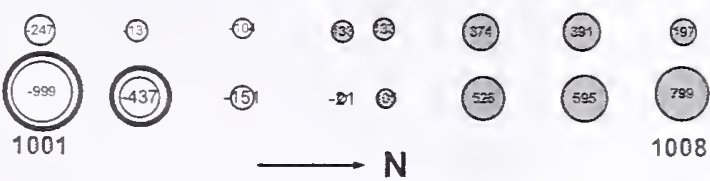

(b) Before splices 1001 and 1002 fail $(\mathrm{t}=40 \mathrm{~min}$ )

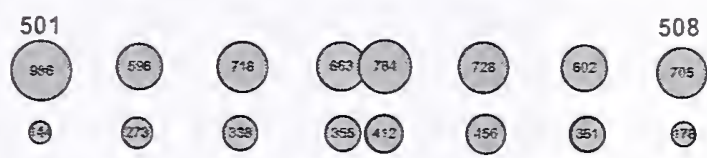

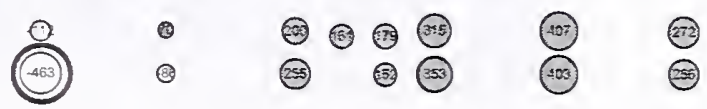

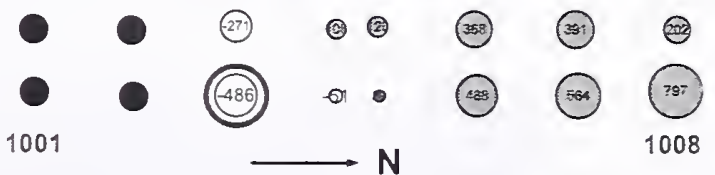
(d) After splices 901 and 902 fail $(\mathrm{t}=40 \mathrm{~min})\left(2^{\mathrm{nd}}\right.$ iteration $)$

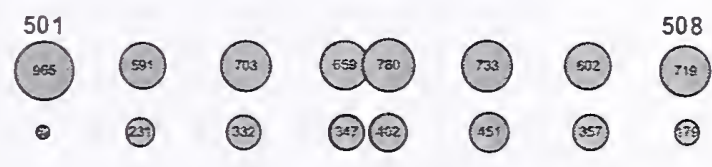

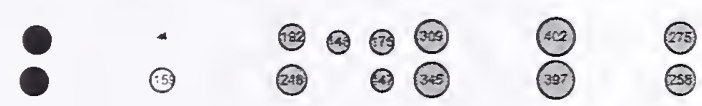

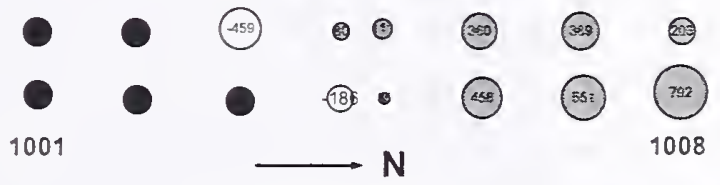
(f) After splice 701 fails $(\mathrm{t}=40 \mathrm{~min})\left(4^{\text {th }}\right.$ iteration $)$

(e) After splices 801 and 1003 fail $(t=40 \mathrm{~min})\left(3^{\text {rd }}\right.$ iteration $)$

Already failed splices

Figure 4-126. Progressive failure of core column splices at Floor 105 of WTC 2 (compression is positive; values are in kip). 


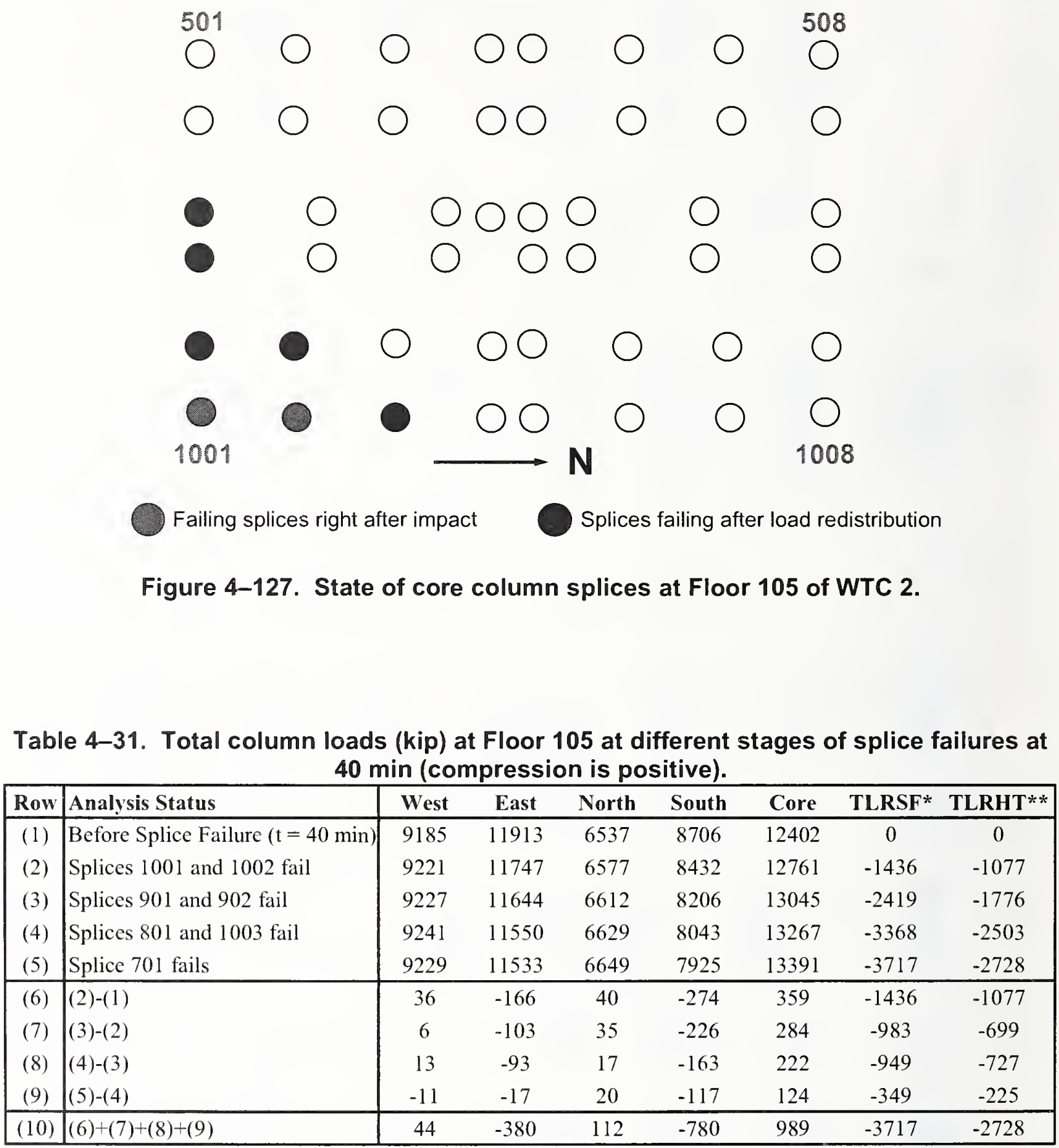

Table 4-31. Total column loads (kip) at Floor 105 at different stages of splice failures at

* Total load released by the splice failure

** Total load retransferred to the hat-truss 


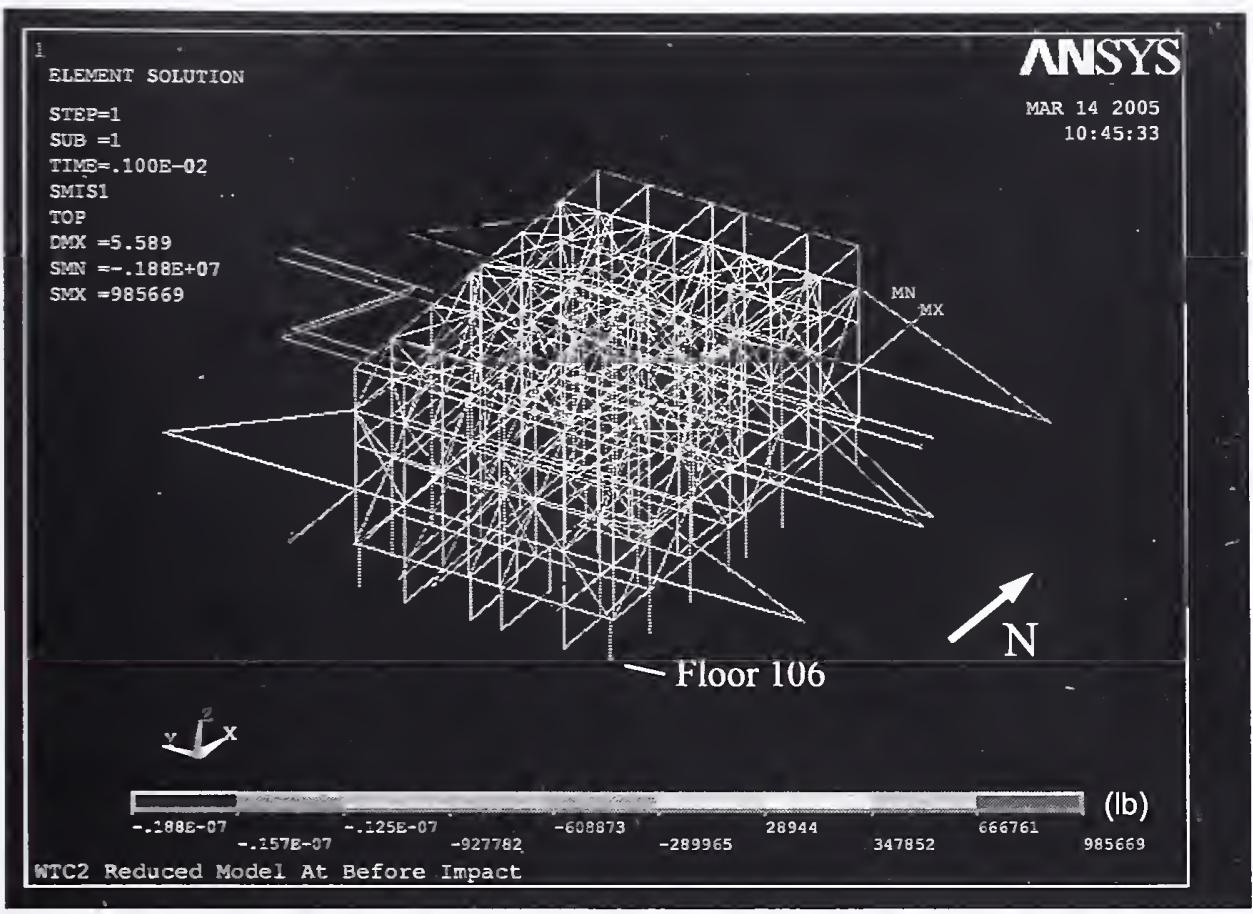

(a) Before impact

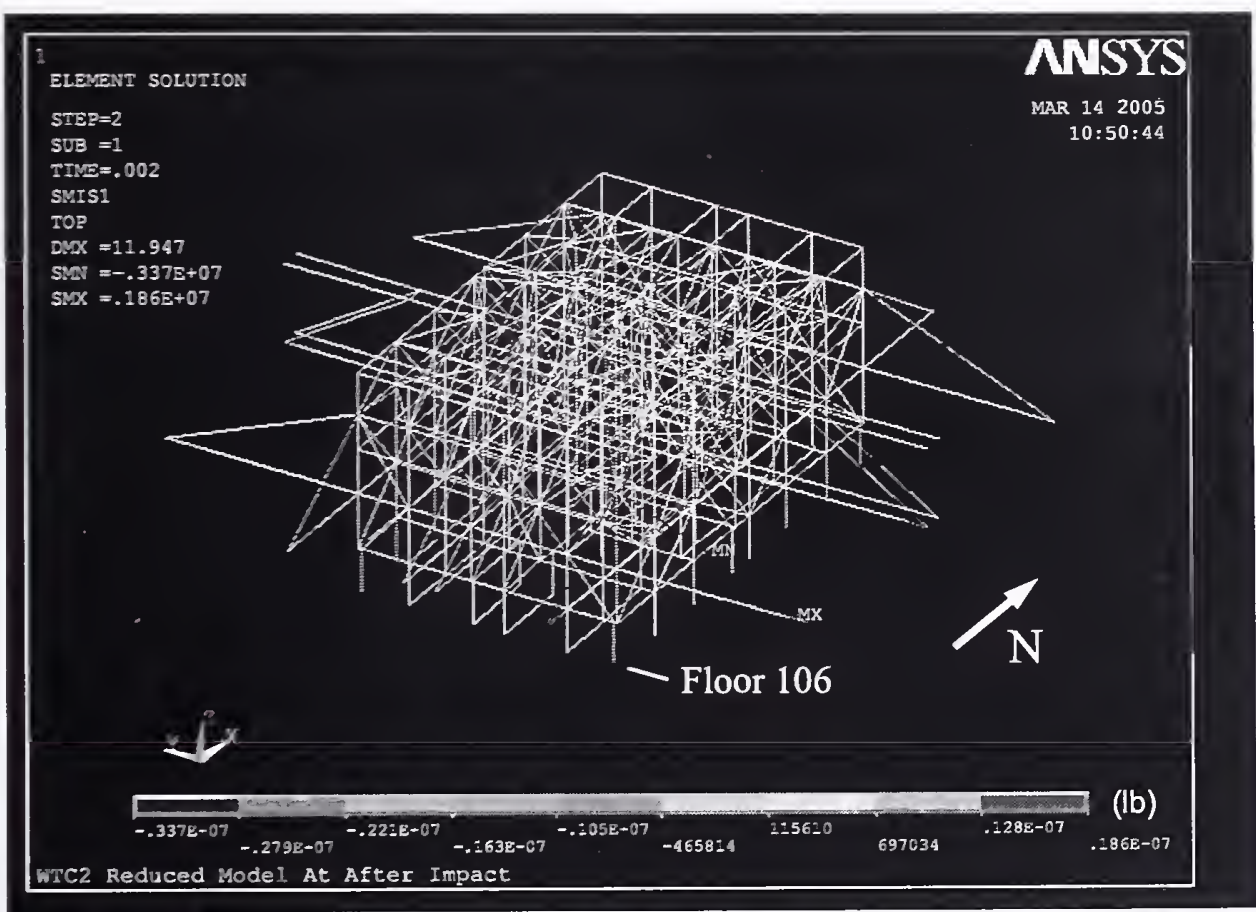

(b) After impact

Figure 4-128. Axial force in hat truss members of WTC 2 for Case D conditions (tension is positive). 


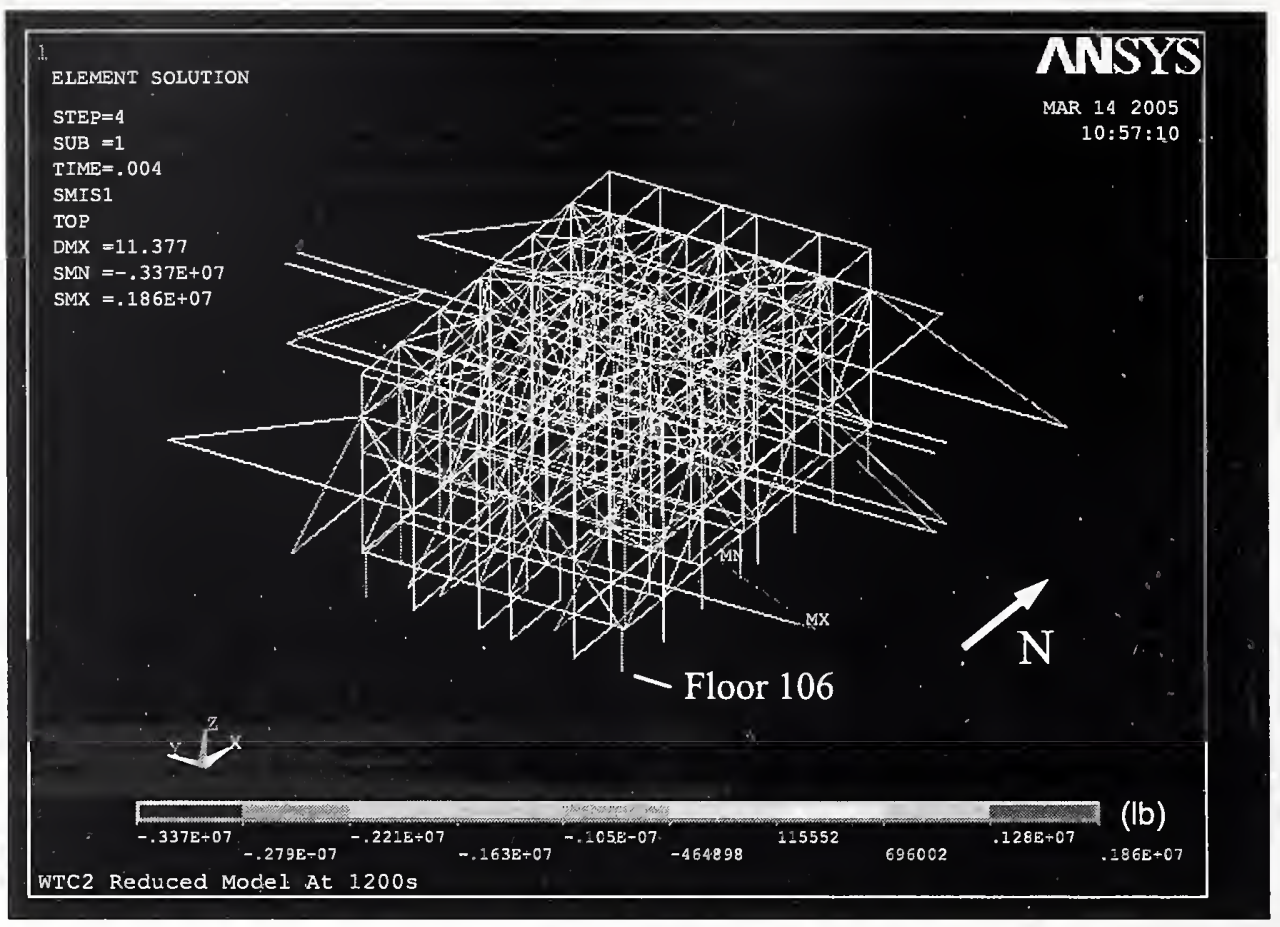

(a) $20 \mathrm{~min}$

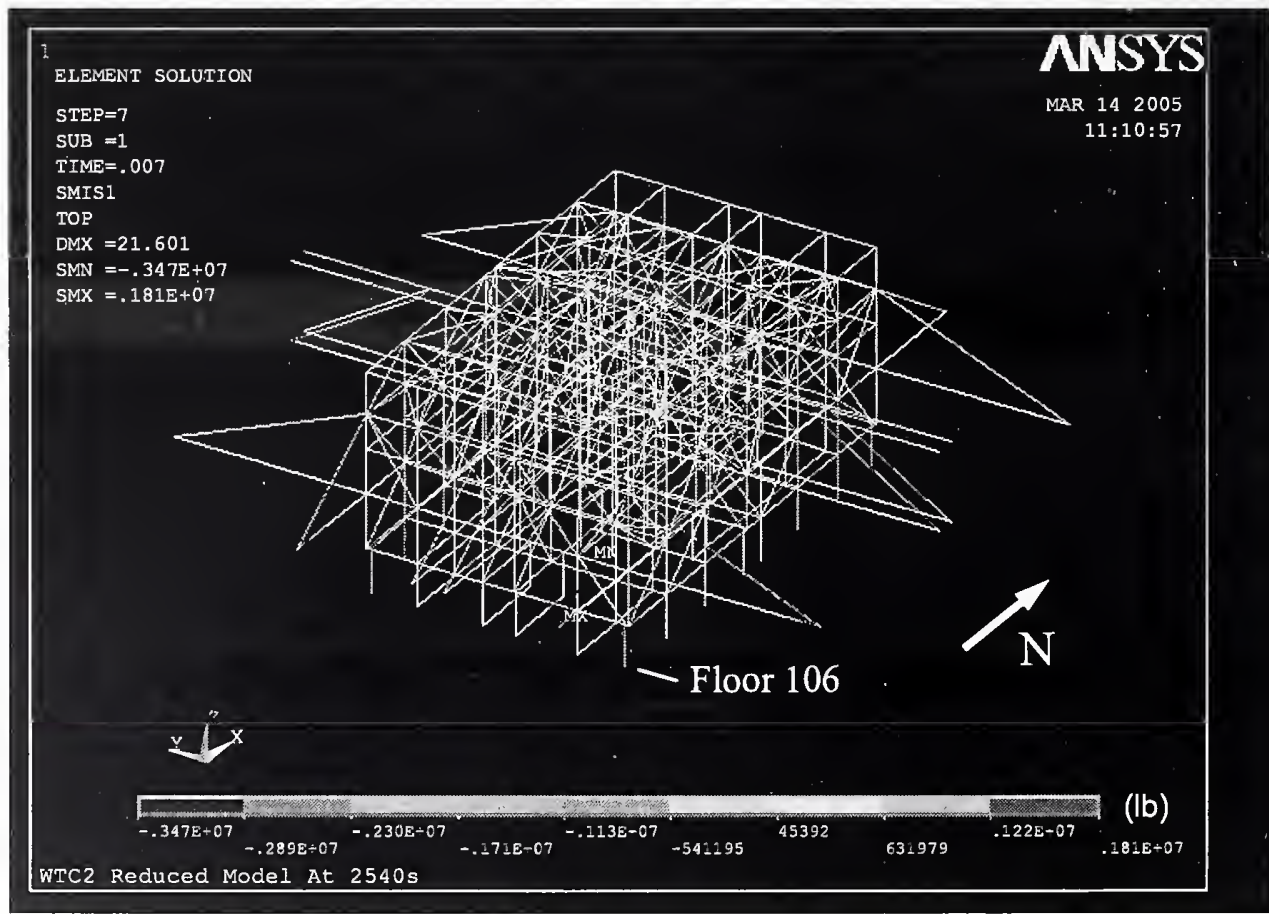

(b) $43 \mathrm{~min}$

Figure 4-129. Axial force in hat truss members of WTC 2 for Case D conditions (tension is positive). 


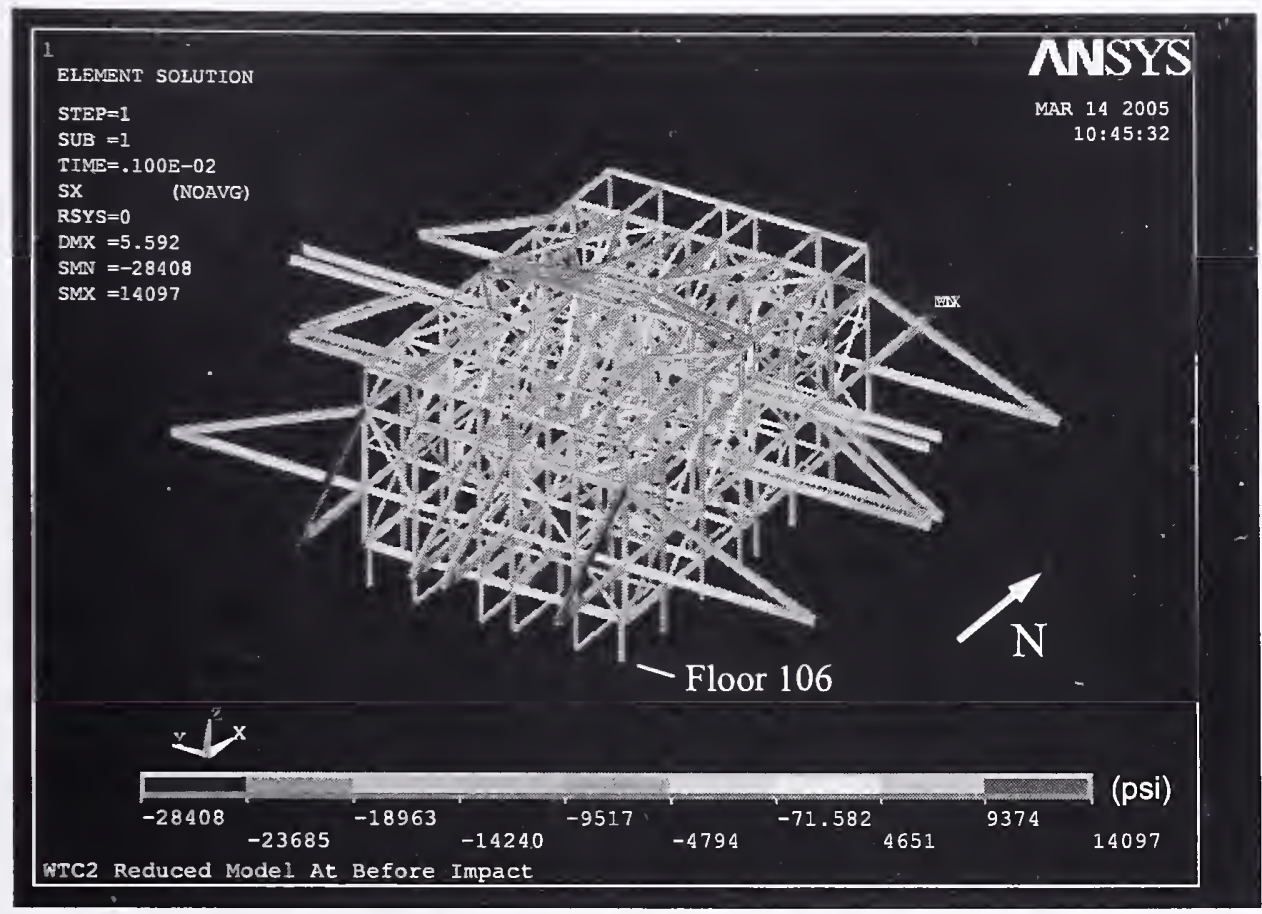

(a) Before impact

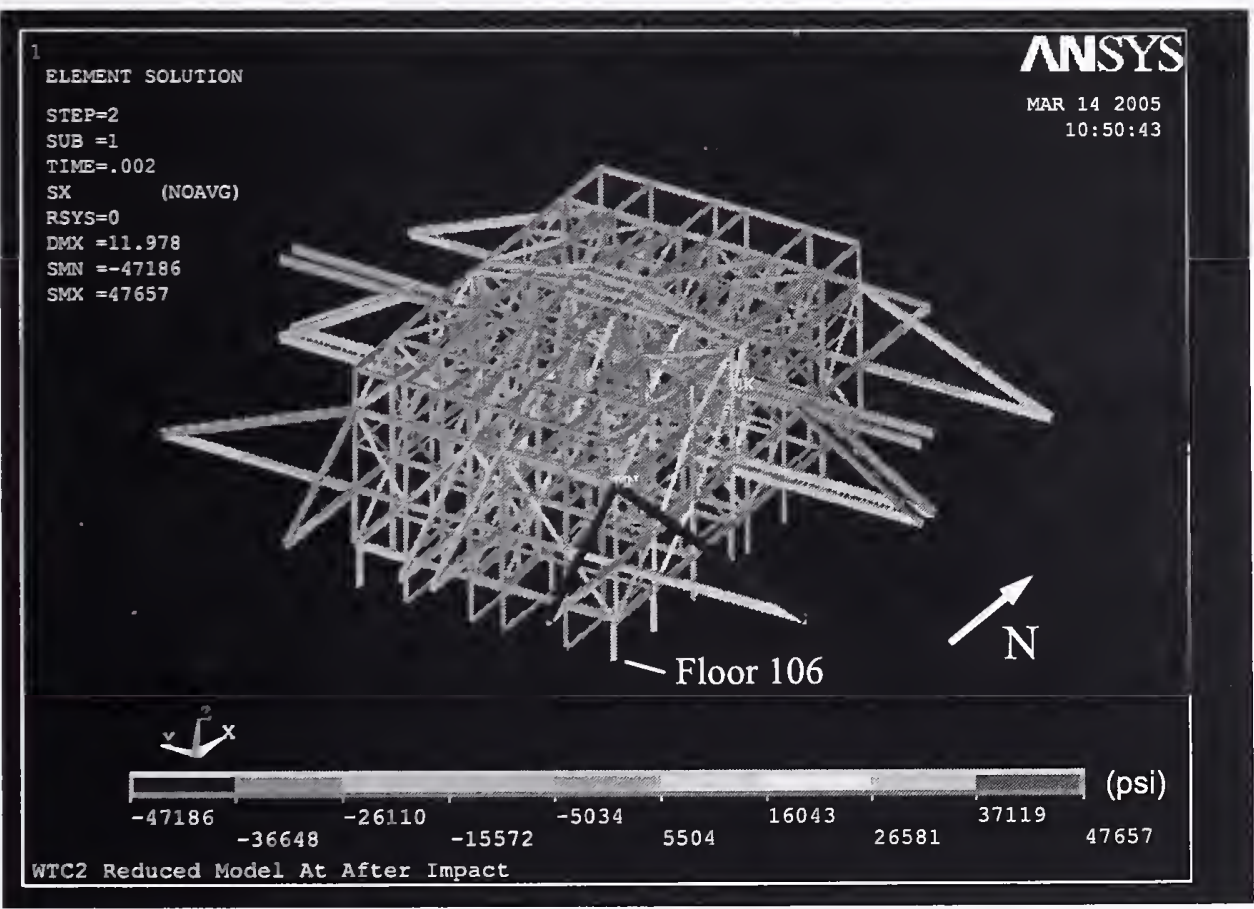

(b) After impact

Figure 4-130. Axial stress in hat truss members of WTC 2 for Case D conditions (tension is positive). 


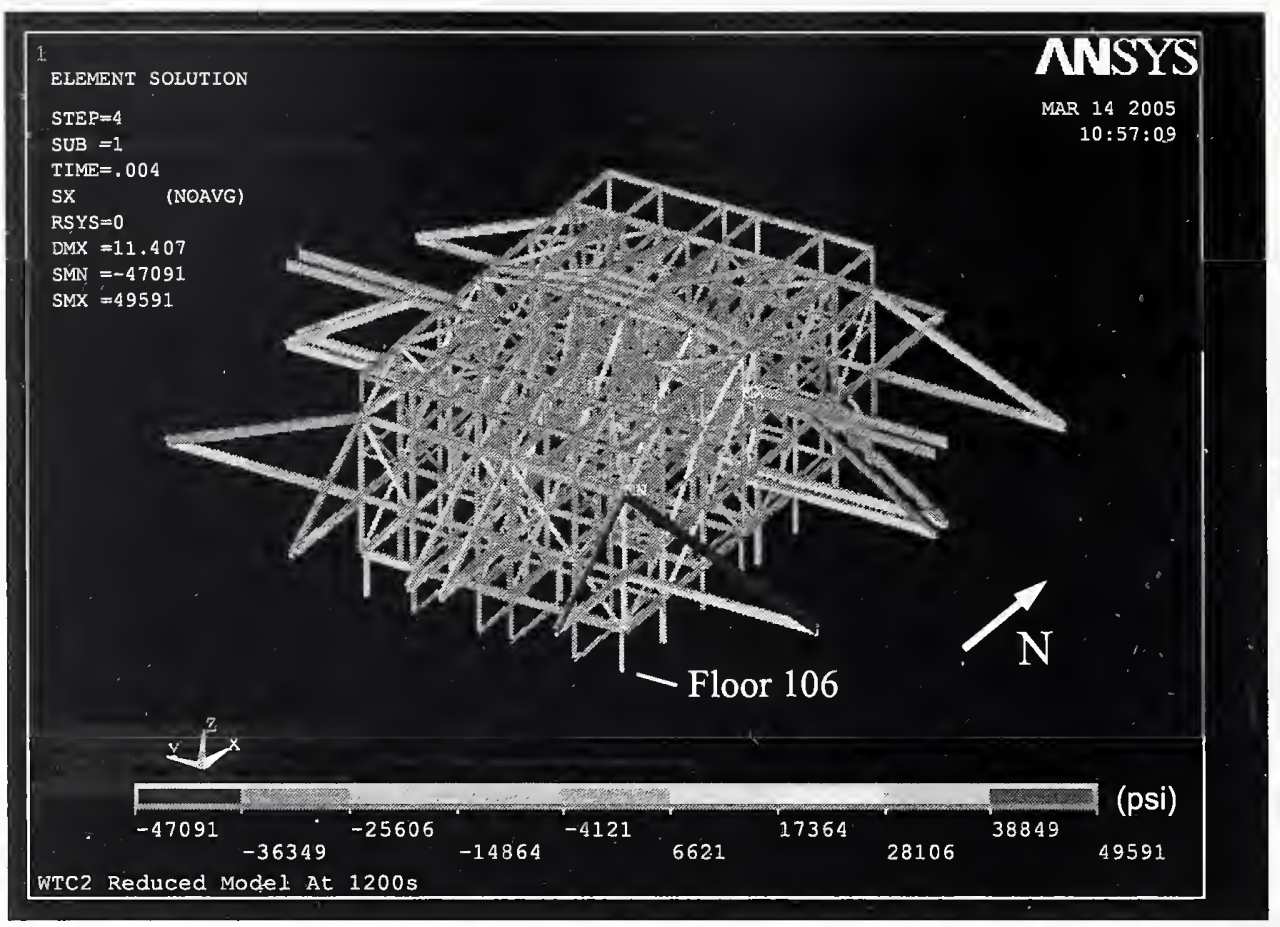

(a) $20 \mathrm{~min}$

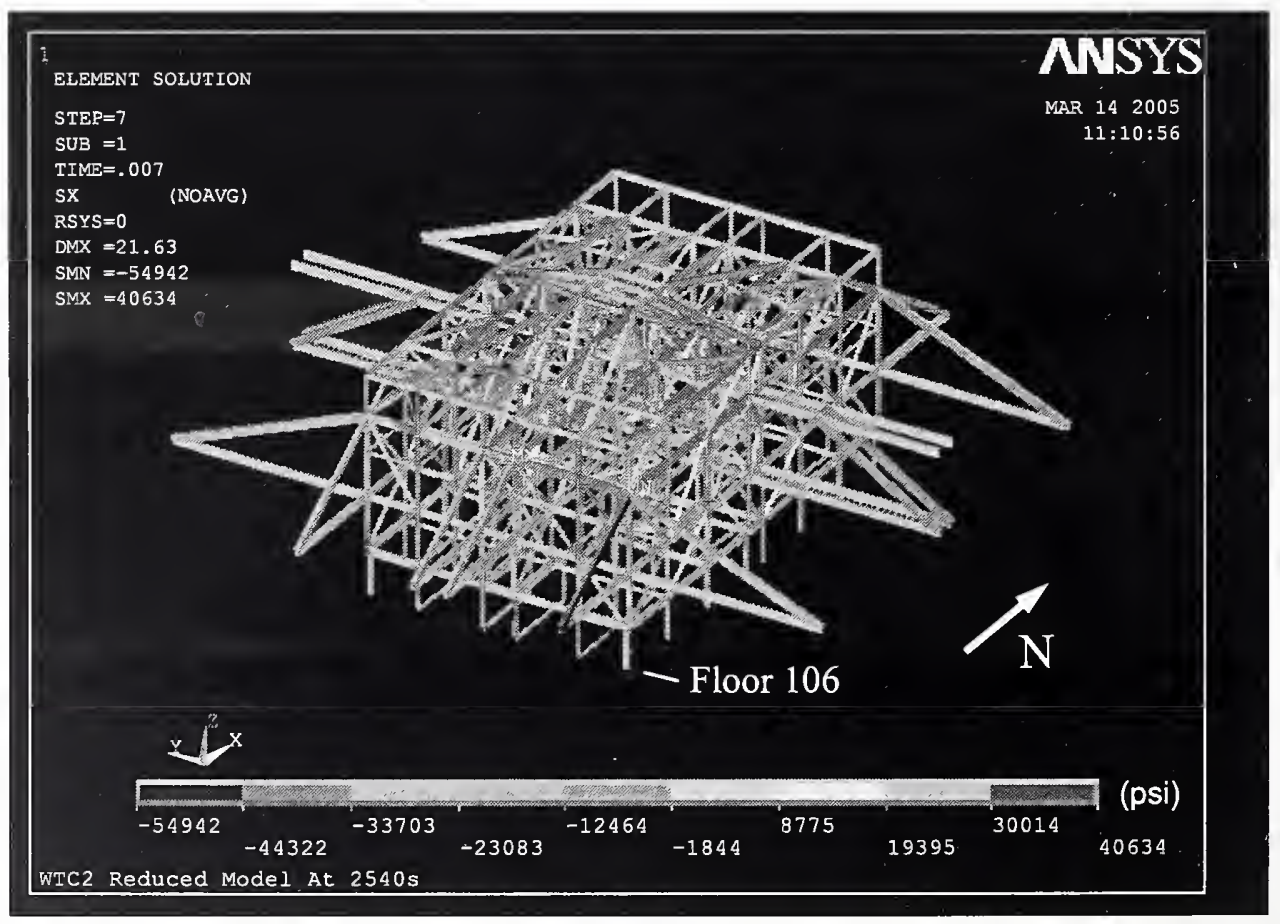

(b) $43 \mathrm{~min}$

Figure 4-131. Axial stress in hat truss members of WTC 2 for Case D conditions (tension is positive). 
Table 4-32. Demand-to-capacity ratios for outriggers of WTC 2 for Case D conditions (outrigger IDs are shown in Fig. 4-3).

\begin{tabular}{|c|c|c|c|c|c|c|c|c|c|}
\hline Outrigger ID & Bfr. Imp. & Aftr. Imp. & $10 \mathrm{~min}$ & $20 \mathrm{~min}$ & $30 \mathrm{~min}$ & $40 \mathrm{~min}$ & $40 * \min$ & $40 * * \min$ & $43 \mathrm{~min}$ \\
\hline \multicolumn{10}{|c|}{\begin{tabular}{|l|l|} 
West & \\
\end{tabular}} \\
\hline$A$ & 0.23 & 0.61 & 0.61 & 0.64 & 0.66 & 0.63 & 0.63 & 0.38 & 0.25 \\
\hline B & 0.19 & 0.30 & 0.30 & 0.32 & 0.33 & 0.31 & 0.31 & 0.25 & $-0.03 * * *$ \\
\hline $\mathrm{C}$ & 0.19 & 0.27 & 0.27 & 0.28 & 0.30 & 0.27 & 0.28 & 0.22 & -0.07 \\
\hline D & 0.21 & 0.18 & 0.18 & 0.19 & 0.21 & 0.18 & 0.20 & 0.19 & -0.29 \\
\hline \multicolumn{10}{|l|}{ North } \\
\hline $\mathbf{E}$ & 0.29 & 0.11 & 0.10 & 0.10 & 0.13 & 0.14 & 0.17 & 0.09 & -0.01 \\
\hline F & 0.21 & 0.10 & 0.08 & 0.08 & 0.10 & 0.12 & 0.13 & 0.11 & 0.08 \\
\hline G & 0.20 & 0.11 & 0.08 & 0.08 & 0.11 & 0.13 & 0.14 & 0.13 & 0.10 \\
\hline $\mathbf{H}$ & 0.30 & 0.21 & 0.16 & 0.18 & 0.19 & 0.18 & 0.19 & 0.24 & 0.24 \\
\hline \multicolumn{10}{|l|}{ East } \\
\hline 1 & 0.22 & 0.39 & 0.35 & 0.34 & 0.45 & 0.43 & 0.42 & 0.41 & -0.18 \\
\hline $\mathbf{J}$ & 0.18 & 0.56 & 0.52 & 0.53 & 0.66 & 0.65 & 0.61 & 0.71 & 0.02 \\
\hline $\mathbf{K}$ & 0.18 & 0.62 & 0.58 & 0.59 & 0.72 & 0.72 & 0.66 & 0.79 & 0.09 \\
\hline L & 0.22 & 1.12 & 1.09 & 1.12 & 1.24 & 1.30 & 1.11 & 0.00 & 0.72 \\
\hline \multicolumn{10}{|l|}{ South } \\
\hline$M$ & 0.30 & 0.68 & 0.64 & 0.64 & 0.70 & 0.75 & 0.52 & 0.72 & 0.87 \\
\hline $\mathrm{N}$ & 0.22 & 0.34 & 0.32 & 0.33 & 0.36 & 0.38 & 0.28 & 0.34 & 0.40 \\
\hline O & 0.22 & 0.30 & 0.28 & 0.30 & 0.32 & 0.33 & 0.27 & 0.31 & 0.33 \\
\hline $\mathrm{P}$ & 0.31 & 0.51 & 0.50 & 0.52 & 0.53 & 0.53 & 0.50 & 0.57 & 0.49 \\
\hline
\end{tabular}

* After load redistribution due to core column splice failures.

** After Outrigger L was removed.

*** Negative value indicates tension 
Table 4-33. Demand to capacity ratios for columns supporting outriggers at Floor 107 of WTC 2 for Case D conditions.

\begin{tabular}{|c|c|c|c|c|c|c|c|}
\hline Column ID & Bfr. Inıp. & Aftr. Imp. & $10 \mathrm{~min}$ & $20 \mathrm{~min}$ & $30 \mathrm{~min}$ & $40 \mathrm{~min}$ & $43 \mathrm{~min}$ \\
\hline \multicolumn{8}{|l|}{ West } \\
\hline 110 & 0.26 & 0.46 & 0.46 & 0.47 & 0.48 & 0.47 & 0.28 \\
\hline 111 & 0.26 & 0.39 & 0.39 & 0.40 & 0.41 & 0.40 & 0.26 \\
\hline 129 & 0.27 & 0.37 & 0.37 & 0.38 & 0.40 & 0.37 & 0.06 \\
\hline 130 & 0.31 & 0.39 & 0.39 & 0.40 & 0.41 & 0.39 & 0.09 \\
\hline 131 & 0.27 & 0.35 & 0.34 & 0.36 & 0.37 & 0.35 & 0.05 \\
\hline 149 & 0.25 & 0.24 & 0.24 & 0.25 & 0.25 & 0.24 & 0.08 \\
\hline 150 & 0.25 & 0.23 & 0.23 & 0.23 & 0.24 & 0.23 & 0.00 \\
\hline \multicolumn{8}{|l|}{ North } \\
\hline 217 & 0.17 & 0.11 & 0.11 & 0.11 & 0.12 & 0.12 & 0.07 \\
\hline 218 & 0.23 & 0.12 & 0.11 & 0.11 & 0.13 & 0.14 & 0.05 \\
\hline 228 & 0.27 & 0.17 & 0.15 & 0.15 & 0.17 & 0.19 & 0.15 \\
\hline 229 & 0.22 & 0.14 & 0.12 & 0.12 & 0.14 & 0.16 & 0.13 \\
\hline 231 & 0.26 & 0.18 & 0.15 & 0.15 & 0.18 & 0.19 & 0.17 \\
\hline 232 & 0.26 & 0.18 & 0.15 & 0.15 & 0.18 & 0.20 & 0.18 \\
\hline 242 & 0.20 & 0.15 & 0.12 & 0.13 & 0.14 & 0.14 & 0.17 \\
\hline 243 & 0.19 & 0.16 & 0.14 & 0.14 & 0.15 & 0.14 & 0.17 \\
\hline \multicolumn{8}{|l|}{ East } \\
\hline 310 & 0.25 & 0.34 & 0.32 & 0.31 & 0.36 & 0.36 & 0.05 \\
\hline 311 & 0.27 & 0.33 & 0.32 & 0.31 & 0.35 & 0.35 & 0.13 \\
\hline 329 & 0.26 & 0.61 & 0.57 & 0.58 & 0.69 & 0.69 & 0.12 \\
\hline 330 & 0.31 & 0.68 & 0.65 & 0.66 & 0.77 & 0.77 & 0.19 \\
\hline 331 & 0.27 & 0.63 & 0.60 & 0.61 & 0.72 & 0.72 & 0.16 \\
\hline 349 & 0.26 & 0.56 & 0.55 & 0.56 & 0.60 & 0.62 & 0.42 \\
\hline 350 & 0.26 & 0.71 & 0.69 & 0.71 & 0.77 & 0.81 & 0.51 \\
\hline \multicolumn{8}{|l|}{ South } \\
\hline 417 & 0.18 & 0.30 & 0.29 & 0.29 & 0.31 & 0.33 & 0.37 \\
\hline 418 & 0.24 & 0.46 & 0.43 & 0.44 & 0.47 & 0.50 & 0.57 \\
\hline 428 & 0.28 & 0.38 & 0.36 & 0.37 & 0.39 & 0.41 & 0.43 \\
\hline 429 & 0.23 & 0.30 & 0.29 & 0.30 & 0.32 & 0.33 & 0.34 \\
\hline 431 & 0.26 & 0.33 & 0.31 & 0.32 & 0.34 & 0.36 & 0.36 \\
\hline 432 & 0.27 & 0.35 & 0.34 & 0.35 & 0.37 & 0.38 & 0.38 \\
\hline 442 & 0.21 & 0.32 & 0.31 & 0.32 & 0.33 & 0.33 & 0.31 \\
\hline 443 & 0.20 & 0.28 & 0.28 & 0.28 & 0.29 & 0.29 & 0.27 \\
\hline
\end{tabular}




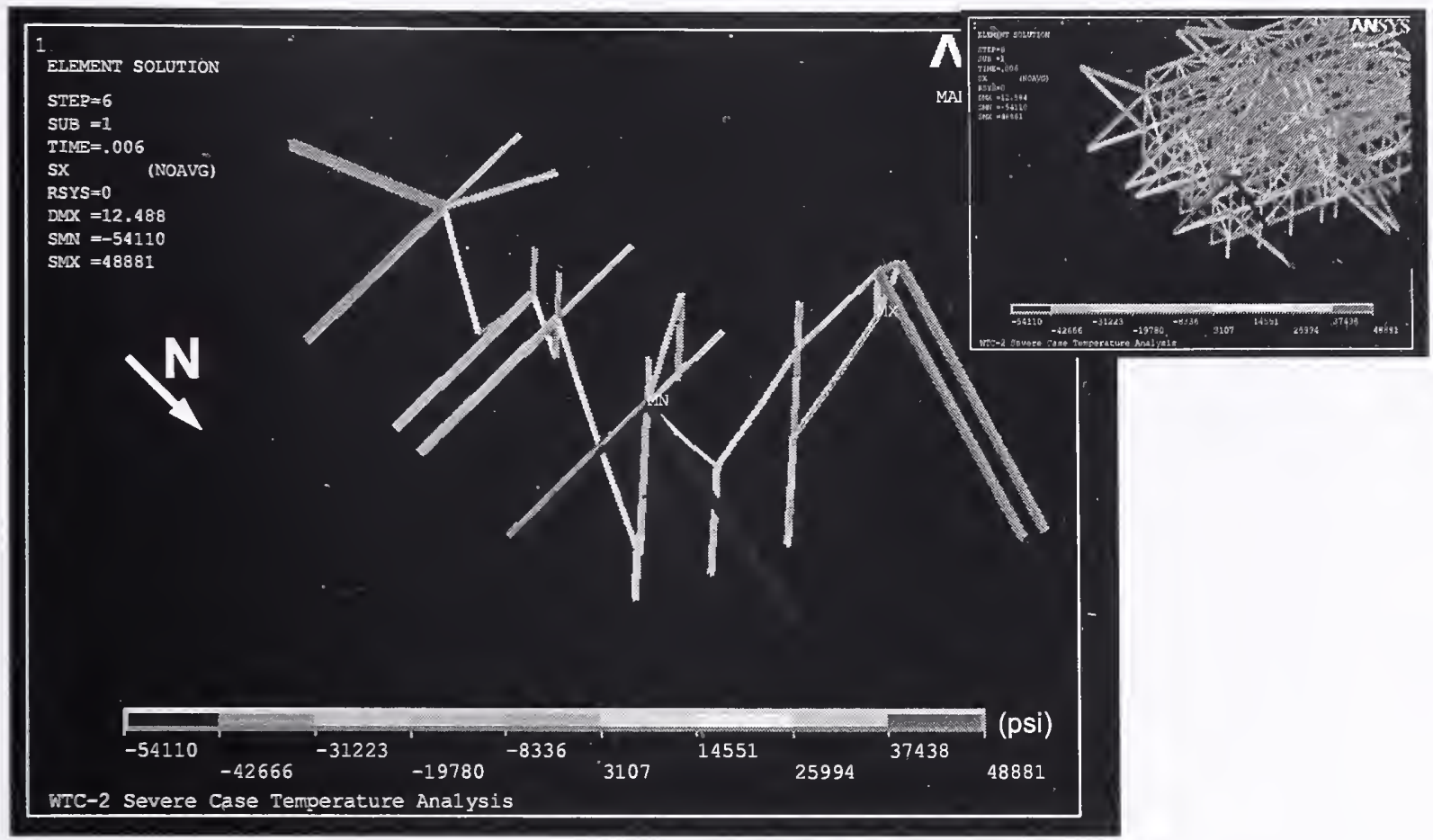

Figure 4-132. Primary load path within the hat truss of WTC 2.

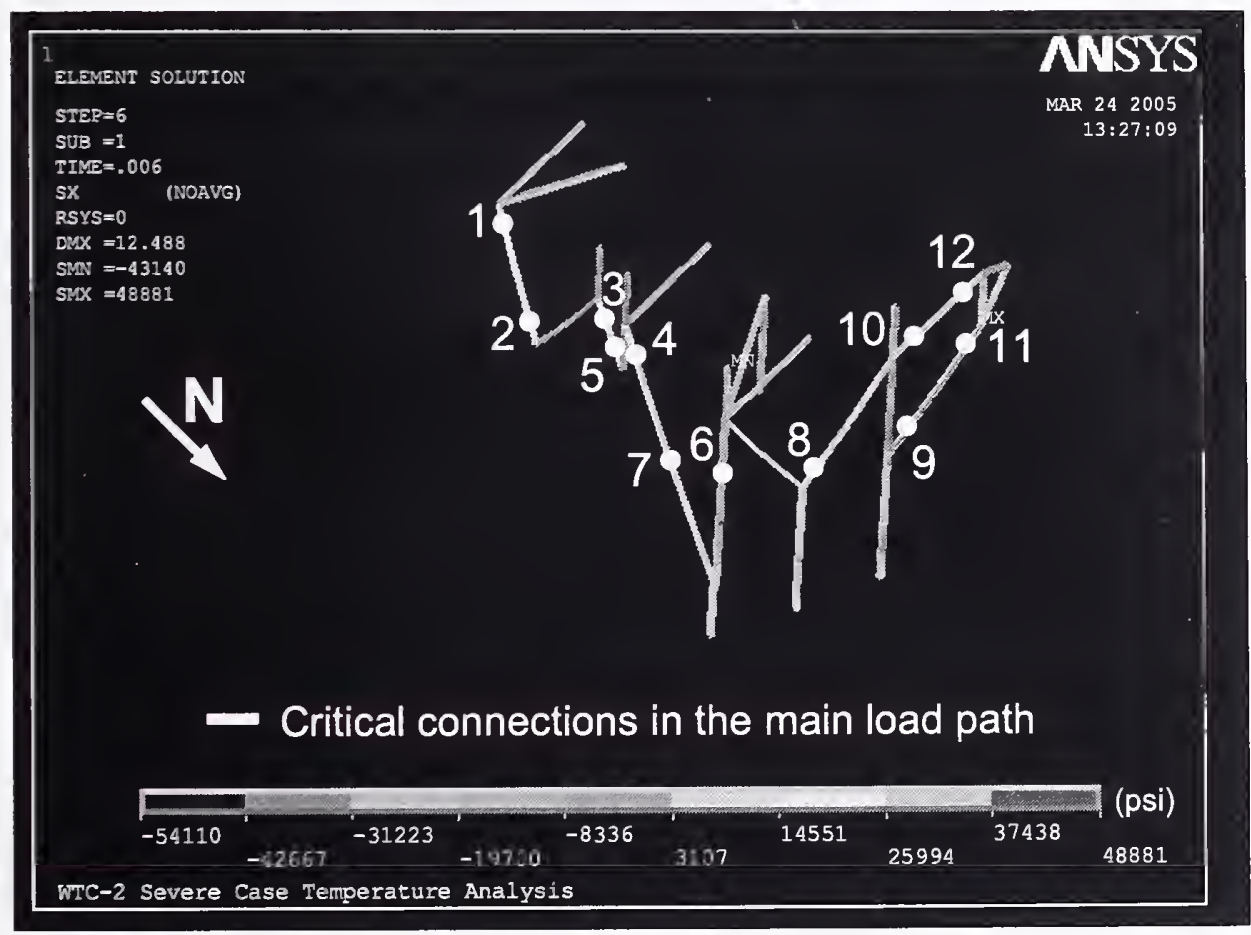

Figure 4-133. Location of hat truss connections that were in the primary load path. 
Table 4-34. Demand and capacity of the hat truss connections (kip) in the primary load path at 40 min (connection IDs are shown in Fig. 4-133).

\begin{tabular}{|c|c|c|c|c|c|c|c|c|}
\hline \multirow{2}{*}{$\begin{array}{c}\text { Con. } \\
\text { ID }\end{array}$} & \multicolumn{2}{|c|}{ Location } & \multicolumn{2}{|c|}{ Tension Capacity } & \multicolumn{2}{c|}{ Demand Force (tension) } & \multicolumn{2}{|c|}{ Status } \\
\cline { 2 - 9 } & Floor & Column & Yield & Ultimate & Before Redist. & After Redist. & Before Redist. & After Redist. \\
2 & 110 & 501 & 840 & 1,100 & 567 & 512 & Safe & Safe \\
3 & 108 & 601 & 840 & 1,100 & 567 & 512 & Safe & Safe \\
4 & 110 & 701 & 630 & 870 & 575 & 370 & Safe & Safe \\
5 & 110 & 801 & 840 & 1,100 & 950 & 510 & Yielded & Safe \\
6 & 109 & 801 & 630 & 870 & 575 & 370 & Safe & Safe \\
7 & 108 & 1001 & 760 & 870 & 1,370 & 760 & Failed & Safe \\
8 & 108 & 1002 & 1,250 & 1,250 & 440 & 110 & Safe & Safe \\
9 & 108 & 1003 & 1,470 & 1,470 & 1,000 & 700 & Safe & Safe \\
10 & 110 & 1003 & 1,250 & 1,250 & 750 & 550 & Safe & Safe \\
11 & 110 & 1004 & 1,470 & 1,470 & 1,000 & 700 & Safe & Safe \\
12 & 111 & 1004 & 1,250 & 1,250 & 750 & 550 & Safe & Safe \\
\hline
\end{tabular}

Table 4-35. Total column loads at Floor 83 of WTC 2 for Case D conditions.

\begin{tabular}{|c|l|c|c|c|c|c|c|}
\hline Row & Analysis Step & West & East & North & South & Core & Sum \\
\hline$(1)$ & Before Impact & 18,065 & 18,114 & 13,567 & 13,284 & 61,828 & 124,857 \\
$(2)$ & After Impact & 18,670 & 22,481 & 12,193 & 13,511 & 57,821 & 124,676 \\
$(3)$ & $10 \mathrm{~min}$ & 18,728 & 22,226 & 11,896 & 13,358 & 58,413 & 124,621 \\
$(4)$ & $20 \mathrm{~min}$ & 18,914 & 22,208 & 12,052 & 13,318 & 58,124 & 124,616 \\
$(5)$ & $30 \mathrm{~min}$ & 18,876 & 23,681 & 11,770 & 13,365 & 56,967 & 124,659 \\
$(6)$ & $40 \mathrm{~min}$ & 18,531 & 23,682 & 11,906 & 13,473 & 56,825 & 124,418 \\
$(7)$ & $43 \mathrm{~min}$ & 15,667 & 15,143 & 14,215 & 16,292 & 62,422 & 123,738 \\
\hline$(8)$ & $(2)-(1)$ & 604 & 4,368 & $-1,374$ & 227 & $-4,007$ & -181 \\
$(9)$ & $(3)-(1)$ & 662 & 4,112 & $-1,670$ & 74 & $-3,415$ & -236 \\
$(10)$ & $(4)-(1)$ & 849 & 4,094 & $-1,515$ & 35 & $-3,704$ & -241 \\
$(11)$ & $(5)-(1)$ & 811 & 5,567 & $-1,797$ & 81 & $-4,861$ & -199 \\
$(12)$ & $(6)-(1)$ & 466 & 5,568 & $-1,661$ & 190 & $-5,003$ & -439 \\
$(13)$ & $(7)-(1)$ & $-2,398$ & $-2,971$ & 648 & 3,009 & 594 & $-1,119$ \\
\hline
\end{tabular}

Note: Compression is positive. Units are in kip. 
Table 4-36. Total column loads at Floor 105 of WTC 2 for Case D conditions.

\begin{tabular}{|c|l|c|c|c|c|c|c|}
\hline Row & Analysis Step & West & East & North & South & Core & Sum \\
\hline$(1)$ & Before Impact & 8,497 & 8,572 & 7,382 & 7,169 & 17,123 & 48,742 \\
$(2)$ & After Impact & 9,170 & 11,272 & 6,487 & 8,432 & 13,382 & 48,742 \\
$(3)$ & $10 \mathrm{~min}$ & 9,182 & 11,061 & 6,250 & 8,275 & 13,975 & 48,742 \\
$(4)$ & $20 \mathrm{~min}$ & 9,279 & 11,120 & 6,311 & 8,351 & 13,682 & 48,742 \\
$(5)$ & $30 \mathrm{~min}$ & 9,370 & 11,859 & 6,416 & 8,553 & 12,544 & 48,742 \\
$(6)$ & $40 \mathrm{~min}$ & 9,198 & 11,927 & 6,524 & 8,691 & 12,402 & 48,742 \\
$(7)$ & $43 \mathrm{~min}$ & 7,086 & 8,026 & 6,546 & 9,169 & 17,915 & 48,742 \\
\hline$(8)$ & $(2)-(1)$ & 674 & 2,699 & -895 & 1,263 & $-3,741$ & 0 \\
$(9)$ & $(3)-(1)$ & 685 & 2,489 & $-1,132$ & 1,106 & $-3,148$ & 0 \\
$(10)$ & $(4)-(1)$ & 783 & 2,547 & $-1,071$ & 1,182 & $-3,441$ & 0 \\
$(11)$ & $(5)-(1)$ & 873 & 3,287 & -965 & 1,384 & $-4,579$ & 0 \\
$(12)$ & $(6)-(1)$ & 702 & 3,355 & -858 & 1,522 & $-4,721$ & 0 \\
$(13)$ & $(7)-(1)$ & $-1,411$ & -547 & -835 & 2,000 & 792 & 0 \\
\hline
\end{tabular}

Note: Compression is positive. Units are in kip.

Table 4-37. Change in total column loads before and after aircraft impact. (Loads After Impact) - (Loads Before Impact)

\begin{tabular}{|c|c|c|c|c|c|c|}
\hline Row & Floor & West & East & North & South & Core \\
\hline$(1)$ & 83 & 604 & 4,368 & $-1,374$ & 227 & $-4,007$ \\
$(2)$ & 105 & 674 & 2,699 & -895 & 1,263 & $-3,741$ \\
\hline$(3)$ & $(2)-(1)$ & 69 & $-1,668$ & 479 & 1,035 & 266 \\
\hline
\end{tabular}

Note: Increase in compression is shown as positive. Units are in kip.

Table 4-38. Change in total column loads between $40 \mathrm{~min}$ and $43 \mathrm{~min}$. (Loads at $43 \mathrm{~min}$ ) - (Loads at $40 \mathrm{~min}$ )

\begin{tabular}{|c|c|c|c|c|c|c|}
\hline Row & Floor & West & East & North & South & Core \\
\hline$(1)$ & 83 & $-2,864$ & $-8,539$ & 2,309 & 2,819 & 5,596 \\
$(2)$ & 105 & $-2,112$ & $-3,901$ & 23 & 479 & 5,513 \\
\hline$(3)$ & $(2)-(1)$ & 752 & 4,637 & $-2,286$ & $-2,340$ & -84 \\
\hline
\end{tabular}

Note: Increase in compression is shown as positive. Units are in kip. 


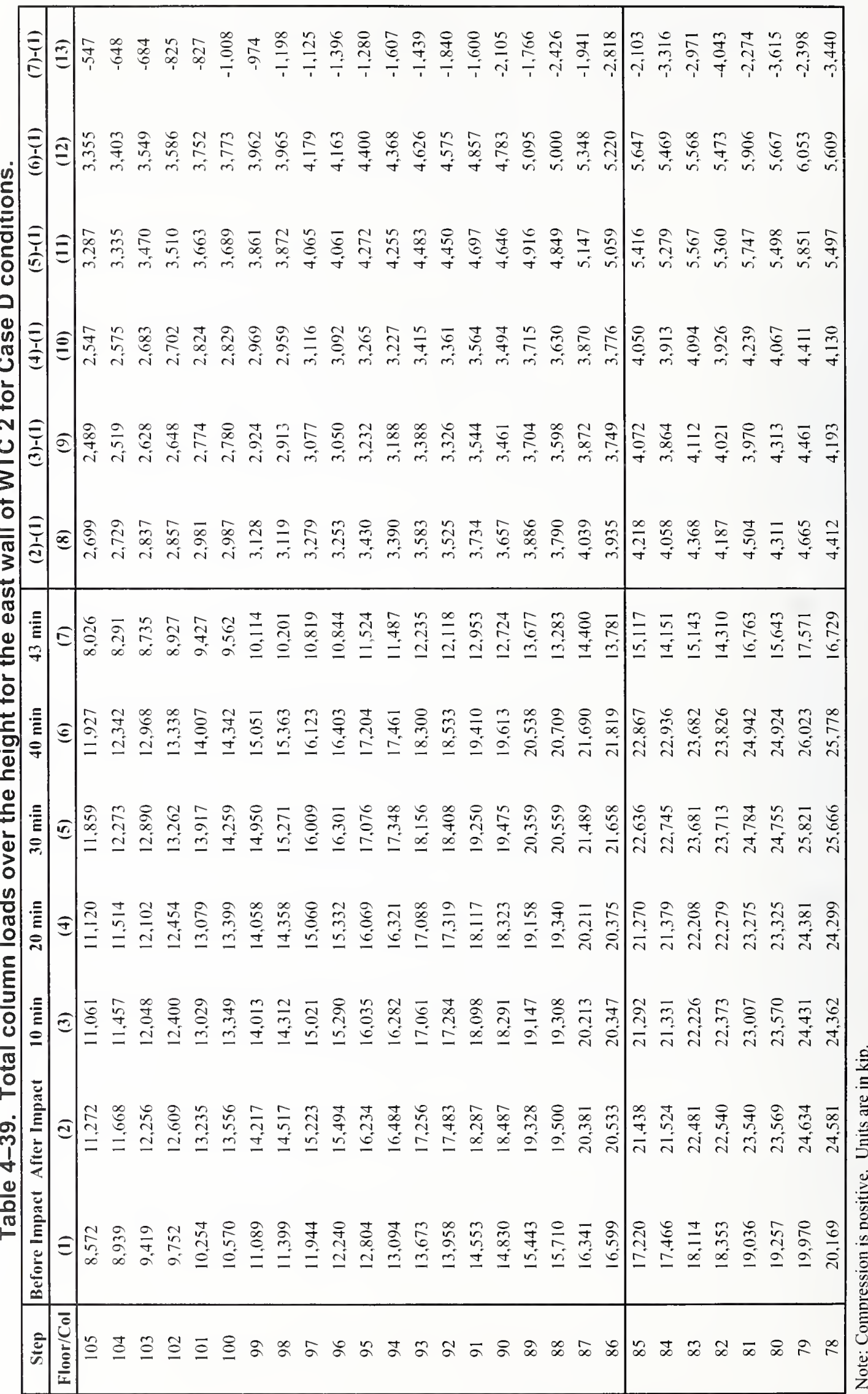




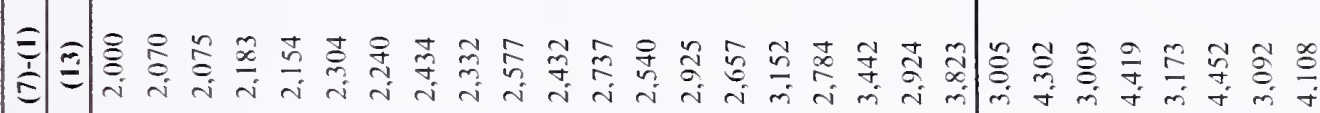

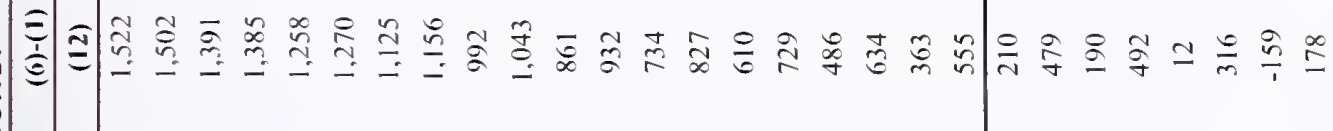

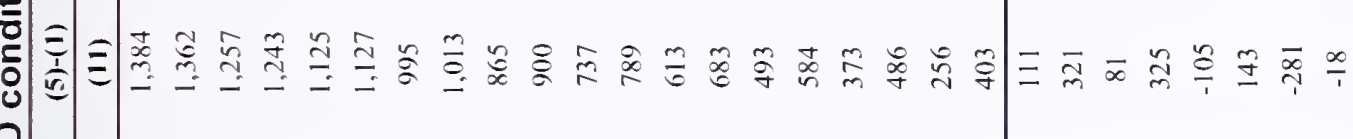

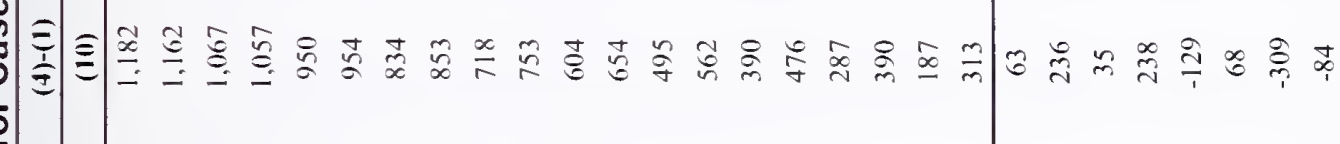

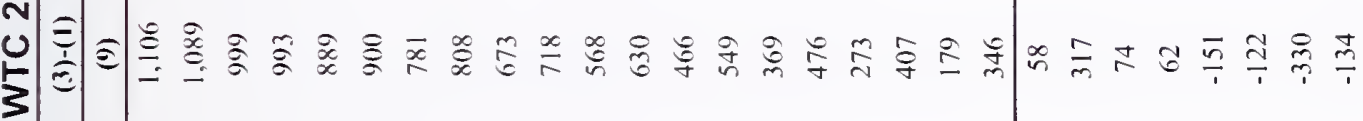

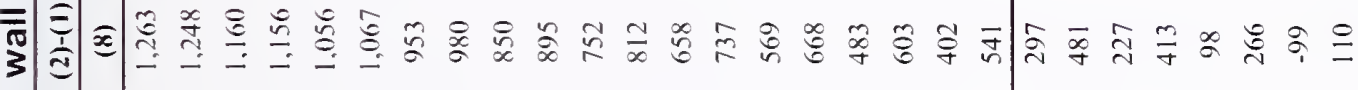

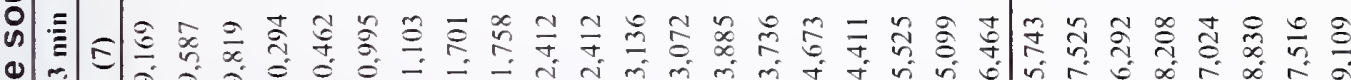

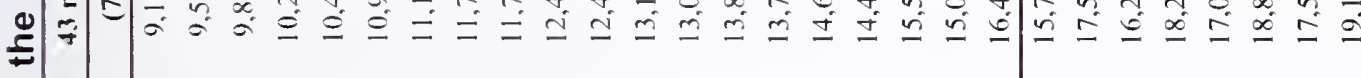

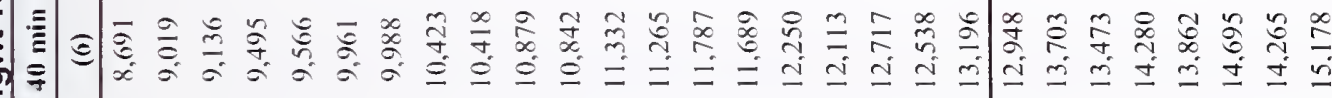

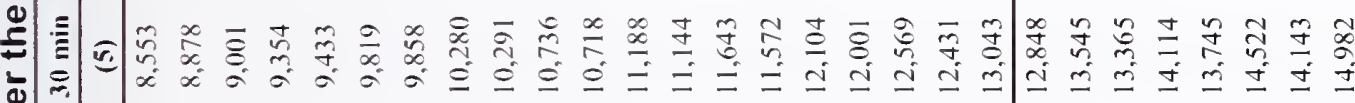

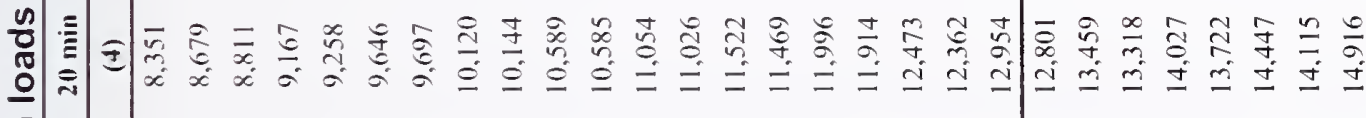

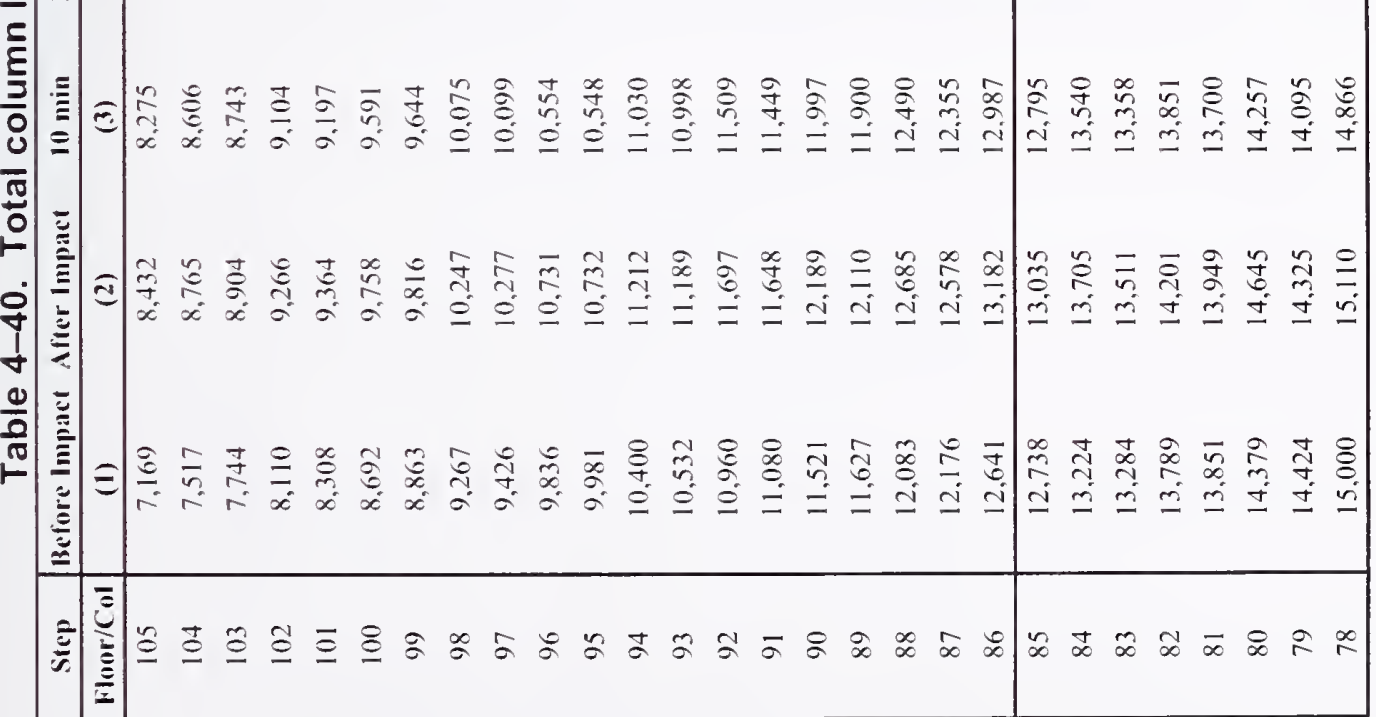




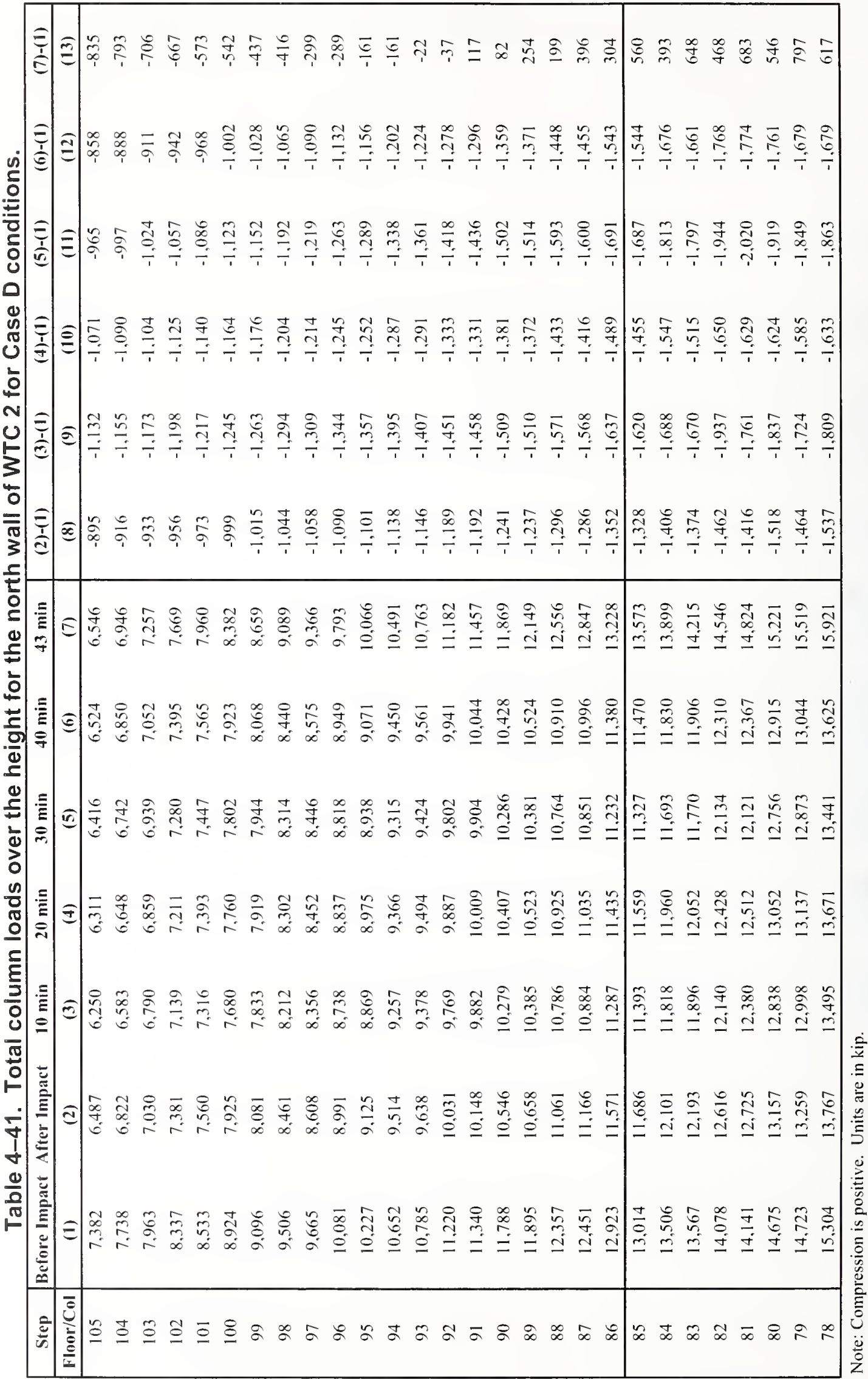




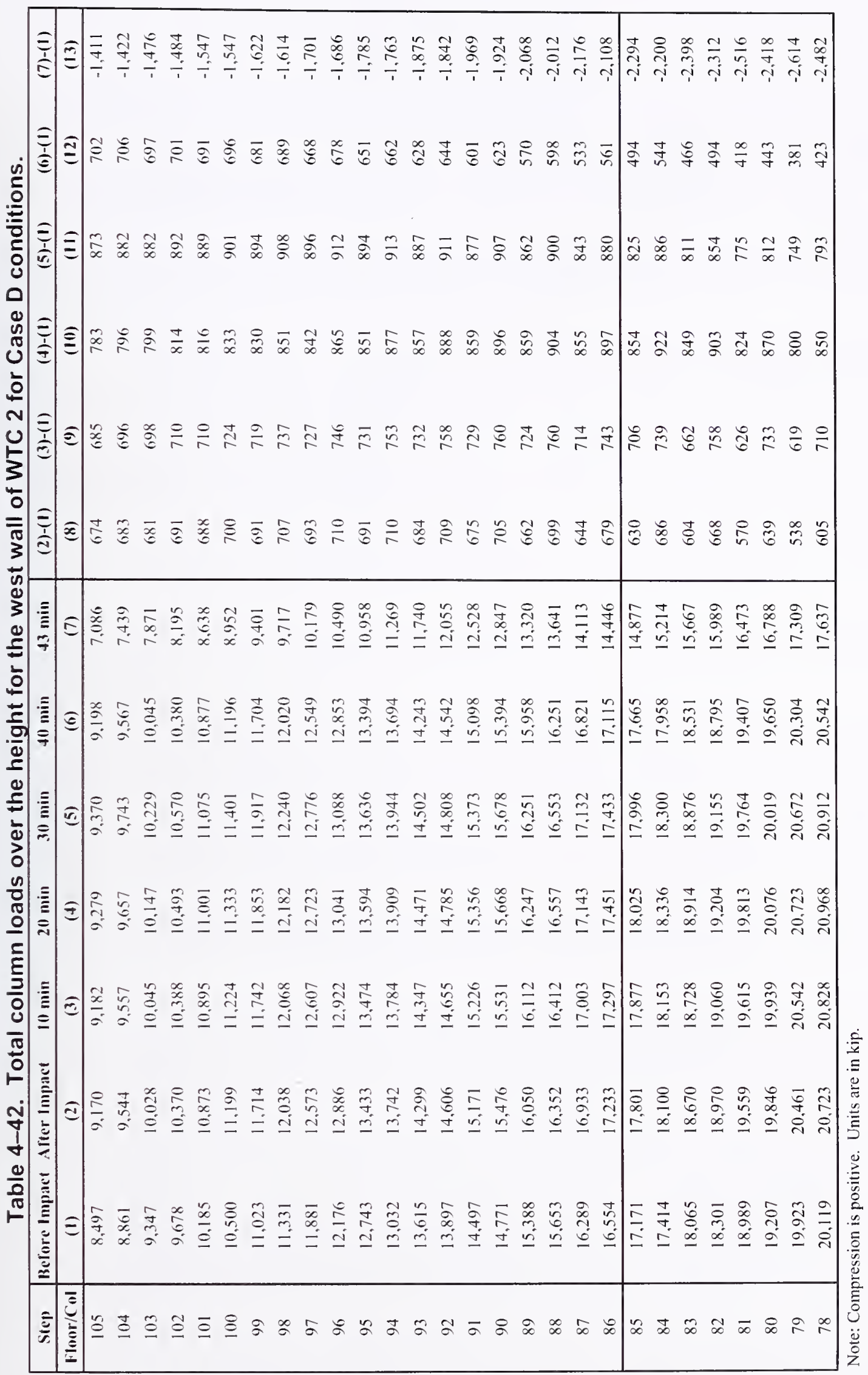




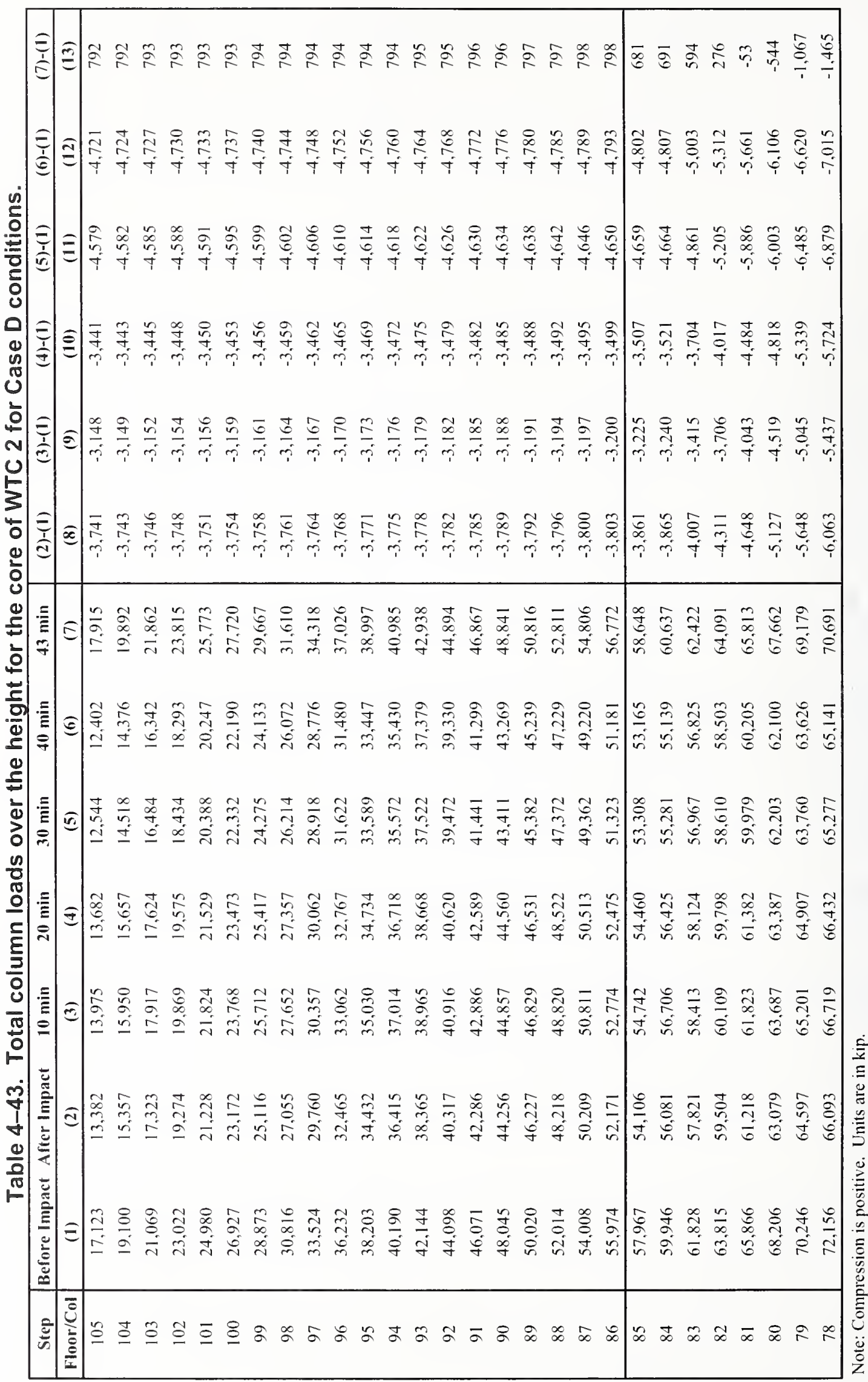




\section{Chapter 5 \\ Collapse Sequence}

\section{$5.1 \quad$ INTRODUCTION}

This chapter summarizes the sequences of collapse of WTC 1 and WTC 2 from the analyses of global models with creep. The basis of the conclusions derived here are as follows:

- Analyses of subcomponents, such as connections (truss seats and knuckles), components (trusses and columns), subsystems (full floors and exterior walls), preliminary global models, and isolated parts of the final global models (core and exterior wall) subjected to tcmperature loads from fire, were performed to identify failure modes and failure loads. These models included large deflection effects and temperaturc-dependent material nonlinearities due to plasticity and creep. Break elements were developed to capture various failure modes such as truss seat failure and web diagonal buckling (NIST NCSTAR 1-6C). As they became larger, the models were gradually reduced in complexity to enhance computational efficiency, while ensuring that key failure modes and failure sequences were captured.

- The final global models included large deflection, and temperature-dependent plasticity and creep.

- The final global models were developed based on the following assumptions:

- Floors were modeled by plate elements with elastic properties without the ability to simulate sagging and its effect on the development of pull-in forces at floor/wall disconnections. Full floor analyses found that floors initially expanded outward but then pulled inward as the floor sag increased. Pull-in forces resulting from floor sagging and floor/wall disconnections were determined based on the results of full floor models and isolated wall models and were modified by visual observations. They were input in the global model analyses at different times as fire-induced damage.

- Spandrels were modeled by beam elements. The axial degrce of freedom of the beam elements was released to enhance numerical efficiency and avoid thcrmally-induced buckling; spandrel elements continued to transfer shear and bending moments. The exterior wall subsystem analysis showed that large deformations and buckling of spandrels would not affect the stability of exterior columns significantly.

- Columns were modeled to capture inelastic buckling, but not the kink-type buckling that is initiated by the local buckling of plates and results in significant distortion of the cross section. The analysis of columns showed that when buckling occurred in a column that spanned several floors and was at high temperatures, inelastic buckling, rather than kinktype buckling, governed its load deformation characteristic. 
- The building sections below the impact zones were removed, and the vertical stiffness of the removed sections was replaced with equivalent vertical springs. Preliminary analyses of the global models showed that building sections below the impact zone did not contribute much to the overall behavior of the towers.

- Construction sequence was not considered to enhance computational efficiency. A comparative study showed that the total column load on each face of the exterior wall increased by 7 percent to 15 percent and the total column load on the core decreased by about 10 percent when the construction sequence was not included.

- Structural members that were severed or heavily damaged by aircraft impact were removed from the final global models before gravity loads were applied.

- Break elements were not uscd in the final global models to represent component failures such as failure of column splices. However, the results of the global model analyses were examined to detcrmine whether any component failure occurred and to what extent its failure impacted the collapse sequence.

The key structural events common to both towers are discussed below.

- Floor sagging sufficient to cause the observed inward bowing of the exterior wall was caused by the elevated steel temperatures resulting from loss of thermal insulation. The elevated temperature caused buckling of the truss web diagonals, as shown in Fig. 5-1 (NIST NCSTAR 16C), which caused the floor sag to increase significantly and to approach a catenary shape. The catenary action in this study refers to the combined action that results when the bending capacity of the truss is exceeded and additional load is carried by the floor system acting as a tensile structure (Fig. 5-2). Note that in Fig. 5-2, $M$ refers to the residual moment capacity in the floor with highly deformed truss. Sagging of the floor resulted in pull-in forces at floor/exterior wall connections, and led to inward bowing of the exterior wall

- Bowing and buckling of the entire exterior wall of a tower occurred under the combined effects of temperature, redistributed gravity load, pull-in force from sagging floors, and loss of lateral support due to sagging or floor/wall disconnections. Floors with large sag did not restrain the exterior wall columns from buckling.

- Downward displacement of the core was due to severed core columns from the aircraft impact and redistributing column loads to non-severed core columns and due to shortening of the core columns caused by buckling, plasticity, and creep of core columns at elevated temperatures.

- Redistribution of gravity loads among exterior and interior columns resulted from aircraft impact damage, restrained thermal expansion, shortening of core columns, tilting of the tower above the impact area, and bowing and buckling of exterior walls. Gravity loads were redistributed from columns with aircraft impact or fire-induced damage, both in the core and exterior walls, primarily to neighboring columns. Loads were redistributed from the core to the exterior walls and from the exterior walls to the core primarily through the hat truss. Loads were redistributed between adjacent exterior walls primarily through the spandrels, and to a lesser extent through the hat truss. Major load redistribution mechanisms wcre as follows: 
- Aircraft impact reduced the load on the impacted wall and on the opposite wall through the hat truss and redistributed the load to the side walls.

- Restrained thermal expansion caused increased loads in the heated elements.

- Shortening of the core columns caused a redistribution of the load from the core to the exterior walls.

- Tilting of the tower redistributed the load among the exterior walls, resulting in increased load on the compressed part of the exterior walls.

The collapse sequence of each tower is presented in detail below.

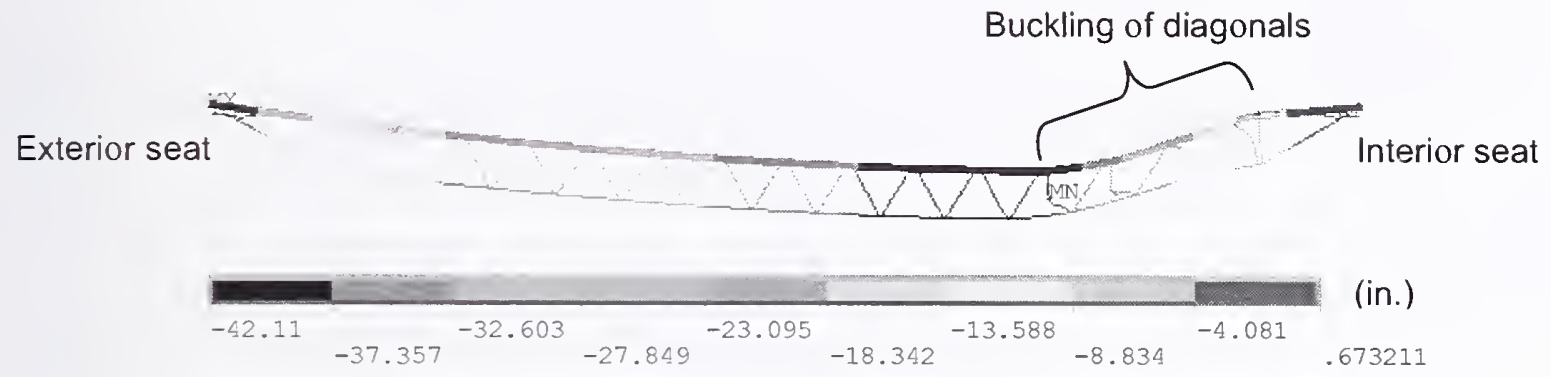

Figure 5-1. Vertical displacement of the truss model under thermal loading.

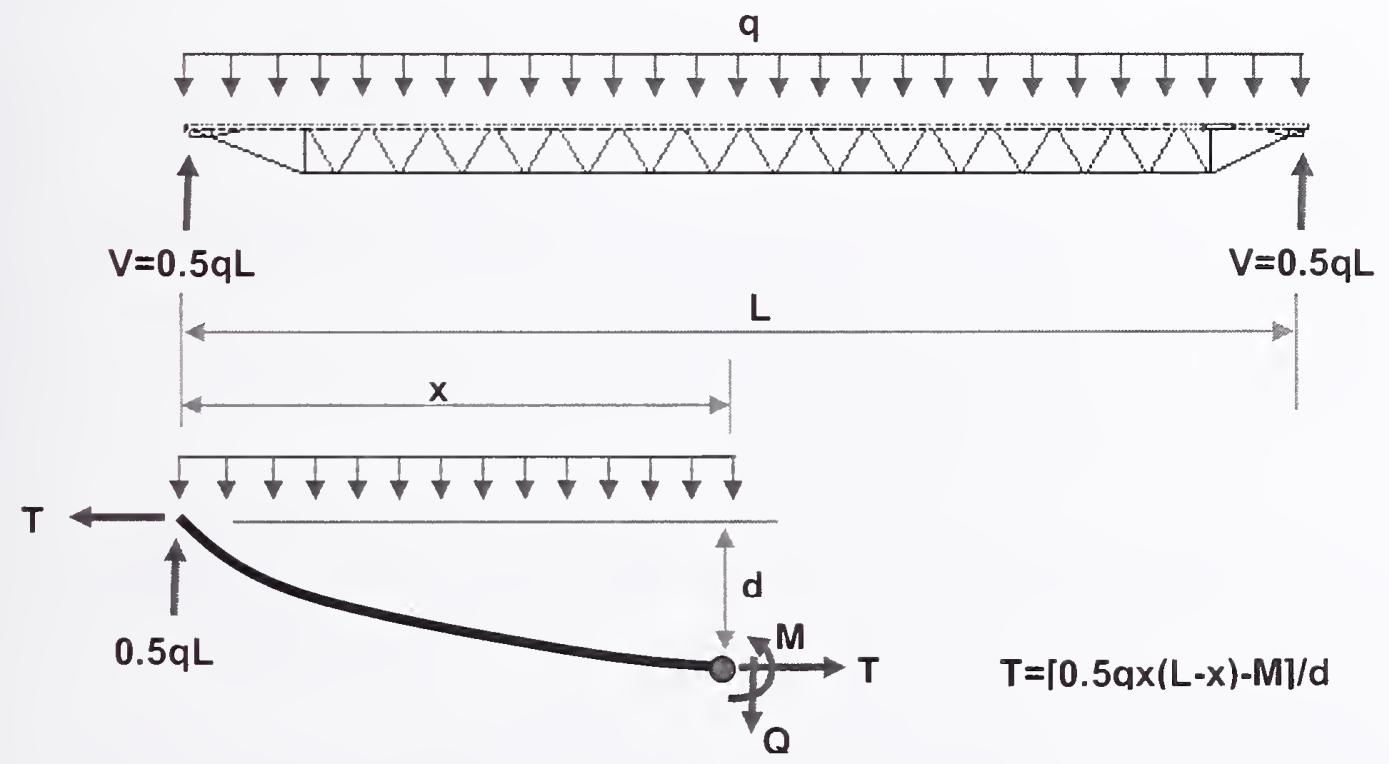

Figure 5-2. Catenary action in the floor system. 


\subsection{WTC 1 COLLAPSE SEQUENCE}

The aircraft impacted the north wall of WTC 1 at 8:46 a.m. The aircraft severed exterior columns and floors on the north side of the tower and core columns and floor members between Floor 93 and Floor 98. The subsequent fires weakened structural subsystems, including the core columns, floors, and exterior walls. The core displaced downward, the floors sagged, and the south exterior wall bowed inward. At 10:28 a.m., about $102 \mathrm{~min}$ after the aircraft impact, WTC 1 began to collapse.

The sequence of main structural events that led to the collapse of WTC 1, starting from aircraft impact, and the causes and effects of these structural events along with key observations, are discussed below.

The WTC 1 collapse sequence consists of five main events, listed in Table 5-1, which are discussed below. Actual observations are summarized in Table 5-2, which are based on NIST's examination of photos and videos (NIST NCSTAR 1-6).

Table 5-1. Summary of main events that led to the collapse of WTC 1.

\begin{tabular}{|c|l|}
\hline Event Number & \multicolumn{1}{|c|}{ Event } \\
\hline 1 & Aircraft impact \\
\hline 2 & Unloading of core \\
\hline 3 & Sagging of floors and floor/wall disconnections \\
\hline 4 & Bowing of south wall \\
\hline 5 & Buckling of south wall and collapse initiation \\
\hline
\end{tabular}

Table 5-2. Observations on WTC 1 provided by NIST.

\begin{tabular}{|c|c|l|}
\hline Time & $\begin{array}{c}\text { Time from } \\
\text { Impact } \\
\text { (min) }\end{array}$ & \multicolumn{1}{|c|}{ Observation } \\
\hline $8: 46: 26$ & 0 & $\begin{array}{l}\text { Aircraft impact on the north wall of WTC 1 between Floor 93 and } \\
\text { Floor 99 and Columns 112 and 151. }\end{array}$ \\
\hline $9: 25: 28$ & 39 & Fire on west side of south wall. \\
\hline $9: 40$ & 54 & $\begin{array}{l}\text { No bowing of columns was observed between Columns 301 and 323 on } \\
\text { the east side of south wall. }\end{array}$ \\
\hline $10: 18: 43$ & 92 & $\begin{array}{l}\text { Smoke suddenly expelled on Floor 92 north wall, Floor 94 east side of } \\
\text { north wall, Floor 95 to Floor 98 on west side of north wall, Floor 95 } \\
\text { and Floor 98 on north side of west wall, lower floor on south side. }\end{array}$ \\
\hline $10: 22: 59$ & 97 & $\begin{array}{l}\text { Inward bowing from Floor 95 to about Floor 99 between Columns 308 } \\
\text { and 326 (maybe to 340) on the south wall, maximum amplitude } \\
\text { approximately 55 in. at Floor 97. }\end{array}$ \\
\hline $10: 28: 18$ & 102 & $\begin{array}{l}\text { Smoke puff out of north edge and center of west wall; smoke and } \\
\text { debris clouds out of the north, east, and west walls on Floor 98. Fire } \\
\text { out of windows on the north, east, west, and south walls between } \\
\text { Floor 92 and Floor 98, and on Floor 104. }\end{array}$ \\
\hline $10: 28: 20$ & 102 & $\begin{array}{l}\text { WTC 1 began to collapse. First exterior sign of collapse was at } \\
\text { Floor 98. Rotation of at least 8 degrees to the south occurred before the } \\
\text { building section began to fall vertically under gravity. }\end{array}$ \\
\hline
\end{tabular}




\section{Aircraft Impact}

The aircraft impacted WTC 1 at the north wall. The aircraft severed or heavily damaged Columns 112 to 151 between Floors 94 and 98 on the north wall. After breaching the building's perimeter, the aircraft continued to penetrate into the building. The north office area floor system sustained severe structural damage between Columns 112 and 145 at Floors 94 to 98. Core Columns 503, 504, 505, 506, 604, 704, 706. 805, and 904 were severed or heavily damaged between Floor 92 and Floor 97 . The aircraft also severed a single exterior panel at the center of the south wall from Columns 329 to 331 between Floor 93 and Floor 96 . In summary, 38 of 59 columns of the north wall, three of 59 columns of the south wall, and nine of 47 core columns were severed or heavily damaged. In addition, thermal insulation on floor framing and columns was also damaged from the impact area to the south perimeter wall, primarily through the center of WTC 1 and over one-third to one-half of the core width. Figures 2-2, 2-14, and 218 summarize aircraft impact damage to exterior and core columns and floors of WTC 1.

Gravity loads in the columns that were severed were redistributed, mostly to the neighboring columns. Due to the severe impact damage to the north wall, the wall section above the impact zone moved downward as shown in Figs. 4-9 and 4-13. The hat truss resisted the downward movement of the north wall and rotated about its east-west axis, which reduced the load on the south wall. As a result, the north and south walls each carried about 7 percent less gravity loads at Floor 98 after impact, the east and west walls each carried about 7 percent more loads, and the core carried about 1 percent more gravity loads at Floor 98 after impact (Table 5-3). Column 705 buckled, and Columns 605 and 804 showed minor buckling.

\section{Unloading of Core}

Temperatures in the core area rose quickly, and thermal expansion of the core was greater than the thermal expansion of the exterior walls in early stages of the fire. This increased the gravity loads in the core columns until 10 min after impact (Table 5-3). The additional gravity loads from adjacent severed columns and high temperatures caused high plastic and creep strains to develop in the core columns in early stages of the fire. More columns buckled inelastically due to high temperatures. Creep strain continued to increase to the point of collapse (see Fig. 4-81). By $30 \mathrm{~min}$, the plastic-plus-creep strains exceeded thermal expansion strains. Due to high plastic and creep strains and inelastic buckling of core columns, the core columns shortened, and the core displaced downward. At $100 \mathrm{~min}$, the downward displacement of the core at Floor 99 became 2.0 in. on the average, as shown in Fig. 4-37.

The shortening of core columns was resisted by the hat truss, which unloaded the core over time and redistributed the gravity loads from the core to the exterior walls, as can be seen in Table 5-3. As a result, the north, east, south, and west walls at Floor 98 carried about 12 percent, 27 percent, 10 percent, and 22 percent more gravity loads, respectively, at $80 \mathrm{~min}$ than the state after the impact, and the core carried about 20 percent less loads as shown in Table 5-3. The net increase in the total column load on the south wall, where exterior wall failure initiated, was only about 10 percent due to the downward displacement of the core (see Fig. 5-3). At $80 \mathrm{~min}$, the total core column loads reached their maximum. As the floor pulled in starting at $80 \mathrm{~min}$ on in the south side, the south exterior wall began to shed load to adjacent walls and the core. 


\section{Sagging of Floors and Floor/Wall Disconnections}

The long-span trusses of Floor 95 through Floor 99 sagged due to high temperatures. While the fires were on the north side and the floors on the north side sagged first, the fires later reached the south side, and the floors on the south side sagged. Figure 5-4 shows vertical displacements of Floors 95 through 98 determined by the full floor models at $100 \mathrm{~min}$. Full floor models underestimated the extent of sagging because cracking and spalling of concrete and creep in steel under high temperatures were not included in the floor models, and because the cxtent of insulation damage was conservatively estimated. The sagging floors pulled in the south wall columns over Floors 95 to 99. In addition, the exterior seats on the south wall in the hot zone of Floors 97 and 98 began to fail due to their reduced vertical shear capacity at around $80 \mathrm{~min}$, and by $100 \mathrm{~min}$ about 20 percent of the exterior seats on the south wall of Floors 97 and 98 failed, as shown in Figs. 5-4 and 5-5. Partial collapse of the floor may have occurred at Floors 97 and 98 , resulting from the exterior seat failures, as indicated by the observed smoke puff at $92 \mathrm{~min}(10: 19$ a.m.) in Table 5-2, but this phenomenon was not modeled.

\section{Bowing of South Wall}

The exterior columns on the south wall bowed inward as they were subjected to high temperatures, pull-in forces from the floors beginning at $80 \mathrm{~min}$, and additional gravity loads redistributed from the core. Figure 5-6 shows the observed and the estimated inward bowing of the south wall at 97 min after impact (10:23 a.m.). Since no bowing was observed on the south wall at $69 \mathrm{~min}$ (9:55 a.m.), as shown in Table $5-2$, it is estimated that the south wall began to bow inward at around $80 \mathrm{~min}$ when the floors on the south side began to substantially sag. The inward bowing of the south wall increased with time due to continuing floor sagging and increased temperatures on the south wall as shown in Figs. 4-42 and 5-7. At 97 min (10:23 a.m.), the maximum bowing observed was about 55 in. (see Fig. 5-6).

\section{Buckling of South Wall and Collapse Initiation}

With continuously increased bowing, as more columns buckled, the entire width of the south wall buckled inward. Instability started at the center of the south wall and rapidly progressed horizontally toward the sides. As a result of the buckling of the south wall, the south wall significantly unloaded (Fig. 5-3), redistributing its load to the softened core through the hat truss and to the south side of the east and west walls through the spandrels. The onset of this load redistribution can be found in the total column loads in the WTC 1 global model at $100 \mathrm{~min}$ in the bottom line of Table 5-3. At $100 \mathrm{~min}$, the north, east, and west walls at Floor 98 carried about 7 percent, 35 percent, and 30 percent more gravity loads than the state after impact, and the south wall and the core carried about 7 percent and 20 percent less loads, respectively. The section of the building above the impact zone tilted to the south (observed at about $8^{\circ}$, Table 5-2) as column instability progressed rapidly from the south wall to the adjacent east and west walls (see Fig. 5-8), resulting in increased gravity load on the core columns. The release of potential energy due to downward movement of building mass above the buckled columns exceeded the strain energy that could be absorbed by the structure. Global collapse ensued. 
Table 5-3. Total column loads at Floor 98 and Floor 105 of WTC 1 global model for Case $\mathrm{B}$ conditions.

\begin{tabular}{|c|c|c|c|c|c|c|c|c|c|c|c|}
\hline Row & $\begin{array}{c}\text { Analysis } \\
\text { Step }\end{array}$ & $\begin{array}{c}\text { North Wal } \\
\text { Floor } 98\end{array}$ & Floor 105 & $\begin{array}{r}\text { East Wall } \\
\text { Floor } 98 \\
\end{array}$ & Floor 105 & $\begin{array}{c}\text { South Wall } \\
\text { Floor } 98\end{array}$ & Floor 105 & $\begin{array}{c}\text { West Wall } \\
\text { Floor } 98\end{array}$ & Floor 105 & $\begin{array}{l}\text { Core } \\
\text { Floor } 98 \\
\end{array}$ & Floor 105 \\
\hline (1) & Beforc lmpact & 10.974 & 8.026 & 8.545 & 6.562 & 11.025 & 8,092 & 8.572 & 6.604 & 34,029 & $20,36 I$ \\
\hline (2) & After lmpact & 10.137 & 7.294 & 9.071 & 7,028 & 10,356 & 7,488 & 9,146 & 7,076 & 34,429 & 20,761 \\
\hline (3) & 10 min & 9.796 & 6,944 & 8.490 & 6,461 & 9.848 & 6,981 & 8,536 & 6,469 & 36,473 & 22,790 \\
\hline (4) & $20 \mathrm{~min}$ & 10.437 & 7.551 & 9.108 & 7.075 & 9,900 & 7,057 & 9.202 & 7,158 & 34,495 & 20,806 \\
\hline (5) & $30 \mathrm{~min}$ & 10.913 & 8.020 & 10,034 & 7.998 & 10,420 & 7,569 & 9,715 & 7,685 & 32,060 & 18,377 \\
\hline (6) & $40 \mathrm{~min}$ & 11.068 & 8.193 & 10.599 & 8.571 & 11,004 & 8,129 & 10,178 & 8.147 & 30,294 & 16,608 \\
\hline (7) & $50 \mathrm{~min}$ & 11,149 & 8.285 & 10,908 & 8,878 & 11.192 & 8,315 & 10,458 & 8,428 & 29.435 & 15,743 \\
\hline (8) & $60 \mathrm{~min}$ & 11.205 & 8.351 & 11.168 & 9.130 & 11.285 & 8,414 & 10,716 & 8.687 & 28,766 & 15,069 \\
\hline (9) & $70 \mathrm{~min}$ & 11.286 & 8.435 & 11.366 & 9,319 & 11,343 & 8,481 & 10.939 & 8,914 & 28,205 & 14,502 \\
\hline$(10)$ & $80 \mathrm{~min}$ & 11.376 & 8.528 & 11.555 & 9,497 & 11,409 & 8,551 & 11.119 & 9.097 & 27,681 & 13,978 \\
\hline (11) & $90 \mathrm{~min}$ & 10.916 & 8.096 & 11,991 & 9.847 & 9.949 & 7,327 & 11.657 & 9.506 & 28,587 & 14,876 \\
\hline$(12)$ & $100 \mathrm{~min}$ & 10.828 & 8.023 & 12.249 & 10.076 & 9.638 & 7,066 & 11,905 & 9.720 & 28,478 & 14,767 \\
\hline (13) & $(2)-(1)$ & -837 & -732 & 526 & 466 & -668 & -604 & 574 & 472 & 400 & 400 \\
\hline (14) & $(10)-(2)$ & 1.239 & 1.234 & 2.484 & 2.470 & 1,052 & 1.063 & 1,973 & 2,021 & $-6,748$ & $-6,783$ \\
\hline (15) & $(12)-(2)$ & 692 & 730 & 3.178 & 3,048 & -719 & -422 & 2,759 & 2,644 & $-5,951$ & $-5,993$ \\
\hline (I6) & $(12)-(10)$ & -548 & -504 & 694 & 579 & -1.771 & -1.485 & 786 & 623 & 797 & 790 \\
\hline
\end{tabular}

Note : Compression is positive. Units are in kip.

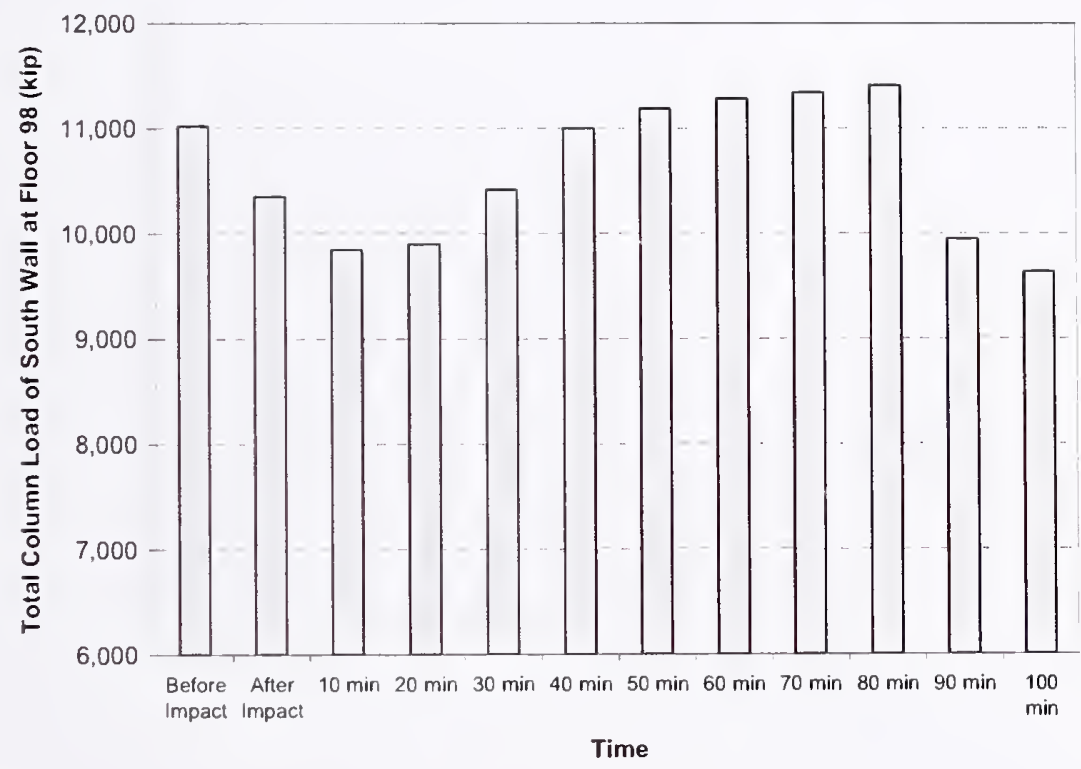

Figure 5-3. Total column loads at Floor 98 of the south wall of WTC 2 global model for Case $B$ conditions (compression is positive). 


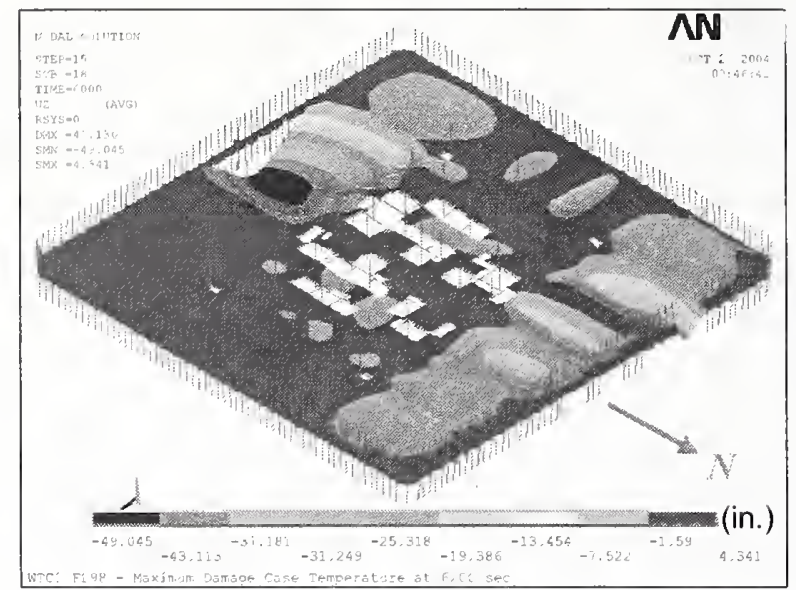

(a) Floor 98

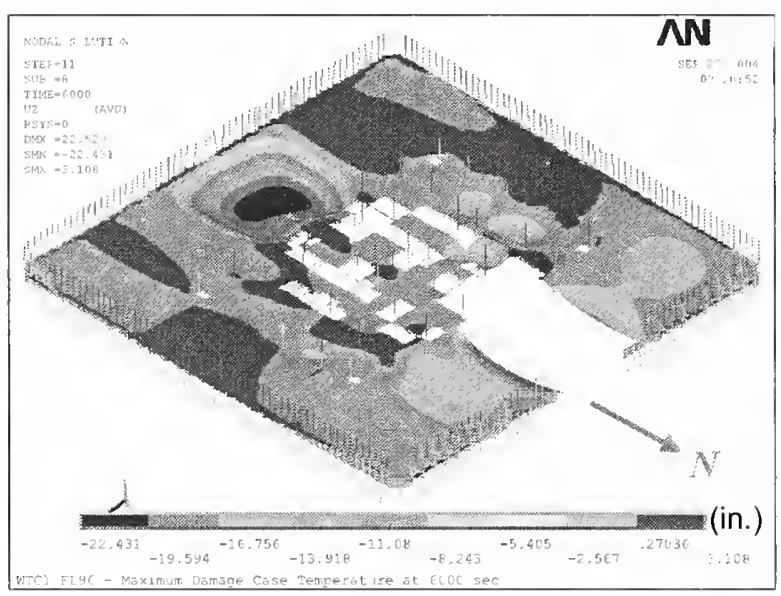

(c) Floor 96

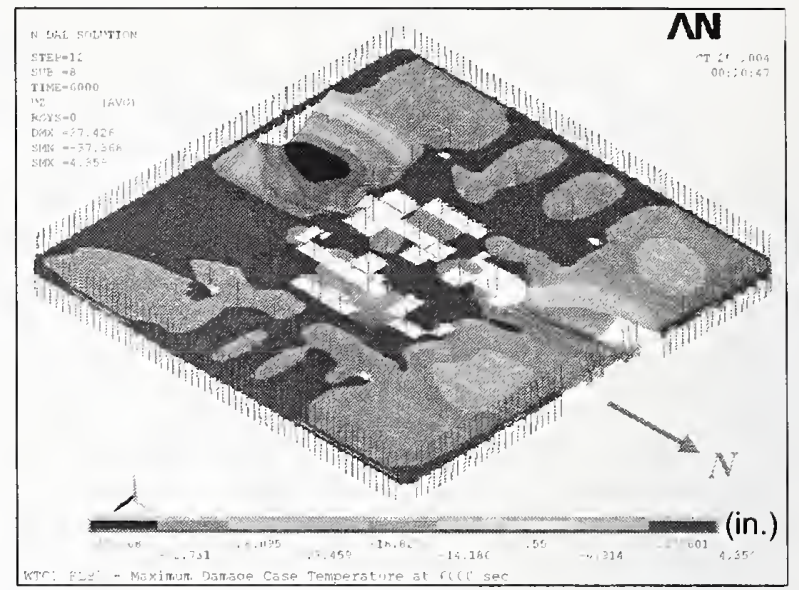

(b) Floor 97

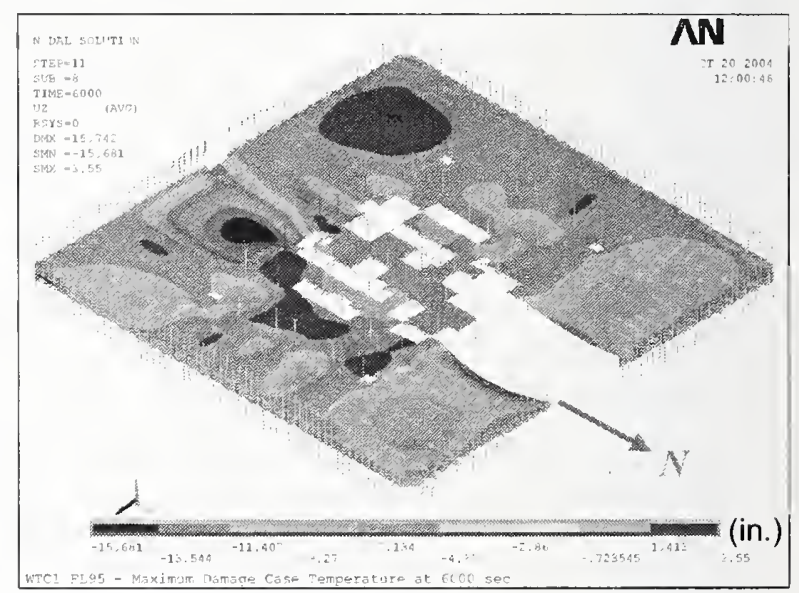

(d) Floor 95

Figure 5-4. Vertical displacements of full floor models of WTC 1 for Case $B_{i}$ temperature condition at $100 \mathrm{~min}$ (downward displacement is negative).

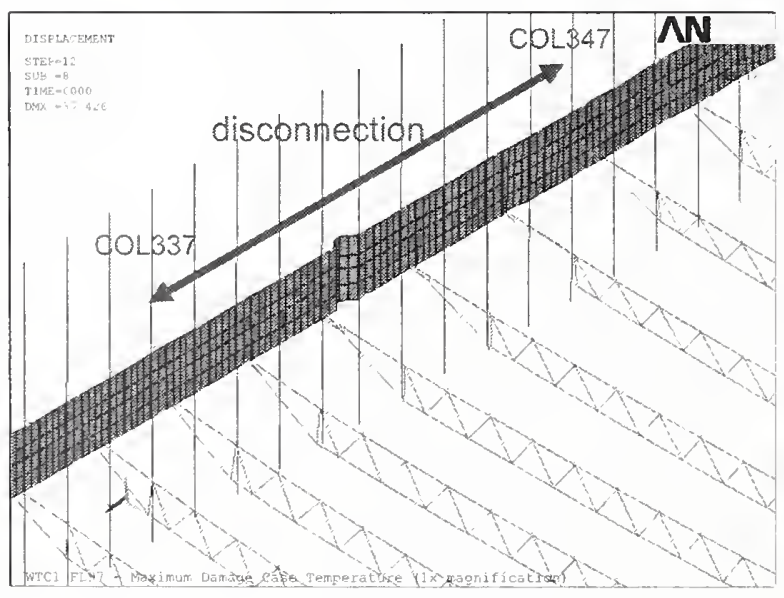

(a) Floor 97

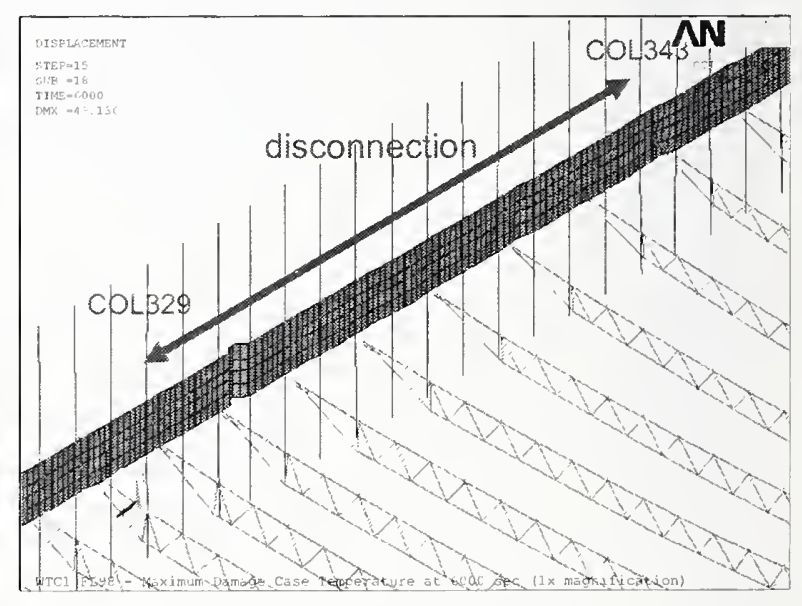

(b) Floor 98

Figure 5-5. Loss of vertical supports obtained in Floor 97 and Floor 98 full floor models of WTC 1 for Case $B_{i}$ temperature condition at $100 \mathrm{~min}$ (1x displacement magnification). 


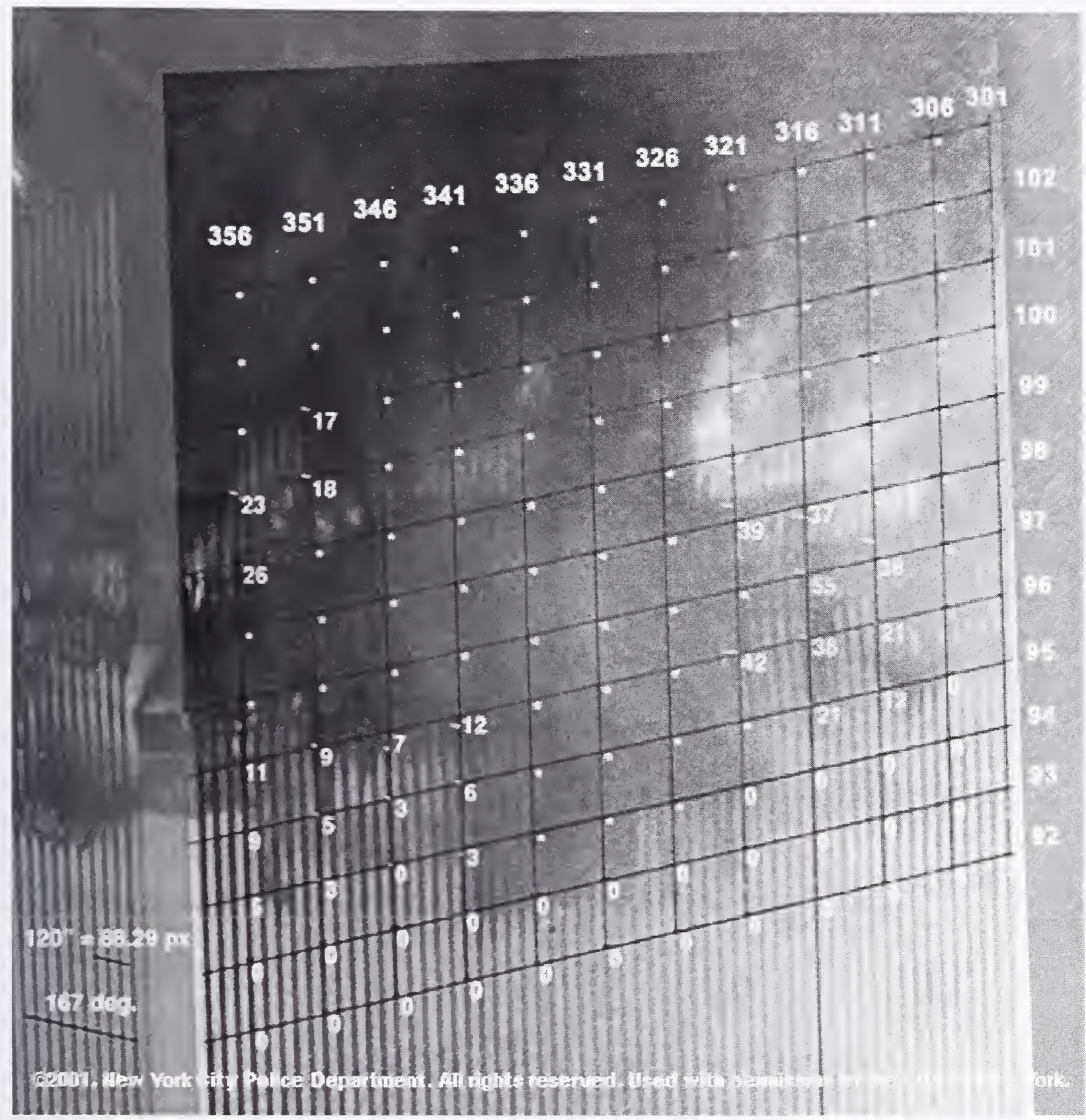

Figure 5-6. Inward bowing of exterior columns of the South wall of WTC 1 at 10:23 a.m. (97 min after impact). Displacements were estimated by NIST from the analysis of this photograph. 


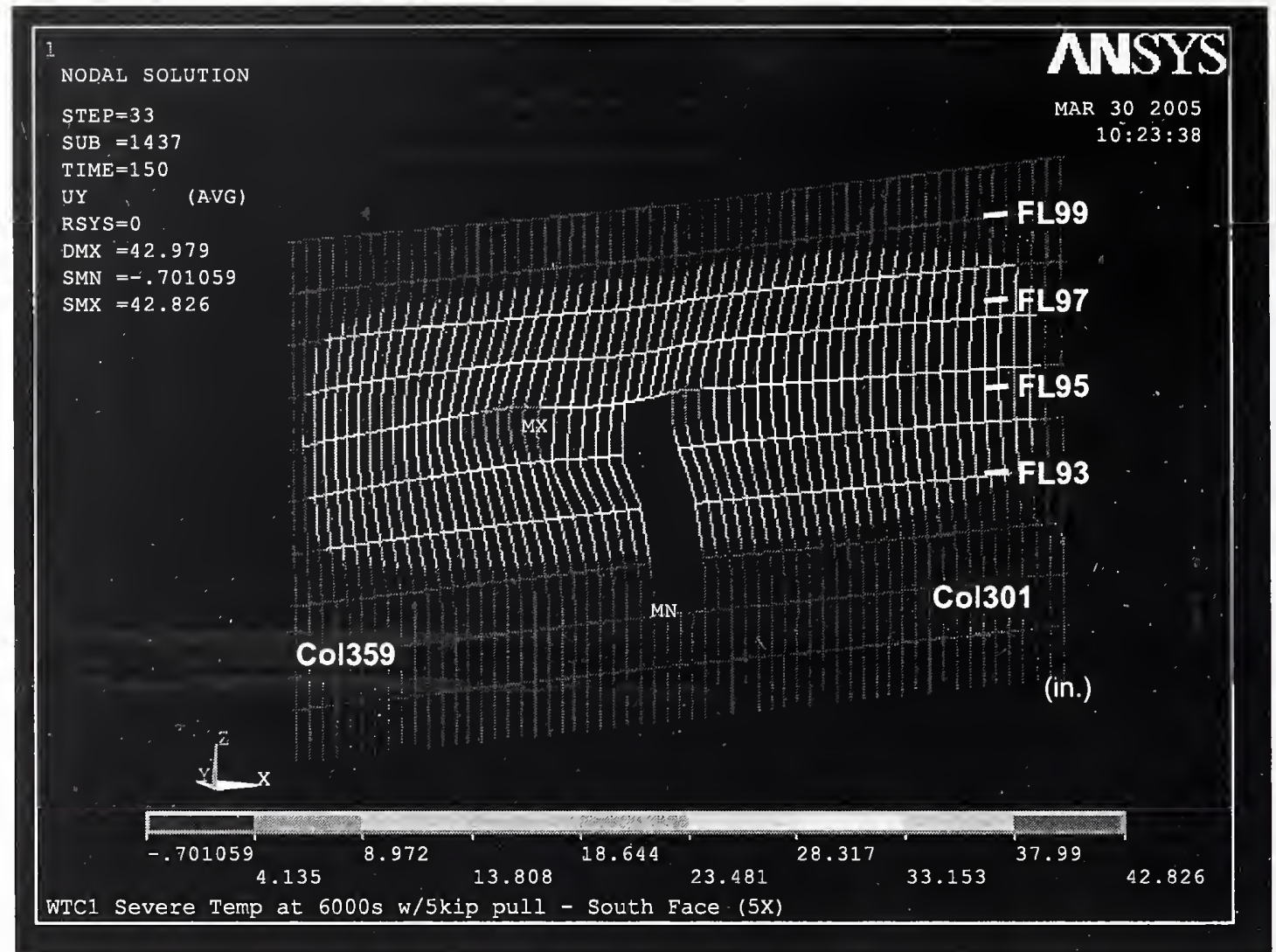

Figure 5-7. Inward bowing of south wall of WTC 1 global model with creep at $100 \mathrm{~min}$ for Case B conditions with 5 kip pull-in forces ( $5 x$ displacement magnification).

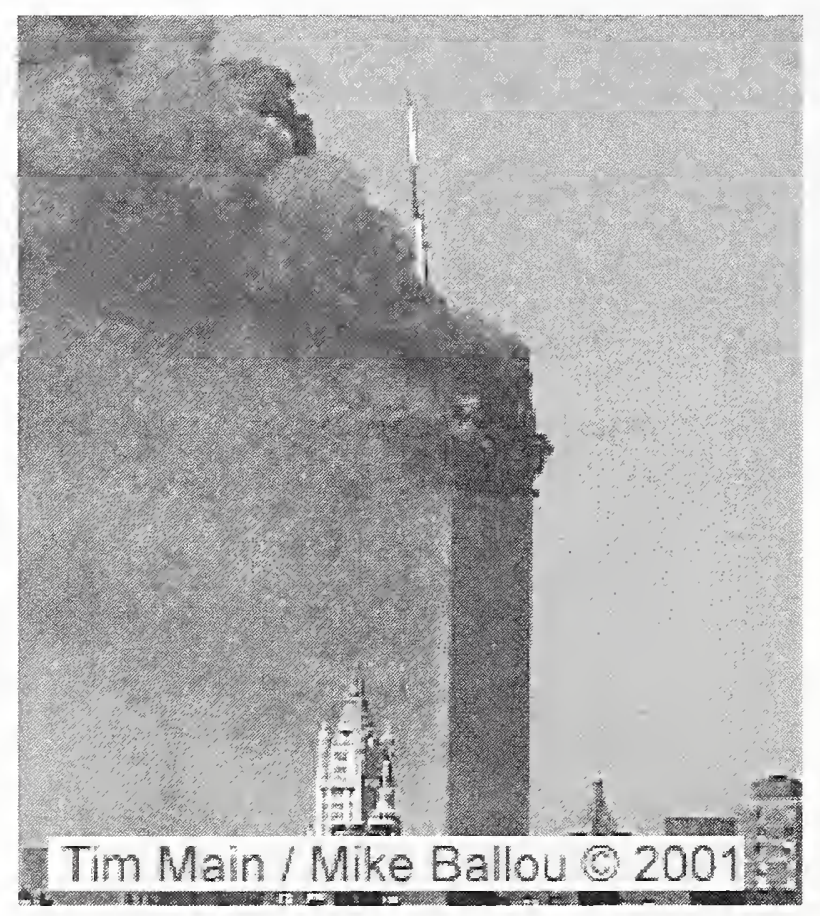

Figure 5-8. Collapse initiation and tilting of WTC 1 (view from the northeast). 


\section{$5.3 \quad$ WTC 2 COLLAPSE SEQUENCE}

The aircraft traveling in a north-northeast direction impacted the south wall of WTC 2 at 9:03 a.m. The aircraft mostly severed columns and floors that were on the east side of the building between Floor 78 and Floor 84. The subsequent fires were also on the east side of the building. At 9:59 a.m., about 56 min after the aircraft impact, the building started to collapse with the east wall buckling inward, followed by tilting of the building above Floor 82 to the east and south.

The section below discusses the sequence of main structural events that led to the collapse of WTC 2, and the causes and effects of these structural events along with key observations, starting from aircraft impact.

The WTC 2 collapse sequence consisted of five main structural events. These events are chronologically listed in Table 5-4 and discussed below. Actual observations for WTC 2 are summarized in Table 5-5, which are based on NIST's examination of photos and videos (NIST NCSTAR 1-6).

Table 5-4. Summary of main events that led to the collapse of
\begin{tabular}{|c|l|}
\hline Event number & \multicolumn{1}{c|}{ Event } \\
\hline 1 & Aircraft impact \\
2 & Sagging of floors and floor/wall disconnections \\
3 & Bowing of east wall \\
4 & Unloading and leaning of core \\
5 & Buckling of east wall and collapse initiation \\
\hline
\end{tabular}

Table 5-5. Observations on WTC 2 provided by NIST.

\begin{tabular}{|c|c|c|}
\hline Time & $\begin{array}{l}\text { Time } \\
\text { from } \\
\text { Impact } \\
\text { (min) }\end{array}$ & Observation \\
\hline 9:03 & 0 & Aircraft impact on the south wall of WTC 2 between Floors 77 and 85, Columns 404 to 443 . \\
\hline $9: 21$ & 18 & $\begin{array}{l}\text { Columns of the east wall bowed inward over the entire width of Floors } 78 \text { to } 83 \text {; maximum of } \\
7-9 \text { in. at Floor } 80 .\end{array}$ \\
\hline $9: 38$ & 35 & Floor 83 disconnections on the east wall appeared to extend. \\
\hline 9:54 & 51 & $\begin{array}{l}\text { Columns of the east wall bowed inward between Floor } 78 \text { and Floor } 84,12-20 \text { in. at } \\
\text { Floor } 80 \text {. } \\
\text { East side of Floor } 83 \text { draped between Columns } 310 \text { to } 342 \text {. }\end{array}$ \\
\hline 9:59 & 56 & $\begin{array}{l}\text { WTC } 2 \text { began to collapse. } \\
\text { Column splices failed at every third panel and columns sprung back from inward bowing as } \\
\text { collapse initiated on the east wall near the northeast corner } \\
\text { Smoke and debris clouds were expelled from Floor } 81 \text { on the east, north, and west walls of } \\
\text { the building. } \\
\text { WTC } 2 \text { appeared to tilt around the base of Floor } 82 \text { and initial downward motion was visible } \\
\text { at the same location. } \\
\text { Tilt of approximately } 3 \text { to } 4 \text { degrees to the south and } 7 \text { to } 8 \text { degrees to the east occurred } \\
\text { before building section fell. } \\
\text { Kink (change in slope) on the southeast corner near Floor } 94 \text { (halfway along building section } \\
\text { above failure). } \\
\text { Kink (change in slope) and offset about at the Floor } 106 \text {. }\end{array}$ \\
\hline
\end{tabular}




\section{Aircraft Impact}

The aircraft impacted the south wall of WTC 2, severing a number of exterior columns on the south wall from Floor 78 to Floor 84 . The south office area floor system sustained severe structural damage between Columns 410 and 436 from Floor 79 to Floor 83. Core columns 701, 702, 801, 802, 803, 901, 903, 1001, 1002, 1003, and 1004 were severed or heavily damaged between Floor 77 and Floor 84 . The aircraft also severed Column 253 of the north wall. The aircraft damaged the floor framing and core columns at the southeast corner of the core. In summary, 32 of 59 columns of the south wall, two of 59 columns of the north wall, and 11 of 47 core columns were severed or heavily damaged. Thermal insulation was damaged from the impact area through the east half of the core to the north and east exterior walls. The floor truss seat connections over about one-quarter to one-half of the east side of the core were severed on Floor 80 and Floor 81 and over about one-third of the east wall on Floor 83. Figures 2-3, 2-15, and 2-19 summarize aircraft impact damage to the exterior wall and core columns and floors of WTC 2.

Gravity loads in the columns that were severed on the south wall and in the southeast corner of the core were redistributed to adjacent intact columns and also to the columns on the east wall (see Table 5-6). In this redistribution, the total axial load on the core columns reduced by 6 percent, and the total axial load on the north wall columns reduced by 10 percent. The total axial load on the east wall columns increased by 24 percent, and the total axial load on the west and south wall columns increased by 2 percent to 3 percent. The large increases in loads on the east wall resulted from their proximity to the severed core columns at the southeast comer of the core. The total load on the south wall at Floor 83 did not change, as some of the loads from the corc area were redistributed to that wall through the hat truss.

At Floor 105, splices in the columns at the southeast corner of the core failed (Columns 1001 and 1002 and most likely Columns 701, 801, 901, 902, and 1003). This increased the core tendency to lean toward southeast and also increased the vertical downward displacement of the core at the impact zone. After the core column splices failed, 73 percent of the loads released from the failing core columns were redistributed through the hat truss to the exterior walls.

As can be seen in Table 5-6 for Floor 83, about 20 percent (= $227 \mathrm{kip} / 1,263 \mathrm{kip}$ ) of the redistributed load at the hat truss level of the south wall was transferred through columns, and the rest of the load (about 1,000 kip) was transferred to the columns of the east and west walls through the spandrels.

After load redistribution following impact, the core was prevented from tilting excessively toward the east by the north and south exterior walls through the action of the floors and the hat truss.

\section{Sagging of Floors and Floor/Wall Disconnections}

Aircraft impact and high temperatures due to subsequent fires caused Floor 79 through Floor 83 to sag. The sag was greater at Floor 80 and Floor 81 where the truss seats on the east side of the core failed at aircraft impact (see Fig. 5-9). High temperatures weakened the truss seats on the east exterior wall and caused truss seats to fail at Floor 83 and Floor 82 (see Fig. 5-10) which in turn increased the sag in those floors. Floor sagging induced pull-in forces on the east wall columns, beginning approximately $10 \mathrm{~min}$ after impact and increasing with time. 


\section{Bowing of East Wall}

The east wall columns bowed inward as a result of increasing temperatures (reduced strength and stiffness) and pull-in forces induced by sagging floors (see Fig. 3-98). The inward bowing in the east wall increased with time due to the combined effects of pull-in from sagging floors (see Fig. 4-101), increased axial loads, and a continuous increase in plastic and creep strains. As columns bowed, they shed load to adjacent unbowed columns, but the total column load on the east wall did not change significantly after impact until buckling of the east wall started near the collapse time (see Fig. 5-11).

\section{Unloading and Leaning of Core}

With increasing time and temperatures, the core columns developed high plastic and creep strains, especially on the east side of the core. Plastic and creep strains exceeded the thermal expansion strains beginning about 30 min after the aircraft impact (see Fig. 5-12). High plastic and creep strains caused unloading on the east side core columns. This increased leaning of the core toward the east and transferred more loads to the east wall (Table 5-6). Calculations showed that resistance to core leaning was provided by the north and south exterior walls, partly through the floors and partly through the hat truss. Leaning of the core resulted in tilting of the upper part of the tower as the east wall buckled.

\section{Buckling of East Wall and Collapse Initiation}

With continuously increased bowing and axial loads, the entire width of the east wall buckled inward. The instability started at the center of the wall and rapidly progressed horizontally toward the sides. As a result of the buckling of the east wall, the east wall significantly unloaded, redistributing its load to the softened core through the hat truss and to the east side of the south and north walls through the spandrels (see Figs. 5-13 and 5-14 and Table 4-38). The section of tower above the buckled wall suddenly moved downward, and the building tilted toward the east (see Fig. 5-15).

The section of the building above the impact zone tilted to the east and south (observed at about $7^{\circ}$ to $8^{\circ}$ to east and about $3^{\circ}$ to $4^{\circ}$ to south, Fig. 5-16) as column instability progressed from the east wall to the adjacent south and north walls. The release of potential energy due to downward movement of the building mass above the buckled columns exceeded the strain energy that could be absorbed by the structure. Global collapse ensued. 
Table 5-6. Total column loads at Floor 83 and Floor 105 of WTC 2 for Case D conditions.

\begin{tabular}{|c|c|c|c|c|c|c|c|c|c|c|c|}
\hline \multirow[t]{2}{*}{ Row } & \multirow{2}{*}{$\begin{array}{c}\text { Analysis } \\
\text { Step }\end{array}$} & \multicolumn{2}{|c|}{ West Wall } & \multicolumn{2}{|l|}{ East Wall } & \multicolumn{2}{|c|}{ North Wall } & \multicolumn{2}{|c|}{ South Wall } & \multicolumn{2}{|l|}{ Core } \\
\hline & & Floor 83 & Floor 105 & Floor 83 & Floor 105 & Floor 83 & Floor 105 & Floor 83 & Floor 105 & Floor 83 & Floor 105 \\
\hline (1) & Before Impact & 18,065 & 8,497 & 18,114 & 8,572 & 13,567 & 7,382 & 13,284 & 7,169 & 61,828 & 17,123 \\
\hline (2) & After Impact & 18,670 & 9,170 & 22,481 & 11,272 & 12,193 & 6,487 & 13,511 & 8,432 & 57,821 & 13,382 \\
\hline (3) & $10 \mathrm{~min}$ & 18,728 & 9,182 & 22,226 & 11,061 & 11,896 & 6,250 & 13,358 & 8.275 & 58,413 & 13,975 \\
\hline (4) & $20 \mathrm{~min}$ & 18,914 & 9,279 & 22,208 & 11,120 & 12,052 & 6,311 & 13,318 & 8,351 & 58,124 & 13,682 \\
\hline (5) & $30 \mathrm{~min}$ & 18,876 & 9,370 & 23,681 & 11,859 & 11,770 & 6,416 & 13,365 & 8,553 & 56,967 & 12,544 \\
\hline (6) & $40 \mathrm{~min}$ & 18,531 & 9,198 & 23,682 & 11,927 & 11,906 & 6,524 & 13,473 & 8,691 & 56,825 & 12,402 \\
\hline (7) & $43 \mathrm{~min}$ & 15,667 & 7,086 & 15,143 & 8,026 & 14,215 & 6,546 & 16,292 & 9,169 & 62,422 & 17,915 \\
\hline (8) & $(2)-(1)$ & 604 & 674 & 4,368 & 2,699 & $-1,374$ & -895 & 227 & 1,263 & $-4,007$ & $-3,741$ \\
\hline (9) & $(6)-(2)$ & -138 & 28 & 1,201 & 656 & -287 & 37 & -38 & 259 & -996 & -980 \\
\hline (10) & $(7)-(6)$ & $-2,864$ & $-2,112$ & $-8,539$ & $-3,901$ & 2,309 & 23 & 2,819 & 479 & 5,596 & 5,513 \\
\hline
\end{tabular}

Note: Compression is positive. Units are in kip. 


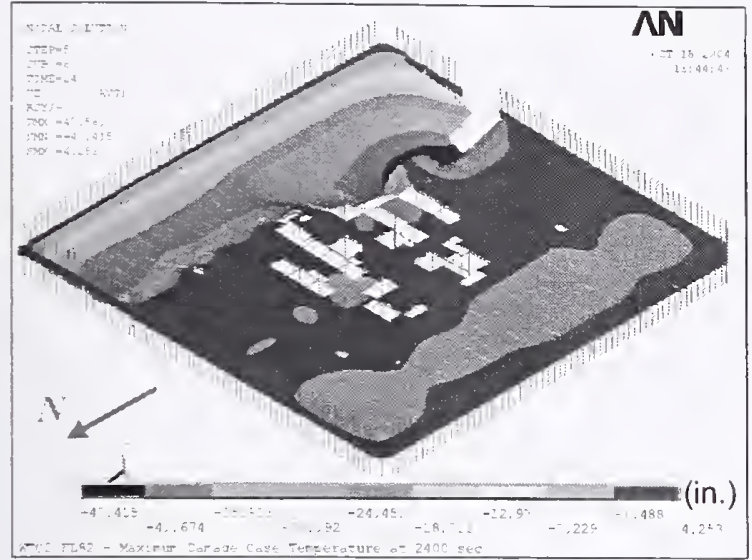

(a) Floor 82

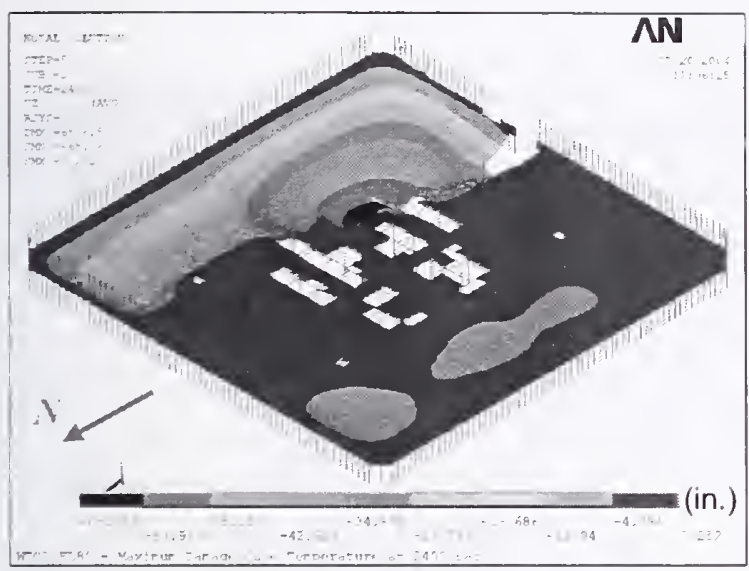

(c) Floor 80

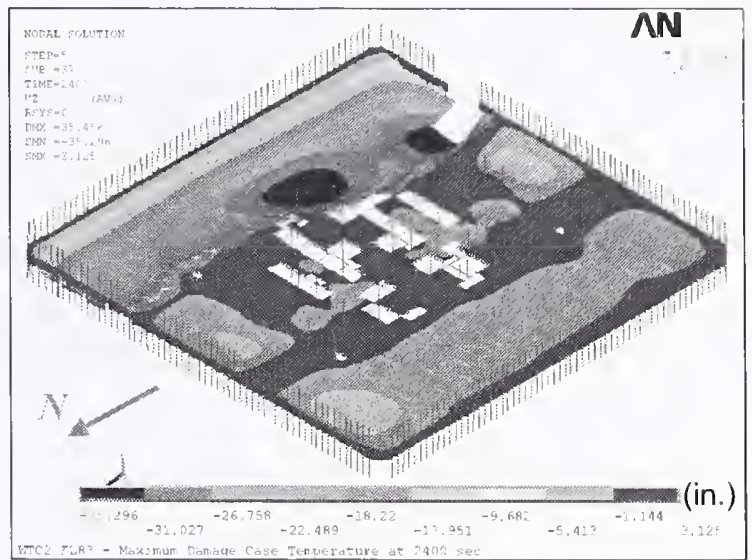

(b) Floor 83

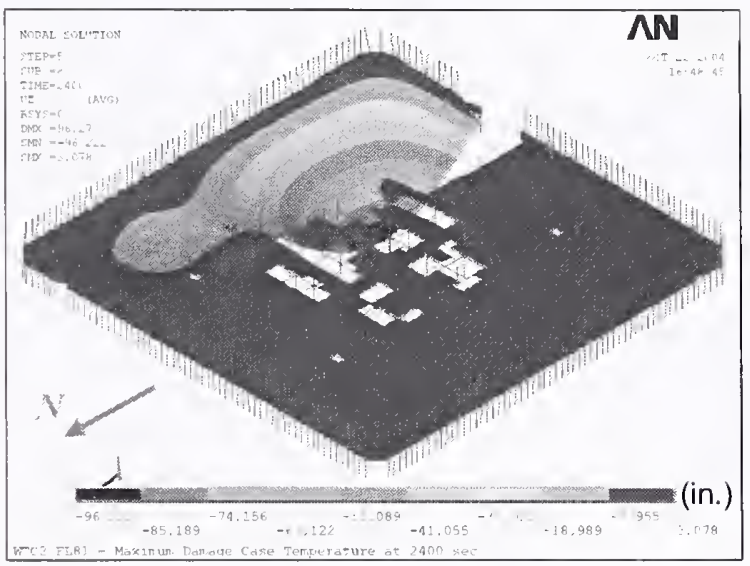

(d) Floor 81

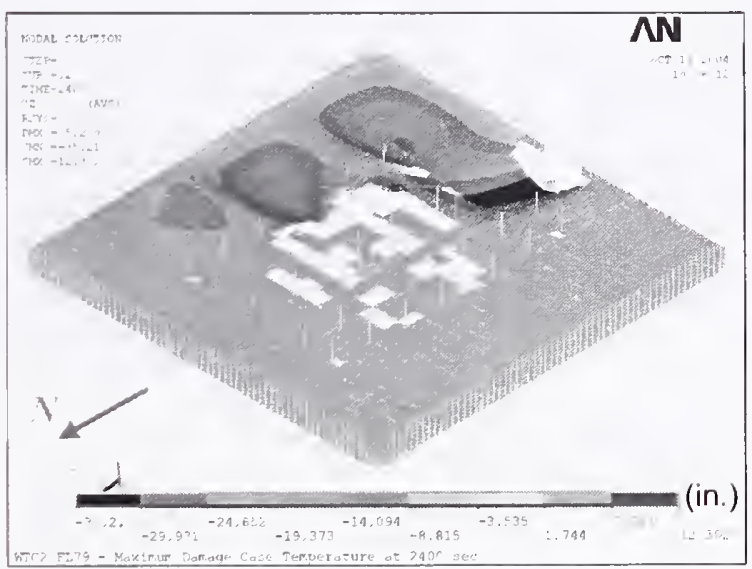

(e) Floor 79

Figure 5-9. Vertical displacements of full floor models of WTC 2 for Case $D_{i}$ temperature condition at $\mathbf{4 0} \mathrm{min}$ (downward displacement is negative). 


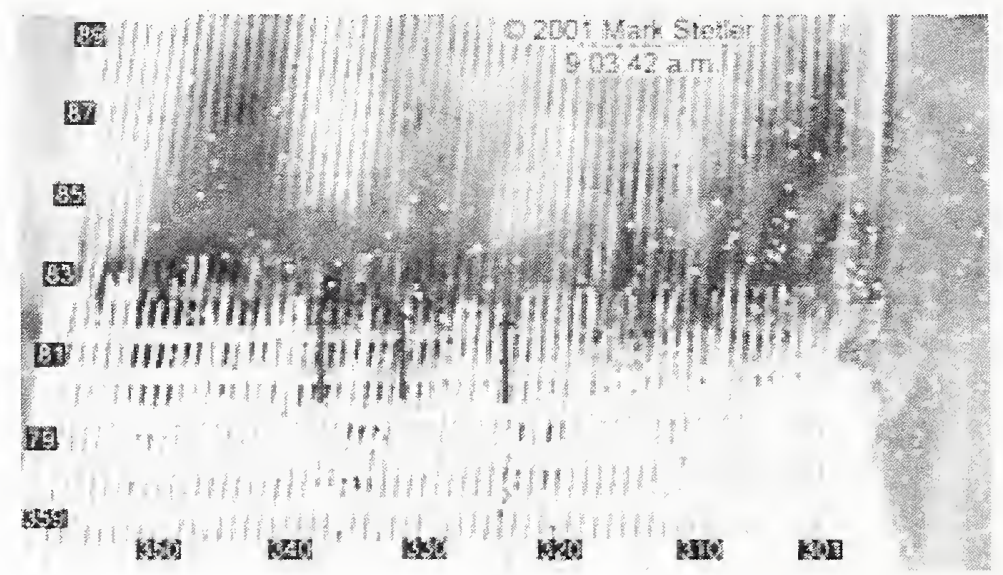

(a) After impact damage

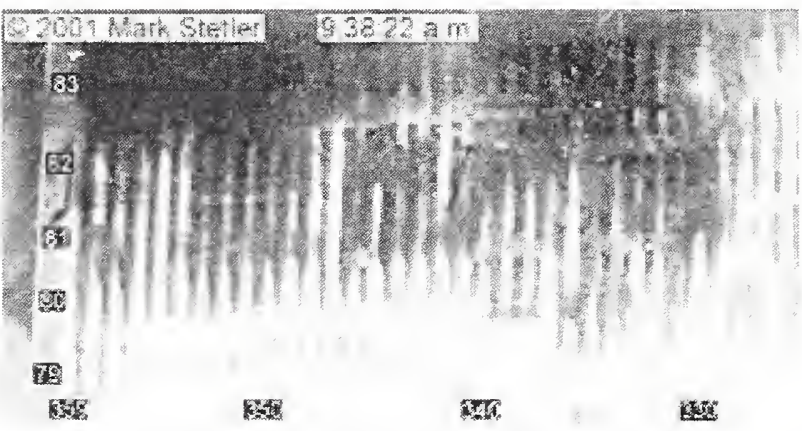

(b) South side at 9:38 a.m. (35 min after impact)

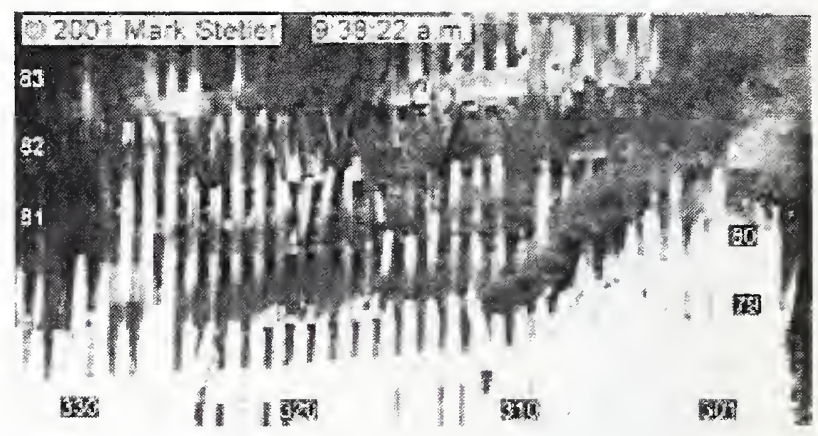

(c) North side at 9:38 a.m. (35 min after impact)

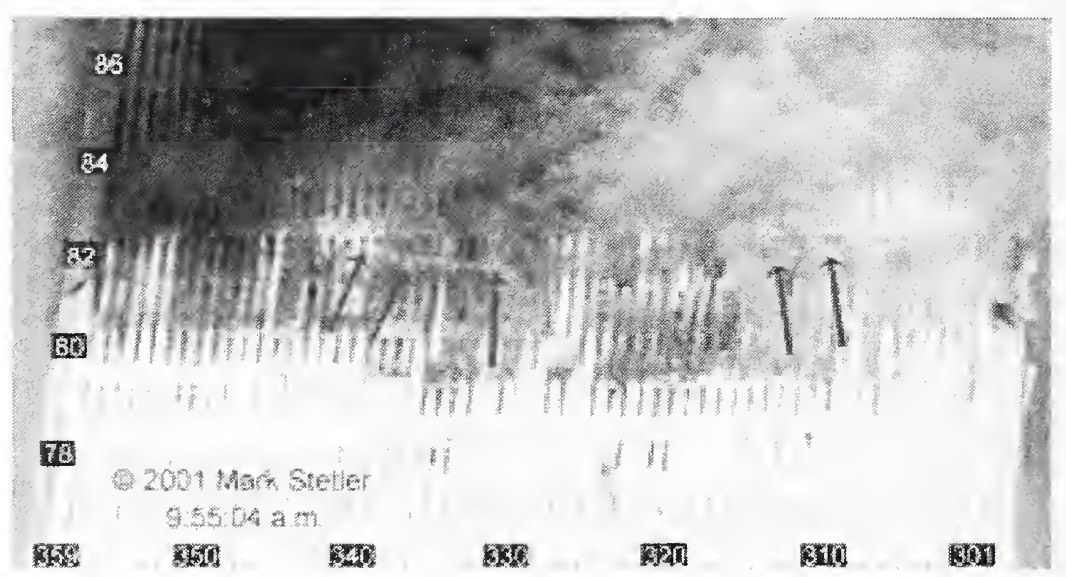

(d) At 9:54 a.m.

(51 min after impact)

Figure 5-10. Floor sagging observed on the east wall of WTC 2 at different times. 


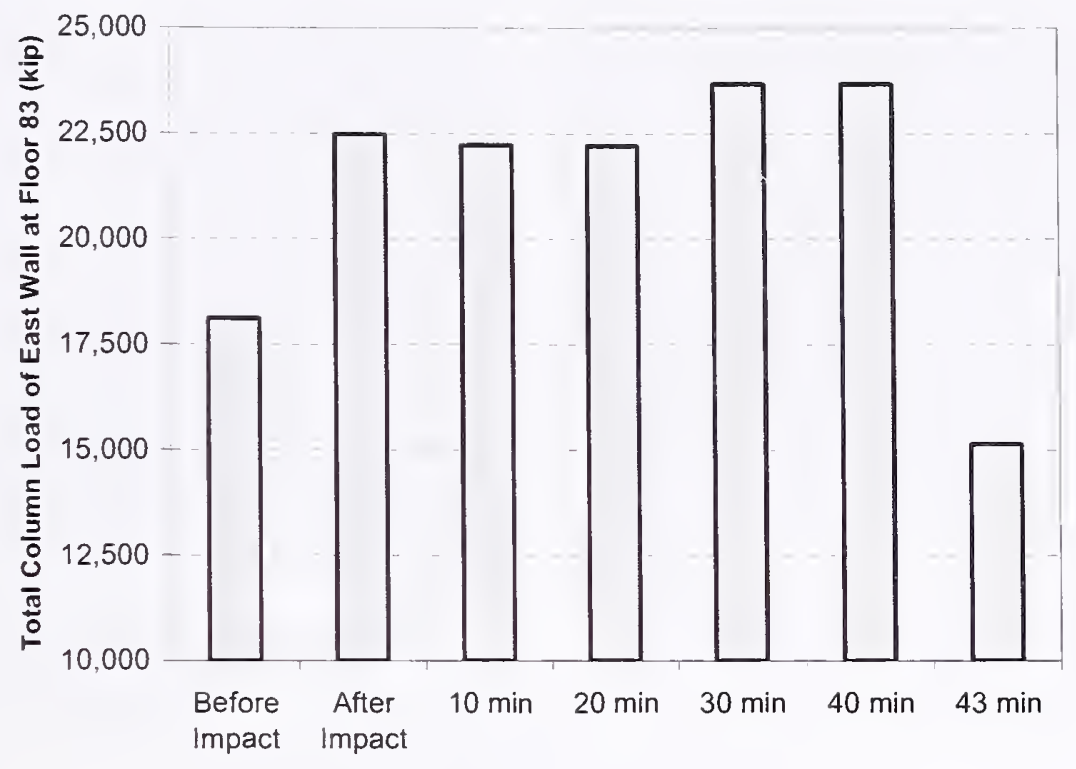

Time

Figure 5-11. Total column loads at Floor 83 of the east wall of WTC 2 global model for Case $\mathrm{D}$ conditions (compression is positive). 


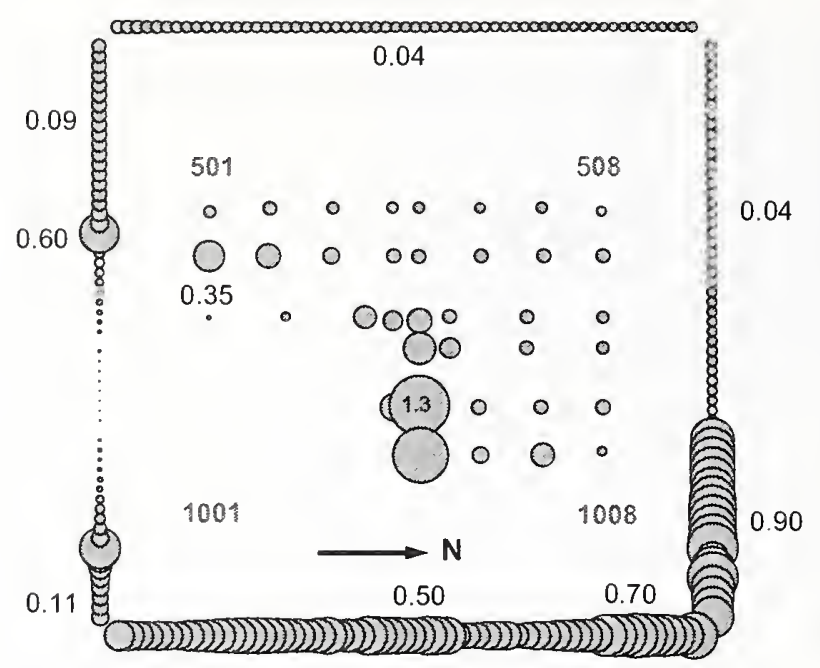

(a) At $20 \mathrm{~min}$

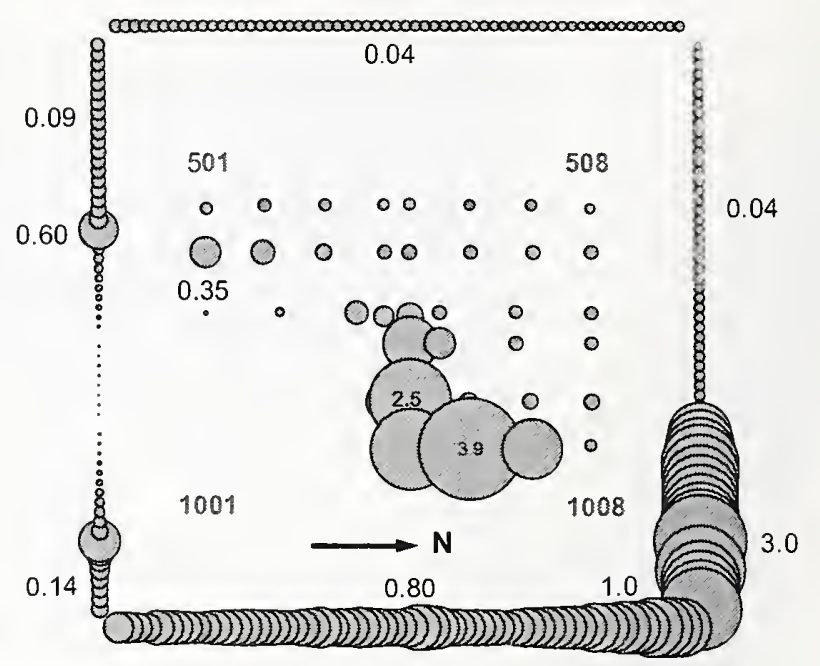

(b) At $30 \mathrm{~min}$

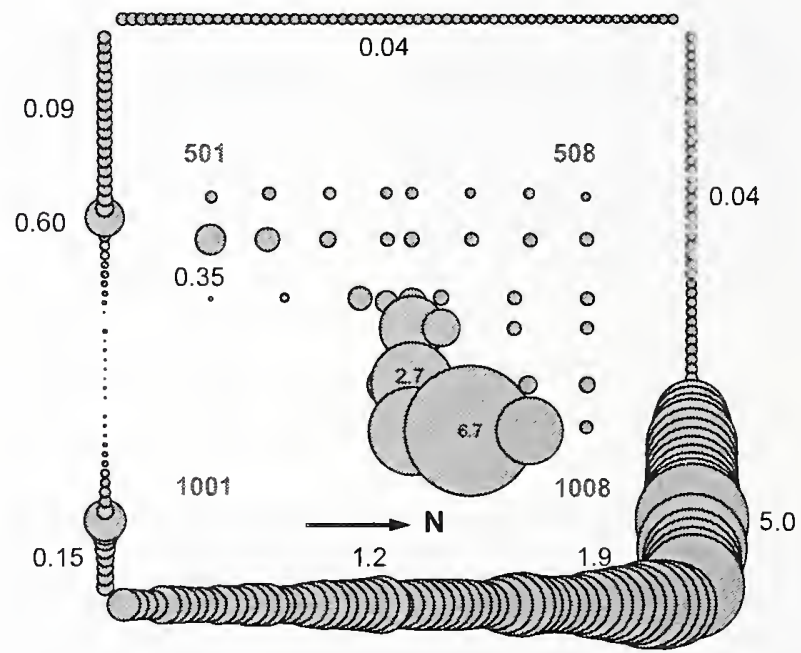

(c) At $40 \mathrm{~min}$

Figure 5-12. Maximum elastic + plastic + creep strain magnitudes for columns between Floor 78 and Floor 83 of WTC 2 global model for Case D conditions at $20 \mathrm{~min}, 30 \mathrm{~min}$, and $40 \mathrm{~min}$ (compressive strain is positive; strain values are in percent). 


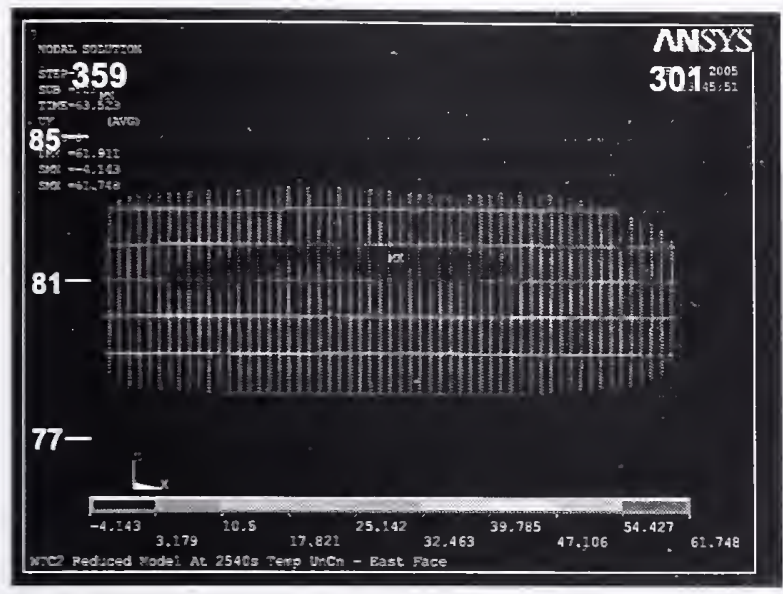

(a) View from east

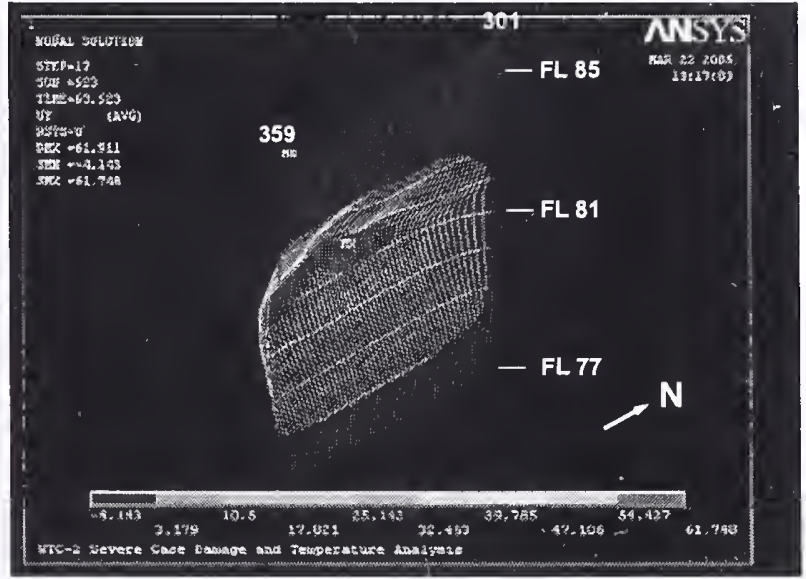

(b) View from southeast

Figure 5-13. Inward bowing of the east wall of WTC 2 global model for Case D conditions at $43 \mathrm{~min}$ at the instant of collapse initiation (deformed shape scaled four times).

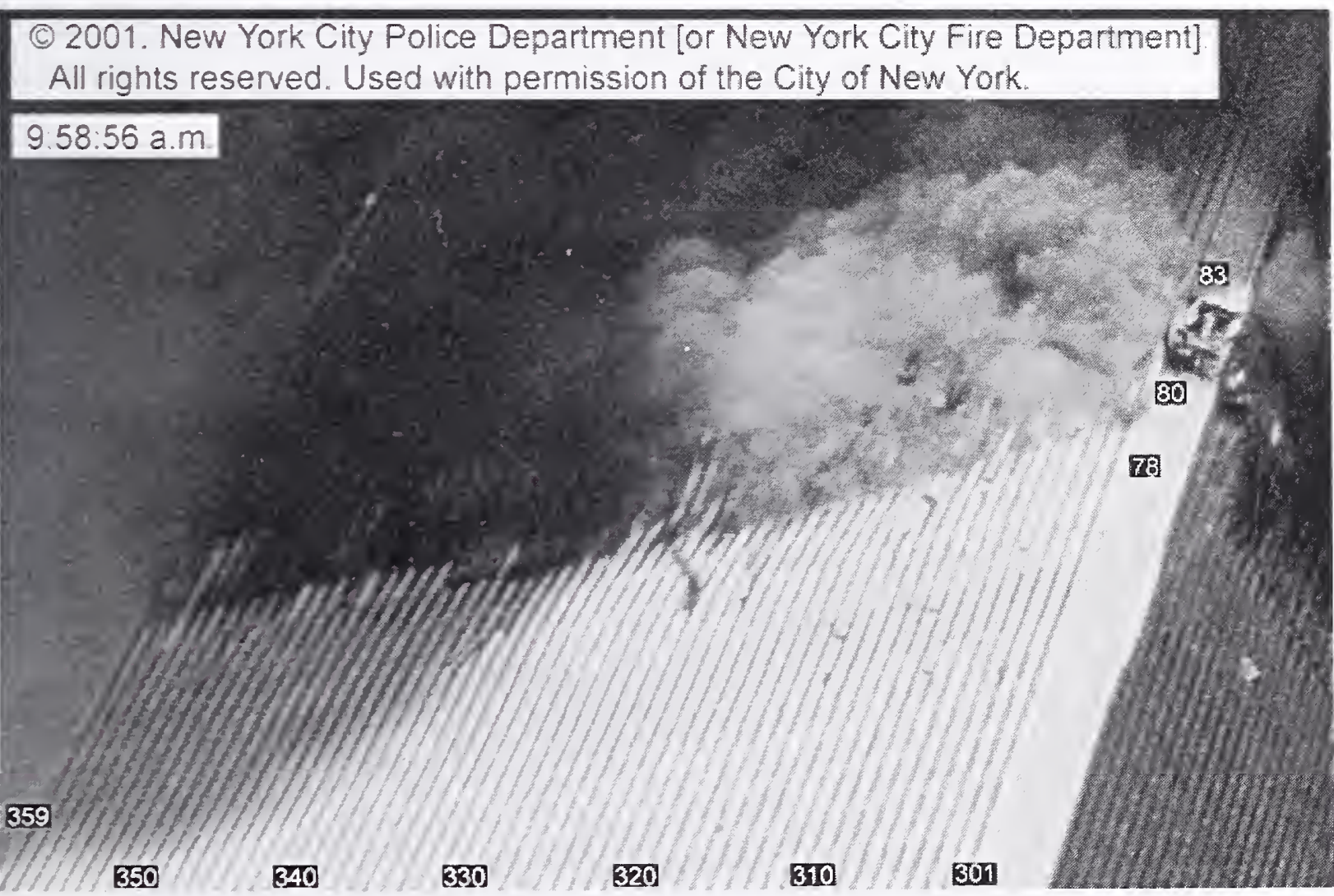

Figure 5-14. Inward bowing of exterior columns of the west wall of WTC 2 just before collapse. 


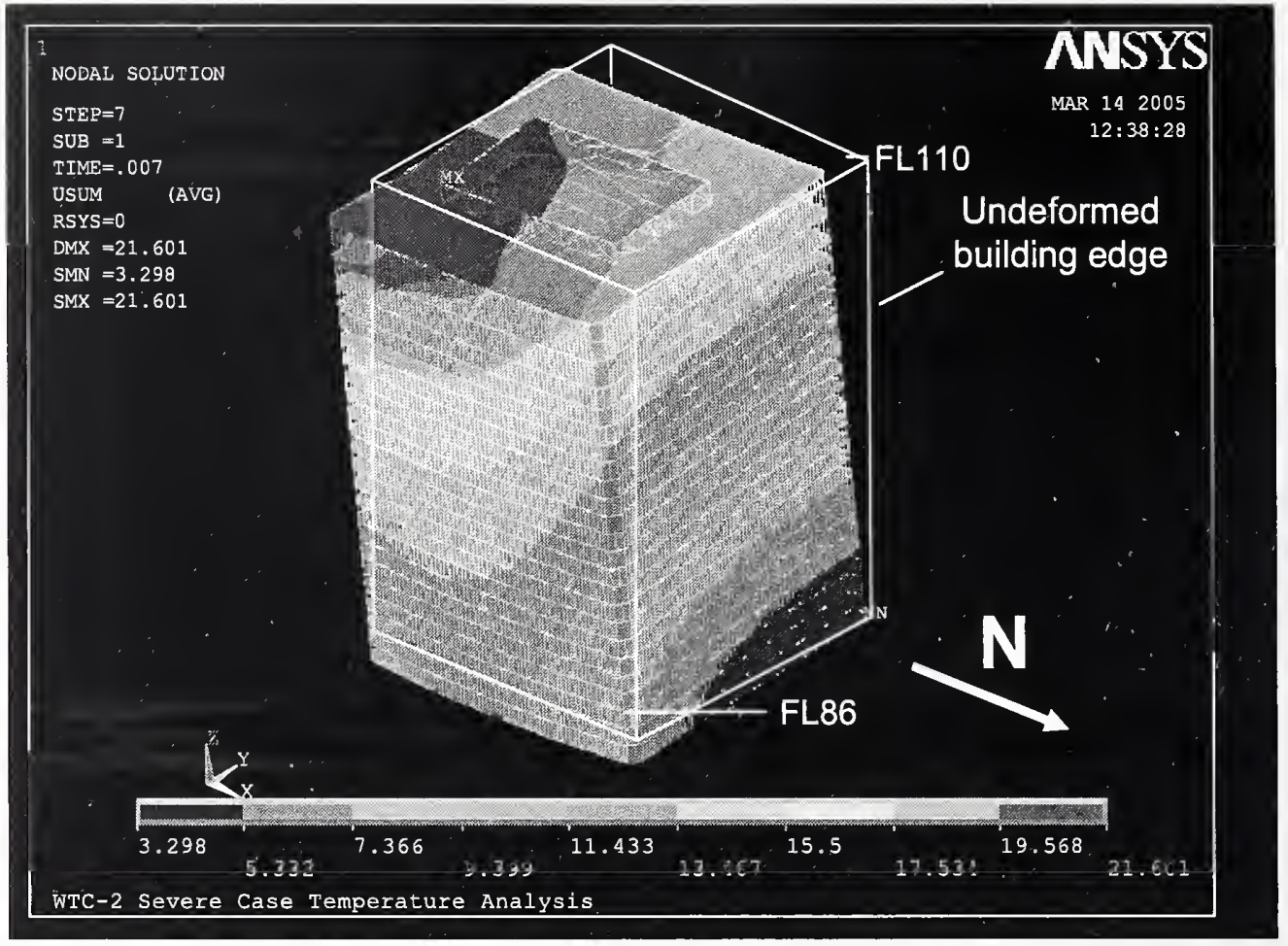

Figure 5-15. Total displacements of WTC 2 global model above Floor 86 for Case D conditions at $\mathbf{4 3} \mathrm{min}$ at collapse initiation (note the tilt toward east and south; deformed shape magnified 20 times).

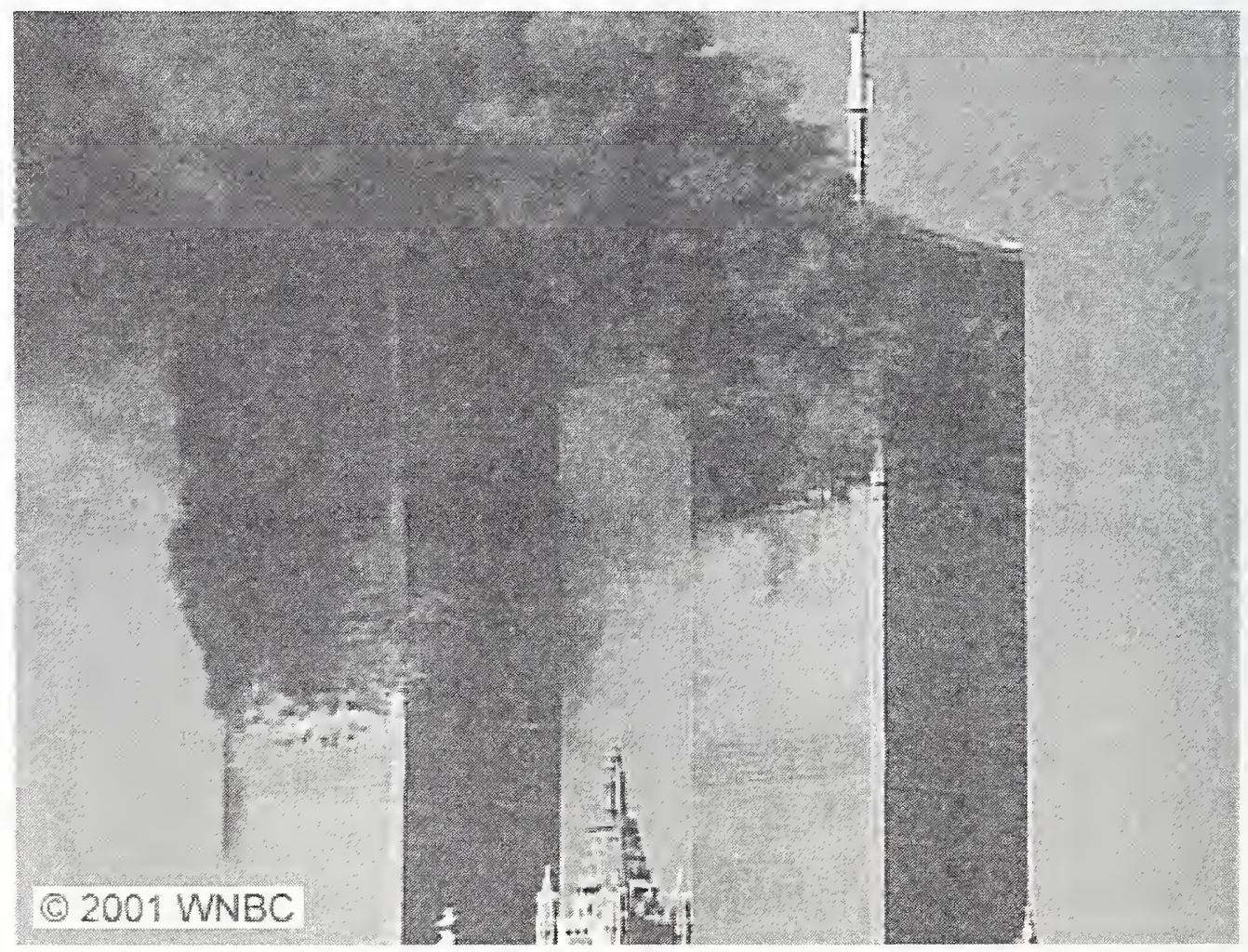

Figure 5-16. Initiation of Collapse of WTC 2. Note the tilt toward east and south. 


\subsection{DISCUSSION}

The structural analyses of this study on components, subsystems, isolated exterior walls and corcs, and global models of WTC 1 and WTC 2, as well as observations from photos and videos taken during the event, showed that the tower collapses were caused by the combined effects of the structural and thermal insulation damage from aircraft impact and the subsequent intense fires.

The structural impact damage alone did not cause the collapse of the towers, as they stood for pcriods of time, and collapsed after fire-induced weakening of the cores, floor systems and exterior walls. In the absence of impact damage, there would have been no insulation damage, and the likelihood of collapse of towers under the intense fires would have been very small.

The damage to insulation was a result of direct impact by debris from the aircraft impact, which knocked down walls and partitions and removed the insulation (NIST NCSTAR 1-6). Insulation is a key fire protection system for steel structural members. Without insulation, steel members may be heated during the "effective duration" of the fires to an extent that causes significant stiffness and strength reduction. According to the results of fire dynamics simulations by NIST (NIST NCSTAR 1-5), fire moved from location to location, and the "effective duration" of the fire at any one location was roughly about 20 minutes, which was much shorter than the time to collapse. It was found in the results of the thermal analysis conducted by NIST that temperatures of floor trusses and columns with insulation were lower than $400{ }^{\circ} \mathrm{C}$ during the Case B fire (100 min long) for WTC 1 . On the other hand, temperatures of steel members that lost insulation were found to be higher than $600{ }^{\circ} \mathrm{C}$ and even higher than $800{ }^{\circ} \mathrm{C}$.

Reductions in modulus of elasticity, yield strength, and ultimate tensile strength of steel in the WTC towers were found to be 13 percent, 20 percent, and 10 percent at $400{ }^{\circ} \mathrm{C}$, and 35 percent, 92 pcrcent, and 80 percent at $700{ }^{\circ} \mathrm{C}$. In addition, creep in steel may become more significant than plastic strain when the steel is exposed to temperatures higher than $500{ }^{\circ} \mathrm{C}$ under high stress.

The temperatures in the steel members without insulation damage were lower than those with insulation damage for the same fire (see Fig. 5-17 (c)), and the lower temperatures resulted in much reduced crecp, plasticity, and buckling. Without insulation damage, floor sag would have been significantly reduced (see Figs. 5-17 (d) and (e)), and consequently the floors could not and would not have pulled inward on the exterior wall. Without insulation damage, the core columns and the exterior wall would not be significantly weakened, and the likelihood of the exterior wall buckling would be negligibly small. Without insulation damage, the likelihood of collapse of the towers would have been very small. 


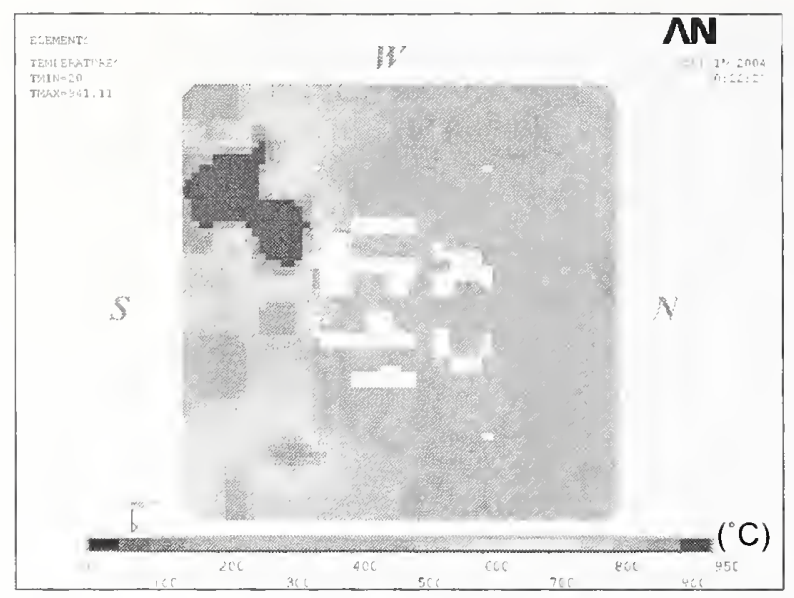

(a) Temperatures on the top surface of the slab

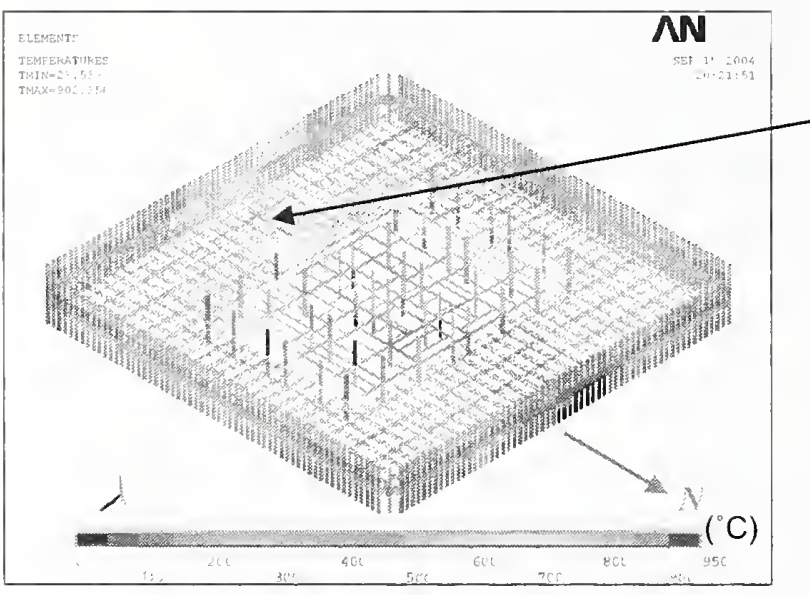

(c) Temperatures of steel members

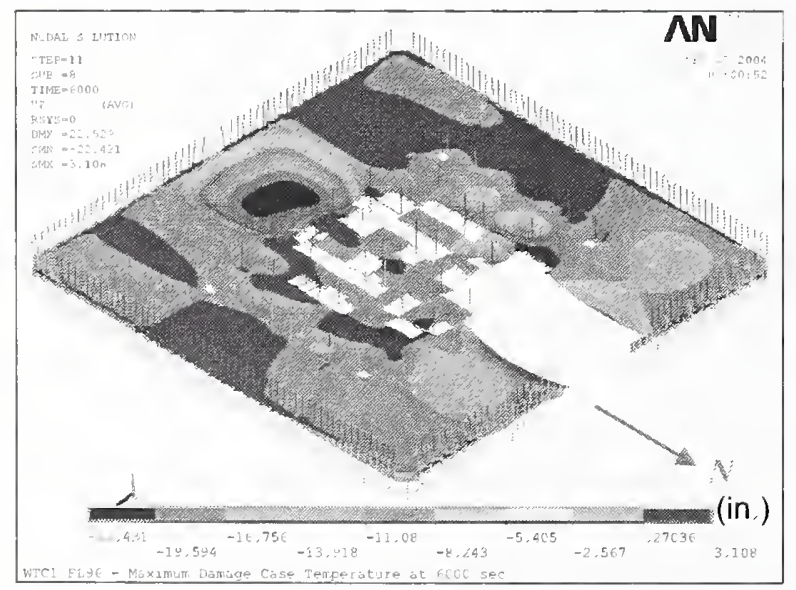

(d) Vertícal displacement (entire model)

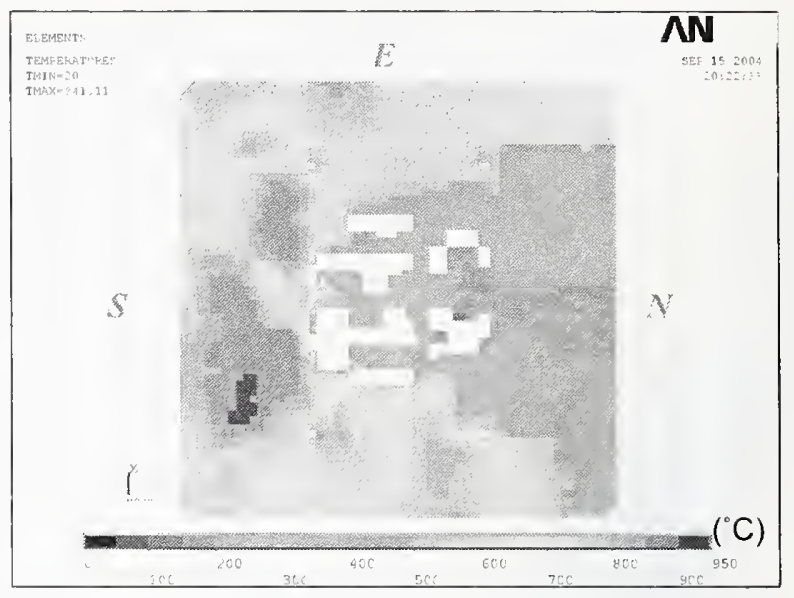

(b) Temperatures on the bottom surface of the slab

Area of insulation damage

Note: Structural members that were severed by aircraft impact are also shown in temperature plots (a), (b), and (c)

Figure 5-17. Full floor model of Floor 96 of WTC 1 for Case $B_{i}$ temperature condition at $100 \mathrm{~min}$. 


\section{Appendix A \\ FEA OF FLOORS}

\section{A.1 MODEL DESCRIPTION}

A full floor model includes 1) both exterior and core columns extending from one floor below to one floor above, 2) spandrels of the floor of interest, 3) floor slab, 4) floor trusses including primary and bridging trusses, 5) strap anchors, 6) core beams, 7) deck support angles, and 8) break elements to capture failure modes including truss web diagonal buckling and weld failure, exterior and interior seat failure, stud failure, strap anchor weld failure, connection failure between primary and bridging trusses, and connection failure between long-span and transfer trusses. A full floor model without impact damage is shown in Fig. A-1.

The members listed below were found to fail in the early stage of thermal loading and cause large nonlinearities in the subsequent stages; therefore, they were removed from the model to enhance computational efficiency. Figure A-2 shows the full floor model with these members removed. Deck support angles and bridging trusses buckled due to their thermal expansion in the one-way area. Shear studs and welds between strap anchors and truss top chords failed due to shear force caused by the difference in thermal expansion between the floor and the exterior wall in the direction transverse to the primary trusses. Although these members did not exist in the floor model, they were not expected to control the stability and ultimate failure mode of the full floor system under fire. Analyses performed after removal of the following members showed no premature failure mode such as torsional buckling of primary trusses:

- Deck support angles

- Bridging trusses except in the two-way zone

- Shear studs connecting the slab and the spandrel

- Strap anchors

The visco-elastic dampers that connected the truss bottom chords to the spandrels were not included in the full floor model because dampers were expccted to be soft when subjected to slow loading rates.

Truss members (top and bottom chords and web diagonals) were modeled by BEAM1 88 elements. Columns were modeled by BEAM1 89 elements. Spandrels wcre modeled by SHELL181 elements. The concrete slab was modeled by SHELL181 elements with four layers through the thickness. Each layer of the shell element for the slab had one integration point.

Nonlinear steel material properties were assigned to each structural member according to the drawings. A bilinear model with a yield point at its compressive strength was used for the concrete slab, where the yield strength was the same in both tension and compression. Creep in the steel was not included in any of the full floor analyses. It was found that creep in BEAM188/189 elements would cause severe convergence problems when those elements experience thermally-induced buckling. 
Both core and exterior columns were fixed in the vertical direction at the bottom. Core columns were free in horizontal directions and fixed in all rotations at the top and bottom. Exterior columns were fixed in translation perpendicular to the face of building and in rotation about the axis parallel to the face of the building at the top and bottom. They were also fixed in torsion at the top and bottom.

Elements corresponding to severed members due to impact were removed from the model in the beginning of the analysis based on the results of the aircraft impact analysis (NIST NCSTAR 1-2 and Chapter 5 of NCSTAR 1-6).

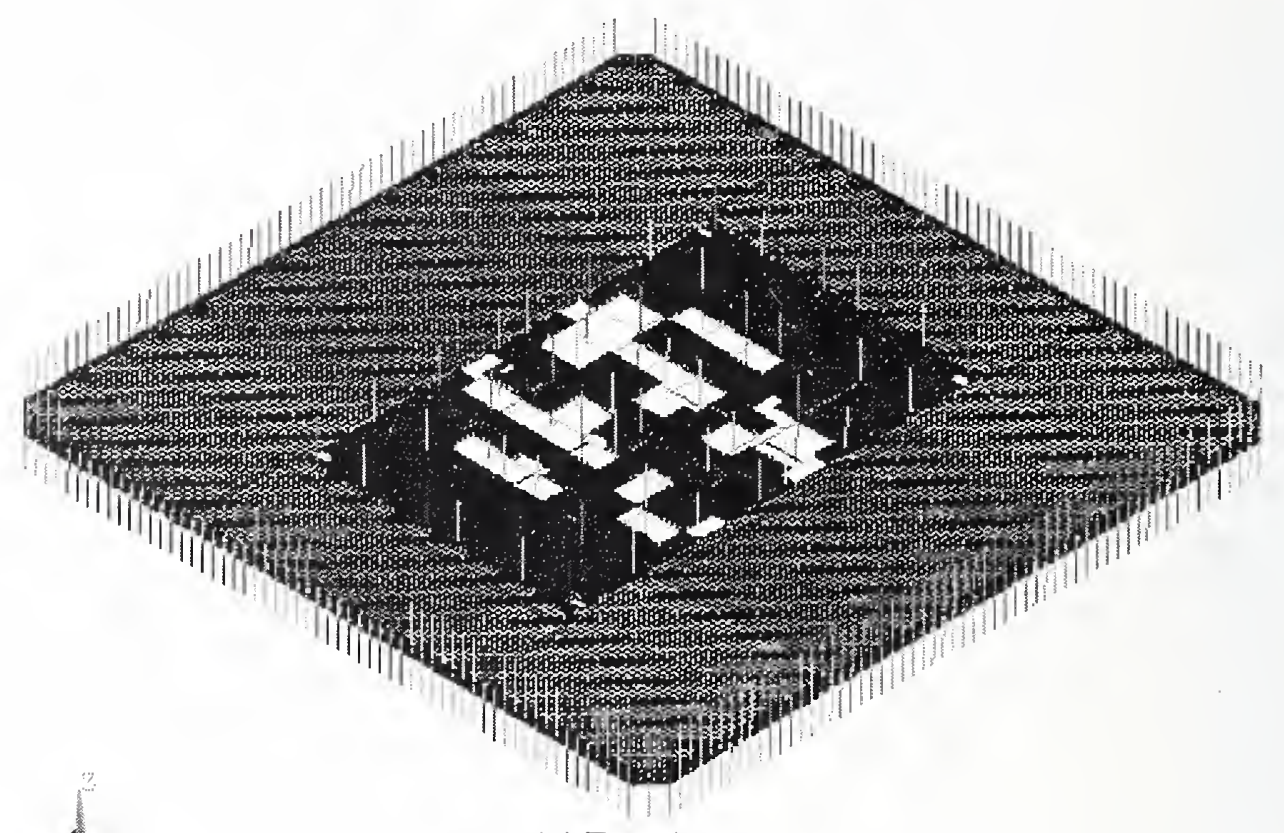

(a) Top view

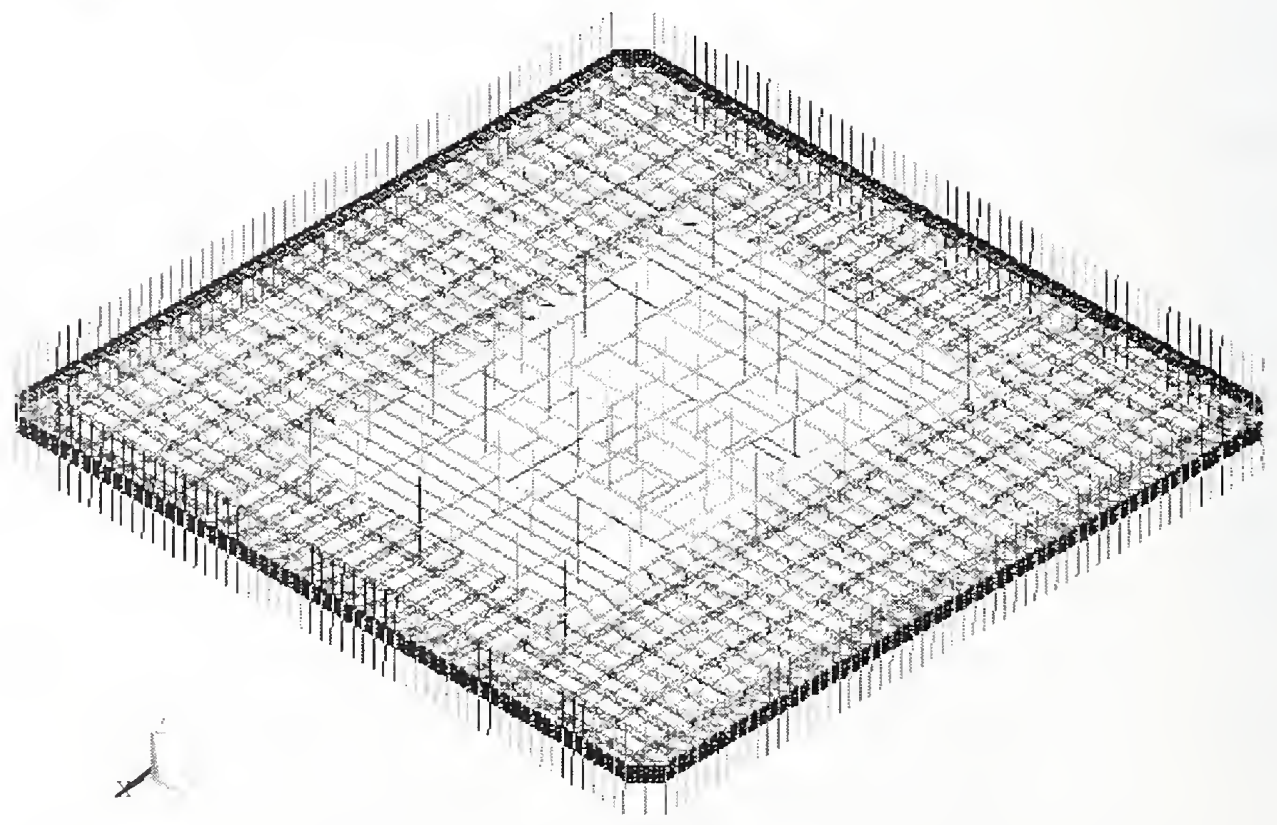

(b) Top view without concrete slab

Figure A-1. Full floor model without impact damage. 
The full floor model was first analyzed for dead and live loads, and then thermal loads were applied to simulate the path-dependent nonlinear response. Dead and live loads consisted of self-weight, 8 psf superimposed dead load, and 25 percent of design live loads. Design live loads varied from 55 psf to $85 \mathrm{psf}$ in the model. Vertical loads were not applied to columns. For thermal loads, NIST provided two sets of temperature time histories for each building. These temperature cases were designated as "Case $\mathrm{A}_{i}$ temperature condition" and "Case $\mathrm{B}_{\mathrm{i}}$ temperature condition" for WTC 1, and "Case $\mathrm{C}_{\mathrm{i}}$ temperature condition" and "Case $\mathrm{D}_{\mathrm{i}}$ temperature condition" for WTC 2. NIST later refined these temperatures, and refined cases were referred to as "Case A temperature condition" and "Case B temperature condition" for WTC 1, and "Case C temperature condition" and "Case D temperature condition" for WTC 2. Since the largest change in thermal insulation damage and temperatures occurred for WTC 1 Floor 97, Case $A_{i}$ and Case A temperature conditions were used to analyze the difference in the floor behavior for the two cases. Since results from the WTC 1 Floor 97 analysis under Case A temperature condition was very similar to those from the analysis with Case $\mathrm{A}_{\mathrm{i}}$ condition, it was concluded that the refined temperature cases would not change the floor behavior significantly. Therefore, analysis of other floors were not conducted with the refined temperature conditions.

Temperature data sets were provided for every structural node at $10 \mathrm{~min}$ intervals up to $100 \mathrm{~min}$ for WTC 1 and up to $60 \mathrm{~min}$ for WTC 2 for each temperature case. In the first step of the thermal loading, temperature was linearly ramped up from room temperature to the temperature specified at $10 \mathrm{~min}$. After the first step, the temperature was linearly interpolated between two data sets. Figures $A-3$ to $A-8$ show NIST temperature distributions for WTC 1 Floor 97 for Case $A_{i}$ temperature condition, Case A temperature condition, and $\mathrm{Case} \mathrm{B}_{\mathrm{i}}$ temperature condition. For each case, temperatures were plotted at only $10 \mathrm{~min}, 50 \mathrm{~min}$, and $100 \mathrm{~min}$. Figures A-9 to A-12 show NIST temperature distributions for WTC 2 Floor 82 for Case $C_{i}$ and Case $D_{1}$ temperature conditions. In the figures, severed members were also shown; however, the severed members were removed from the floor models and did not exist in the analysis.

Temperatures for truss members and spandrels were assigned at node locations and did not change within the cross section. Temperatures for columns were assigned at node locations and had a gradient within the cross section. Temperatures for the slab were assigned at node locations, and there were five points to define the temperature distribution through the thickness at each node location. 


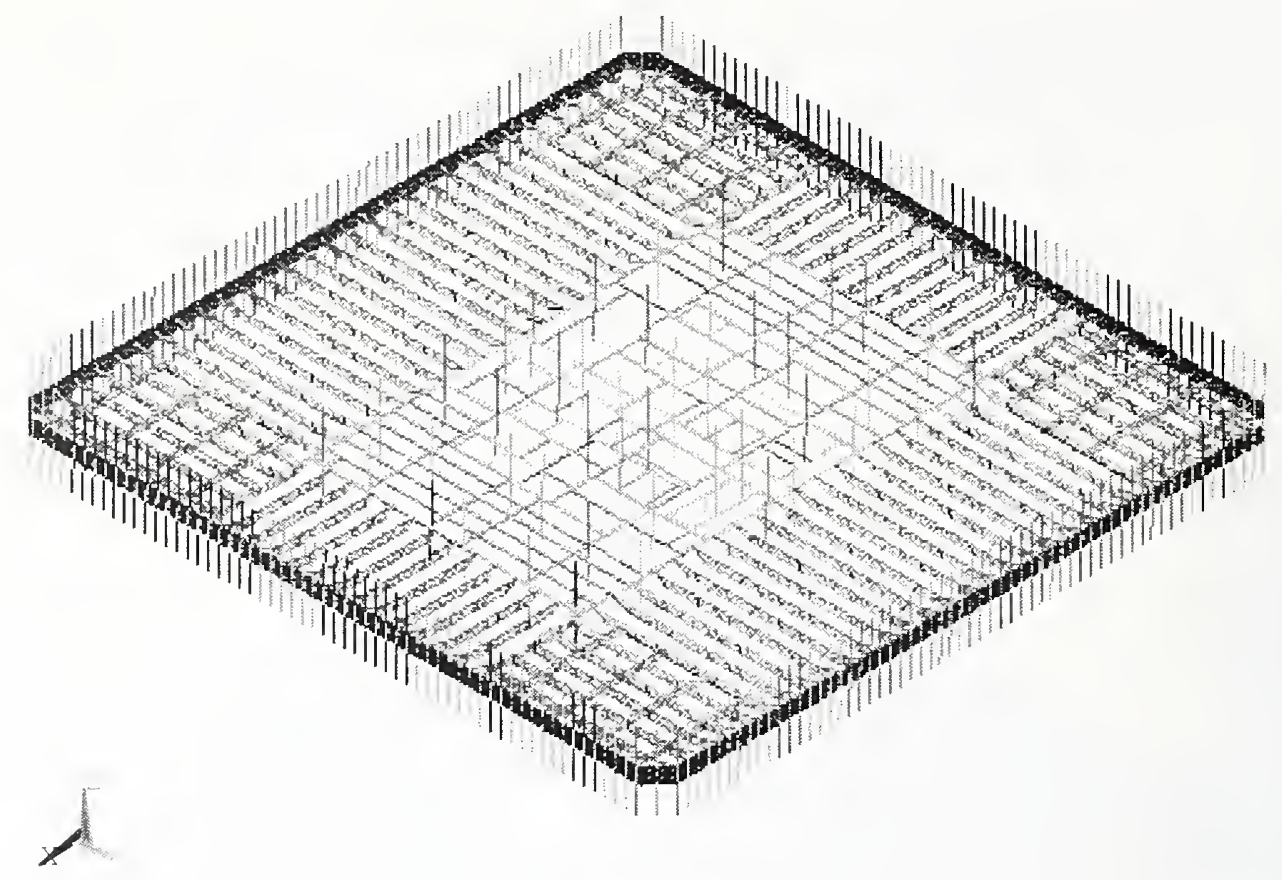

Figure A-2. Full floor model after removal of deck support angles, spandrel studs, bridging trusses outside of two-way zones, and strap anchors. 


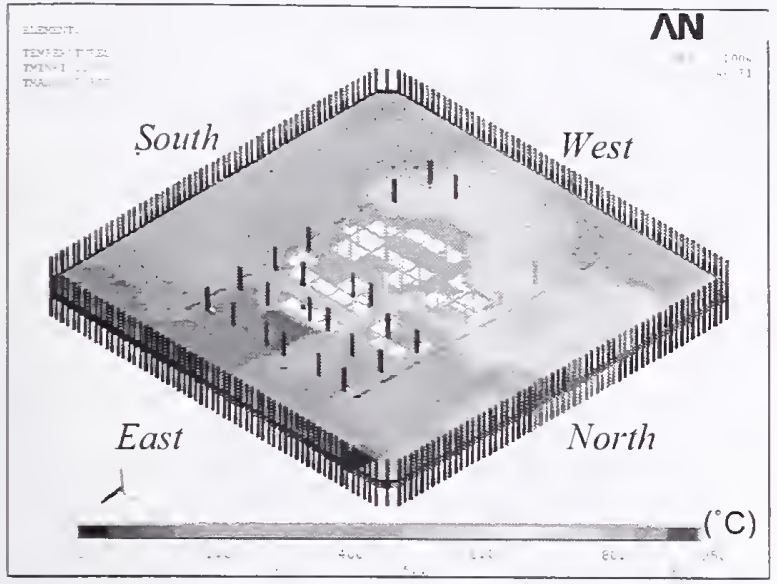

(a) Top view at $10 \mathrm{~min}$

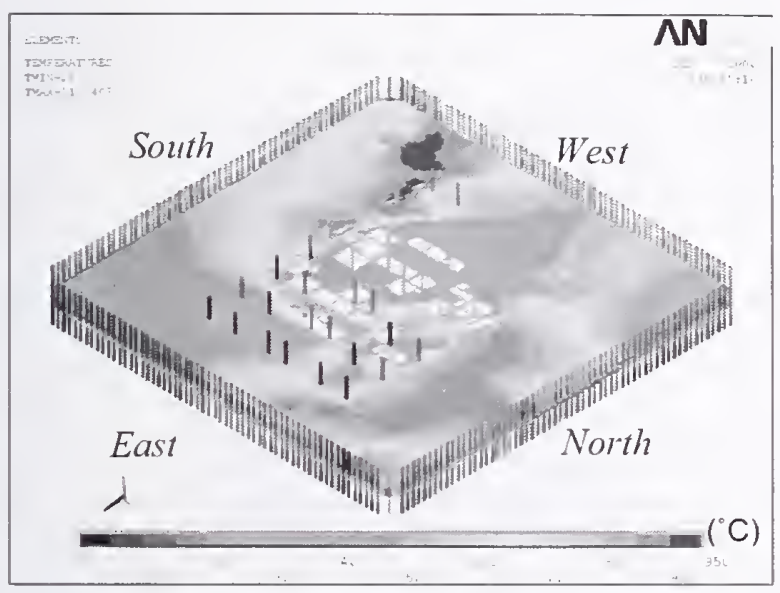

(c) Top view at $50 \mathrm{~min}$

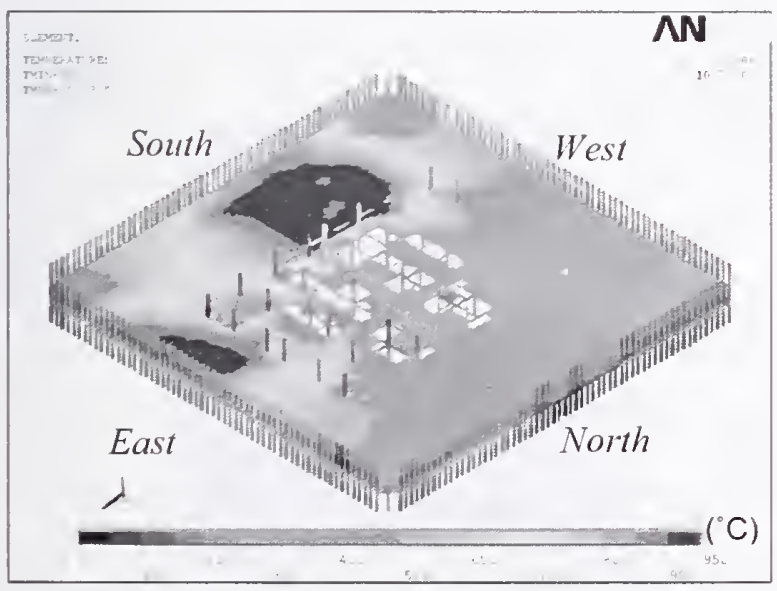

(e) Top view at $100 \mathrm{~min}$

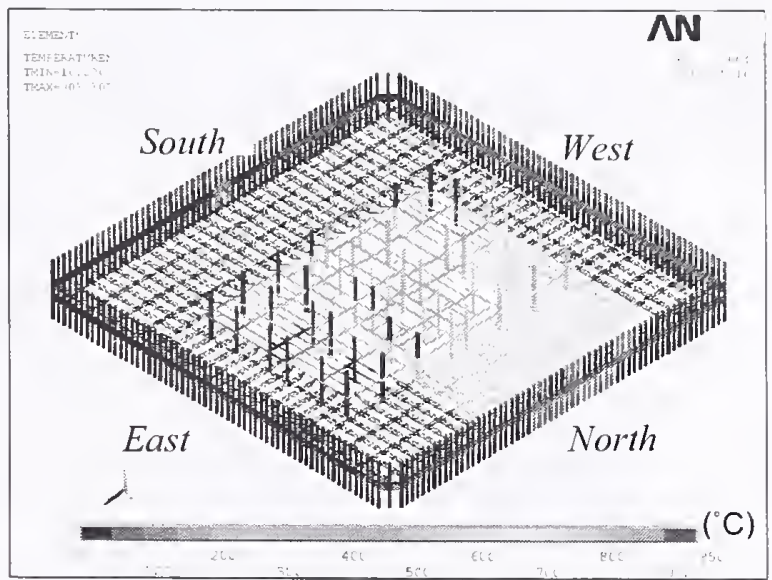

(b) Top view without concrete slab at $10 \mathrm{~min}$

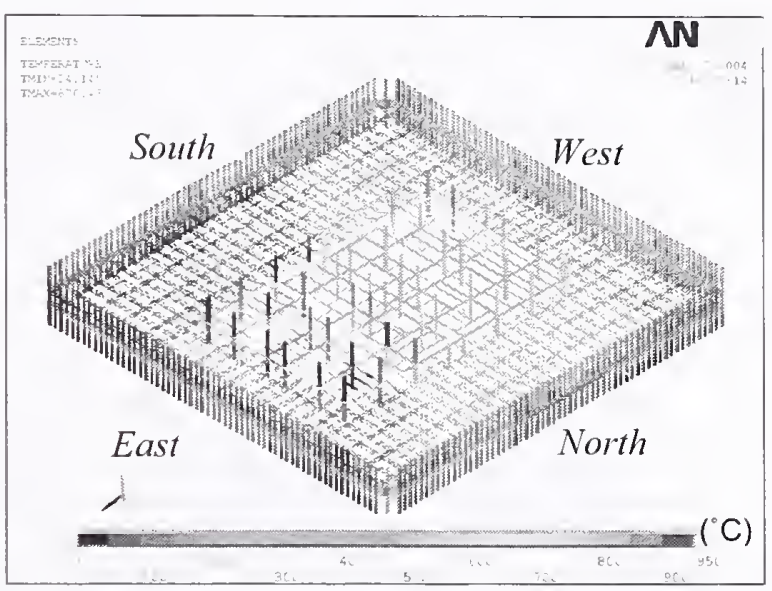

(d) Top view without concrete slab at $50 \mathrm{~min}$

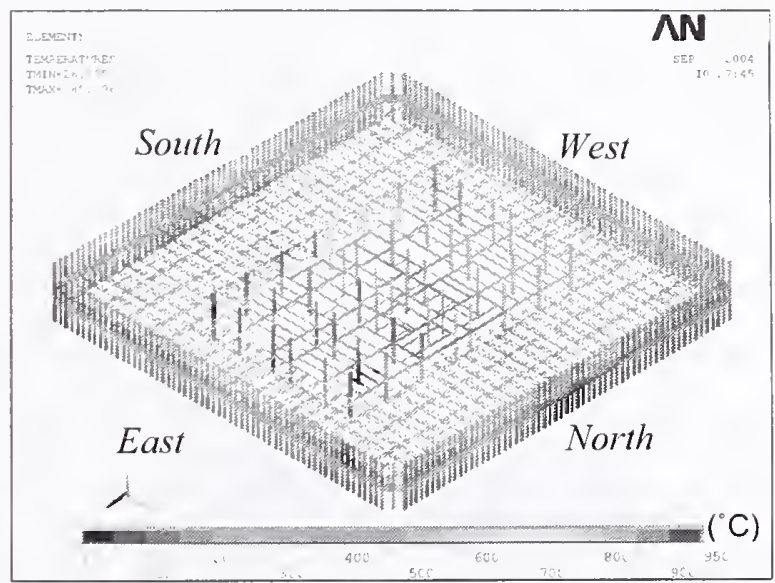

(f) Top view without concrete slab at $100 \mathrm{~min}$

Figure A-3. Case $A_{i}$ temperature distribution for Floor 97 of WTC 1. 


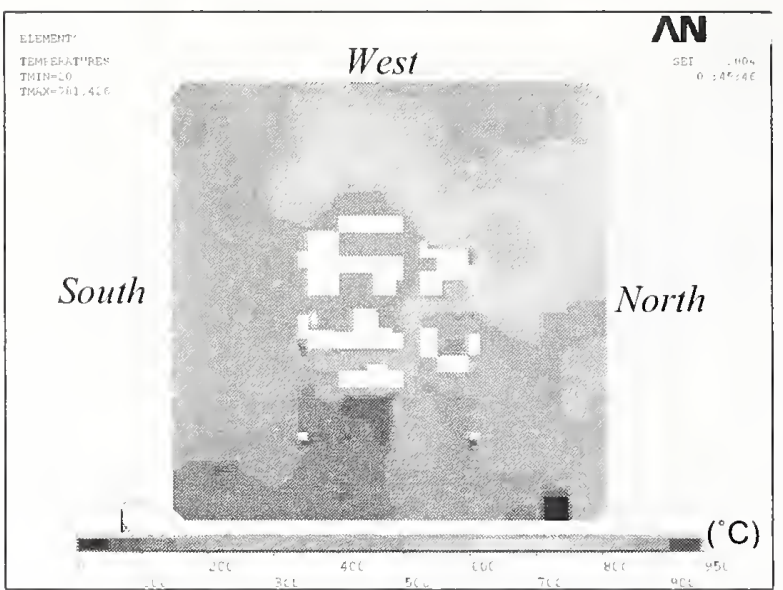

(a) Top surface at $10 \mathrm{~min}$

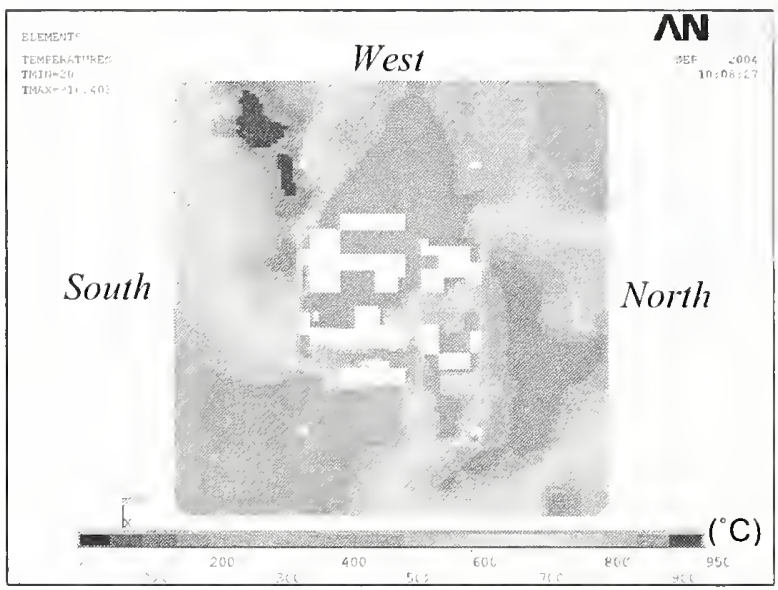

(c) Top surface at $50 \mathrm{~min}$

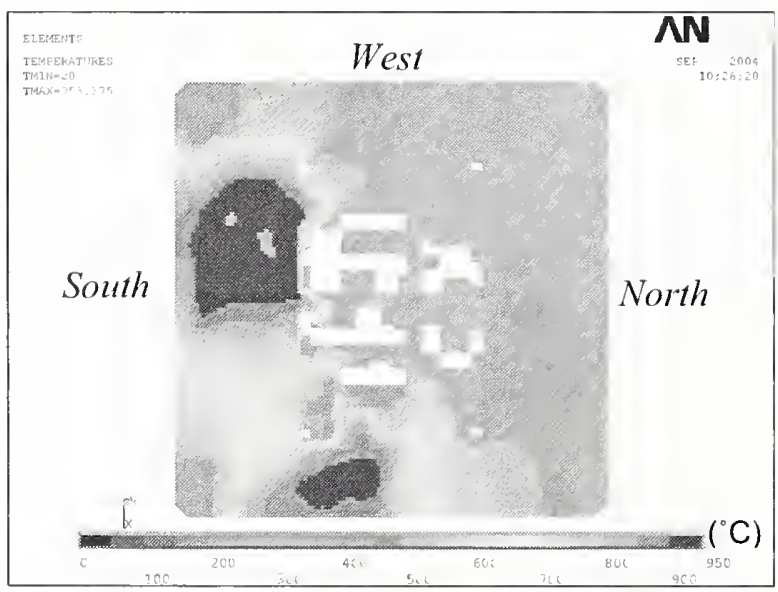

(e) Top surface at $100 \mathrm{~min}$

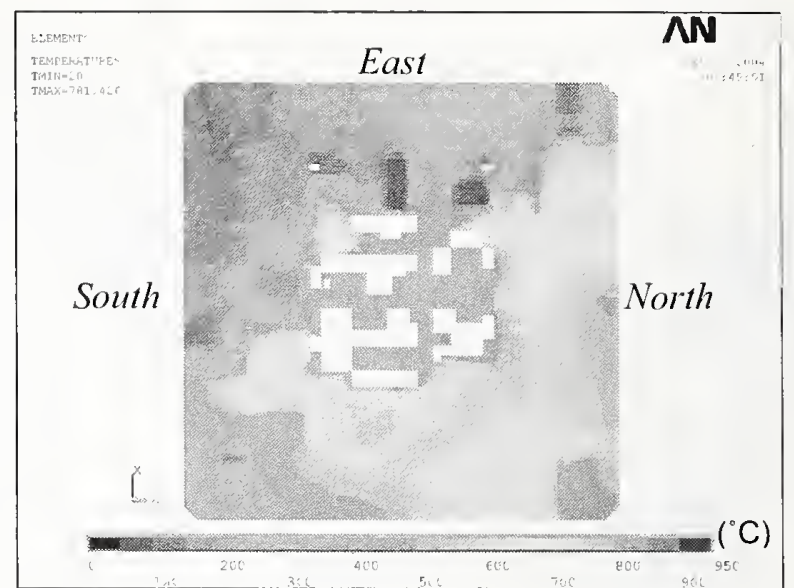

(b) Bottom surface at $10 \mathrm{~min}$

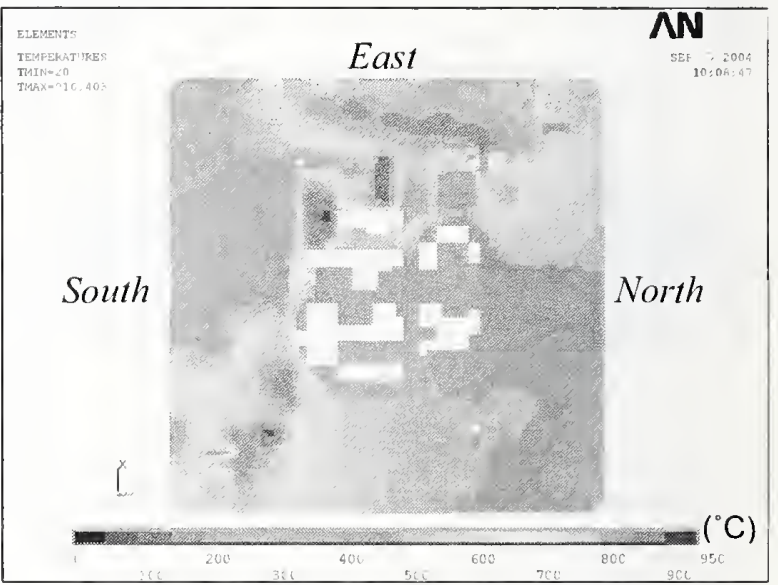

(d) Bottom surface at 50 min

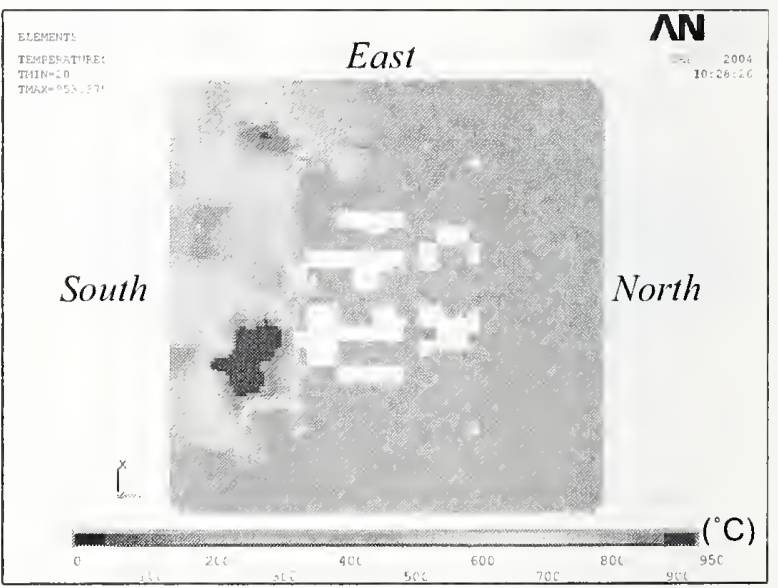

(f) Bottom surface at $100 \mathrm{~min}$

Figure A-4. Case $A_{i}$ temperature distribution in the slab for Floor 97 of WTC 1. 


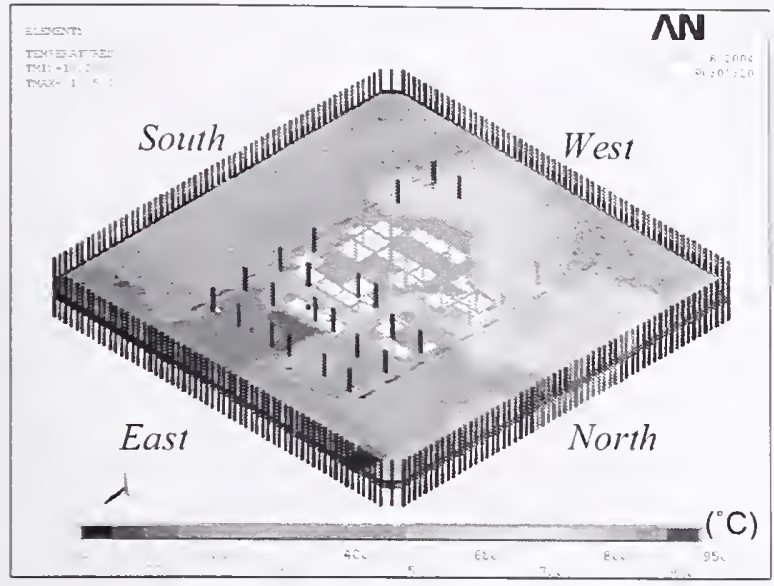

(a) Top view at $10 \mathrm{~min}$

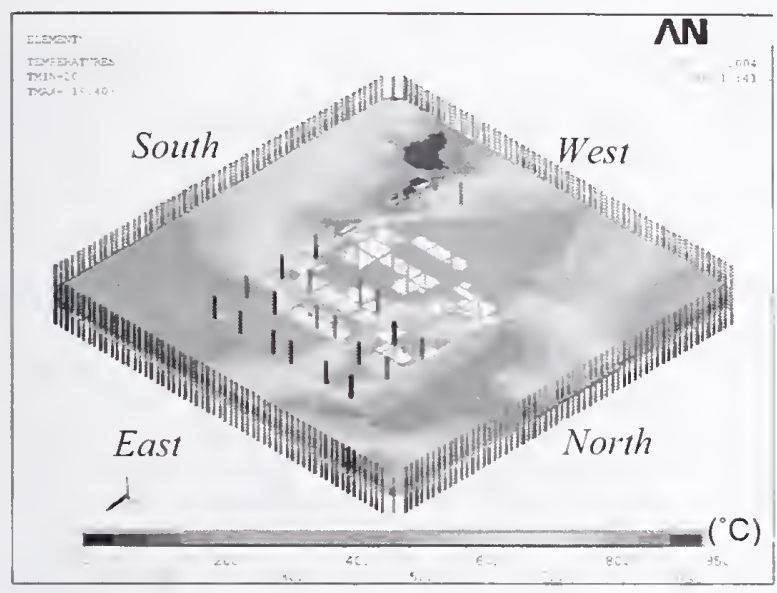

(c) Top view at $50 \mathrm{~min}$

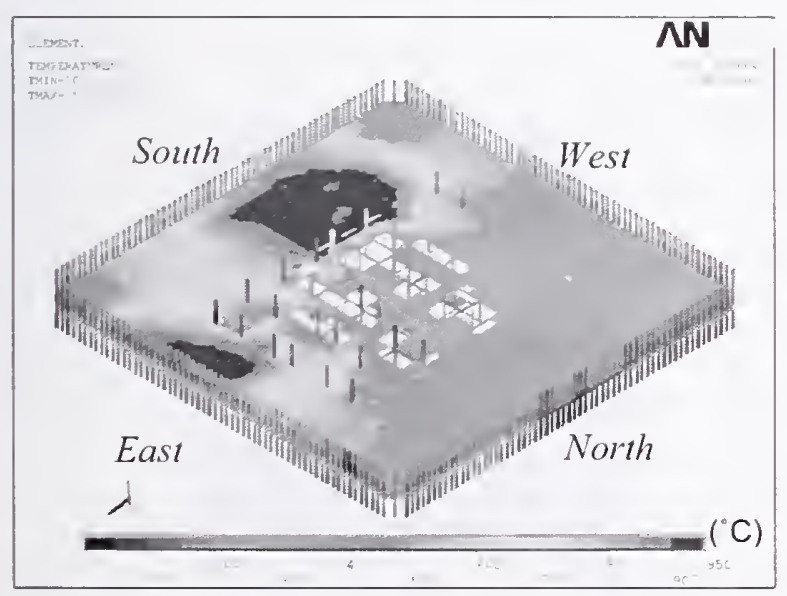

(e) Top view at $100 \mathrm{~min}$

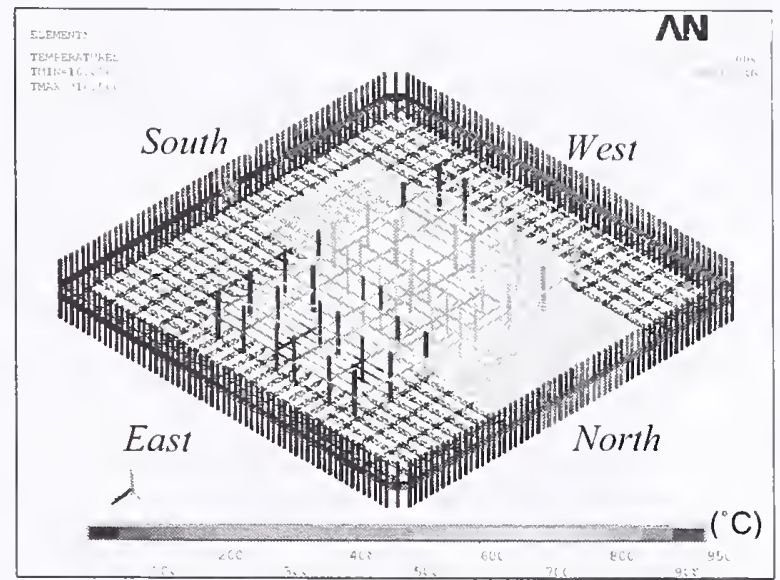

(b) Top view without concrete slab at $10 \mathrm{~min}$

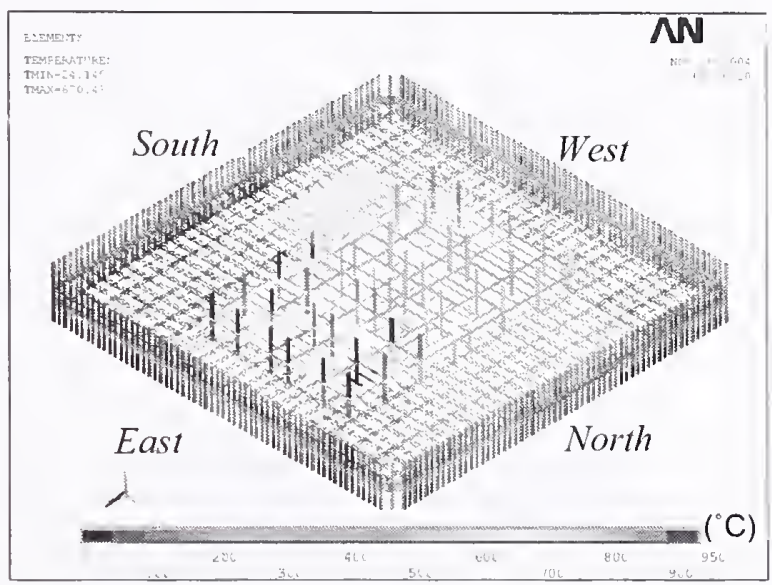

(d) Top view without concrete slab at $50 \mathrm{~min}$

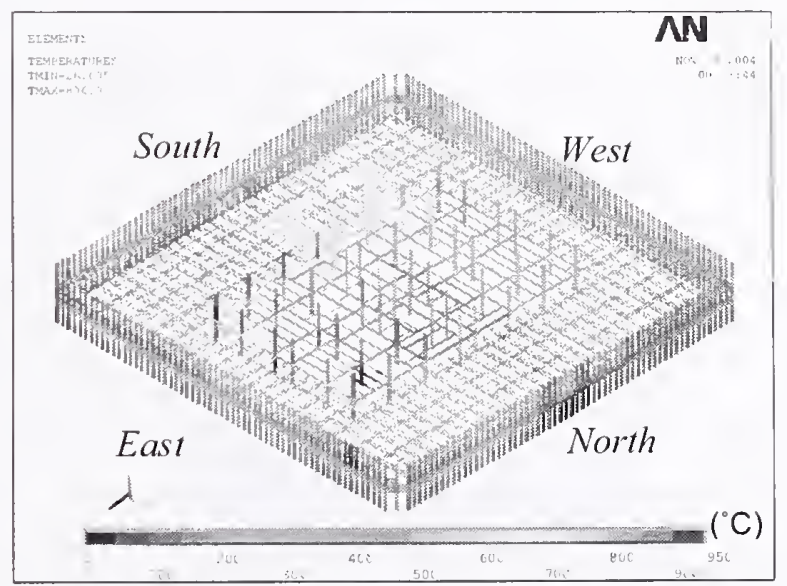

(f) Top view without concrete slab at $100 \mathrm{~min}$

Figure A-5. Case A temperature distribution for Floor 97 of WTC 1. 


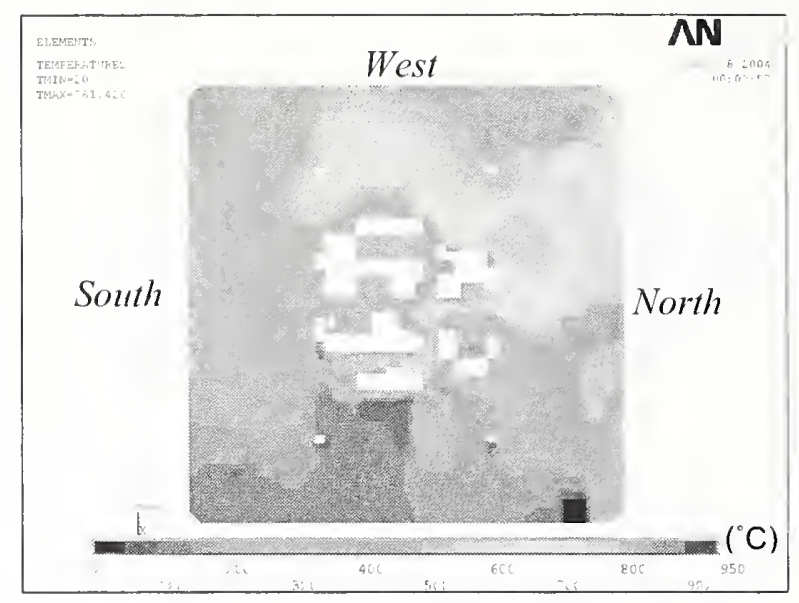

(a) Top surface at $10 \mathrm{~min}$

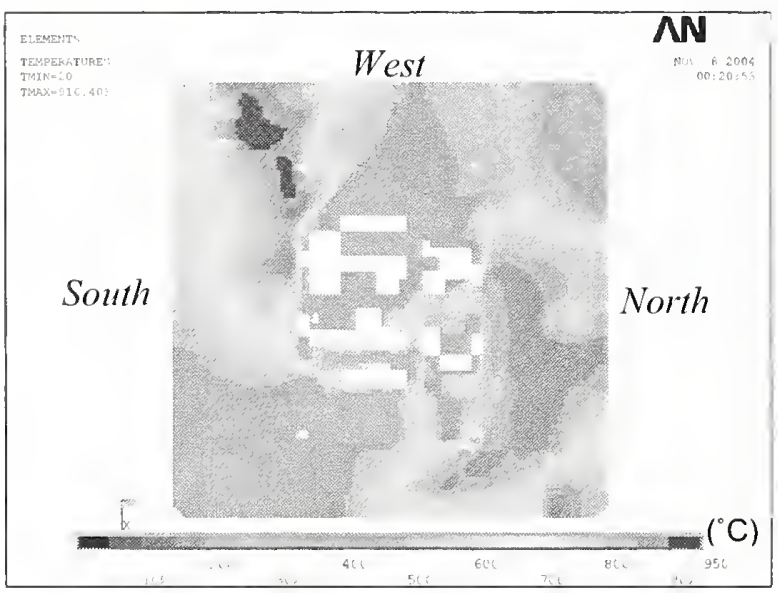

(c) Top surface at 50 min

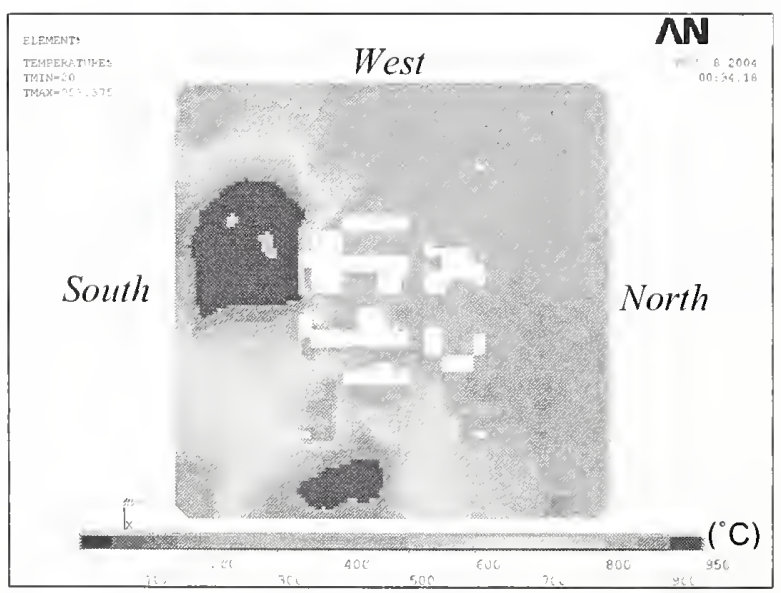

(e) Top surface at $100 \mathrm{~min}$

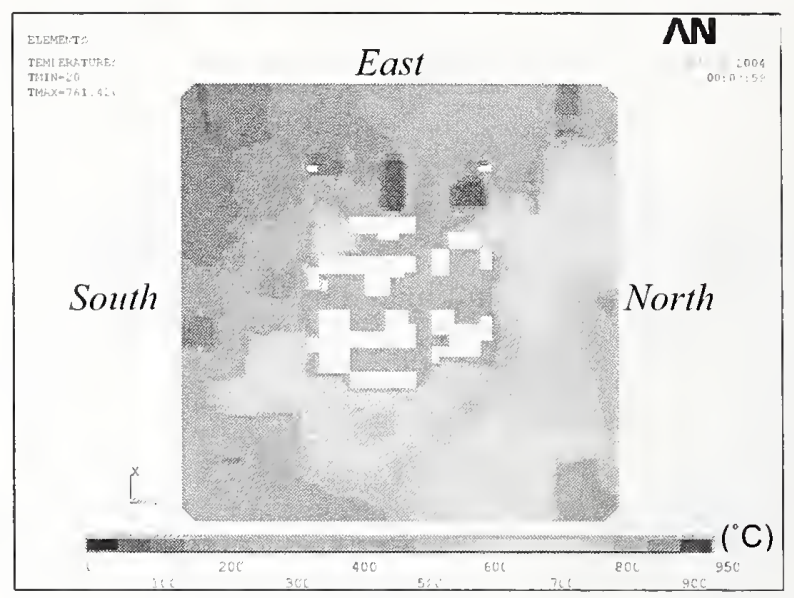

(b) Bottom surface at $10 \mathrm{~min}$

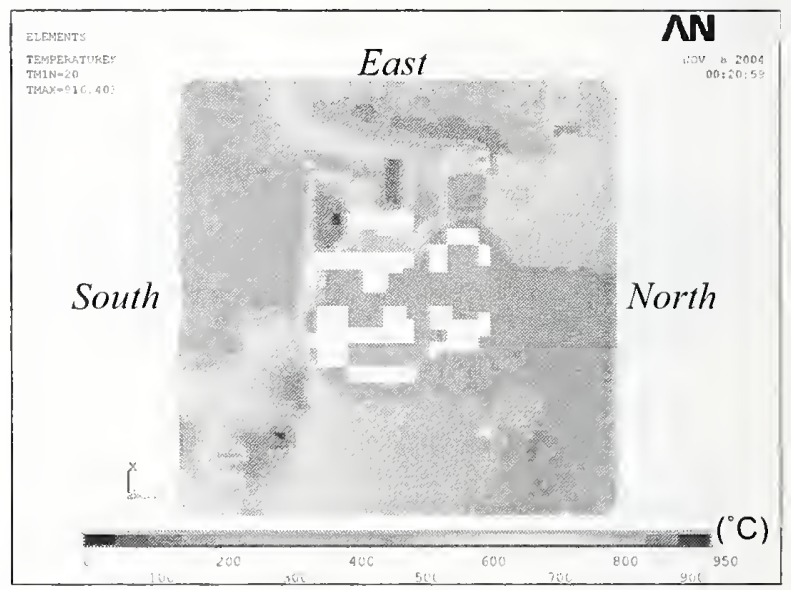

(d) Bottom surface at $50 \mathrm{~min}$

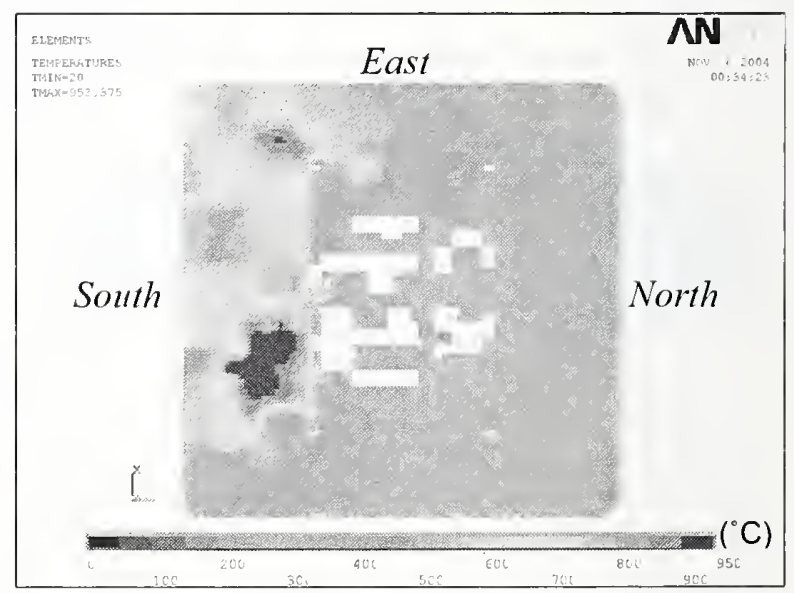

(f) Bottom surface at $100 \mathrm{~min}$

Figure A-6. Case A temperature distribution in the slab for Floor 97 of WTC 1. 


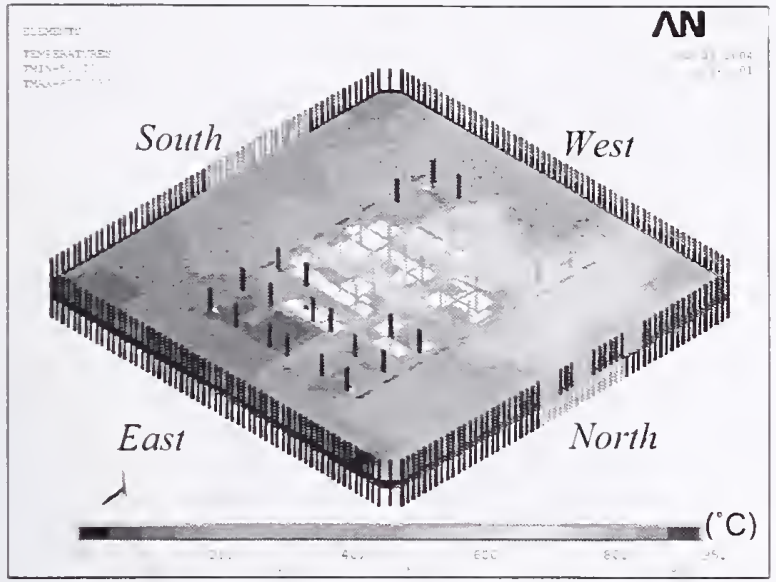

(a) Top view at $10 \mathrm{~min}$

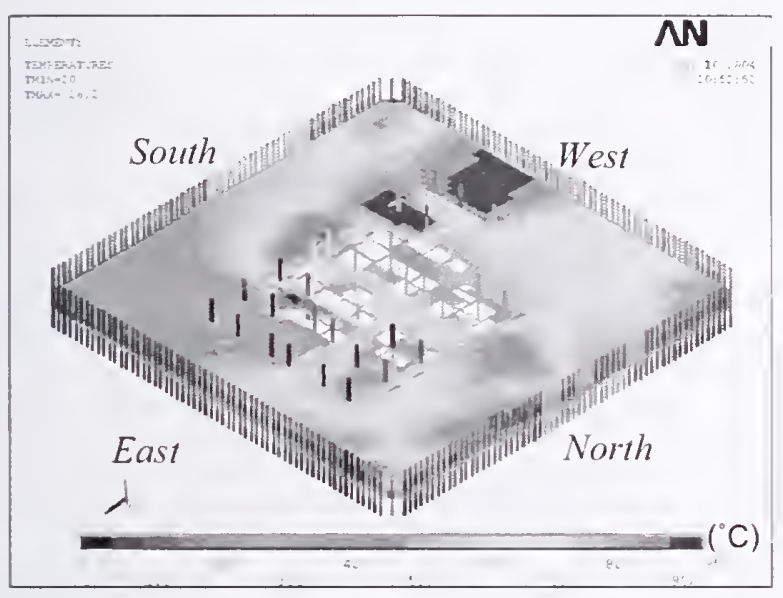

(c) Top view at $50 \mathrm{~min}$

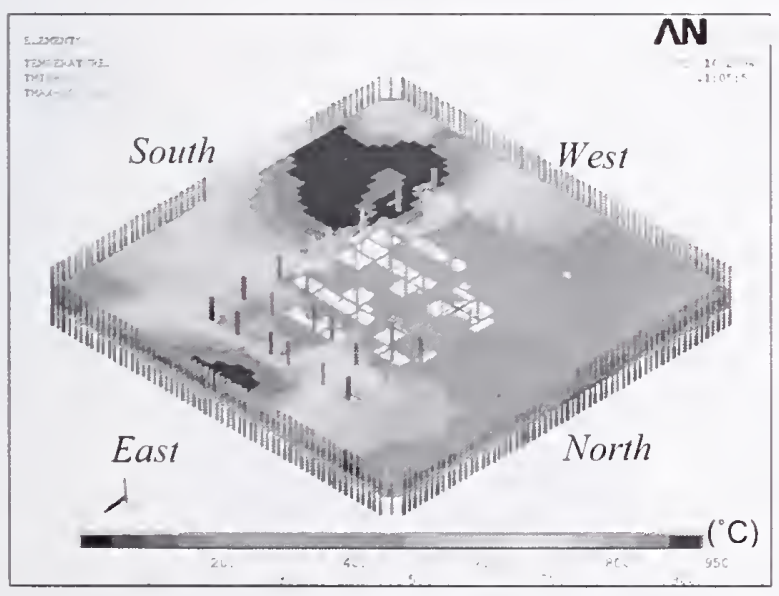

(e) Top view at $100 \mathrm{~min}$

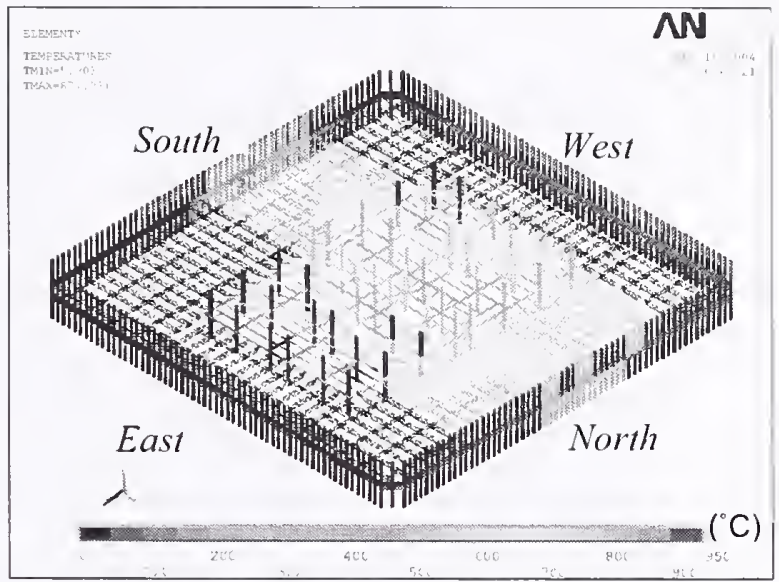

(b) Top view without concrete slab at $10 \mathrm{~min}$

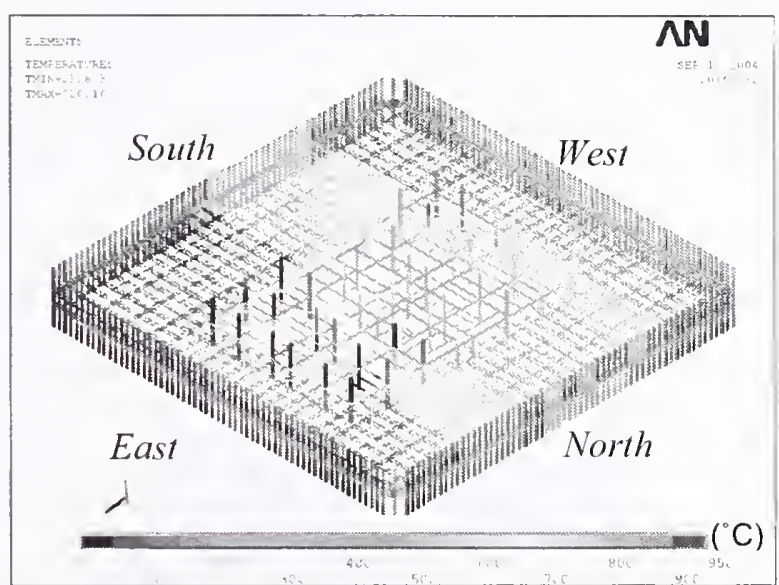

(d) Top view without concrete slab at $50 \mathrm{~min}$

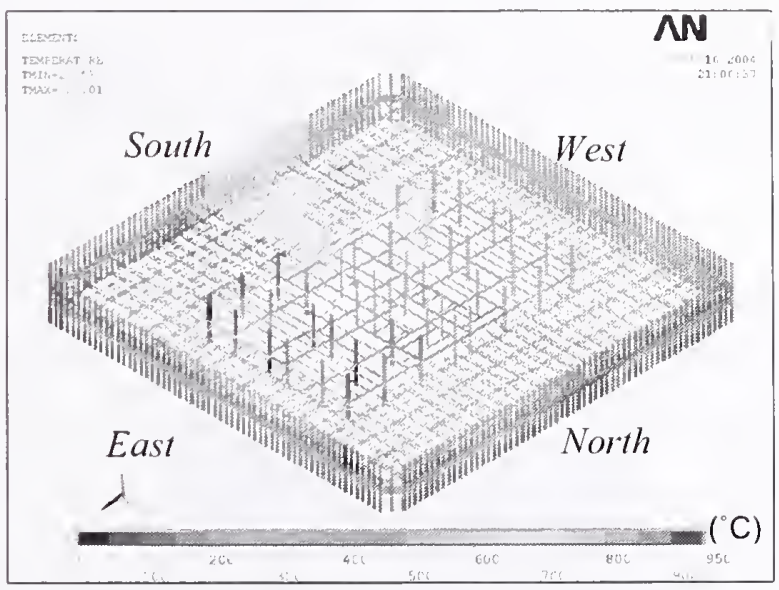

(f) Top view without concrete slab at $100 \mathrm{~min}$

Figure A-7. Case $B_{i}$ temperature distribution for Floor 97 of WTC 1. 


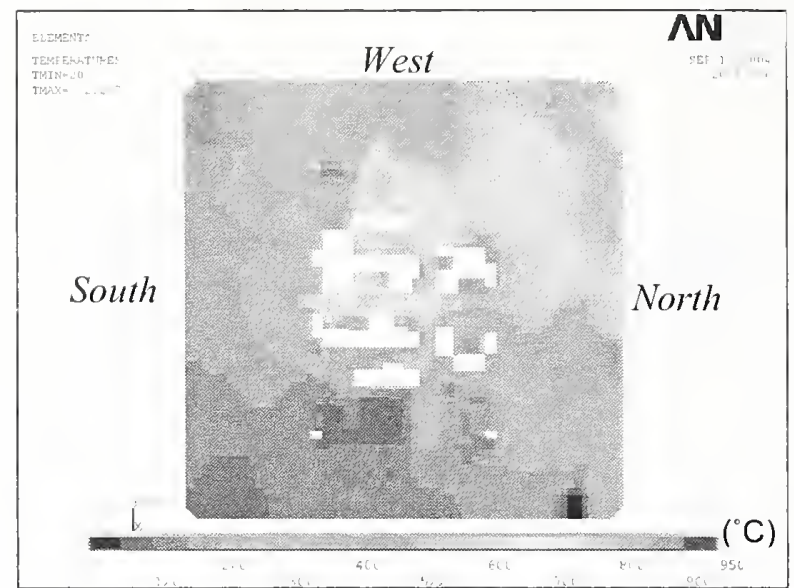

(a) Top surface at $10 \mathrm{~min}$

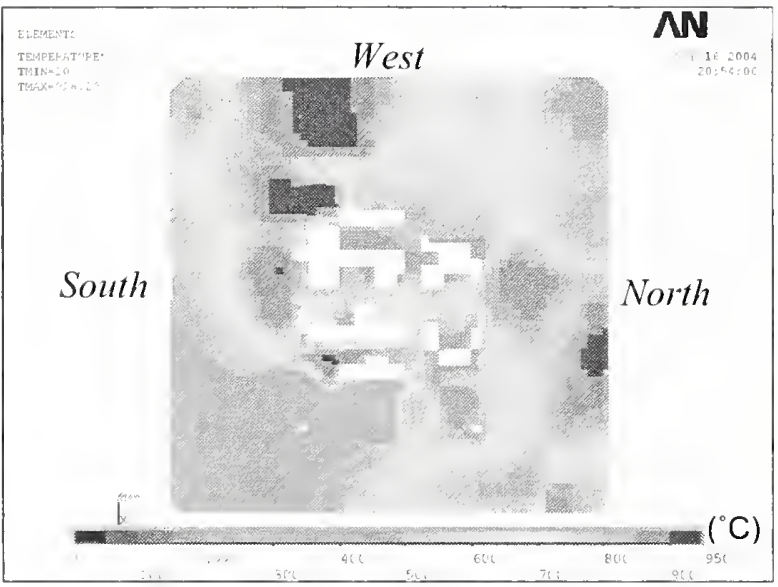

(c) Top surface at $50 \mathrm{~min}$

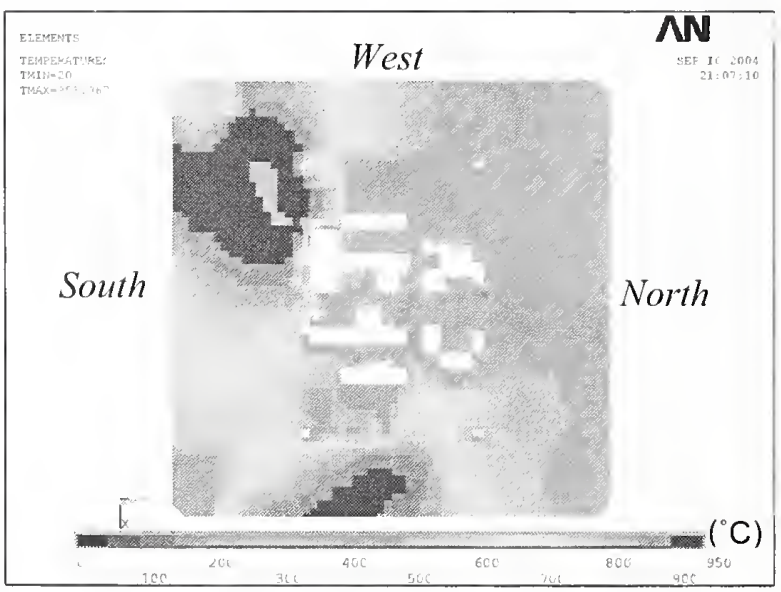

(e) Top surface at $100 \mathrm{~min}$

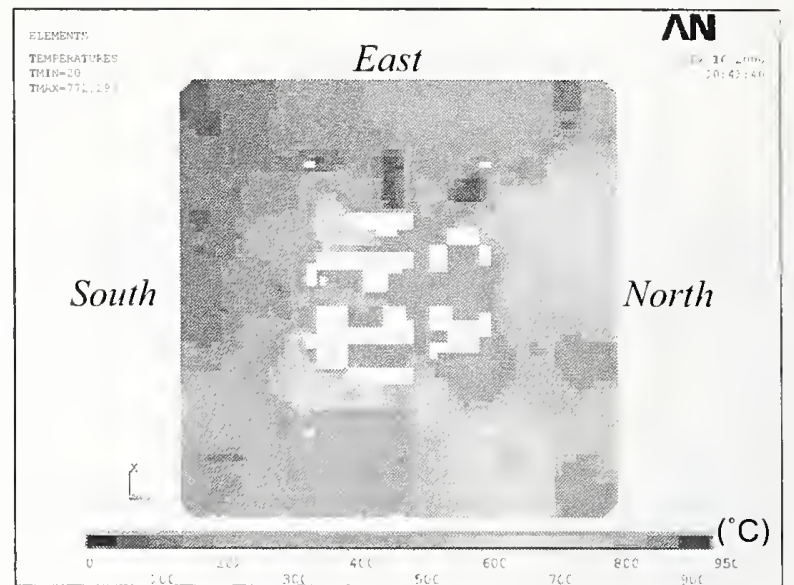

(b) Bottom surface at $10 \mathrm{~min}$

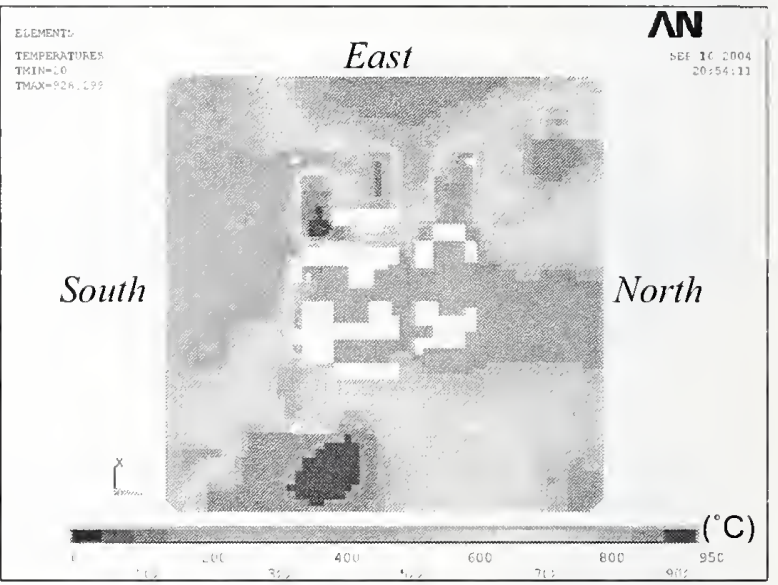

(d) Bottom surface at 50 min

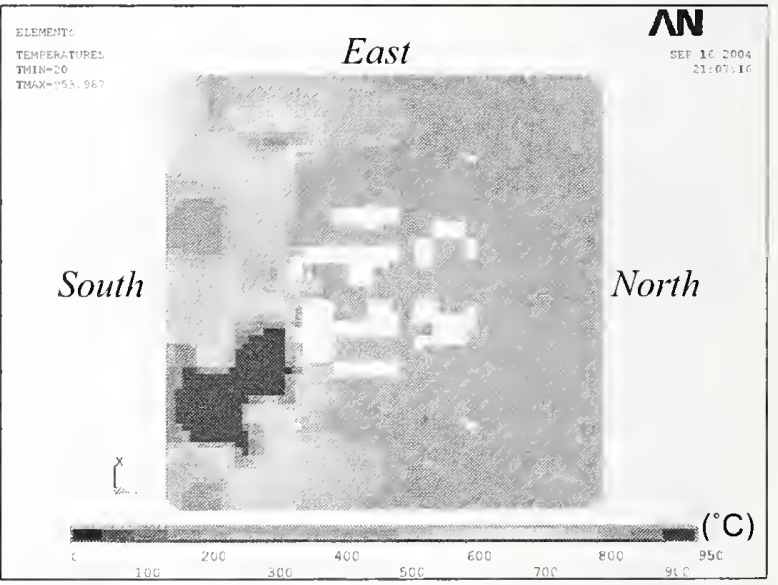

(f) Bottom surface at $100 \mathrm{~min}$

Figure A-8. Case $B_{i}$ temperature distribution in the slab for Floor 97 of WTC 1. 


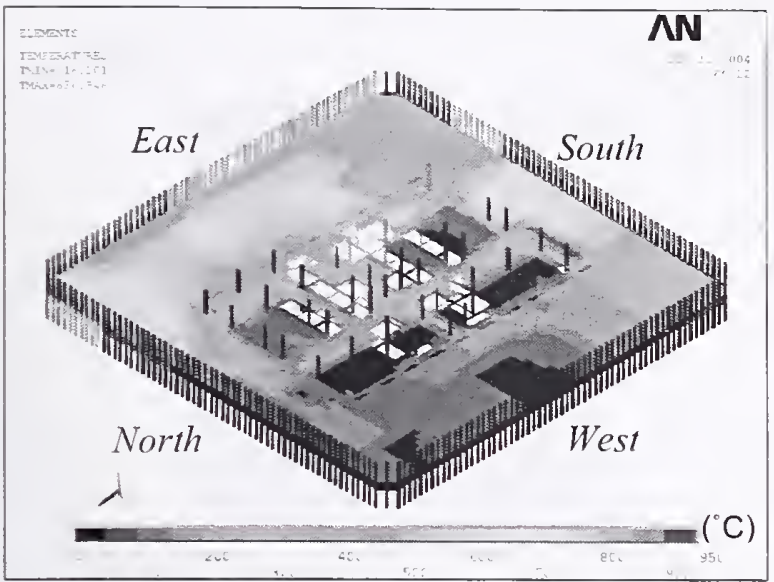

(a) Top view at $10 \mathrm{~min}$

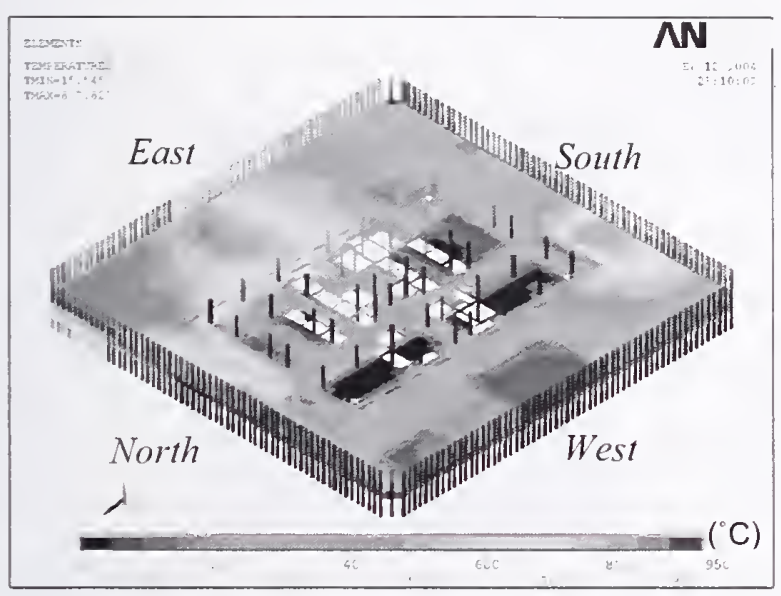

(c) Top view at $30 \mathrm{~min}$

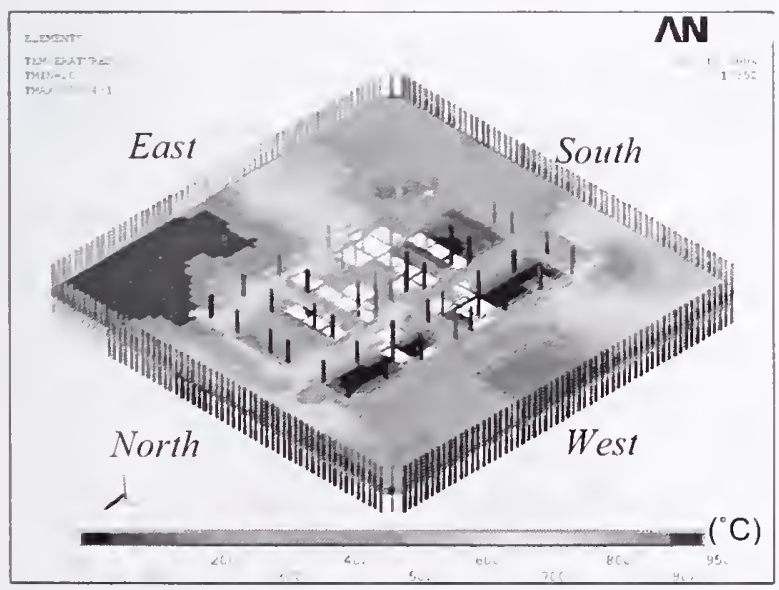

(e) Top view at $60 \mathrm{~min}$

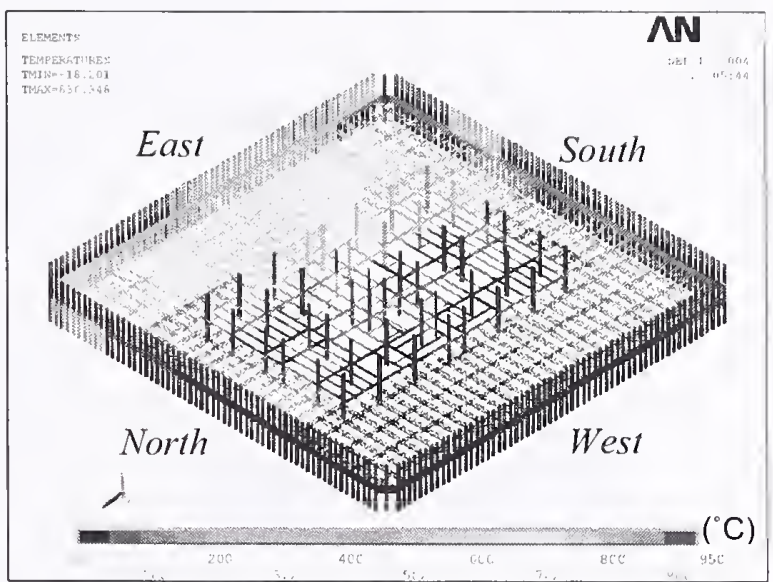

(b) Top view without concrete slab at $10 \mathrm{~min}$

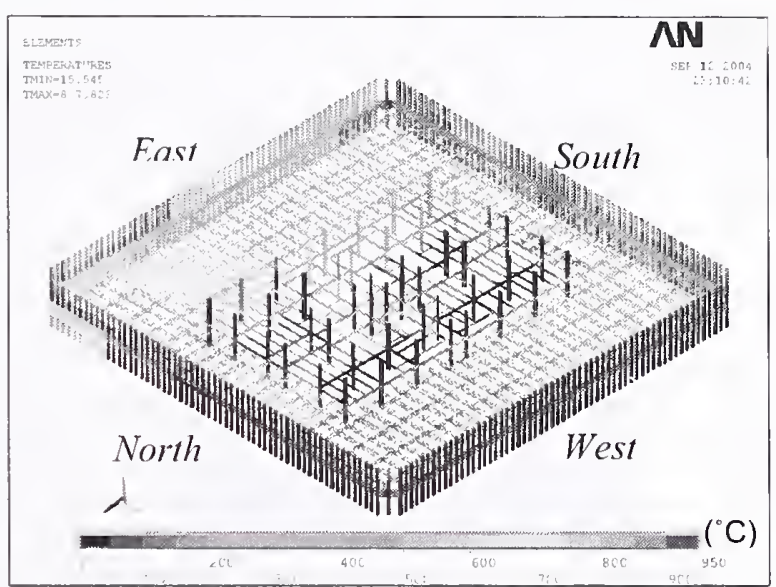

(d) Top view without concrete slab at $30 \mathrm{~min}$

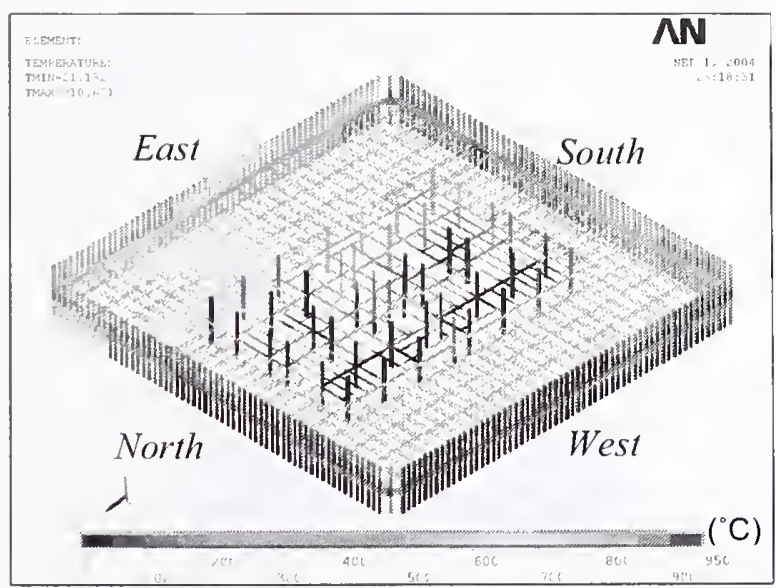

(f) Top view without concrete slab at $60 \mathrm{~min}$

Figure A-9. Case $C_{i}$ temperature distribution for Floor 82 of WTC 2. 


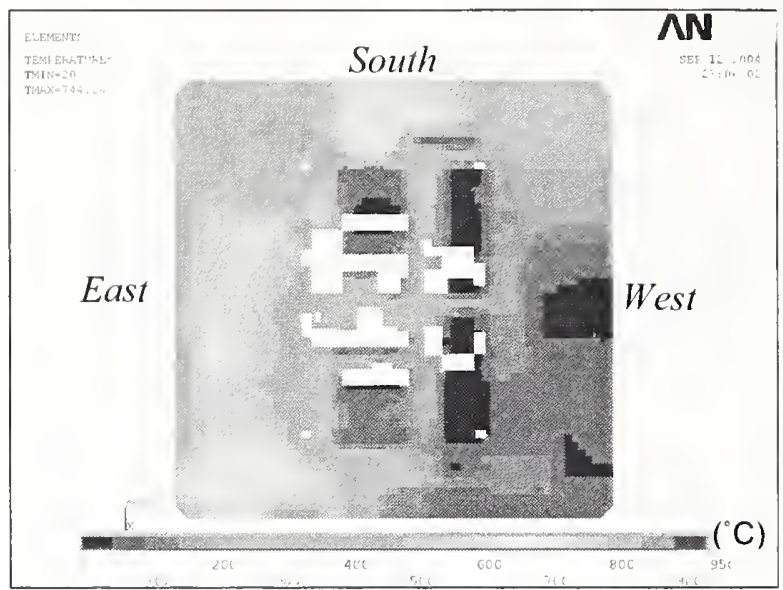

(a) Top surface at $10 \mathrm{~min}$

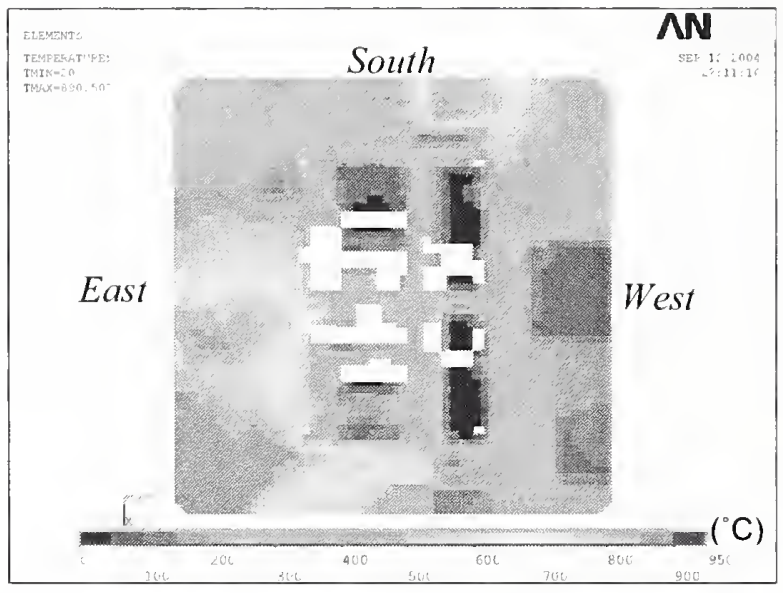

(c) Top surface at $30 \mathrm{~min}$

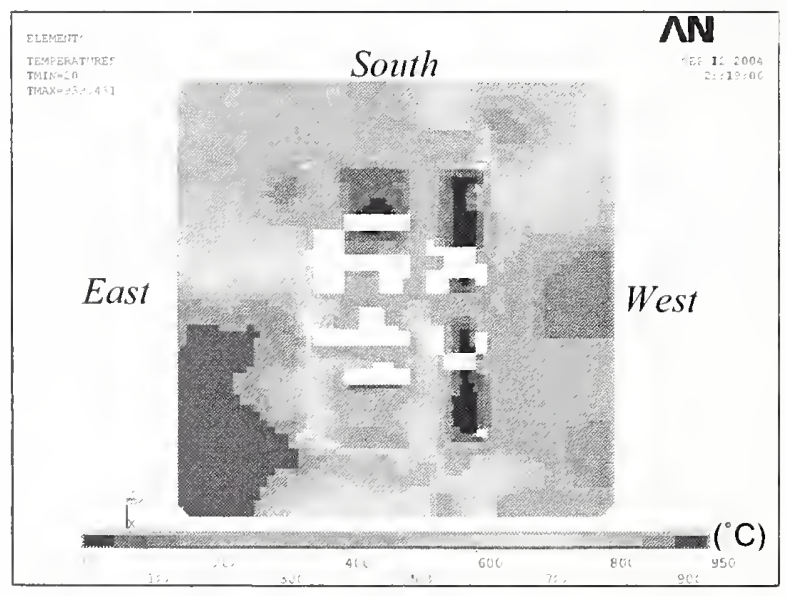

(e) Top surface at $60 \mathrm{~min}$

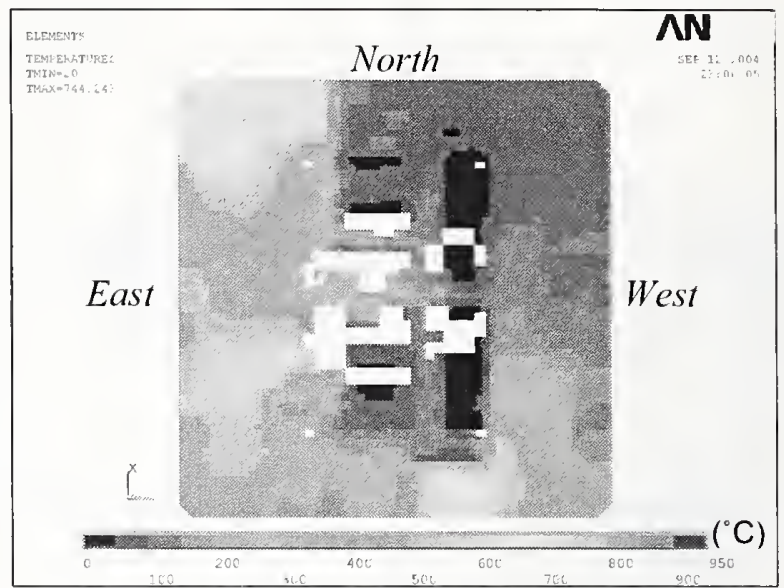

(b) Bottom surface at $10 \mathrm{~min}$

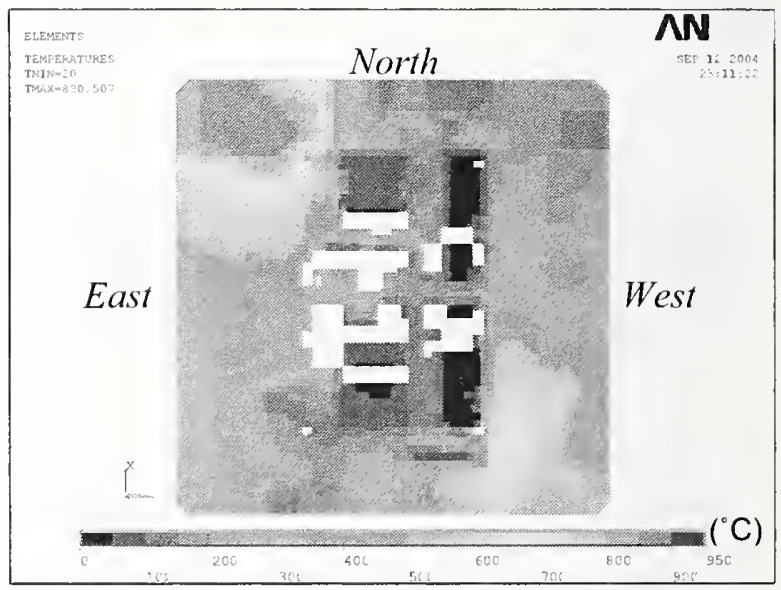

(d) Bottom surface at $30 \mathrm{~min}$

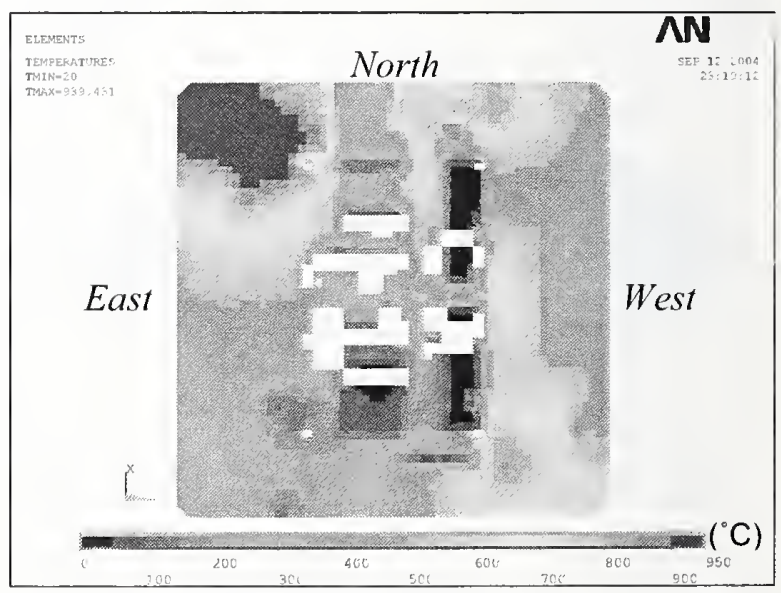

(f) Bottom surface at $60 \mathrm{~min}$

Figure A-10. Case $C_{i}$ temperature distribution in the slab for Floor 82 of WTC 2. 


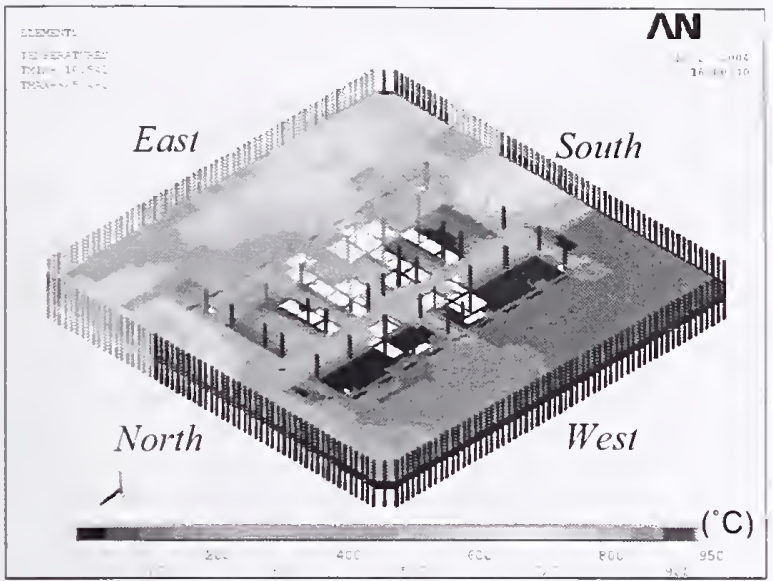

(a) Top view at $10 \mathrm{~min}$

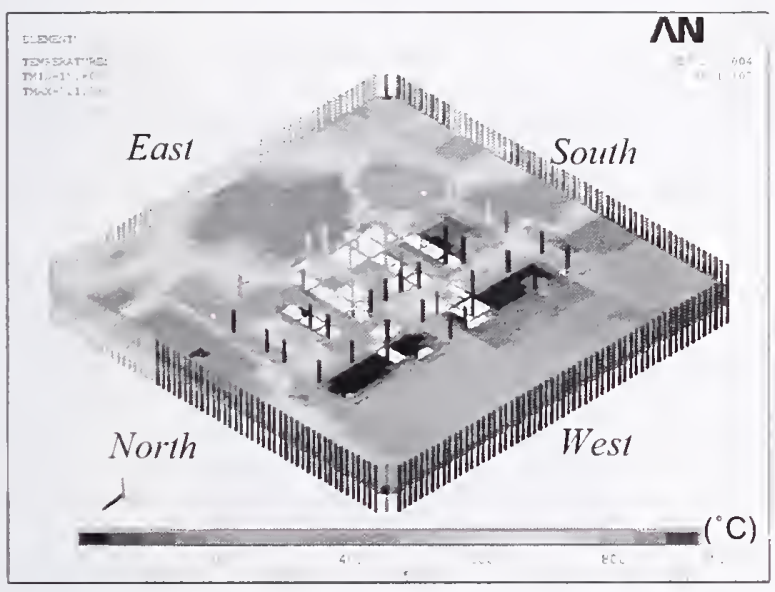

(c) Top view at $30 \mathrm{~min}$

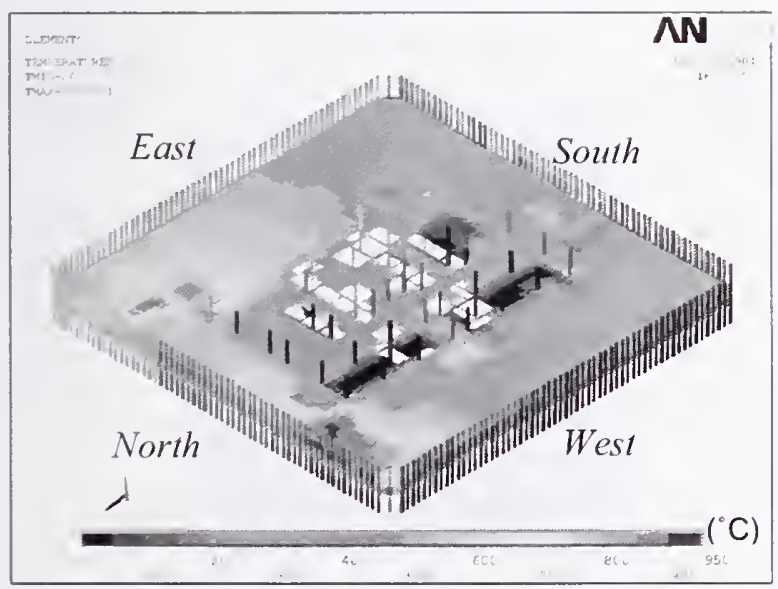

(e) Top view at $60 \mathrm{~min}$

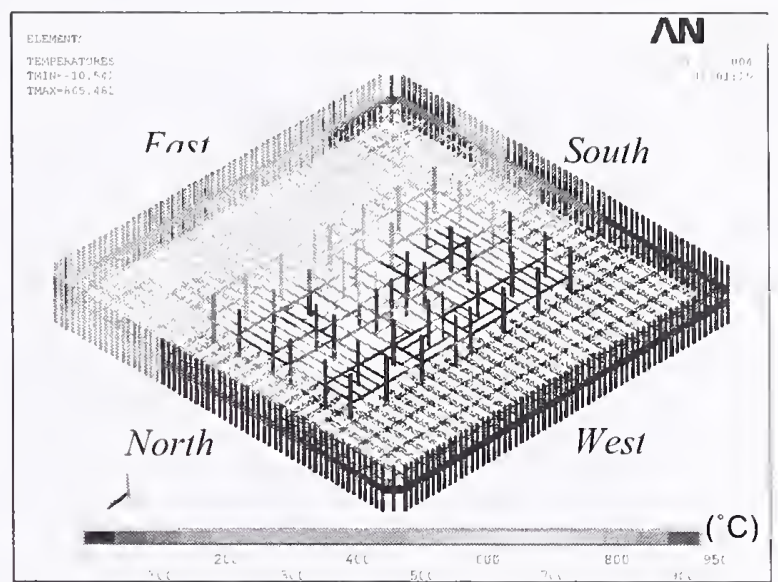

(b) Top view without concrete slab at $10 \mathrm{~min}$

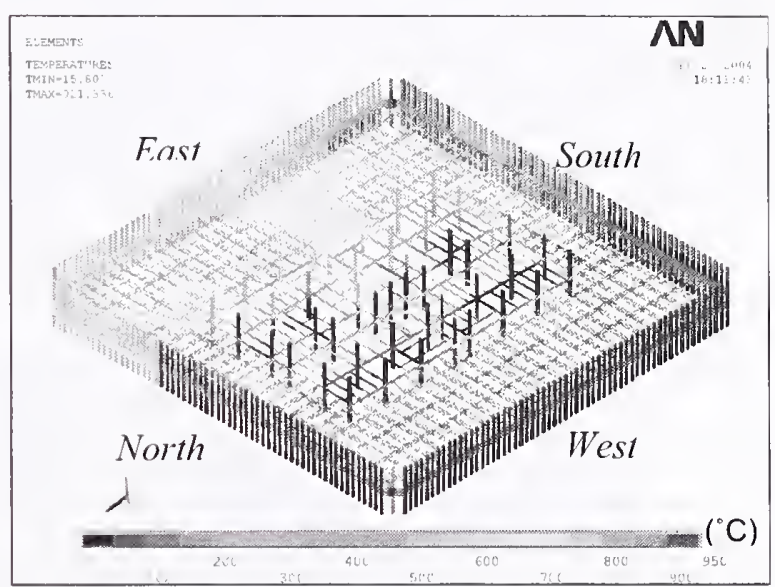

(d) Top view without concrete slab at $30 \mathrm{~min}$

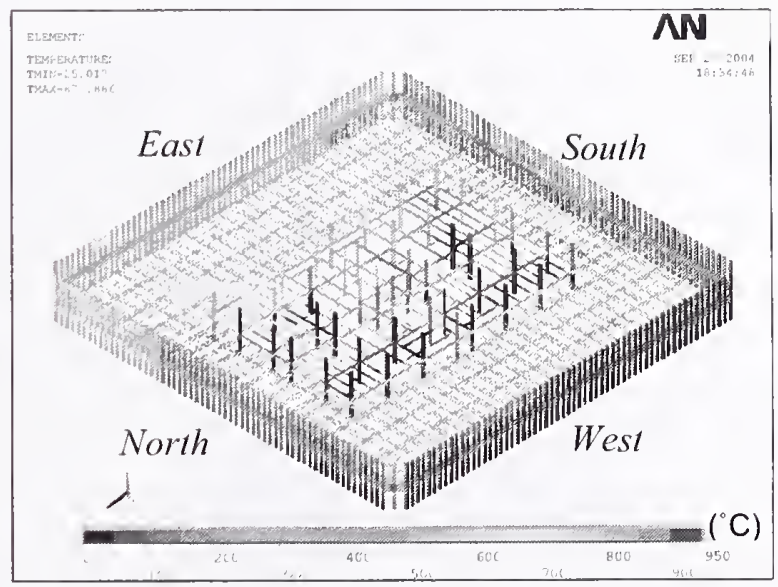

(f) Top view without concrete slab at $60 \mathrm{~min}$

Figure A-11. Case $D_{i}$ temperature distribution for Floor 82 of WTC 2. 


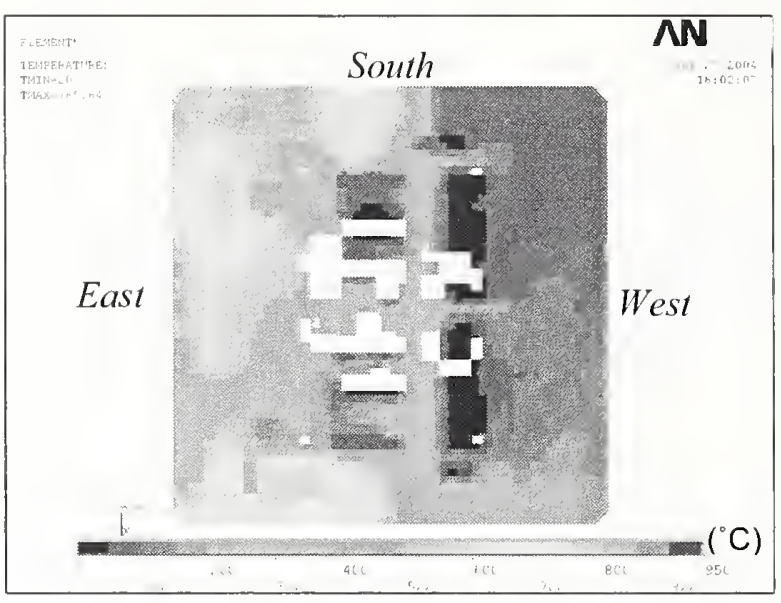

(a) Top surface at $10 \mathrm{~min}$

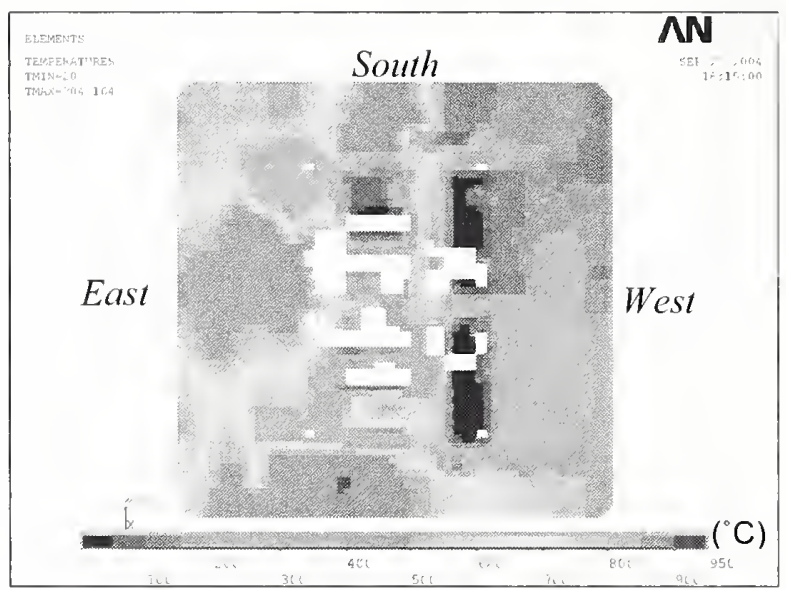

(c) Top surface at $\mathbf{3 0}$ min

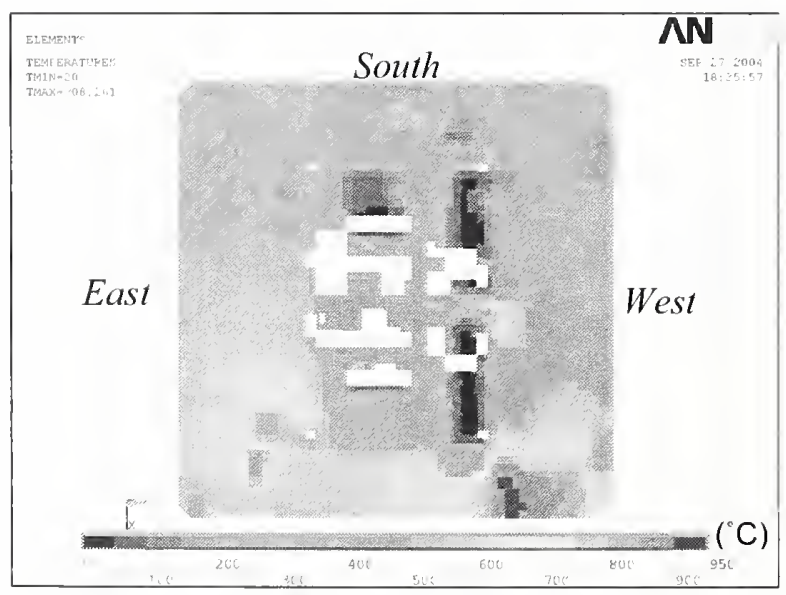

(e) Top surface at $60 \mathrm{~min}$

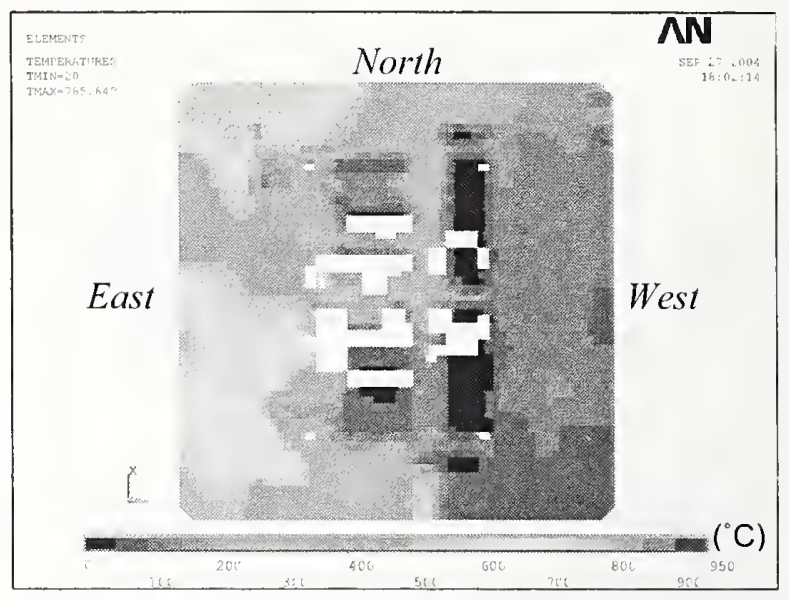

(b) Bottom surface at $10 \mathrm{~min}$

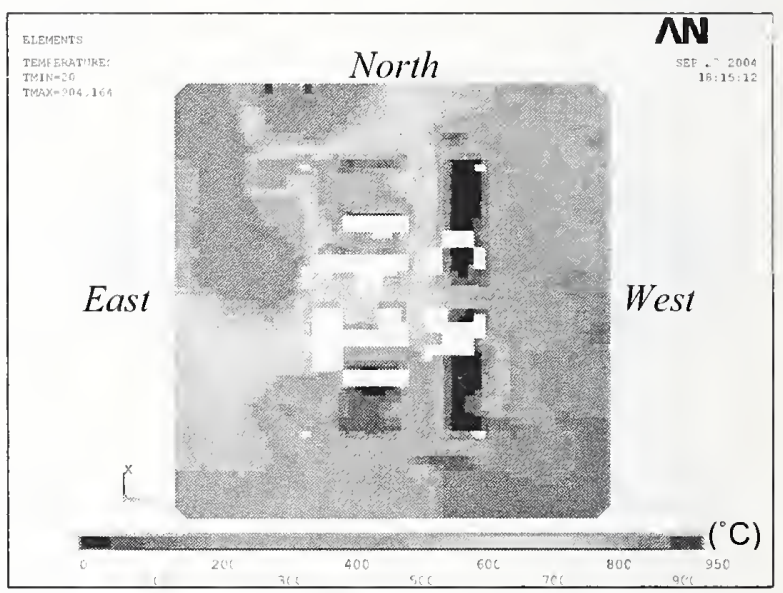

(d) Bottom surface at $30 \mathrm{~min}$

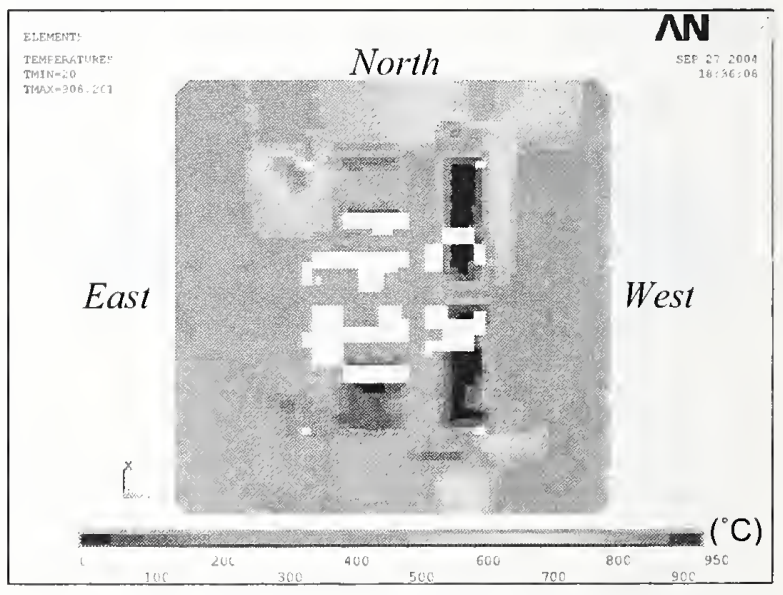

(f) Bottom surface at $60 \mathrm{~min}$

Figure A-12. Case $D_{i}$ temperature distribution in the slab for Floor 82 of WTC 2. 


\section{A.2 WTC 1 FLOORS}

\section{A.2.1 Case $A_{i}$ Temperature Condition}

Table A-1 gives the maximum vertical displacement of WTC 1 floors, and Figs. A-13 to A-19 show the vertical displacements of the floors when the maximum displacement was obtained. Floor 95 to Floor 98 showed a significant vertical displacement in the north office area near the impact damage. The maximum vertical displacement of all floors was $32 \mathrm{in}$. for Floor 97 at $60 \mathrm{~min}$. The vertical displacement in the south office area was found to be insignificant on all the floors throughout the thermal loading. Figure A-20 shows the average total thermal expansion of floors over the entire width of the building at $100 \mathrm{~min}$ in two orthogonal directions. The total floor expansion ranged from $4 \mathrm{in}$. to $8 \mathrm{in}$. Many web diagonals of Floor 95 to Floor 98 buckled in the hot zones of the north office area. Although gusset plates fractured at several locations, a complete disconnection of the floor from the exterior wall was not observed. Figure A-21 shows horizontal reaction force at individual columns of the north and south faces of Floor 97. A positive reaction force in the figure means that the column is pulled inward by the floor, and a negative reactions force means that the column is pushed out by the floor. Since columns that were not at floor trusses were not connected to the floor in the model because of the removal of strap anchors and studs, reaction forces at those columns were small, and the plots became jagged. As can be seen in the figure. almost all the columns were pushed out by the floor. This was also the case for other floors.

Table A-1. Maximum vertical displacement of WTC 1 floors for Case $A_{i}$ temperature condition.

\begin{tabular}{|c|c|c|}
\hline Floor & $\begin{array}{c}\text { Max. Displacement } \\
\text { (in.) }\end{array}$ & $\begin{array}{c}\text { Time at the Maximum } \\
\text { (min) }\end{array}$ \\
\hline 93 & 5.4 & 30 \\
\hline 94 & 13.5 & 100 \\
\hline 95 & 30.9 & 10 \\
\hline 96 & 23.3 & 10 \\
\hline 97 & 31.5 & 60 \\
\hline 98 & 26.4 & 30 \\
\hline 99 & 7.0 & 50 \\
\hline
\end{tabular}

Note: Downward displacement is positive. 


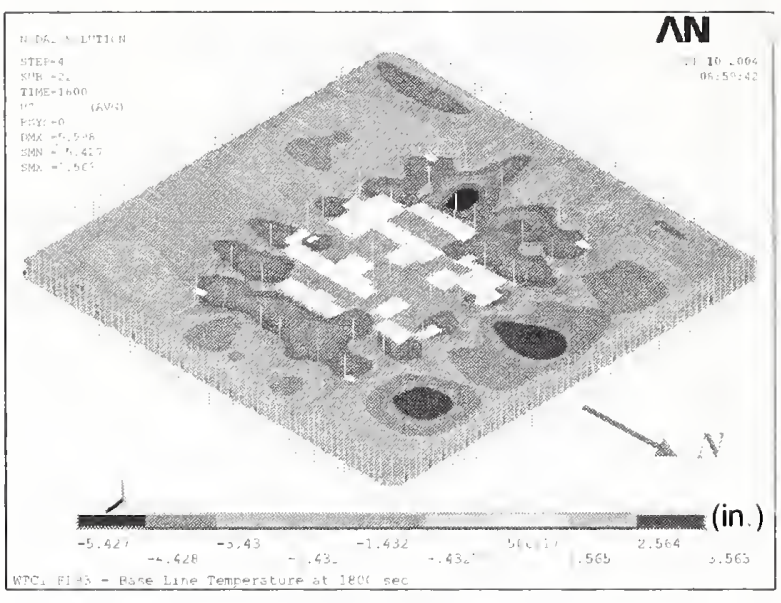

(a) Entire model

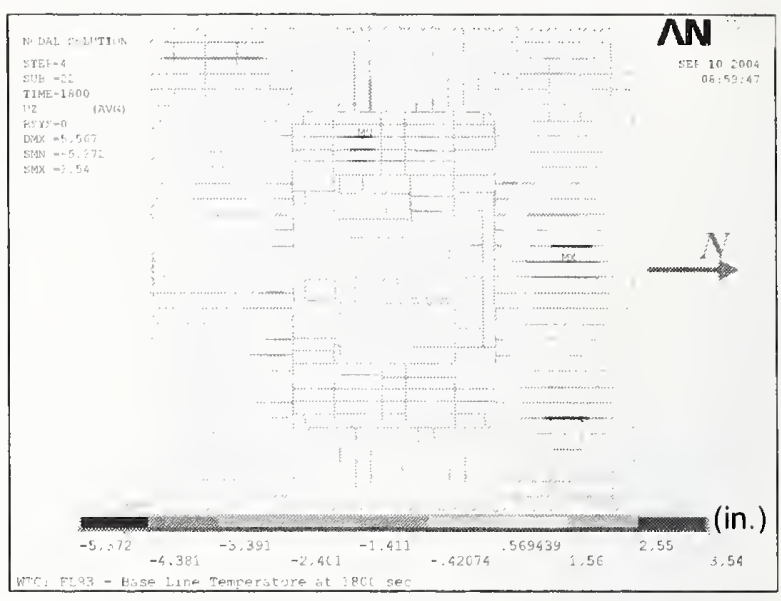

(b) Top view without slab

Figure A-13. Vertical displacement of WTC 1 Floor 93 for Case $A_{i}$ temperature condition at $30 \mathrm{~min}$ (downward displacement is negative; 5x displacement magnification).

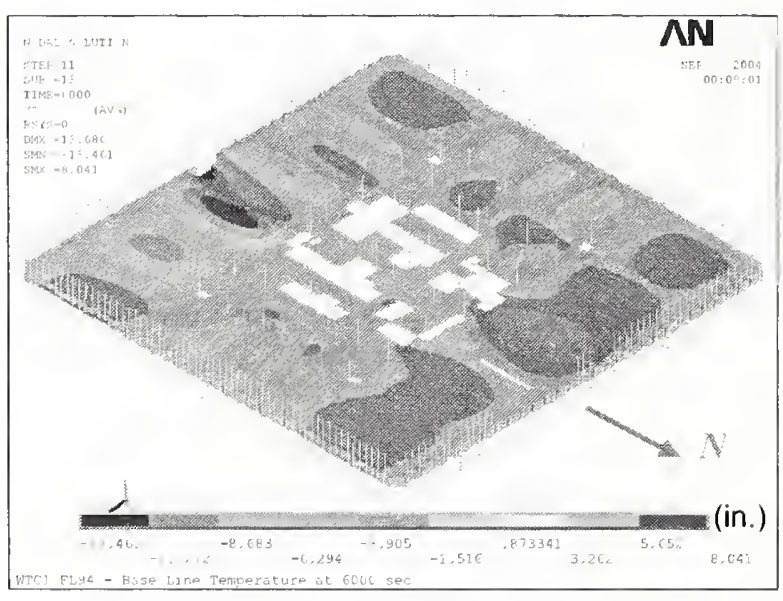

(a) Entire model

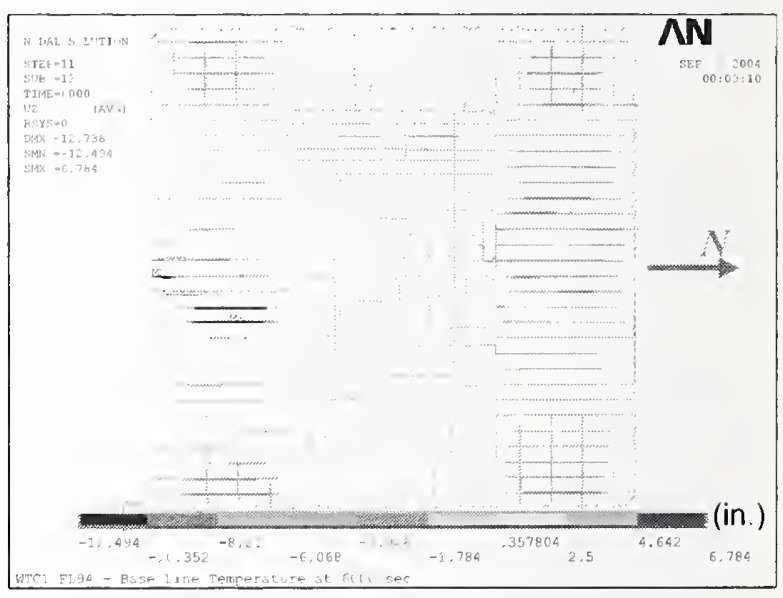

(b) Top view without slab

Figure A-14. Vertical displacement of WTC 1 Floor 94 for Case $A_{i}$ temperature condition at $100 \mathrm{~min}$ (downward displacement is negative; $\mathbf{5 x}$ displacement magnification). 


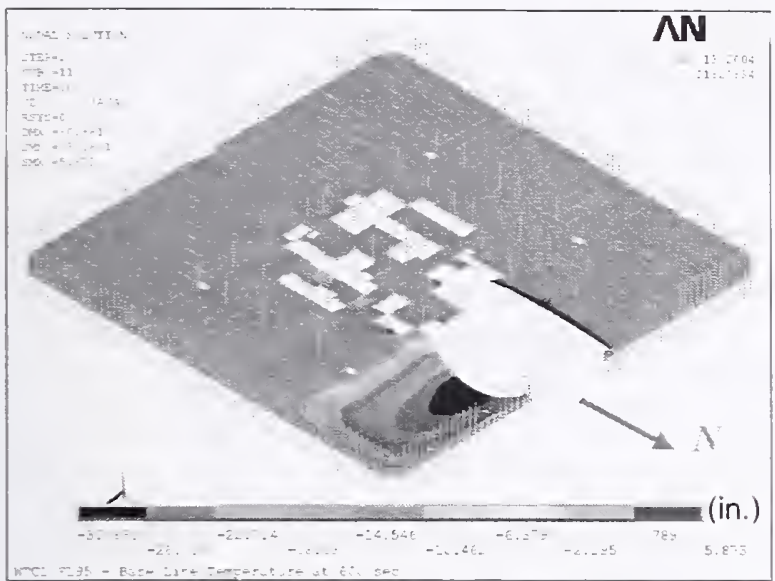

(a) Entire model

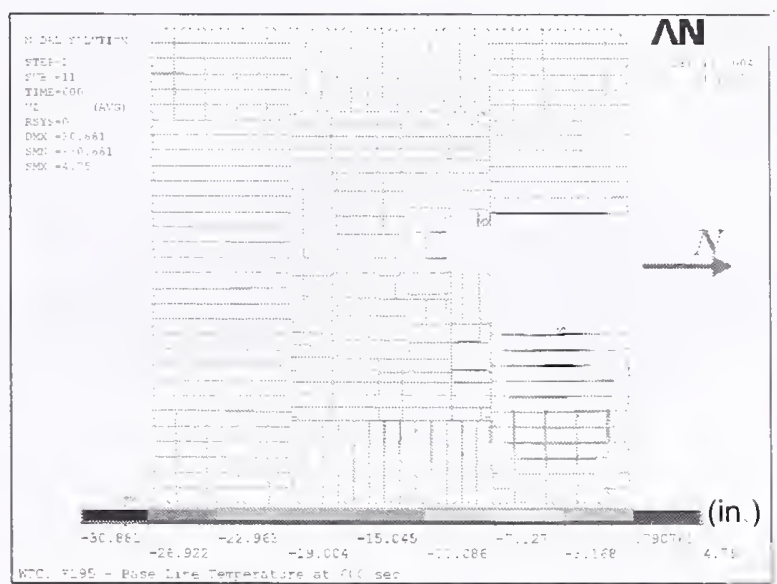

(b) Top view without slab

Figure A-15. Vertical displacement of WTC 1 Floor 95 for Case $A_{i}$ temperature condition at $10 \mathrm{~min}$ (downward displacement is negative; $5 \mathrm{x}$ displacement magnification).

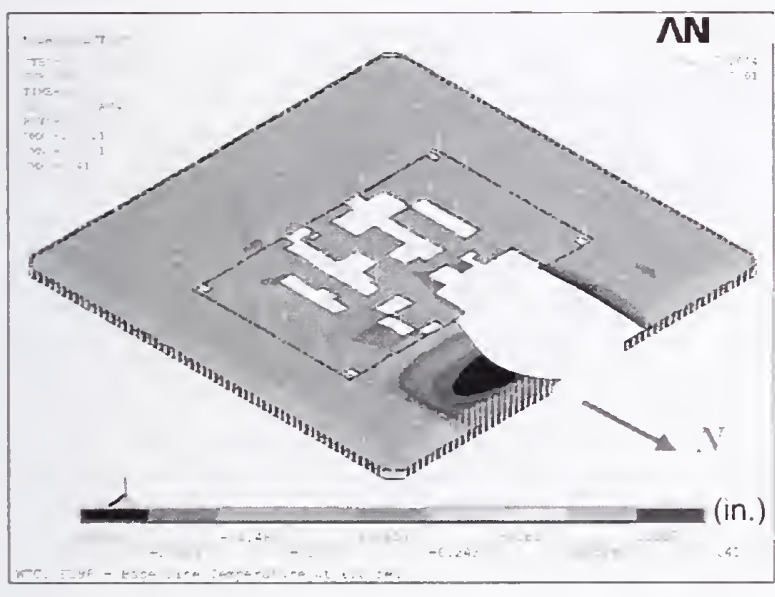

(a) Entire model

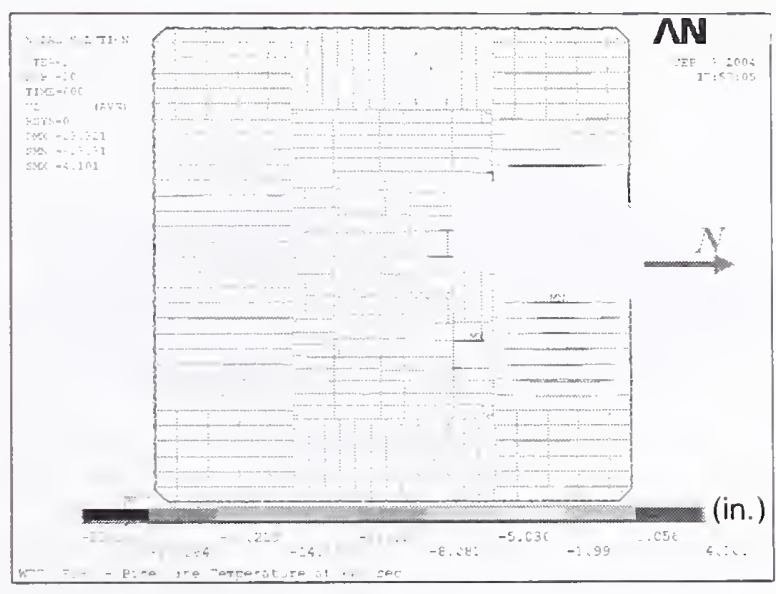

(b) Top view without slab

Figure A-16. Vertical displacement of WTC 1 Floor 96 for Case $A_{i}$ temperature condition at $10 \mathrm{~min}$ (downward displacement is negative; $5 x$ displacement magnification). 


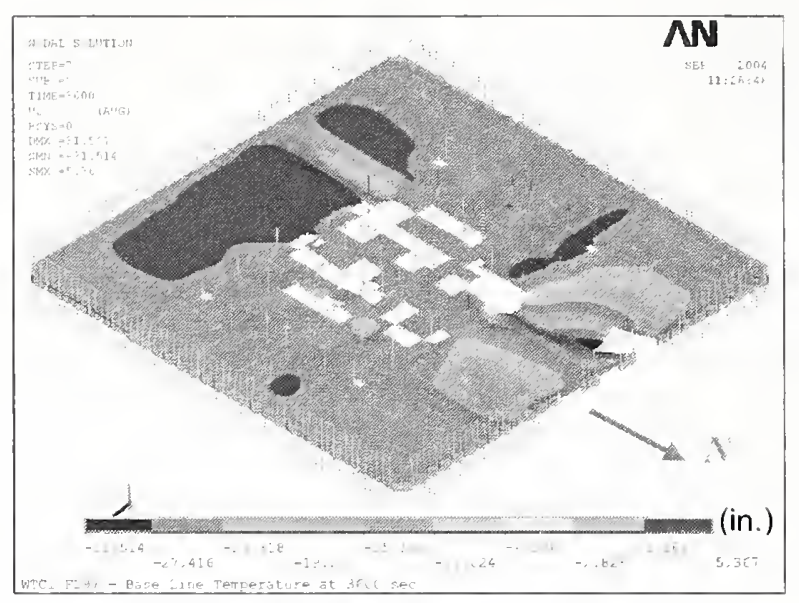

(a) Entire model

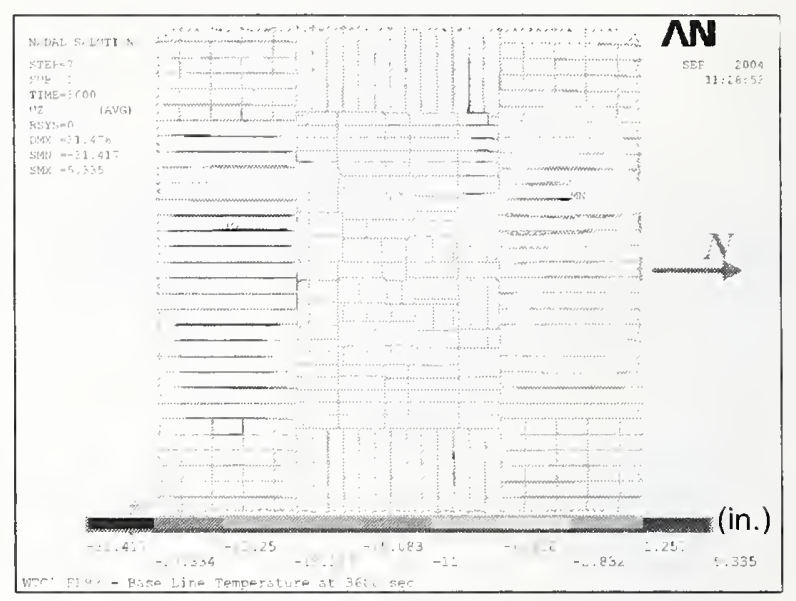

(b) Top view without slab

Figure A-17. Vertical displacement of WTC 1 Floor 97 for Case $A_{i}$ temperature condition at $60 \mathrm{~min}$ (downward displacement is negative; $5 \mathrm{x}$ displacement magnification).

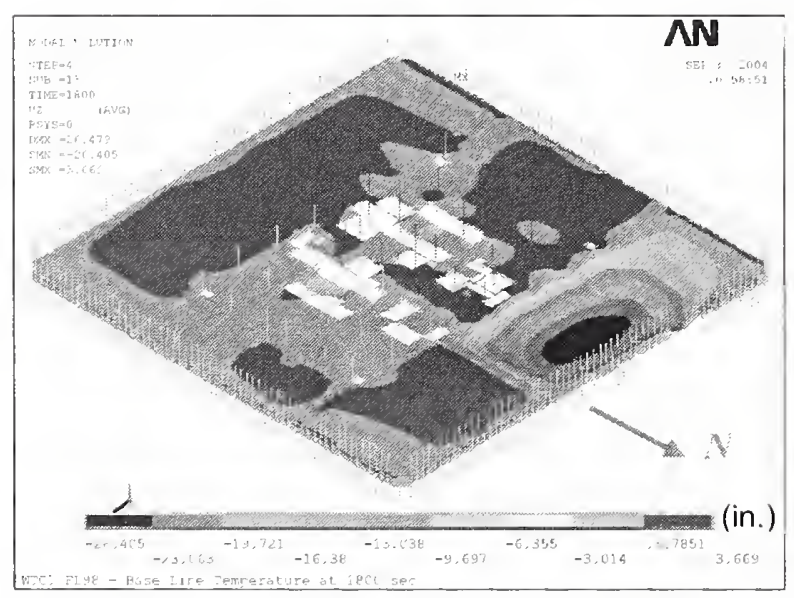

(a) Entire model

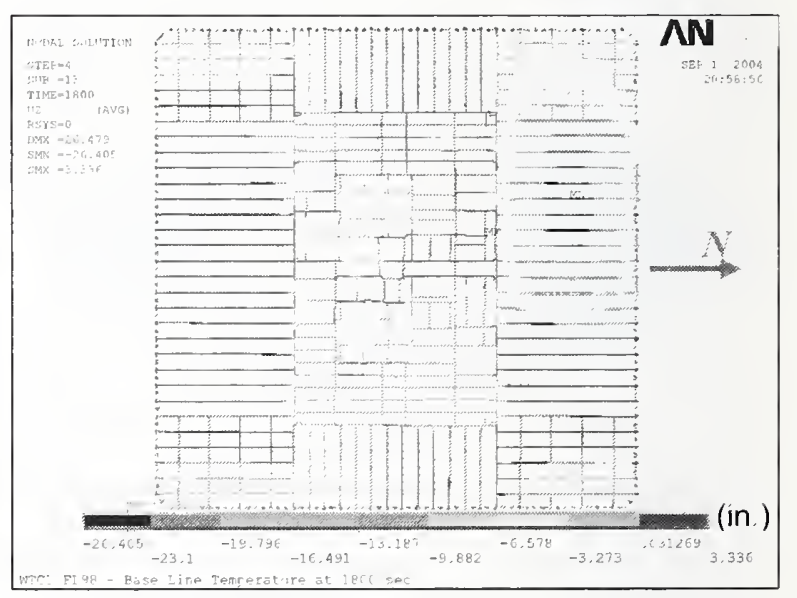

(b) Top view without slab

Figure A-18. Vertical displacement of WTC 1 Floor 98 for Case $A_{i}$ temperature condition at $30 \mathrm{~min}$ (downward displacement is negative; $5 x$ displacement magnification). 


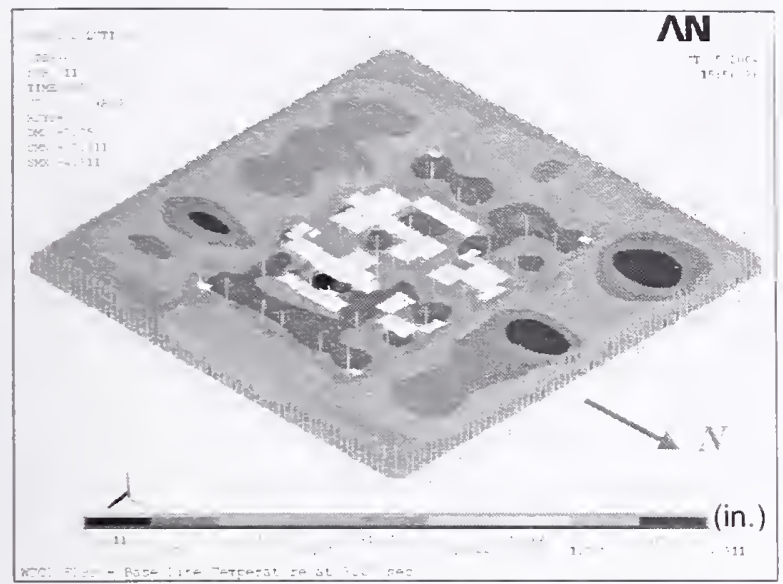

(a) Entire model

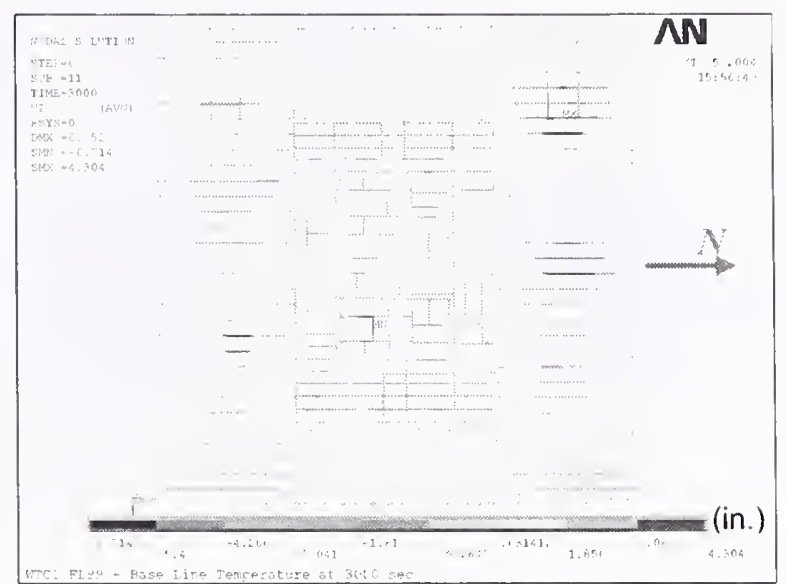

(b) Top view without slab

Figure A-19. Vertical displacement of WTC 1 Floor 99 for Case $A_{i}$ temperature condition at $50 \mathrm{~min}$ (downward displacement is negative; $5 x$ displacement magnification).

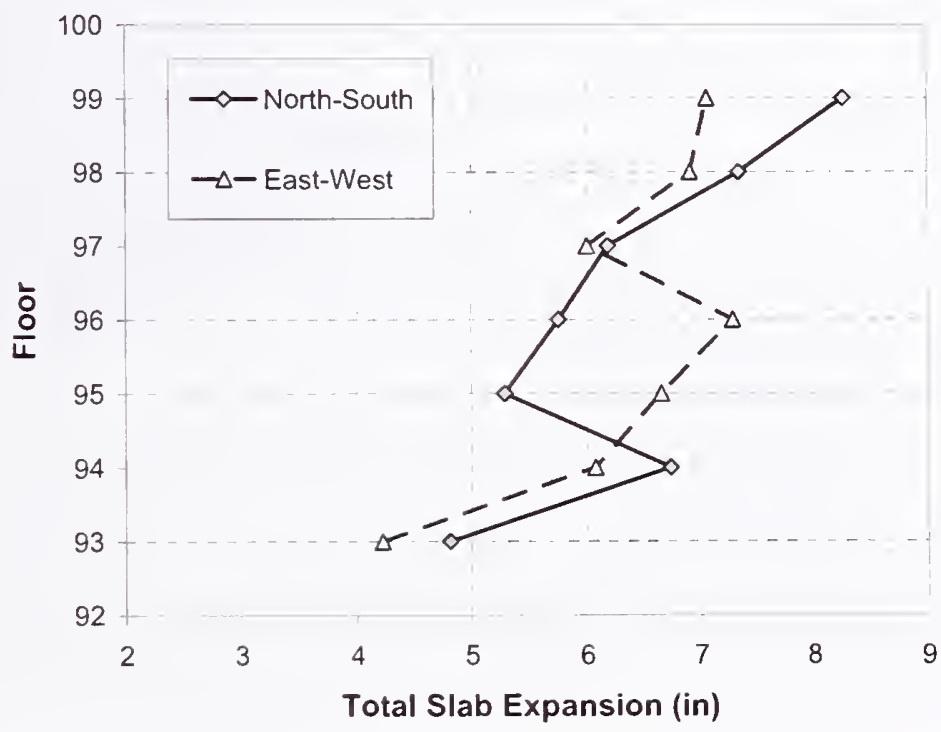

Figure A-20. Thermal expansion of floors of WTC 1 for Case $A_{i}$ temperature condition at $100 \mathrm{~min}$. 


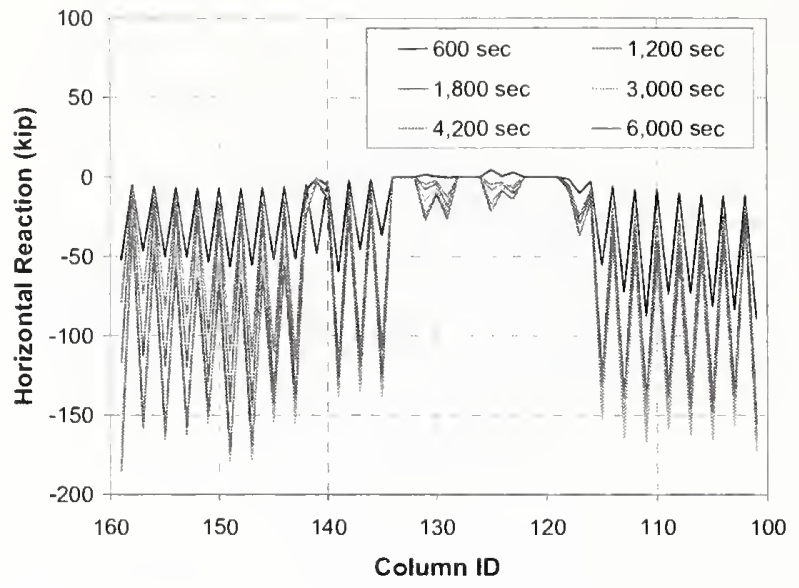

(a) North face

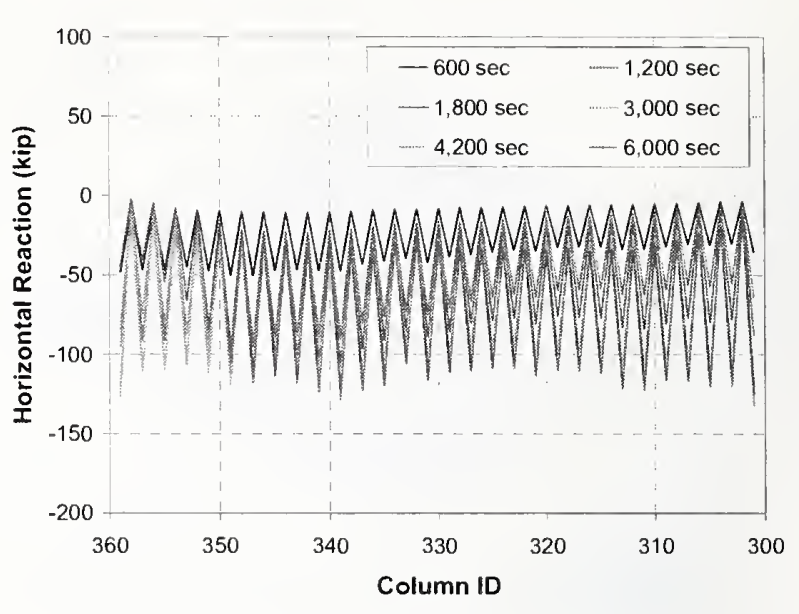

(b) South face

Figure A-21. Horizontal reaction force per column of WTC 1 Floor 97 for Case $A_{i}$ temperature condition.

\section{A.2.2 Case $B_{i}$ Temperature Condition}

Table A-2 gives the maximum vertical displacement of WTC 1 floors for Case $\mathrm{B}_{1}$ temperature condition, and Figs. A-22 to A-28 show the vertical displacements when the maximum displacement was obtained. The maximum vertical displacement of Floor 95 to Floor 98 increased due to higher temperature when compared to Case $\mathrm{A}_{\mathrm{i}}$ temperature condition. Especially, the vertical displacement in the south office area of those floors became significant. The maximum among the floors was $49 \mathrm{in}$. in the south office area of Floor 98 as shown in Fig. A-27. The large displacement on the south side of Floor 98 was caused by the exterior seat failure between Column 329 and Column 343 that started at 90 min (see Fig. A-29).

Exterior seats between Column 337 and Column 347 of Floor 97 also failed, which caused a vertical displacement of $37 \mathrm{in}$. in the south office area (see Fig. A-29). These exterior seats failed due to loss of vertical shear strength under the extreme temperatures encountered. Figure A-30 shows the average total thermal expansion of floors over the entire width of the building at 100 min of Case $\mathrm{B}_{\mathrm{i}}$ temperature condition. The total floor expansion ranged from $5 \mathrm{in}$. to $8.5 \mathrm{in}$. Figure A-31 shows horizontal reaction force at individual columns of the north and south faces of Floor 98 . It can be seen in the figure that reaction forces of columns between Column 329 and Column 343 became close to zero after the trusses attached to these column lost their vertical support at the exterior seats. Almost all the columns were found to be pushed out by the floor. This was also the case for other floors. 
Table A-2. Maximum vertical displacement of WTC 1 floors for Case $B_{i}$ temperature condition.

\begin{tabular}{|c|c|c|}
\hline Floor & $\begin{array}{c}\text { Max. Displacement } \\
\text { (in.) }\end{array}$ & $\begin{array}{c}\text { Time at the Maximum } \\
(\mathbf{m i n})\end{array}$ \\
\hline 93 & -5.8 & 100 \\
\hline 94 & 12.7 & 100 \\
\hline 95 & 29.2 & 10 \\
\hline 96 & 28.6 & 10 \\
\hline 97 & 37.4 & 100 \\
\hline 98 & 49.0 & 100 \\
\hline 99 & 6.8 & 100 \\
\hline
\end{tabular}

Note: Downward displacement is positive.

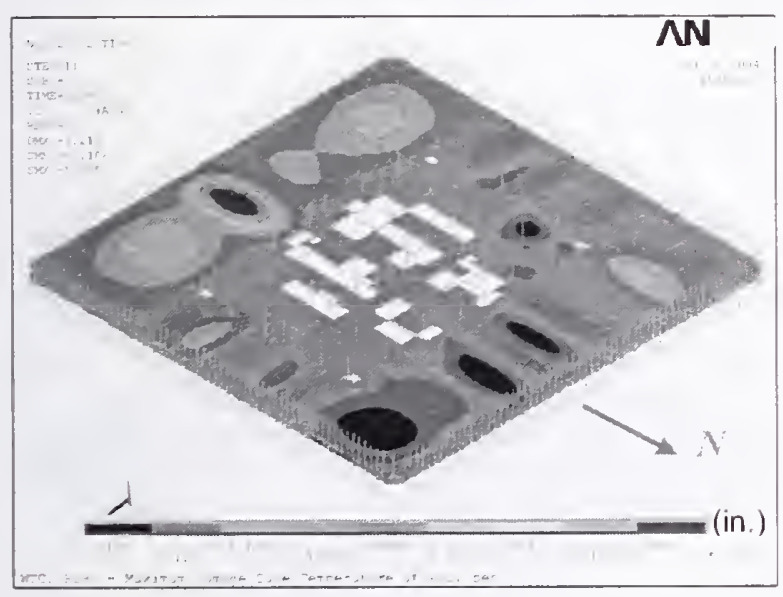

(a) Entire model

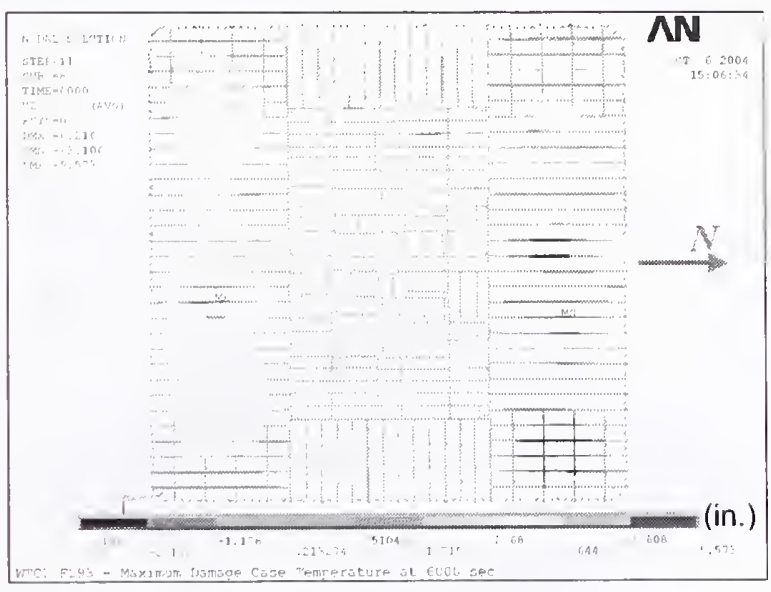

(b) Top view without slab

Figure A-22. Vertical displacement of WTC 1 Floor 93 for Case $B_{i}$ temperature condition at $100 \mathrm{~min}$ (downward displacement is negative; $5 x$ displacement magnification). 


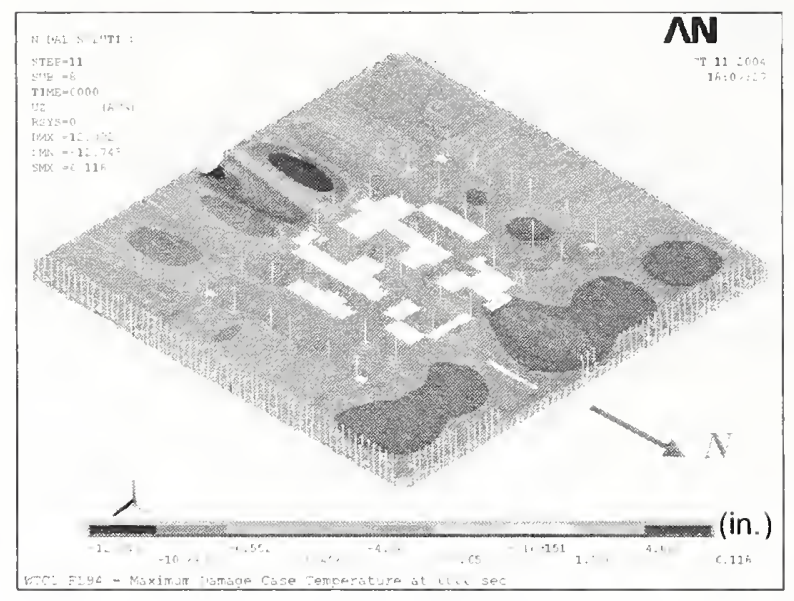

(a) Entire model

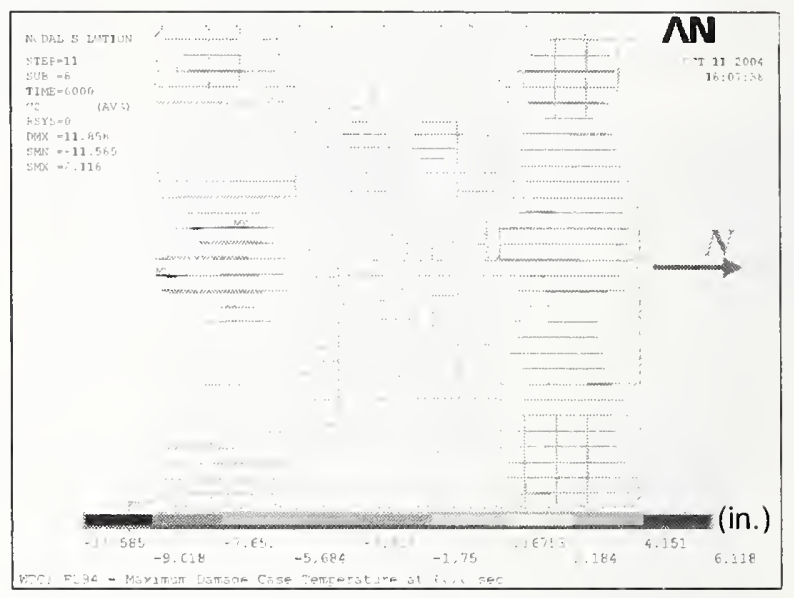

(b) Top view without slab

Figure A-23. Vertical displacement of WTC 1 Floor 94 for Case $B_{i}$ temperature condition at $100 \mathrm{~min}$ (downward displacement is negative; $5 x$ displacement magnification).

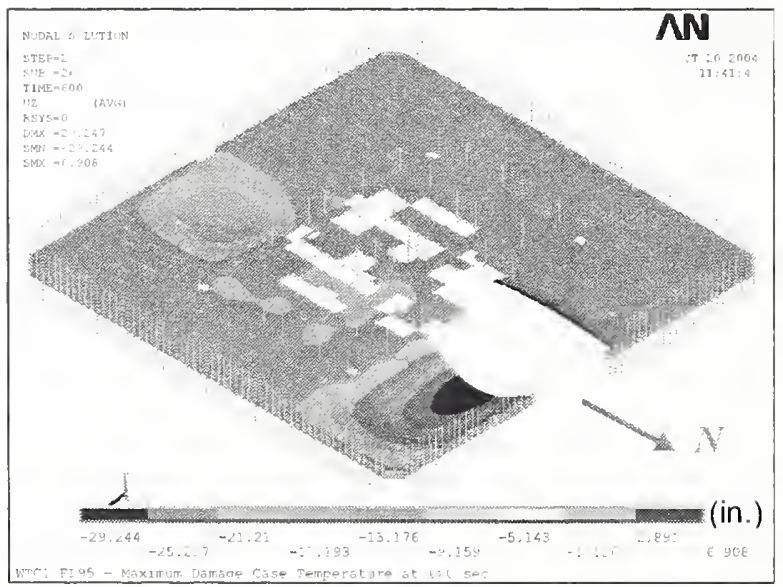

(a) Entire model

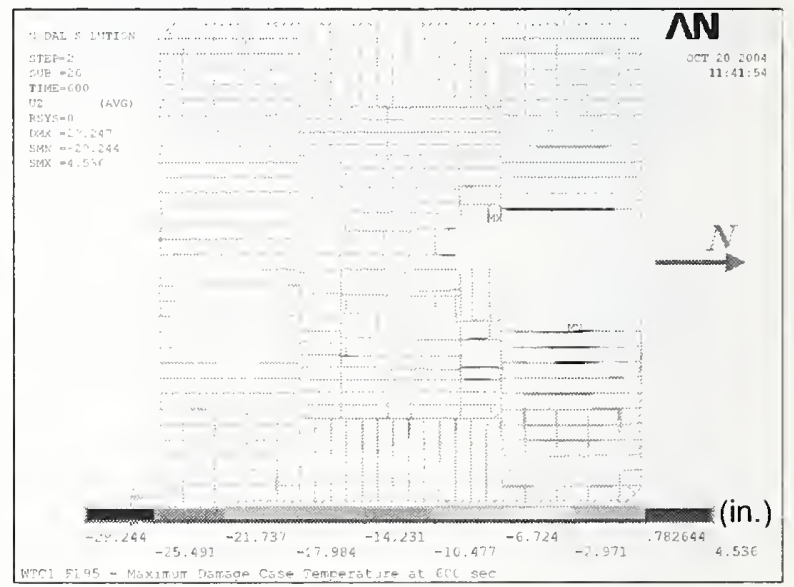

(b) Top view without slab

Figure A-24. Vertical displacement of WTC 1 Floor 95 for Case $B_{i}$ temperature condition at $10 \mathrm{~min}$ (downward displacement is negative; $5 x$ displacement magnification). 


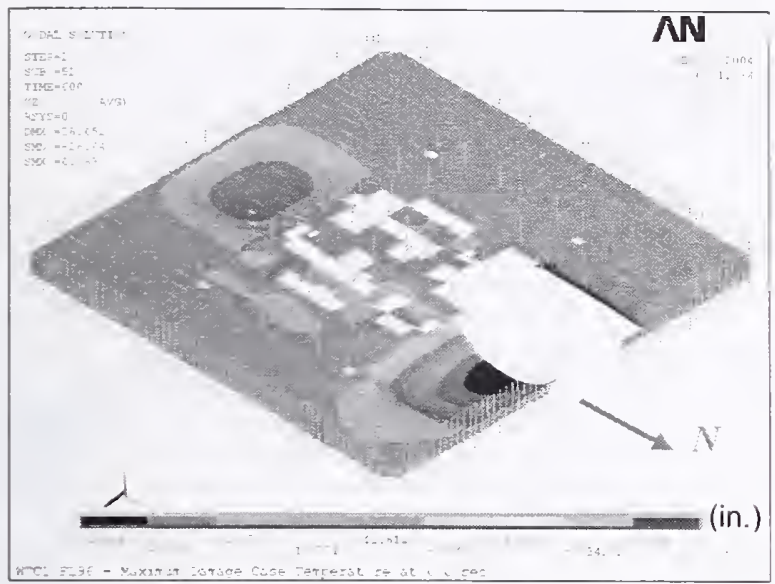

(a) Entire model

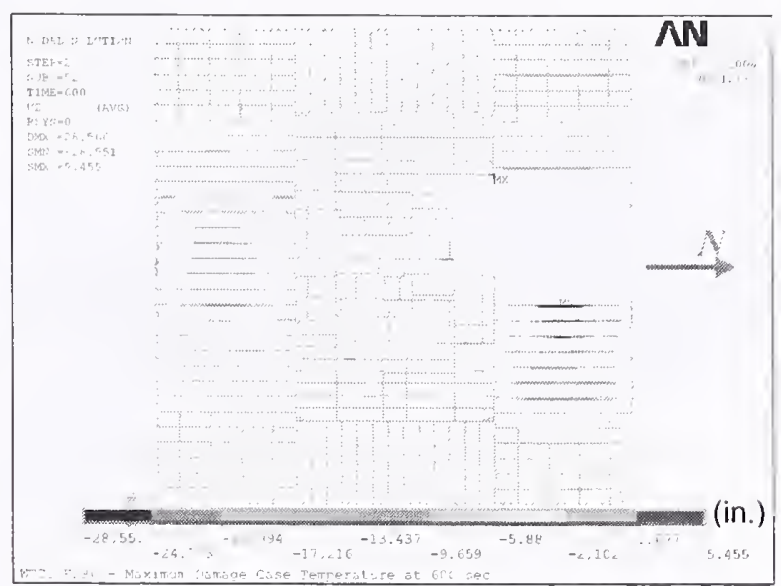

(b) Top view without slab

Figure A-25. Vertical displacement of WTC 1 Floor 96 for Case $B_{i}$ temperature condition at $10 \mathrm{~min}$ (downward displacement is negative; $5 x$ displacement magnification).

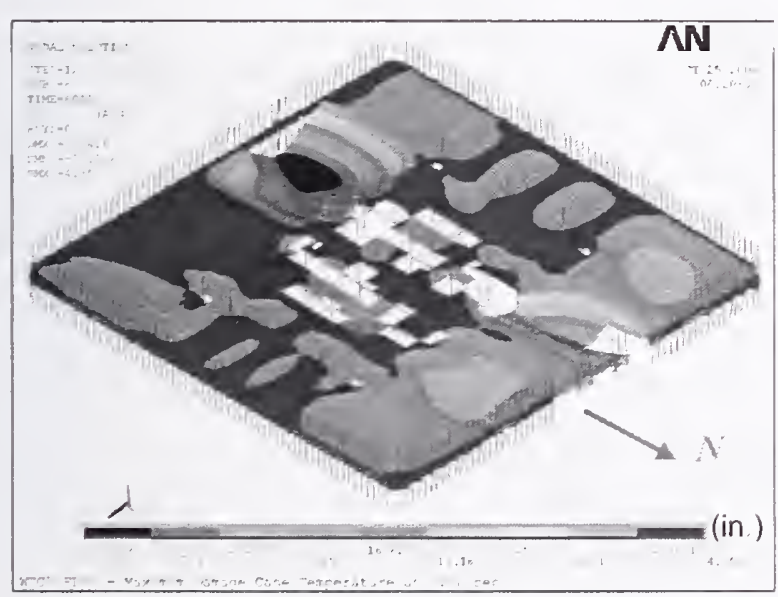

(a) Entire model

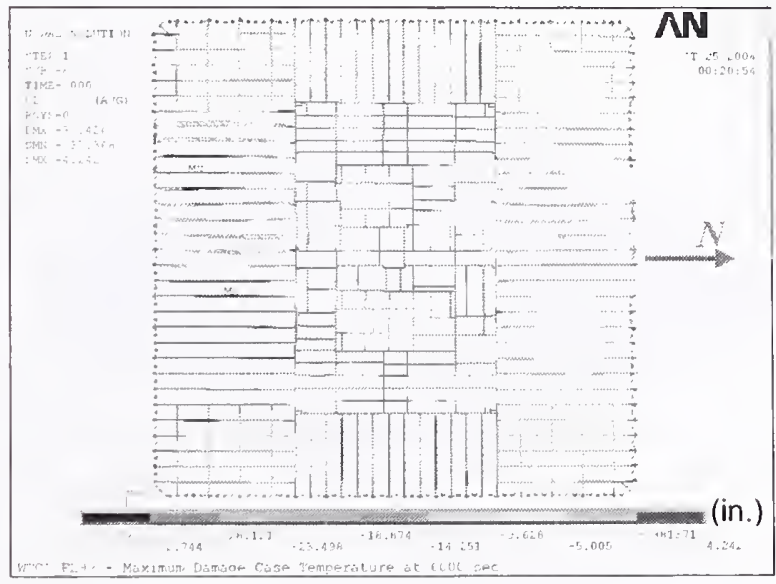

(b) Top view without slab

Figure A-26. Vertical displacement of WTC 1 Floor 97 for Case $B_{i}$ temperature condition at $100 \mathrm{~min}$ (downward displacement is negative; $5 \mathrm{x}$ displacement magnification). 


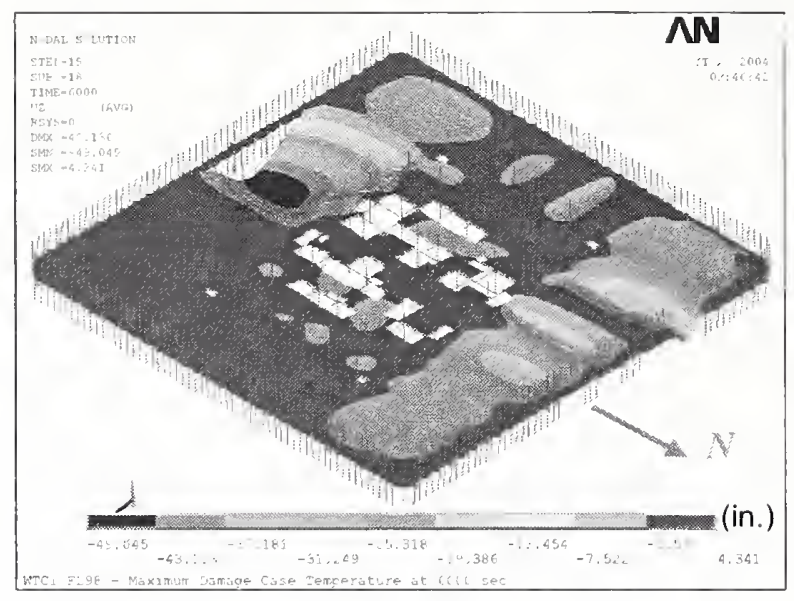

(a) Entire model

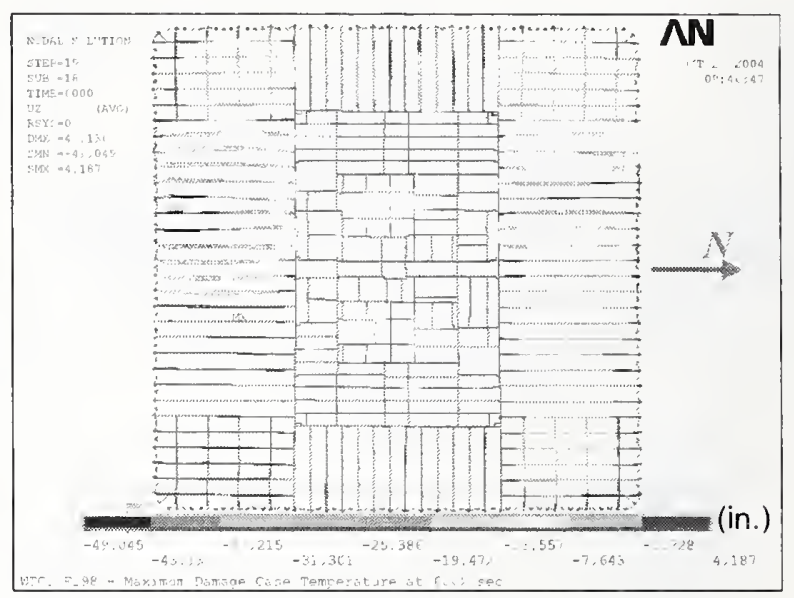

(b) Top view without slab

Figure A-27. Vertical displacement of WTC 1 Floor 98 for Case $B_{i}$ temperature condition at $100 \mathrm{~min}$ (downward displacement is negative; $5 x$ displacement magnification).

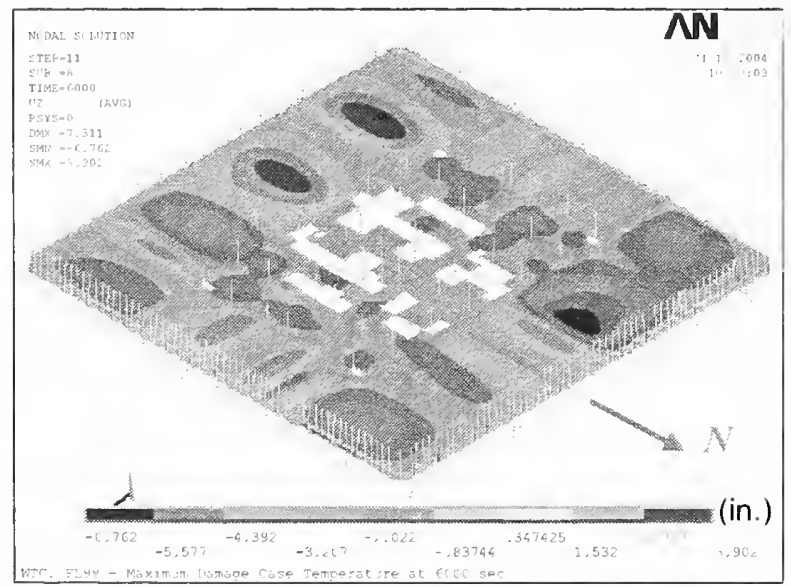

(a) Entire model

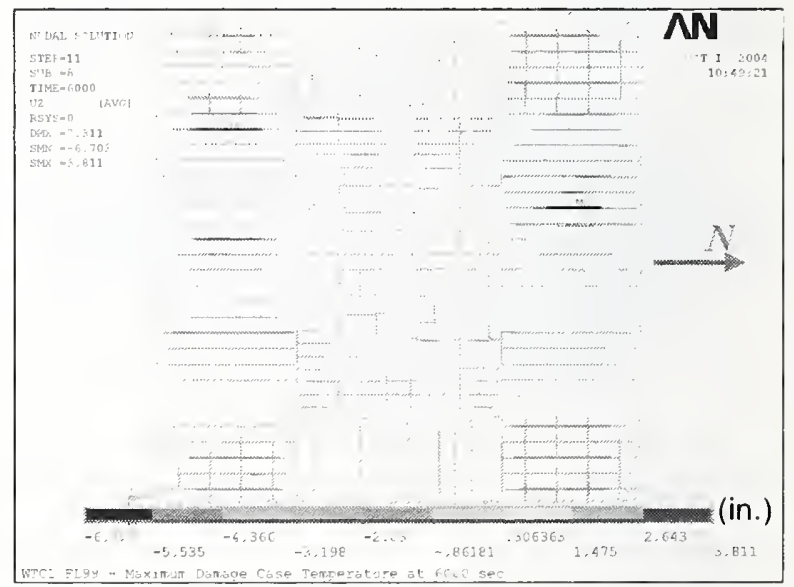

(b) Top view without slab

Figure A-28. Vertical displacement of WTC 1 Floor 99 for Case $B_{i}$ temperature condition at $100 \mathrm{~min}$ (downward displacement is negative; $5 x$ displacement magnification). 


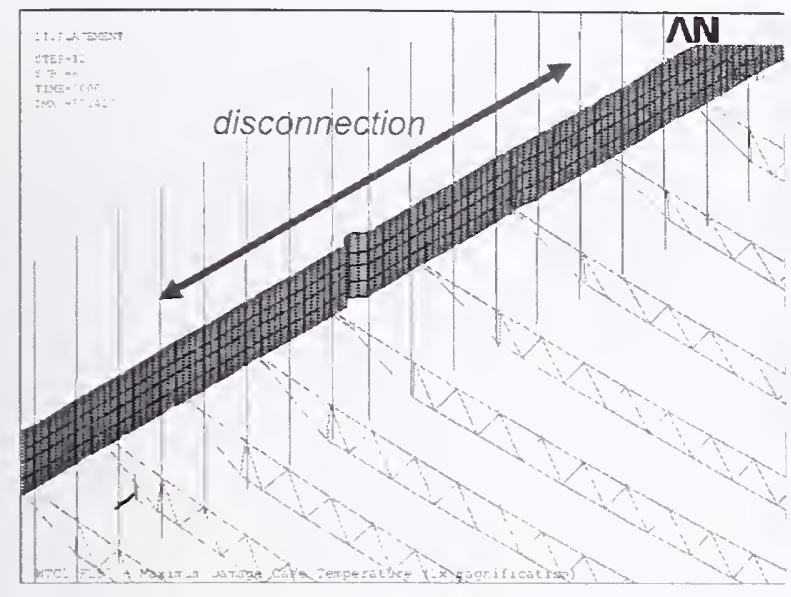

(a) Floor 97

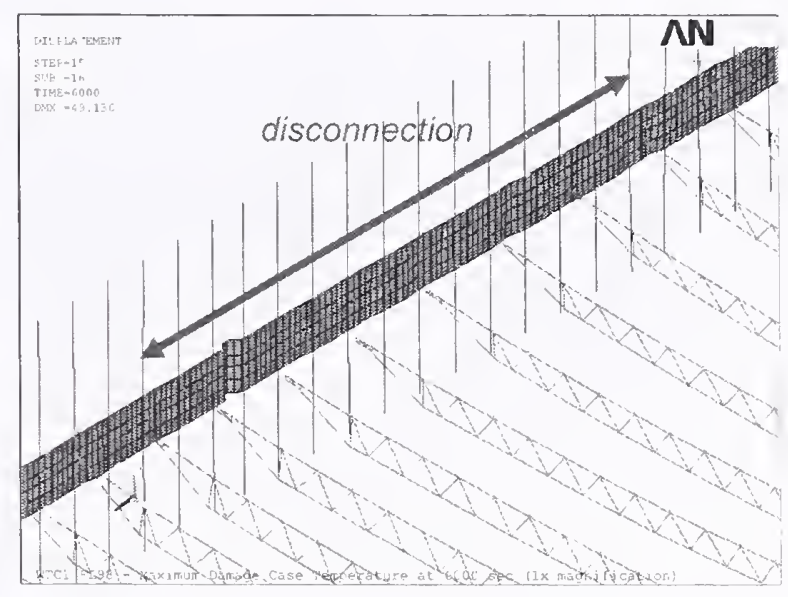

(b) Floor 98

Figure A-29. Loss of vertical supports in WTC 1 Floor 97 and Floor 98 for Case $B_{i}$ temperature condition at $100 \mathrm{~min}$ (1x displacement magnification).

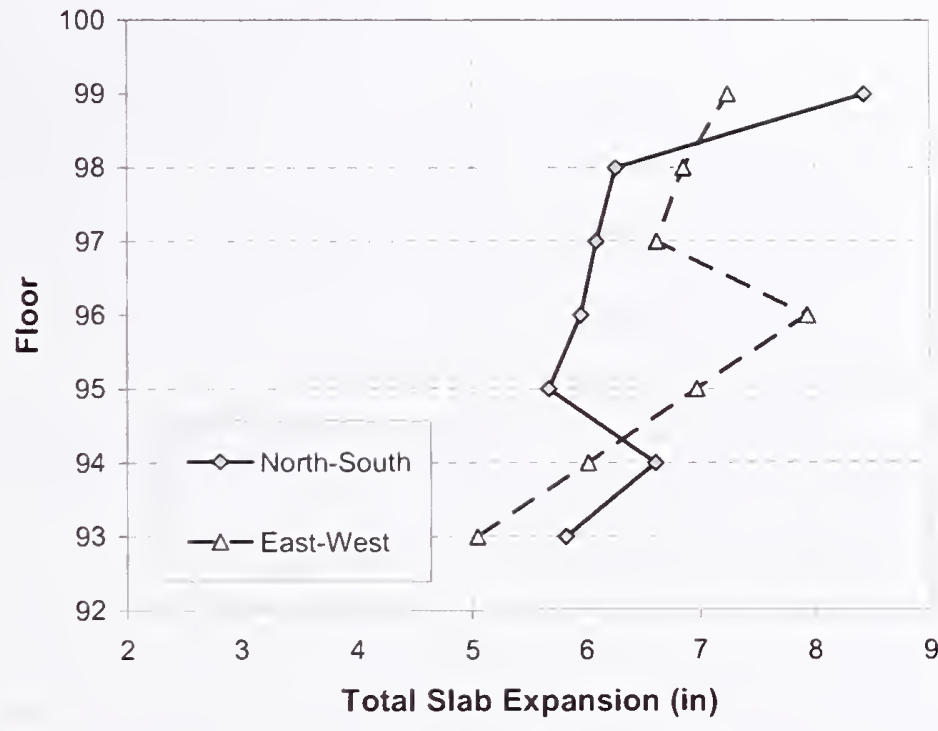

Figure A-30. Thermal expansion of floors of WTC 1 for Case $B_{i}$ temperature condition at $100 \mathrm{~min}$. 


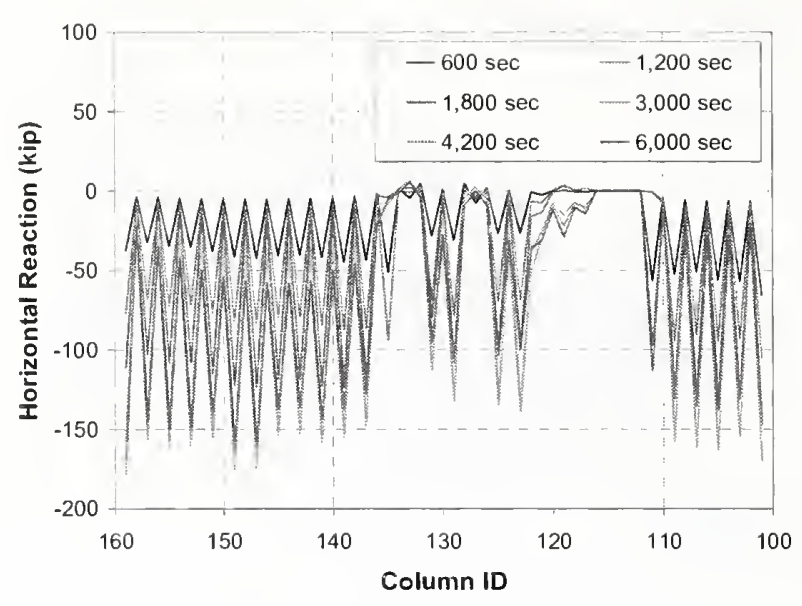

(a) North face

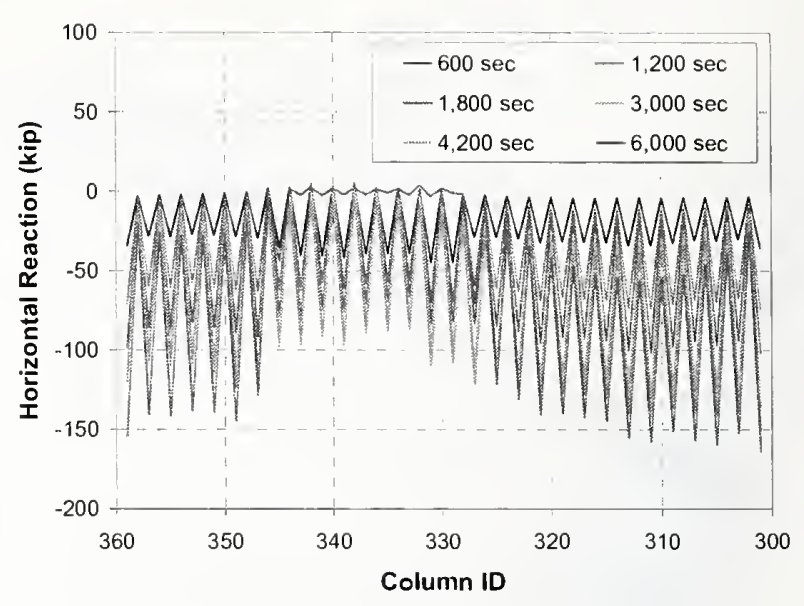

(b) South face

Figure A-31. Horizontal reaction force per column of WTC 1 Floor 98 for Case $B_{i}$ temperature condition.

\section{A.3 WTC 2 FLOORS}

\section{A.3.1 Case $\mathrm{C}_{\mathrm{i}}$ Temperature Condition}

Table A-3 gives the maximum vertical displacement of WTC 2 floors for Case $\mathrm{C}_{\mathrm{i}}$ temperature condition, and Figs. A-32 to A-36 show the vertical displacements when the maximum displacement was obtained. Floor 79 to Floor 83, except Floor 82, had the maximum vertical displacement in the southeast area near the impact damage. Floor 82 had the maximum vertical displacement in the northeast area. The maximum displacement was projected to occur at $60 \mathrm{~min}$ on all floors. The vertical displacement in the west office area was found to be insignificant on all the floors throughout the thermal loading. Floor 80 had a fair amount of vertical displacement in the north half of the west office area although the maximum displacement was 15 in. A significant amount of web diagonals of Floor 82 to Floor 83 buckled in the hot zones of the east office area. Seat failures were not observed on Floor 79 to Floor 81. Trusses at Column 301 to Column 317 on the east face of Floor 82 and at Column 325 to Column 333 on the east face of Floor 83 lost their vertical support at the exterior seats at $50 \mathrm{~min}$ as shown in Fig. A-37. Figure A-38 shows the average total thermal expansion of floors over the entire width of the building at $60 \mathrm{~min}$ in two orthogonal directions. The total floor expansion ranged from 2.5 in. to 5.5 in. Figure A-39 shows horizontal reaction force at individual columns of the north and south faces of Floor 81 . When the reaction force is positive, the column is pulled inward by the floor. Columns between Column 353 and Column 359 were pulled in by the floor. This was caused by the floor sagging in the southeast area that was primarily due to the impact damage to the interior truss seats. A few columns at the southeast corner of Floor 80 and Floor 83 were also pulled in by the floor. 
Table A-3. Maximum vertical displacement of WTC 2 floors for Case $C_{i}$ temperature condition.

\begin{tabular}{|c|c|c|}
\hline Floor & $\begin{array}{c}\text { Max. Displacement } \\
\text { (in.) }\end{array}$ & $\begin{array}{c}\text { Time at the Maximum } \\
\text { (min) }\end{array}$ \\
\hline 79 & 19.0 & 60 \\
\hline 80 & 30.1 & 60 \\
\hline 81 & 31.0 & 60 \\
\hline 82 & 45.2 & 60 \\
\hline 83 & 38.9 & 60 \\
\hline
\end{tabular}

Note: Downward displacement is positive.

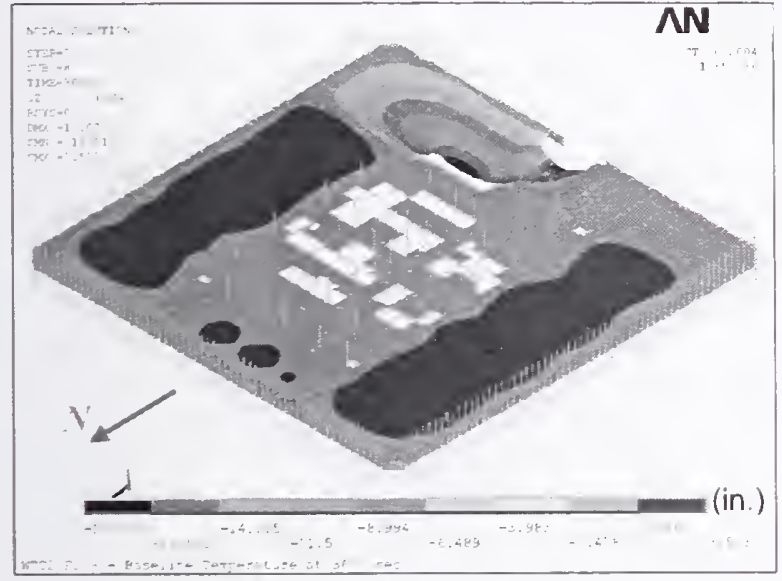

(a) Entire model

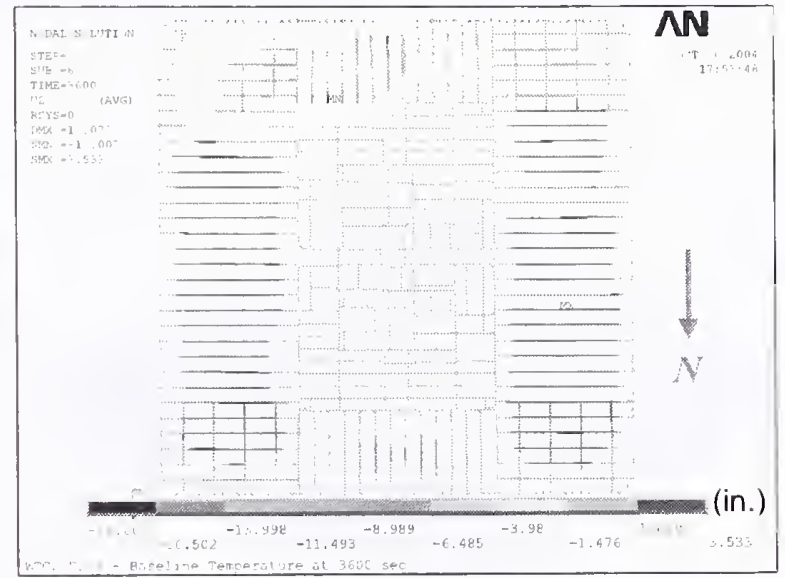

(b) Top view without slab

Figure A-32. Vertical displacement of WTC 2 Floor 79 for Case $C_{i}$ temperature condition at $60 \mathrm{~min}$ (downward displacement is negative; $5 \mathrm{x}$ displacement magnification). 


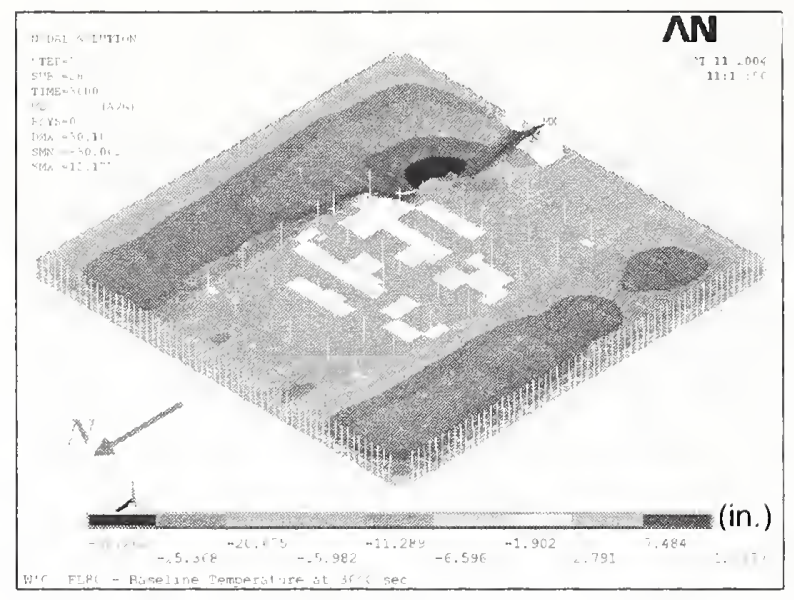

(a) Entire model

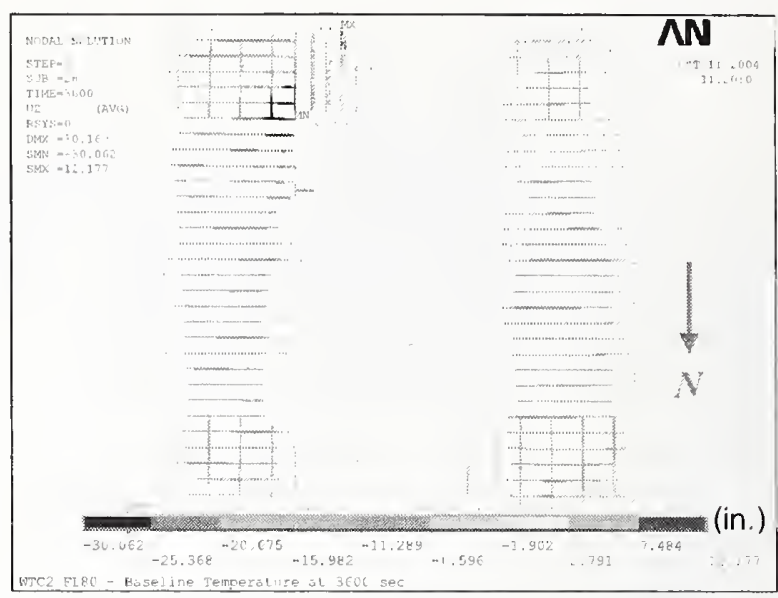

(b) Top view without slab

Figure A-33. Vertical displacement of WTC 2 Floor 80 for Case $C_{i}$ temperature condition at $60 \mathrm{~min}$ (downward displacement is negative; $5 x$ displacement magnification).

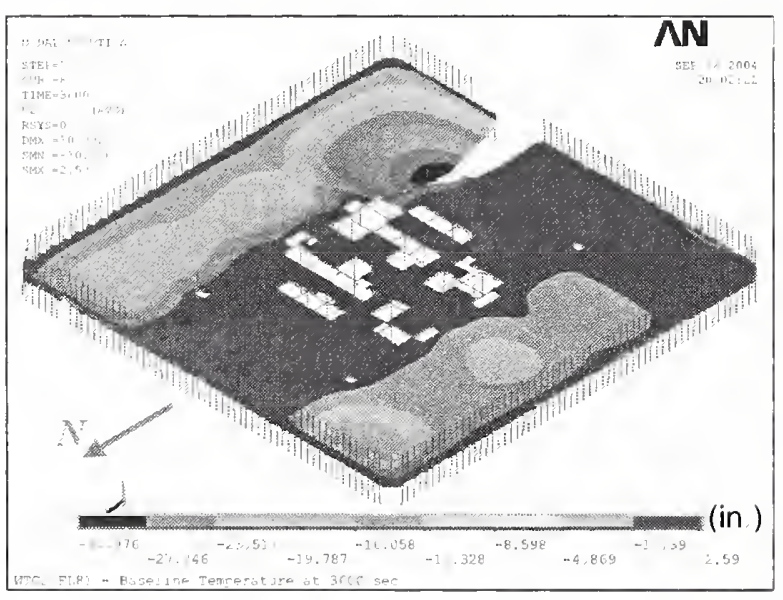

(a) Entire model

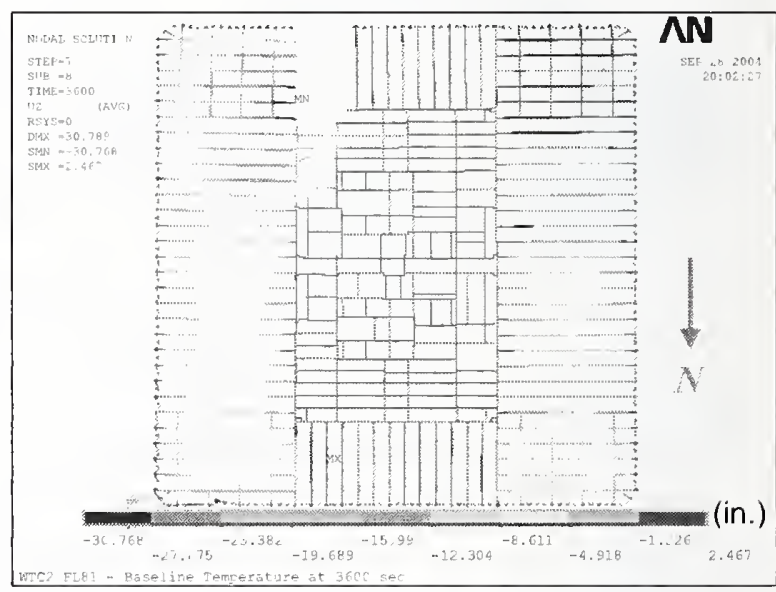

(b) Top view without slab

Figure A-34. Vertical displacement of WTC 2 Floor 81 for Case $C_{i}$ temperature condition at $60 \mathrm{~min}$ (downward displacement is negative; $5 x$ displacement magnification). 


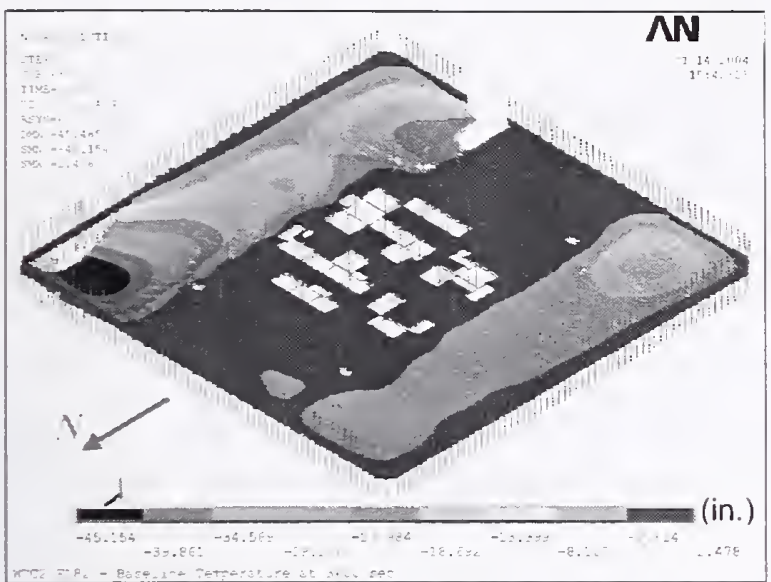

(a) Entire model

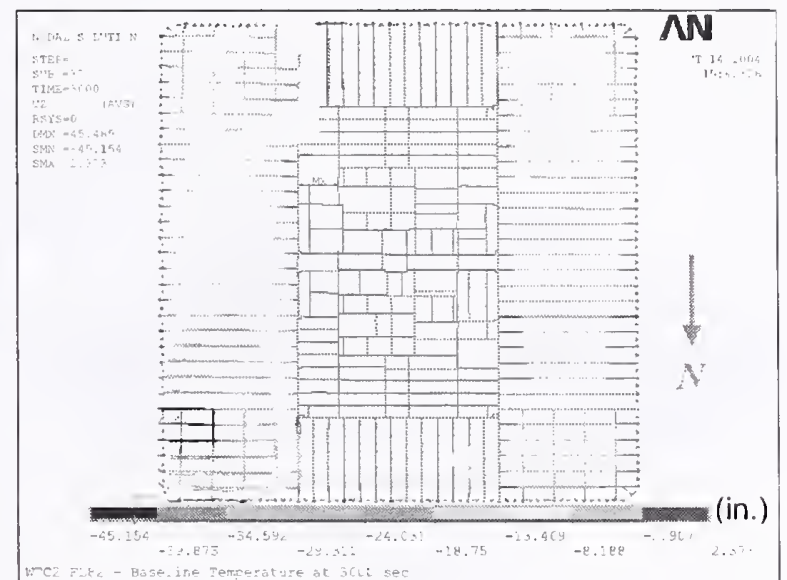

(b) Top view without slab

Figure A-35. Vertical displacement of WTC 2 Floor 82 for Case $C_{i}$ temperature condition at $60 \mathrm{~min}$ (downward displacement is negative; $5 x$ displacement magnification). 


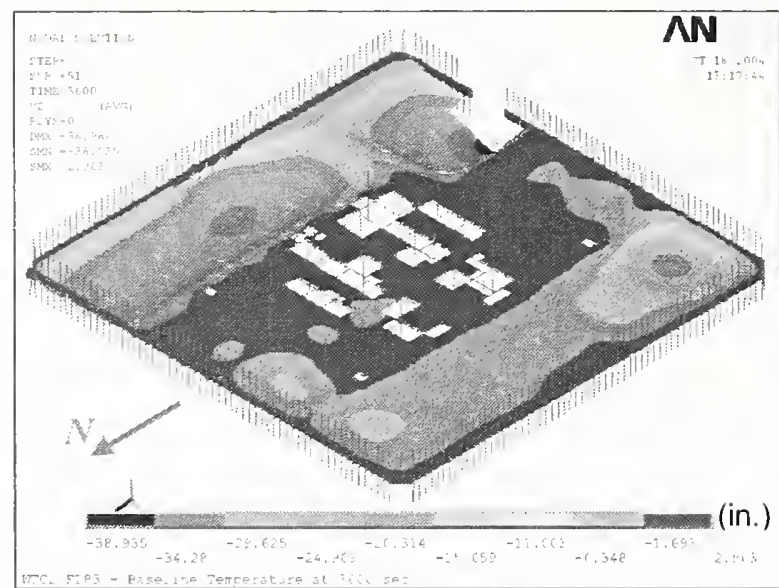

(a) Entire model

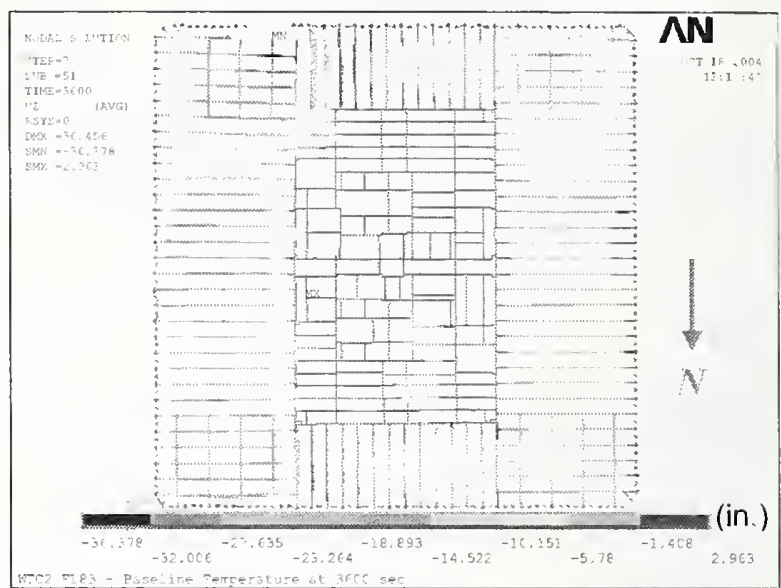

(b) Top view without slab

Figure A-36. Vertical displacement of WTC 2 Floor 83 for Case $C_{i}$ temperature condition at $60 \mathrm{~min}$ (downward displacement is negative; 5x displacement magnification).

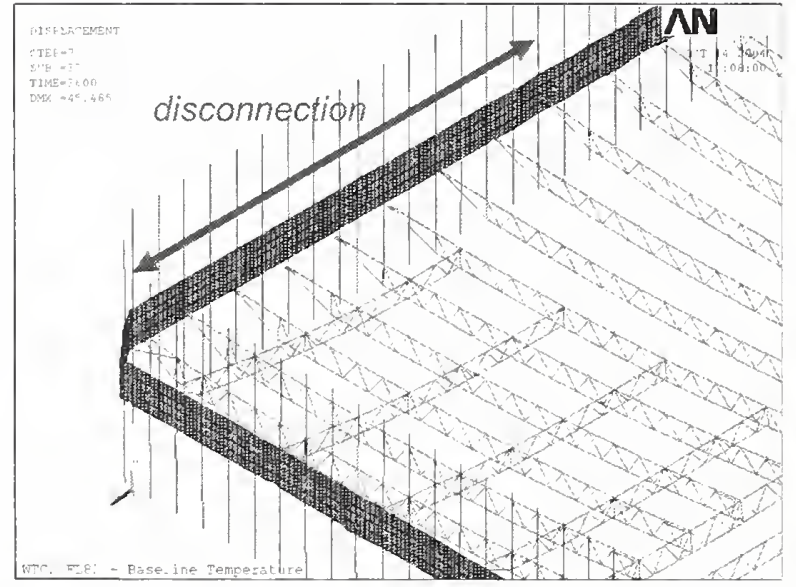

(a) Floor 82

(1X displacement magnification)

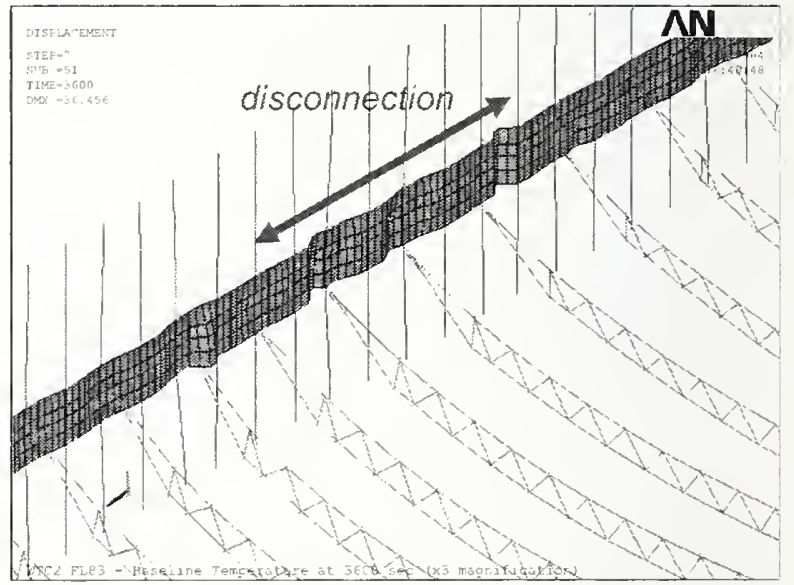

(b) Floor 83

(3X displacement magnification)

Figure A-37. Loss of vertical supports in WTC 2 Floor 82 and Floor 83 for Case $C_{i}$ temperature condition at $60 \mathrm{~min}$. 


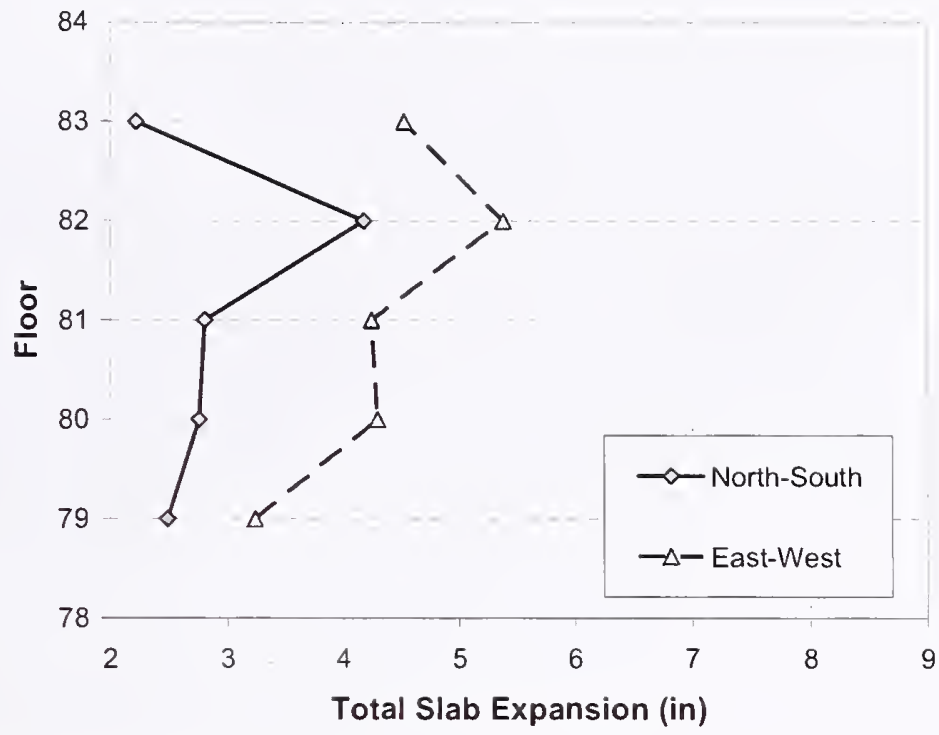

Figure A-38. Thermal expansion of floors of WTC 2 for Case $C_{i}$ temperature condition at $60 \mathrm{~min}$.

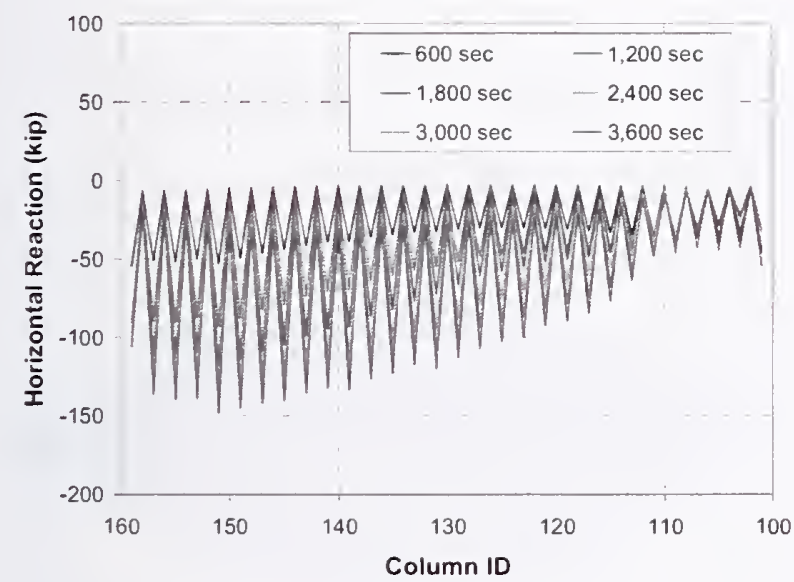

(a) West face

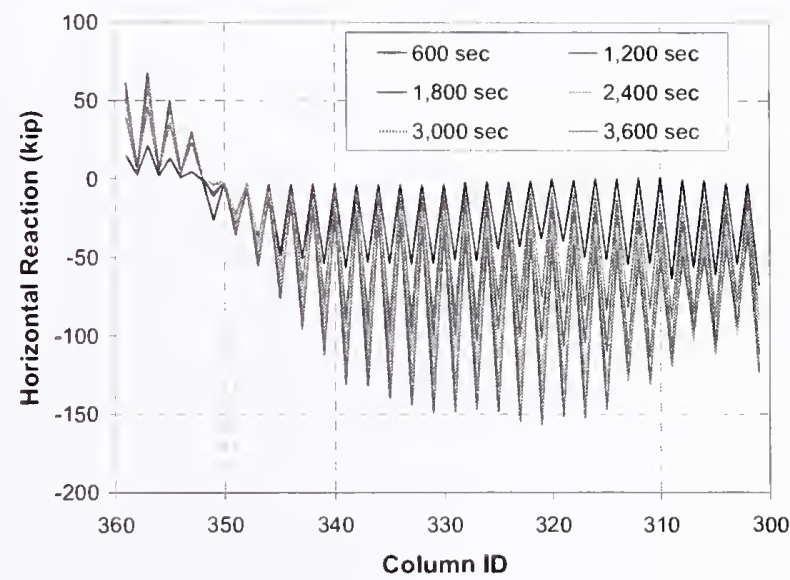

(b) East face

Figure A-39. Horizontal reaction force per column of WTC 2 Floor 81 for Case $C_{i}$ temperature condition.

\section{A.3.2 Case $D_{i}$ Temperature Condition}

Table A-4 gives the maximum vertical displacement of WTC 2 floors for Case $D_{i}$ temperature condition, and Figs. A -40 to $A-44$ show the vertical displacements when the maximum displacement was obtained. Due to more impact damage and different temperature histories than Case $\mathrm{C}_{\mathrm{i}}$ temperature condition, all floors had larger vertical displacement. Floor 80 and Floor 81 suffered damage to many interior seats in the east office area due to the aircraft impact. Bridging trusses that had been removed in Case $\mathrm{C}_{\mathrm{i}}$ temperature condition were replaced in these two floors in order to support primary trusses of the east 
office area after the impact. The maximum vertical displacement of $97 \mathrm{in}$. was calculated at Floor 81 near the impact damage at $50 \mathrm{~min}$ as shown in Fig. A-42. Significant sagging of the floor was observed at Floor 79 to Floor 83. Gusset plates and bolts at more than 75 percent of all the exterior seats of the east face of Floor 82 and Floor 83 failed due to horizontal shear force that was caused by the difference in the thermal expansion between the floor and the exterior wall in the direction transverse to the primary trusses. The truss at Column 357 of Floor 81 was the only truss that lost its vertical support at the exterior seat among all the floors. This truss walked off the truss seat. Figure A-45 shows the average total thermal expansion of floors over the entire width of the building at $60 \mathrm{~min}$ of Case $\mathrm{D}_{\mathrm{i}}$ temperature condition. The total floor expansion ranged from 1 in. to 5 in. Figures A-46 and A-47 show horizontal reaction force at individual columns of the north and south faces of Floor 80 and Floor 82, respectively. Column 101 to Column 111 on the west face and Column 347 to Column 359 on the east face were pulled in by the floor at $60 \mathrm{~min}$ on Floor 80 because of the floor sagging occurring in the southeast area. Since core columns were not restrained in the horizontal directions, when the floor pulled in one face of exterior wall, the opposite face of the exterior wall would also be pulled in. Columns at the southeast corner were pulled in by the floor at Floor 79 and Floor 81. Many columns of the west face of Floor 82 were pulled inward, while reaction forces at many columns of the east face were close to zero. As described above, gusset plates and seat bolts failed at a number trusses on the east face of Floor 82. Because columns at these locations were not supported in the horizontal direction by the floor, the reaction force became close to zero at these columns.

Table A-4. Maximum vertical displacement of WTC 2 floors for Case $D_{i}$ temperature condition.

\begin{tabular}{|c|c|c|}
\hline Floor & $\begin{array}{c}\text { Max. Displacement } \\
(\text { in. })\end{array}$ & $\begin{array}{c}\text { Time at the Maximum } \\
(\mathbf{m i n})\end{array}$ \\
\hline 79 & 35.8 & 60 \\
\hline 80 & 65.6 & 40 \\
\hline 81 & 96.7 & 50 \\
\hline 82 & 49.4 & 60 \\
\hline 83 & 44.6 & 60 \\
\hline
\end{tabular}

Note: Downward displacement is positive.

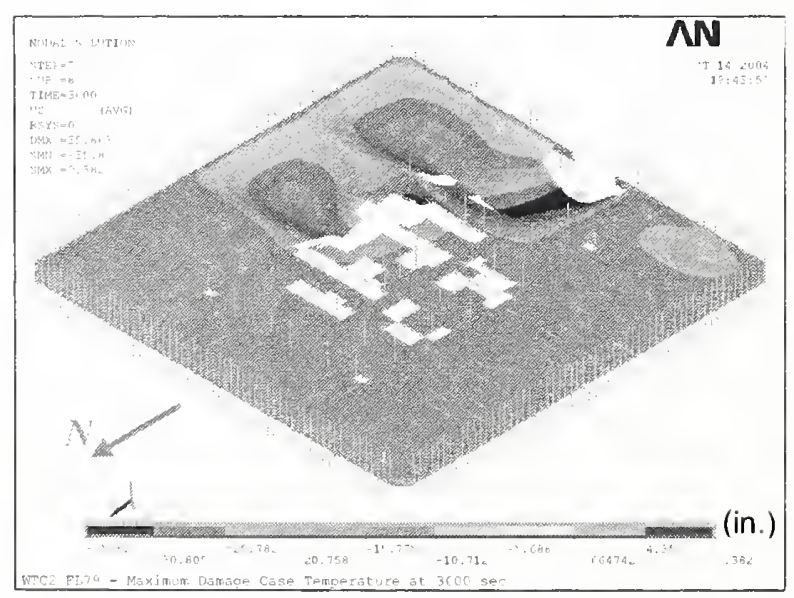

(a) Entire model

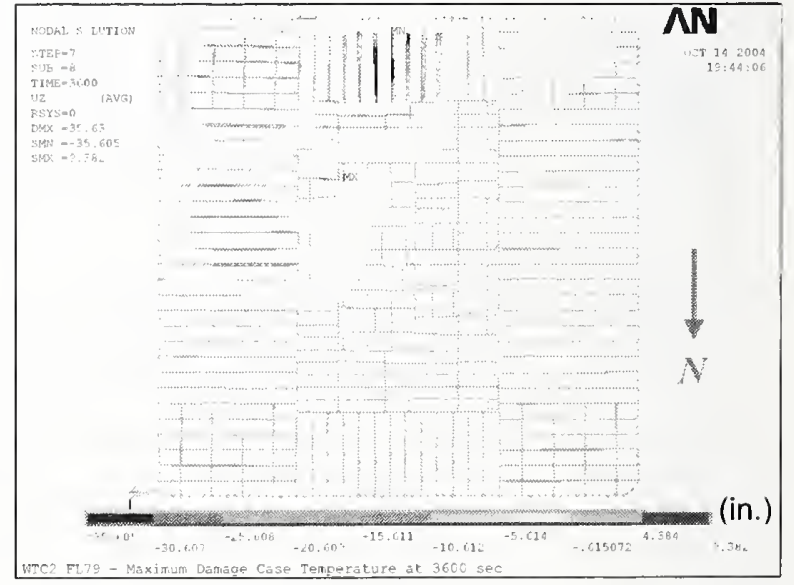

(b) Top view without slab

Figure A-40. Vertical displacement of WTC 2 Floor 79 for Case $D_{i}$ temperature condition at $60 \mathrm{~min}$ (downward displacement is negative; 5x displacement magnification). 


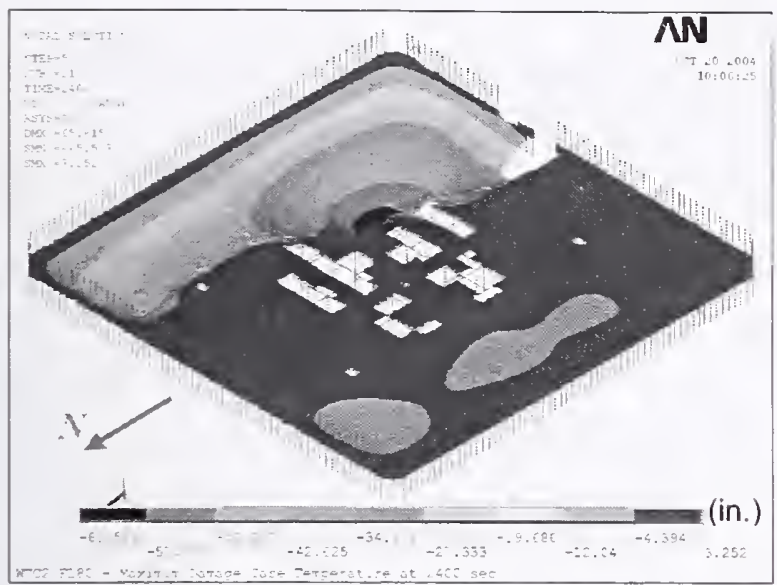

(a) Entire model

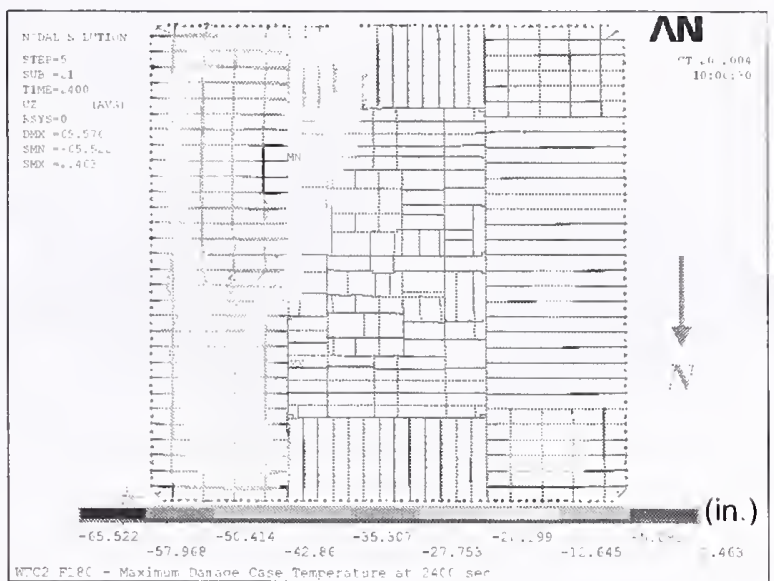

(b) Top view without slab

Figure A-41. Vertical displacement of WTC 2 Floor 80 for Case $D_{i}$ temperature condition at 40 min (downward displacement is negative; $5 x$ displacement magnification).

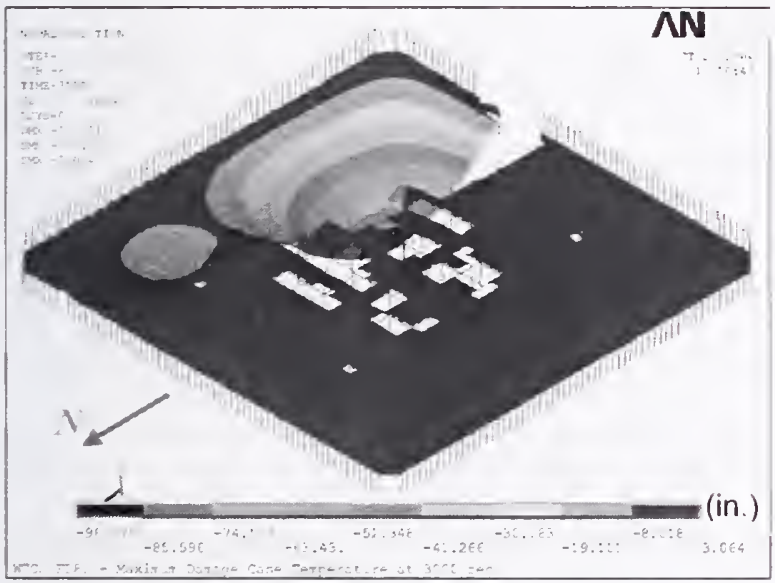

(a) Entire model

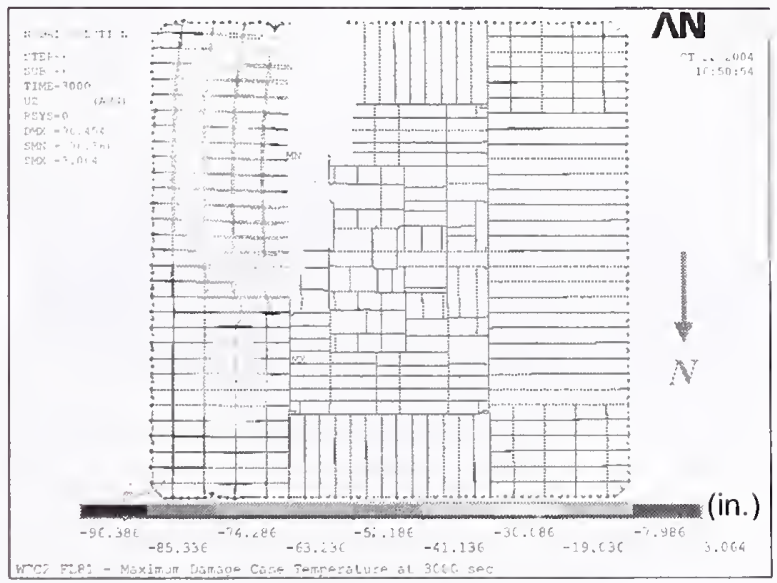

(b) Top view without slab

Figure A-42. Vertical displacement of WTC 2 Floor 81 for Case $D_{i}$ temperature condition at $50 \mathrm{~min}$ (downward displacement is negative; $5 x$ displacement magnification). 


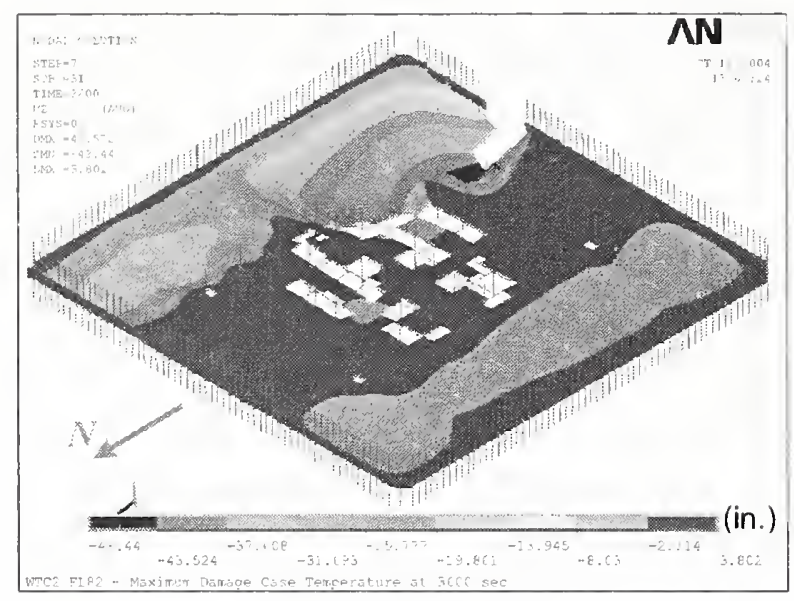

(a) Entire model

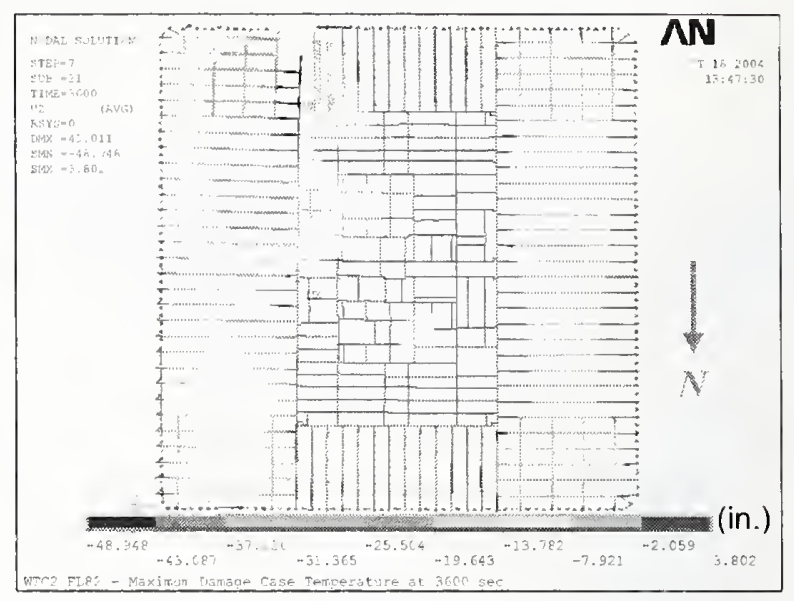

(b) Top view without slab

Figure A-43. Vertical displacement of WTC 2 Floor 82 for Case $D_{i}$ temperature condition at $60 \mathrm{~min}$ (downward displacement is negative; $5 x$ displacement magnification).

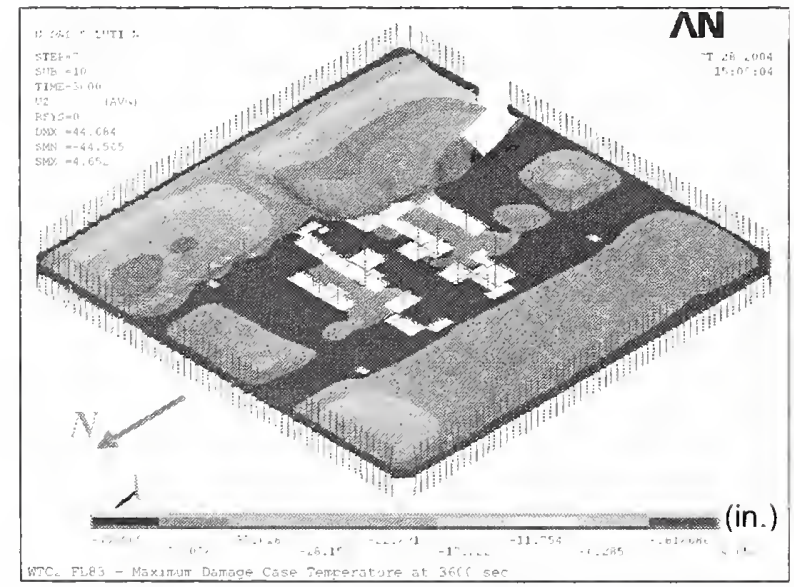

(a) Entire model

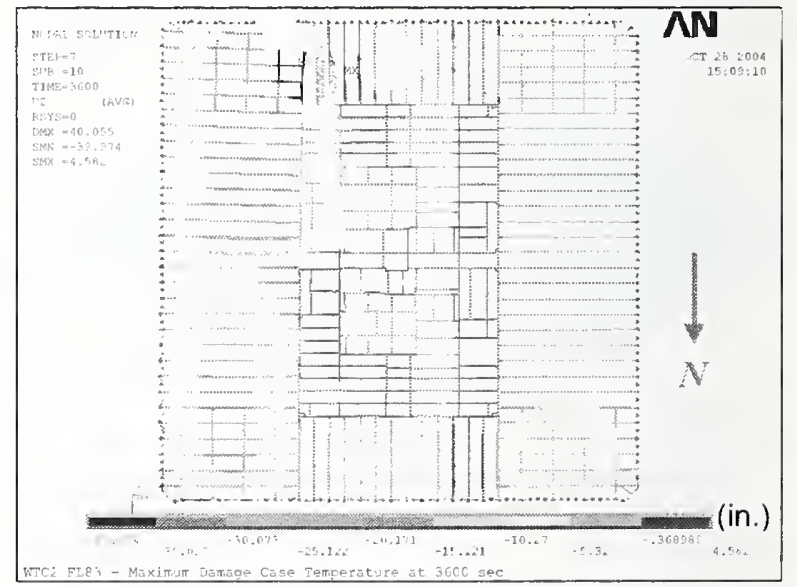

(b) Top view without slab

Figure A-44. Vertical displacement of WTC 2 Floor 83 for Case $D_{i}$ temperature condition at $60 \mathrm{~min}$ (downward displacement is negative; 5x displacement magnification). 


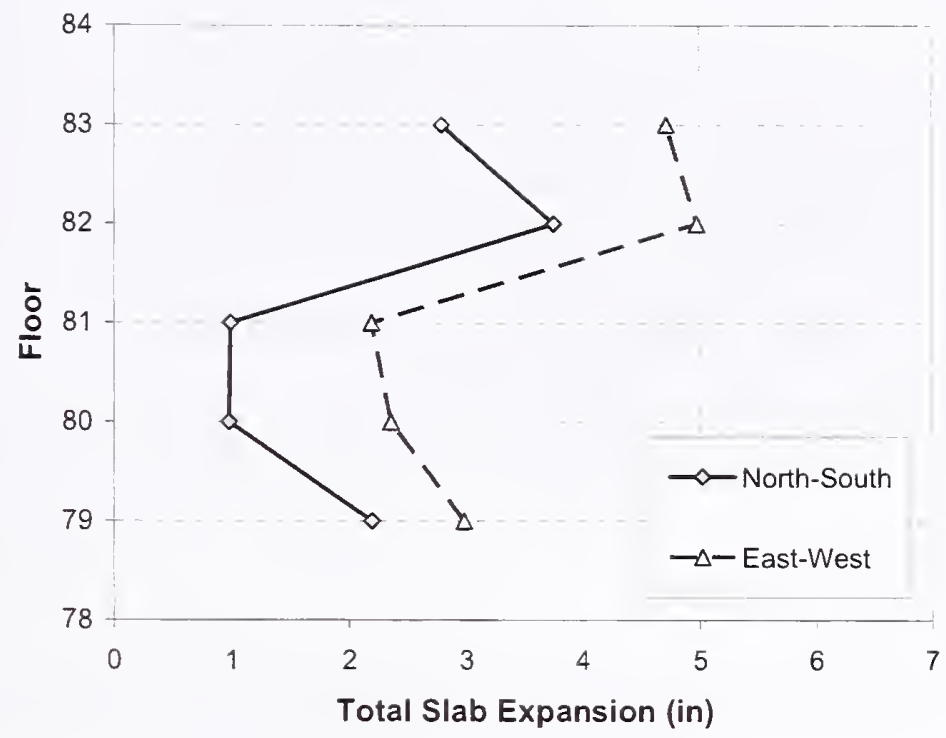

Figure A-45. Thermal expansion of floors of WTC 2 for Case $D_{i}$ temperature condition at $60 \mathrm{~min}$.

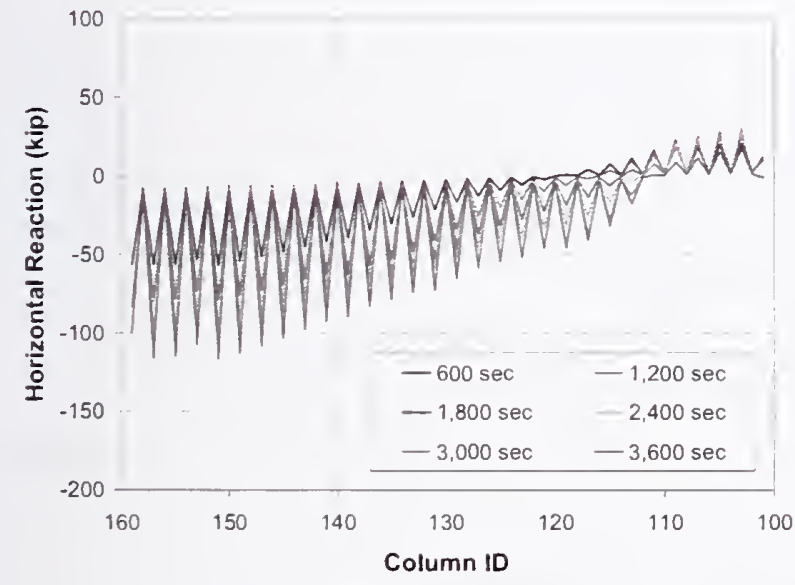

(a) West face

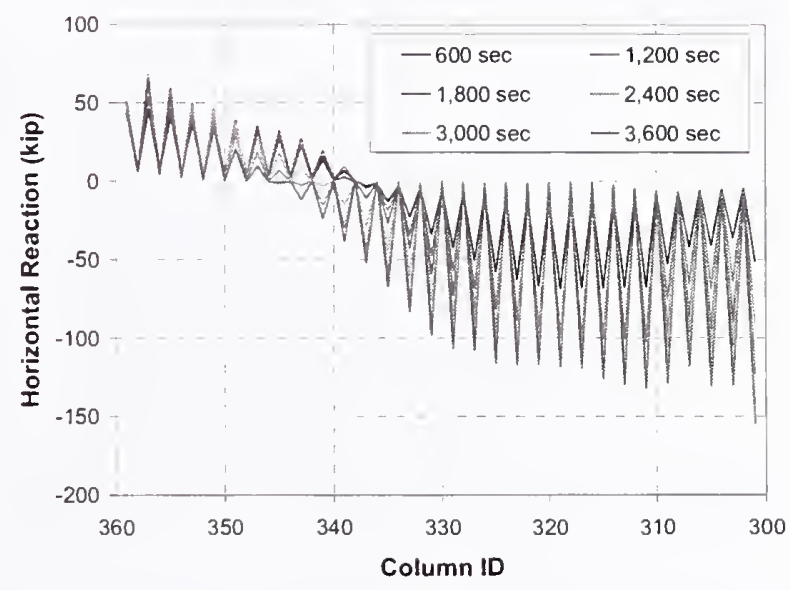

(b) East face

Figure A-46. Horizontal reaction force per column of WTC 2 Floor 80 for Case $D_{i}$ temperature condition. 


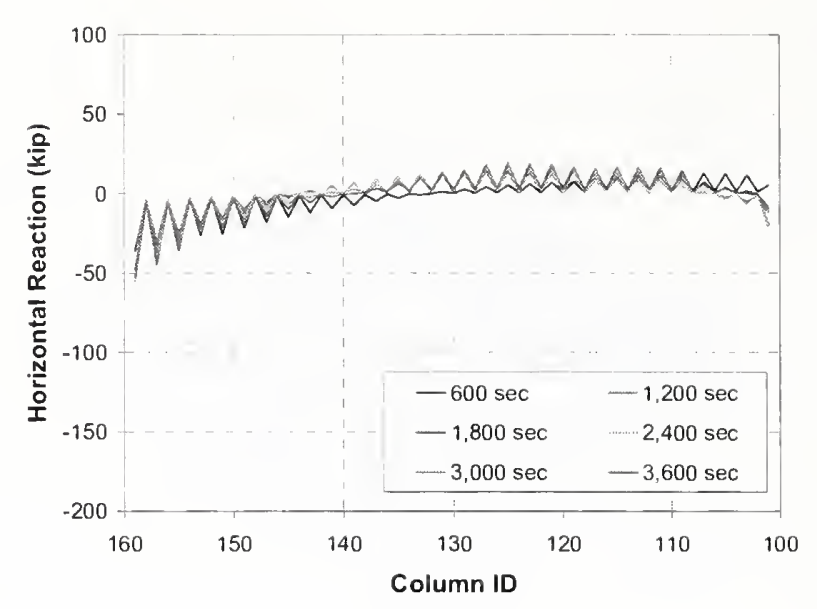

(a) West face

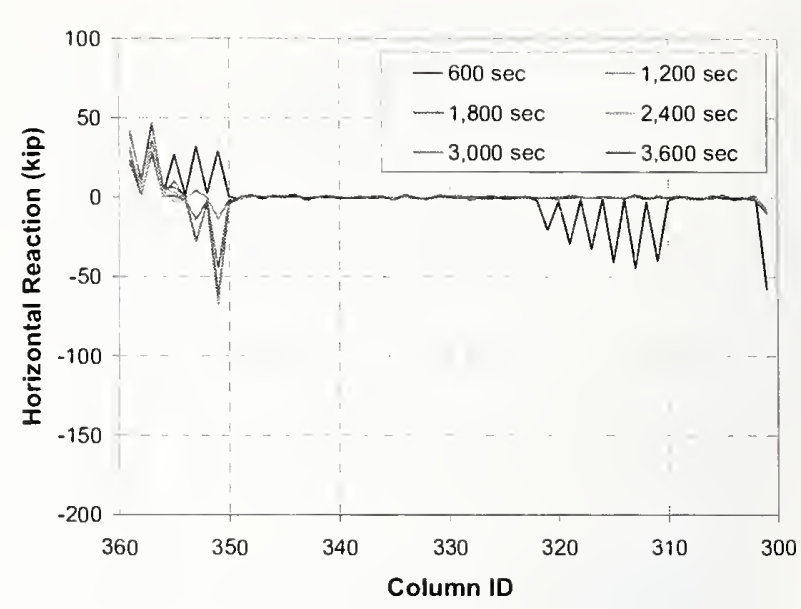

(b) East face

\section{Figure A-47. Horizontal reaction force per column of WTC 2 Floor 82 for Case $D_{i}$ temperature condition.}

\section{A.4 SUMMARY OF RESULTS}

Figures $\mathrm{A}-48$ and $\mathrm{A}-49$ show conditions of the connections between the exterior wall and the floors predicted by the WTC 1 floor models at $100 \mathrm{~min}$ for Case $\mathrm{A}_{i}$ and Case $\mathrm{B}_{1}$ temperature conditions, and Figs. A-50 and A-51 show conditions of the connections between the exterior wall and the floors predicted by the WTC 2 floor models at $60 \mathrm{~min}$ for Case $C_{i}$ and $C$ ase $D_{i}$ temperature conditions. The figures show the following conditions:

- Condition 1: gusset platc failure + seat failure due to vertical shear (loss of vertical support)

- Condition 2: gusset plate failure + bolt shear-off + truss walk-off (loss of vertical support)

- Condition 3: gusset plate failure + bolt shear-off + significant displacement $(>25$ in. $)$ of the floor in that area (floor remains vertically supported)

- Condition 4: tensile force between the exterior wall and the floor system (floor remains vertically connected, but exerts pull-in force on the exterior wall)

Conditions 1 and 2 were treated as the case of floor/wall disconnections. Conditions 3 and 4 were treated as the case where the floor pulled in the exterior wall.

The behaviors of the floor system found in the full floor models subjected to impact damage and elevated temperatures from the fires can be summarized as follows:

- Bridging trusses subjected to elevated temperatures buckled between primary trusses.

- When significant differences in thermal expansion of floors and exterior walls in the direction transverse to the axes of primary trusses occurred near the corners, studs, diagonal strap anchors, gusset plates, and seat bolts at exterior truss seats failed due to the lateral shear.

- Web diagonals of floor trusses with damaged thermal insulation buckled. 
- Floors sagged into catenary forms.

- Truss seats disconnected from the exterior walls.

Pull-in forces were expected to develop whenever the floor sagged. Although the floor sagging was captured by the floor models in the heated area, the pull-in force on the exterior columns was not captured in most of the full floor model analyses. To accurately calculate pull-in forces between the floor and the exterior columns in the full floor model, much more detailed modeling would be required. Such modeling includes accurate boundary conditions on columns, creep in steel, friction at the truss seats, accurate evaluation of failure of strap anchors and studs, and concrete cracking and spalling. In addition, temperature time histories that were used in the full floor model analyses may be different if improved estimates of impact damage to insulation were obtained. Further discussion on the pull-in force can be found in Section 2.5.2 of this report. 

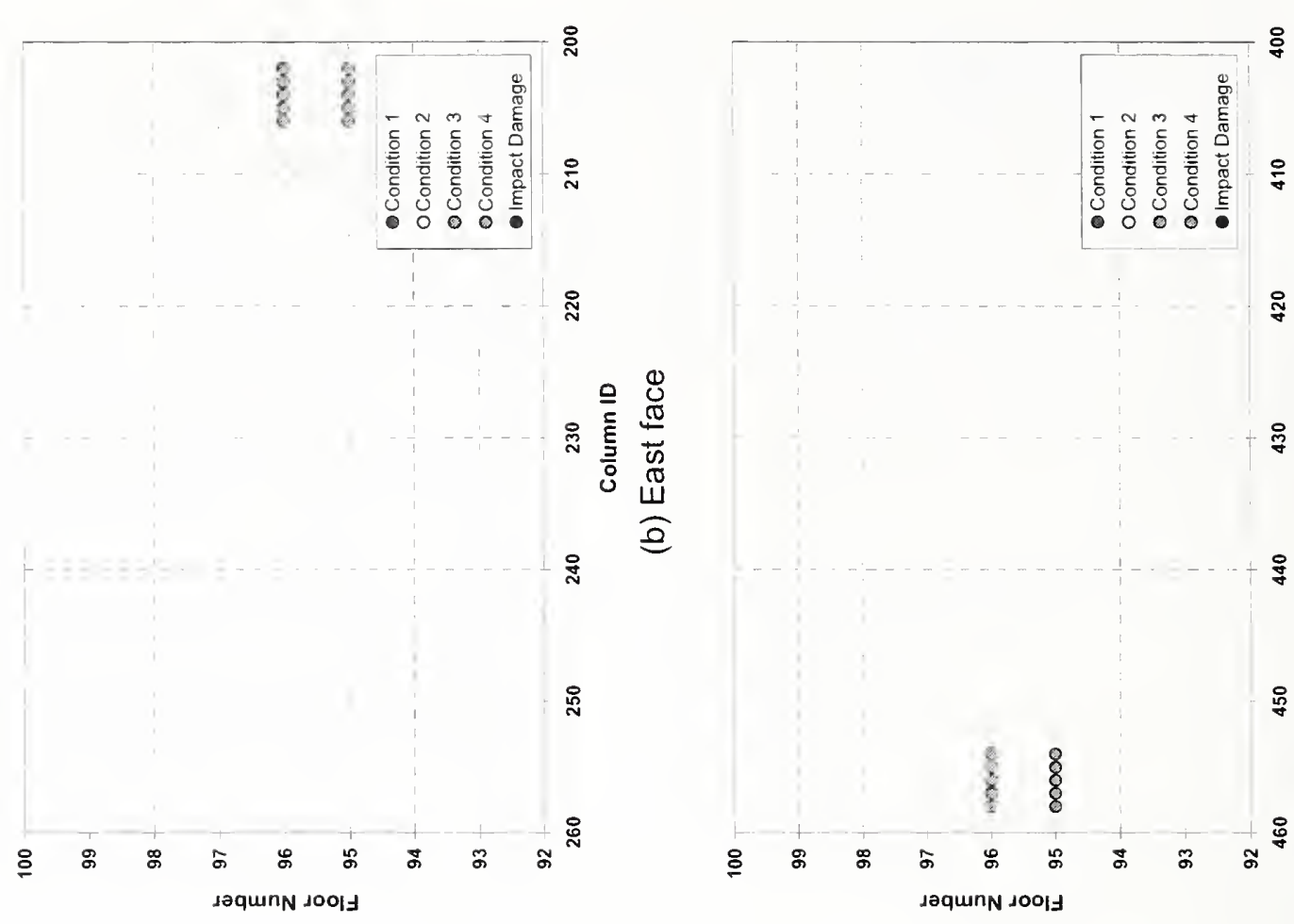

$\bar{x}$

월

$\frac{10}{2}$

:

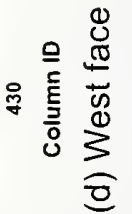

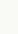

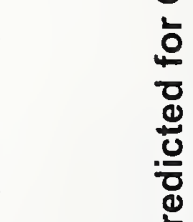

을
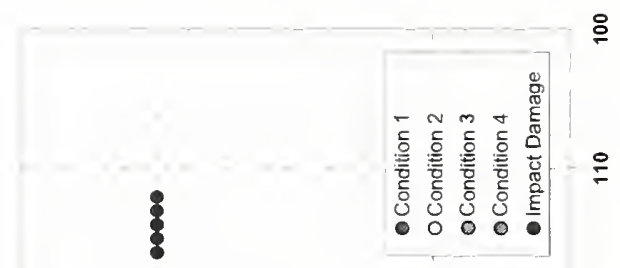

$\stackrel{8}{\circ}$

8

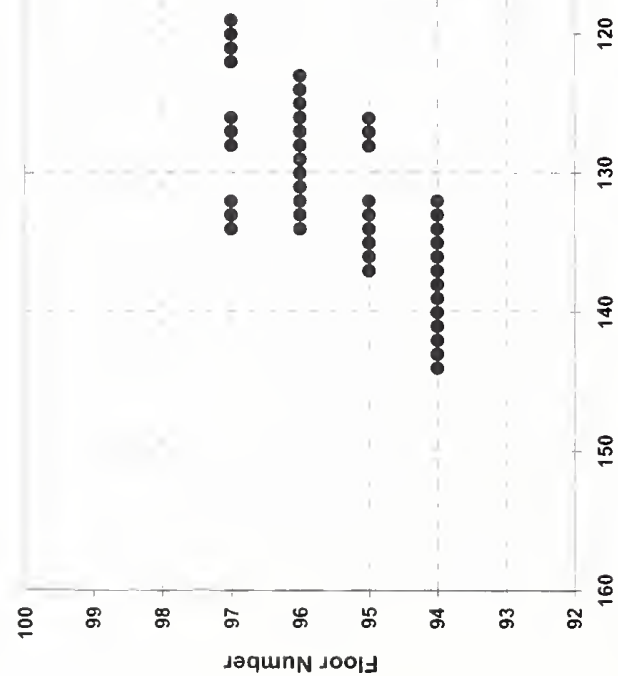

$\cong$

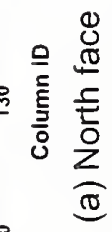

$\frac{1}{2}$

$\stackrel{8}{\circ}$

$\stackrel{8}{\circ}$

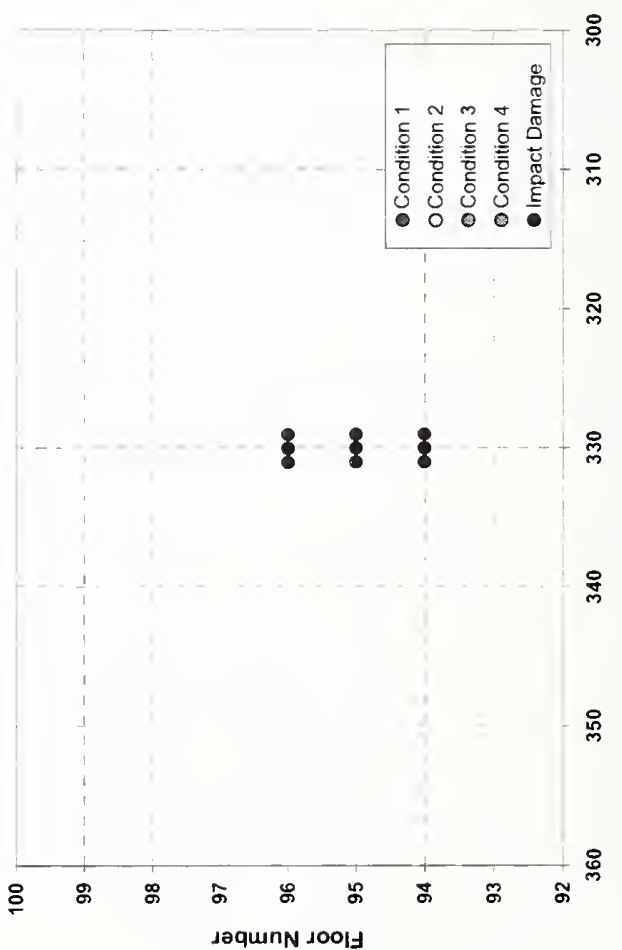

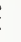

응

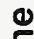

들

응

उั

으동

这

(1)

ᄃ

든

ఏ

这

อ ํㅡㄹ

o

ธิ

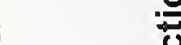

ग

응

章吉

ฮิ

ن

$\stackrel{\infty}{+}$

ํํํ 


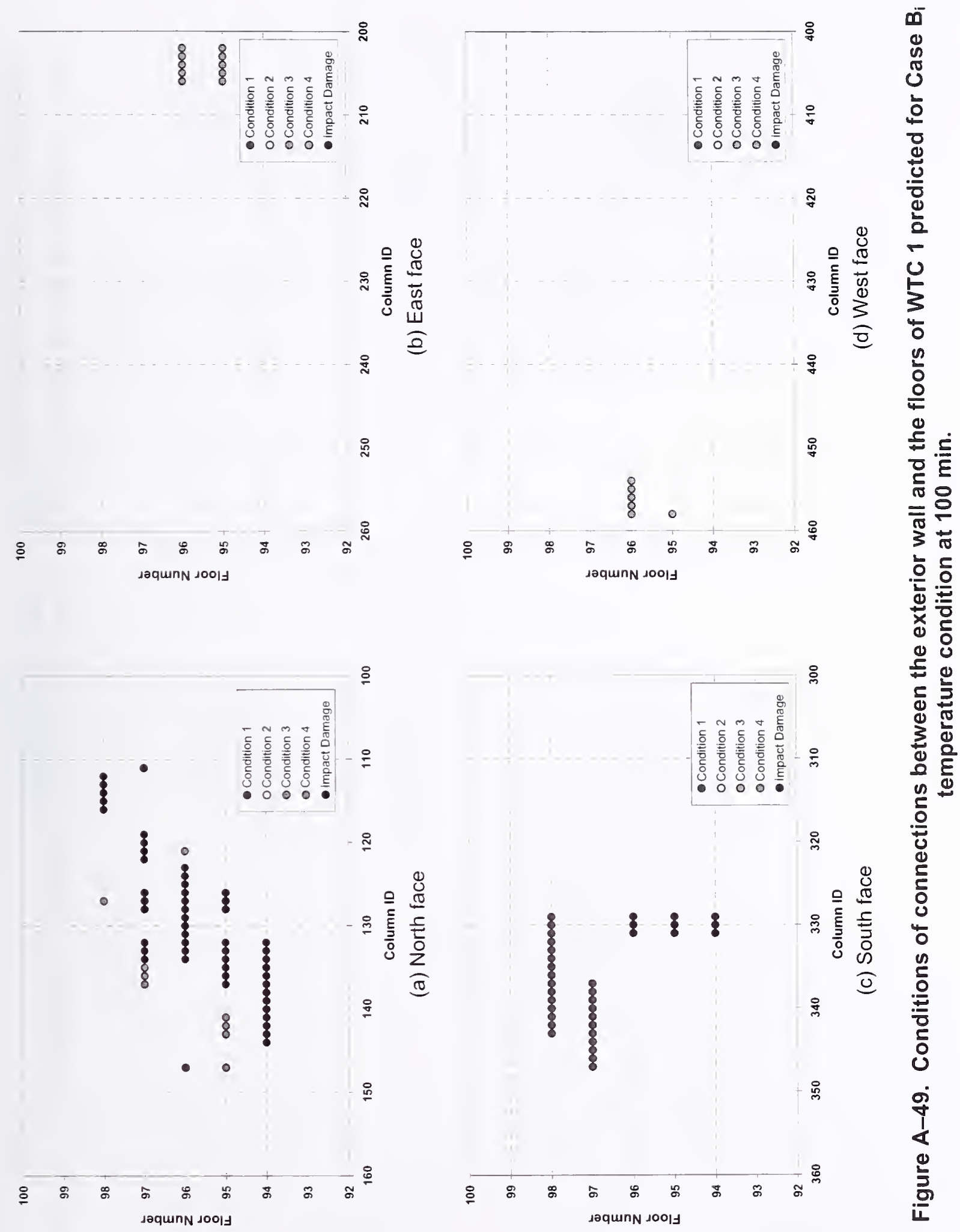



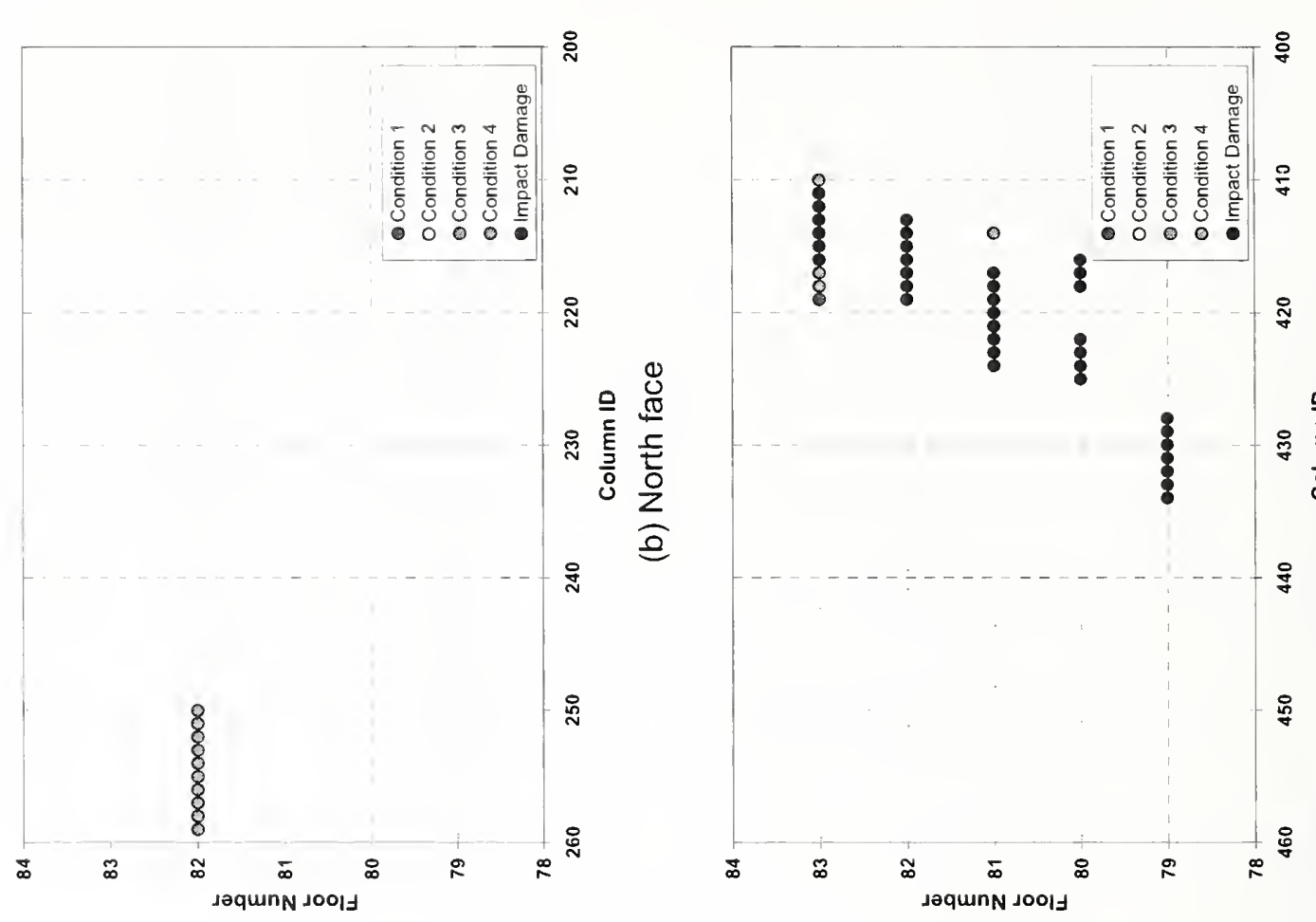

u

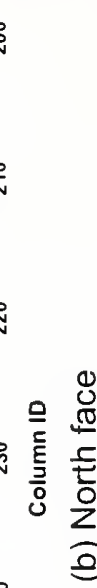

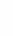

q

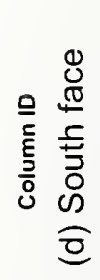

ธุ้

ก

$\frac{0}{5}$

응

융
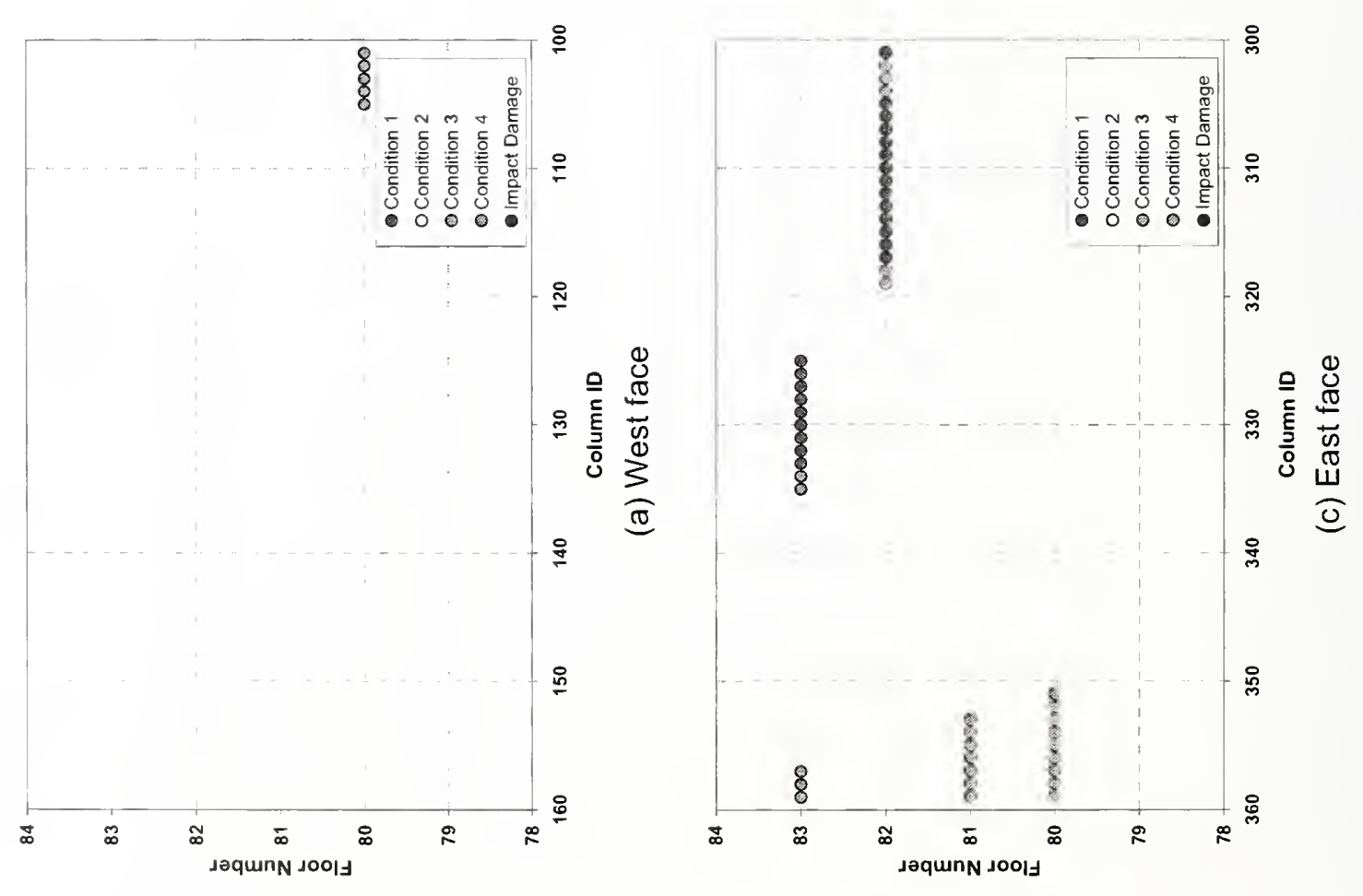

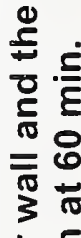

는 은 

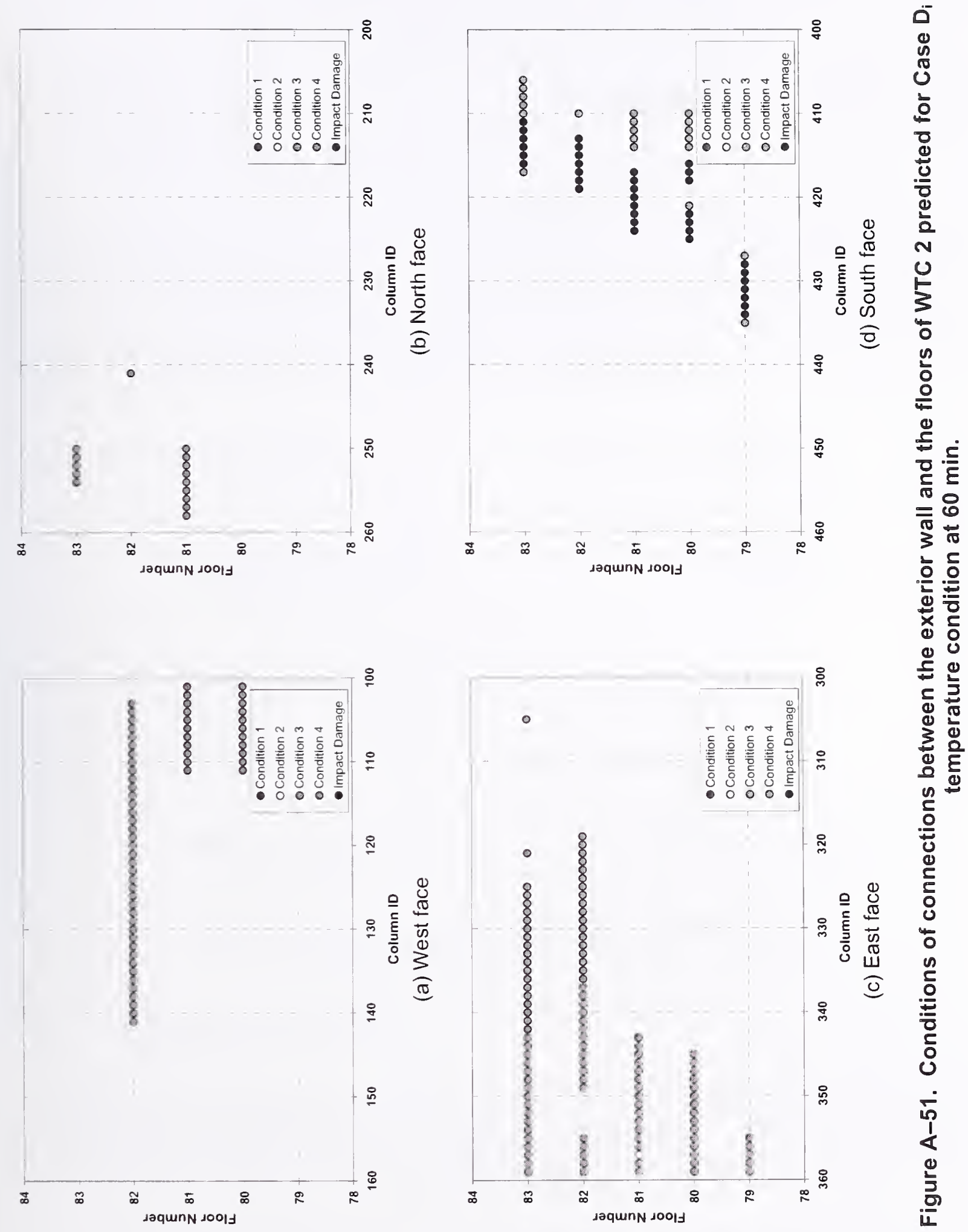

告 
This page intentionally left blank. 


\section{Appendix B \\ FLOOR TRUSS DYNAMIC RESPONSE \\ DUE TO IMPACT OF DROPPING FLOOR}

\section{B.1 IMPACT OF DROPPING FLOOR}

The failure of dropping floor may occur due to thermal and/or additional debris weight on the truss, and/or as a result of the aircraft impact. A floor truss or a group of floor trusses could lose support at both the exterior and interior supporting ends and drop onto the floor below. This failure mode, which is shown in Fig. B-1, will be referred to as full truss drop. Alternatively, a floor truss or a group of floor trusses could lose support on one side and drop down to impact the target floor below. This failure mode, which is also shown in Fig. B-1, will be referred to as partial truss drop.

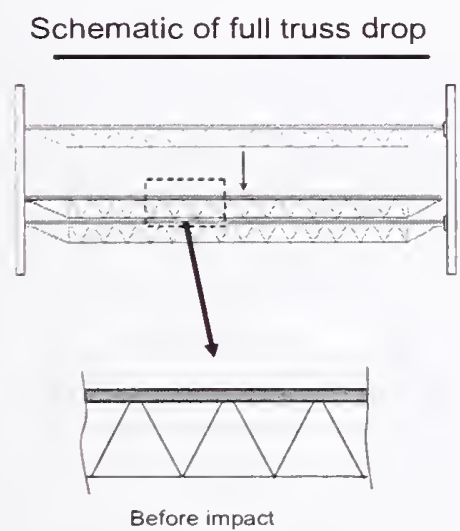

Before impact

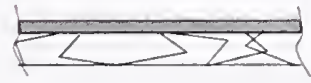

After impact
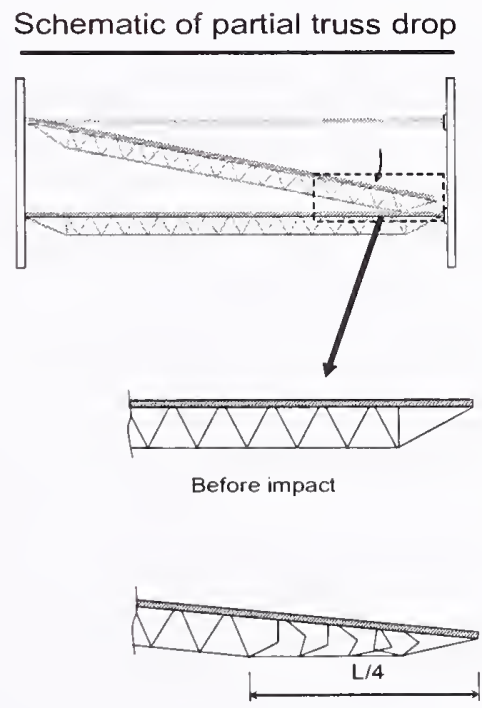

After impact

Figure B-1. Schematic of full truss or partial truss drop and web diagonal crushing at impact.

\section{B.2 PURPOSE AND SCOPE}

The purpose of this study is to determine the dynamic response of the target truss from the impact of full and partial truss drop, to determine whether the target truss seats can resist such an impact load, and to determine whether the target truss will lose its composite action, sag significantly, and consequently fail to restrain the exterior column to which it is connected against instability. 


\section{B.3 METHOD OF ANALYSIS}

The simulation of a floor drop is idealized with a truss drop. This has inherent assumptions that all seats for the floor fail simultaneously to cause a full or partial drop. The dynamic response of the target truss from the impact of a dropping truss is calculated using conservation of energy principle. The potential energy of the truss just before drop, which is a function of drop height, converts to the kinetic energy of the truss just before impact. As the dropping truss starts to impact the target truss, the web diagonal members of the dropping truss are assumed to deform plastically to absorb some of the kinetic energy. The energy absorption due to crushing of the furniture and partitions are neglected in this study. The energy absorption due to web diagonal member crushing reduces the kinetic energy available at impact to deform the target truss. All the web diagonal members are assumed to deform plastically for the full truss drop case, while only one quarter of the web diagonal members are assumed to deform plastically for partial truss drop, representing one quarter of the length of the truss that may come in contact at impact with the floor below. The kinetic energy loss at the time of impact of the dropping truss and the target truss is calculated based on conservation of momentum. The two trusses are assumed to travel together after the impact, at one-half of the velocity of the dropping floor before impact.

The dynamic load due to the impact of the dropping truss onto the target truss will cause the target truss to deform plastically beyond the static load due to the weight of the two trusses. The maximum dynamic deformation of the trusses is calculated by conservation of energy principle assuming that the resistance of the truss is a bilinear function of displaccment. This assumption is based on fitting the accelerationdeflection relationship of target truss, calculated with finite element analysis, as shown in Fig. B-2.

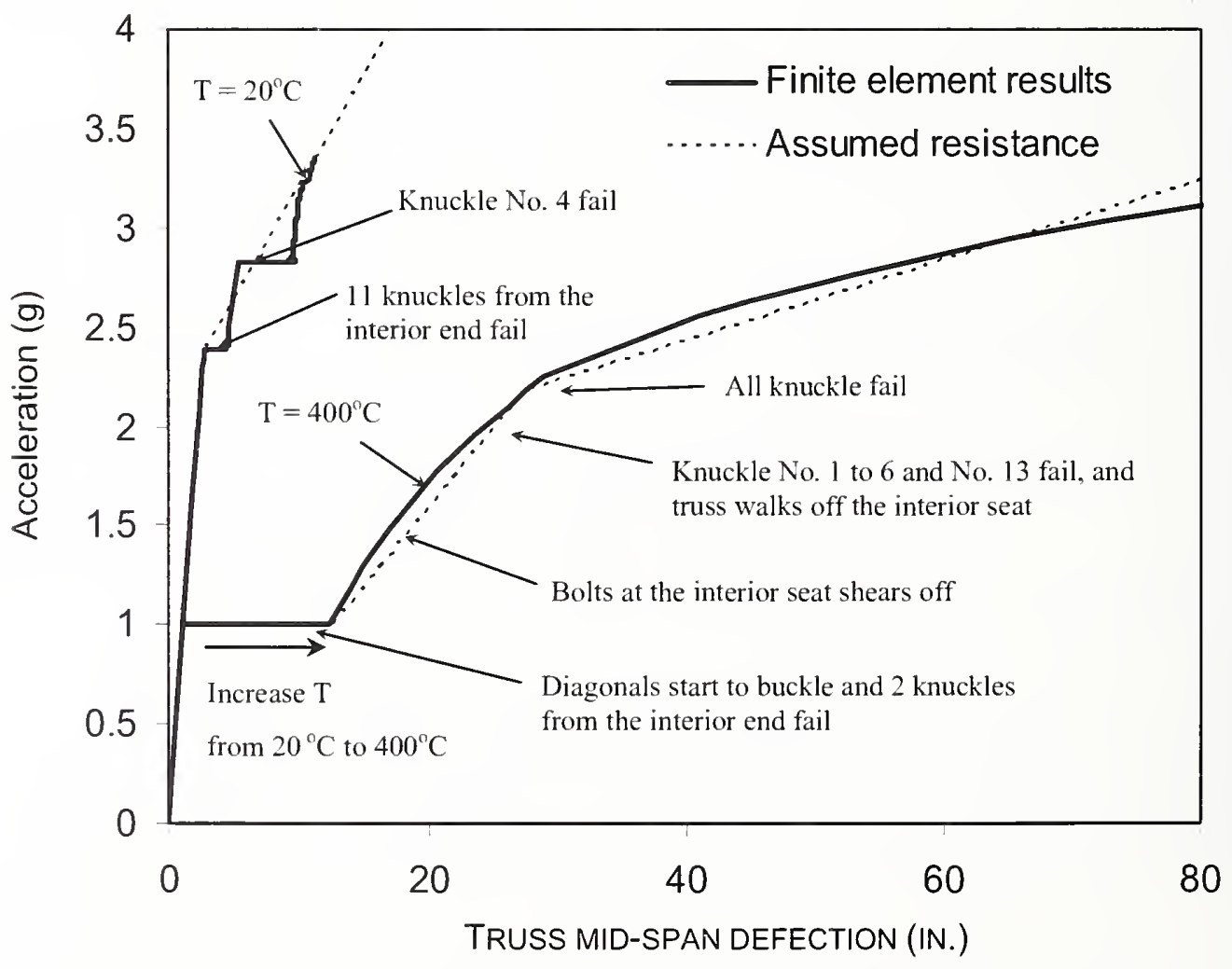

Figure B-2. Target truss resistance against increasing acceleration. 


\section{B.4 RESULTS}

The ratios of demand-to-seat capacity for the gravity loads of the dropped and impacted trusses moving together for temperatures of $20^{\circ} \mathrm{C}, 400{ }^{\circ} \mathrm{C}, 600{ }^{\circ} \mathrm{C}$, and $700{ }^{\circ} \mathrm{C}$, and the gravity plus dynamic impact loads for temperatures of $20^{\circ} \mathrm{C}$ and $400{ }^{\circ} \mathrm{C}$, are calculated. A demand-to-capacity ratio of less than one shows that the truss seat has sufficient capacity to resist the load, and a demand-to-capacity ratio of larger than one implies that the seat could fail. The range of the demand-to-capacity ratios is due to the different assumptions for the amount of energy loss due to crushing of the web diagonal members of the dropped truss.

The demand-to-capacity ratio of the long-span truss for gravity loads is shown in Table B-1 and for gravity plus impact loads is shown in Table B-2. The result for gravity loads alone shows the both the exterior and interior truss seats have sufficient capacity to support the weight of two floors for all temperatures considered. The result for gravity plus impact loads shows that at temperatures below $400{ }^{\circ} \mathrm{C}$ neither the exterior nor interior truss seats is expected to fail. Peak deflection response due to gravity and the dynamic impact of the dropping truss is given in Table B-3. The results show that at room temperature, and more so at $400{ }^{\circ} \mathrm{C}$, the impacted truss will deflect to an extent that it loses composite action and becomes a catenary. At $400{ }^{\circ} \mathrm{C}$ the truss walks off the interior seat. Obviously, a catenary truss is not able to restrain the exterior column against transverse movement and cannot restrain it from instability. Although a truss response to increasing acceleration at $700{ }^{\circ} \mathrm{C}$ was not developed, the strength reduction of the truss seats clearly indicates that the failure of truss seats will occur.

Table B-1. Demand-to-capacity ratio of long-span truss for static gravity load.

\begin{tabular}{|c|c|c|c|c|c|}
\hline \multirow{2}{*}{$\begin{array}{c}\text { Temp. } \\
\left({ }^{\circ} \mathrm{C}\right)\end{array}$} & \multirow{2}{*}{$\begin{array}{c}\text { Demand } \\
\text { (kip) }\end{array}$} & \multicolumn{2}{|c|}{ Capacity (kip) } & \multicolumn{2}{c|}{ Demand/Capacity } \\
\cline { 3 - 6 } & Int. Seat & Ext. Seat & Int. Seat & Ext. Seat \\
\hline 20 & 26.4 & 187.3 & 140.0 & 0.14 & 0.19 \\
\hline 400 & 26.4 & 166.9 & 125.7 & 0.16 & 0.21 \\
\hline 600 & 26.4 & 81.6 & 77.8 & 0.32 & 0.34 \\
\hline 700 & 26.4 & 37.2 & 35.5 & 0.71 & 0.74 \\
\hline
\end{tabular}

Table B-2. Demand-to-capacity ratio of long-span truss for dynamic impact load from full truss drop.

\begin{tabular}{|c|c|c|c|c|c|c|c|c|c|c|c|}
\hline \multirow{3}{*}{$\begin{array}{c}\text { Temp. } \\
\left({ }^{\circ} \mathrm{C}\right) \\
20\end{array}$} & \multirow{2}{*}{\multicolumn{3}{|c|}{ Demand (kip) }} & \multicolumn{2}{|c|}{ Capacity (kip) } & \multicolumn{6}{|c|}{ Demand / Capacity } \\
\hline & & & & \multirow{2}{*}{$\begin{array}{c}\text { Int. Seat } \\
187.3\end{array}$} & \multirow{2}{*}{$\frac{\text { Ext. Seat }}{140.0}$} & \multicolumn{3}{|c|}{ Int. Seat } & \multicolumn{3}{|c|}{ Ext. Seat } \\
\hline & 38.6 & - & 65.3 & & & 0.21 & - & 0.35 & 0.28 & - & 0.47 \\
\hline 400 & 39.1 & - & 45.2 & 166.9 & 125.7 & 0.23 & - & 0.27 & 0.31 & - & 0.36 \\
\hline
\end{tabular}

Table B-3. Peak deflection response due to static gravity and dynamic impact.

\begin{tabular}{|c|c|c|c|c|}
\hline $\begin{array}{c}\text { Temp. } \\
\text { ('C) }\end{array}$ & $\begin{array}{c}\text { Static } \\
\text { Deflection (in.) }\end{array}$ & \multicolumn{3}{|c|}{$\begin{array}{c}\text { Dynamic } \\
\text { Deflection (in.) }\end{array}$} \\
\hline 20 & 2.3 & 7.6 & - & 25.4 \\
\hline 400 & 24.2 & 66.4 & - & 89.6 \\
\hline
\end{tabular}




\section{B.5 CONCLUSIONS}

At room temperature, the impact of a dropping truss will not cause failure of truss seats, but will cause the impacted truss to deform into a catenary. At $400{ }^{\circ} \mathrm{C}$, the impacted truss will walk off the interior seat. In either case the impacted floor will not restrain the exterior column against transverse movement and instability. The impact of a dropping truss at $700{ }^{\circ} \mathrm{C}$ will cause failure of truss seats. 


\section{Appendix C \\ Global Analysis Without Creep}

The analysis results presented in this Appendix were obtained from the preliminary global analyses that were performed neglecting the effects of creep and inelastic buckling of columns. These analyses were primarily used to study the interaction between various structural components in the overall global response of the towers. Because of the significant role that creep played in the collapse process, the results from these analyses are presented without making any conclusive remarks about the collapse sequence of the towers.

\section{C.1 WTC 1 GLOBAL ANALYSIS WITHOUT CREEP}

The WTC 1 ANSYS model without creep was analyzed with Case $A_{i}$ structural damage condition and subjected to Case $\mathrm{A}_{\mathrm{i}}$ temperature time histories. Temperature-dependent plasticity and the nonlinear geometry were the major sources of nonlinearity in the model. Table $\mathrm{C}-1$ summarizes the sequence of analyses that were conducted with the WTC 1 ANSYS model with Case $A_{i}$ conditions (see Section 2.2 for Case $A_{i}$ structural damage condition). The results of each analysis step were used as the initial conditions for the next. Case $\mathrm{B}_{\mathrm{i}}$ was not considered for preliminary global analysis, as the lack of creep and inelastic buckling behaviors limited the usefulness of repeating this analysis.

\section{Table C-1. Analysis Steps of WTC 1 ANSYS global model for Case $A_{i}$ conditions.}

\begin{tabular}{|c|l|}
\hline $\begin{array}{c}\text { Analysis } \\
\text { Step }\end{array}$ & \multicolumn{1}{|c|}{ Description } \\
\hline 1 & WTC 1 model below Floor 106 was analyzed under its own self-weight. \\
\hline 2 & $\begin{array}{l}\text { Structures above Floor } 106 \text { were added in a stress-free state, and the model was analyzed for dead } \\
\text { load including those above Floor } 106 .\end{array}$ \\
\hline 3 & Superimposed dead load and 25 percent of the design live loads were superimposed on the model. \\
\hline 4 & $\begin{array}{l}\text { Columns, spandrels, and floor elements that were severed during aircraft impact were removed, } \\
\text { and the model was analyzed. }\end{array}$ \\
\hline 5 & Column and spandrel temperatures were ramped up linearly to temperatures at 10 min. \\
\hline 6 & Column and spandrel temperatures were changed linearly from temperatures at 10 min to 20 min. \\
\hline 7 & Column and spandrel temperatures were changed linearly from temperatures at 20 min to 30 min. \\
\hline 8 & Column and spandrel temperatures were changed linearly from temperatures at 30 min to 50 min. \\
\hline 9 & Column and spandrel temperatures were changed linearly from temperatures at 50 min to 100 min. \\
\hline
\end{tabular}

The model was first analyzed to capture the effects of the construction sequence (analysis steps 1, 2, and 3). The results of analysis for Step 3 represent the state before the aircraft impact. In analysis step 4, the columns, spandrels, and floor elements that were severed during the aircraft impact were removed from the model, and the building was reanalyzed to redistribute the load to the non-severed members. The results of this analysis represent the state of the building after the aircraft impact. After load redistribution, the column and spandrel temperatures provided by NIST at $10 \mathrm{~min}$ intervals were applied to the model (analysis steps 5 through 9). Tcmperatures were calculated by linear interpolation for times in between 10 min intervals. In order to reduce computation time, the temperature data sets for which 
temperatures remained approximately linear with time during the interval from the previous and the following temperature data sets were eliminated from the analyses. Based on this elimination, temperature analyses were performed at $10 \mathrm{~min}, 20 \mathrm{~min}, 30 \mathrm{~min}, 50 \mathrm{~min}$, and $100 \mathrm{~min}$. Temperatures were not applied to the floor elements to prevent unrealistic buckling of floors. As discussed earlier, the floors in the global models did not include the floor trusses and were modeled by plate elements to match their membrane stiffncss; the bending stiffness of the floors was not modeled accurately.

The results of the WTC 1 global model analyses for Case $\mathrm{A}_{\mathrm{i}}$ conditions are summarized in Figs. C-1 through C -9 .

Figure $\mathrm{C}-1$ shows the total displacement at different analysis steps. The maximum displacement was obtained right after aircraft impact and was equal to $4.9 \mathrm{in}$. This displacement was gradually reduced to $4.1 \mathrm{in}$. at the end of $100 \mathrm{~min}$ due to thermal expansion of core and exterior columns between Floor 93 and Floor 98. Figures $\mathrm{C}-2$ and $\mathrm{C}-3$ show the total displacements for north, south, and east exterior walls before and after aircraft impact and at $50 \mathrm{~min}$ and $100 \mathrm{~min}$. The total displacements for the west exterior wall are not shown as they were similar to the east cxterior wall. The displacements were typically at their maximum right after aircraft impact around the hat truss and gradually decreased with time, reaching their minimum value at $50 \mathrm{~min}$ to $100 \mathrm{~min}$ in the aircraft damage zone.

Figures $\mathrm{C}-4$ and $\mathrm{C}-5$ show the axial load variations over north, south, and east exterior wall columns and spandrels before and after aircraft impact and at $50 \mathrm{~min}$ and $100 \mathrm{~min}$. After the aircraft impact, the loads carried by the severed columns of the north wall were primarily redistributed to the remaining columns of the north wall and also to the core and other exterior walls. As a result of this redistribution, the maximum column load incrcased from $265 \mathrm{kip}$ to $1,200 \mathrm{kip}$ on the north exterior wall and from $268 \mathrm{kip}$ to $380 \mathrm{kip}$ on the south exterior wall. The 1,200 kip load occurred in a column adjacent to the impact damage area. The column loads on the east and west walls slightly increased in the south side of the walls. With increasing time, the maximum column load in the south wall increased from 380 kip after aircraft impact to $670 \mathrm{kip}$ at the end of $100 \mathrm{~min}$. This increase was primarily due to the steady increase of column temperatures on the south wall.

Figure C -6 shows the total column loads in the core columns and the north, south, east, and west exterior walls for several floors at the end of each analysis step. Due to the removal of severed columns, spandrels, and floor elements, the total column loads below Floor 98 after aircraft impact did not equal the total column loads before the aircraft impact.

After the aircraft impact, the total load in core columns increased about 1,000 kip, in the east exterior wall columns about $200 \mathrm{kip}$, and in the west exterior wall columns about $150 \mathrm{kip}$, whereas the total load decreased in the north wall columns about 1,150 kip and in the south wall columns about 200 kip. With increasing time, the temperatures in the core and the south exterior wall columns increased more than the other exterior wall columns, causing relative thermal expansion. Relative thermal expansion caused the core and the south exterior wall columns to attract more load, and the exterior walls on the north, east, and west sides to unload. The increase in loads due to thermal expansion of the core columns increased the plastic strains with time and the resulting shortening caused the unloading of the core columns at $30 \mathrm{~min}$. As time approaches $100 \mathrm{~min}$, the temperatures of all columns dropped down, and the effects of thermal expansion decreased. This can be seen in the loads at $100 \mathrm{~min}$, where core columns unloaded and redistributed load to each exterior wall (about 3,500 kip). About 32 percent of the redistributed load was 
transferred to each of the east and west exterior wall columns, and 18 percent to each of the north and south exterior wall columns.

Figures $\mathrm{C}-7 . \mathrm{C}-8$, and $\mathrm{C}-9$ show the ratio of elastic-plus-plastic strain to the temperature-dependent yield strain of the core columns between Floor 96 and Floor 97 before and after aircraft impact and at $10 \mathrm{~min}$, $20 \mathrm{~min}, 50 \mathrm{~min}$, and $100 \mathrm{~min}$. As can be noticed, the elastic-plus-plastic strain in the core columns before aircraft impact was typically 50 percent to 60 percent less than the yield strains at room temperature. After the aircraft impact, except for Column 504, the maximum strain ratio did not get beyond 1.0, indicating that almost all core columns remained in the elastic range. With increasing time and temperatures, the strain ratios started to increase, especially in the west and the south sides of the core. Strain ratios reached their maximum at the end of $50 \mathrm{~min}$ and remained almost constant till the end at about 100 min. Higher strain ratios were clustered around west and south side core columns, indicating a tendency to tilt toward the south and west. 


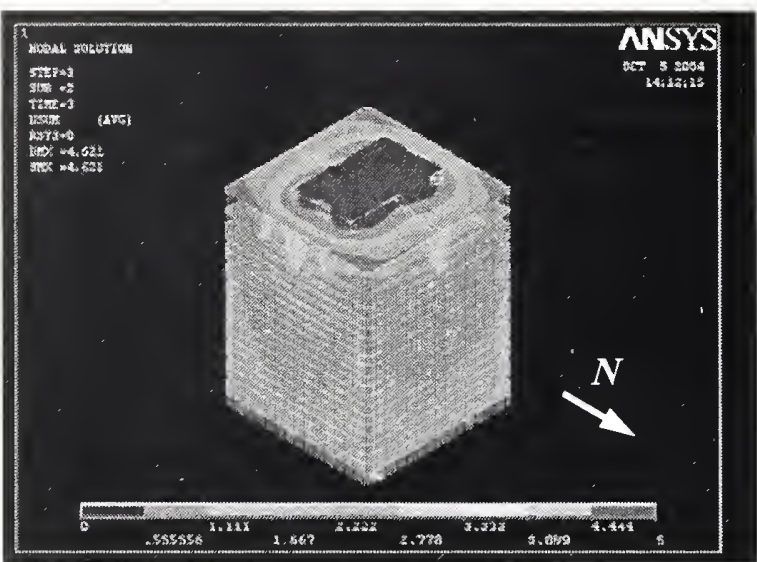

(a) Before impact

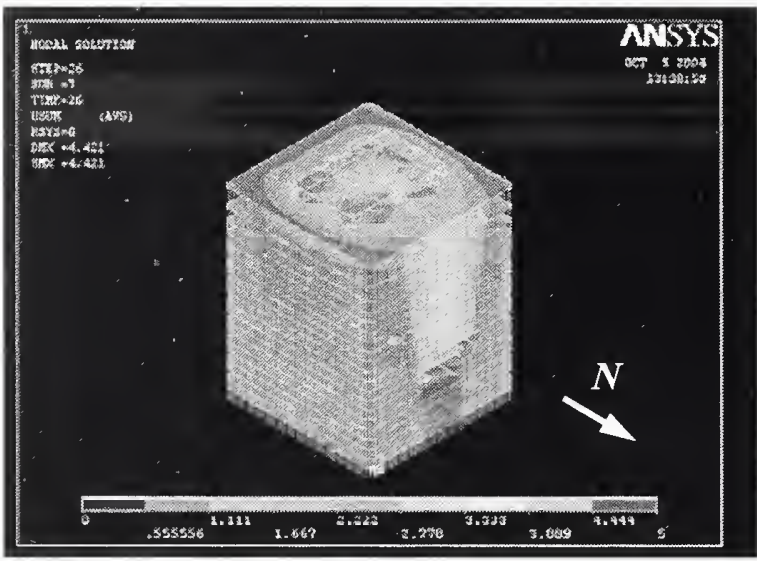

(c) At $10 \mathrm{~min}$

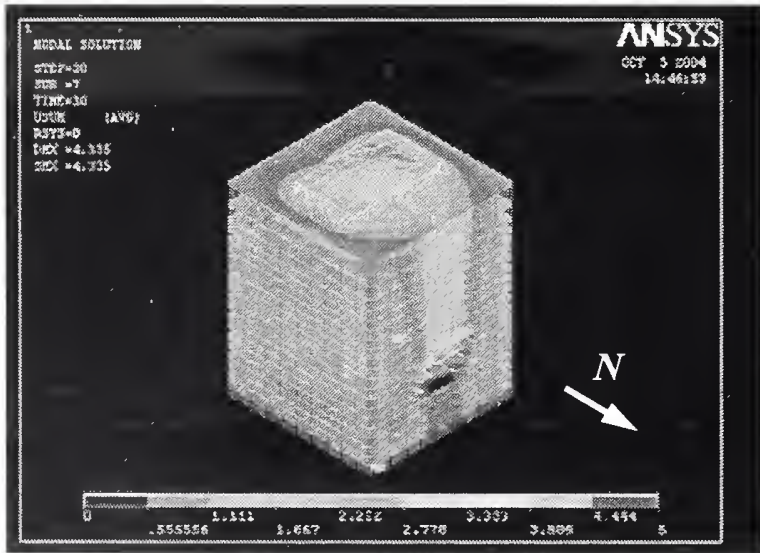

(e) At $50 \mathrm{~min}$

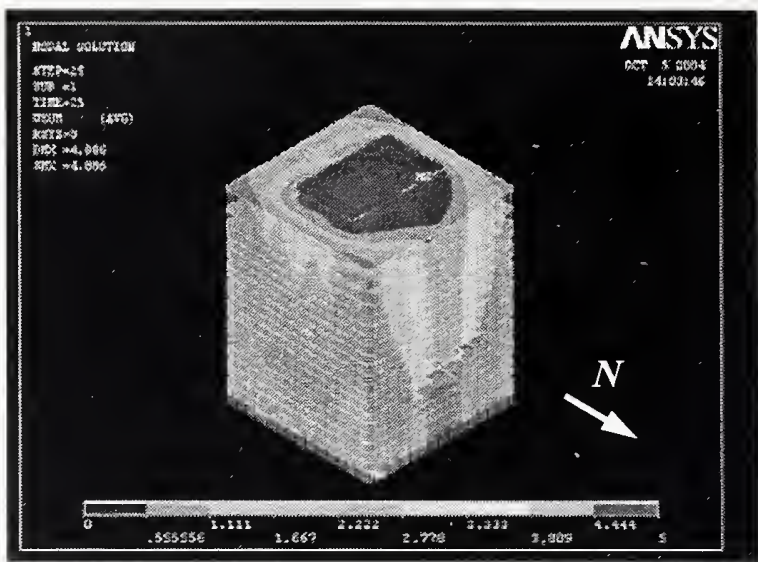

(b) After impact

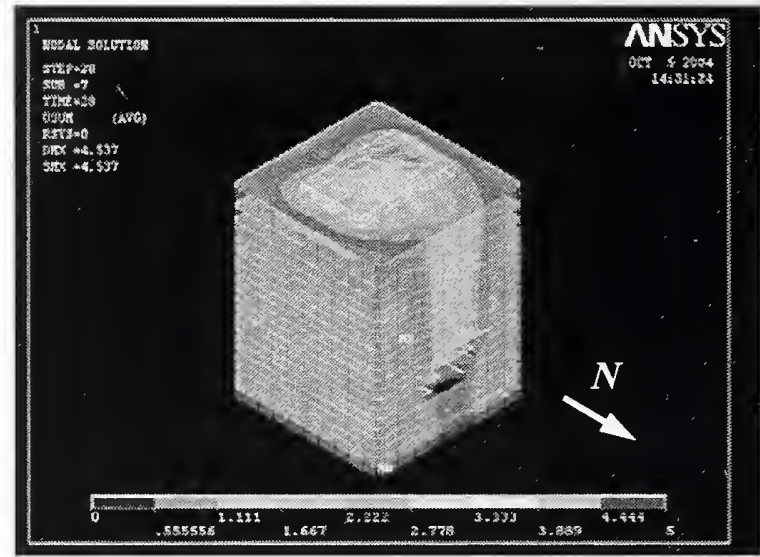

(d) At $30 \mathrm{~min}$

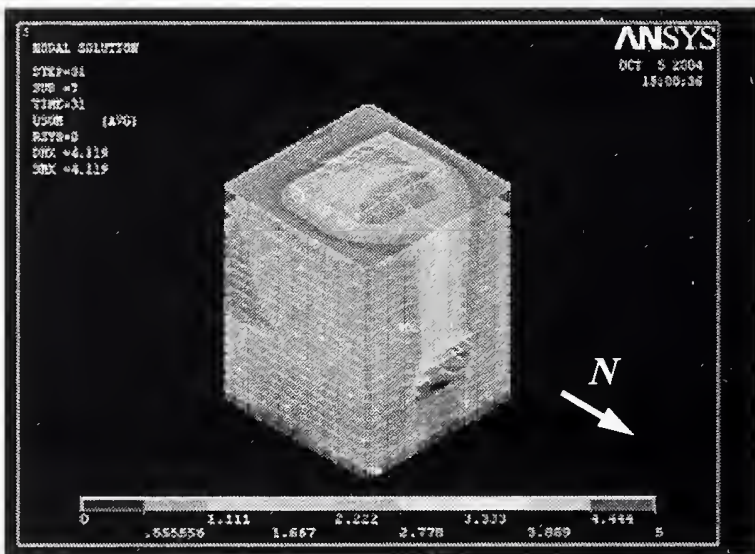

(f) At $100 \mathrm{~min}$

Figure $\mathbf{C}-1$. Total displacement at different stages of WTC 1 for Case $A_{i}$ conditions. 


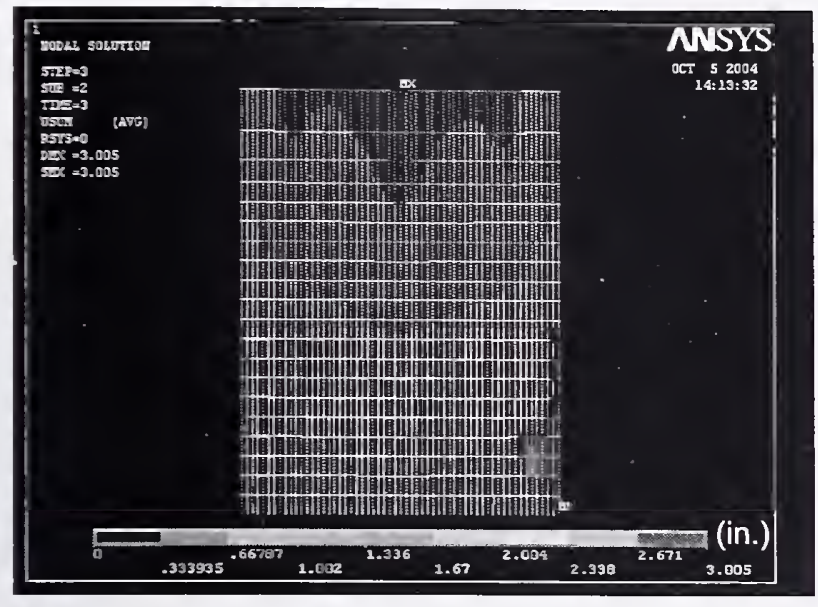

(a) North wall before impact

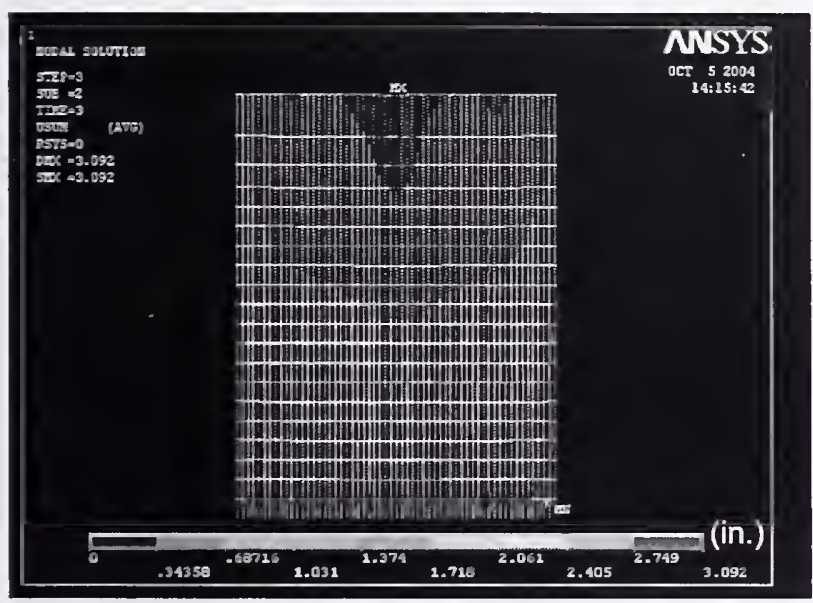

(c) South wall before impact

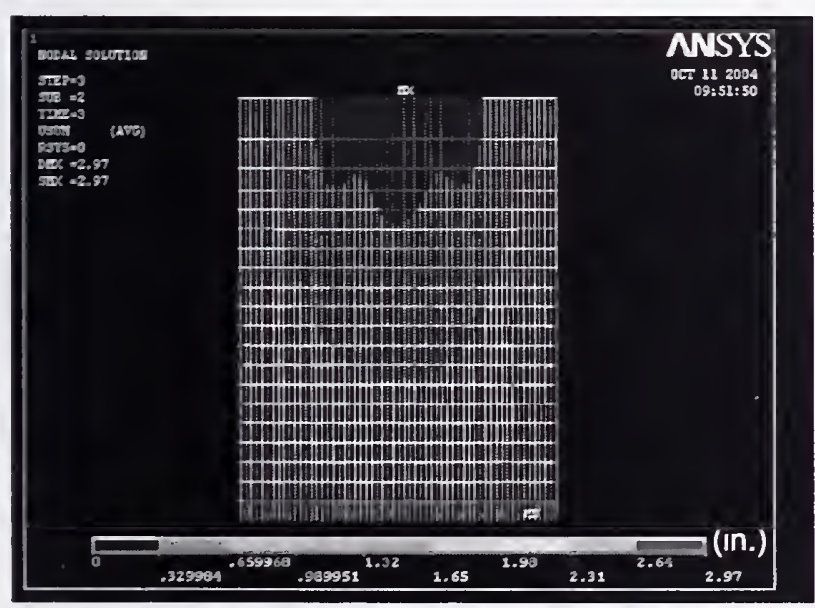

(e) East wall before impact

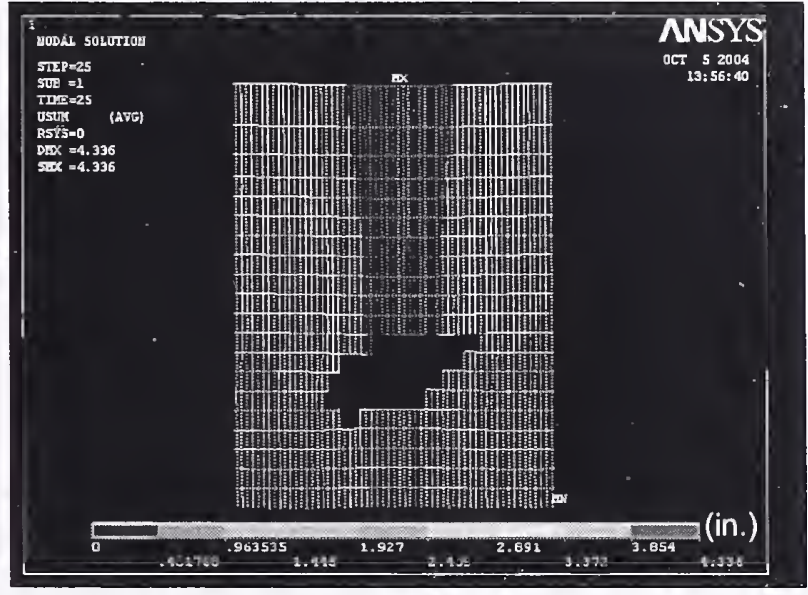

(b) North wall after impact

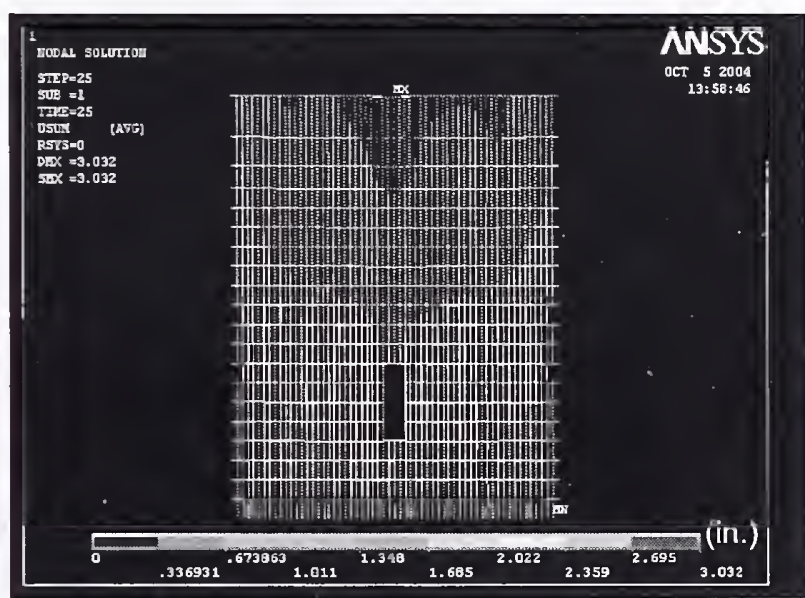

(d) South wall after impact

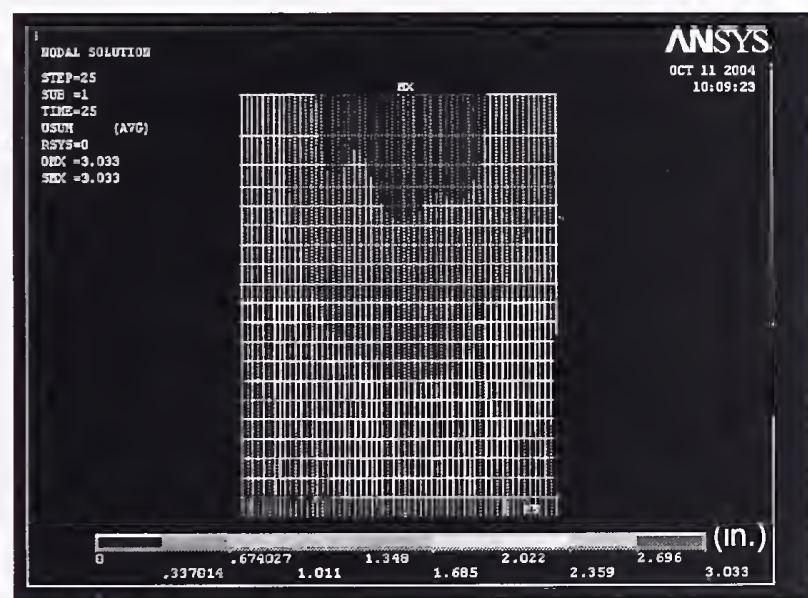

(f) East wall after impact

Figure C-2. Total displacement on the north, south, and east faces of the WTC 1 before and after aircraft impact (looking from outside). 


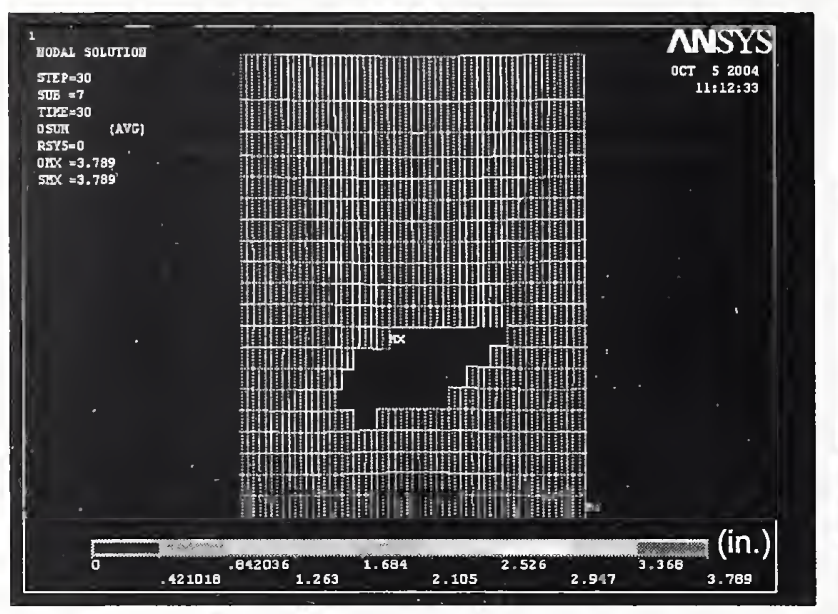

(a) North wall at $50 \mathrm{~min}$

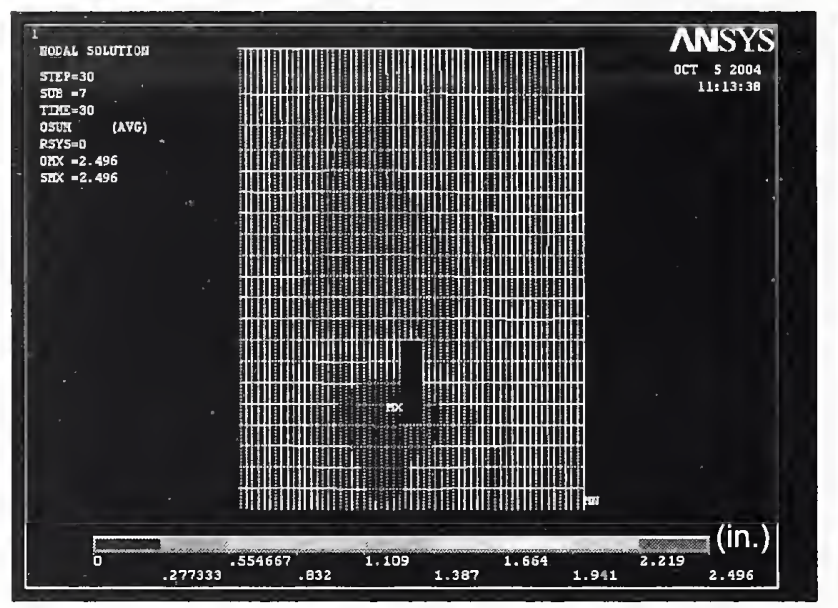

(c) South wall at $50 \mathrm{~min}$

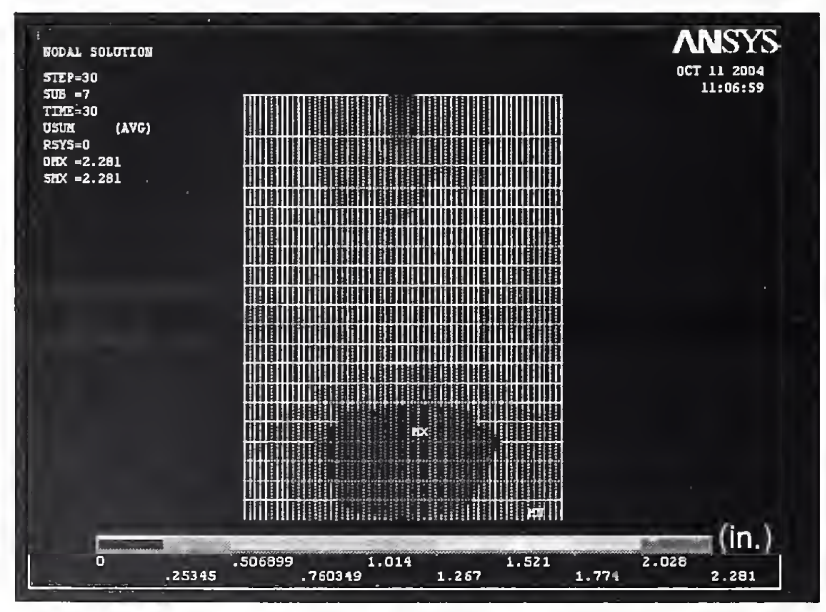

(e) East wall at 50 min

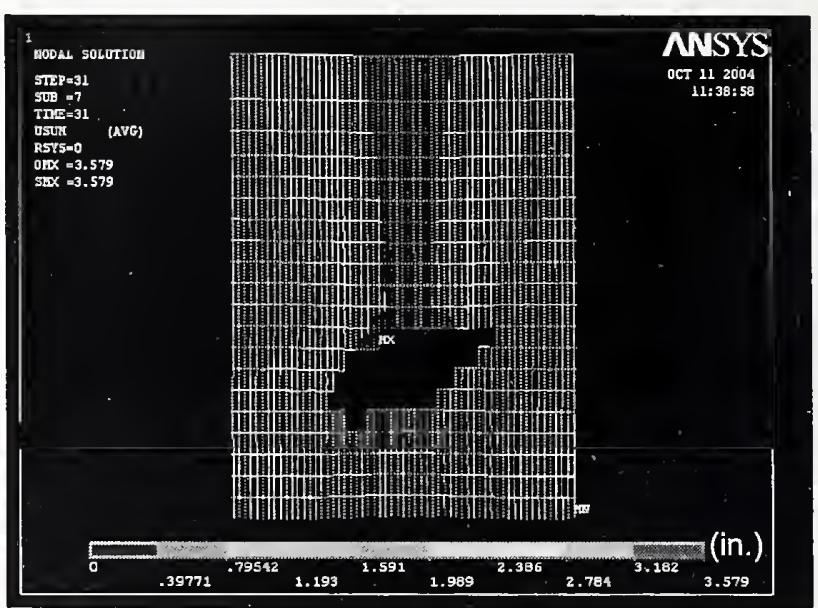

(b) North wall at $100 \mathrm{~min}$

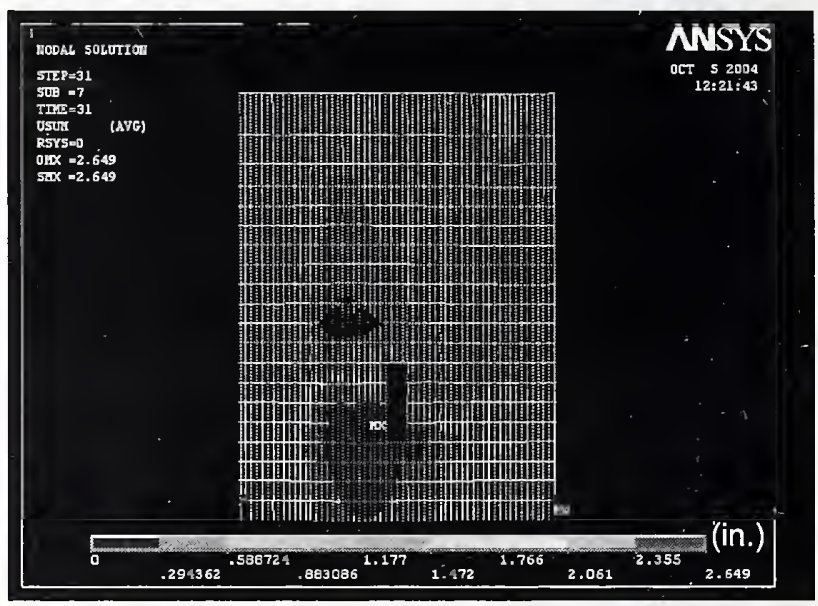

(d) South wall at $100 \mathrm{~min}$

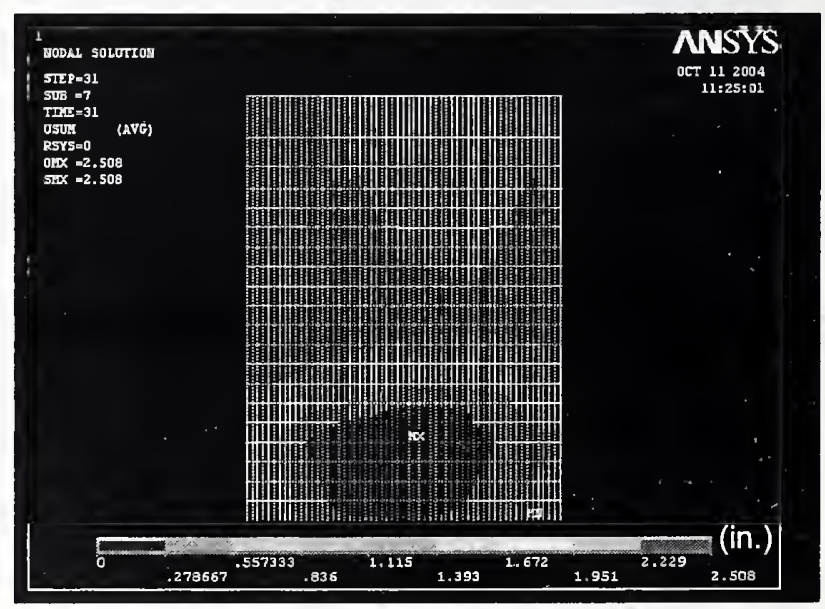

(f) East wall at $100 \mathrm{~min}$

Figure C-3. Total displacement on the north, south, and east faces of the WTC 1 at $50 \mathrm{~min}$ and $100 \mathrm{~min}$ of Case $A_{i}$ conditions (looking from outside). 


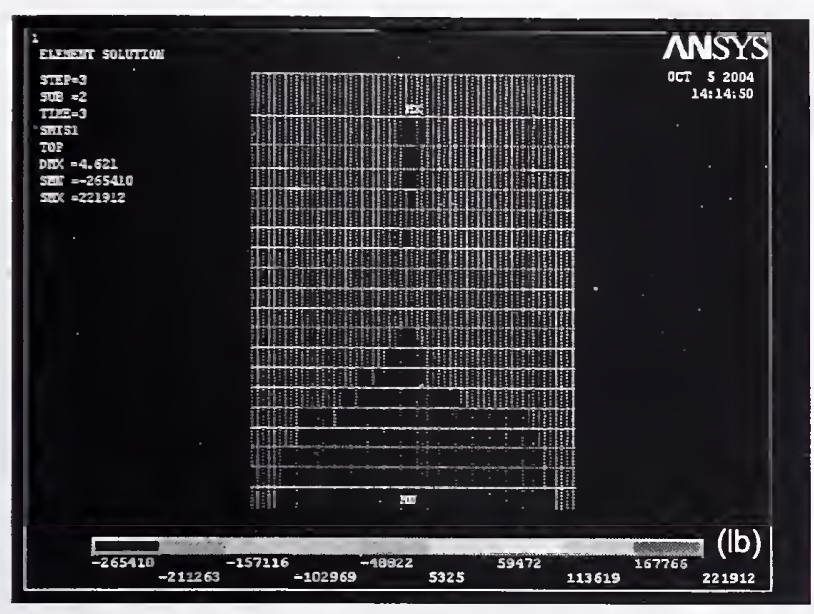

(a) North wall before impact

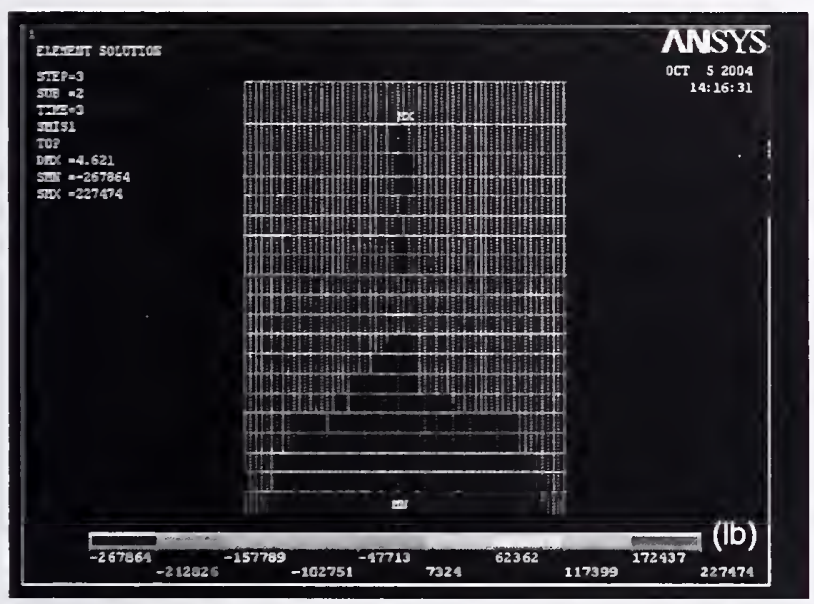

(c) South wall before impact

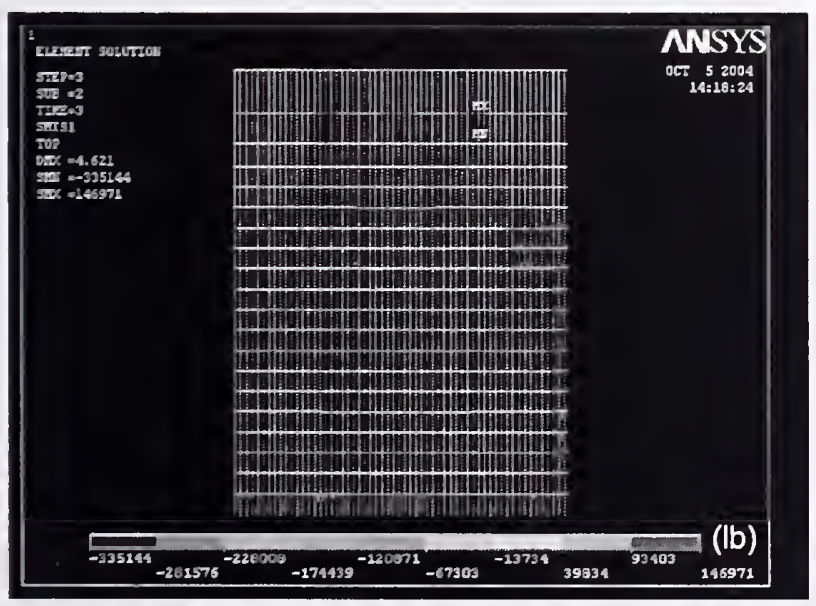

(e) East wall before impact

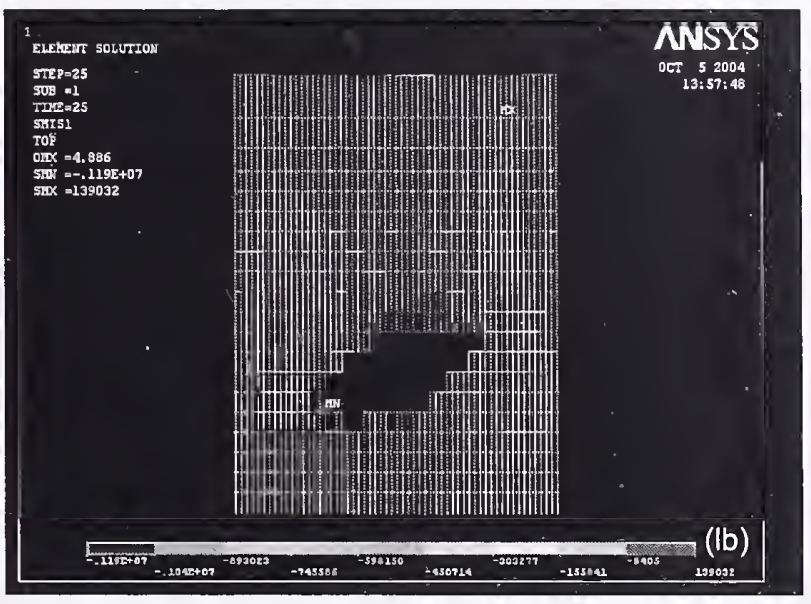

(b) North wall after impact

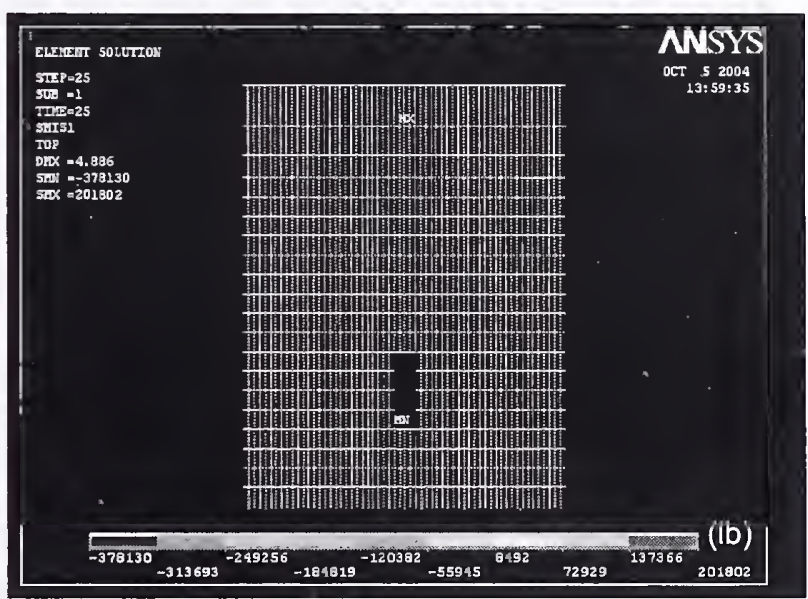

(d) South wall after impact

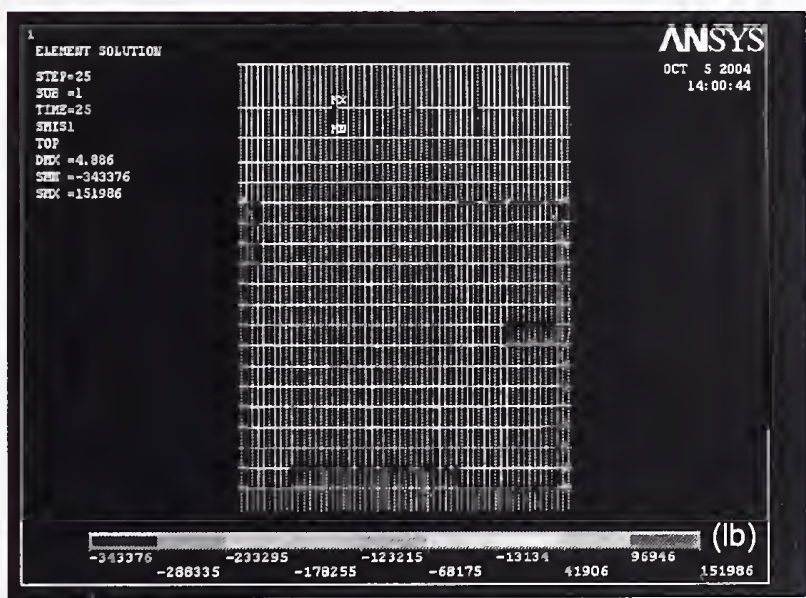

(f) East wall after impact

Figure C-4. Axial load variation on the north, south, and east faces of the WTC 1 before and after aircraft impact (looking from outside; compression is negative). 


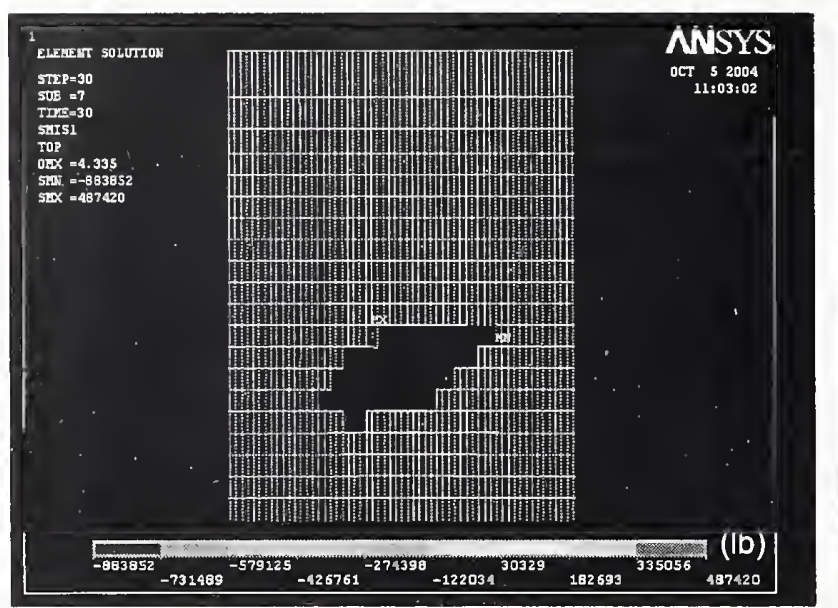

(a) North wall at $50 \mathrm{~min}$

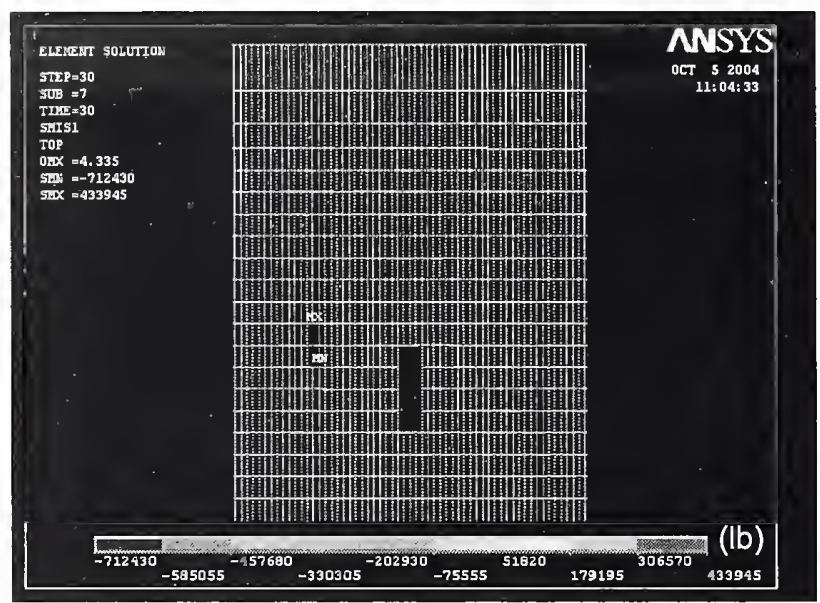

(c) South wall at $50 \mathrm{~min}$

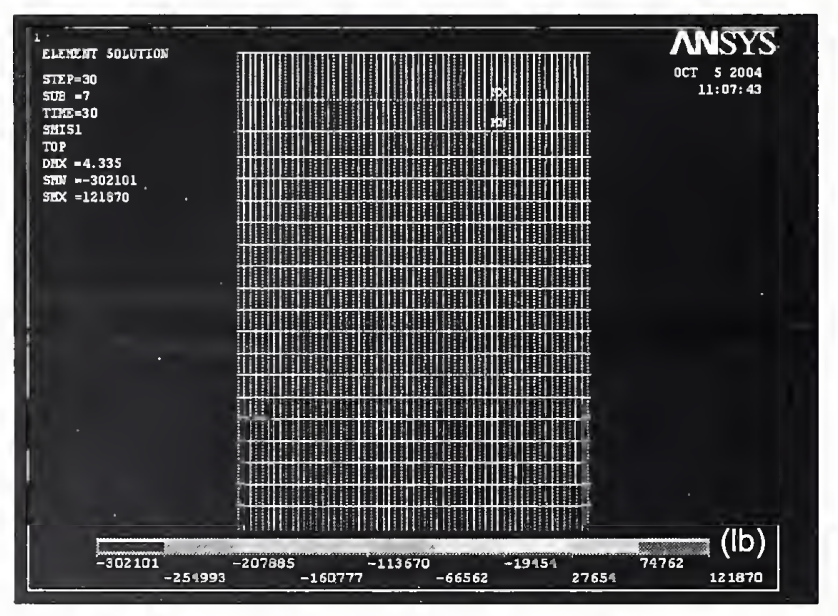

(e) East wall at $50 \mathrm{~min}$

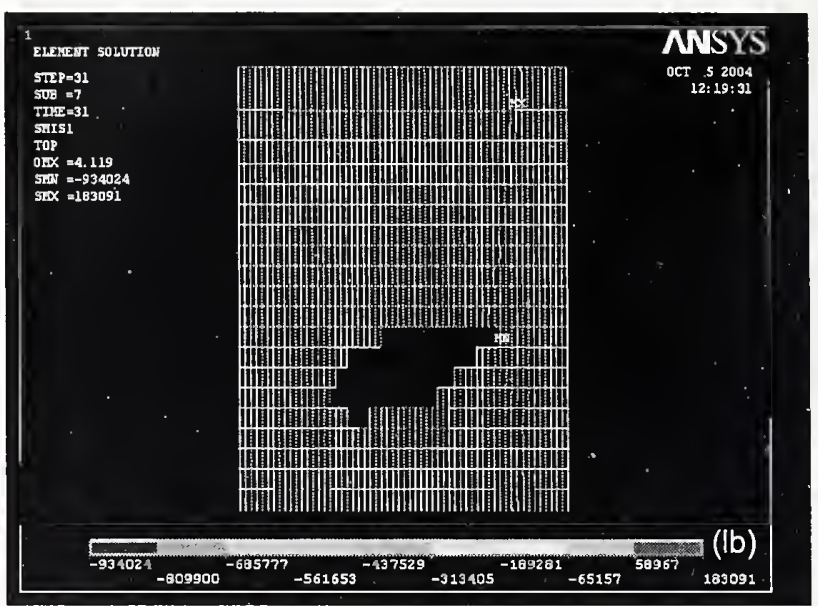

(b) North wall at $100 \mathrm{~min}$

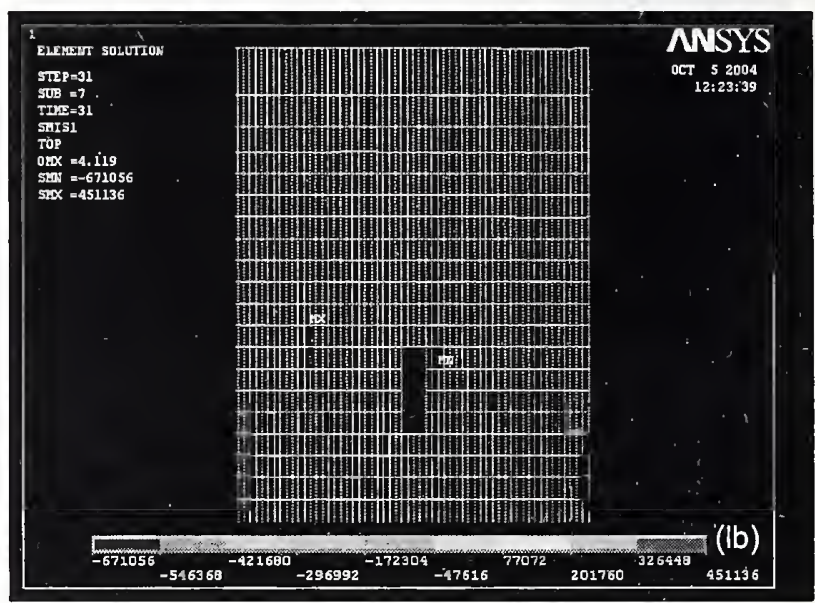

(d) South wall at $100 \mathrm{~min}$

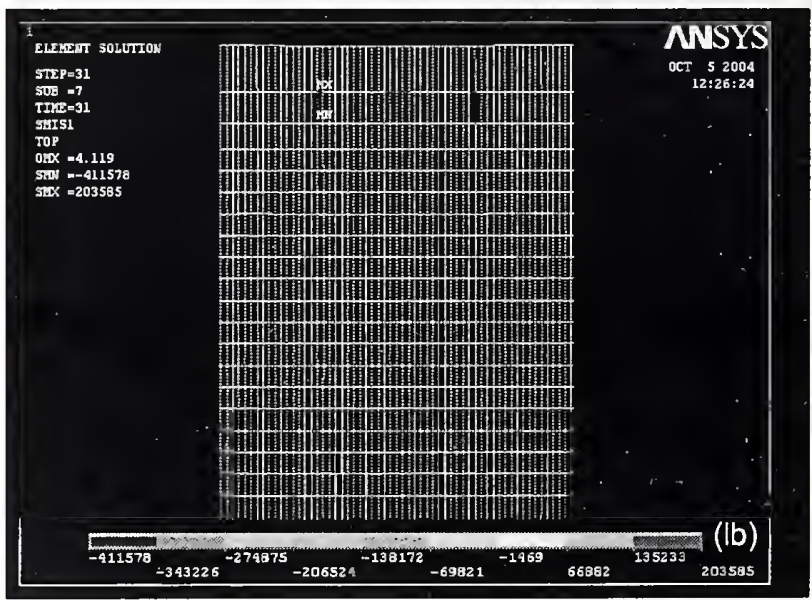

(f) East wall at $100 \mathrm{~min}$

Figure C-5. Axial load variation on the north, south, and east faces of the WTC 1 at $50 \mathrm{~min}$ and $100 \mathrm{~min}$ of Case $A_{i}$ conditions (looking from outside; compression is negative). 


\begin{tabular}{|c|l|c|c|c|c|c|c|}
\hline Row & Analysis Stage & North & South & East & West & Core & Sum \\
\hline$(1)$ & Before Impact & 12375 & 12459 & 9006 & 9045 & 47101 & 89986 \\
$(2)$ & After Impact & 10330 & 12151 & 9222 & 9242 & 47812 & 88757 \\
$(3)$ & $10 \mathrm{~min}$ & 9649 & 11714 & 8429 & 8142 & 50762 & 88696 \\
$(4)$ & $20 \mathrm{~min}$ & 9528 & 11761 & 8260 & 8023 & 51185 & 88757 \\
$(5)$ & $30 \mathrm{~min}$ & 9564 & 11931 & 8421 & 8060 & 50781 & 88757 \\
$(6)$ & $50 \mathrm{~min}$ & 9852 & 12228 & 9065 & 8714 & 48898 & 88757 \\
$(7)$ & $100 \mathrm{~min}$ & 10492 & 12880 & 10067 & 9880 & 45438 & 88757 \\
\hline
\end{tabular}

(a) Above Floor 93

\begin{tabular}{|c|l|c|c|c|c|c|c|}
\hline Row & Analysis Stage & North & South & East & West & Core & Sum \\
\hline$(1)$ & Before Impact & 11119 & 11204 & 8126 & 8170 & 41216 & 79835 \\
$(2)$ & After Impact & 9598 & 11004 & 8328 & 8339 & 42174 & 79443 \\
$(3)$ & $10 \mathrm{~min}$ & 8898 & 10573 & 7546 & 7237 & 45137 & 79391 \\
$(4)$ & $20 \mathrm{~min}$ & 8757 & 10620 & 7387 & 7128 & 45552 & 79444 \\
$(5)$ & $30 \mathrm{~min}$ & 8795 & 10780 & 7545 & 7176 & 45148 & 79444 \\
$(6)$ & $50 \mathrm{~min}$ & 9094 & 11068 & 8179 & 7840 & 43263 & 79444 \\
$(7)$ & $100 \mathrm{~min}$ & 9771 & 11668 & 9192 & 9014 & 39799 & 79444 \\
\hline
\end{tabular}

(b) Above Floor 96

\begin{tabular}{|c|l|c|c|c|c|c|c|}
\hline Row & Analysis Stage & North & South & East & West & Core & Sum \\
\hline$(1)$ & Before lmpact & 10264 & 10352 & 7564 & 7611 & 37299 & 73090 \\
$(2)$ & After Impact & 9090 & 10171 & 7761 & 7770 & 38295 & 73087 \\
$(3)$ & $10 \mathrm{~min}$ & 8397 & 9739 & 6983 & 6661 & 41262 & 73042 \\
$(4)$ & $20 \mathrm{~min}$ & 8261 & 9783 & 6828 & 6546 & 41669 & 73087 \\
$(5)$ & $30 \mathrm{~min}$ & 8299 & 9938 & 6990 & 6594 & 41266 & 73087 \\
$(6)$ & $50 \mathrm{~min}$ & 8588 & 10223 & 7625 & 7270 & 39381 & 73087 \\
$(7)$ & $100 \mathrm{~min}$ & 9250 & 10824 & 8628 & 8466 & 35919 & 73087 \\
\hline
\end{tabular}

(c) Above Floor 98

Figure C-6. Total column loads in the core and exterior walls of WTC 1 at different floors and at different times (compression is positive). 


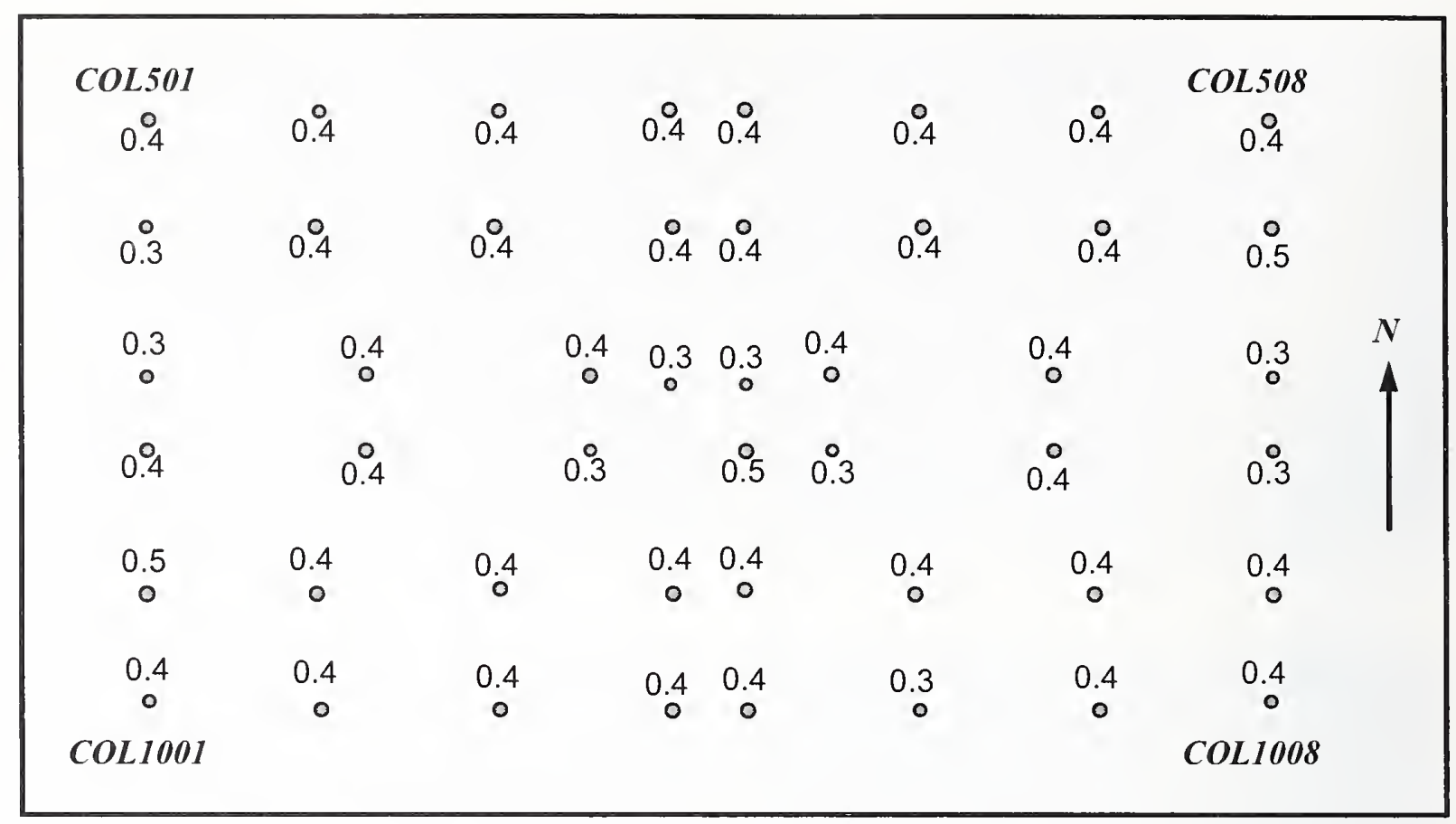

(a) Before impact

COL501

0.4

0.3

0.3

0

$\stackrel{0}{0.4}$

0.5

0.4

COL1001
0.4

0.5

0.5

0.5

0.4

0.4

0.4

0.4
0

0.4

0.4

4

$\begin{array}{cc}0 & 0 \\ 1.2 & 0.7\end{array}$

$\begin{array}{cc}0.1 & 0 \\ 0.7\end{array}$

$$
\begin{array}{cccc}
0.5 & 0.4 & 0.4 & 0.2 \\
0 & 0 & 0 & 0
\end{array}
$$

0.3

0.5

0.4

$\begin{array}{cc}0.5 & 0.4 \\ 0 & 0\end{array}$

$\begin{array}{cc}0.4 & 0.4 \\ 0 & 0\end{array}$

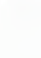

0.4

0.4

\section{COL508}

0.4

0.5

0.5

0
0.5 


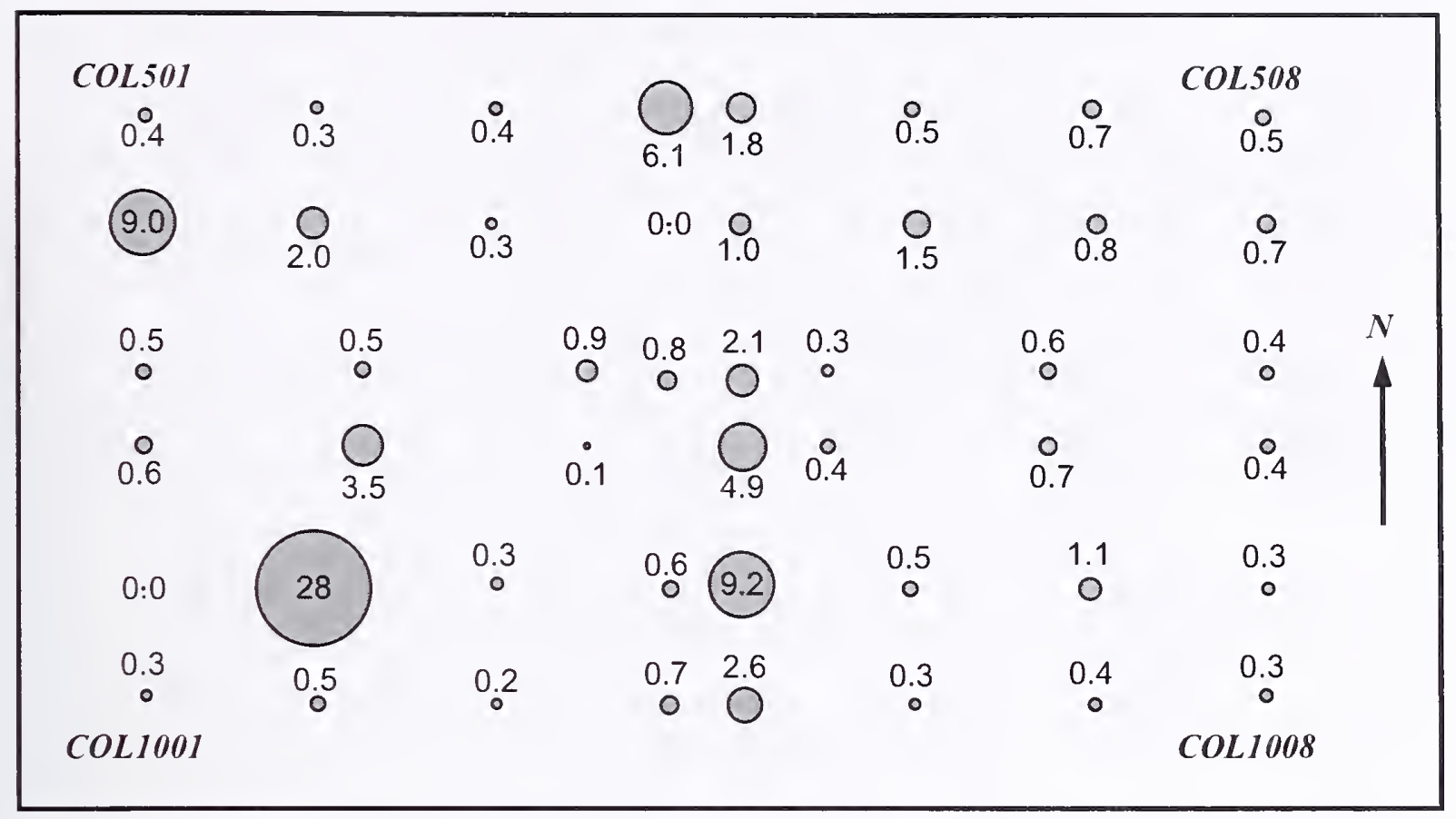

(a) At $10 \mathrm{~min}$

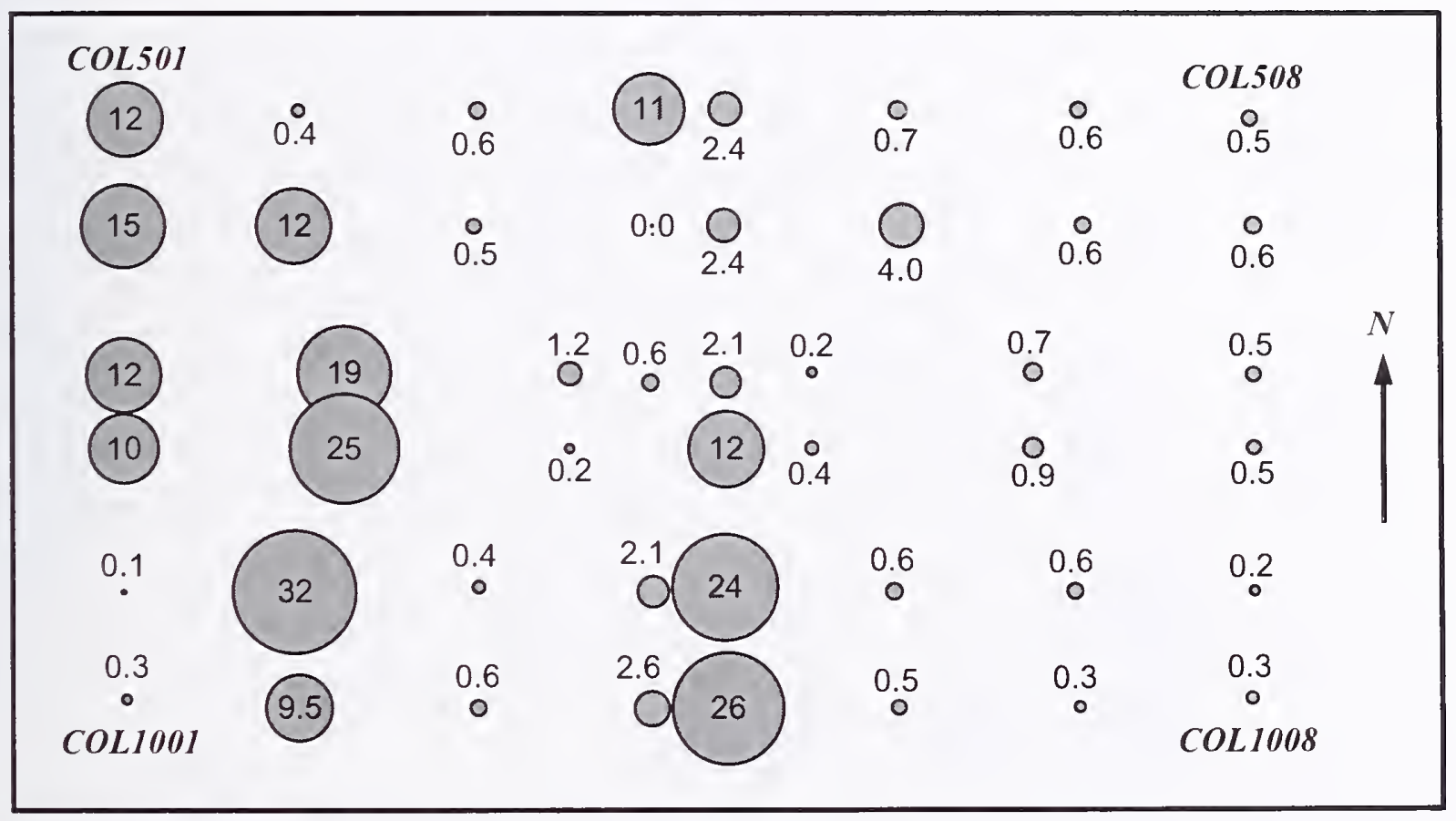

(b) At $20 \mathrm{~min}$

Figure C-8. Ratios of axial elastic-plus-plastic strain to temperature-dependent yield strain for the core columns at Floor 96 of WTC 1 at $10 \mathrm{~min}$ and $20 \mathrm{~min}$ for Case $A_{i}$ conditions. 


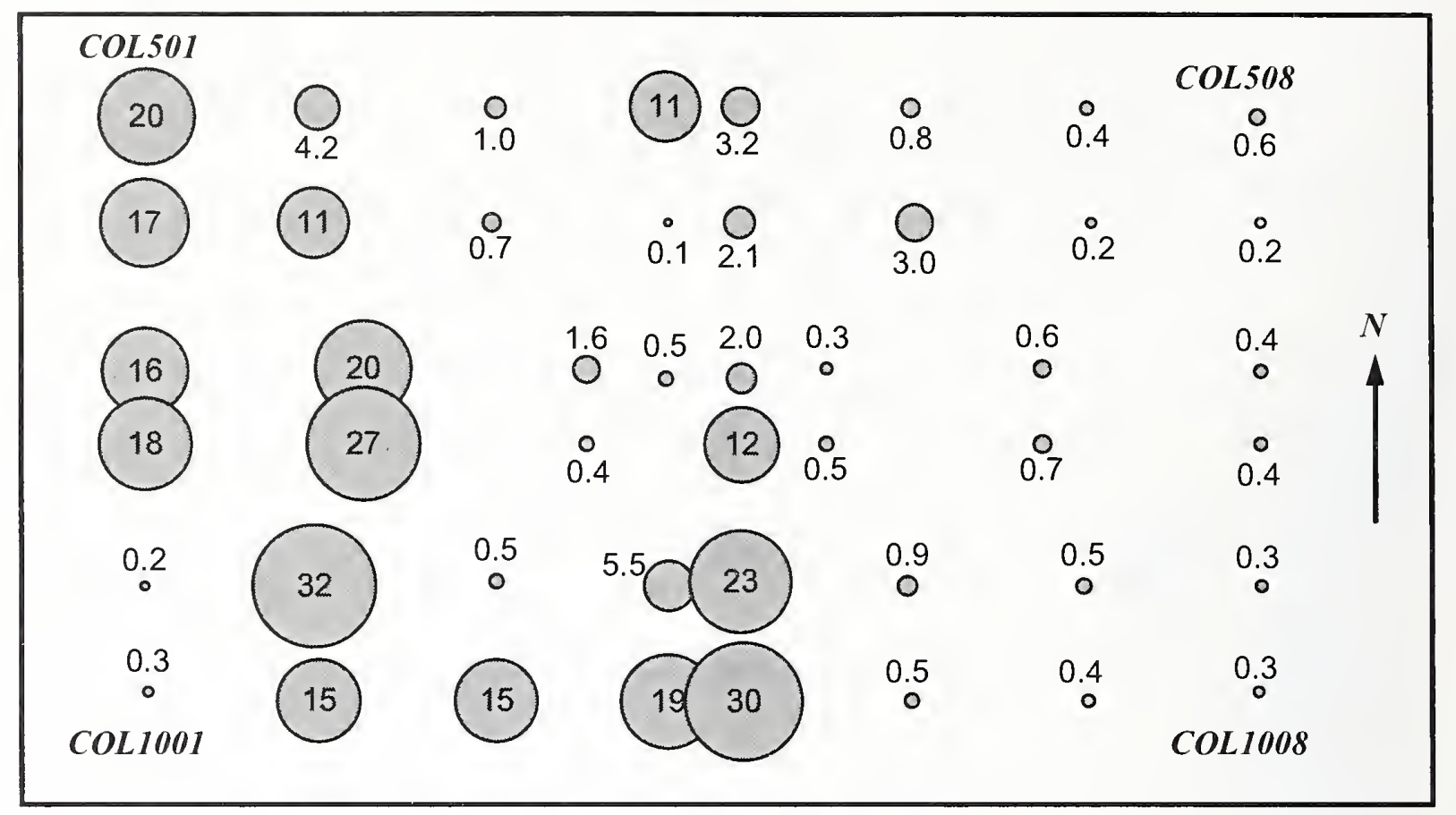

(a) At $50 \mathrm{~min}$

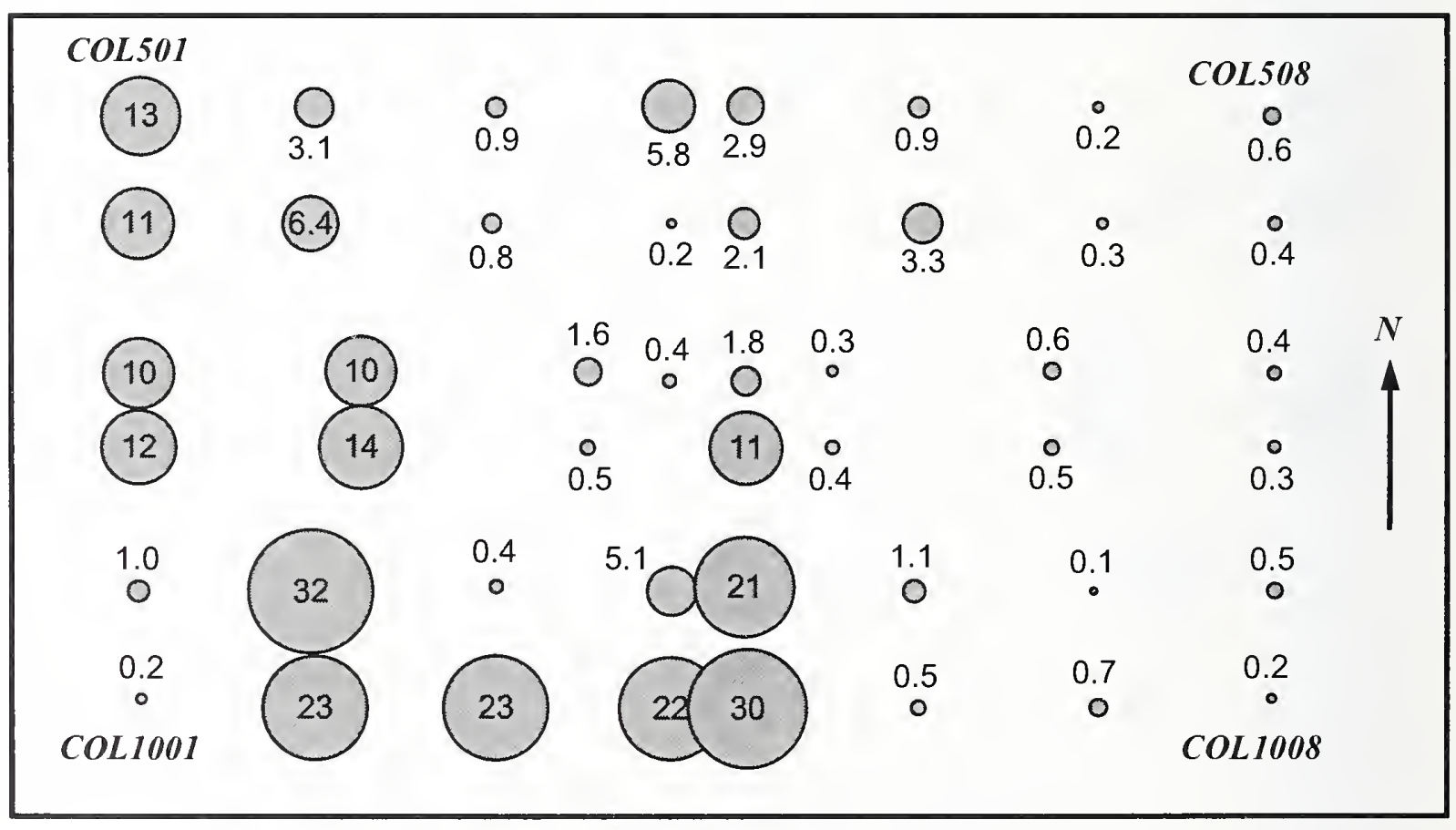

(b) At $100 \mathrm{~min}$

Figure C-9. Ratios of axial elastic-plus-plastic strain to temperature-dependent yield strain for the core columns at Floor 96 of WTC 1 at $50 \mathrm{~min}$ and $100 \mathrm{~min}$ for Case $A_{i}$ conditions. 


\section{C.2 WTC 2 GLOBAL ANALYSIS WITHOUT CREEP}

The WTC 2 ANSYS model with no creep and no inelastic buckling of columns was analyzed with Case $C_{i}$ and revised Case $C_{i}$ structural damage condition and subjected to Case $C_{i}$ and Case $D_{i}$ temperature conditions. Temperature-dependent plasticity and the nonlinear geometry were the sources of nonlinearity in the model.

Case $D_{i}$ temperature histories were analyzed with revised Case $C_{i}$ structural damage as there was little structural response to Case $\mathrm{C}_{\mathrm{i}}$ temperature histories, particularly in the core. The application of structural damage to the global model was a lengthy process, hence, only a different temperature history was considered for preliminary analysis purposes.

\section{C.2.1 Case $\mathrm{C}_{\mathrm{i}}$ Structural Damage and Temperature Conditions}

Table C-2 summarizes the sequence of analyses that were performed with the WTC 2 ANSYS global model with Case $C_{i}$ conditions (see Section 2.2 for Case $C_{i}$ structural damage condition). The revised Case $C_{i}$ structural damage condition was provided by NIST in the middle of the analysis for the thermal loading. Additional core columns were removed in analysis step 6 as shown in Table C-2. The results of each analysis step were used as initial conditions for the next analysis step.

Table C-2. Analysis Steps of WTC 2 ANSYS global model with Case $C_{i}$ conditions.

\begin{tabular}{|c|l|}
\hline Analysis Step & \multicolumn{1}{|c|}{ Description } \\
\hline 1 & WTC 2 model below Floor 106 was analyzed under its own self-weight. \\
\hline 2 & $\begin{array}{l}\text { Structures above Floor } 106 \text { were added in a stress-free state, and the model was analyzed for dead } \\
\text { load including those above Floor } 106 .\end{array}$ \\
\hline 3 & Superimposed dead load and 25 percent of the design live loads were superimposed on the model. \\
\hline 4 & $\begin{array}{l}\text { Columns, spandrels, and floor elements severed during aircraft impact were removed, and the } \\
\text { model was analyzed. }\end{array}$ \\
\hline 5 & Column and spandrel temperatures were linearly ramped up to temperatures at 10 min. \\
\hline 6 & $\begin{array}{l}\text { Core Columns } 1003 \text { at Floor } 80 \text { and } 903 \text { between Floors } 77 \text { and } 84 \text { were removed from the model } \\
\text { to represent the additional severed columns that were identified by NIST at later stages of the } \\
\text { investigation (see Section 2.2 for the revised Case C } \mathrm{i}_{\mathrm{i}} \text { structural damage condition). }\end{array}$ \\
\hline 7 & Column and spandrel temperatures were changed linearly from temperatures at 10 min to 20 min. \\
\hline 8 & Column and spandrel temperatures were changed linearly from temperatures at 20 min to 30 min. \\
\hline 10 & Column and spandrel temperatures were changed linearly from temperatures at 30 min to 40 min. \\
\hline 11 & Column and spandrel temperatures were changed linearly from temperatures at 40 min to 50 min. \\
\hline
\end{tabular}

The model was first analyzed to capture the effects of the construction sequence (analysis steps 1, 2, and 3). The results of analysis step 3 represented the structure state before the aircraft impact. In analysis step 4, the columns, spandrels, and floor elements that were severed during the aircraft impact were removed from the model, and the building was reanalyzed to redistribute the load to the non-severed members. This analysis represents the state of the building after the aircraft impact. After load redistribution, the column and spandrel temperatures were applied to the model (analysis steps 5, 7, 8, 9, 10, and 11). The column and spandrel temperatures were provided by NIST at $10 \mathrm{~min}$ intervals. For times between these time intervals, temperatures were calculated by linear interpolation. Analyses were 
conducted at every $10 \mathrm{~min}$ up to $60 \mathrm{~min}$. In Analysis Step 6, after the analysis of $10 \mathrm{~min}$ temperatures, two more core columns that were identified as severed during the aircraft impact were removed from the model. Temperatures were not applied to the floors in the global model to prevent floor buckling, as discussed for the WTC 1 global model without creep.

The results of the WTC 2 global model analyses with Case $C_{i}$ structural damage and temperature conditions are summarized in Figs. C-10 through $\mathrm{C}-20$.

Figure $\mathrm{C}-10$ shows the vertical displacement at different analysis stages. The maximum displacement after the removal of additional severcd core columns at analysis step 6 was equal to $7.2 \mathrm{in}$. This displacement gradually decreased to $7.0 \mathrm{in}$. at the end of $50 \mathrm{~min}$. This reduction was due to thermal expansion of the core and the exterior wall columns between Floor 79 and Floor 83. Figures C-11 and $\mathrm{C}-12$ show the vertical displacements for south and east exterior walls before and after aircraft impact and at $30 \mathrm{~min}$ and $60 \mathrm{~min}$. The displacements were typically at their maximum near the hat truss at analysis step 6, after all severed members were removed, and gradually decreased to their minimum near Floor 77 through Floor 81 at $60 \mathrm{~min}$. Figure $\mathrm{C}-11$ shows that the displacement on the east wall, which was symmetric before the aircraft impact, became greater on the south side of this wall after the aircraft impact. This was due to the load redistribution to the south side of the east wall as a result of the removal of the severed columns on the south wall and in the southeast corner of the core. This was the first sign of tilting of WTC 2 toward the southeast.

Figures $\mathrm{C}-13, \mathrm{C}-14, \mathrm{C}-15$, and $\mathrm{C}-16$ show the axial loads before and after aircraft impact and at $30 \mathrm{~min}$ and 60 min over the south, east, and north exterior wall columns and spandrels and in the 800,900, and 1000 core column series. After the aircraft impact, the load carried by the severed columns of the south exterior wall was primarily redistributed to the remaining columns on the same face and also to the core and other exterior wall columns. As a result of this redistribution, the maximum column load increased from 334 kip to $1,410 \mathrm{kip}$ on the south exterior wall and from $451 \mathrm{kip}$ to $485 \mathrm{kip}$ on the east exterior wall. Similar to displacement, the axial load on the east wall, which was symmetric before the aircraft impact, became greater on the south side of this wall after the aircraft impact. This was due to the load redistribution to the south side of the east wall as a result of the removal of the severed columns on the south wall and in the southeast corner of the core. The axial load in core columns also was significantly affected by the aircraft impact and fire-induced temperatures. The maximum column load increased from $4,930 \mathrm{kip}$ to $5,120 \mathrm{kip}$ in the 1000 core-column series, from 1,600 kip to 2,250 kip in the 900 core column series, and from 1,740 kip to 2,630 kip in the 800 core-column series. After the removal of 1003 and 903 core columns, the column loads increased on the east and south side core columns.

With increasing time, the maximum column load on the east exterior wall columns increased from $485 \mathrm{kip}$ after aircraft impact to $630 \mathrm{kip}$ at the end of $60 \mathrm{~min}$. This increase was primarily due to the steady increase of column temperatures on the east wall as a result of the continuous fires. Similarly, the axial loads in the 1000 core column series steadily increased due to continuous fires on the east side of the core.

Figure $\mathrm{C}-17$ shows the total column loads over the entire core and north, south, east, and west exterior walls for different floors. Due to the removal of severed columns, spandrels, and floor elements, the total column loads below Floor 83 after aircraft impact are not equal to those before the aircraft impact.

Referring to the total column loads above Floor 83 in Fig. C-17, after the aircraft impact (including the effects of additionally removed Column 903 and Column 1003 in the core), the total column load in the 
east exterior wall columns increased about 2,600 kip, whereas the total column load decreased in the core by about 1,200 kip, in the north exterior wall by about $750 \mathrm{kip}$, in the west cxterior wall by about $50 \mathrm{kip}$, and in the south exterior wall by about $600 \mathrm{kip}$. With increasing time, the tempcratures in the east, the north, and the south exterior wall columns increased more than those in the west exterior wall and core columns. The higher temperatures resulted in higher thermal expansions and greater axial loads in the exterior wall columns. In Floor 83, the temperature effect alone resulted in unloading of the corc by $1,820 \mathrm{kip}$ and increased the total column loads by $140 \mathrm{kip}$ on the south exterior wall, by $700 \mathrm{kip}$ on the west exterior wall, by $600 \mathrm{kip}$ on the north exterior wall, and by $380 \mathrm{kip}$ on the east exterior wall.

Figures $\mathrm{C}-18, \mathrm{C}-19$, and $\mathrm{C}-20$ show the ratio of elastic-plus-plastic strain to the temperature-dependent yield strain of the core columns between Floor 82 and Floor 83 before and after aircraft impact and at $10 \mathrm{~min}$ (analysis step 6), $30 \mathrm{~min}, 40 \mathrm{~min}$, and $60 \mathrm{~min}$. As can be noticed, the elastic-plus-plastic strain in the core columns before aircraft impact was typically 60 to 70 percent less than the yield strains at room temperature. After the aircraft impact, the maximum strain ratio reached 0.6 , indicating that all remaining core columns were in their elastic range. After the removal of Core Columns 1003 and 903, Core Columns 1004 and 1005 started to show strain ratios greater than 1.0 (indicating plasticity) with $10 \mathrm{~min}$ temperatures. Strain ratios in Core Columns 1004 and 1005 continued to increase with increasing time and temperatures, reaching their maximum at the end of $60 \mathrm{~min}$. High strains were clustered around the east side core columns, demonstrating possible tilting of the core. 


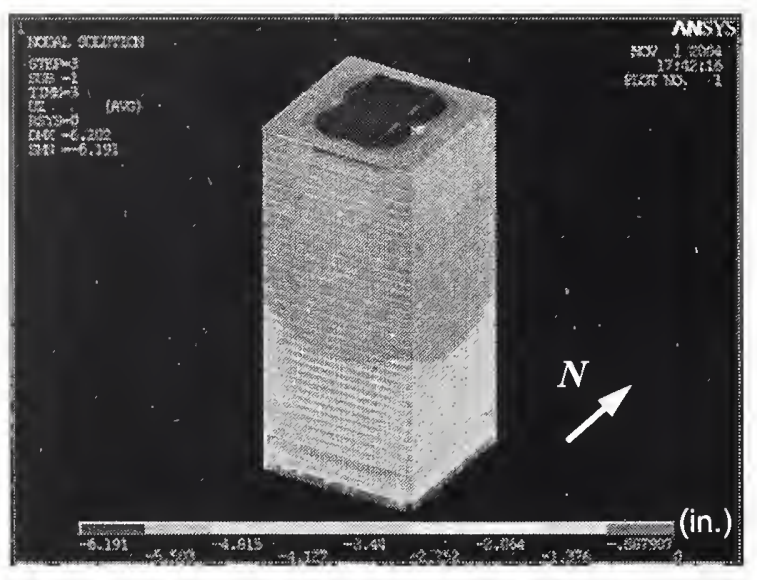

(a) Before impact

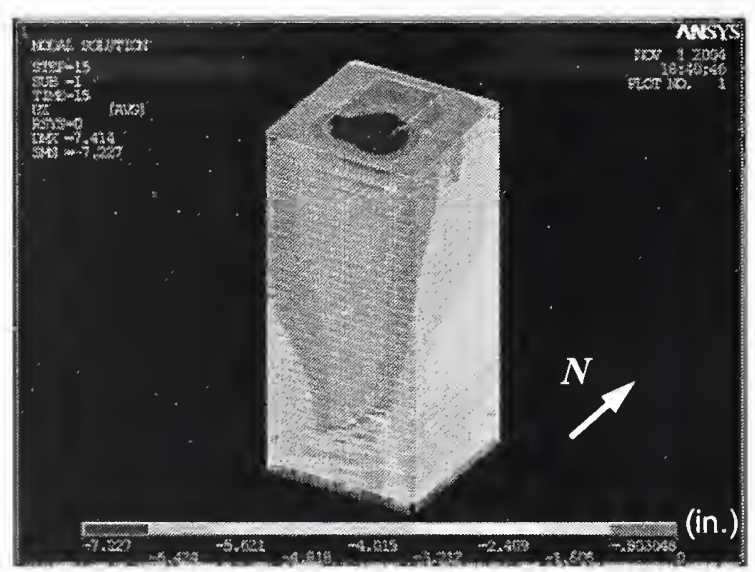

(c) At 10 min (Analysis step 6)

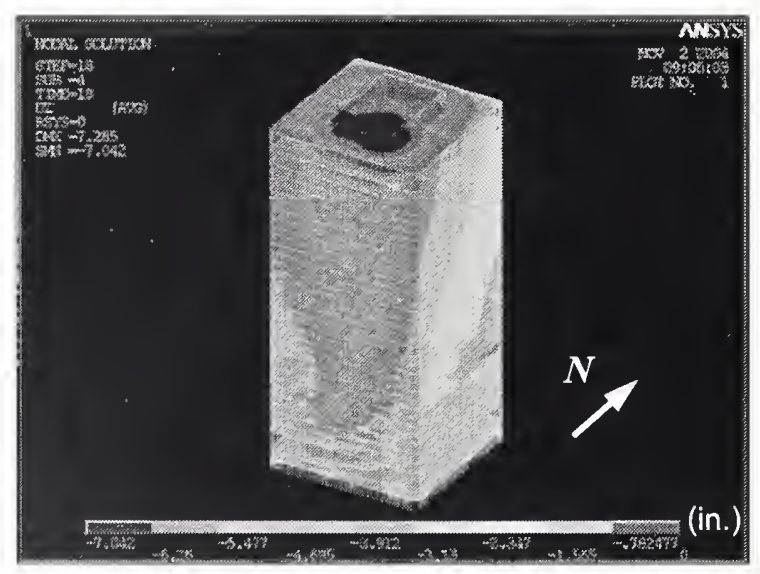

(e) At $40 \mathrm{~min}$

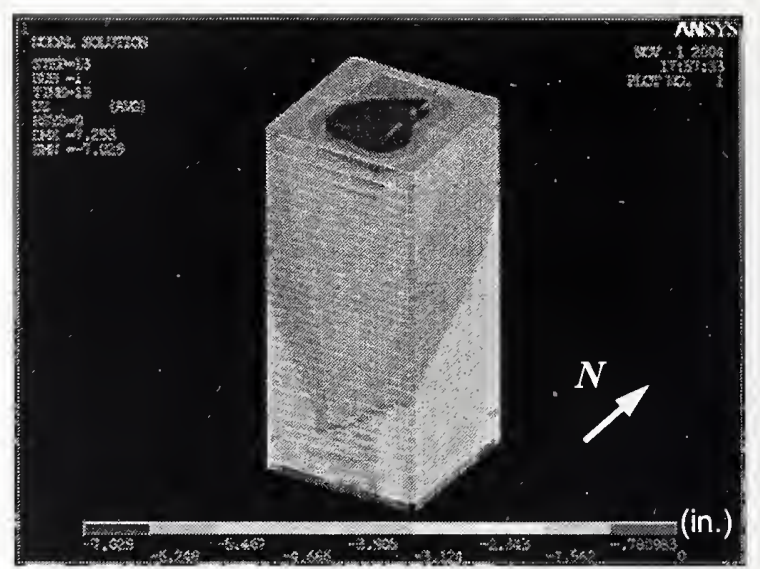

(b) After impact

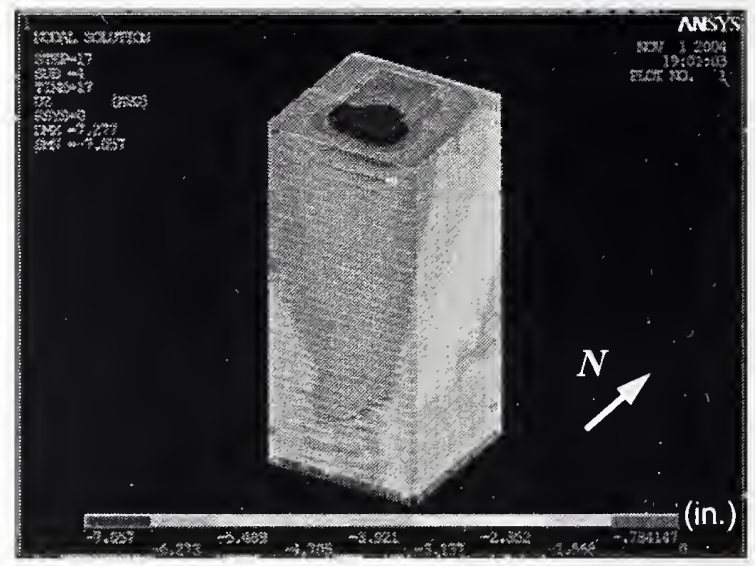

(d) At $30 \mathrm{~min}$

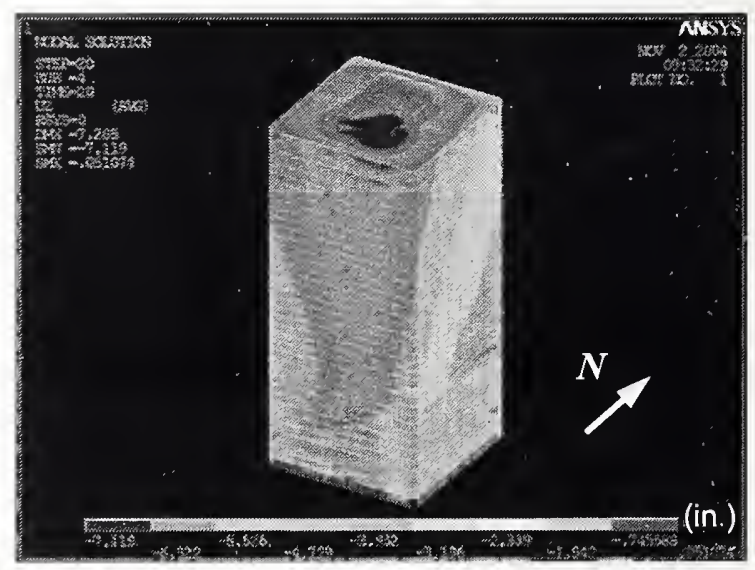

(f) At $60 \mathrm{~min}$

Figure $\mathrm{C}-10$. Vertical displacement at different stages of WTC 2 for Case $\mathrm{C}_{\mathrm{i}}$ conditions. 


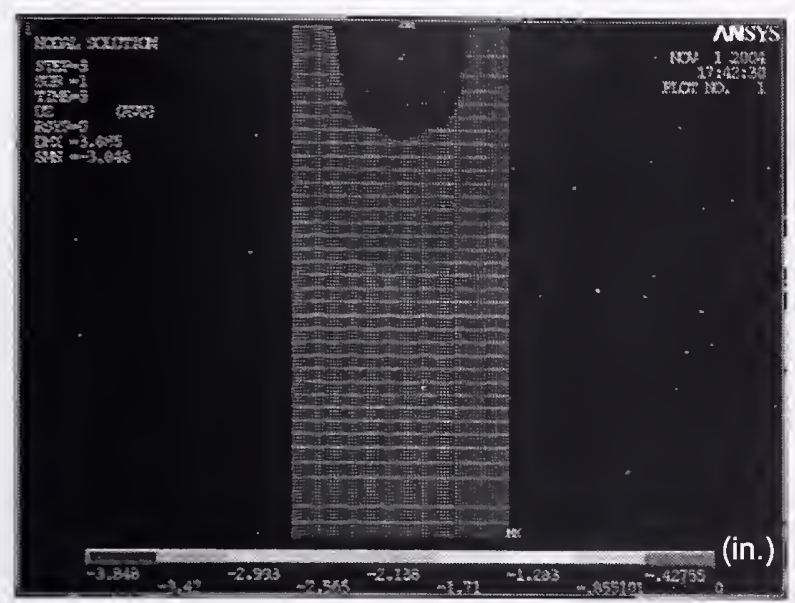

(a) South wall before impact

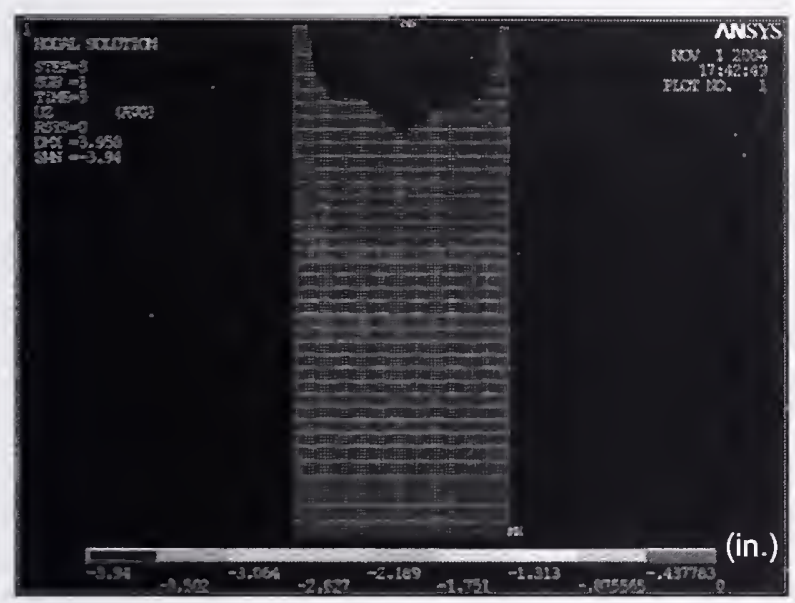

(c) East wall before impact

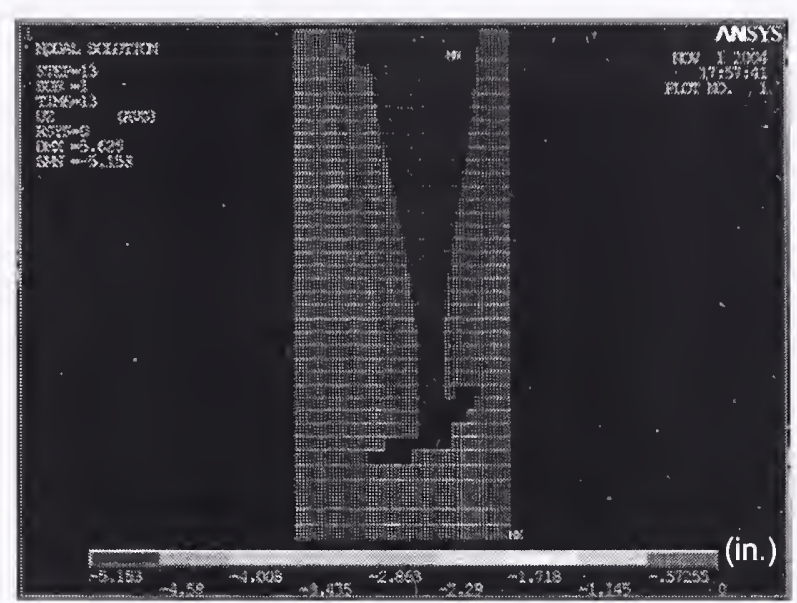

(b) South wall after impact

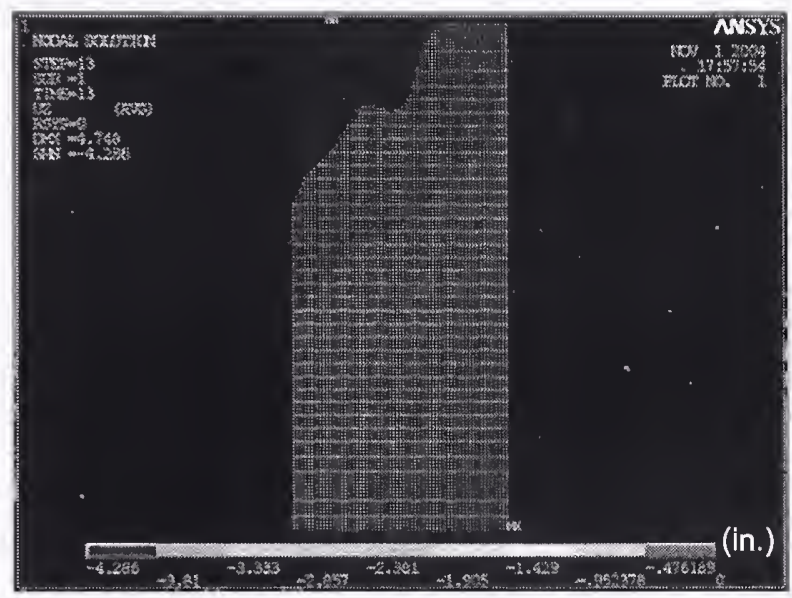

(d) East wall after impact

Figure C-11. Vertical displacement on the south and east faces of WTC 2 before and after aircraft impact (looking from outside; downward displacement is negative). 


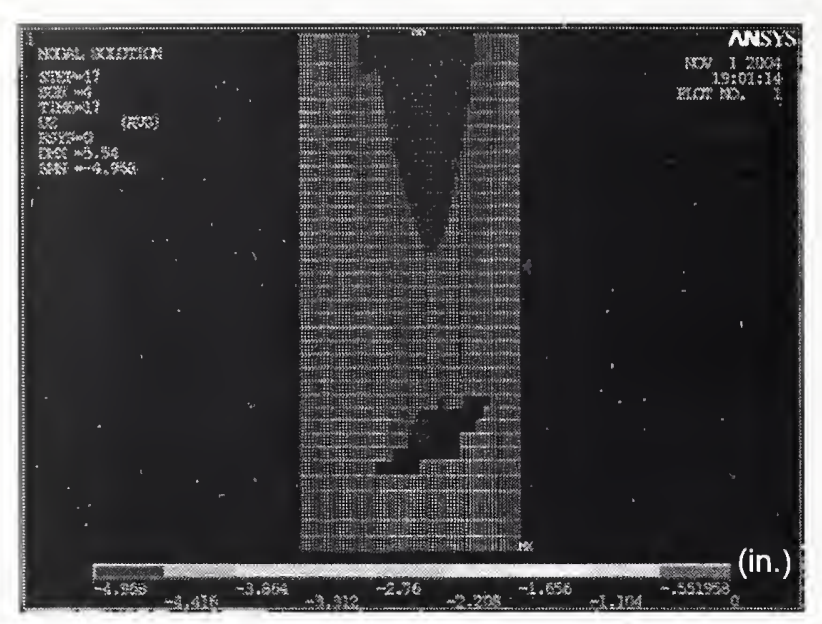

(a) South wall at $30 \mathrm{~min}$

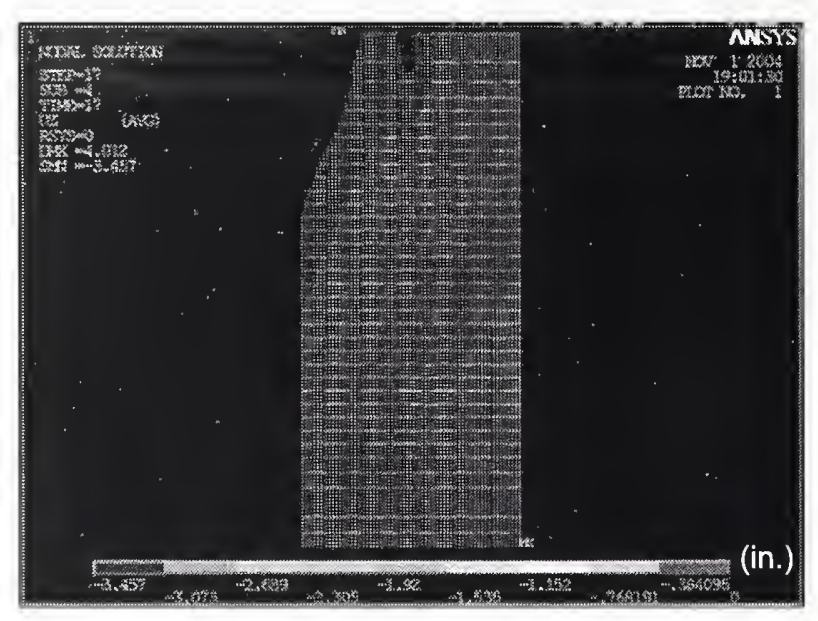

(c) East wall at $30 \mathrm{~min}$

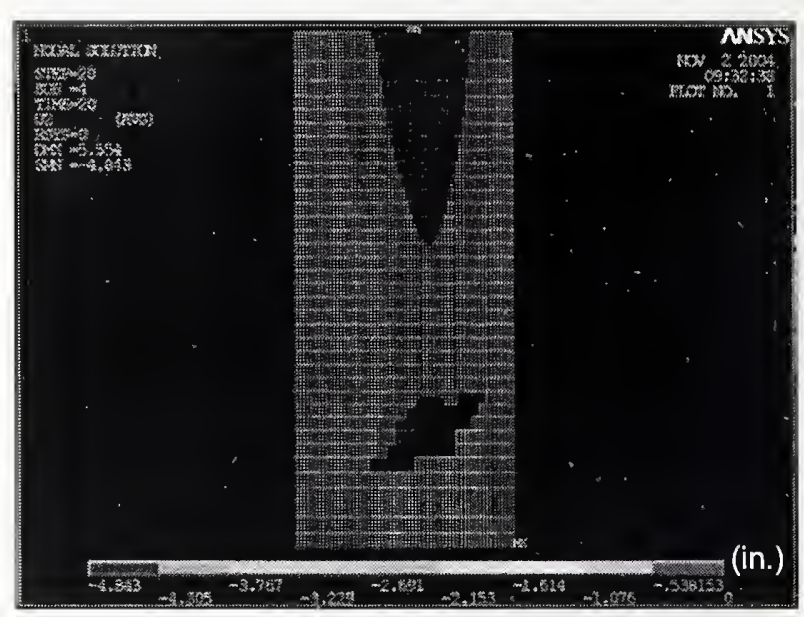

(b) South wall at $60 \mathrm{~min}$

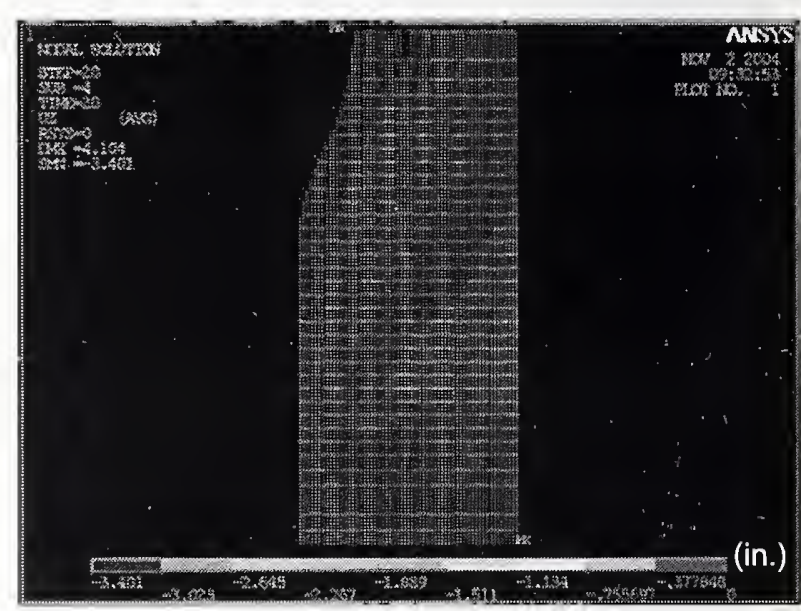

(d) East wall at $60 \mathrm{~min}$

Figure C-12. Vertical displacement on the south and east faces of WTC 2 at 30 min and at 60 min of Case $C_{i}$ conditions (looking from outside; downward displacement is negative). 


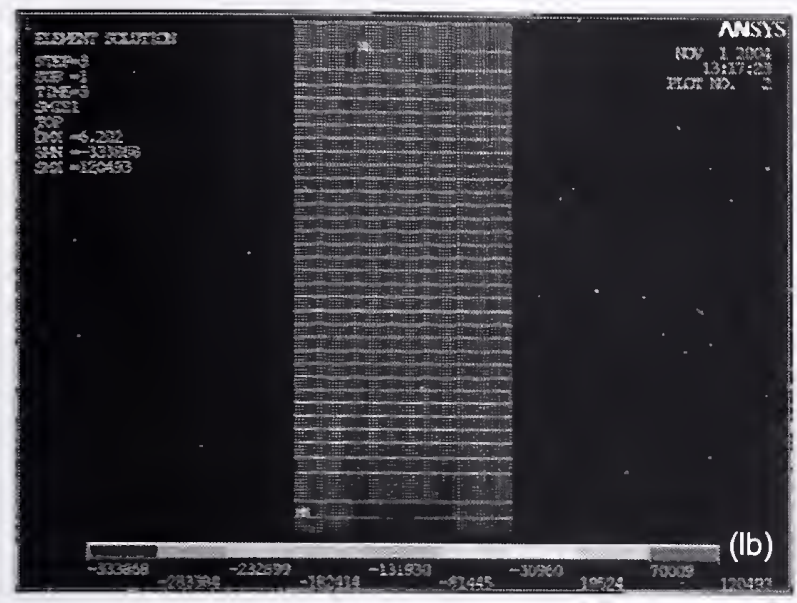

(a) South wall before impact

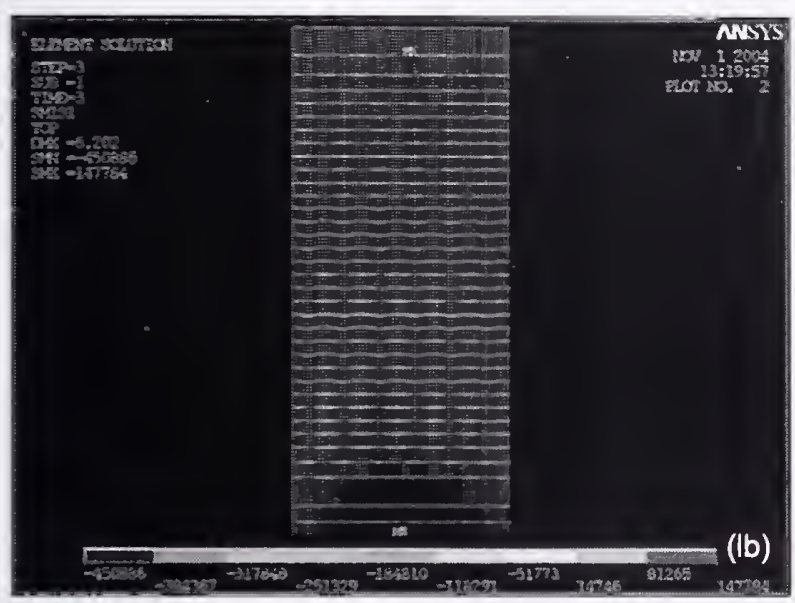

(c) East wall before impact

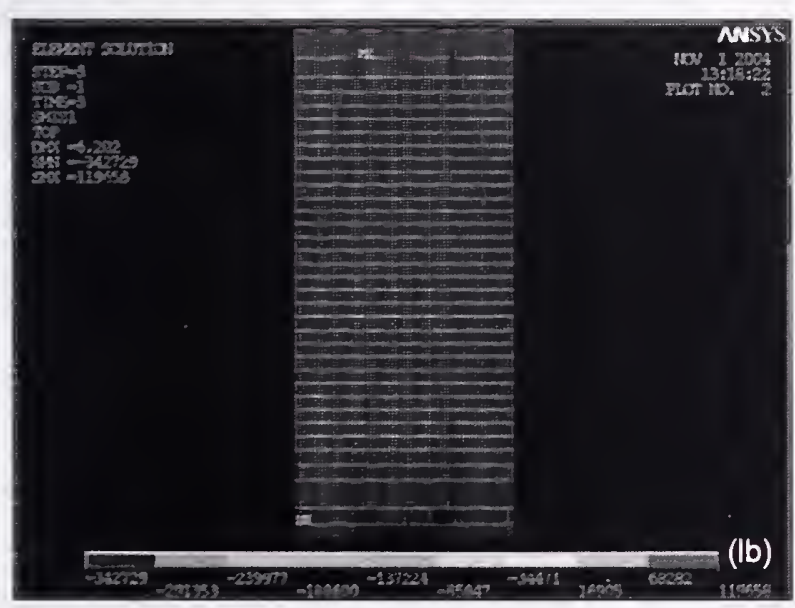

(e) North wall before impact

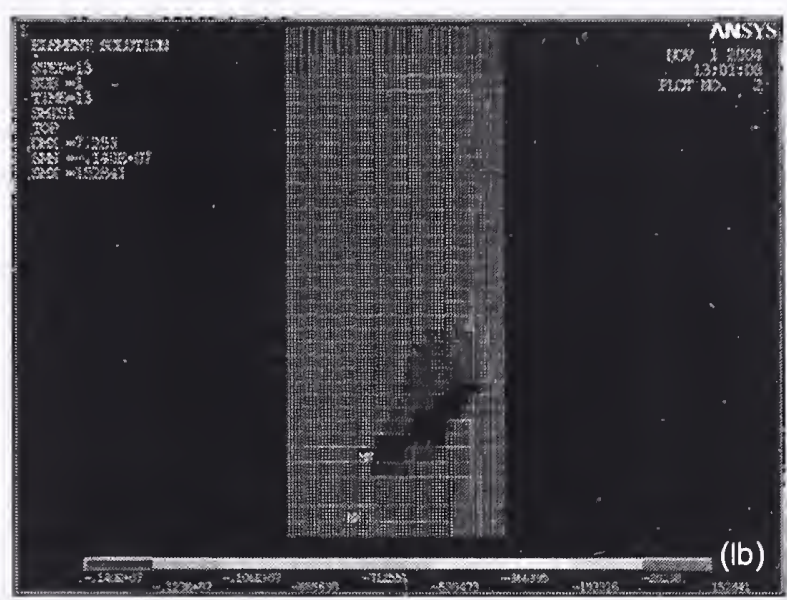

(b) South wall after impact

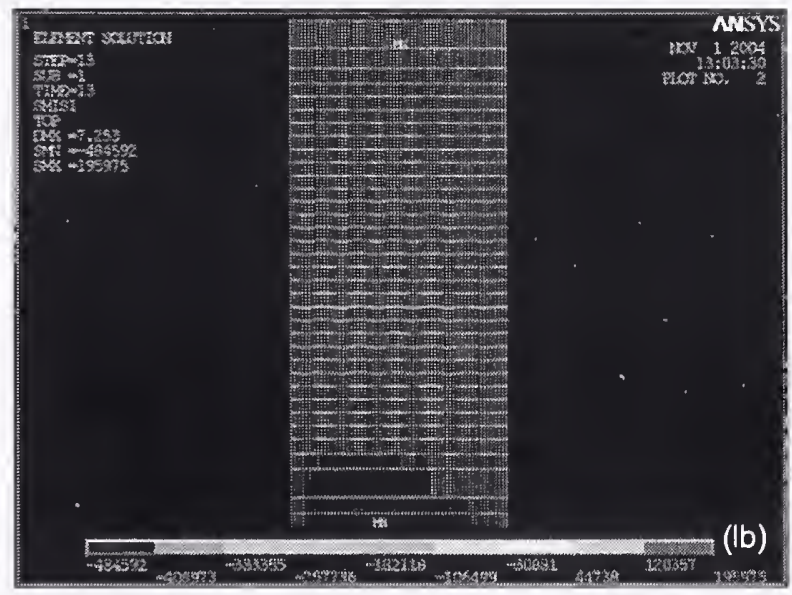

(d) East wall after impact

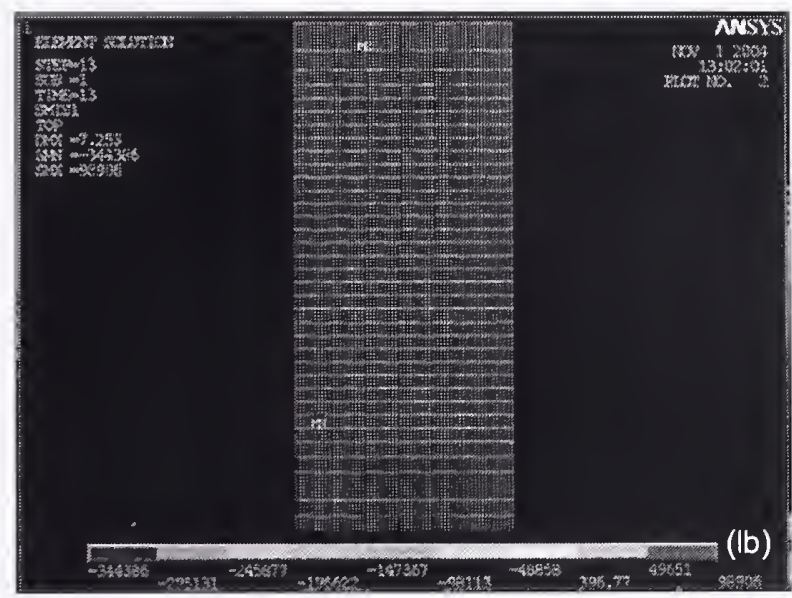

(f) North wall after impact

Figure C-13. Axial force variation on the south, east, and north faces of WTC 2 before and after aircraft impact (looking from outside; compression is negative). 


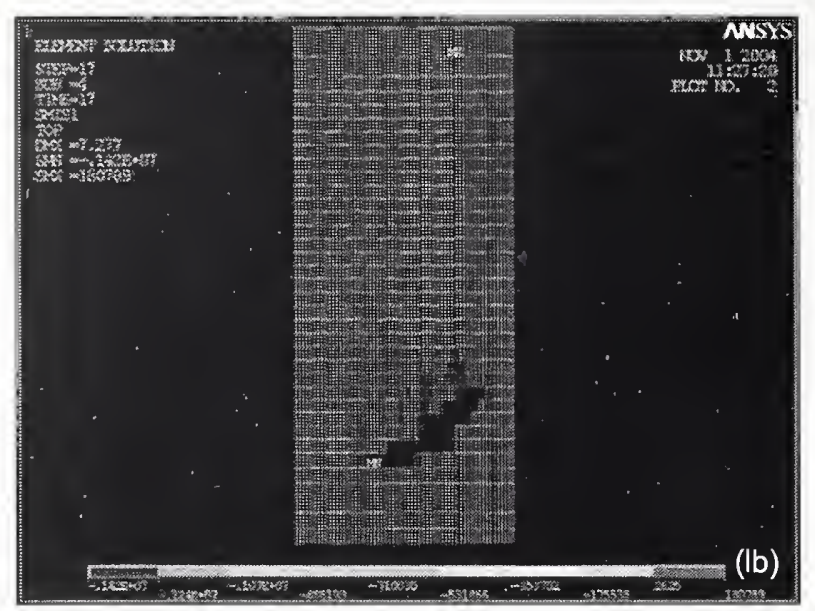

(a) South wall at $30 \mathrm{~min}$

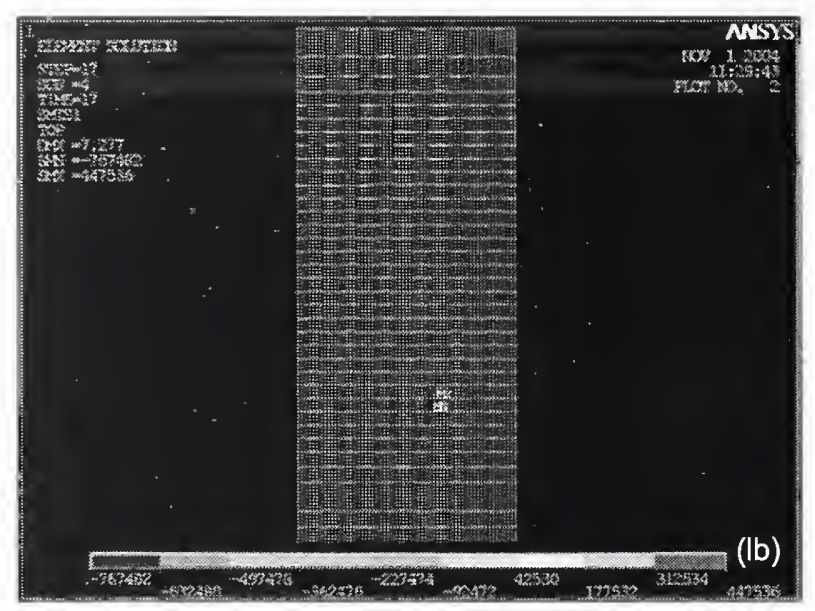

(c) East wall at $30 \mathrm{~min}$

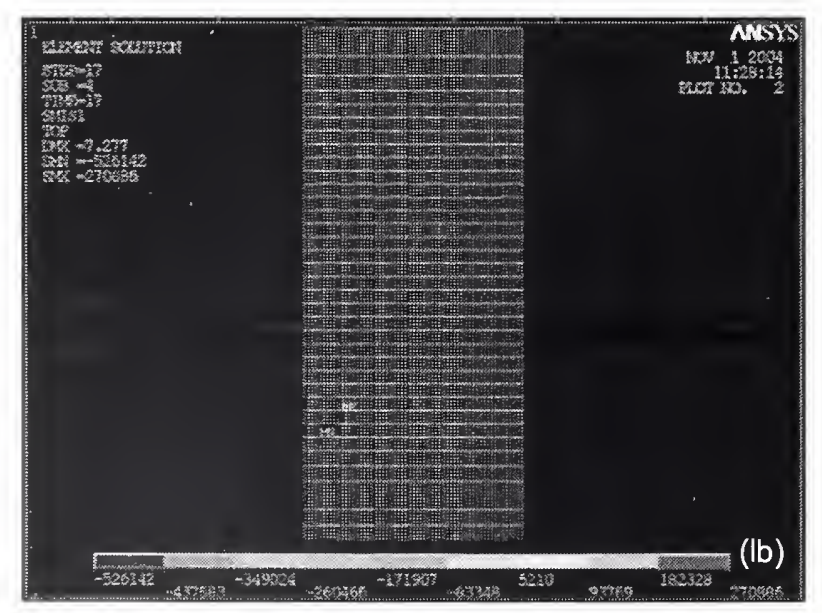

(e) North wall at $30 \mathrm{~min}$

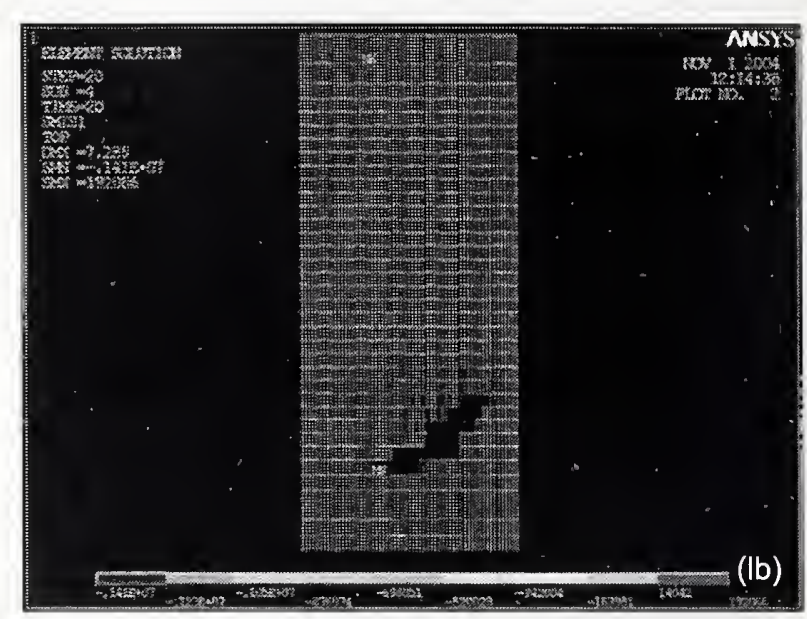

(b) South wall at $60 \mathrm{~min}$

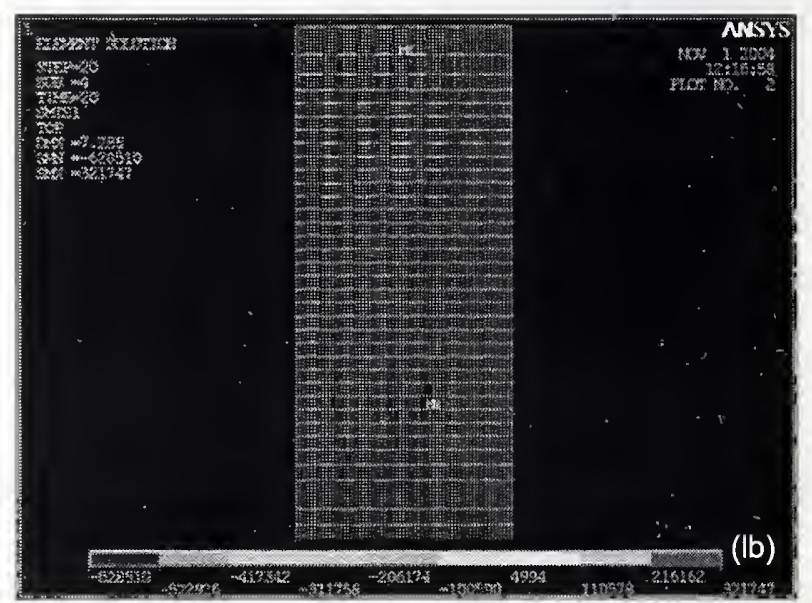

(d) East wall at $60 \mathrm{~min}$

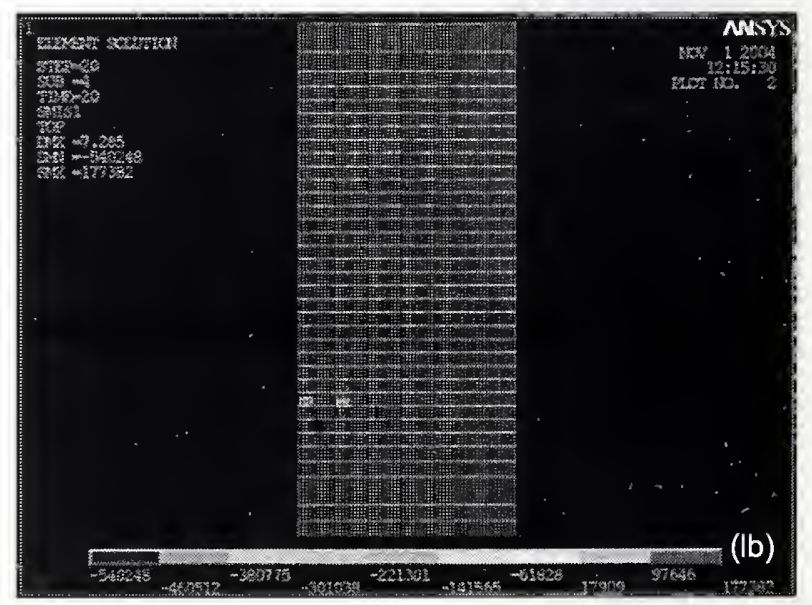

(f) North wall at $60 \mathrm{~min}$

Figure C-14. Axial force variation on the south, east, and north faces of WTC 2 at 30 min and 60 min of Case $C_{i}$ conditions (looking from outside; compression is negative). 


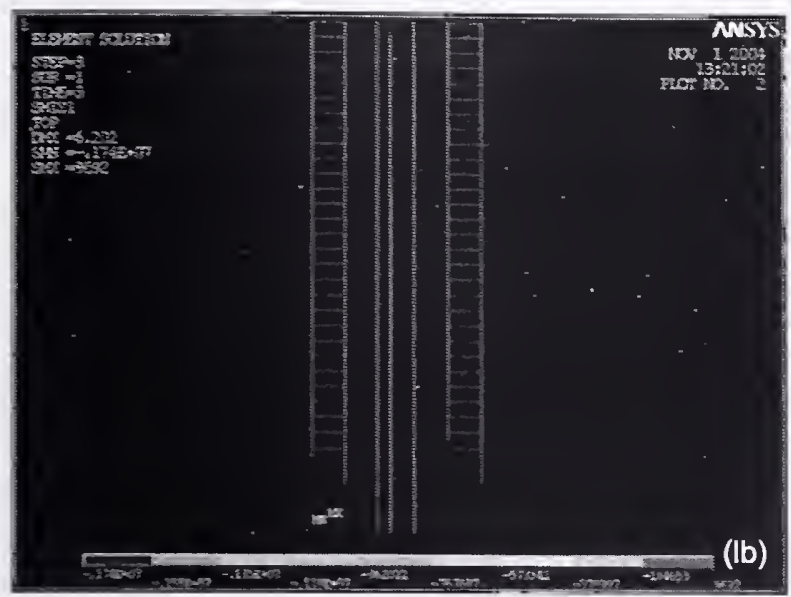

(a) 800 series columns before impact

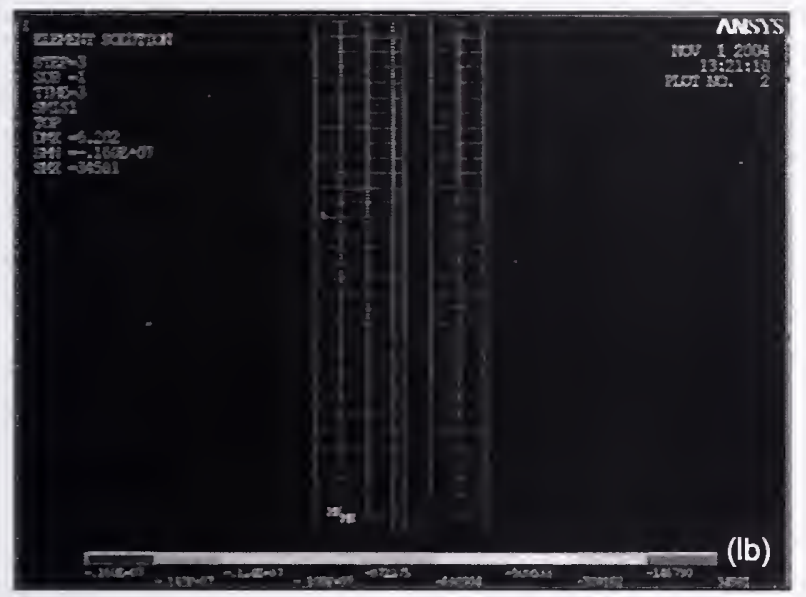

(c) 900 series columns before impact

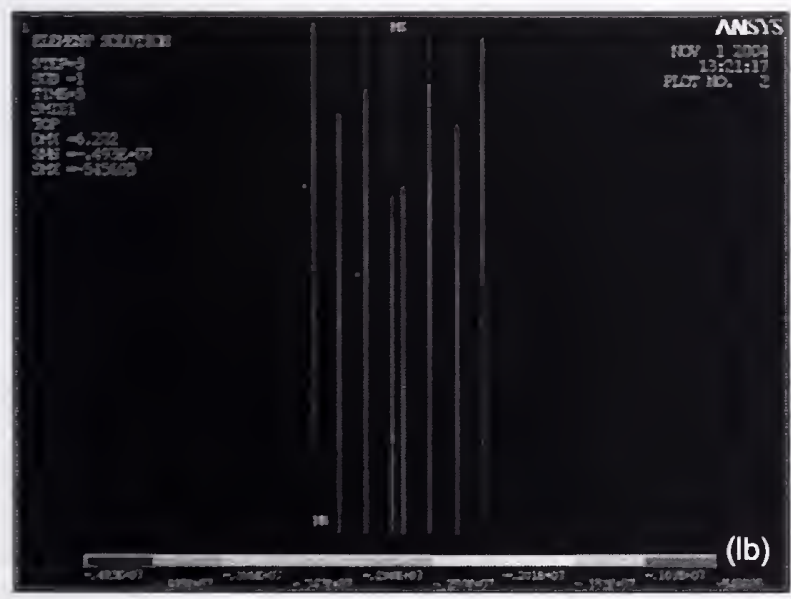

(e) 1000 series columns before impact

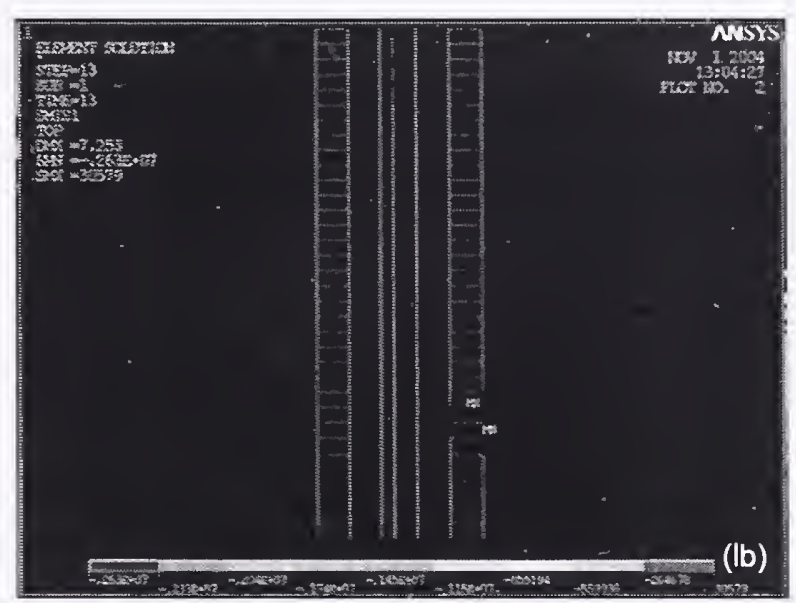

(b) 800 series columns after impact

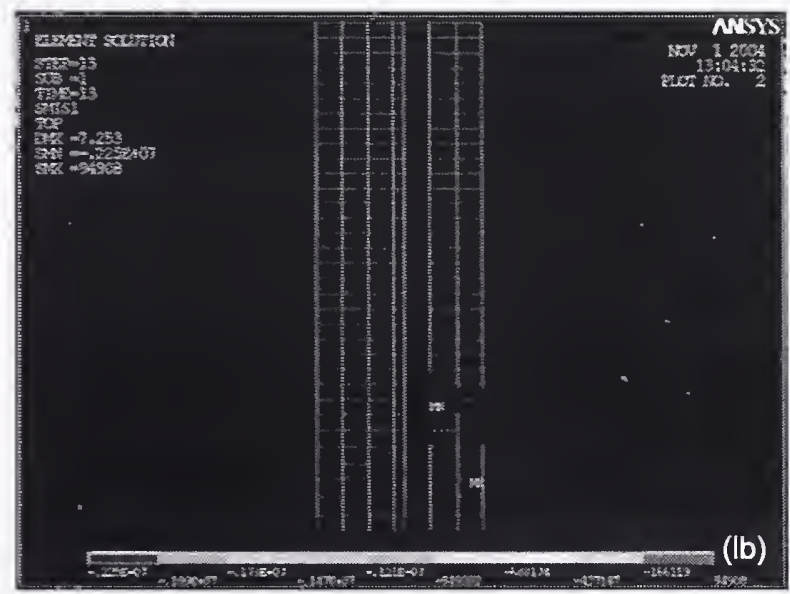

(d) 900 series columns after impact

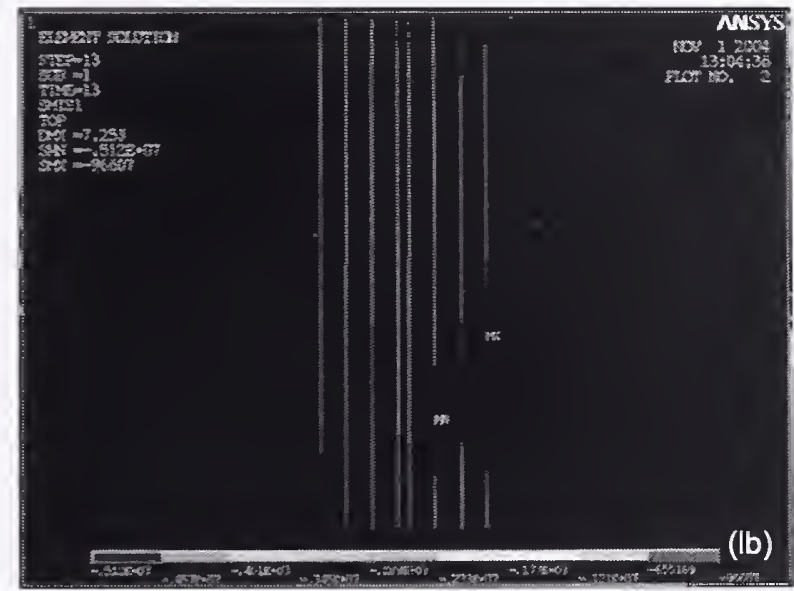

(f) 1000 series columns after impact

Figure C-15. Axial force variation on the 800,900 , and 1000 series core columns of WTC 2 before and after aircraft impact (looking from west; compression is negative). 


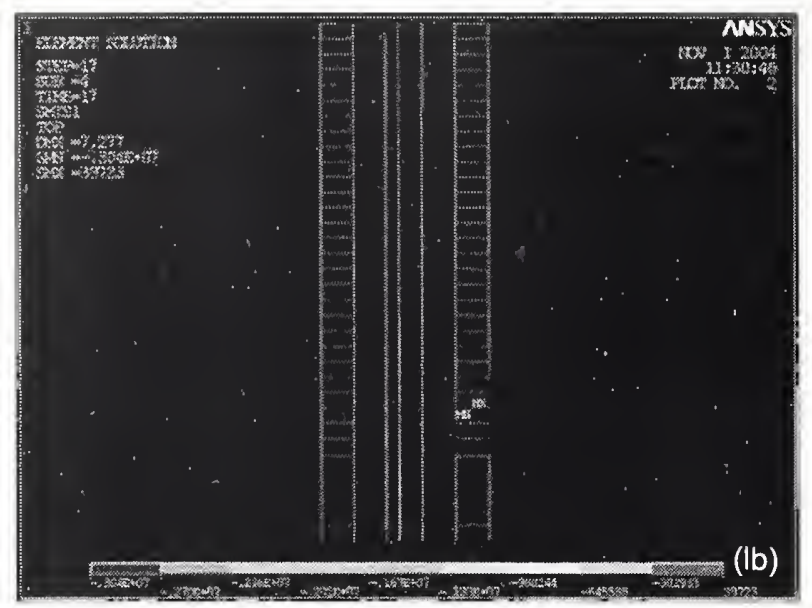

(a) 800 series columns at $30 \mathrm{~min}$

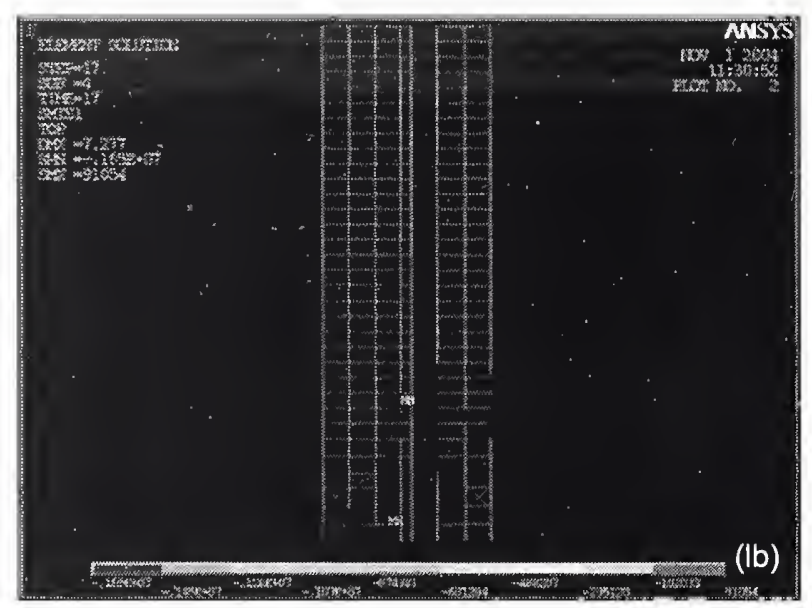

(c) 900 series columns at $30 \mathrm{~min}$

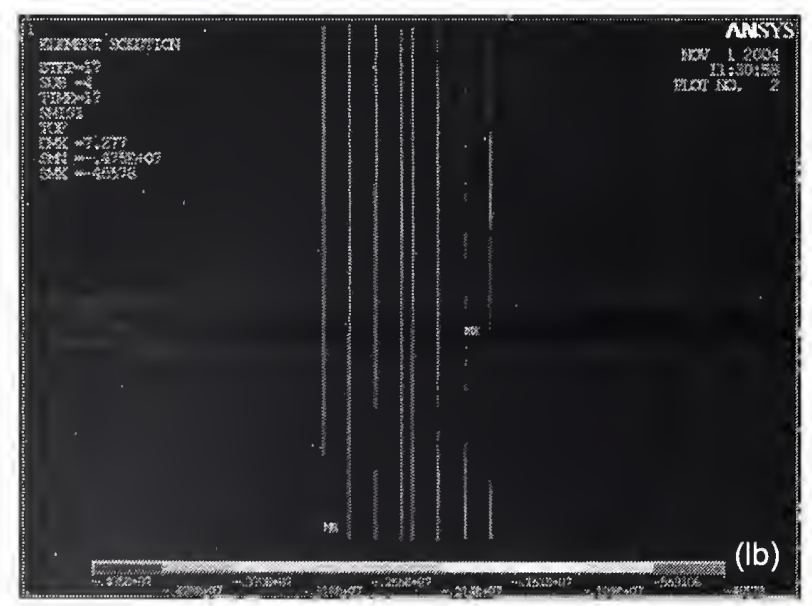

(e) 1000 series columns at $30 \mathrm{~min}$

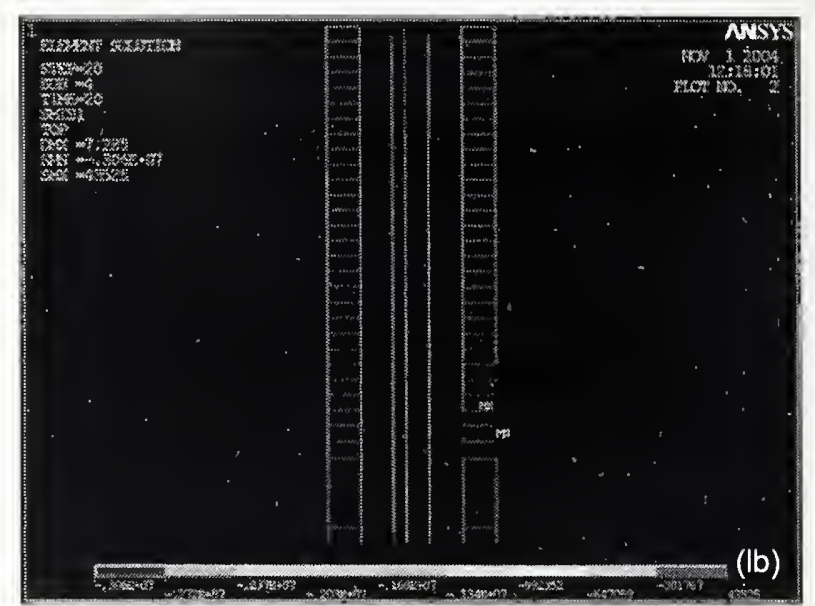

(b) 800 series columns at $60 \mathrm{~min}$

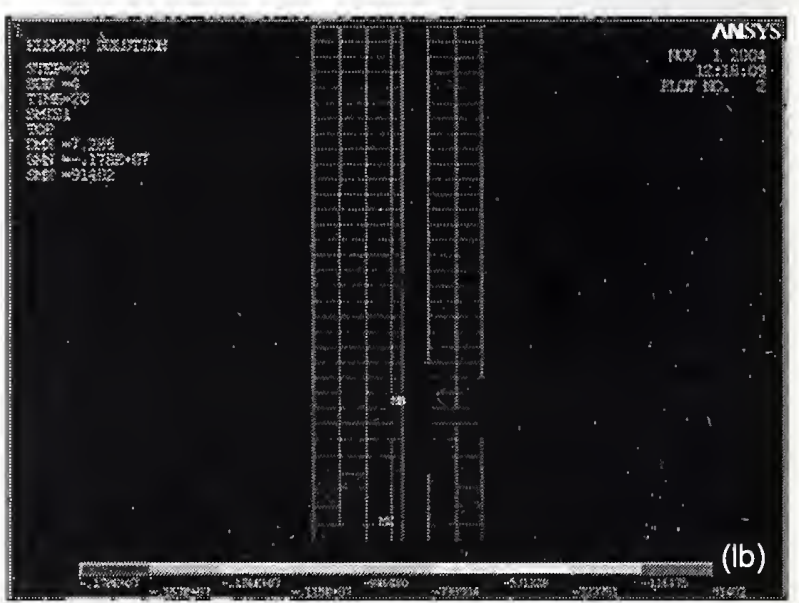

(d) 900 series columns at $60 \mathrm{~min}$

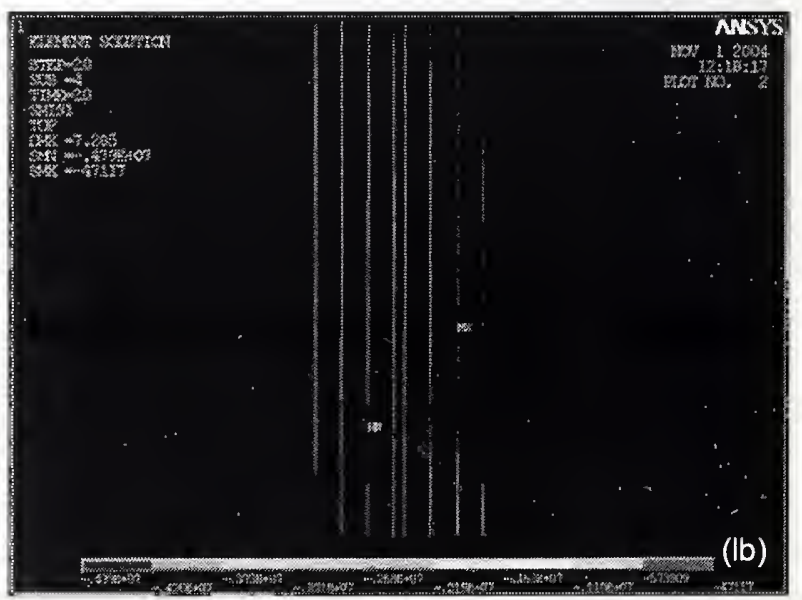

(f) 1000 series columns at $60 \mathrm{~min}$

Figure C-16. Axial force variation on the 800,900 , and 1000 series core columns of WTC 2 at $30 \mathrm{~min}$ and $60 \mathrm{~min}$ of Case $C_{i}$ conditions (looking from west; compression is negative). 


\begin{tabular}{|c|l|c|c|c|c|c|c|}
\hline Row & Analysis Stage & West & East & North & South & Core & Sum \\
\hline$(1)$ & Before Impact & 18779 & 18837 & 13714 & 13529 & 77563 & 142423 \\
$(2)$ & After Impact & 18951 & 20394 & 12892 & 11880 & 76831 & 140949 \\
$(3)$ & After Impact* & 18774 & 21384 & 12800 & 12528 & 75166 & 140652 \\
$(4)$ & $10 \mathrm{~min}$ & 19499 & 20616 & 12997 & 11333 & 76504 & 140949 \\
$(5)$ & $10 \mathrm{~min}(\mathrm{~b})$ & 19322 & 21606 & 12905 & 11981 & 74839 & 140652 \\
$(6)$ & $20 \mathrm{~min}$ & 19471 & 21616 & 12978 & 12130 & 74458 & 140652 \\
$(7)$ & $30 \mathrm{~min}$ & 19469 & 21637 & 13138 & 12309 & 74099 & 140653 \\
$(8)$ & $40 \mathrm{~min}$ & 19462 & 21692 & 13246 & 12427 & 73826 & 140653 \\
$(9)$ & $50 \mathrm{~min}$ & 19472 & 21795 & 13305 & 12506 & 73574 & 140653 \\
$(10)$ & $60 \mathrm{~min}$ & 19500 & 21887 & 13388 & 12538 & 73341 & 140653 \\
\hline
\end{tabular}

* Including the effects of additionally removed Columns 903 and 1003 in the core $(=(2)-(4)+(5))$.

(a) Above Floor 78

\begin{tabular}{|c|l|c|c|c|c|c|c|}
\hline Row & Analysis Stage & West & East & North & South & Core & Sum \\
\hline$(1)$ & Before Impact & 17642 & 17697 & 12558 & 12393 & 71319 & 131609 \\
$(2)$ & After Impact & 17755 & 19295 & 11818 & 10982 & 71249 & 131099 \\
$(3)$ & After Impact* & 17557 & 20305 & 11756 & 11599 & 69754 & 130972 \\
$(4)$ & $10 \mathrm{~min}$ & 18310 & 19529 & 11942 & 10396 & 70924 & 131101 \\
$(5)$ & $10 \mathrm{~min}(\mathrm{~b})$ & 18111 & 20539 & 11881 & 11014 & 69429 & 130974 \\
$(6)$ & $20 \mathrm{~min}$ & 18258 & 20479 & 11960 & 11230 & 69048 & 130974 \\
$(7)$ & $30 \mathrm{~min}$ & 18251 & 20462 & 12137 & 11435 & 68688 & 130973 \\
$(8)$ & $40 \mathrm{~min}$ & 18241 & 20502 & 12245 & 11570 & 68414 & 130972 \\
$(9)$ & $50 \mathrm{~min}$ & 18238 & 20603 & 12309 & 11660 & 68162 & 130972 \\
$(10)$ & $60 \mathrm{~min}$ & 18239 & 20690 & 12414 & 11700 & 67928 & 130972 \\
\hline
\end{tabular}

* Including the effects of additionally removed Columns 903 and 1003 in the core $(=(2)-(4)+(5))$.

(b) Above Floor 81

\begin{tabular}{|c|l|l|l|l|l|l|l|}
\hline Row & Analysis Stage & West & East & North & South & Core & Sum \\
\hline$(1)$ & Before lmpact & 16728 & 16782 & 11972 & 11820 & 67309 & 124611 \\
$(2)$ & After Impact & 16853 & 18337 & 11241 & 10561 & 67499 & 124490 \\
$(3)$ & After Impact* & 16664 & 19328 & 11184 & 11183 & 66115 & 124474 \\
$(4)$ & $10 \mathrm{~min}$ & 17398 & 18604 & 11346 & 9970 & 67172 & 124489 \\
$(5)$ & $10 \mathrm{~min}(\mathrm{~b})$ & 17209 & 19595 & 11288 & 10592 & 65788 & 124473 \\
$(6)$ & $20 \mathrm{~min}$ & 17356 & 19511 & 11365 & 10833 & 65409 & 124473 \\
$(7)$ & $30 \mathrm{~min}$ & 17351 & 19482 & 11537 & 11054 & 65048 & 124473 \\
$(8)$ & $40 \mathrm{~min}$ & 17344 & 19521 & 11644 & 11191 & 64773 & 124473 \\
$(9)$ & $50 \mathrm{~min}$ & 17350 & 19619 & 11702 & 11280 & 64521 & 124473 \\
$(10)$ & $60 \mathrm{~min}$ & 17370 & 19709 & 11787 & 11320 & 64287 & 124473 \\
\hline
\end{tabular}

* Including the effects of additionally removed Columns 903 and 1003 in the core $(=(2)-(4)+(5))$.

(c) Above Floor 83

Figure C-17. Total column loads in the core and exterior walls of WTC 2 at different floors and at different times for Case $C_{i}$ conditions (compression is positive). 


\begin{tabular}{|c|c|c|c|c|c|c|c|c|c|}
\hline $\begin{array}{c}\text { COL501 } \\
0 \\
0.4\end{array}$ & $\stackrel{\circ}{0.4}$ & $\begin{array}{c}0 \\
0.4\end{array}$ & & $\begin{array}{c}0 \\
0.4\end{array}$ & 0.4 & & $\stackrel{0}{0.4}$ & $\stackrel{0}{0.4}$ & $\begin{array}{c}\text { COL508 } \\
0 \\
0.4\end{array}$ \\
\hline $\begin{array}{c}0 \\
0.4\end{array}$ & $\begin{array}{c}0 \\
0.4\end{array}$ & $\begin{array}{c}0 \\
0.4\end{array}$ & & $\begin{array}{c}0 \\
0.4\end{array}$ & $\begin{array}{c}0 \\
0.4\end{array}$ & & $\begin{array}{c}0 \\
0.4\end{array}$ & $\begin{array}{c}0 \\
0.4\end{array}$ & $\begin{array}{c}0 \\
0.4\end{array}$ \\
\hline $\begin{array}{c}0.4 \\
0\end{array}$ & $\begin{array}{c}0.4 \\
0\end{array}$ & & $\begin{array}{c}0.4 \\
0\end{array}$ & $\begin{array}{c}0.4 \\
0\end{array}$ & $\begin{array}{c}0.3 \\
0\end{array}$ & $\begin{array}{c}0.4 \\
0\end{array}$ & & $\begin{array}{c}0.4 \\
0\end{array}$ & $\begin{array}{c}0.4 \\
0\end{array}$ \\
\hline $\begin{array}{c}\circ \\
0.3\end{array}$ & $\begin{array}{c}0 \\
0.4\end{array}$ & & $\begin{array}{c}\circ \\
0.4\end{array}$ & & $\begin{array}{c}0 \\
0.4\end{array}$ & $\begin{array}{c}\circ \\
0.4\end{array}$ & & $\begin{array}{c}0 \\
0.4\end{array}$ & $\begin{array}{c}\circ \\
0.3\end{array}$ \\
\hline $\begin{array}{c}0.4 \\
0\end{array}$ & $\begin{array}{c}0.4 \\
0\end{array}$ & $\begin{array}{c}0.4 \\
0\end{array}$ & & $\begin{array}{c}0.4 \\
0\end{array}$ & $\begin{array}{c}0.3 \\
0\end{array}$ & & $\begin{array}{c}0.4 \\
0\end{array}$ & $\begin{array}{c}0.4 \\
0\end{array}$ & $\begin{array}{c}0.4 \\
0\end{array}$ \\
\hline $\begin{array}{c}0.4 \\
0\end{array}$ & $\begin{array}{c}0.4 \\
0\end{array}$ & $\begin{array}{c}0.4 \\
0\end{array}$ & & $\begin{array}{c}0.4 \\
0\end{array}$ & $\begin{array}{c}0.4 \\
0\end{array}$ & & $\begin{array}{c}0.4 \\
0\end{array}$ & $\begin{array}{c}0.4 \\
0\end{array}$ & $\begin{array}{c}0.4 \\
0\end{array}$ \\
\hline COL1001 & 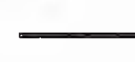 & $N$ & & & & & & & COL 1008 \\
\hline
\end{tabular}

(a) Before impact

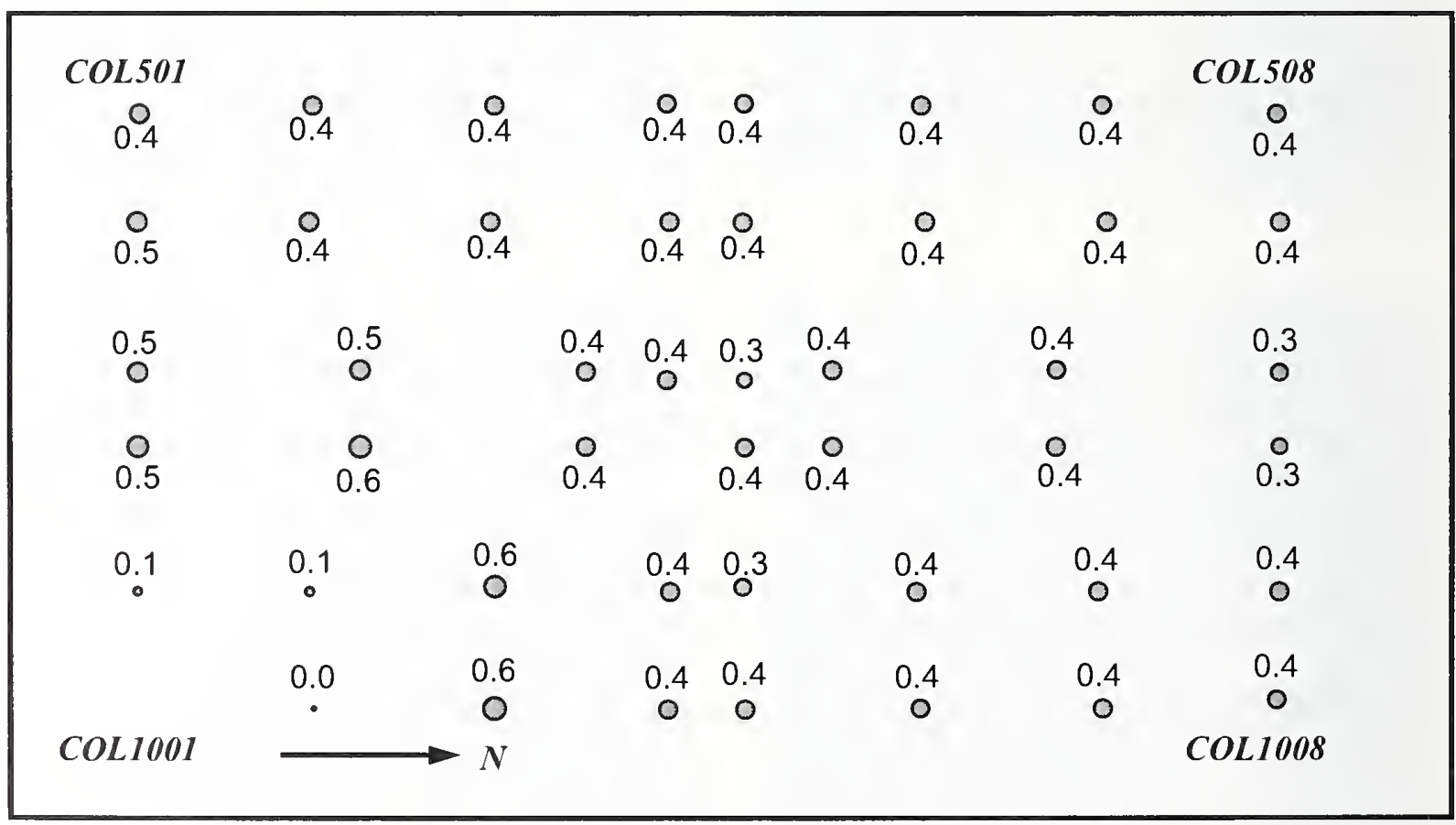

(b) After impact

Figure C-18. Ratios of axial elastic-plus-plastic strain to temperature-dependent yield strain for the core columns at Floor 82 of WTC 2 before and after aircraft impact. 


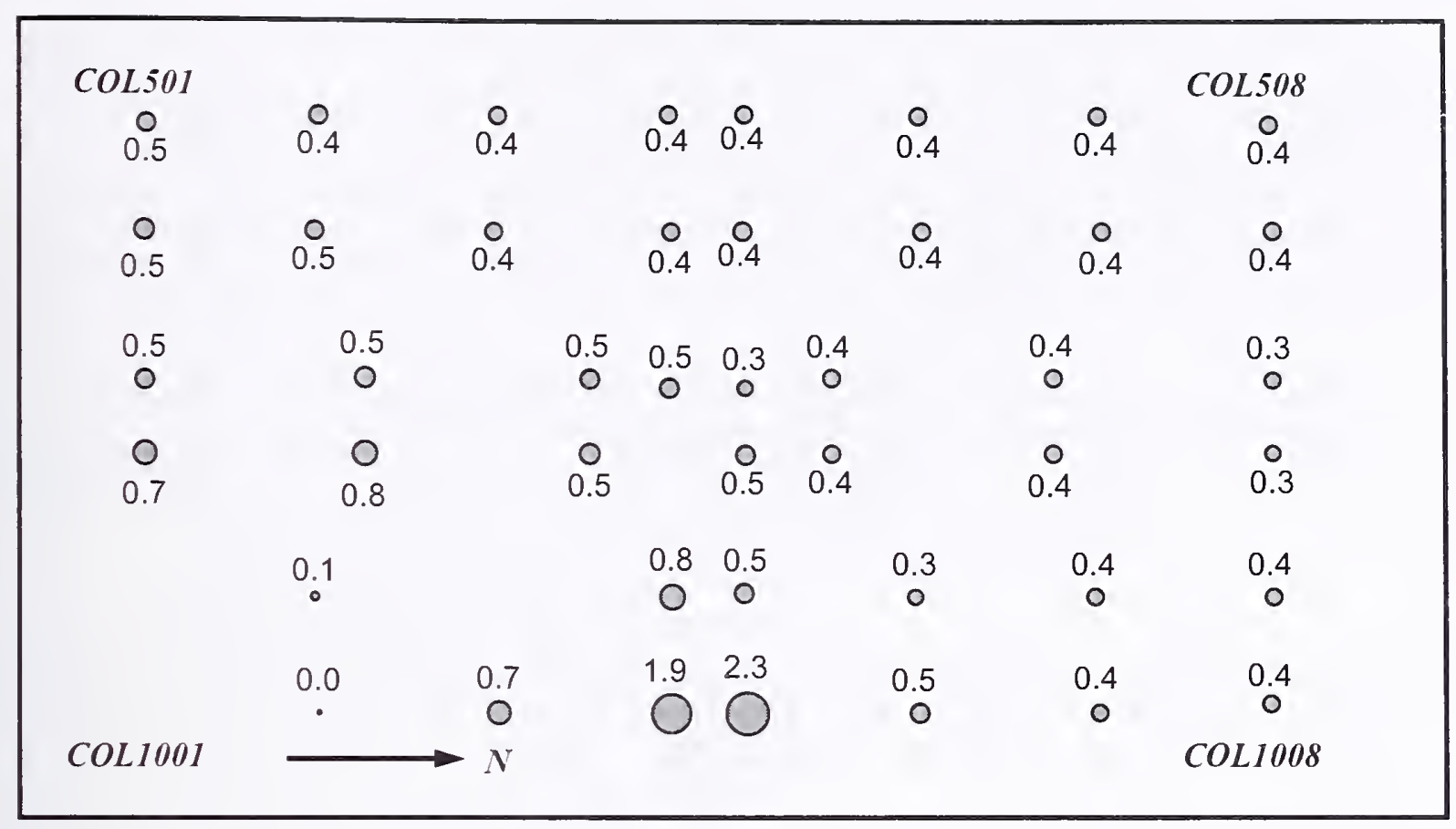

(a) At $10 \min (b)$

\begin{tabular}{|c|c|c|c|c|c|c|c|c|c|}
\hline COL 501 & & & & & & & & & COL 508 \\
\hline $\begin{array}{c}0 \\
0.5\end{array}$ & $\begin{array}{c}0 \\
0.4\end{array}$ & $\begin{array}{c}0 \\
0.4\end{array}$ & & $\stackrel{0}{0.4}$ & $\begin{array}{c}0 \\
0.4\end{array}$ & & $\begin{array}{c}0 \\
0.4\end{array}$ & $\begin{array}{c}0 \\
0.4\end{array}$ & $\begin{array}{c}0 \\
0.4\end{array}$ \\
\hline $\begin{array}{c}0 \\
0.5\end{array}$ & $\begin{array}{c}0 \\
0.4\end{array}$ & $\begin{array}{c}0 \\
0.4\end{array}$ & & $\begin{array}{c}0 \\
0.4\end{array}$ & $\begin{array}{c}0 \\
0.4\end{array}$ & & $\begin{array}{c}0 \\
0.4\end{array}$ & $\begin{array}{c}0 \\
0.4\end{array}$ & $\begin{array}{c}0 \\
0.4\end{array}$ \\
\hline $\begin{array}{c}0.5 \\
0\end{array}$ & $\begin{array}{c}0.5 \\
0\end{array}$ & & $\begin{array}{c}0.5 \\
0\end{array}$ & $\begin{array}{c}0.5 \\
0\end{array}$ & $\begin{array}{c}0.3 \\
0\end{array}$ & $\begin{array}{c}0.4 \\
0\end{array}$ & & $\begin{array}{c}0.4 \\
0\end{array}$ & $\begin{array}{c}0.3 \\
0\end{array}$ \\
\hline \multirow[t]{3}{*}{$\begin{array}{c}0 \\
0.8\end{array}$} & $\begin{array}{c}0 \\
0.7\end{array}$ & & $\begin{array}{c}0 \\
0.5\end{array}$ & & 0.5 & $\begin{array}{l}\circ \\
0.4\end{array}$ & & $\begin{array}{c}0 \\
0.4\end{array}$ & $\begin{array}{l}0 \\
0.3\end{array}$ \\
\hline & $\underset{0}{0.1}$ & & & $\begin{array}{c}0.8 \\
0\end{array}$ & $\begin{array}{c}0.6 \\
0\end{array}$ & & $\begin{array}{c}0.2 \\
0\end{array}$ & 0.3 & $\begin{array}{c}0.3 \\
0\end{array}$ \\
\hline & 0.0 & $\begin{array}{c}0.7 \\
0\end{array}$ & & & 9 & & $\begin{array}{c}0.6 \\
0\end{array}$ & $\begin{array}{c}0.4 \\
0\end{array}$ & $\begin{array}{c}0.3 \\
0\end{array}$ \\
\hline COL1001 & 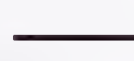 & & & & & & & & COL1008 \\
\hline
\end{tabular}

(b) At $30 \mathrm{~min}$

Figure C-19. Ratios of axial elastic-plus-plastic strain to temperature-dependent yield strain for the core columns at Floor 82 of WTC 2 at $10 \mathrm{~min}$ and $30 \mathrm{~min}$ of Case $C_{i}$ conditions. 


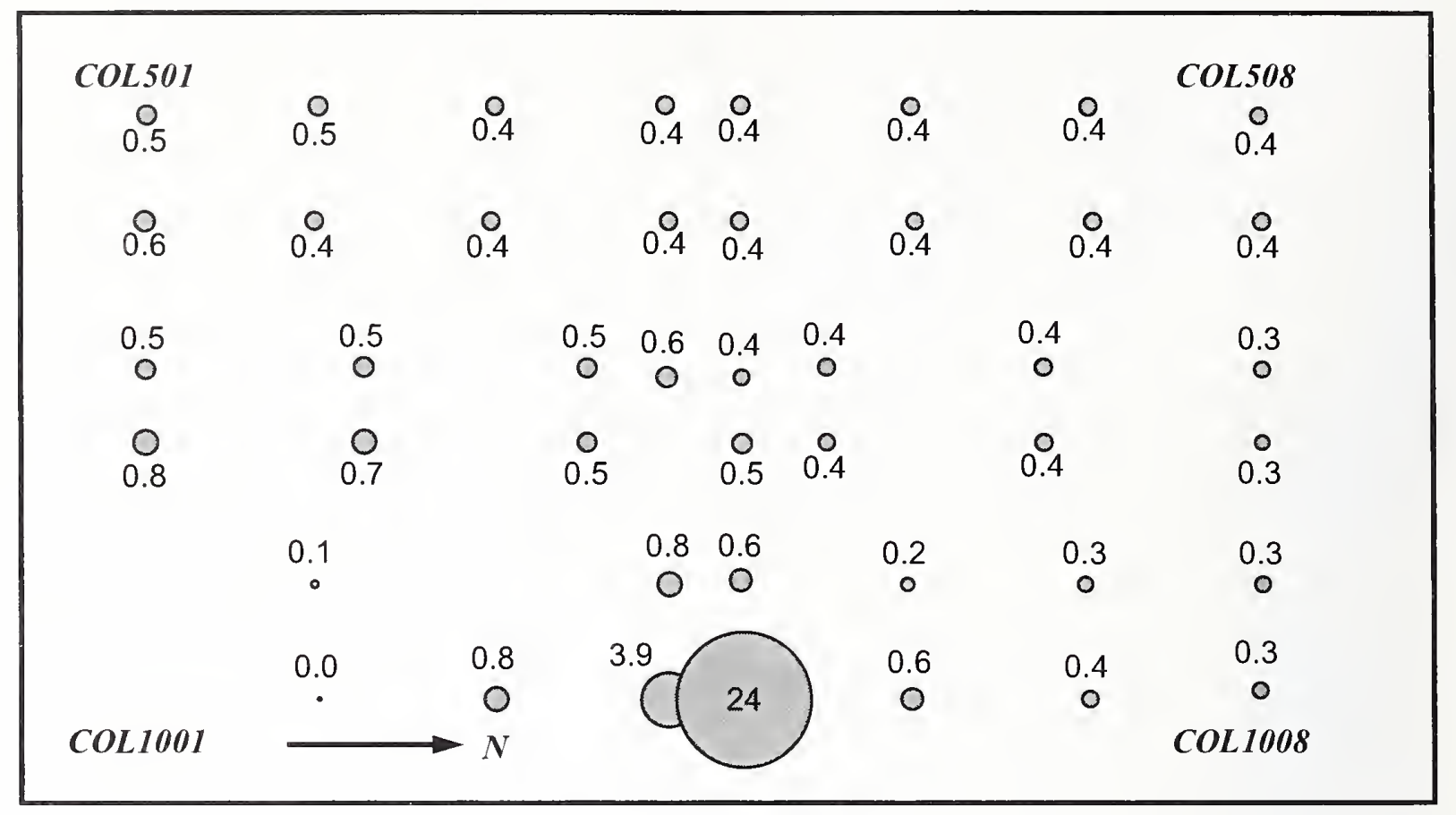

(a) At $40 \mathrm{~min}$

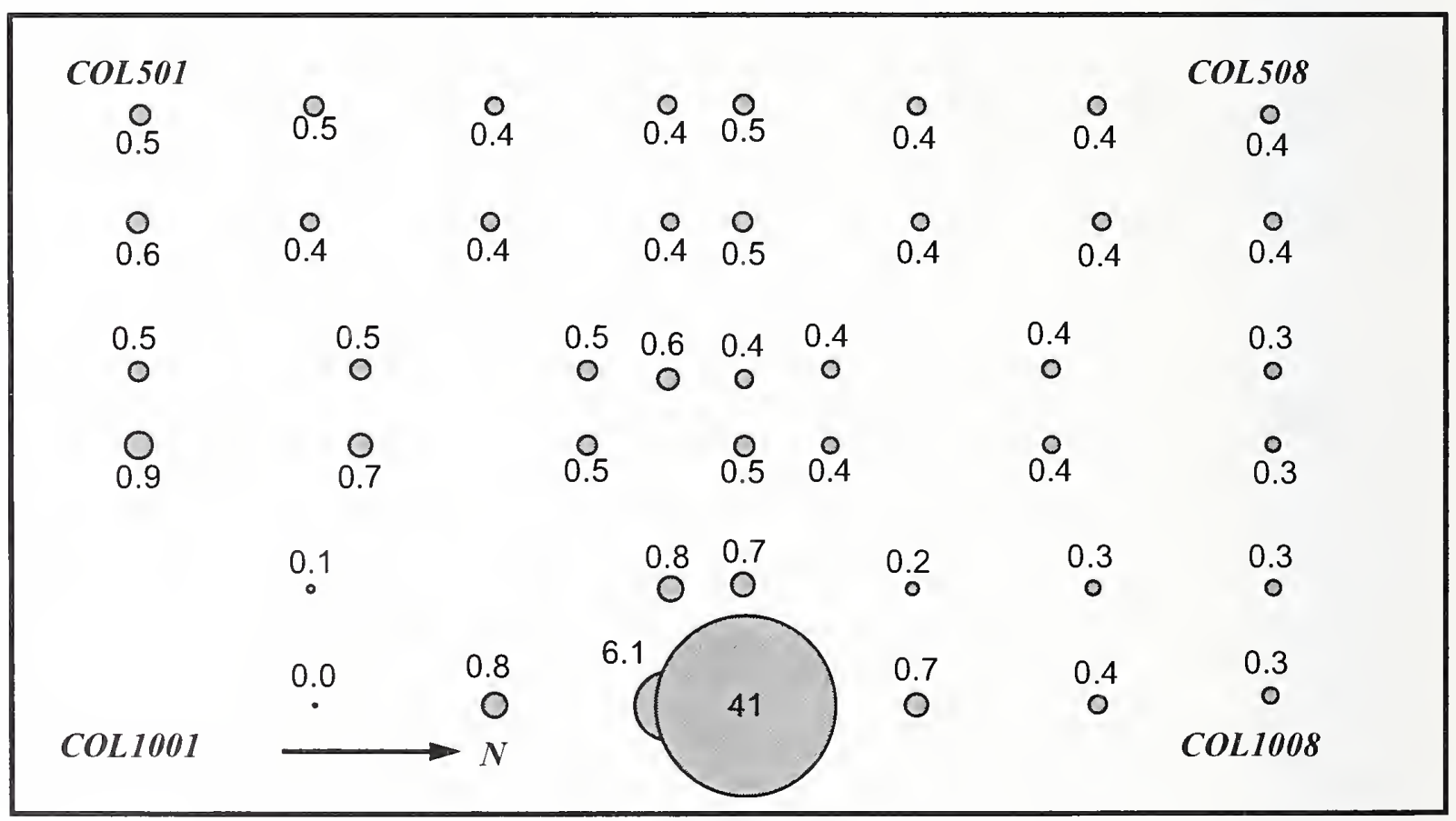

(b) At $60 \mathrm{~min}$

Figure C-20. Ratios of axial elastic-plus-plastic strain to temperature-dependent yield strain for the core columns at Floor 82 of WTC 2 at $40 \mathrm{~min}$ and $60 \mathrm{~min}$ of Case $\mathrm{C}_{i}$ conditions. 


\section{C.2.2 Case $D_{i}$ Temperature Condition and Case $C_{i}$ Structural Damage Condition}

Table C-3 summarizes the sequence of analyses that were performed with the WTC 2 ANSYS global model with the revised Case $C_{i}$ structural damage condition from Step 4, and Casc $D_{i}$ temperature condition. Revised Case $C_{i}$ structural damage refers to the removal of core columns 903 and 1003 in addition to the other core columns that were severed after the aircraft impact as shown in Section 2.2. Case $\mathrm{D}_{\mathrm{i}}$ structural damage condition was not used as this structural damage condition was not available at the time of analyses.

Table C-3. Analysis Steps of WTC 2 ANSYS global model with Case $D_{i}$ temperature condition and Case $\mathrm{C}_{\mathrm{i}}$ structural damage condition.

\begin{tabular}{|c|l|}
\hline Analysis Step & \multicolumn{1}{|c|}{ Description } \\
\hline 1 & WTC 2 model below Floor 106 was analyzed under its own self-weight. \\
\hline 2 & $\begin{array}{l}\text { Structures above Floor } 106 \text { were added in a stress-free state, and the model was analyzed for dead } \\
\text { load including those above Floor } 106 .\end{array}$ \\
\hline 3 & Superimposed dead load plus 25 percent of the design live loads were superimposed on the model. \\
\hline 4 & $\begin{array}{l}\text { Columns (including the } 1003 \text { and } 903 \text { core columns). spandrels, and floor elements that were } \\
\text { severed during aircraft impact were removed, and the model was analyzed (see Section } 2.2 \text { for the } \\
\text { revised Case } C_{\text {i }} \text { structural damage condition). }\end{array}$ \\
\hline 5 & Column and spandrel temperatures were ramped linearly up to temperatures at 10 min. \\
\hline 6 & Column and spandrel temperatures were changed linearly from temperatures at 10 min to 20 min. \\
\hline 7 & Column and spandrel temperatures were changed linearly from temperatures at 20 min to 30 min. \\
\hline 9 & Column and spandrel temperatures were changed linearly from temperatures at 30 min to $40 \mathrm{~min}$. \\
\hline 10 & Column and spandrel temperatures were changed linearly from temperatures at 40 min to 50 min. \\
\hline
\end{tabular}

The model was first analyzed for the effects of the construction sequence (analysis steps 1, 2, and 3). The results of analysis step 3 represent the state of the structure before the aircraft impact. In analysis step 4 , the columns, spandrels, and floor elements that were severed during the aircraft impact were removed, and the structure was reanalyzed to redistribute the loads to other members. This analysis represented the structure state after the aircraft impact. The column and spandrel temperatures were applied to the model (analysis steps 5 through 10). The column and spandrel temperatures were provided by NIST at 10 min intervals. Temperatures were calculated by linear interpolation for times in between 10 min intervals. Temperature analyses were performed at every $10 \mathrm{~min}$ up to $60 \mathrm{~min}$. Temperatures were not applied to the floor elements, as discussed earlier.

The results of the WTC 2 global model analyses with Case $\mathrm{D}_{\mathrm{i}}$ temperature condition and the revised Case $\mathrm{C}_{\mathrm{i}}$ structural damage condition are summarized in Figs. $\mathrm{C}-21$ through $\mathrm{C}-32$.

Figure C-21 shows the vertical displacement at different analysis steps. The maximum vertical displacement after the aircraft impact increased from $7.0 \mathrm{in.} \mathrm{to} 7.7 \mathrm{in.}$ with the inclusion of the additional severed core columns (1003 and 903). The 7.7 in. maximum vertical displacement after aircraft impact gradually increased to $9.2 \mathrm{in}$. at the end of the $60 \mathrm{~min}$ temperature analysis. The exterior columns between Floor 79 and Floor 83 thermally expanded with temperature; however, the core columns shortened due to plastic deformation, and the plastic strains in 1000 series core columns were greater than the thermal expansion strains. Figures $\mathrm{C}-22$ and $\mathrm{C}-23$ show the vertical displacements for the south and east exterior walls after aircraft impact, and at $30 \mathrm{~min}, 50 \mathrm{~min}$, and $60 \mathrm{~min}$. The displacements of these 
exterior walls were typically at their maximum after aircraft impact and gradually decreased to their minimum value with time up to $50 \mathrm{~min}$ and then increased slightly beyond $50 \mathrm{~min}$ up to $60 \mathrm{~min}$, as temperatures reduced.

Figures $\mathrm{C}-24, \mathrm{C}-25$, and $\mathrm{C}-26$ show the axial load variation over the south, east, and north exterior wall columns and spandrels after impact and at $10 \mathrm{~min}, 20 \mathrm{~min}, 30 \mathrm{~min}, 50 \mathrm{~min}$, and $60 \mathrm{~min}$. The maximum column load on the east exterior wall columns increased from 485 kip after aircraft impact to about $880 \mathrm{kip}$ at the end of $30 \mathrm{~min}$ and then dropped down to $800 \mathrm{kip}$ at the end of $60 \mathrm{~min}$. The increase in column forces was mostly concentrated at the center portion of the east wall. The maximum column load on the north exterior wall columns increased from $355 \mathrm{kip}$ after aircraft impact to about $550 \mathrm{kip}$ at the end of $30 \mathrm{~min}$ and then dropped down to $440 \mathrm{kip}$ at $50 \mathrm{~min}$; it then increased again to $700 \mathrm{kip}$ at the end of $60 \mathrm{~min}$.

Figures $\mathrm{C}-27, \mathrm{C}-28$ and $\mathrm{C}-29$ show the axial load variations over the 800,900 , and 1000 core column series after aircraft impact and at $10 \mathrm{~min}, 20 \mathrm{~min}, 30 \mathrm{~min}, 50 \mathrm{~min}$, and $60 \mathrm{~min}$. Over the duration of the fires, the maximum column load after impact increased from 5,200 kip to 6,250 kip in the 1000 core column series and from 1,700 kip to 2,500 kip in the 900 core column series, and decreased slightly from 3,400 kip to $3,200 \mathrm{kip}$ in the 800 core column series.

Figure $\mathrm{C}-30$ shows the total column loads in the core and the north, south, east, and west exterior walls for different floors. Total column loads below Floor 83 after aircraft impact did not add up to the total column loads before the aircraft impact. This difference is equal to the self-weight of severed members and floor loads applied to severed columns.

In Floor 83, the temperature effect alone resulted in an unloading of the core columns by 2,000 kip and increased the total load by $630 \mathrm{kip}$ on the south exterior wall, by $300 \mathrm{kip}$ on the west exterior wall, by 540 kip on the north exterior wall, and by 530 kip on the east exterior wall.

Figures $\mathrm{C}-31$ and $\mathrm{C}-32$ show the ratio of elastic-plus-plastic strain to the temperature-dependent yield strain of the core columns between Floor 82 and Floor 83 at $10 \mathrm{~min}, 20 \mathrm{~min}, 50 \mathrm{~min}$, and $60 \mathrm{~min}$. After $10 \mathrm{~min}$, except for Core Columns 801, 1004, and 1005, the strain ratios in the core columns were all less than 1.0. With increasing time, more columns from east side and center region of the core area started to show strain ratios greater than 1.0, indicating the onset of plasticity. A maximum strain ratio of 41 was reached between $40 \mathrm{~min}$ and $60 \mathrm{~min}$ at Column 1005. Higher strain ratios were clustered around the east side of the core, indicating a tendency of the core to tilt toward east. 


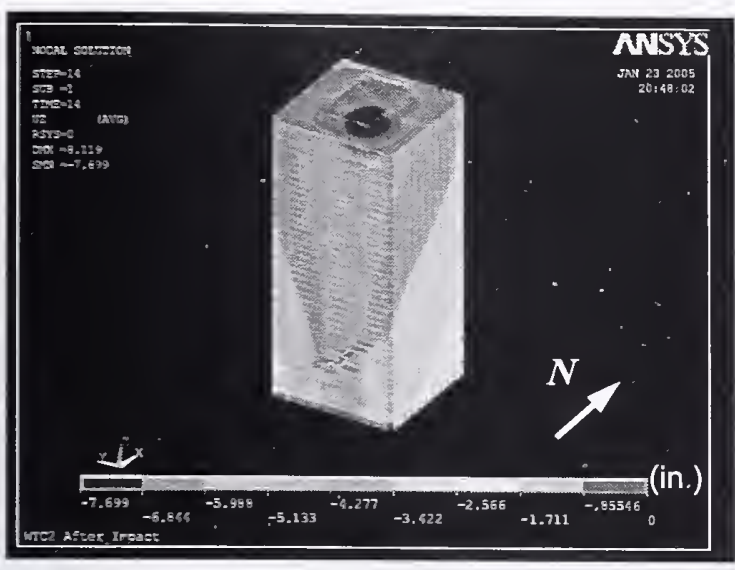

(a) After impact

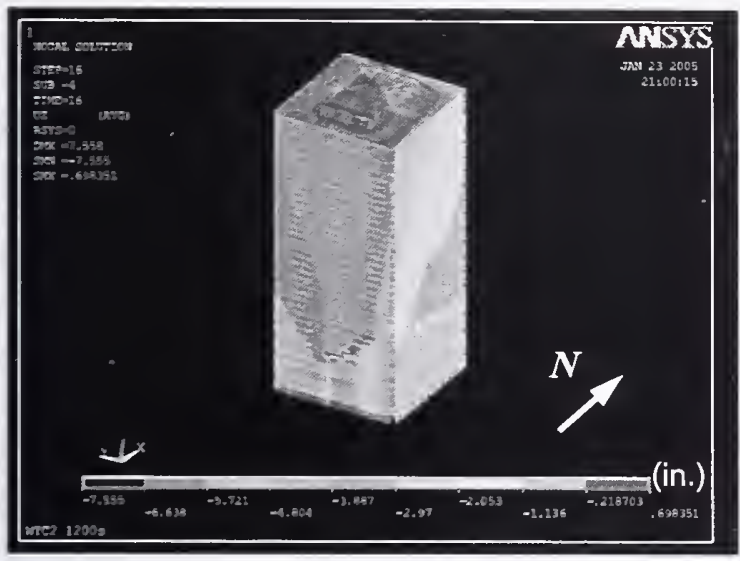

(c) At $20 \mathrm{~min}$

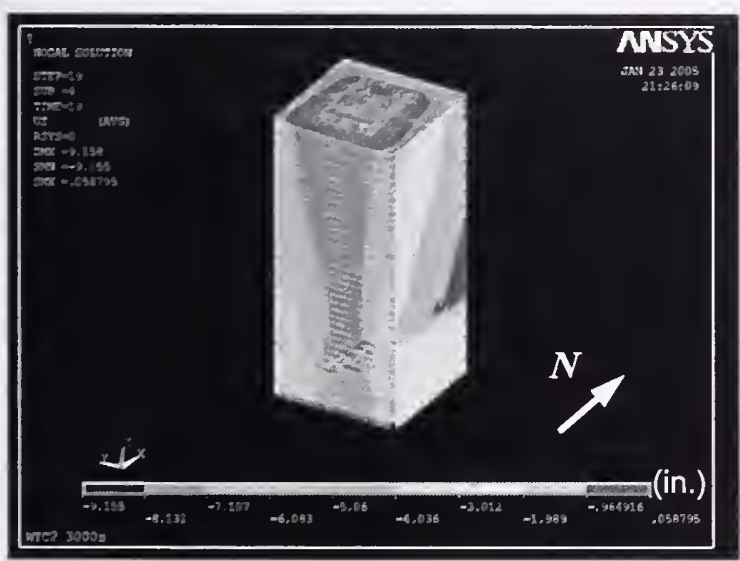

(e) At $50 \mathrm{~min}$

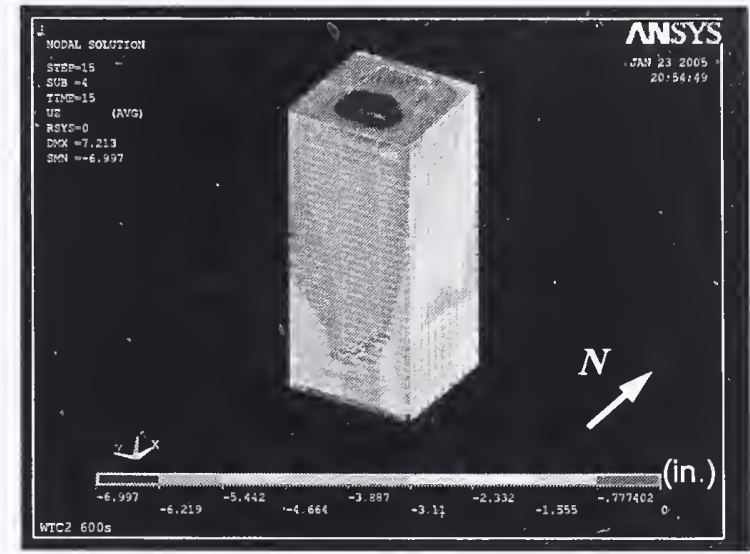

(b) At $10 \mathrm{~min}$

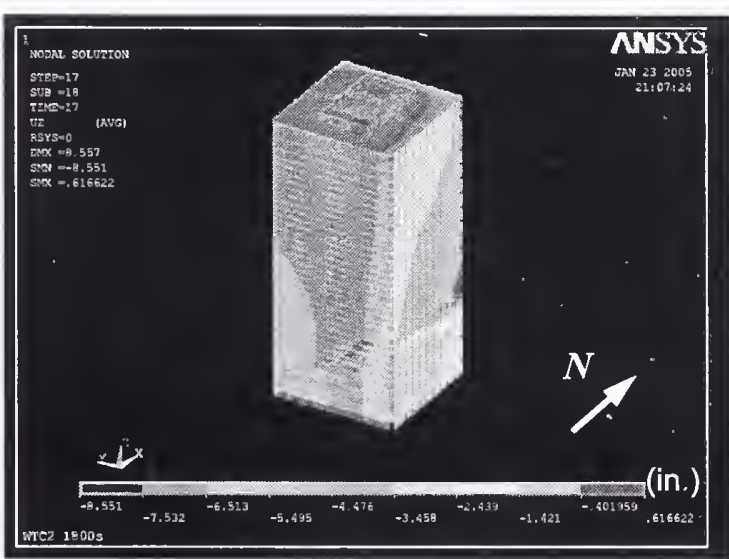

(d) At $30 \mathrm{~min}$

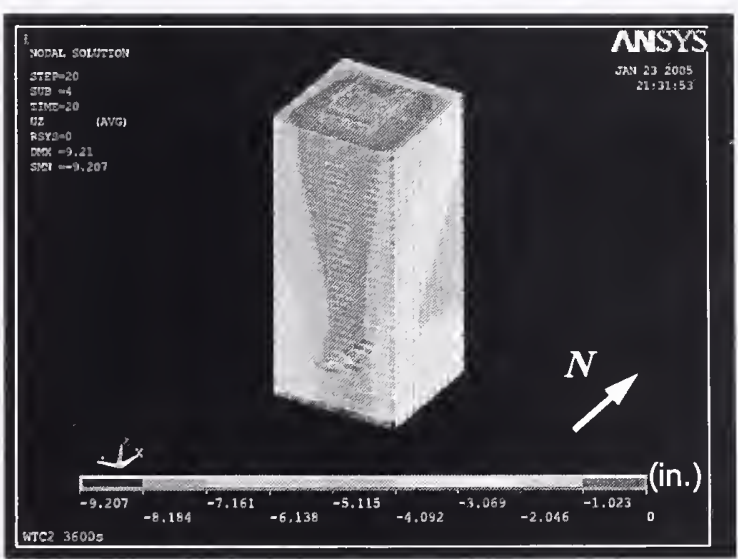

(f) At $60 \mathrm{~min}$

Figure C-21. Vertical displacement at different stages of WTC 2 for Case $D_{i}$ temperature condition and the revised $C_{\text {ase }} C_{i}$ structural damage condition (downward displacement is negative). 


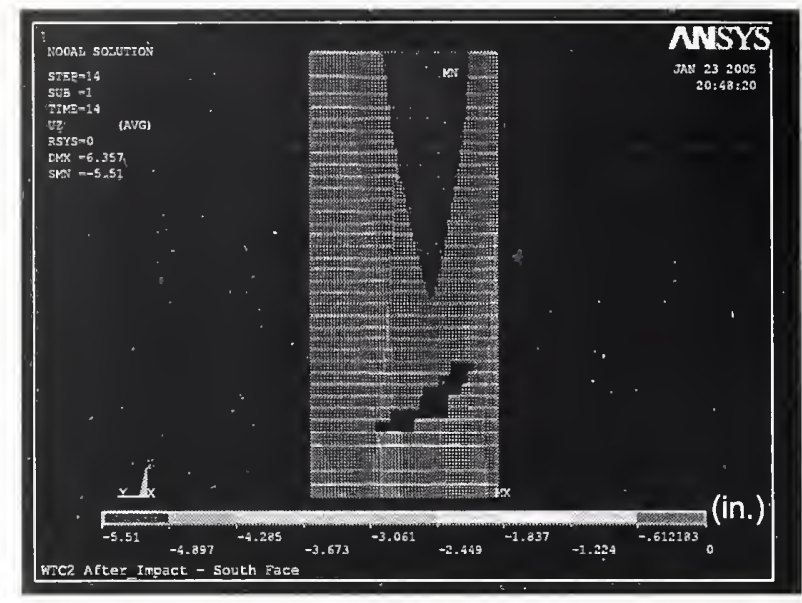

(a) South wall after impact

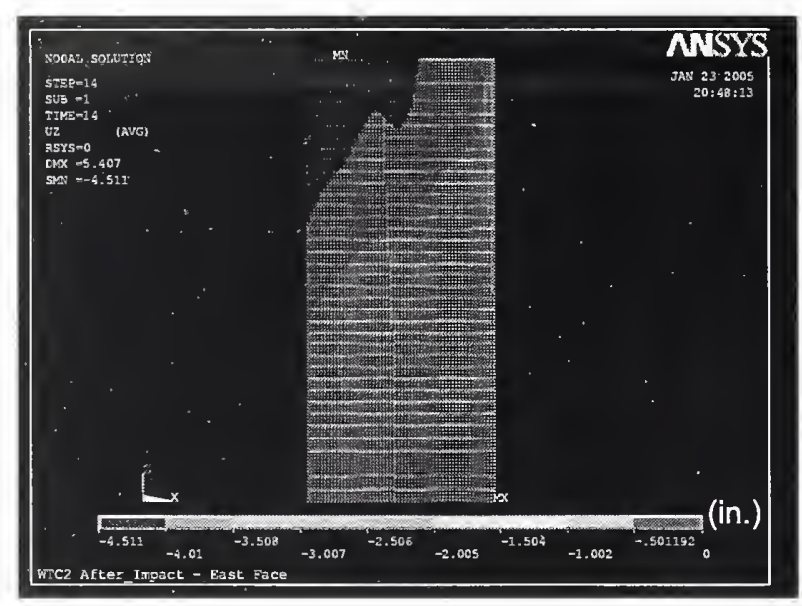

(c) East wall after impact

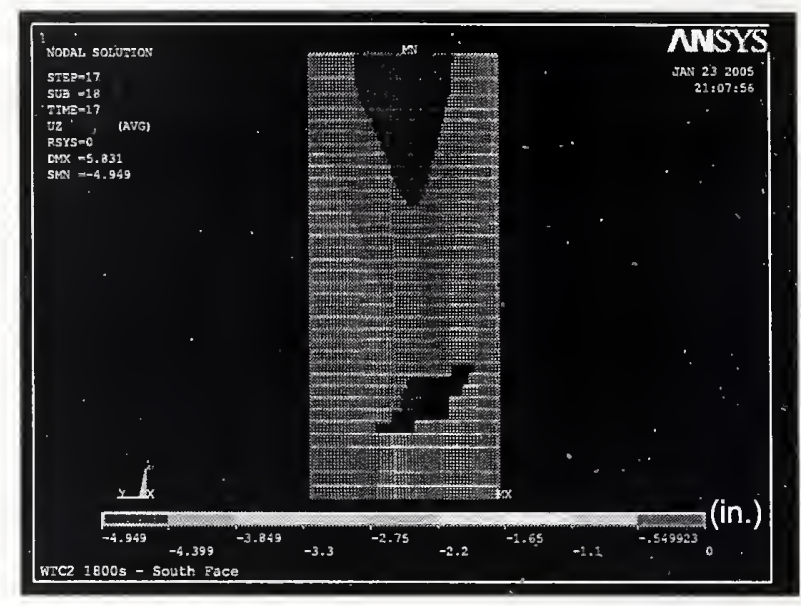

(b) South wall at $30 \mathrm{~min}$

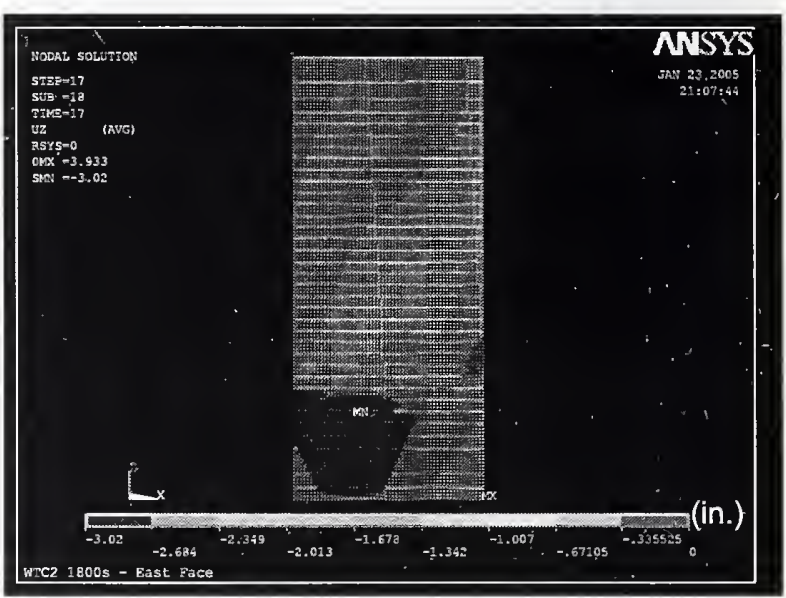

(d) East wall at $30 \mathrm{~min}$

Figure C-22. Vertical displacement on the south and east faces of WTC 2 after aircraft impact and at 30 min of Case $D_{i}$ temperature condition and the revised

Case $C_{i}$ structural damage condition (looking from outside; downward displacement is negative). 


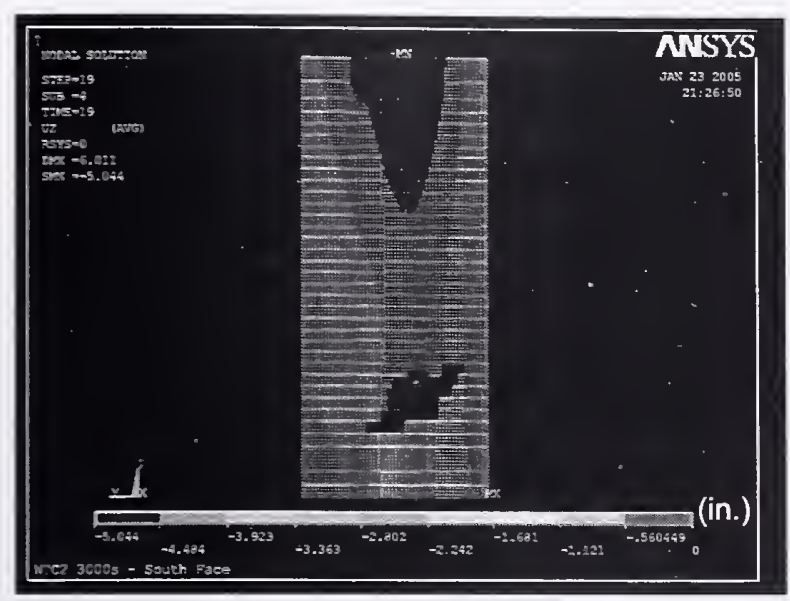

(a) South wall at $50 \mathrm{~min}$

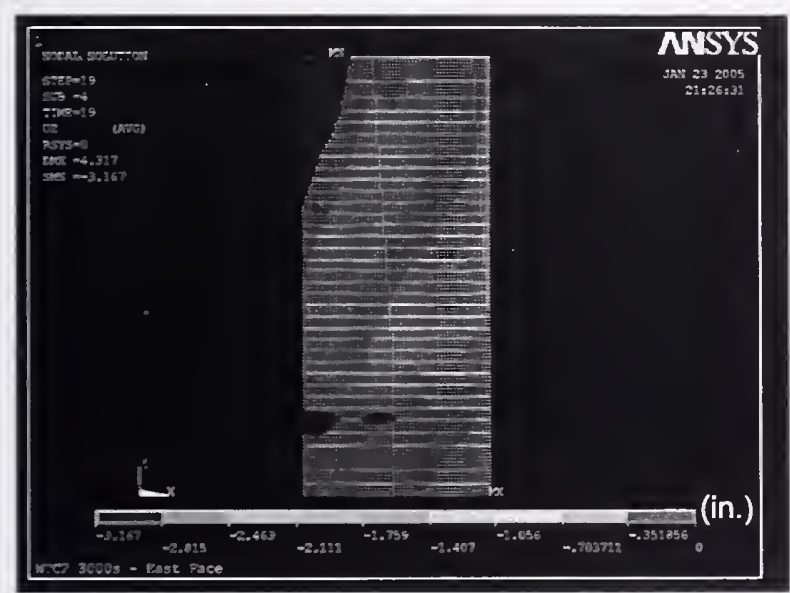

(c) East wall at $50 \mathrm{~min}$

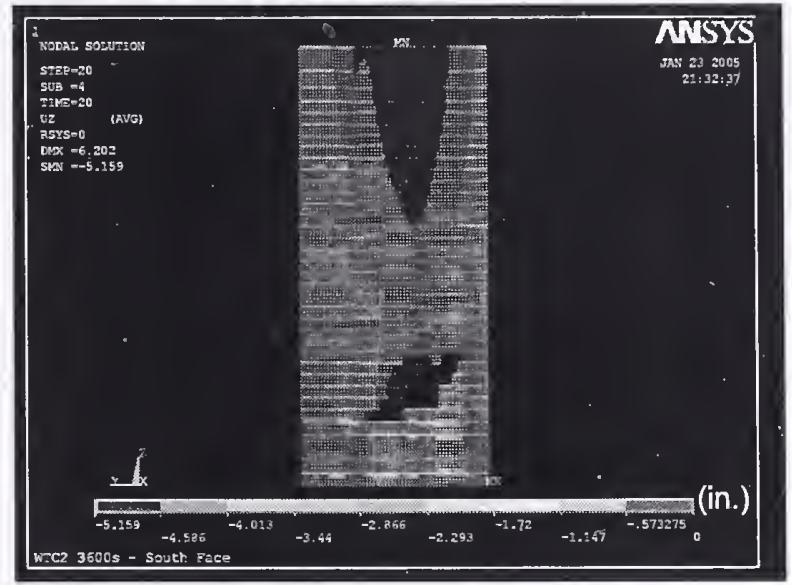

(b) South wall at $60 \mathrm{~min}$

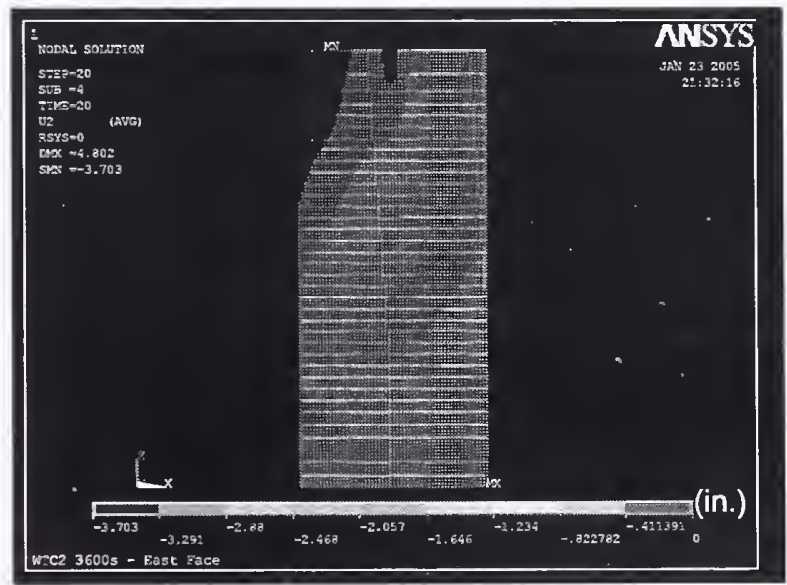

(d) East wall at $60 \mathrm{~min}$

Figure C-23. Vertical displacement on the south and east faces of WTC 2 at 50 min and at 60 min of Case $D_{i}$ temperature condition and the revised Case $C_{i}$ structural damage condition (looking from outside; downward displacement is negative). 


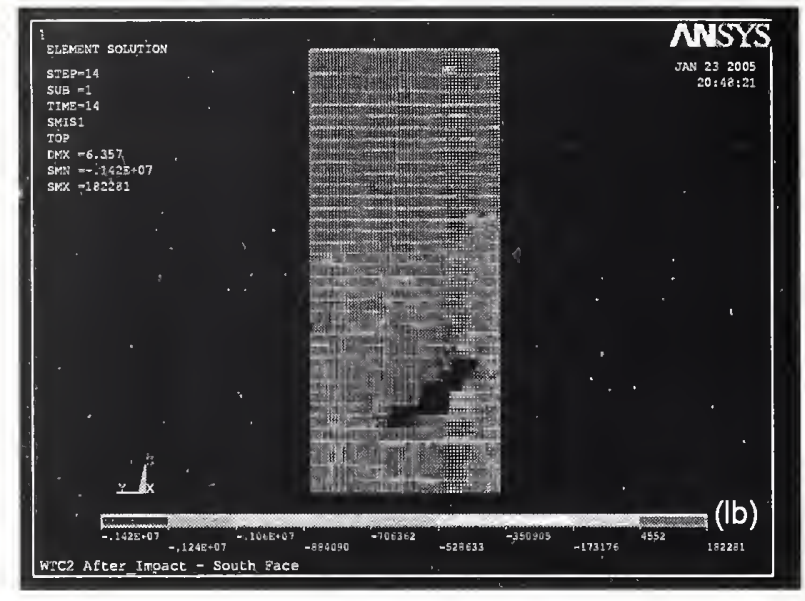

(a) South wall after impact

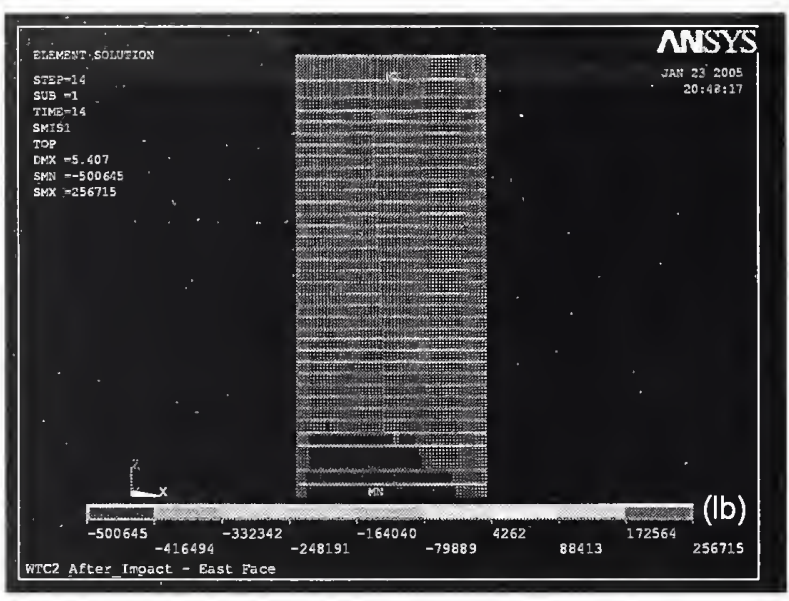

(c) East wall after impact

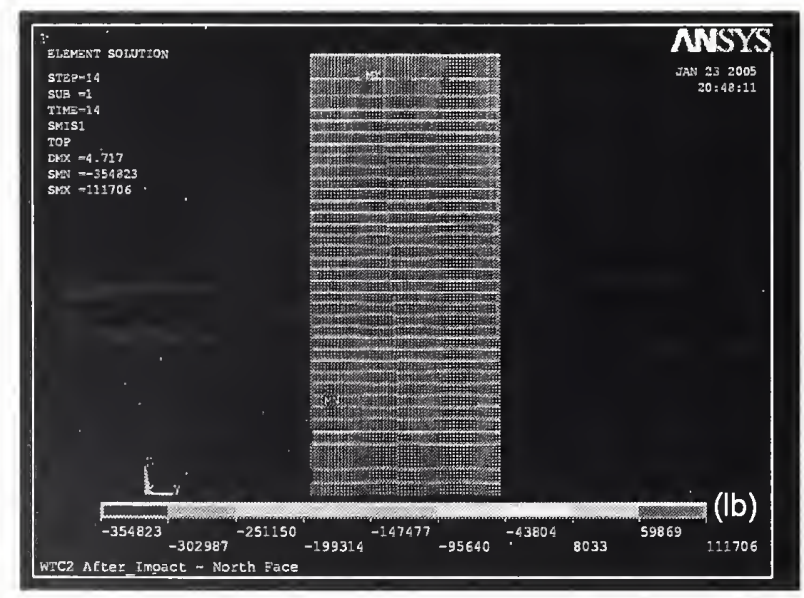

(e) North wall after impact

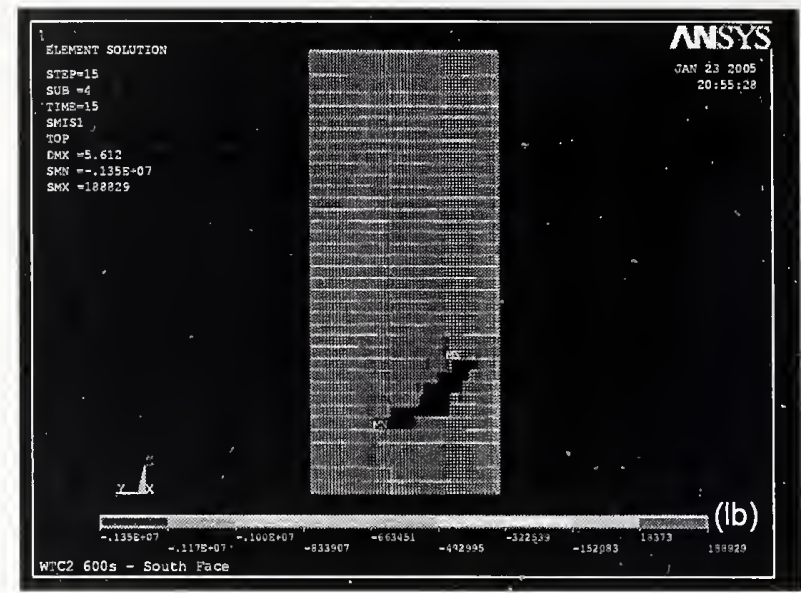

(b) South wall at $10 \mathrm{~min}$

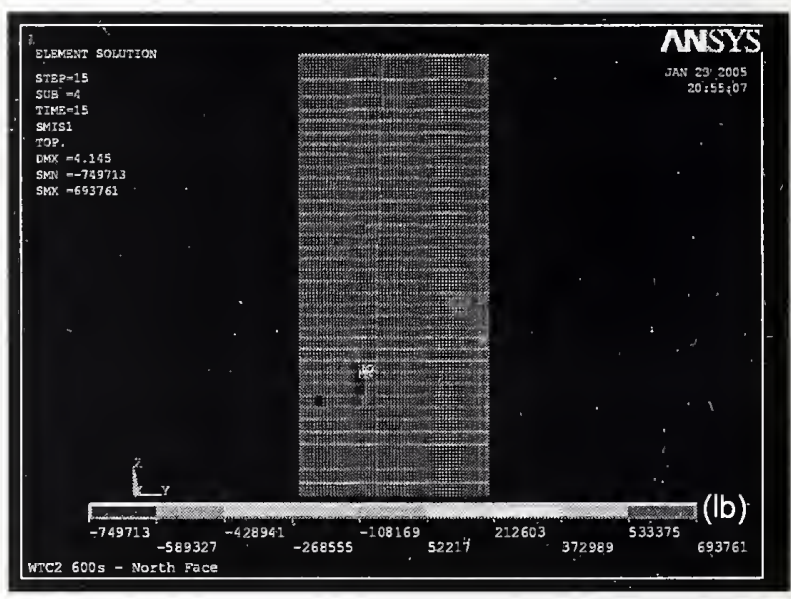

(d) East wall at $10 \mathrm{~min}$

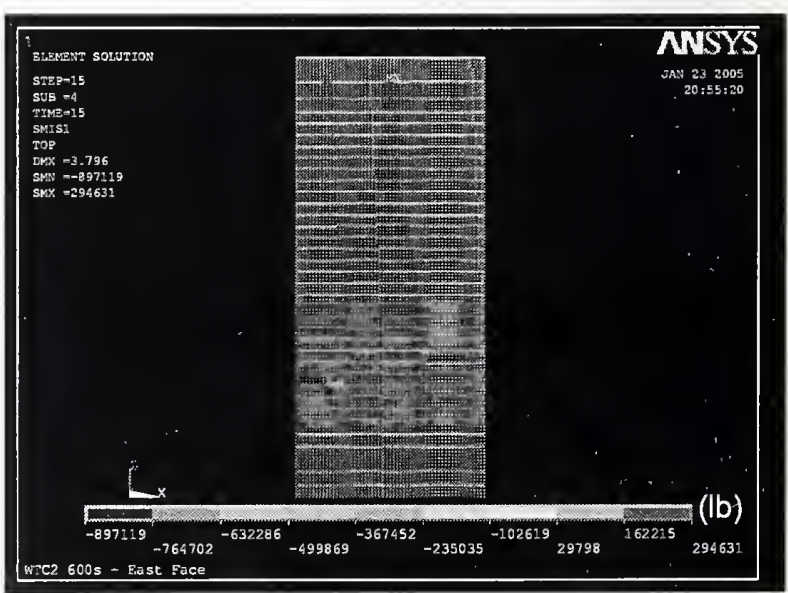

(f) North wall at $10 \mathrm{~min}$

Figure C-24. Axial force variation on the south, east, and north faces of WTC 2 after impact and at $10 \mathrm{~min}$ of Case $D_{i}$ temperature condition and the revised

Case $C_{i}$ structural damage condition (looking from outside; compression is negative). 


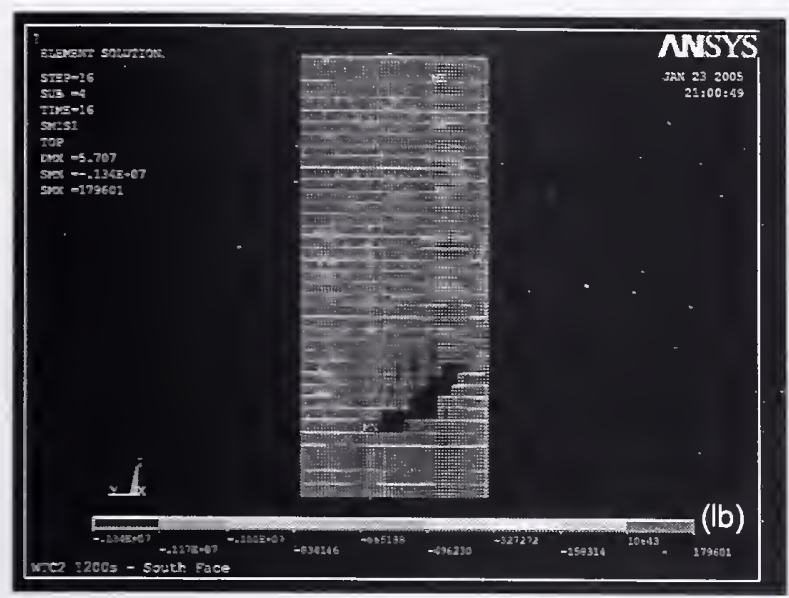

(a) South wall at $20 \mathrm{~min}$

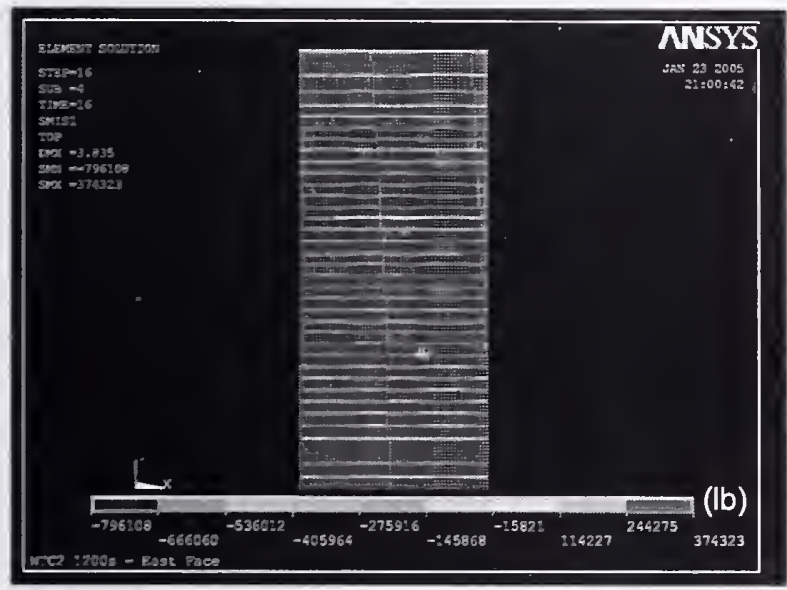

(c) East wall at $20 \mathrm{~min}$

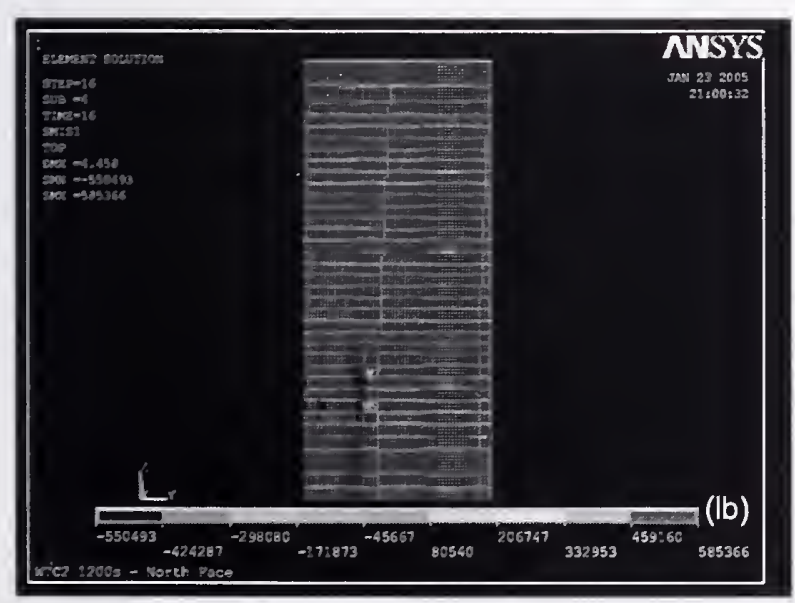

(e) North wall at $20 \mathrm{~min}$

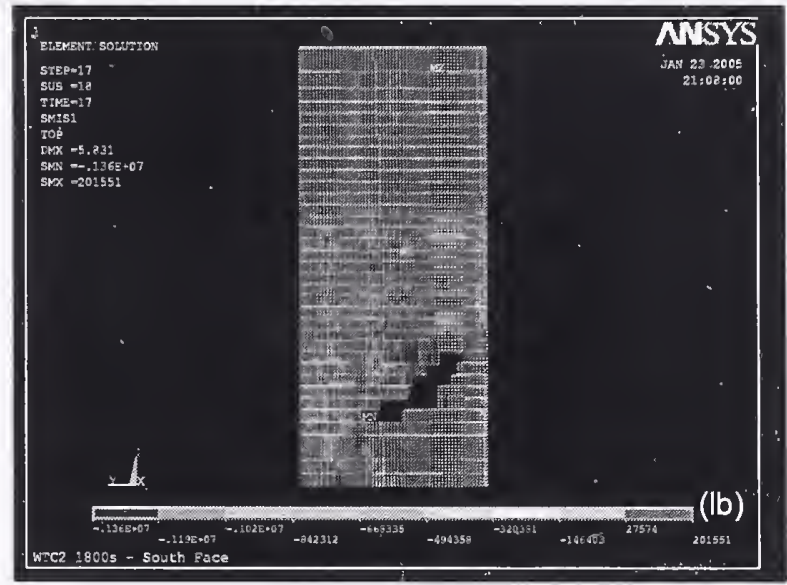

(b) South wall at $30 \mathrm{~min}$

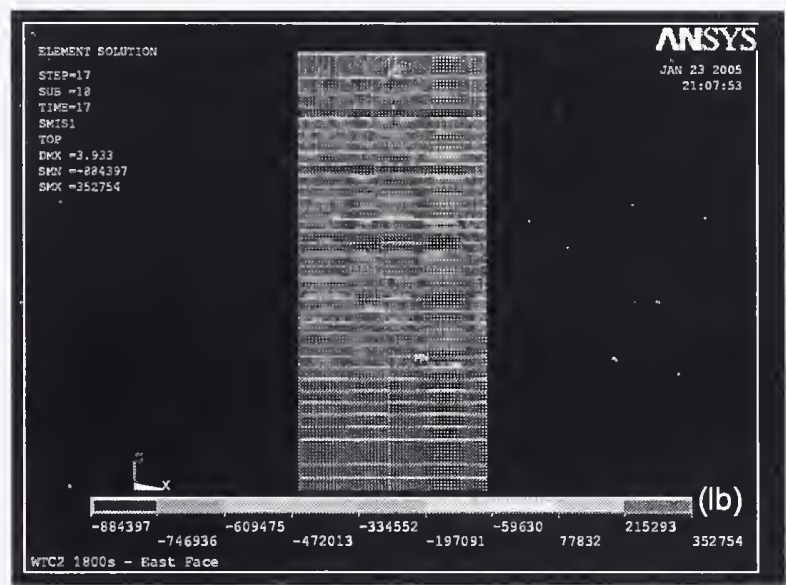

(d) East wall at $30 \mathrm{~min}$

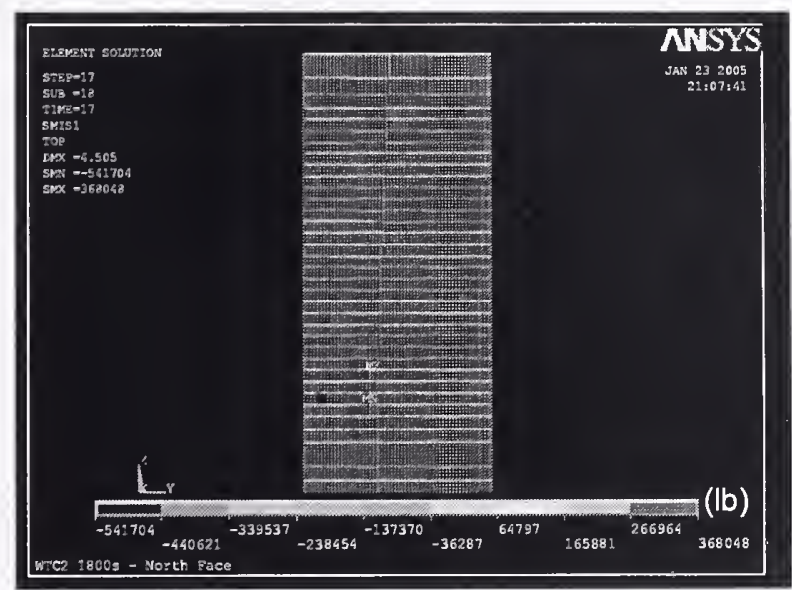

(f) North wall at $30 \mathrm{~min}$

Figure C-25. Axial force variation on the south, east, and north faces of WTC 2 at 20 min and at $30 \mathrm{~min}$ of Case $D_{i}$ temperature condition and the revised

Case $\mathrm{C}_{\mathrm{i}}$ structural damage condition (looking from outside; compression is negative). 


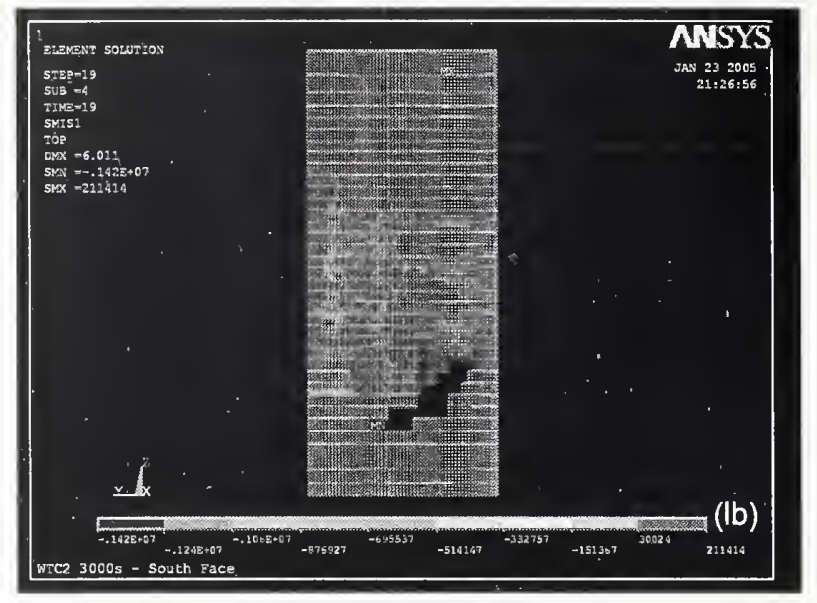

(a) South wall at $50 \mathrm{~min}$

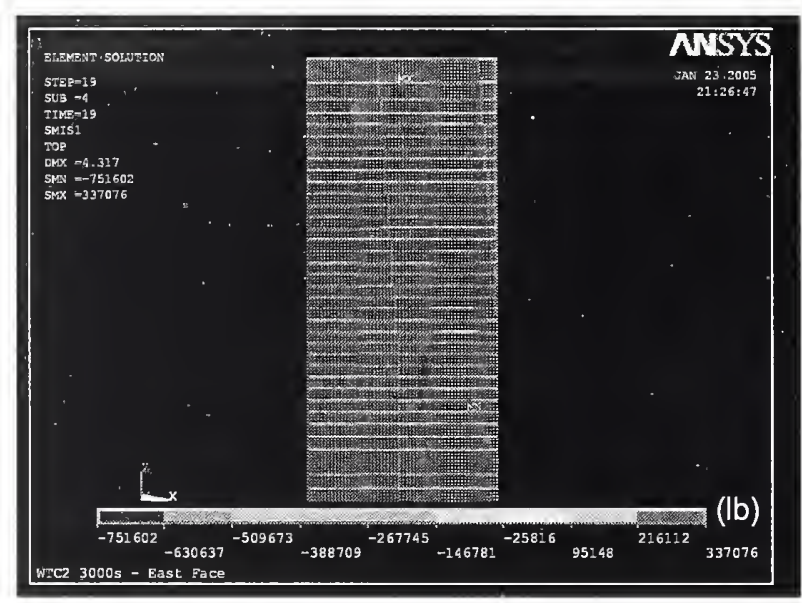

(c) East wall at $50 \mathrm{~min}$

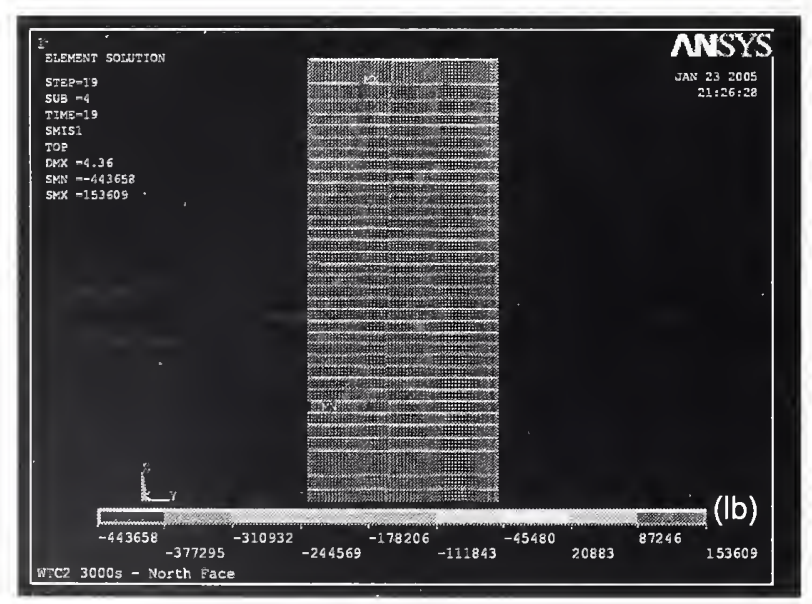

(e) North wall at $50 \mathrm{~min}$

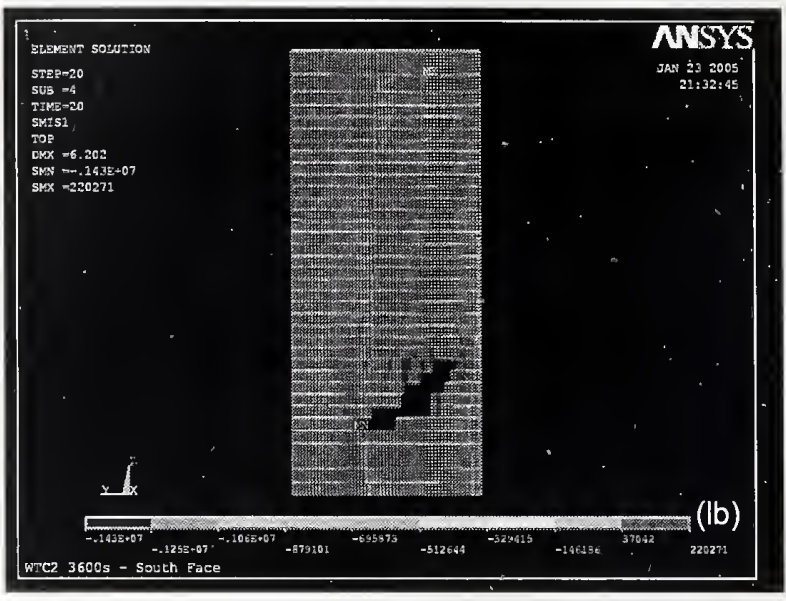

(b) South wall at $60 \mathrm{~min}$

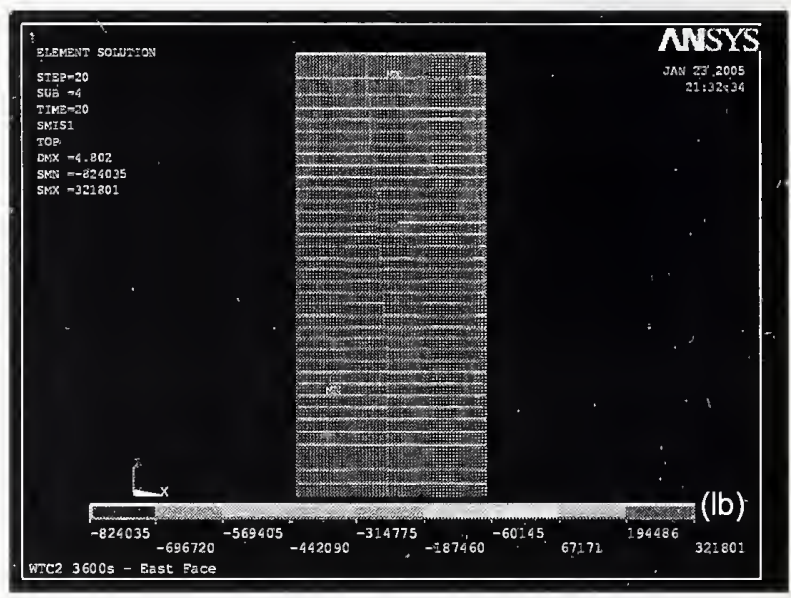

(d) East wall at $60 \mathrm{~min}$

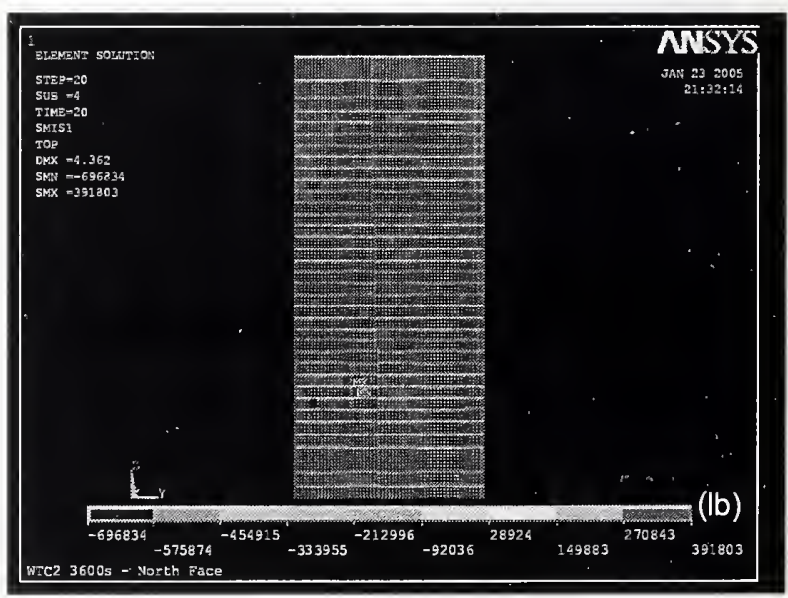

(f) North wall at $60 \mathrm{~min}$

Figure C-26. Axial force variation on the south, east, and north faces of WTC 2 at 50 min and at 60 min of Case $D_{i}$ temperature condition and the revised

Case $C_{i}$ structural damage condition (looking from outside; compression is negative). 


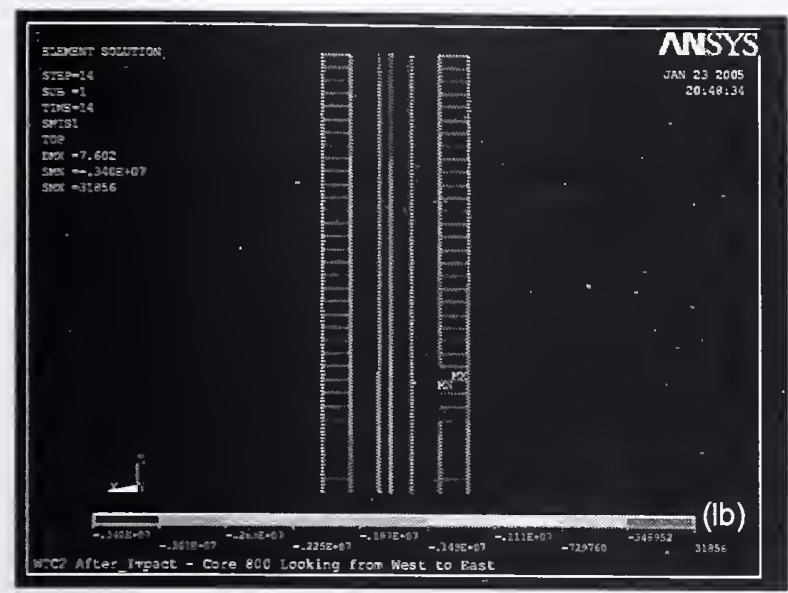

(a) 800 series columns after impact

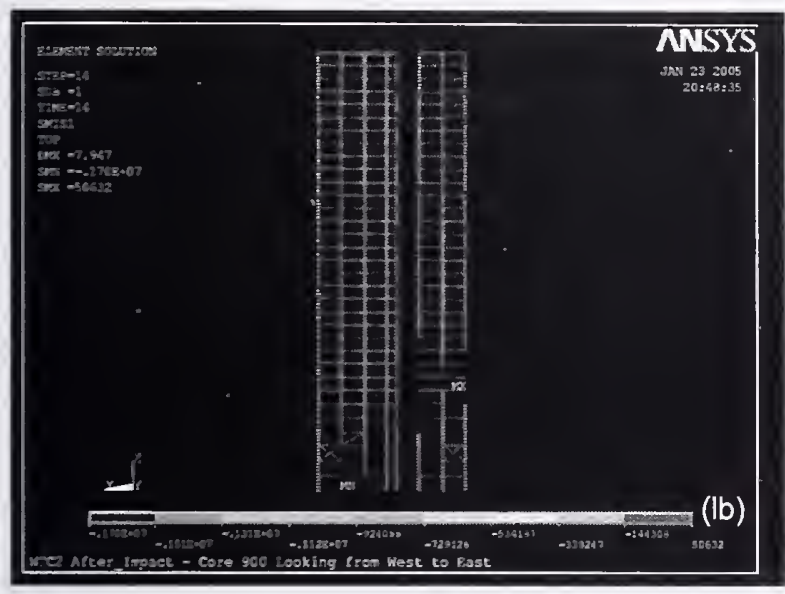

(c) 900 series columns after impact

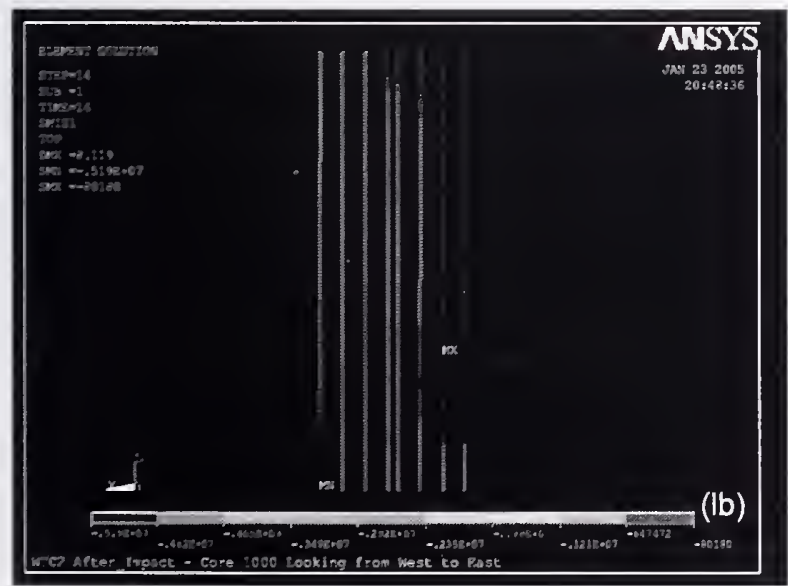

(e) 1000 series columns after impact

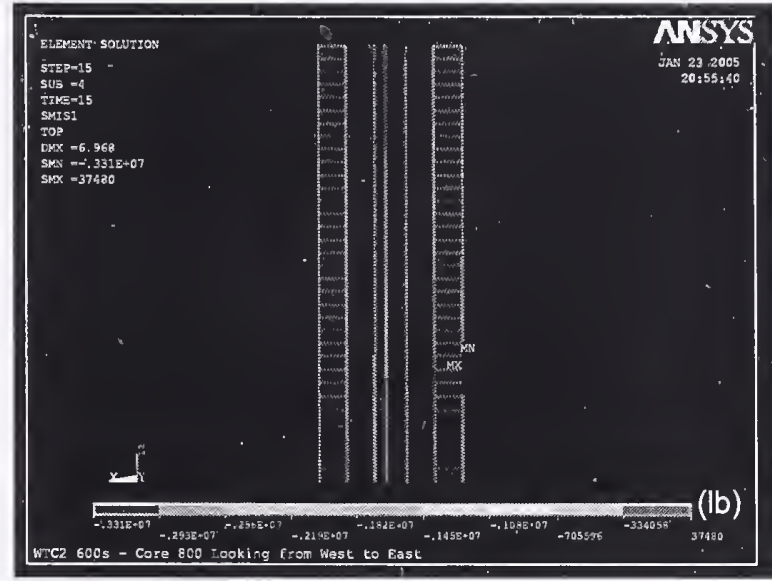

(b) 800 series columns at $10 \mathrm{~min}$

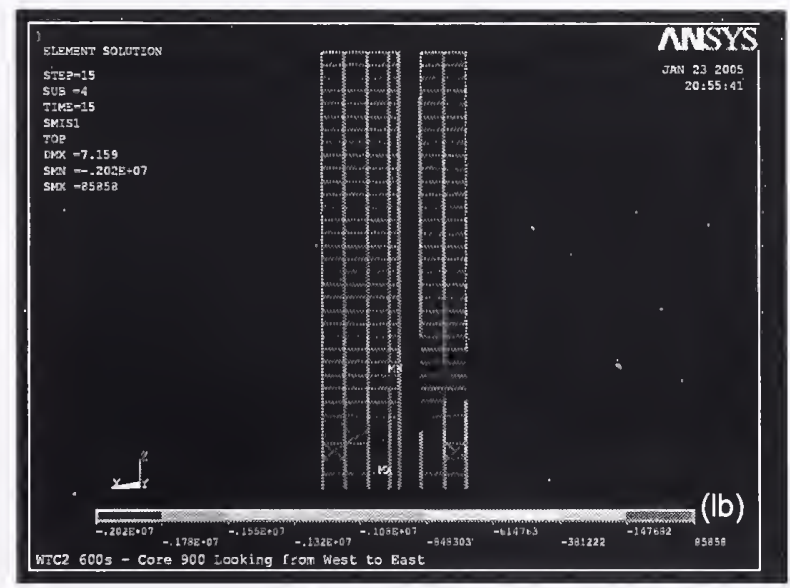

(d) 900 series columns at $10 \mathrm{~min}$

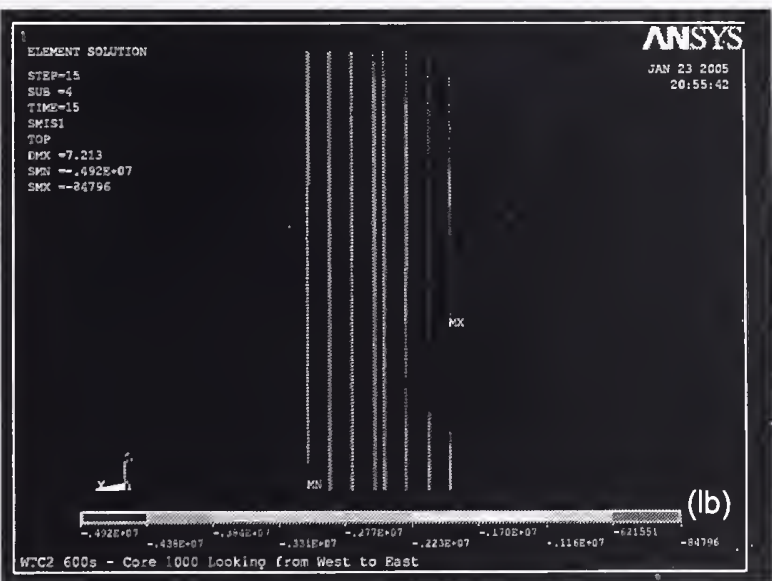

(f) 1000 series columns at $10 \mathrm{~min}$

Figure C-27. Axial force variation on the 800,900 , and 1000 series core columns of WTC 2 after impact and at $10 \mathrm{~min}$ of $C$ ase $D_{i}$ temperature condition and the revised Case $C_{i}$ structural damage condition (looking from west; compression is negative). 


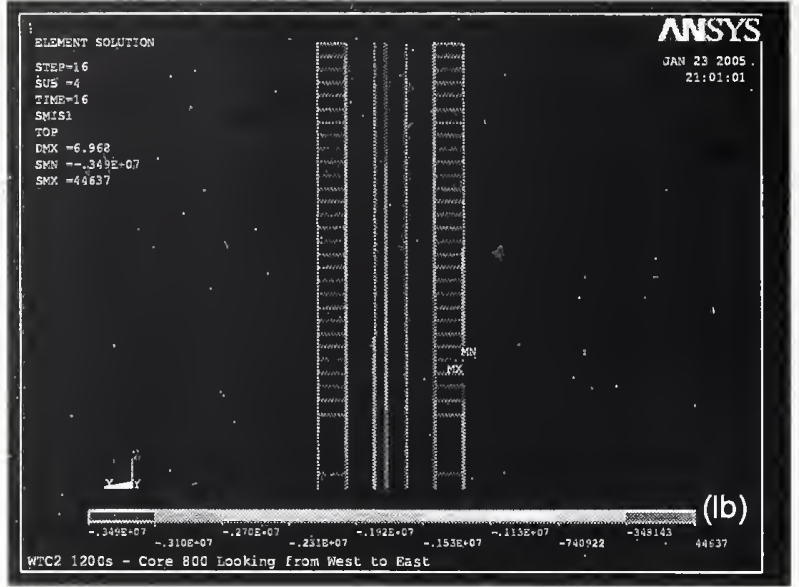

(a) 800 series columns at $20 \mathrm{~min}$

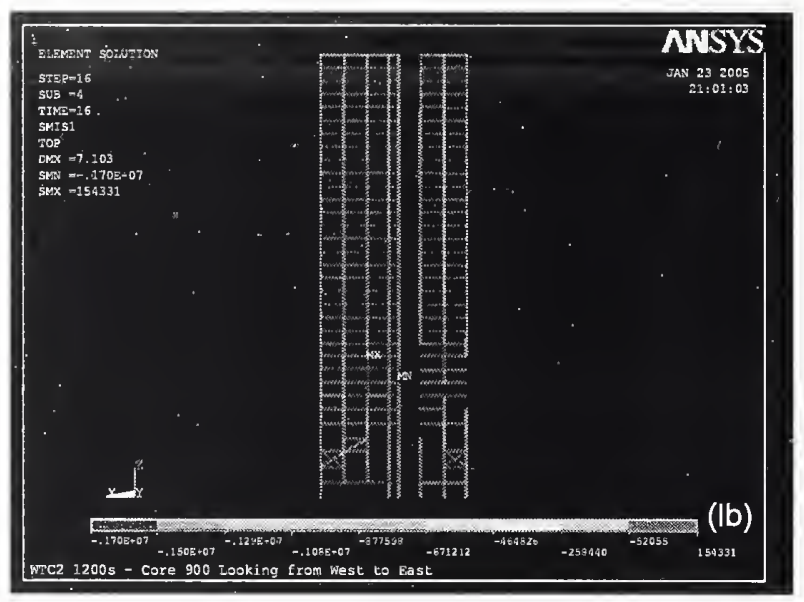

(c) 900 series columns at $20 \mathrm{~min}$

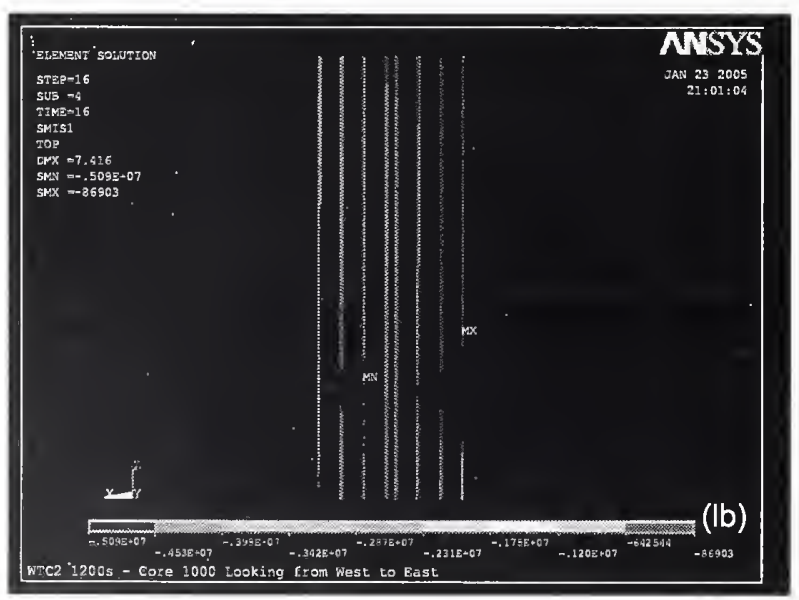

(e) 1000 series columns at $20 \mathrm{~min}$

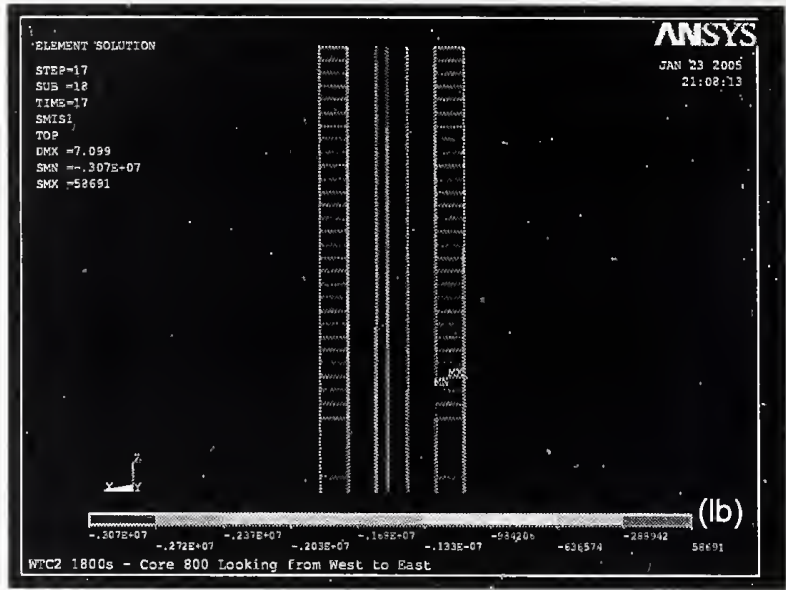

(b) 800 series columns at $30 \mathrm{~min}$

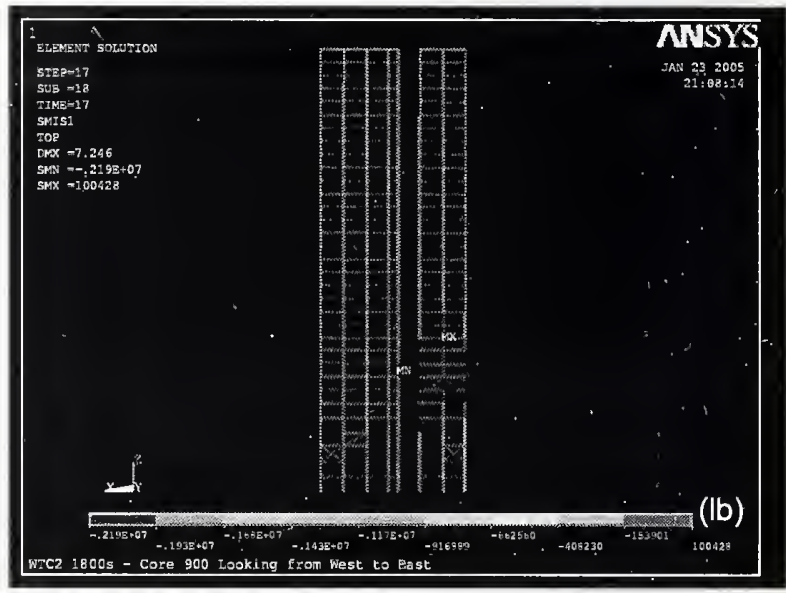

(d) 900 series columns at $30 \mathrm{~min}$

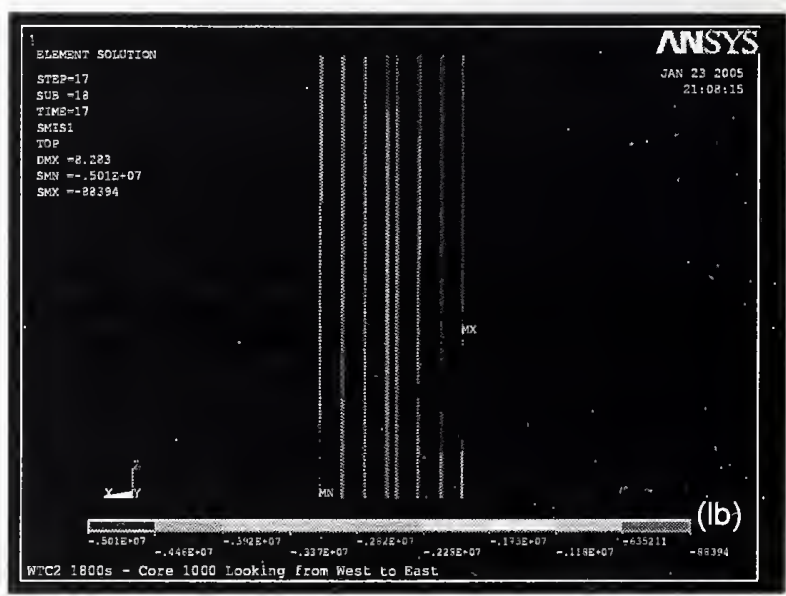

(f) 1000 series columns at $30 \mathrm{~min}$

Figure C-28. Axial force variation on the 800,900 , and 1000 series core columns of WTC 2 at $20 \mathrm{~min}$ and at $30 \mathrm{~min}$ of Case $D_{i}$ temperature condition and the revised Case $\mathrm{C}_{\mathrm{i}}$ structural damage condition (looking from west; compression is negative). 


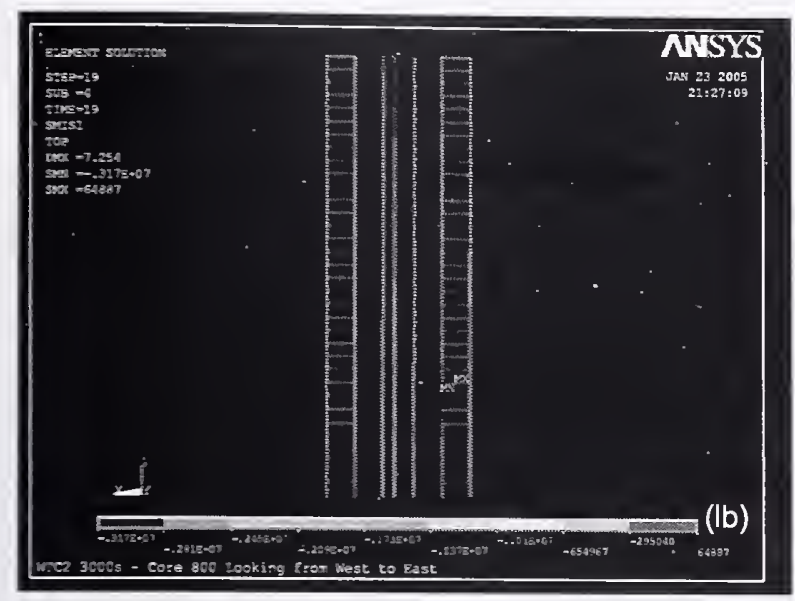

(a) 800 series columns at $50 \mathrm{~min}$

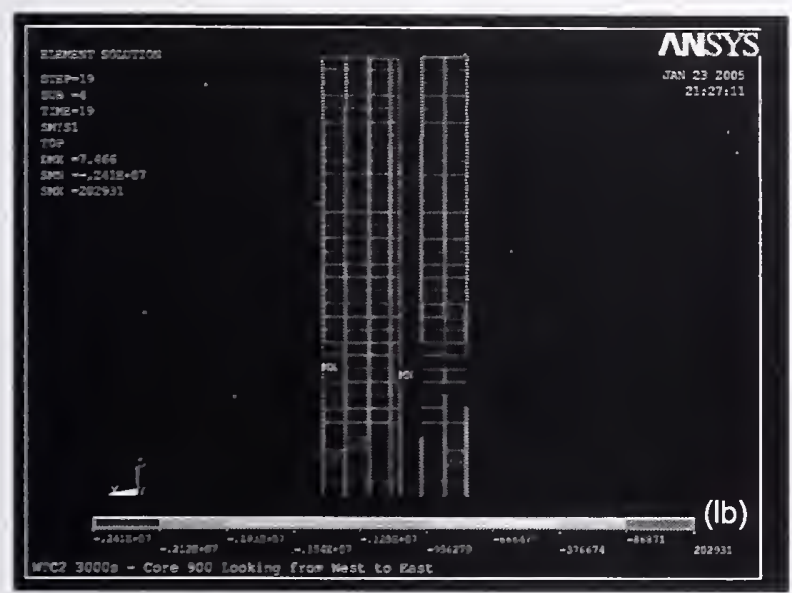

(c) 900 series columns at $50 \mathrm{~min}$

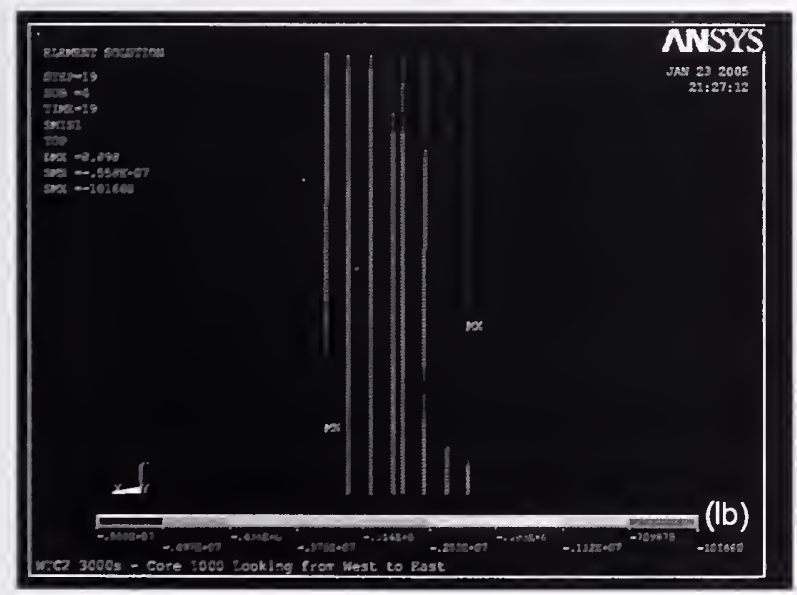

(e) 1000 series columns at $50 \mathrm{~min}$

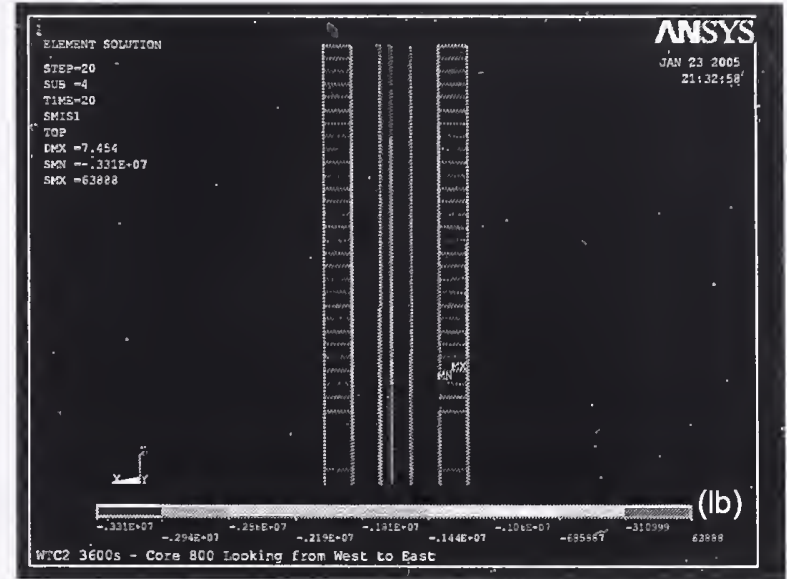

(b) 800 series columns at $60 \mathrm{~min}$

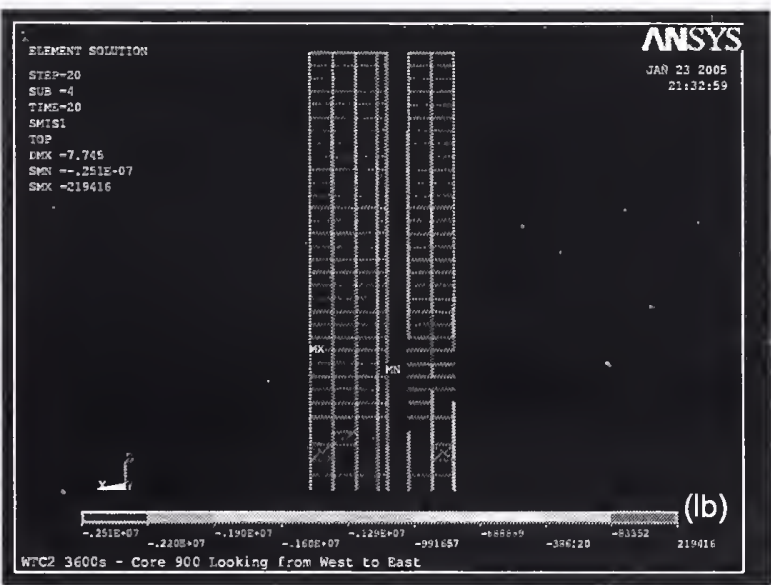

(d) 900 series columns at $60 \mathrm{~min}$

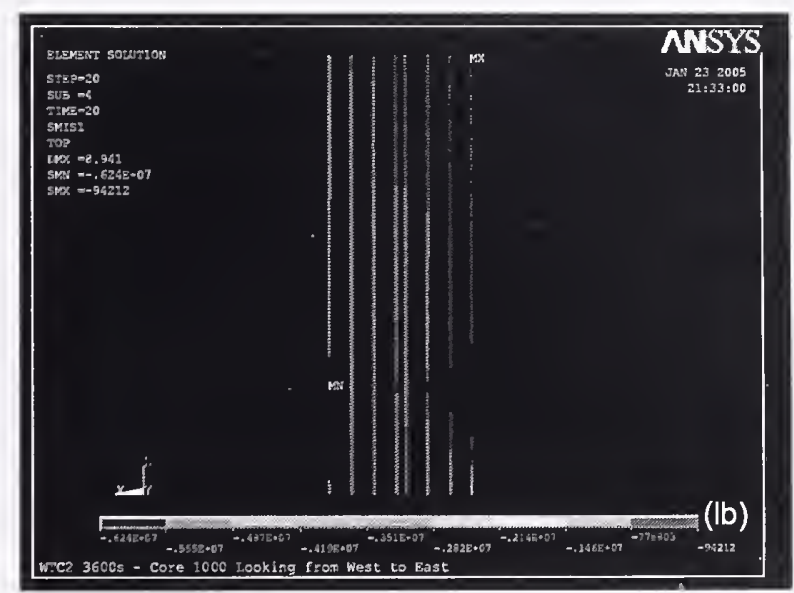

(f) 1000 series columns at $60 \mathrm{~min}$

Figure C-29. Axial force variation on the 800,900 , and 1000 series core columns of WTC 2 at $50 \mathrm{~min}$ and at $60 \mathrm{~min}$ of Case $D_{i}$ temperature condition and the revised Case $C_{i}$ structural damage condition (looking from west; compression is negative). 


\begin{tabular}{|c|l|c|c|c|c|c|c|}
\hline Row & Analysis Stage & West & East & North & South & Core & Sum \\
\hline$(1)$ & Before Impact & 18779 & 18837 & 13714 & 13529 & 77563 & 142423 \\
$(2)$ & After Impact & 18824 & 21170 & 12812 & 12396 & 75450 & 140652 \\
$(3)$ & $10 \mathrm{~min}$ & 19668 & 21636 & 13148 & 11694 & 74506 & 140652 \\
$(4)$ & $20 \mathrm{~min}$ & 19984 & 21951 & 13004 & 11661 & 74053 & 140653 \\
$(5)$ & $30 \mathrm{~min}$ & 20005 & 22426 & 13129 & 11899 & 73195 & 140653 \\
$(6)$ & $40 \mathrm{~min}$ & 19980 & 22260 & 13252 & 12156 & 73005 & 140653 \\
$(7)$ & $50 \mathrm{~min}$ & 19617 & 22048 & 13211 & 12609 & 73168 & 140653 \\
$(8)$ & $60 \mathrm{~min}$ & 19161 & 21803 & 13294 & 12959 & 73436 & 140653 \\
\hline
\end{tabular}

(a) Above Floor 78

\begin{tabular}{|c|l|c|c|c|c|c|c|}
\hline Row & Analysis Stage & West & East & North & South & Core & Sum \\
\hline$(1)$ & Before Impact & 17642 & 17697 & 12558 & 12393 & 71319 & 131609 \\
$(2)$ & After lmpact & 17613 & 20084 & 11761 & 11475 & 70039 & 130972 \\
$(3)$ & $10 \mathrm{~min}$ & 18453 & 20602 & 12152 & 10672 & 69094 & 130973 \\
$(4)$ & $20 \mathrm{~min}$ & 18759 & 20889 & 12005 & 10680 & 68640 & 130973 \\
$(5)$ & $30 \mathrm{~min}$ & 18755 & 21379 & 12146 & 10910 & 67782 & 130972 \\
$(6)$ & $40 \mathrm{~min}$ & 18718 & 21202 & 12290 & 11170 & 67593 & 130972 \\
$(7)$ & $50 \mathrm{~min}$ & 18340 & 20983 & 12237 & 11655 & 67756 & 130971 \\
$(8)$ & $60 \mathrm{~min}$ & 17876 & 20724 & 12316 & 12034 & 68022 & 130972 \\
\hline
\end{tabular}

(b) Above Floor 81

\begin{tabular}{|c|l|c|c|c|c|c|c|}
\hline Row & Analysis Stage & West & East & North & South & Core & Sum \\
\hline$(1)$ & Before Impact & 16728 & 16782 & 11972 & 11820 & 67309 & 124611 \\
$(2)$ & After Impact & 16717 & 19111 & 11188 & 11058 & 66400 & 124474 \\
$(3)$ & $10 \mathrm{~min}$ & 17551 & 19473 & 11596 & 10404 & 65449 & 124473 \\
$(4)$ & $20 \mathrm{~min}$ & 17864 & 19739 & 11443 & 10431 & 64996 & 124472 \\
$(5)$ & $30 \mathrm{~min}$ & 17875 & 20198 & 11573 & 10687 & 64138 & 124471 \\
$(6)$ & $40 \mathrm{~min}$ & 17847 & 20006 & 11709 & 10960 & 63950 & 124471 \\
$(7)$ & $50 \mathrm{~min}$ & 17482 & 19841 & 11651 & 11384 & 64113 & 124472 \\
$(8)$ & $60 \mathrm{~min}$ & 17025 & 19637 & 11734 & 11691 & 64384 & 124471 \\
\hline
\end{tabular}

(c) Above Floor 83

Figure C-30. Total column loads in the core and exterior walls of WTC 2 at different floors and at different times for Case $D_{i}$ temperature condition and the revised Case $C_{i}$ structural damage condition (compression is positive). 
COL501

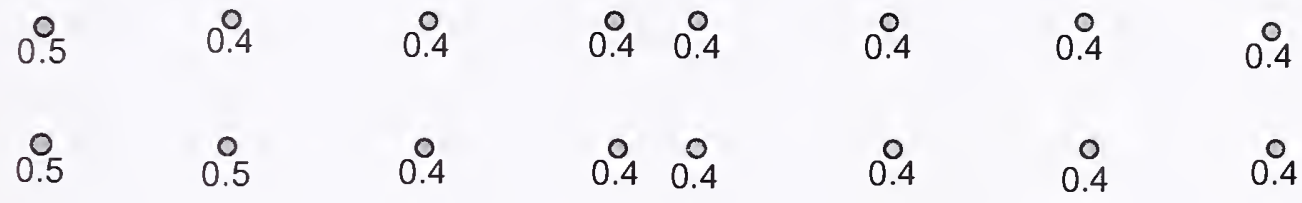

$\begin{array}{ccccccccc}0.4 & 0.5 & 0.4 & 0.4 & 0.2 & 0.3 & 0.4 & 0.3 \\ 0 & 0 & 0 & 0 & 0 & 0 & 0 & 0\end{array}$

$\begin{array}{ccccccc}0 & 0 & 0 & 0 & 0 & 0 & 0 \\ 1.1 & 0.8 & 0.5 & 1.0 & 0.6 & 0.4 & 0.3\end{array}$

\begin{tabular}{|c|c|c|c|c|c|c|c|}
\hline & 0.1 & & $\begin{array}{c}0.5 \\
0\end{array}$ & $\begin{array}{c}0.8 \\
0\end{array}$ & 0.2 & $\begin{array}{c}0.2 \\
0\end{array}$ & $\begin{array}{c}0.3 \\
0\end{array}$ \\
\hline COL1001 & $\begin{array}{r}0.0 \\
\cdot \\
\end{array}$ & $\begin{array}{c}0.1 \\
\dot{0} \\
N\end{array}$ & ${ }^{2.0}$ & 1.9 & $\begin{array}{c}0.6 \\
0\end{array}$ & $\begin{array}{c}0.9 \\
0\end{array}$ & $\begin{array}{c}0.3 \\
0 \\
\text { COL1008 }\end{array}$ \\
\hline
\end{tabular}

(a) At $10 \mathrm{~min}$

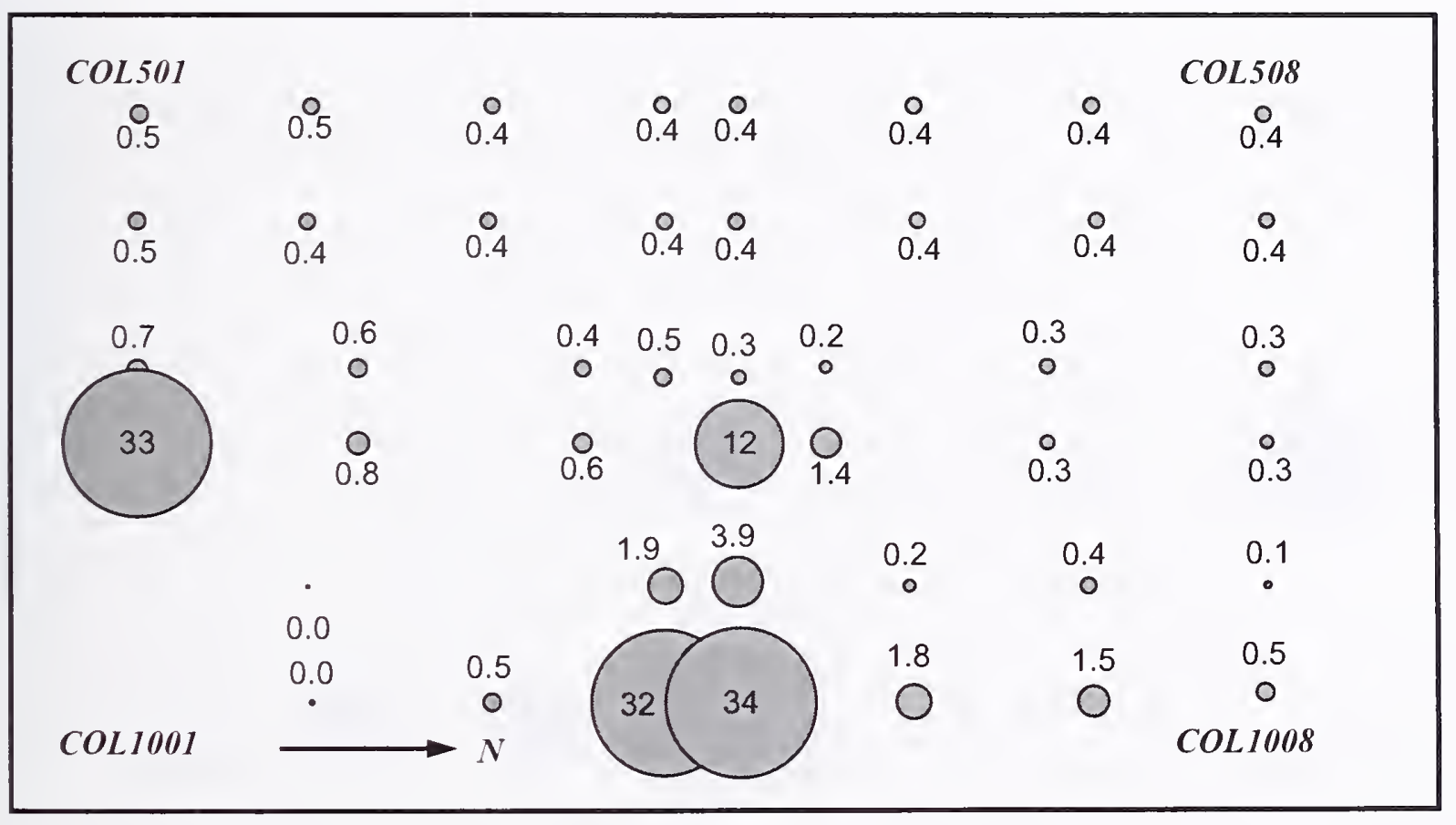

(b) At $30 \mathrm{~min}$

Figure C-31. Ratios of axial elastic-plus-plastic strain to temperature-dependent yield strain for the core columns at Floor 82 of WTC 2 at $10 \mathrm{~min}$ and at $30 \mathrm{~min}$ of Case $D_{i}$ temperature condition and the revised $C$ ase $C_{i}$ structural damage condition. 


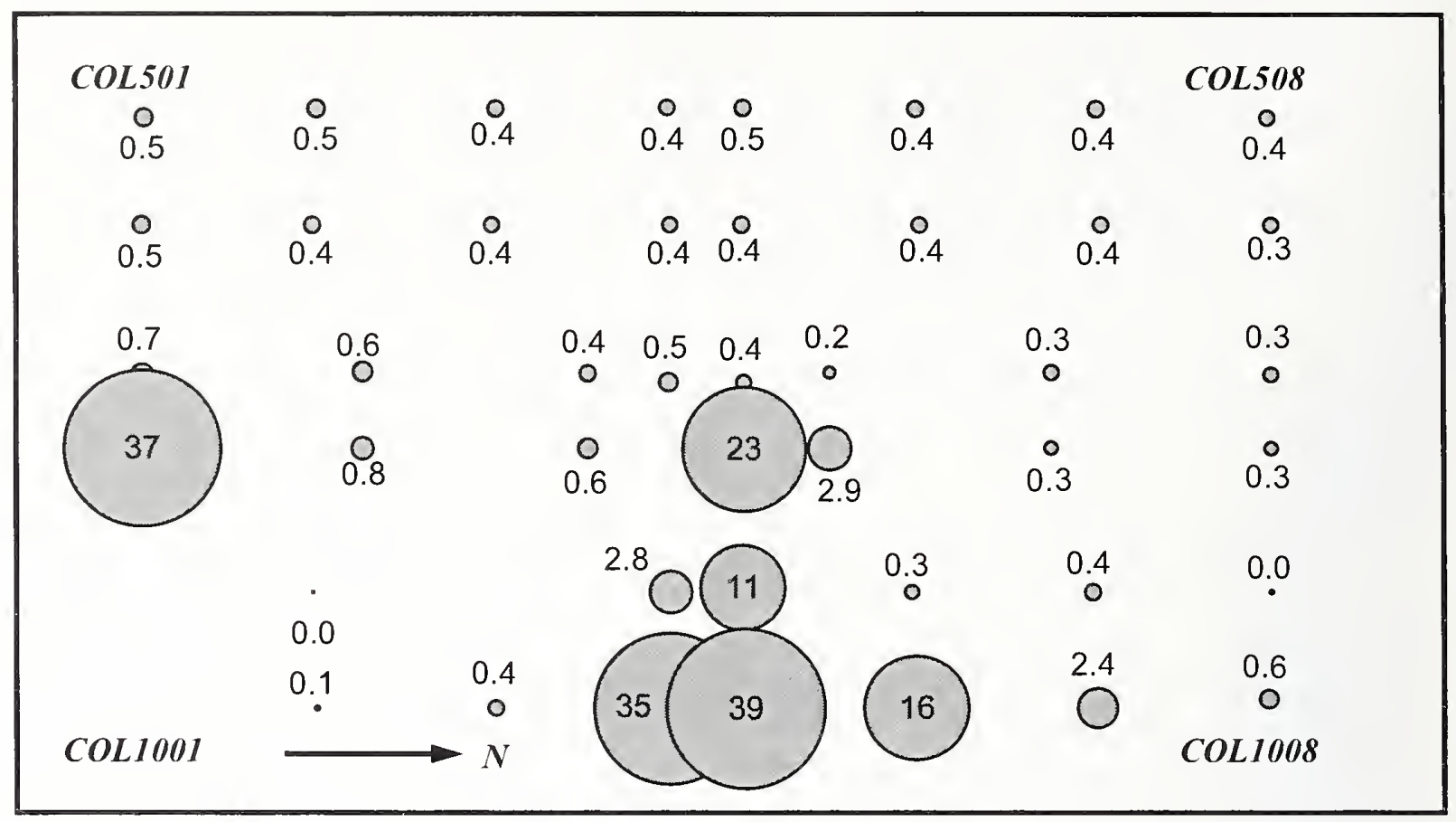

(a) At $40 \mathrm{~min}$

COL501

\begin{tabular}{|c|c|c|c|c|c|c|}
\hline $\begin{array}{c}0 \\
0.5\end{array}$ & $\begin{array}{c}0 \\
0.5\end{array}$ & $\begin{array}{c}0 \\
0.4\end{array}$ & $\begin{array}{c}0 \\
0.4\end{array}$ & 0.5 & $\stackrel{\circ}{0.4}$ & 0.4 \\
\hline $\begin{array}{c}0 \\
0.5\end{array}$ & $\begin{array}{c}\circ \\
0.4\end{array}$ & $\begin{array}{c}0 \\
0.4\end{array}$ & $\begin{array}{c}0 \\
0.3\end{array}$ & $\begin{array}{c}0 \\
0.5\end{array}$ & 0.4 & $\begin{array}{c}\circ \\
0.4\end{array}$ \\
\hline
\end{tabular}

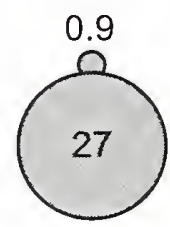

0.6

$\begin{array}{cccc}0.5 & 0.7 & 0.5 & 0.4\end{array}$

0.4
0

0.4

0

000

o

0.8

0
0.7

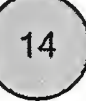

$\bigcirc_{4.0}$

0.3

0.3
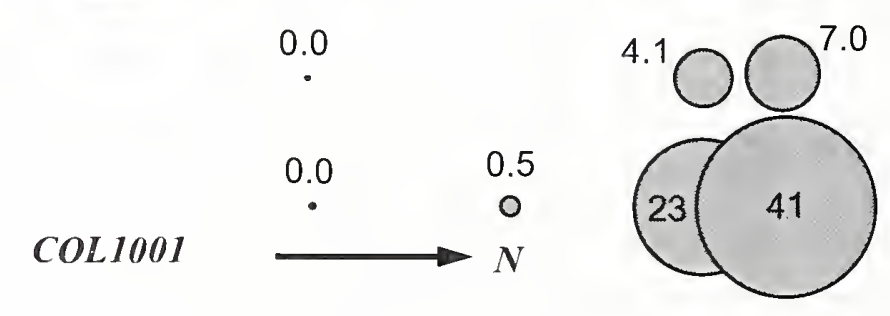

0.7

0.5

0.1

0

13

0.8

13 COL 1008

(b) At $60 \mathrm{~min}$

Figure C-32. Ratios of axial elastic-plus-plastic strain to temperature-dependent yield strain for the core columns at Floor 82 of WTC 2 at $40 \mathrm{~min}$ and at $60 \mathrm{~min}$ of Case $D_{i}$ temperature condition and the revised Case $C_{i}$ structural damage condition. 

